
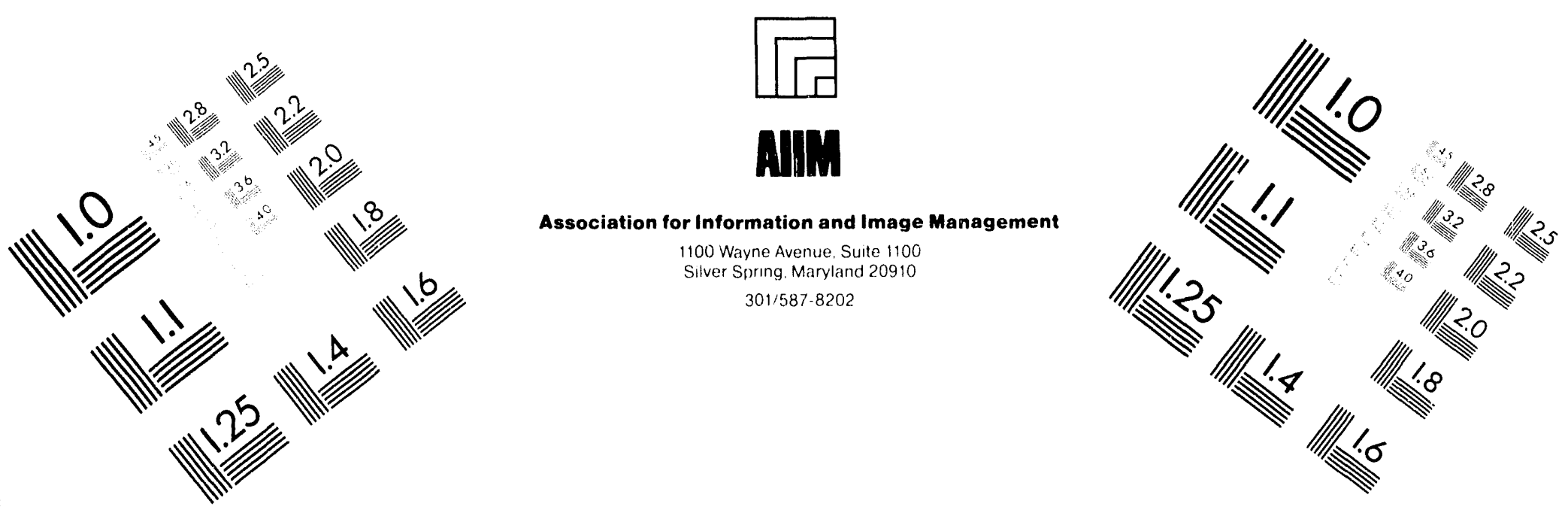

\title{
Centimeter
}

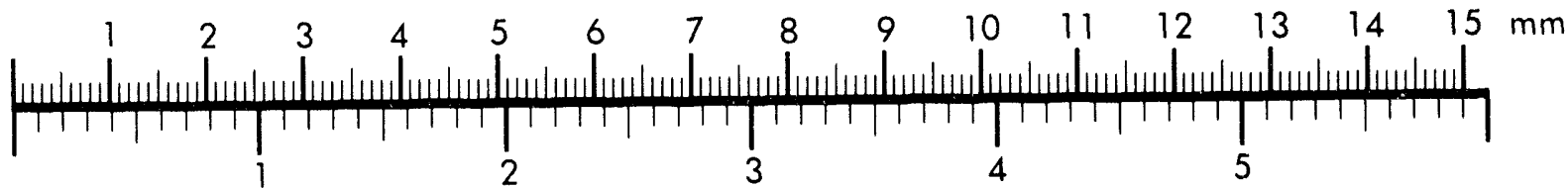

Inches
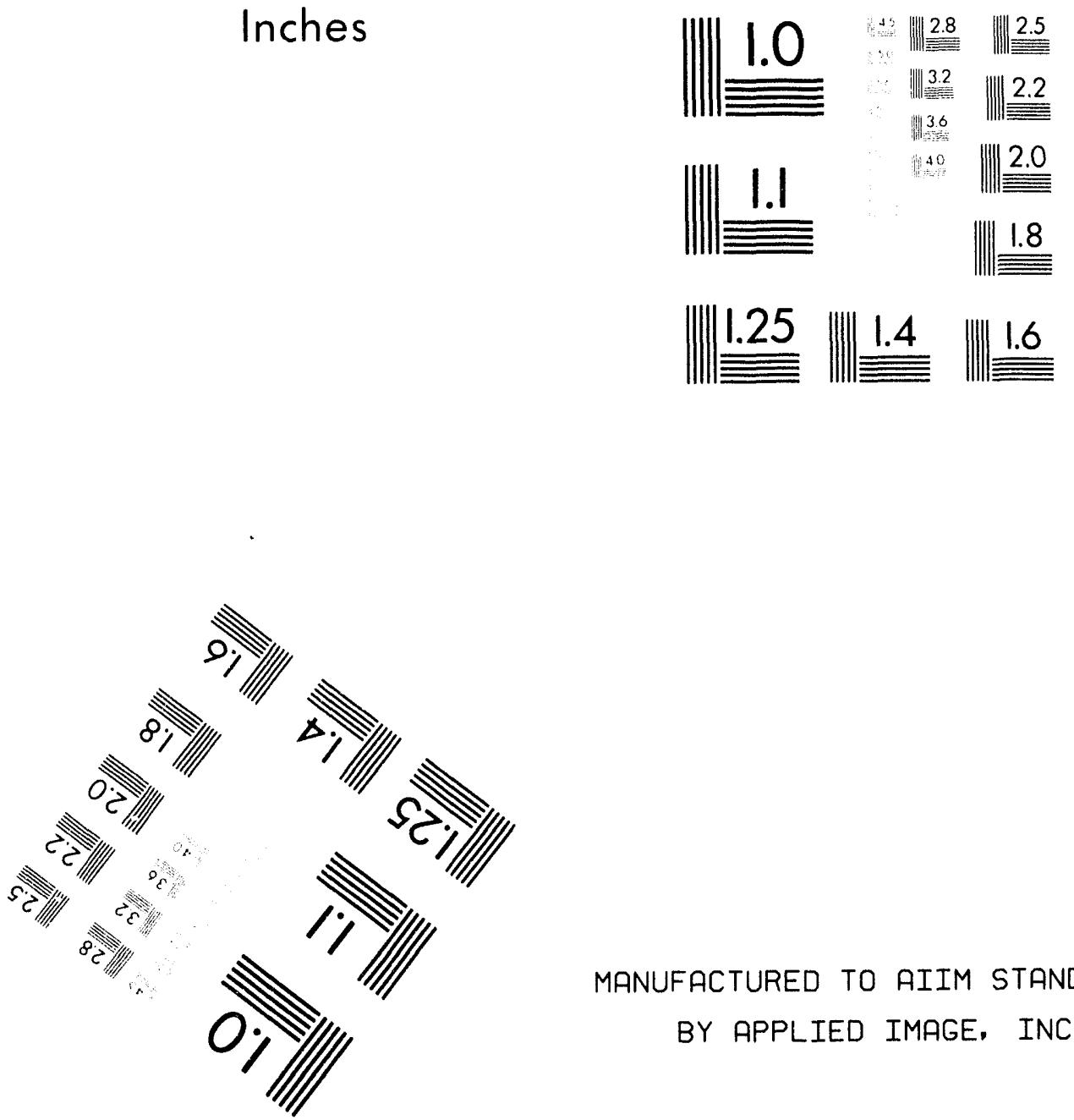

MANUFACTURED TO AIIM STANDARDS

BY APPLIED IMAGE, INC.

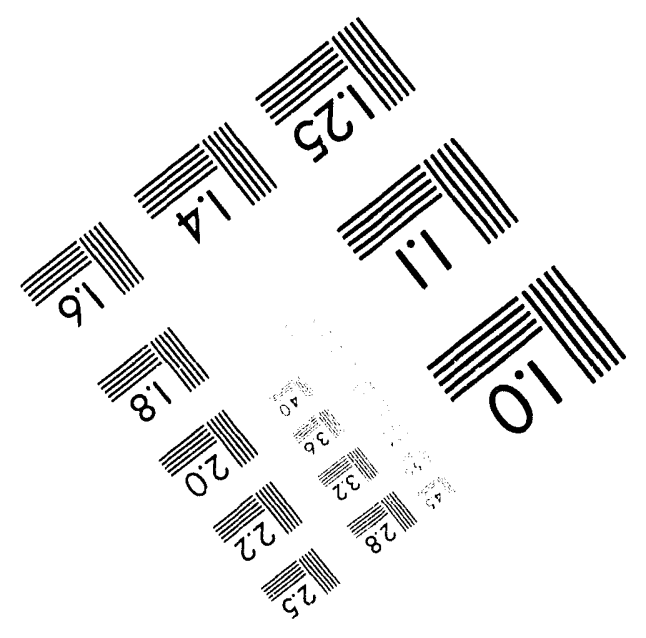



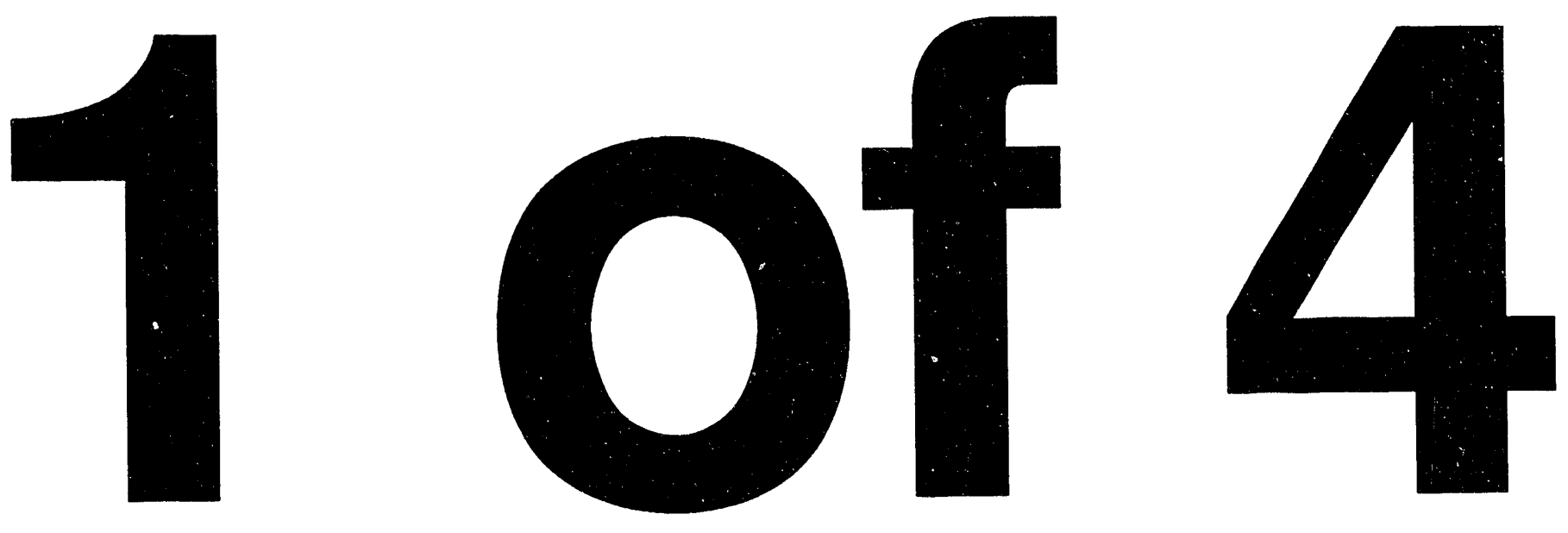
Mr. W. A. Rutherford, Director

Office of Planning and Integration

U.S. Department of Energy

Richland Operations office

Richland, Washington 99352

Dear Mr. Rutherford:

SYSTEMS ENGINEERING FUNCTIONS AND REQUIREMENTS DOCUMENT-ADDENDUM 2 REV. 0

The enclosed document, "Systems Engineering Functions and Requirements for the Hanford Cleanup Mission:First Issue, Addendum 2 Rev. 0," is provided in fulfillment of Milestone SSE-94-003. This document describes the total functional analysis for the Hanford Cleanup Mission but does not replicate the functional analysis for the Environmental Restoration (ER) or Tank Waste Remediation System (TWRS). Functional analys is for ER and TWRS is published as part of the programmatic baseline development, but is integrated with the attached analysis.

If you have any questions on this please contact Mr. J. J. Holmes of my staff on $376-1406$.

Very truly yours,

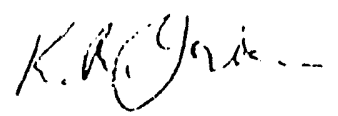

K. R. Jordan, Director

Planning and Systems Integration

$s b$

Enclosure

RL - R. 0. Puthoff ( $w / 0$ enclosure)

J. D. Kautzky

PNL - M. J. Simpson

II. B. Triplett

KEH - R. H. Beers

HEHF - R. H. Ronish

MACTC - W. M. Ritter 
WHC-EP-0722

Addendum 2

Revision 0

\title{
Systems Engineering Functions and Requirements for the Hanford Cleanup Mission: First Issue
}

\author{
J. J. Holmes \\ Date Published \\ January 1994
}

Prepared for the U.S. Department of Energy

Office of Environmental Restoration and

Waste Management

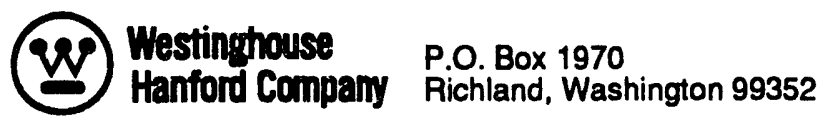

Hantord Operations and Engineering Contractor for the

U.S. Department of Energy under Contract DE-AC06-87RL10930

Approved for Public Release 


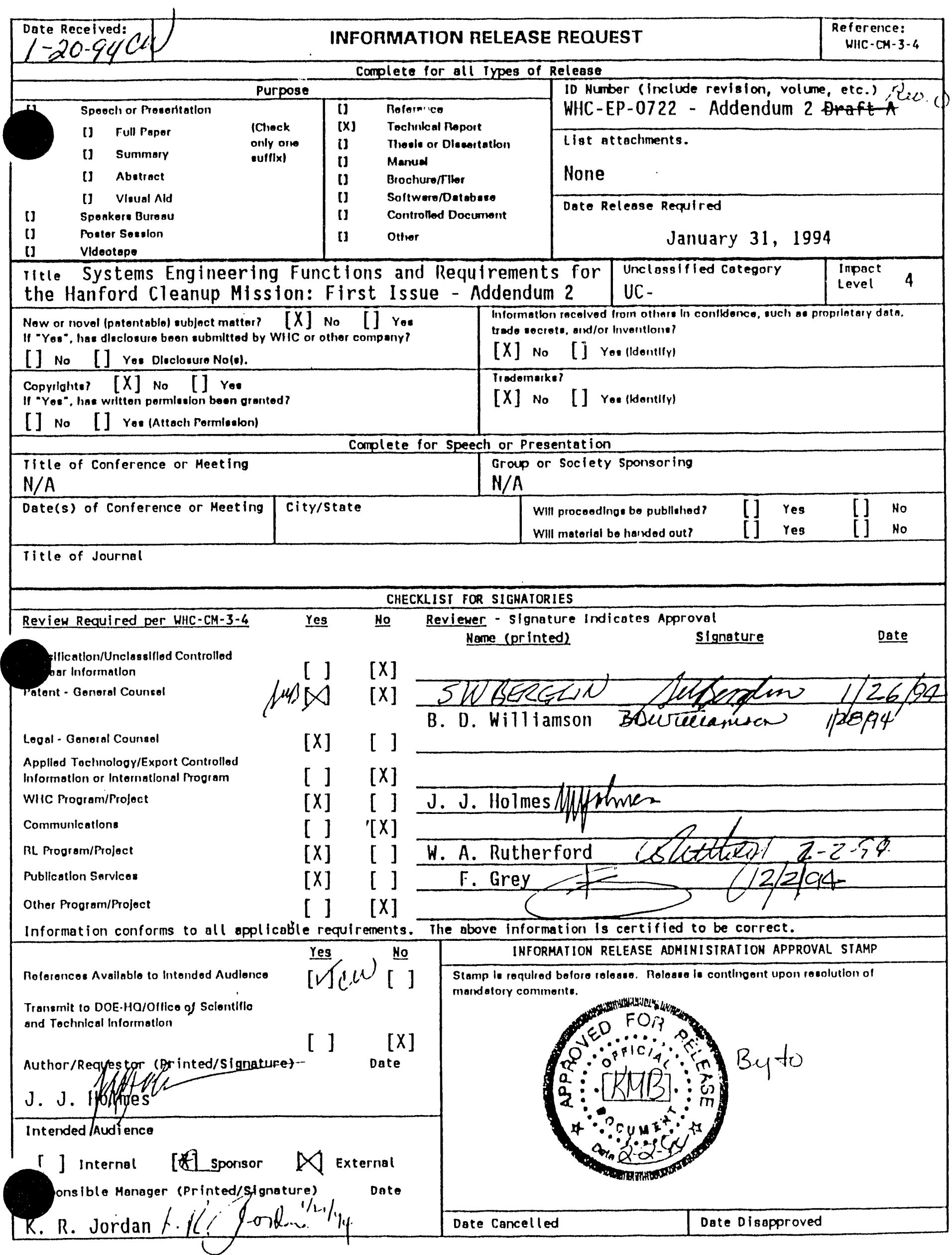




\section{SYSTEMS ENGINEERING FUNCTIONS AND REQUIREMENTS FOR THE HANFORD CLEANUP MISSION: FIRST ISSUE}

\section{J. J. Holmes}

\section{SUNMARY}

This addendum provides the technical detail of a systems engineering functional analysis for the Hanford cleanup mission. Details of the mission analysis including mission statement, scope, problem statement, initial state definition, and final state definition are provided in the parent document ${ }^{1}$. The functional analysis consists of Input Computer Automated Manufacturing Definition (IDEFO) diagrams an definitions, which will be understood by systems engineers, but which may be difficult for others to comprehend. For a more complete explanation of this work, refer to the parent document.

The analysis covers the total Hanford cleanup mission including the decomposition levels at which the various Hanford programs or integrated activities are encountered. Existing and emerging program-specific functional analyses are not replicated in detail in this report. Functional details of specific programs such as the Tank Waste Remediation System and Environmental Restoration programs are published as part of their specific baseline documentation.

${ }^{1}$ Holmes, J. J., 1994, Systems Engineering Functions and Requirements for the Hanford Cleanup Mission: First Issue, WHC-EP-0722, Rev. 0, Westinghouse Hanford Company, Richland, Washington. 
While this functional analysis is intended to emphasize the super system in which programmatic systems are embedded, some program-level functions are included for areas where systems engineering is just starting. As these new areas mature and as the appropriate systems engineering baselines become available the detail included in future updates of this addendum will diminish in favor of a more inclusive set of "program" baselines and associated systems engineering analyses documented as part of specific program planning.

The specific program functional analyses will remain tied to the overall Hanford Cleanup mission through the top-level ("Capstone") functional analysis so that a complete top-to-bottom functional set always exists for all mission activities.

The breakdown of the public acceptance function provided herein differs from the report body. The functions described in this addendum represent an evolution from the earlier work and replace those reported earlier.

This addendum describes work-in-progress, which is 90 to 95 percent complete at the supersystem level. 
WHC-EP-0722 ADDENDUM 2 REV. 0

\section{SECTION 1}

FUNCTION HIERARCHY CHARTS 
FUNCTION HIERARCHY CHARTS

LIST OF FIGURES

Page

o Clean Up Hanford.................................. 1-1

1 Manage Program................................... 1-2

2 Acquire Mission Essential Capabilities.................... 1-3

3 Obtain Public Trust................................. 1-4

4 Remedy Unsafe and Unacceptable Conditions.................. 1-5

4.1 Deactivate Facilities............................ 1-6

4.1.1 Deactivate Facilities With Special Nuclear Materials \&... 1-7 Nuclear Materials (Type 1 Fclty)

4.1.1.1 Maintain Safety \& Compliance Envelope (Type 1 Fclty) 1-8

4.1.1.2 Determine Deactivation Plans \& Negotiate Turnover.... 1-9

4.1.1.3 Stabilize \& Reconfigure Facilities for Minimum ...... 1-10

4.1.1.4 Disposition Currently Identified Radioactive Materials 1-11 Held As A Potential Product \& Special Nuclear Material (Type 1 Fclty)

4.1.2 Deactivate Facilities With Radioactive \& Hazardous...... 1-12 Material (Type 2 Fclty)

4.1.2.1 Maintain Safety \& Compliance Envelope (Type 2 Fclty) 1-13

4.1.2.2 Determine Deactivation Plan \& Negotiate Turnover..... 1-14 Endpoint (Type 2 Fclty)

4.1.2.3 Stabilize \& Reconfigure Facilities For Minimum...... 1-15 Surveillance (Type 2 Fclty)

4.1.3 Deactivate Facilities With Only Hazardous Material, .... 1-16 Including Asbestos (Type 3 Fclty)

4.1.3.1 Maintain Safety \& Compliance Envelope (Type 3 Fclty) 1-17

4.1.3.2 Determine Deactivation Plan \& Negotiate Turnover..... 1-18 Endpoint (Type 3 Fclty)

4.1.3.3 Stabilize \& Reconfigure Facilities for Minimum...... 1-19 Surveillance (Type 3 Fclty)

4.1.4 Deactivate Facilities Without Radioactive Or Hazardous... 1-20 Material (Type 4 Fclty)

4.3 Remedy Solid Waste............................. 1-21

4.3.1 Maintain Solid Waste Safety And Compliance Envelope...... 1-22

4.3.2 Receive Solid Waste........................... 1-23

4.3 .3 Characterize Solid Waste....................... 1-24 


\section{FUNCTION HIERARCHY CHARTS}

\section{LIST OF FIGURES}

Page

4.3.4 Determine Solid Waste Disposition Requirements......... 1-25

4.3.5 Disposition Solid Waste........................ 1-26

4.3.5.1 Treat Solid Waste.......................... 1-27

4.3.5.2 Package Sol id Waste........................ 1-28

4.3.5.5 Ship Solid Waste.......................... 1-29

4.3.5.6 Dispose Solid Waste........................ 1-30

4.5 Manage Aqueous Wastes........................... 1-31

4.7 Store, Treat, and Disposition SNM/NM/NF Materials.......... 1-32

4.7.2 Control SNM, NM, and NF Functions................ 1-33

4.7.3 Handle Incoming Materials....................... 1-34

5 Transition Resources For Beneficial Use..................... 1-35 


\section{Function Hierarchy}

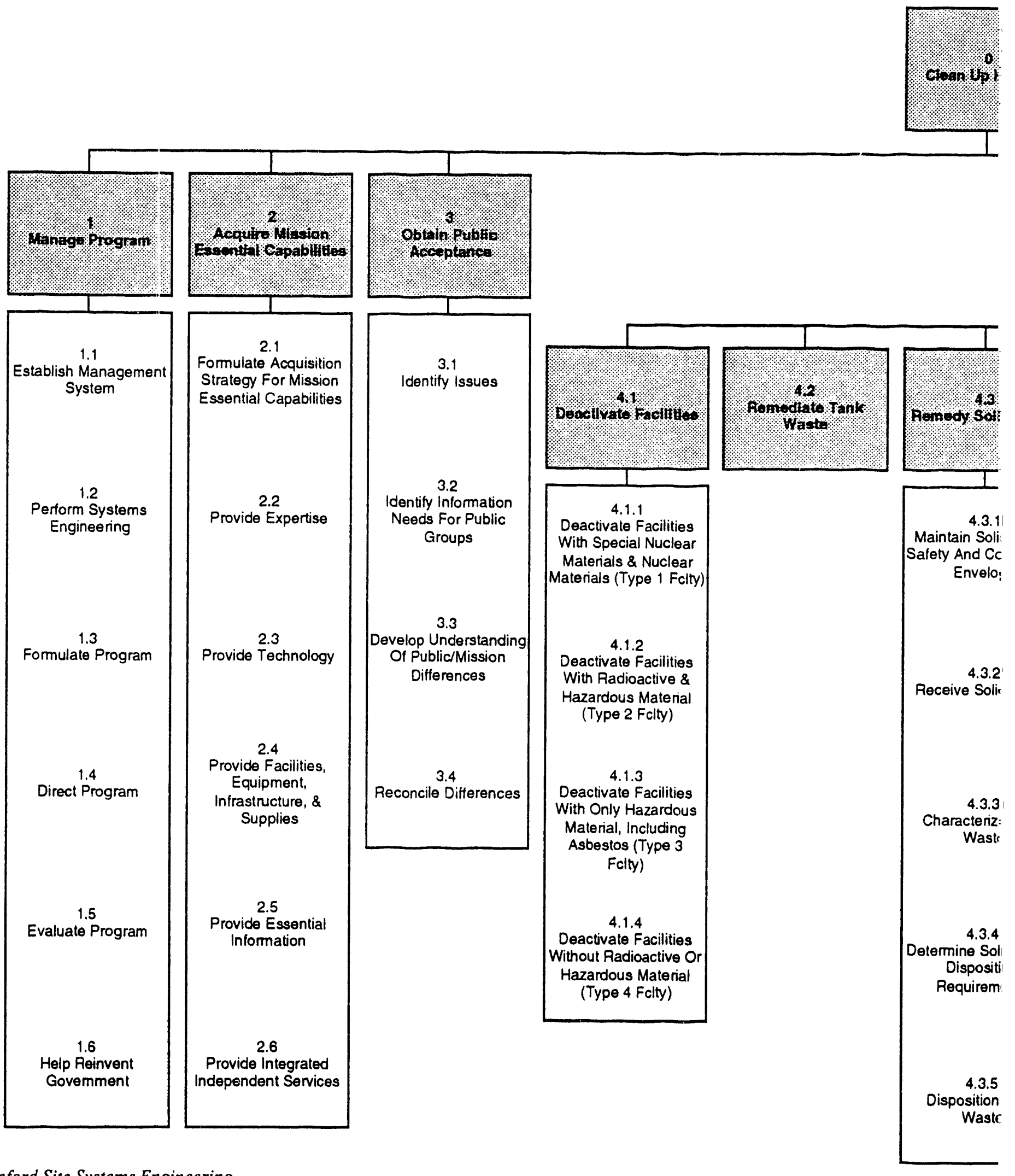




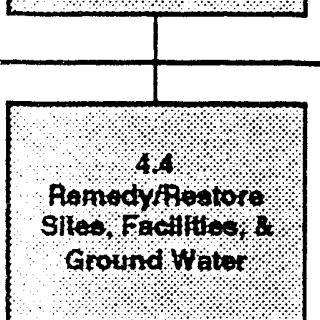

Slles, Factlitios. 2 Ground Wator
Waste
4.5 .2

Develop Aqueous Waste Disposition Plan Wast

$$
\text { 4.5.1 }
$$

Maintain Aqueous Waste Salety and Compliance Envelope (1)
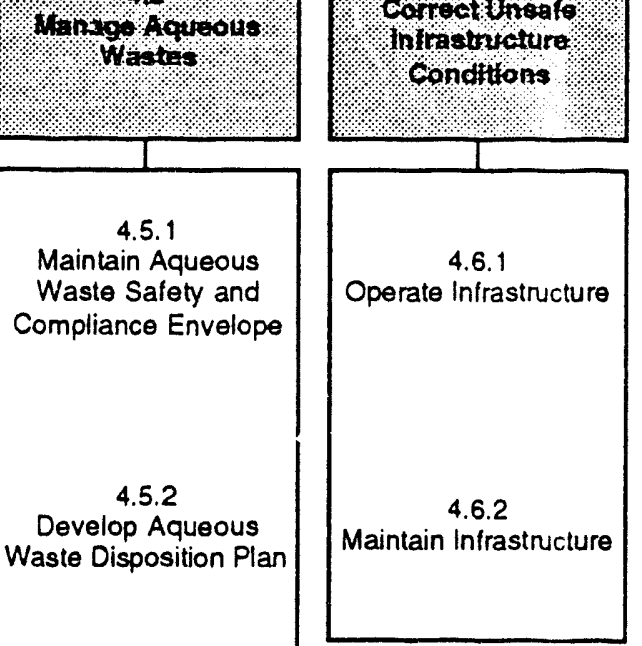

4.5.3

Receive/Transport Aqueous Waste

4.5.4

Treat Aqueous Waste

4.5.5

Disposition Aqueous Waste

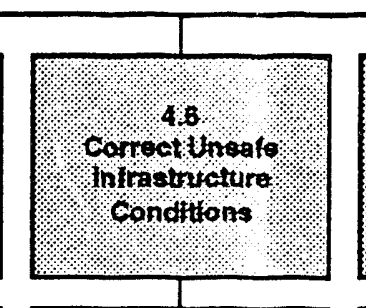

4.6.1

Operate Infrastructure

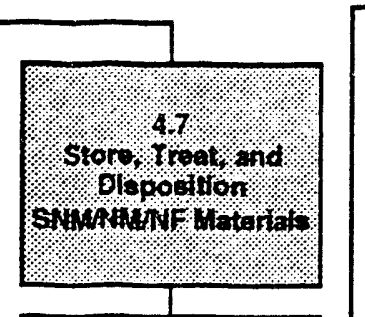

4.7.1

Maintain Safety and Security Envelope (SNM/NM/NF)

4.7 .2

Control SNM, NM, and

NF Functions

Maintain Infrastructure
5.2

Transfer Ownership Of Resources Outside Of Mission

5.3

Transfer Resources

For Use Outside Of Mission 
Function Hierarchy

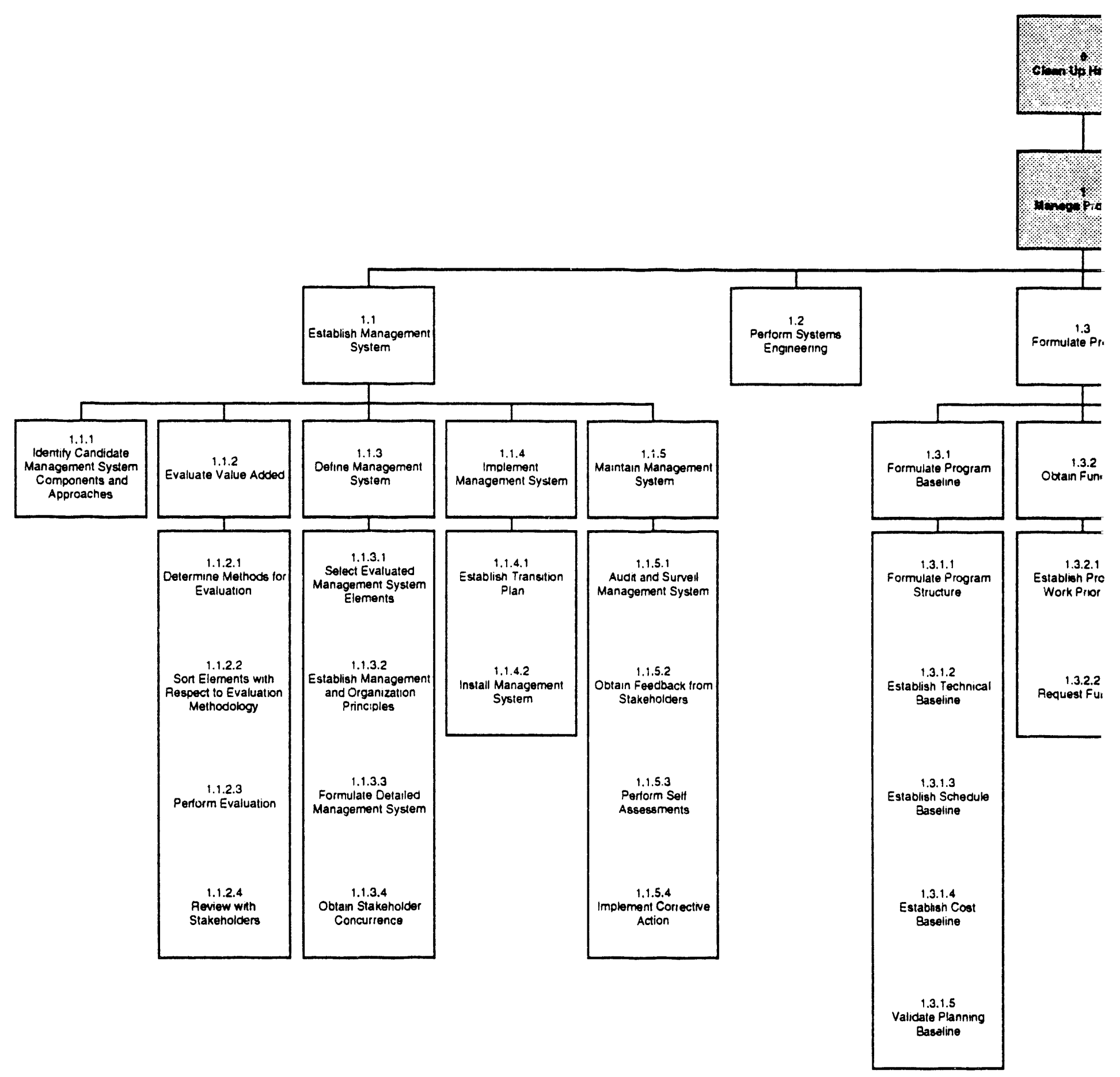




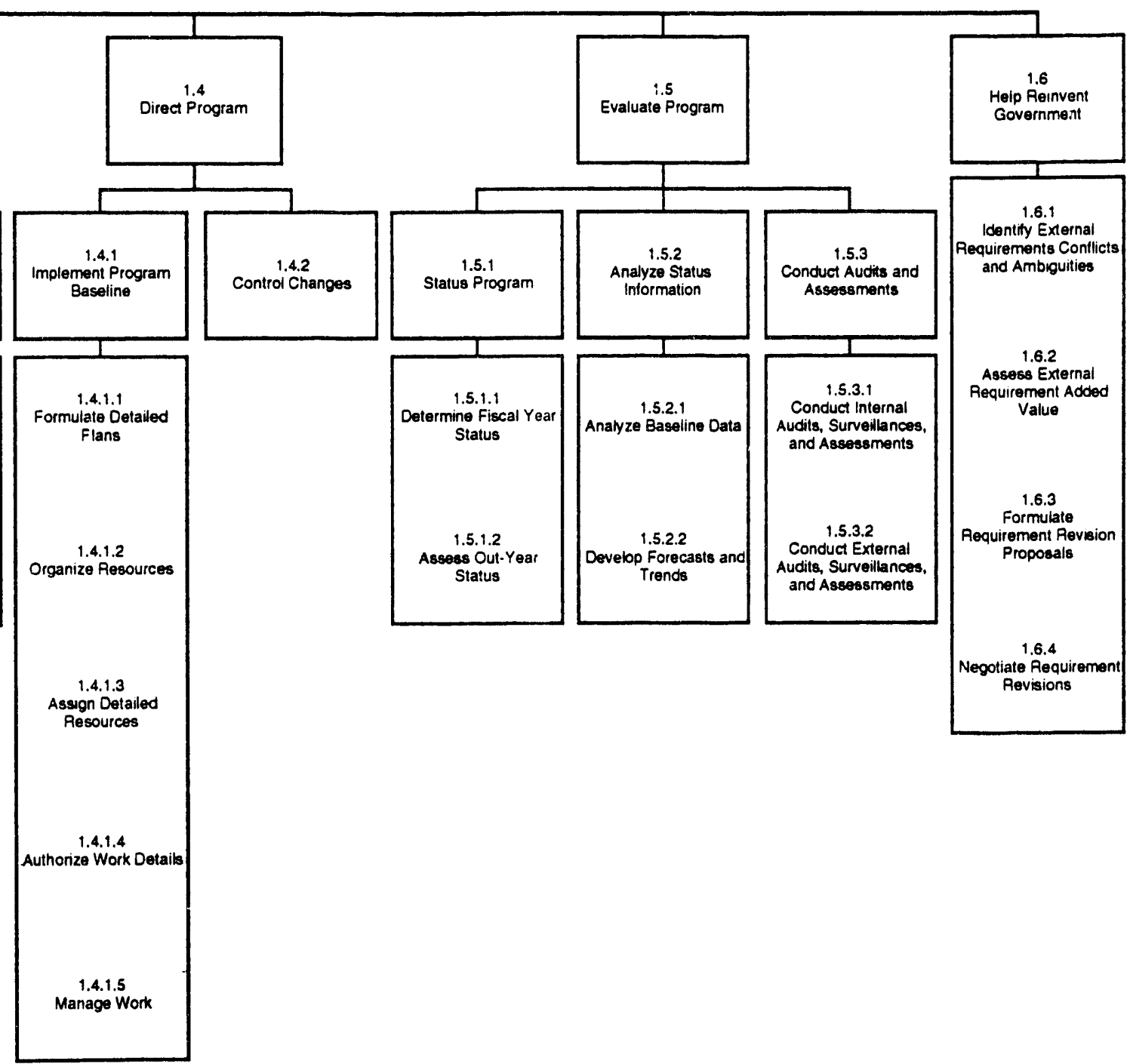


Function Hierarchy

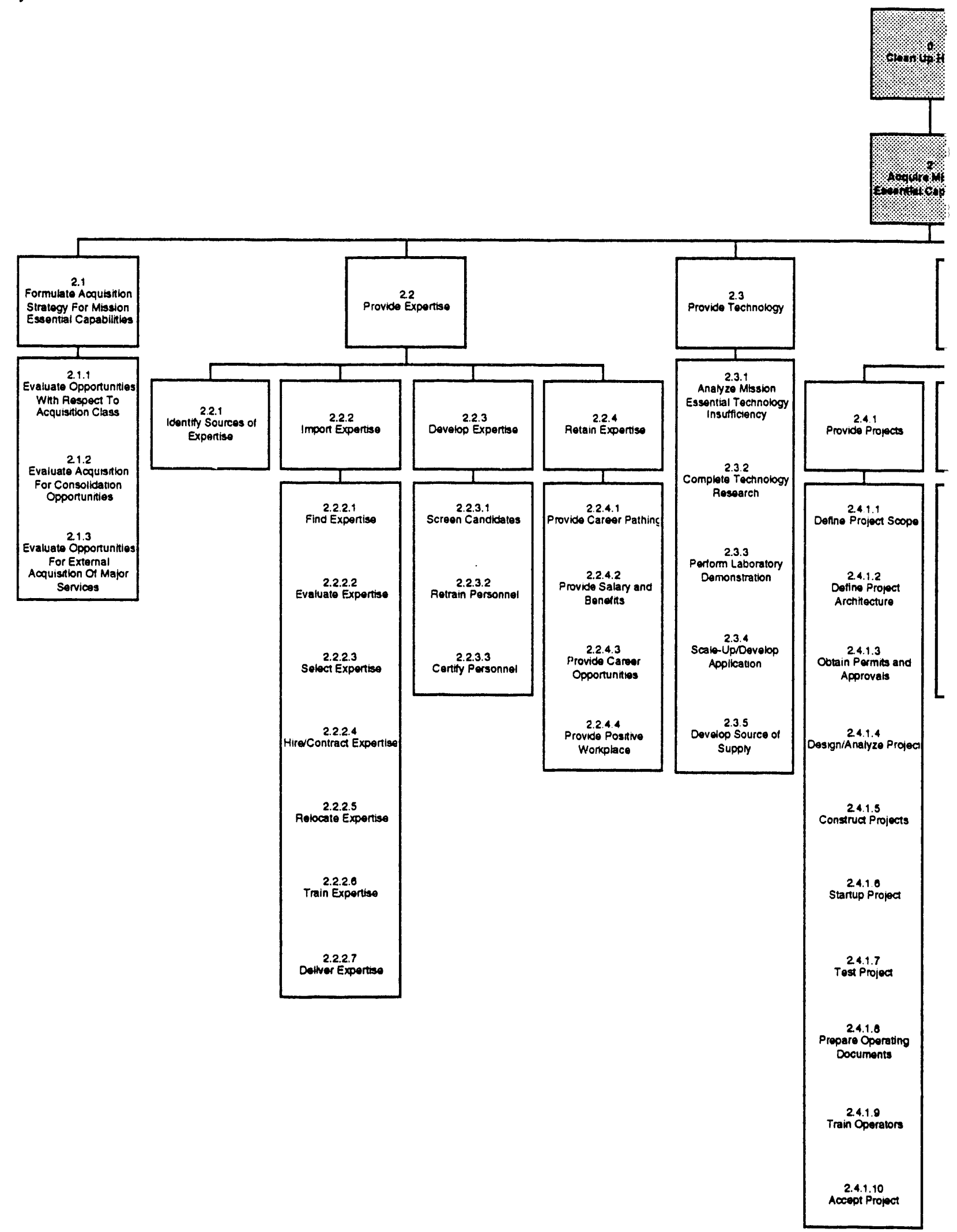




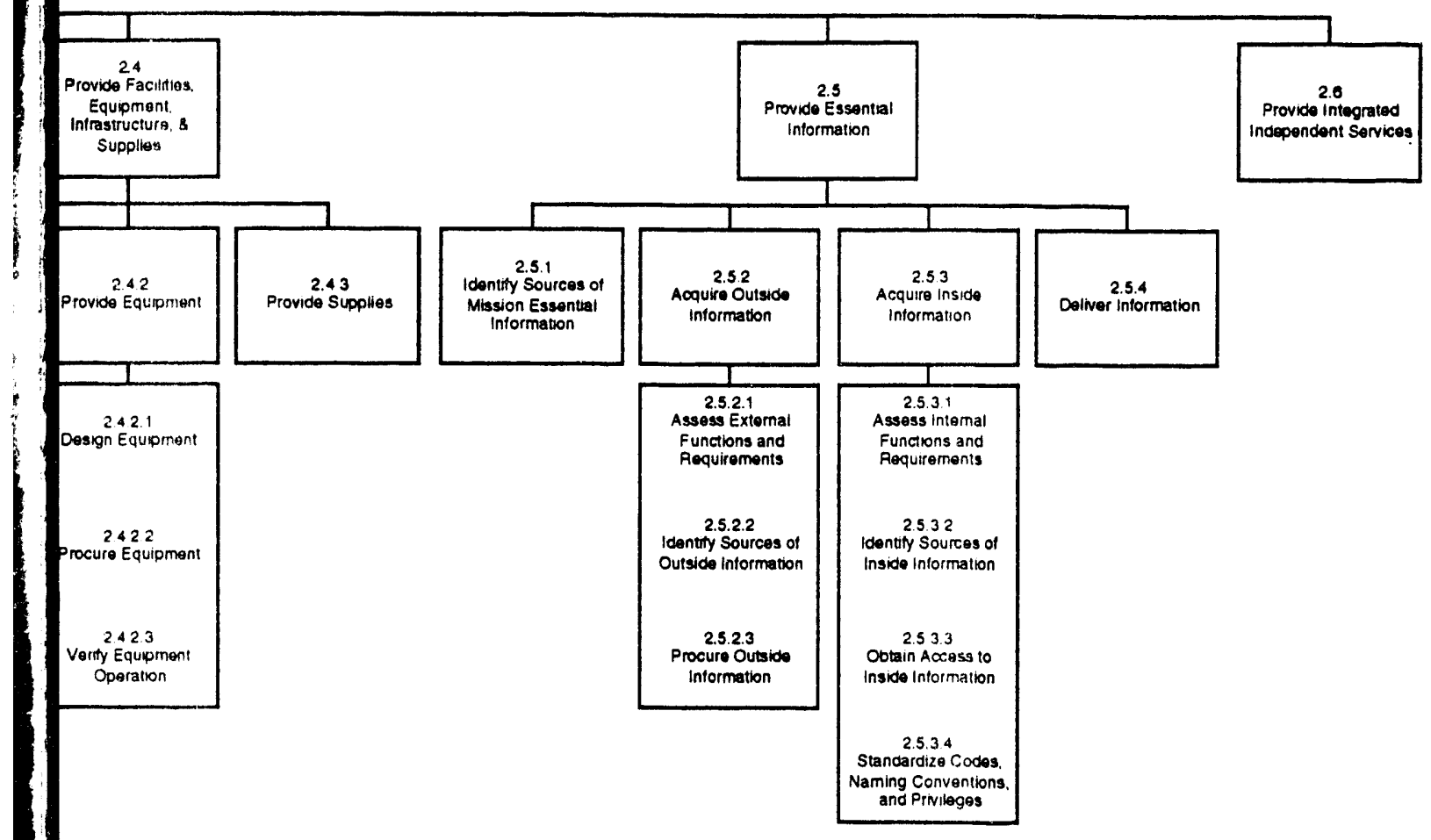


Function Hierarchy

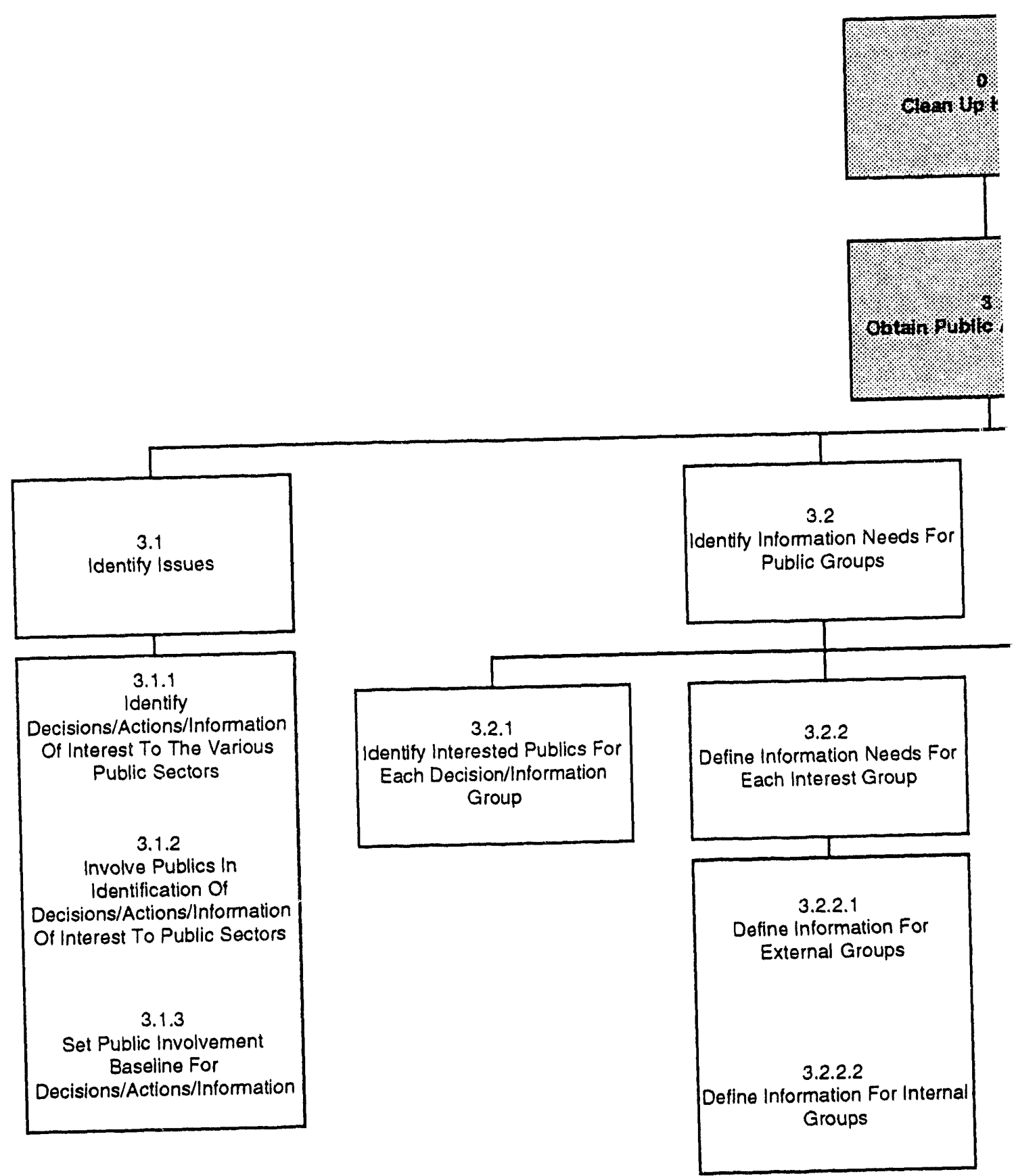


antord

cosptanco

3.3

Develop Understanding of

Public/Mission Differences

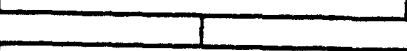

3.3.1

Define Differences in

"Objectives"

Develop Public Involvement Plan For Each Combination of Public

Group/(Decision/Information Area)
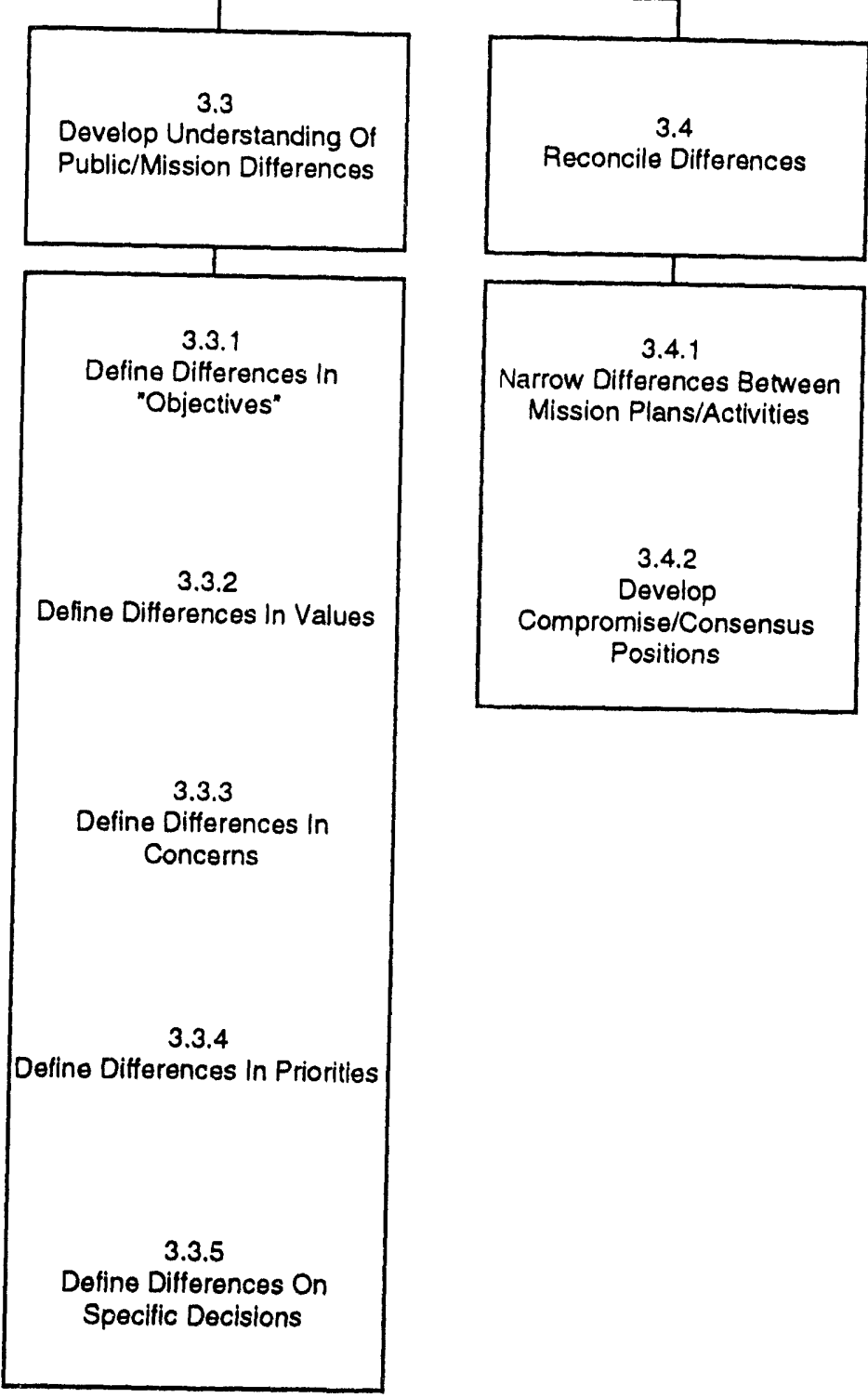
Function Hierarchy

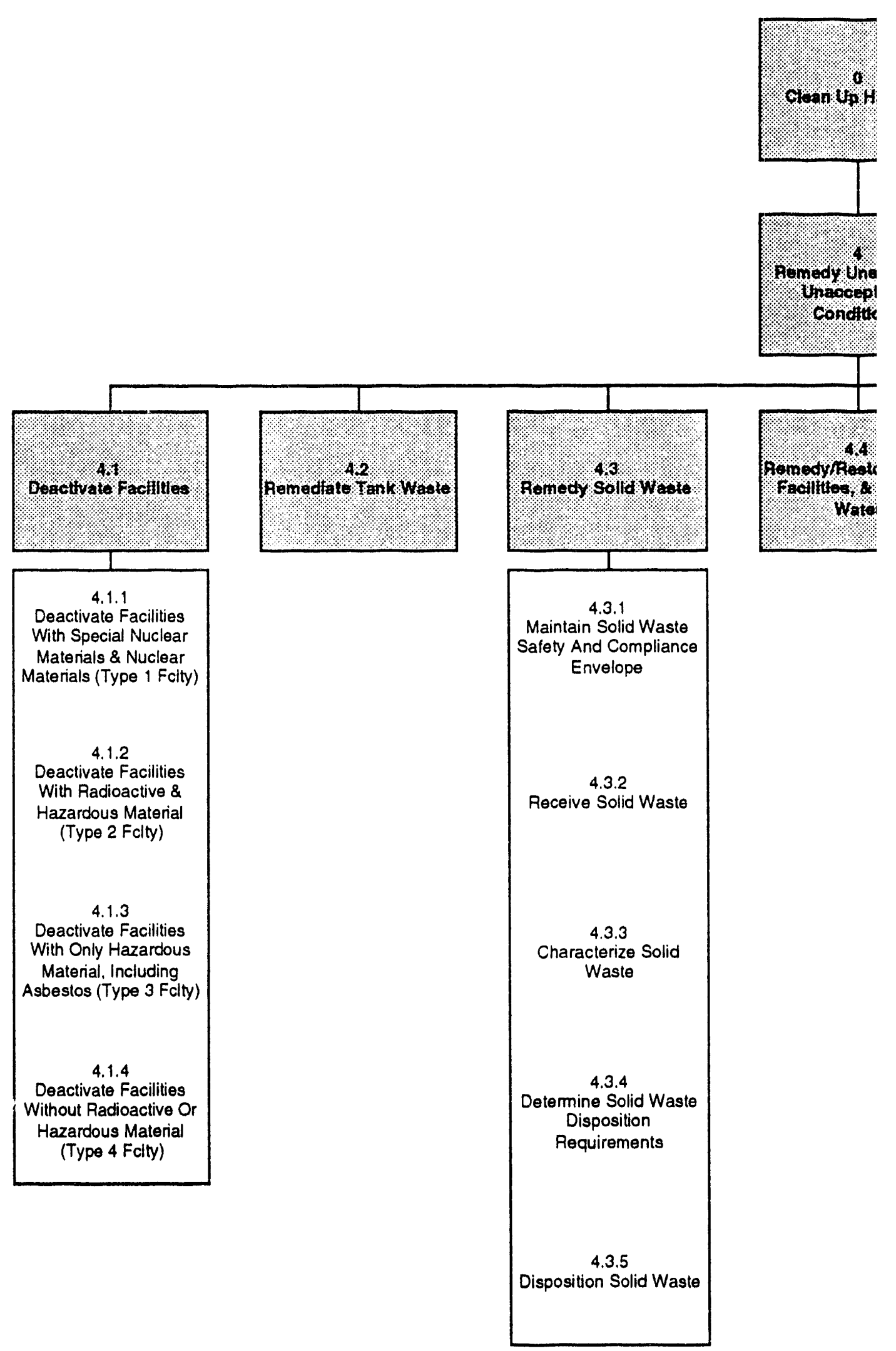




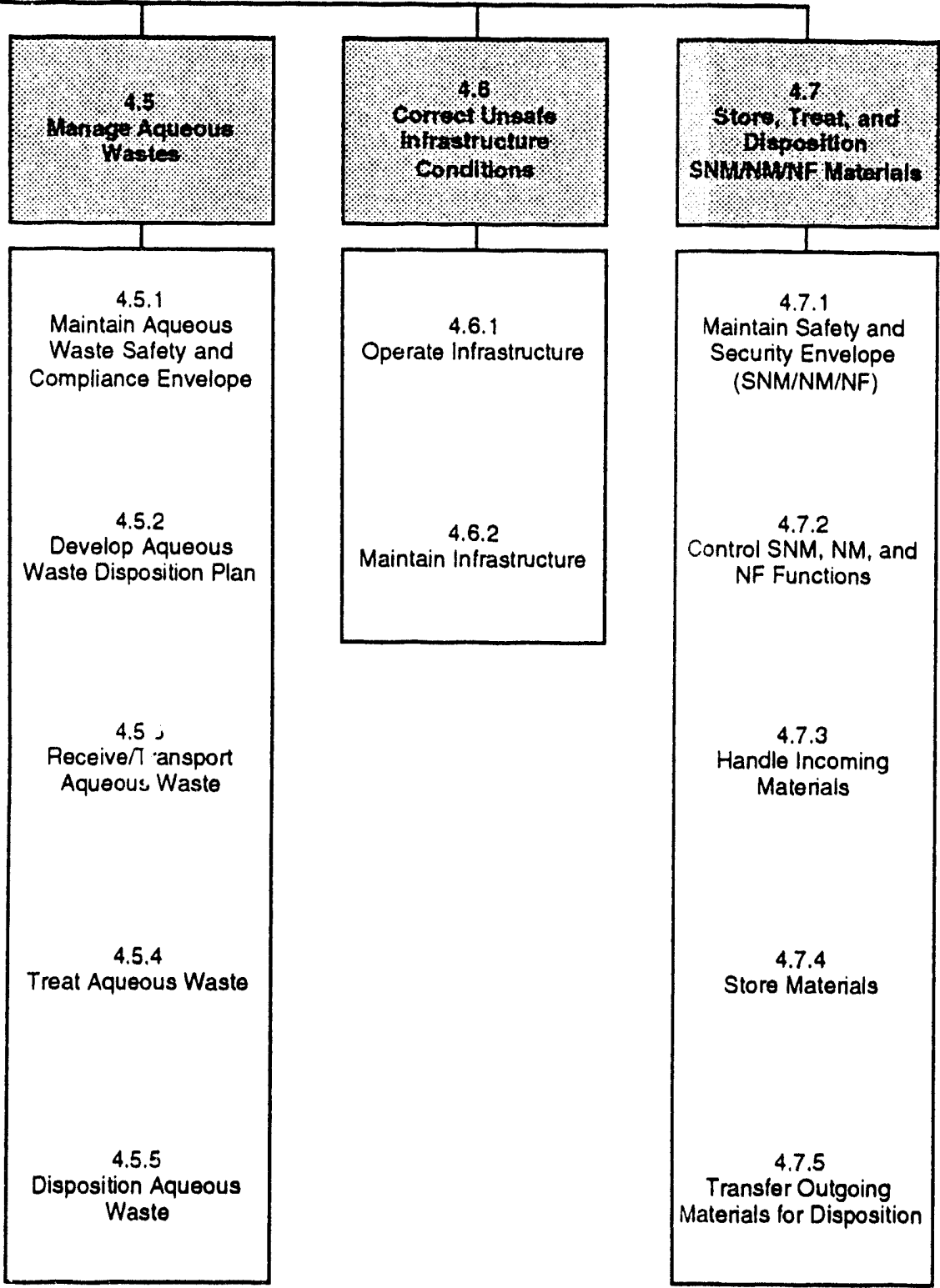




\section{Function Hierarchy}
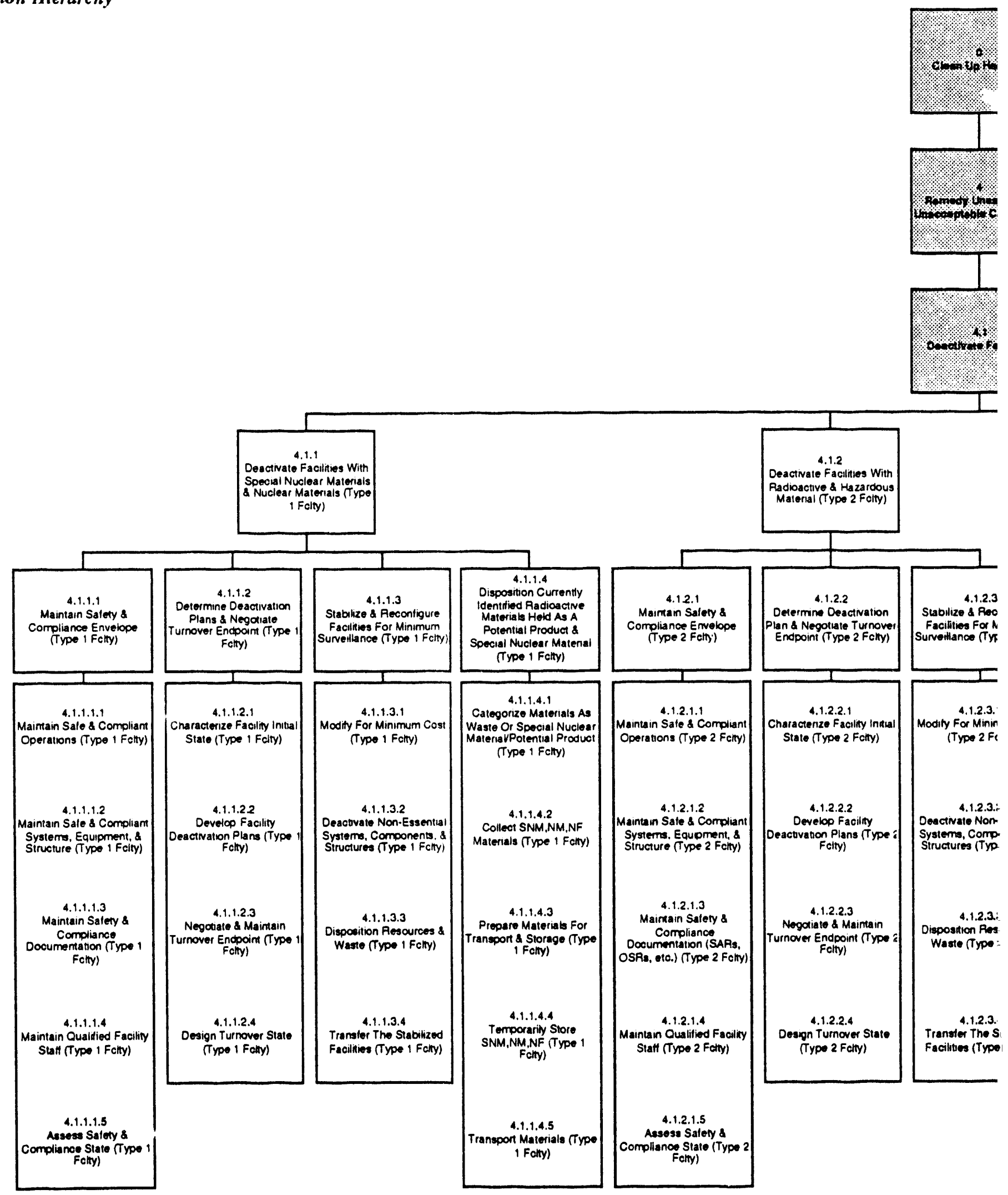

Hanford Site Systems Engineering 


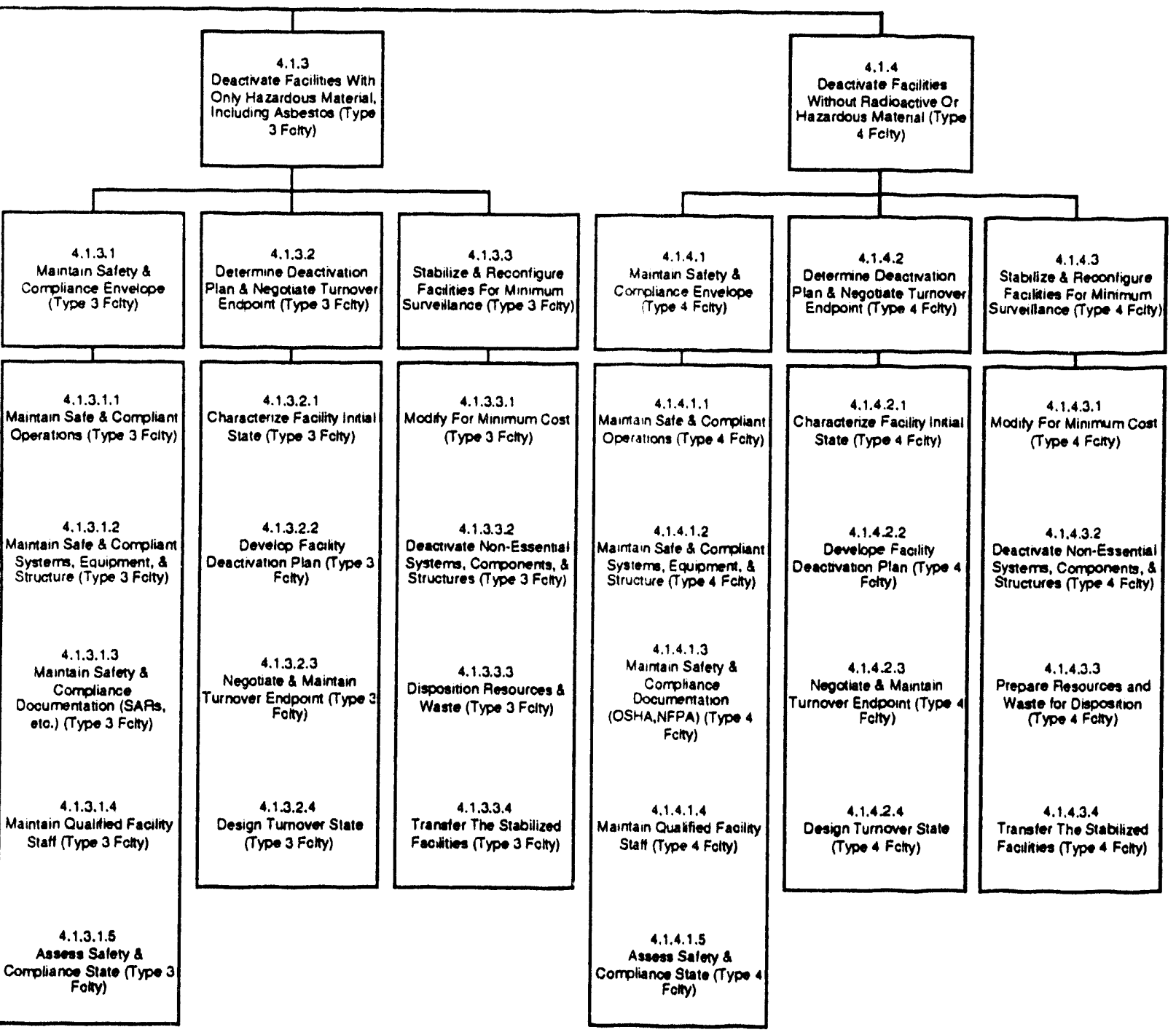


Function Hierarchy

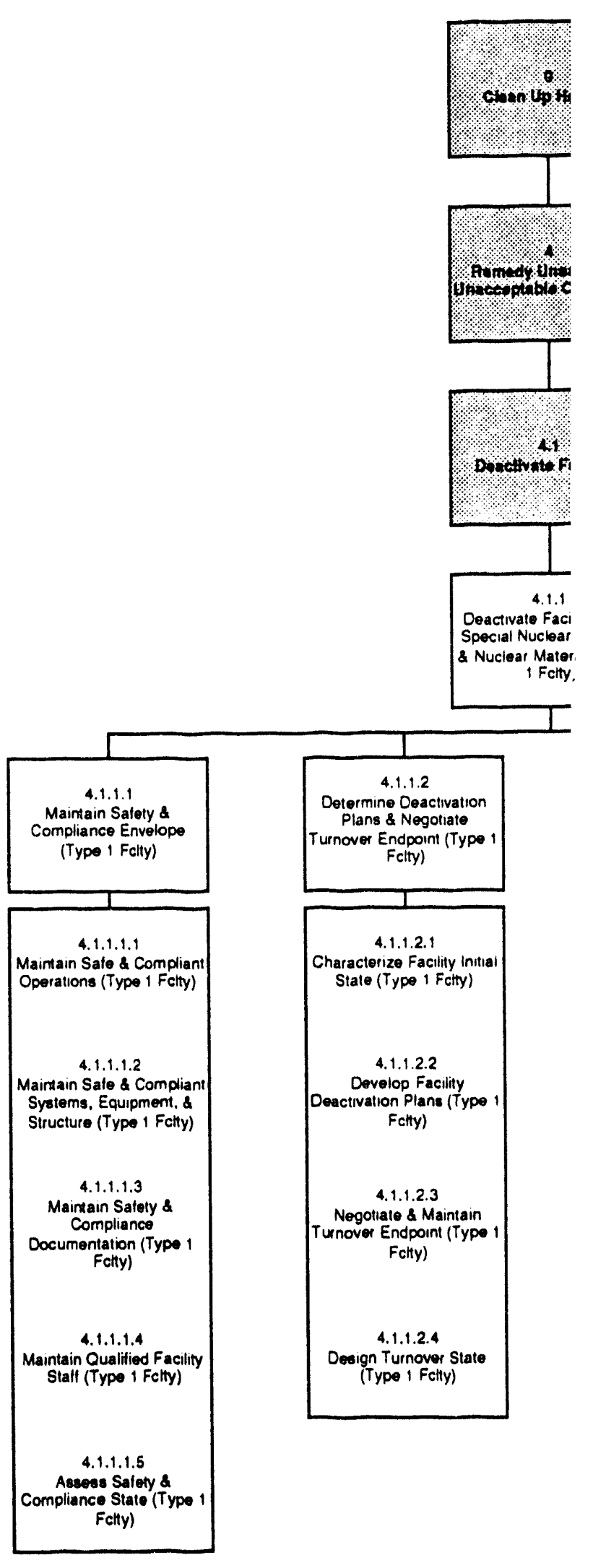




\begin{tabular}{|c|c|}
\hline 4.1.1.3 \\
Stabilize \& Recontigure \\
Facilities For Minimum \\
Surve llance (Type 1 Fcity)
\end{tabular}$\quad \begin{gathered}\text { 4.1.1.4 } \\
\text { Disposition Currently } \\
\text { Ientiliod Radioactive } \\
\text { Malerials Held As A } \\
\text { Potential Product \& } \\
\text { Special Nuclear Material }\end{gathered}$

4.1.1.3.1

Modify For Minimum Cost (Type 1 Fclty)

$$
\text { 4.1.1.3.2 }
$$

Deactivate Non-Essential Systems, Components, \& Structures (Type 1 Fclty)

4.1.1.3.3 Disposition Resources a Waste (Type I Fclty)

4.1.1.3.4

Transter The Stabilized Facilities (Type 1 Fclty)

\subsubsection{1 \\ Categorize Materials As Waste Or Special Nuciear MateriavPotential Product \\ (Type 1 Fclty) \\ 4.1.1,4.2 \\ Collect SNM,NM,NF Materials (Type 1 Fcliy)

$$
\text { 4.1.1.4.3 }
$$ \\ 4.11 .4 .3 poris} Transport \& Storage (Type 1 Felty)

4.1.1.4.4 Temporarily Store SNM,NM,NF (Typo 1 Felty)

4.1.1.4.5 Transport Matonals (Type 1 Feity) 


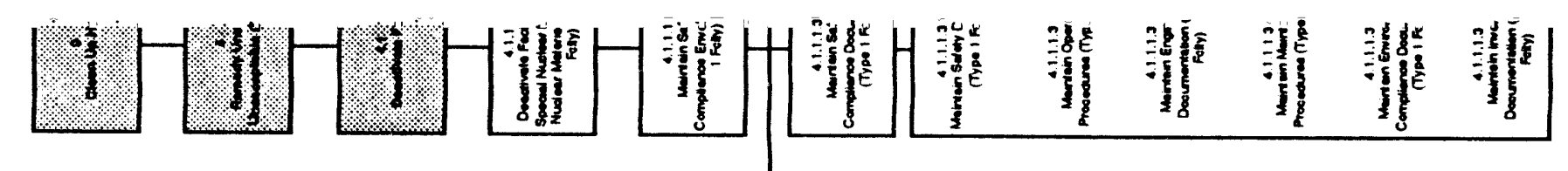

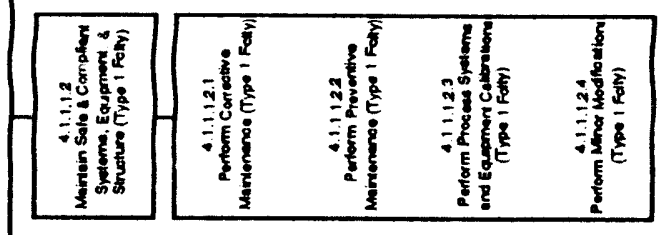

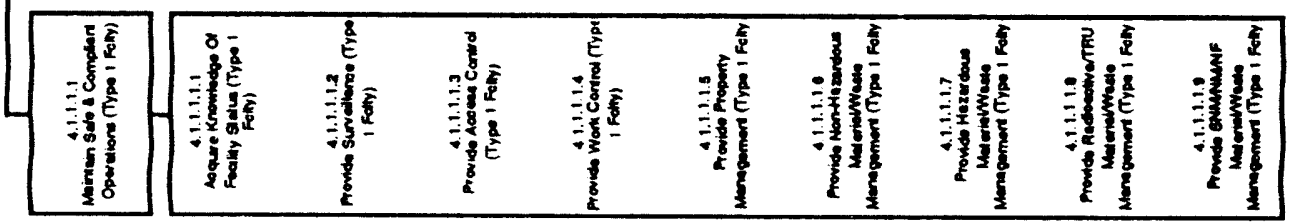

है 
WHC-EP-0722 ADDENDUM 2 REV 0

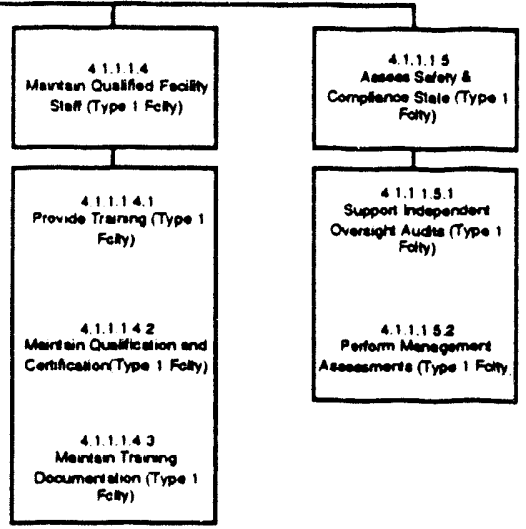


Function Hierarchy

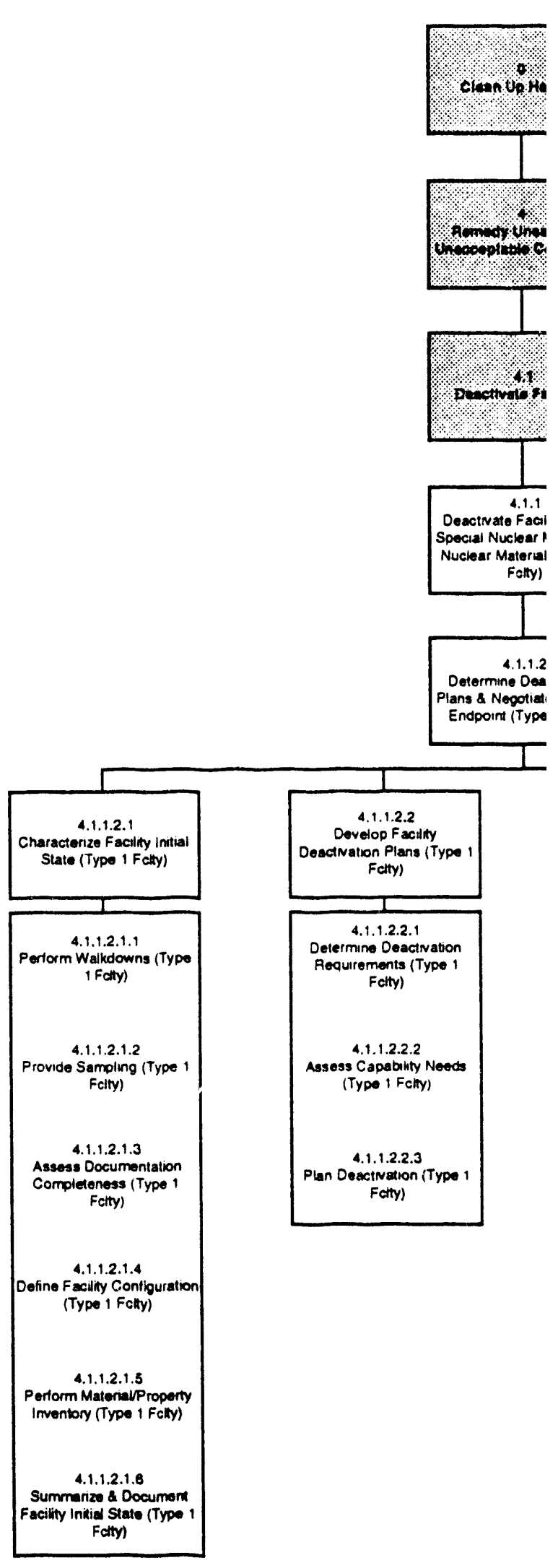




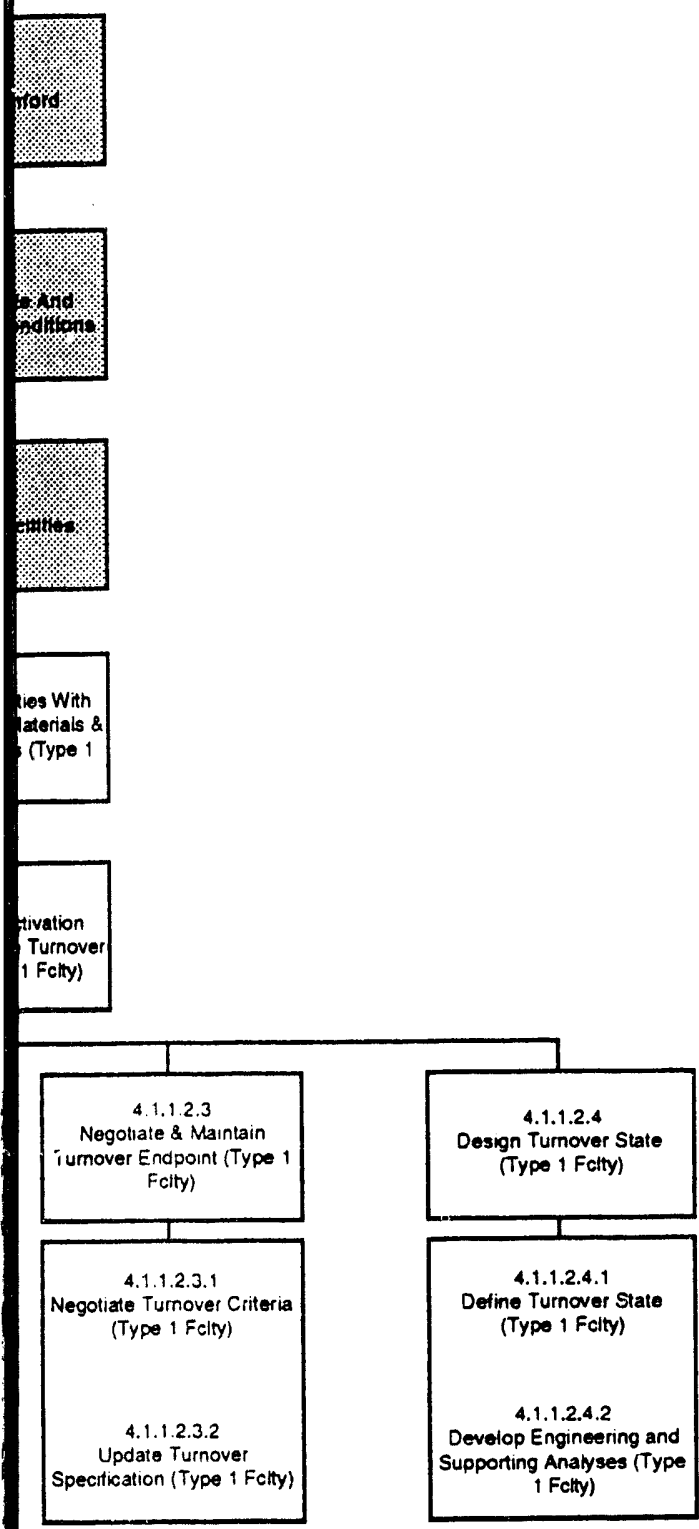




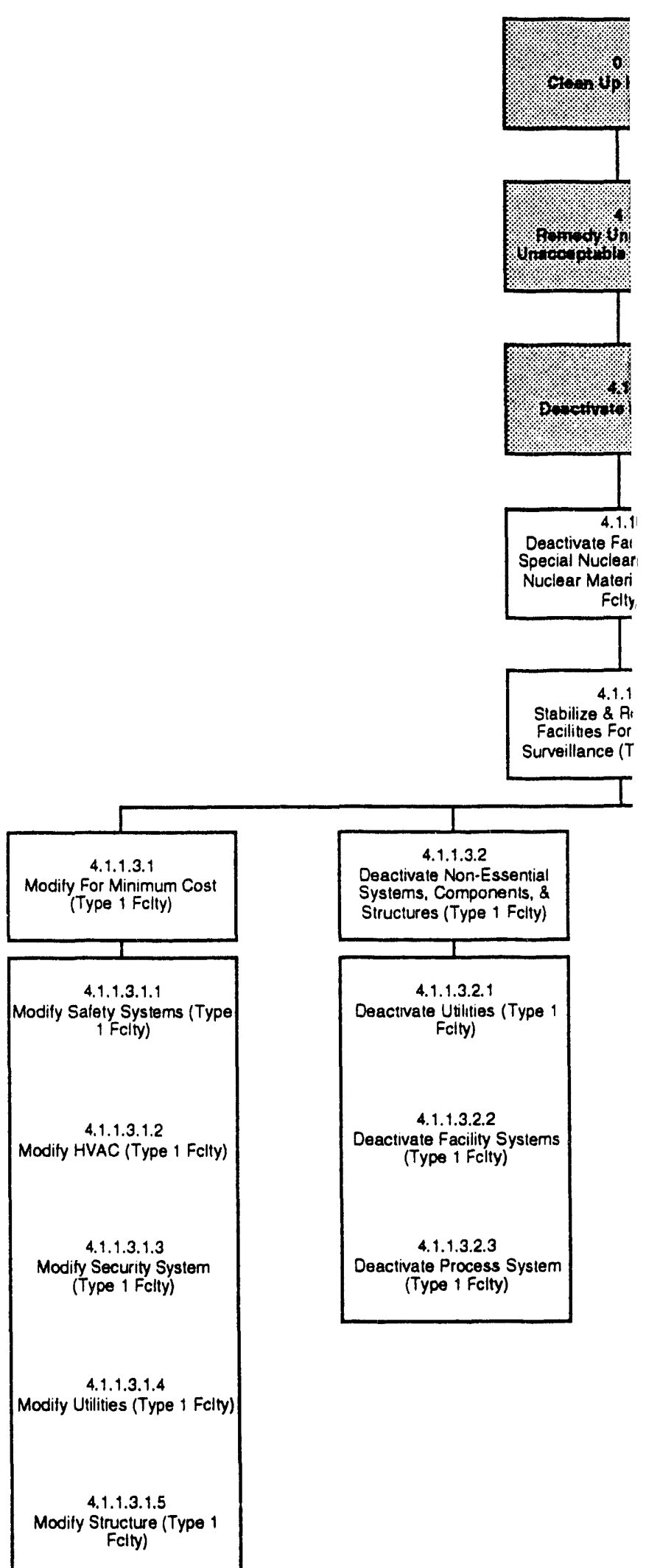



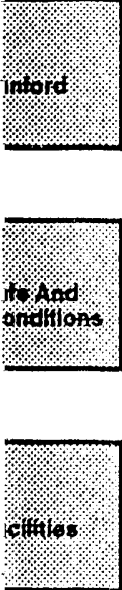

ities With

Materials \&

s (Type 1

\section{onfigure \\ finimum \\ re 1 Fclty)}

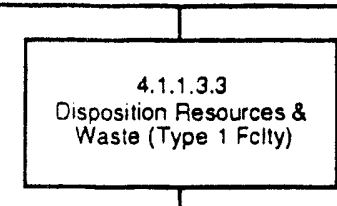

4.1.1.3.3.1

Prepare Resources and

Waste for Disposition (Type

1 Fclty)

4.1.1.3.3.2

Package Oispositloned

Resources \& Waste (Type 1

Folty)

4.1.1.3.3.3

Transport Dispositioned

Pesources \& Waste (Type

Fclity)

4.1.1.3.4

Transfer The Stabstized

Facilities (Type t Fcliy)

4.1.1.3.4.1

Turnover Facilities (Type 1 Fclty

4.1.1.3.4.2

Turnover Facility Intormation

(Type 1 Fclty)

4.1.1.3.4.3

Prepare Turnover Documentation (Type 1 Fcity)

4.1.1.3.3.4

Prepare Resource/Waste Documentation (Type 1 Folty)

4.1.1.3.3.5

Validate Characterization Information (Type 1 Fclty) 

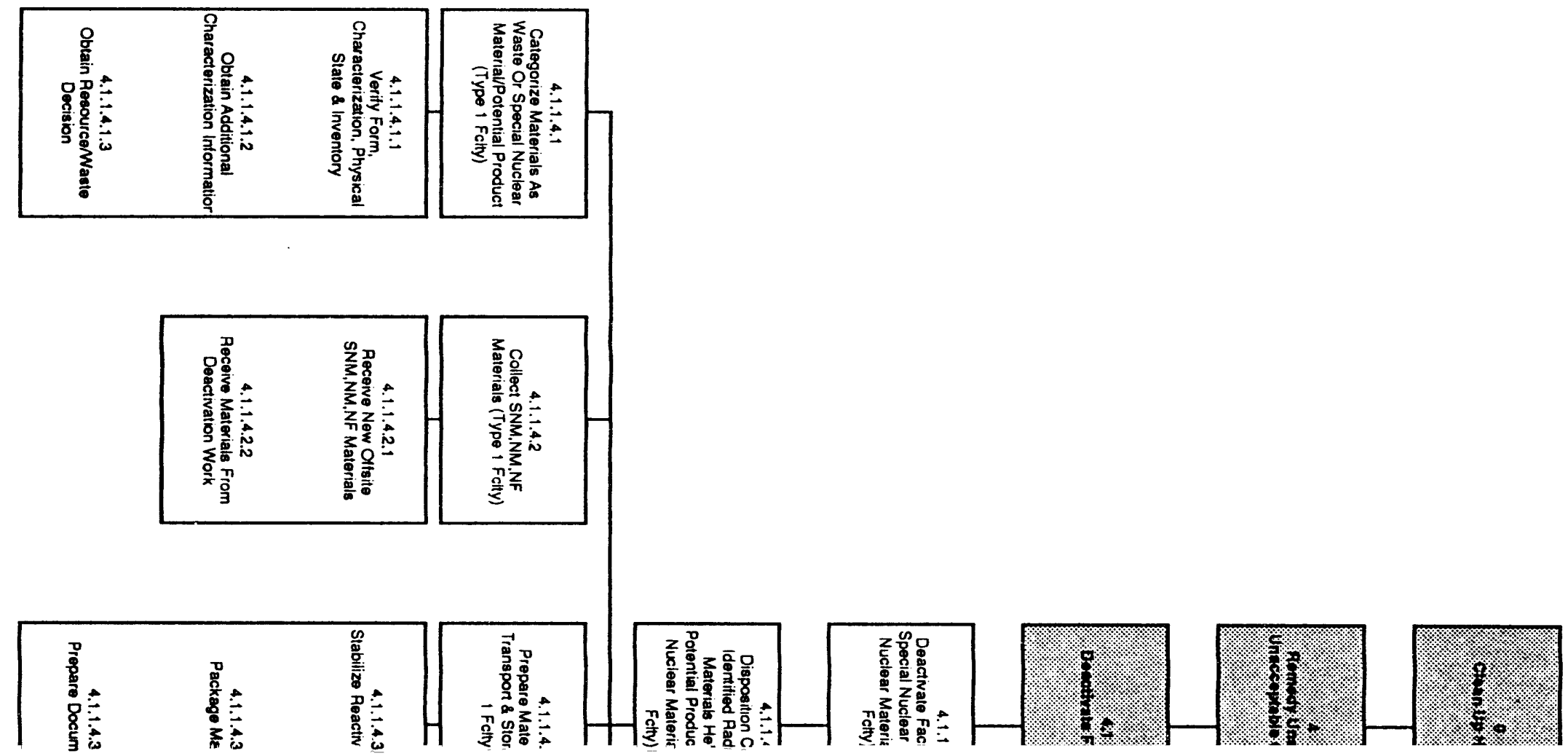


\section{Function Hierarchy}

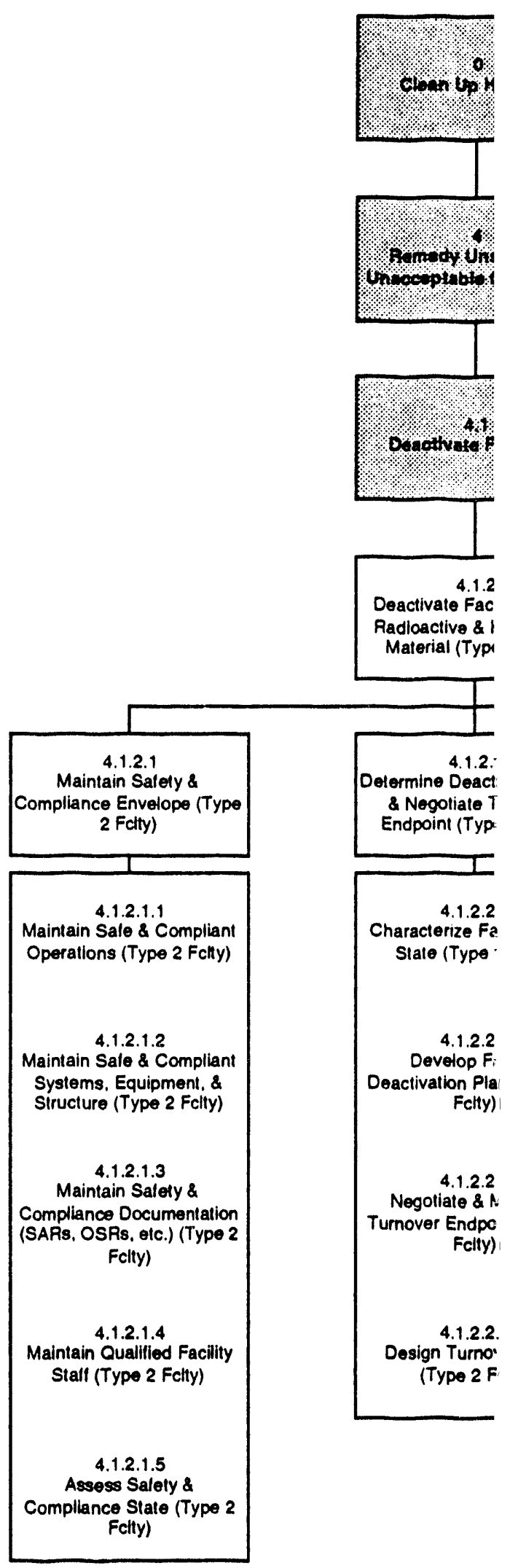


WHC-EP-0722 ADDENDUM 2 REV 0

Facilities For Minimum Surveillance (Type 2 Fcity)

\begin{tabular}{|c|}
\hline \\
4.1 .2 .3 .1 \\
Modify For Minimum Cost \\
(Type 2 Fclty) \\
\\
4.1 .2 .3 .2 \\
Deactivate Non-Essential \\
Systems, Components, \& \\
Siructures (Type 2 Fclty) \\
\\
4.1 .2 .3 .3 \\
Disposition Resources \& \\
Waste (Type 2 Fclty)
\end{tabular}




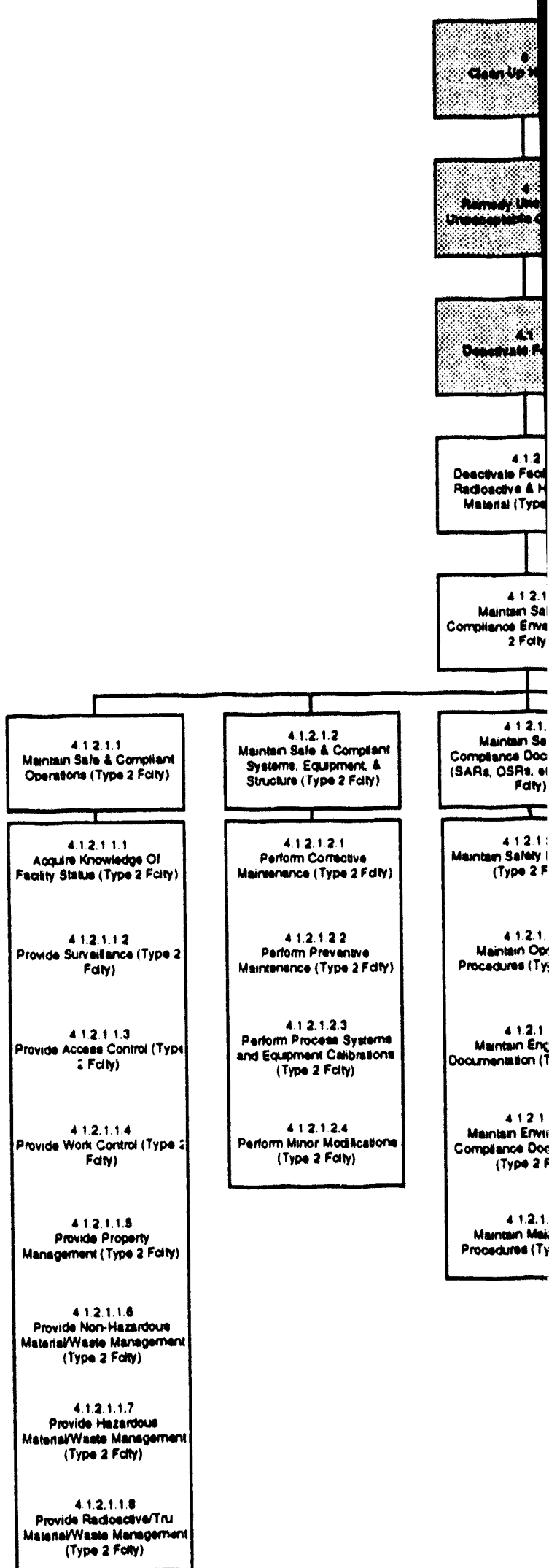


WHC-EP-0722 ADDENDUM 2 REV 0 
Function Hierarchy

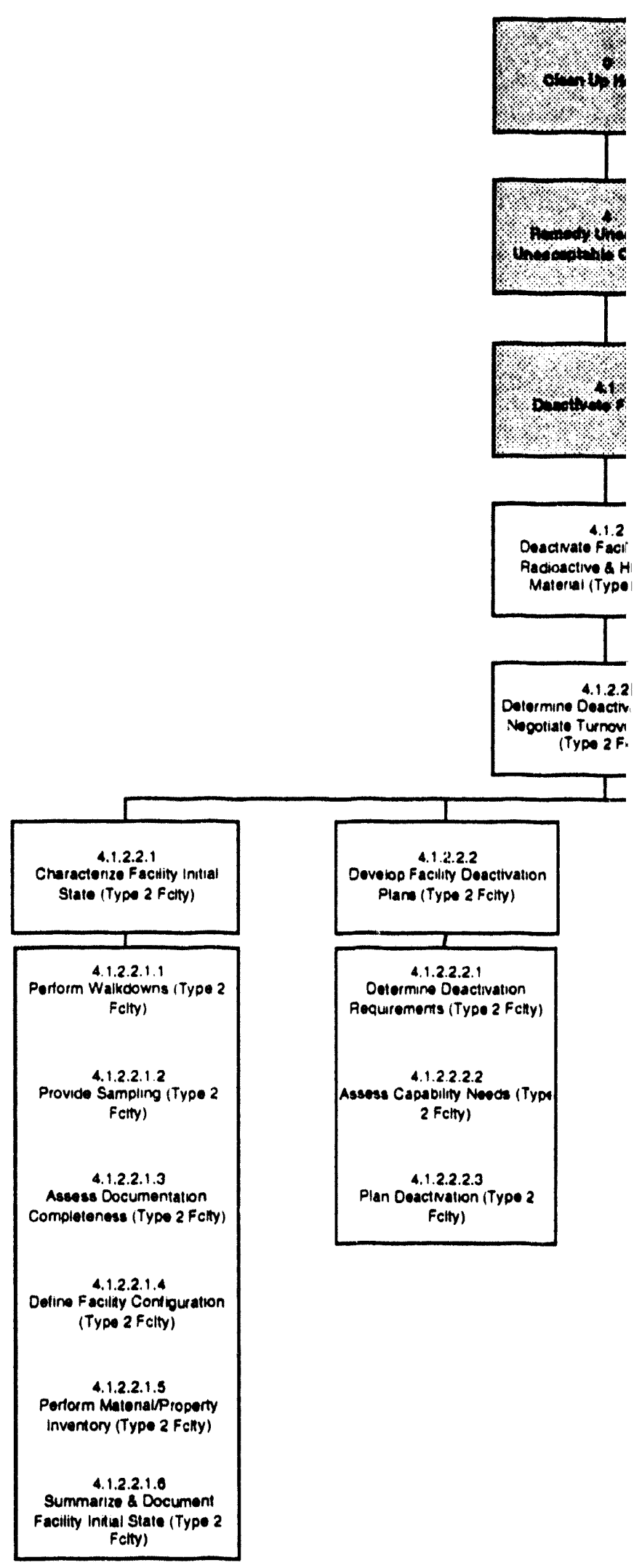




\begin{tabular}{|c|}
\hline $\begin{array}{l}\text { 4.1.2.2.4 } \\
\text { Design Turnover State (Type } 2 \\
\text { Fcly) }\end{array}$ \\
\hline $\begin{array}{l}\text { 4.1.2.2.4.1 } \\
\text { Doline Turnover Slate (Type } 2 \\
\text { Foly) }\end{array}$ \\
\hline $\begin{array}{l}\text { Devel.1.2.2.4.2 } \\
\text { Develop Engineoring and } \\
\text { Supponting Analysess (Typo } 2 \\
\text { Fely) }\end{array}$ \\
\hline
\end{tabular}




\section{Function Hierarchy}

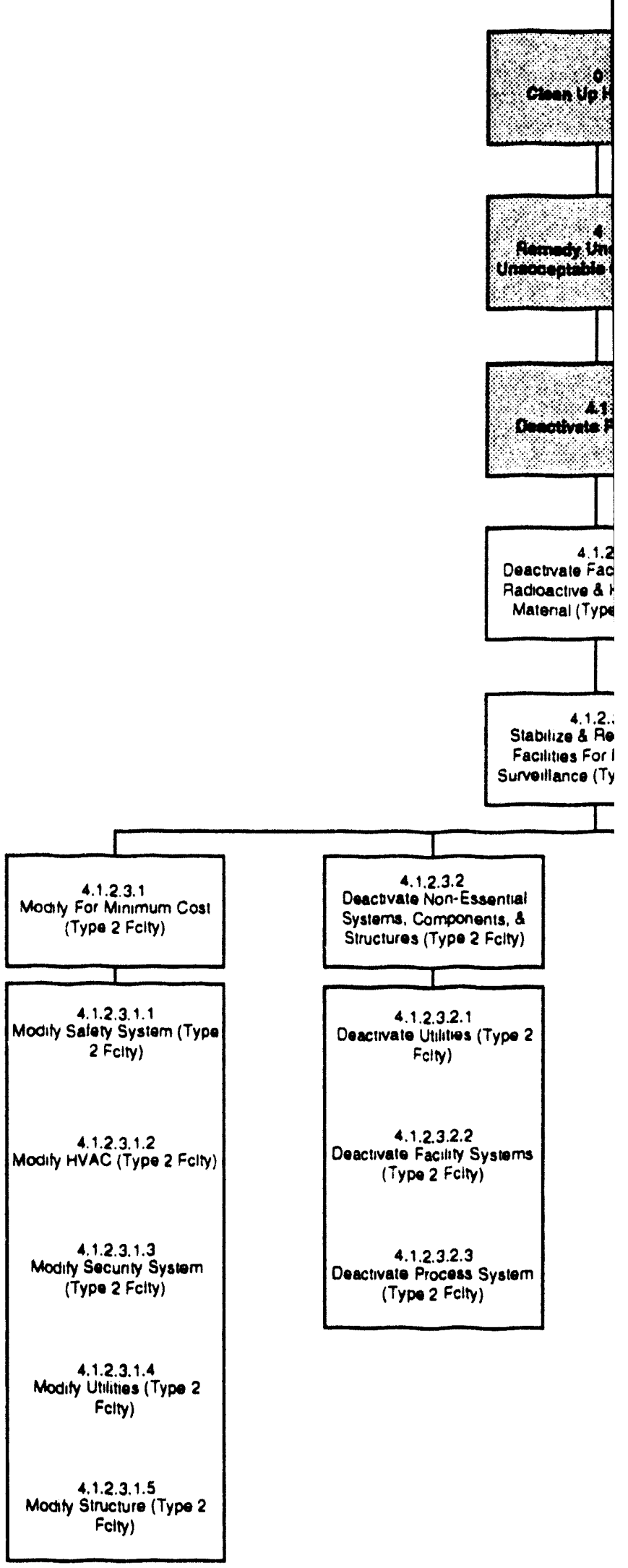




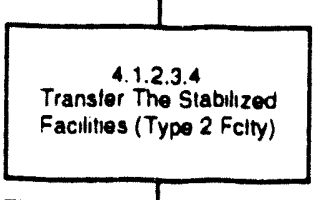

4.1.2.3.3.1

Accumulate. Remove, and

Disposition Resources and

Wasie (Type 2 Fcly)

4. 1.2.3.3.2

Package Dispositioned

Resources \& Waste (Type 2

$$
\text { Foity) }
$$

4.1.2.3.3.3

Transport Dispositioned

Resources \& Waste (Typo 2

Fcity)

4.1.2.3.4.1

Tumover Facilites (Type 2 Fcity)

4.1.2.3.4.2

Turnover Facility iniormation

(Type 2 Fcity)

4.1.2.3.4.?

Prepars Tumover

Documentation (Type 2 Folity)

4.1.2.3.3.4

Prepare Resourcentaste Documentation (Type 2 Fetty) 


\section{Function Hierarchy}

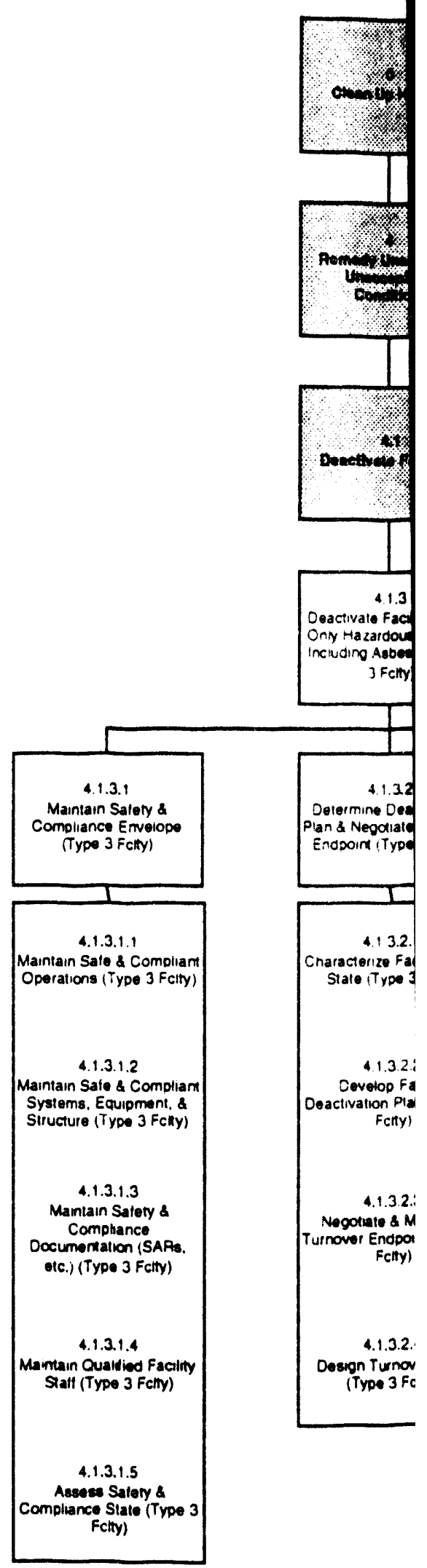



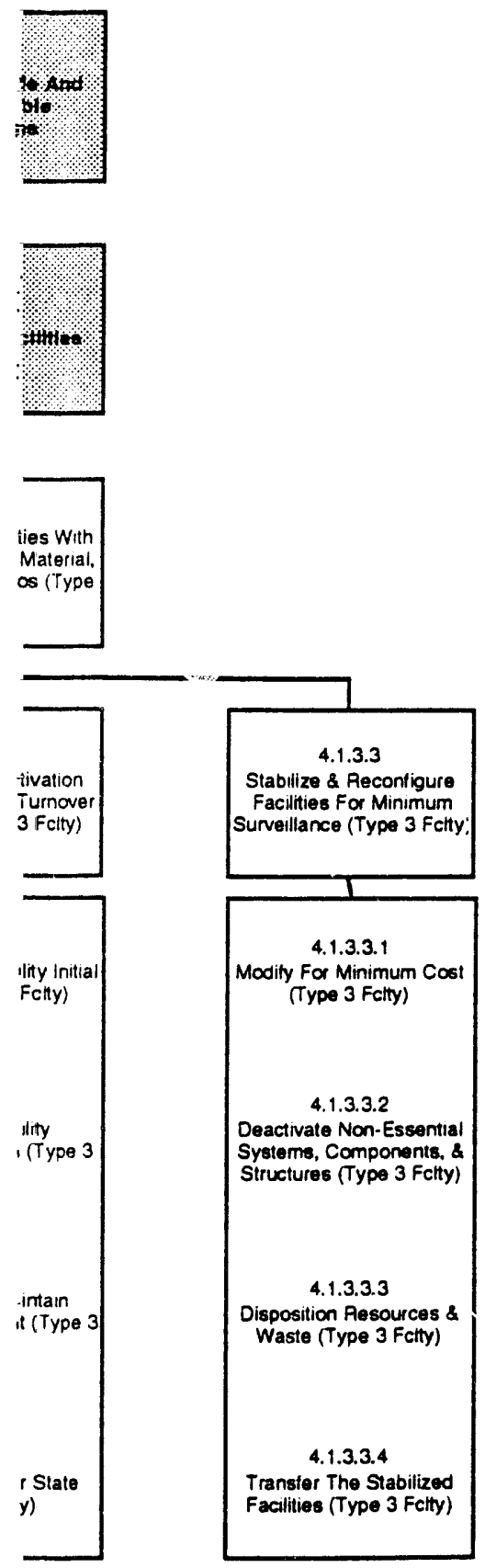


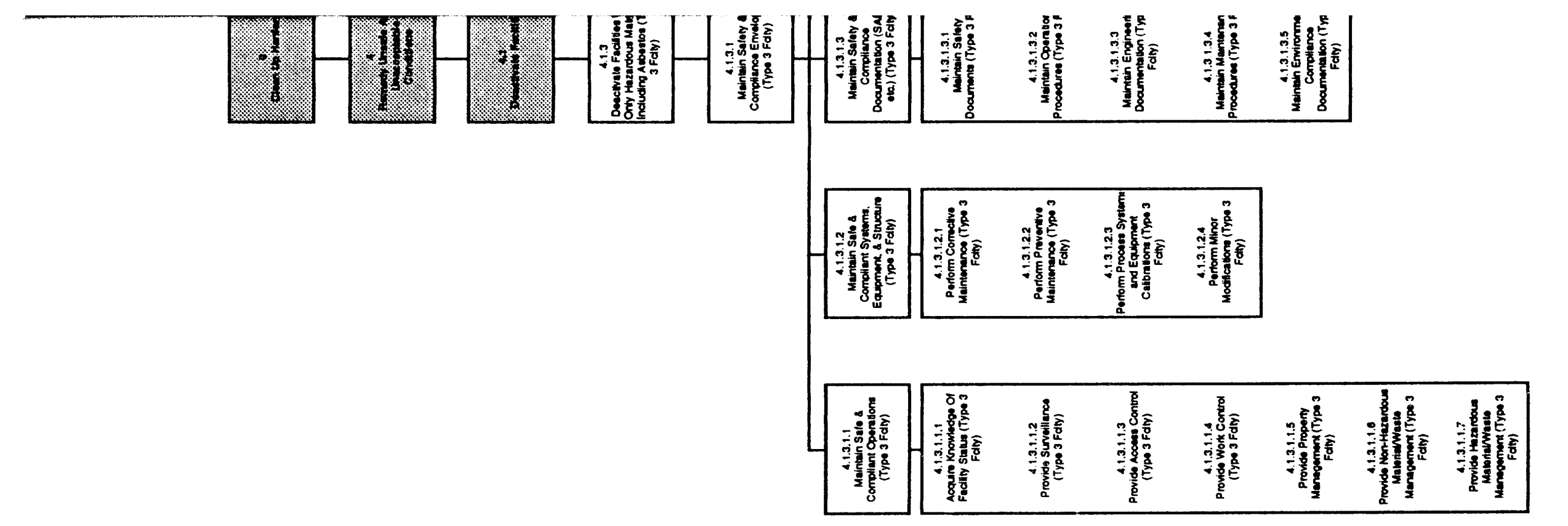




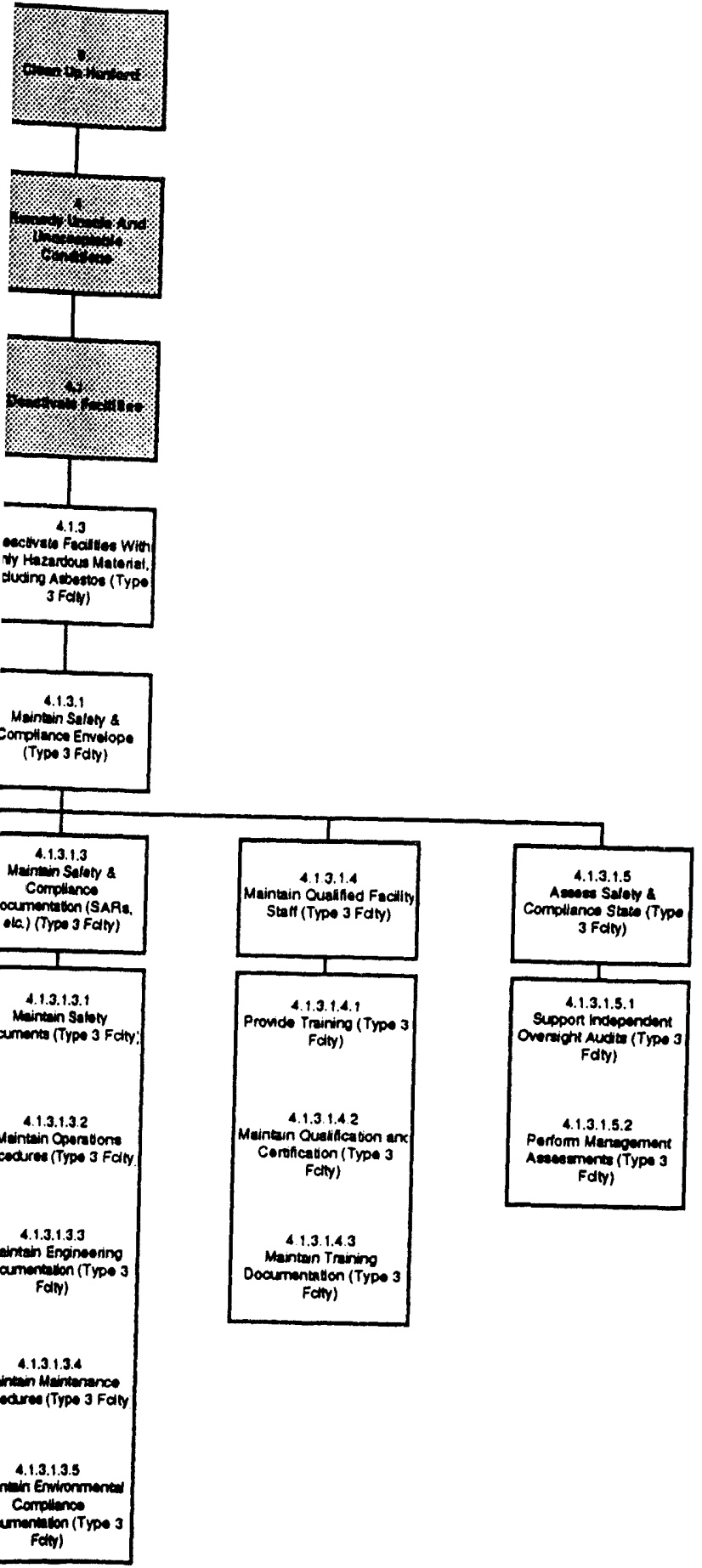




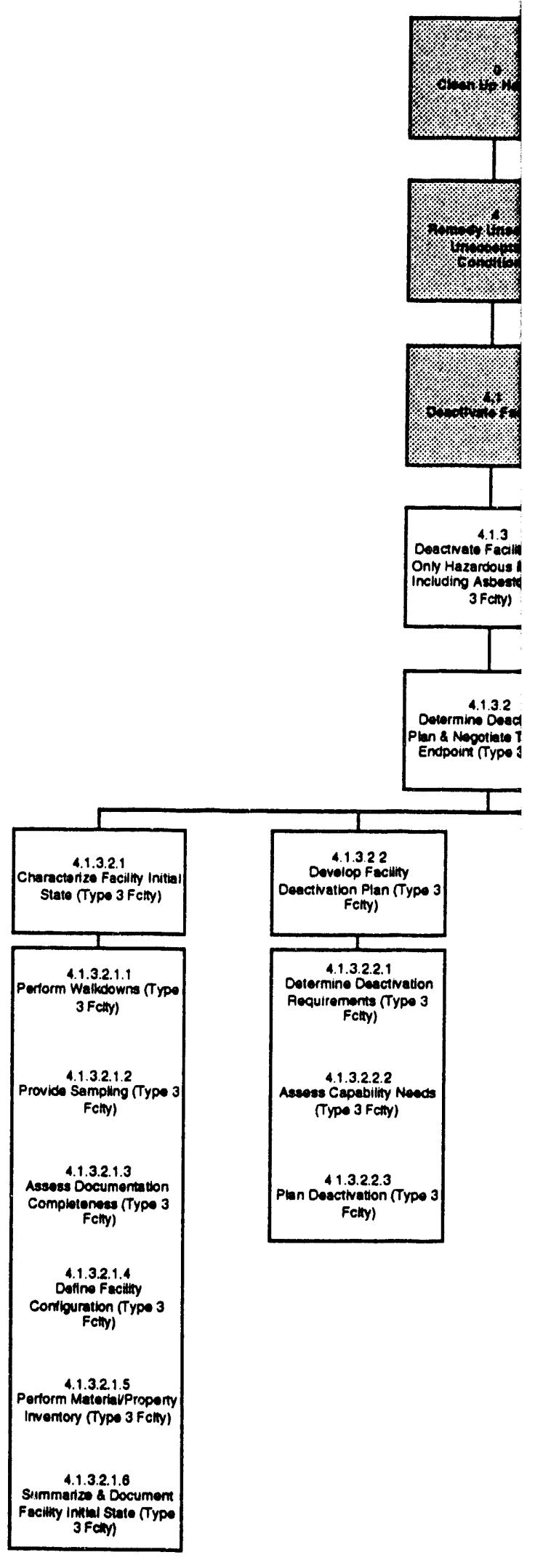




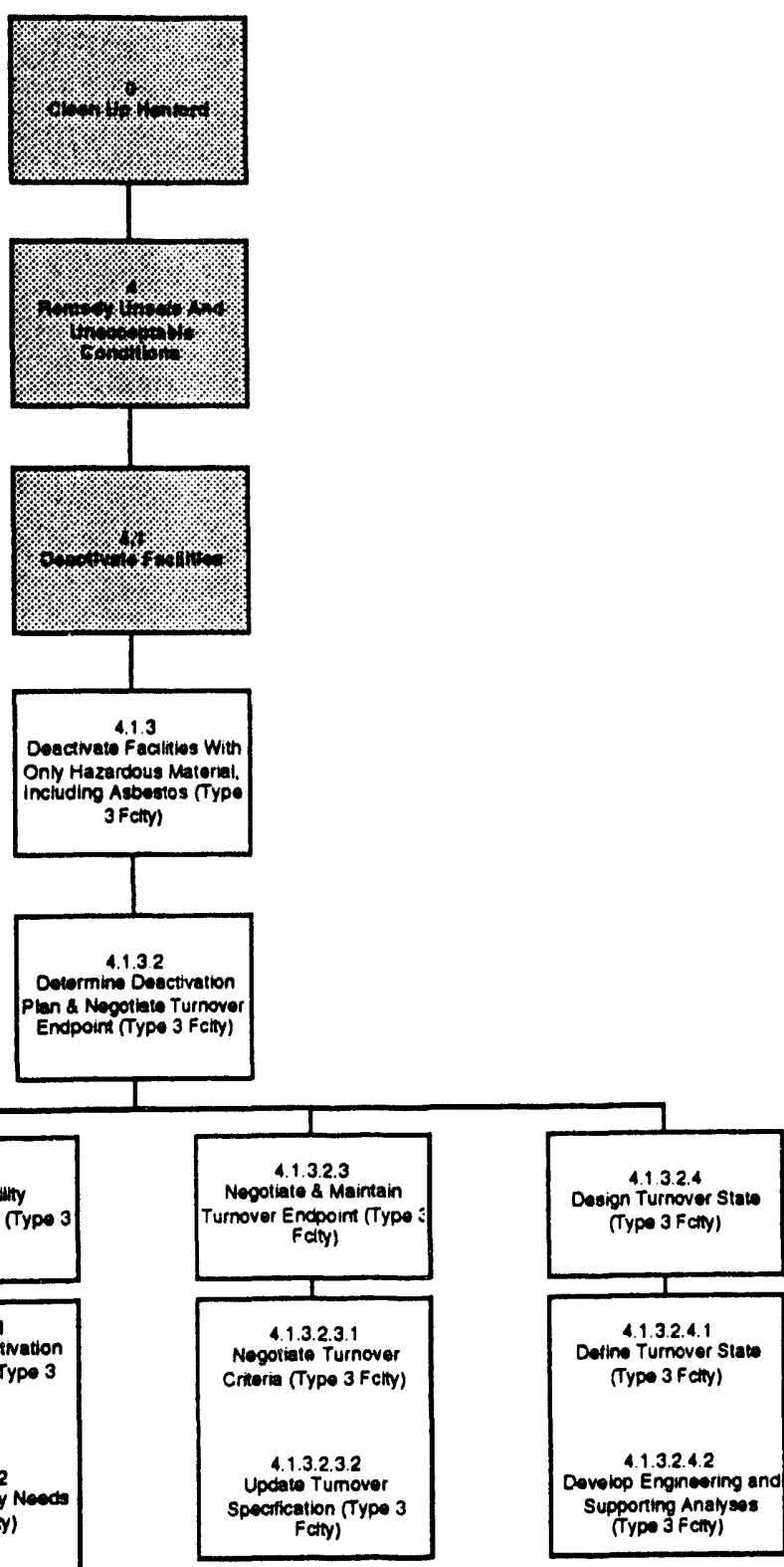




\section{Function Hierarchy}

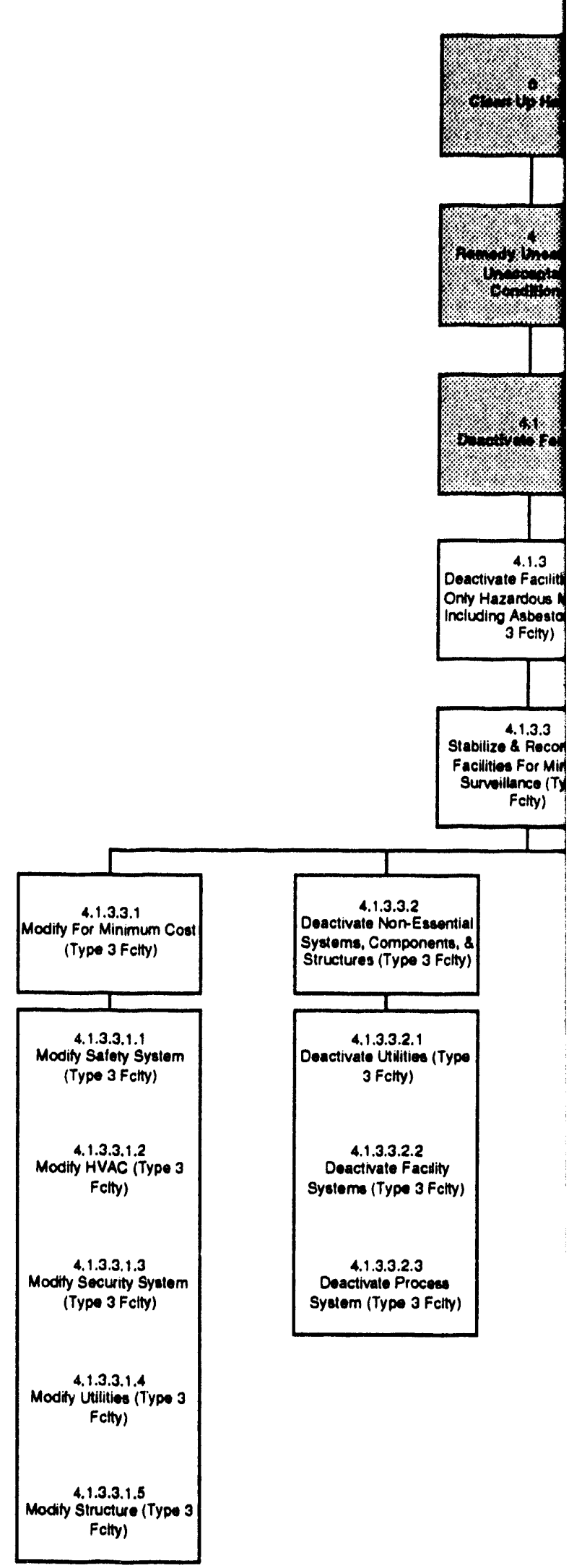



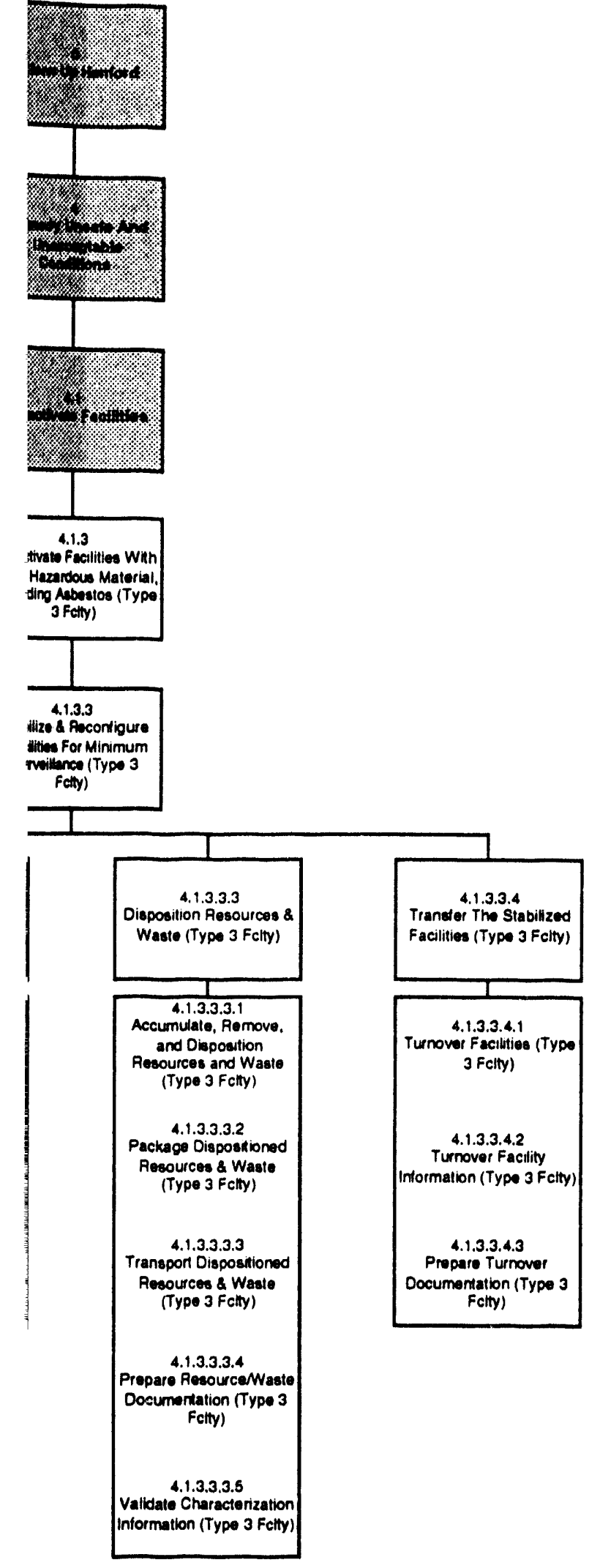


\section{Function Hierarchy}

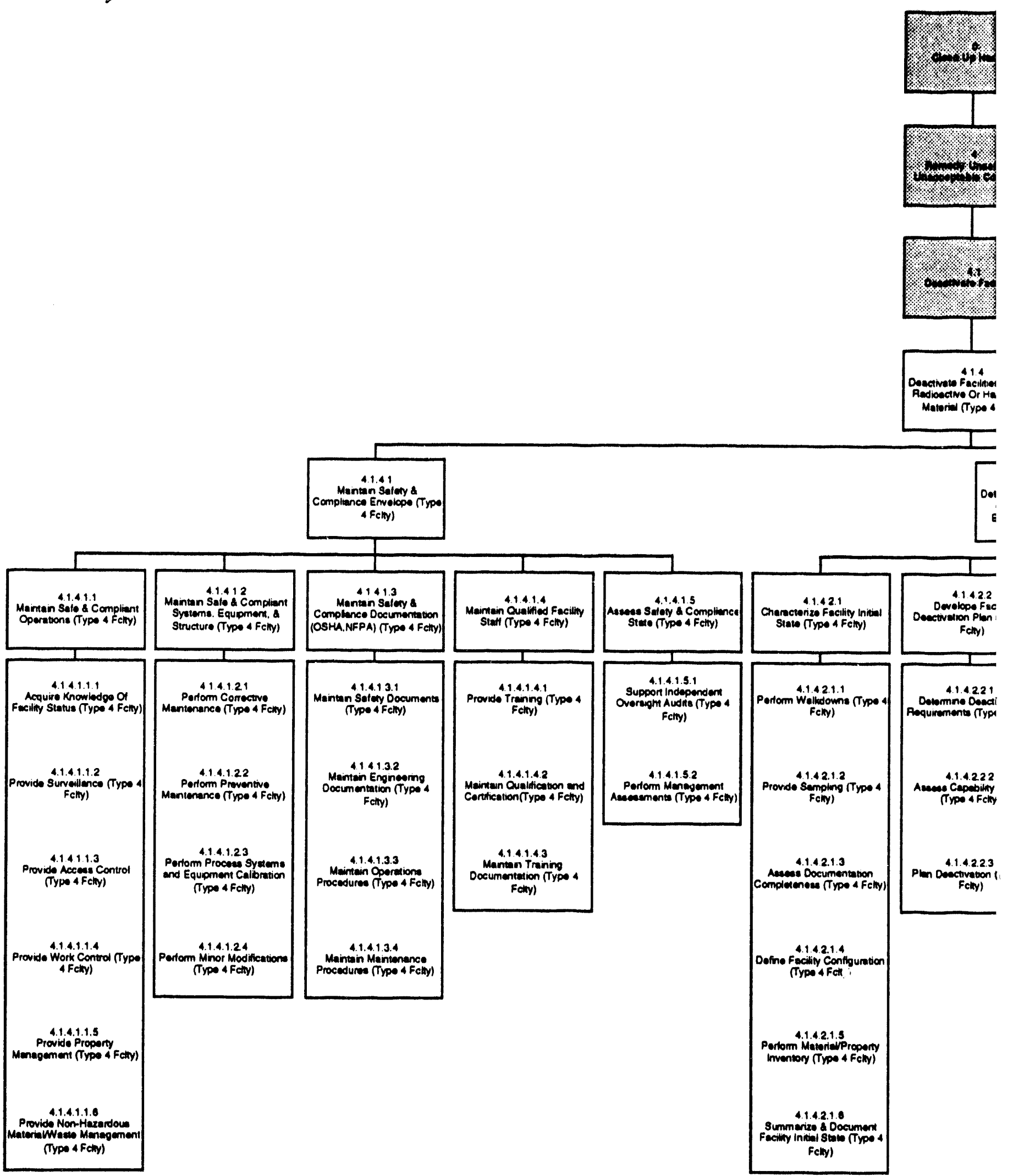

Hanford Site Systems Engineering 


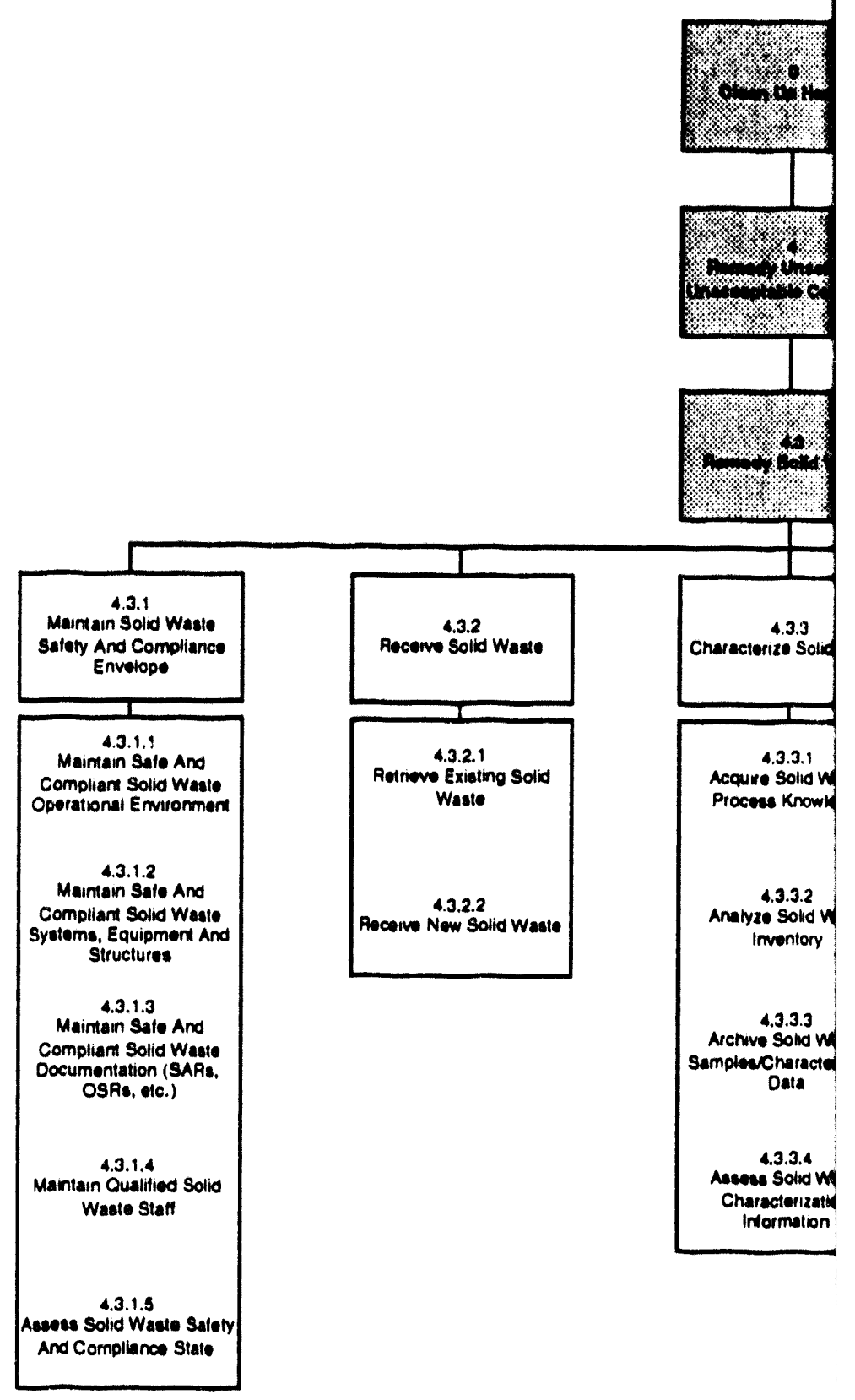




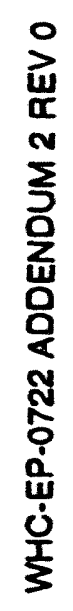

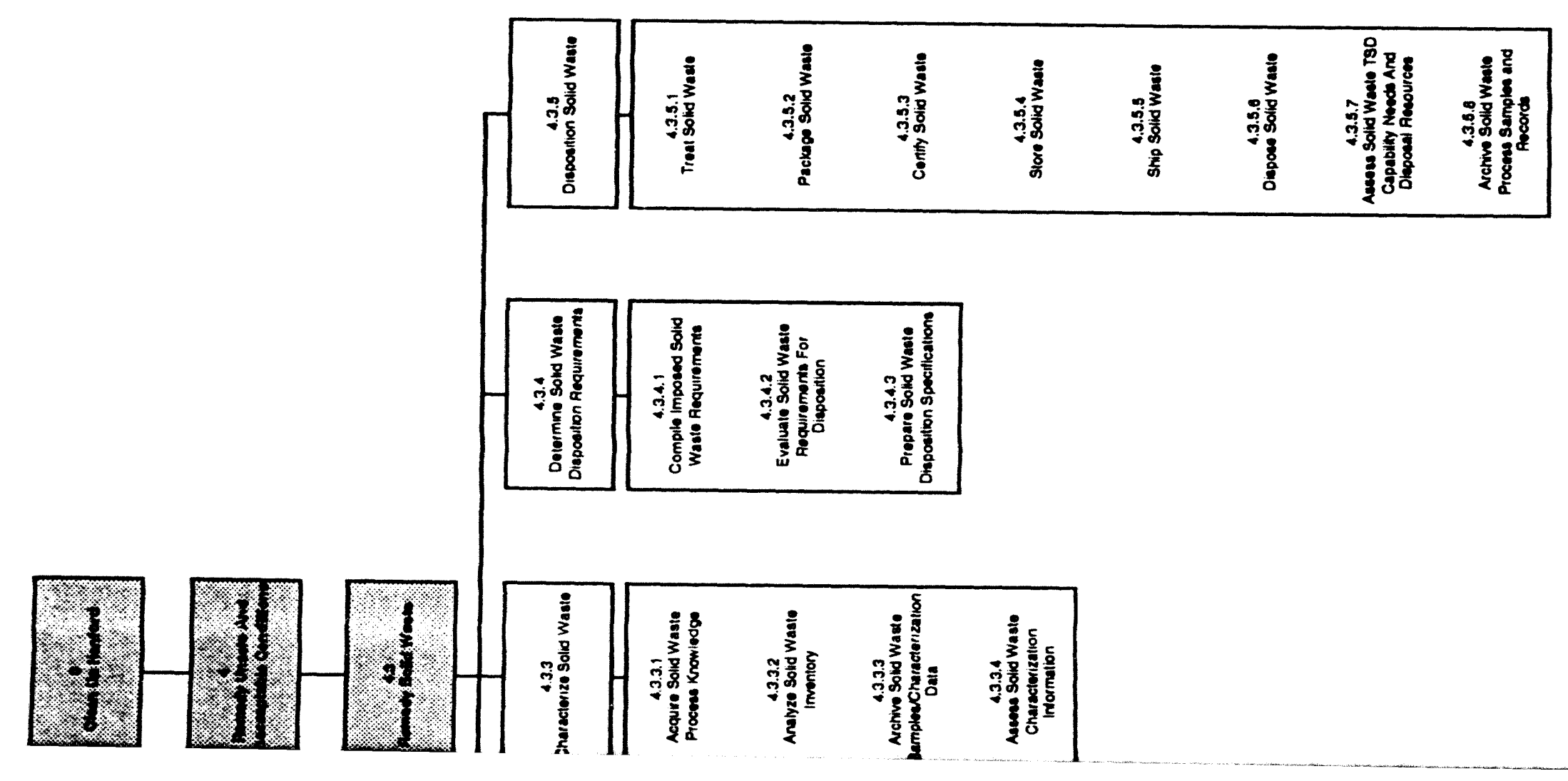




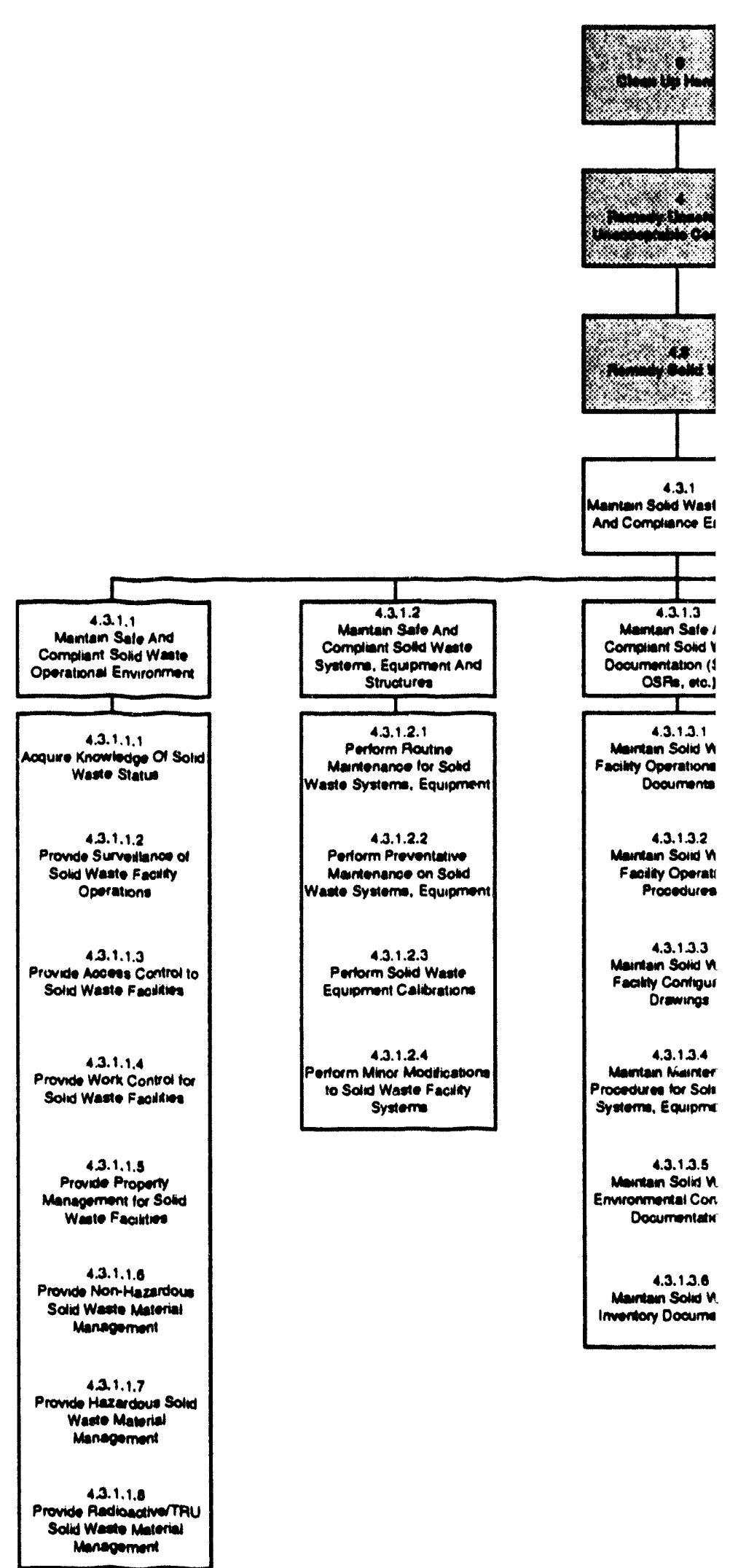


WHC-EP-0722 ADDENDUM 2 REV 0

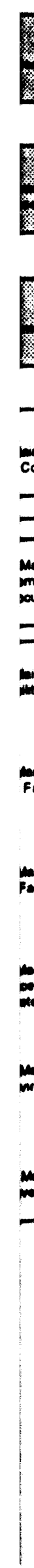

Con Soldwean Seloty

Complienes Envelopo

\begin{tabular}{|c|c|c|}
\hline 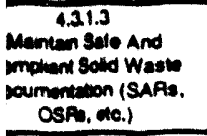 & $\begin{array}{l}\text { Manten Qualitiod Solvd } \\
\text { Warte Staft }\end{array}$ & $\begin{array}{l}4.3 .1 .5 \\
\text { Aeseces Solid Waste Satory } \\
\text { And Compliance State }\end{array}$ \\
\hline 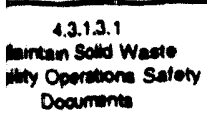 & $\begin{array}{l}\text { 4.3.1.4.1 } \\
\text { Provide Traning of Sold } \\
\text { Weate Feclity Staff }\end{array}$ & $\begin{array}{l}\text { 4.3.1.3.1 } \\
\text { Support Independent } \\
\text { Overeigh Solid Waste } \\
\text { Audts }\end{array}$ \\
\hline 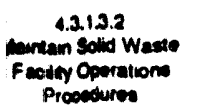 & $\begin{array}{l}\text { 4.3.1.4.2 } \\
\text { Mantain Qualdication of } \\
\text { Sold Waste Feolity Stat }\end{array}$ & $\begin{array}{c}\text { 4.3.1.5.2 } \\
\text { Portorm Solid Waste } \\
\text { Management Aseseasmenta }\end{array}$ \\
\hline $\begin{array}{l}4.3 .13 .3 \\
\text { Partan Colid Waste } \\
\text { Orminguration }\end{array}$ & $\begin{array}{l}\text { 4.3.1.4.3 } \\
\text { Mauman Solv Weate Stan } \\
\text { Traning Oocumentabion }\end{array}$ & \\
\hline
\end{tabular}

4.3.13.4

menten Mortonenoe

podure bor Soldo Waste

tema. Equipment, and

4.3.13.5

Mumean Solid Werte

monmental Compliance

Documentation

4.3.1.3.6

mintan Solid Weate

mertion Documentation

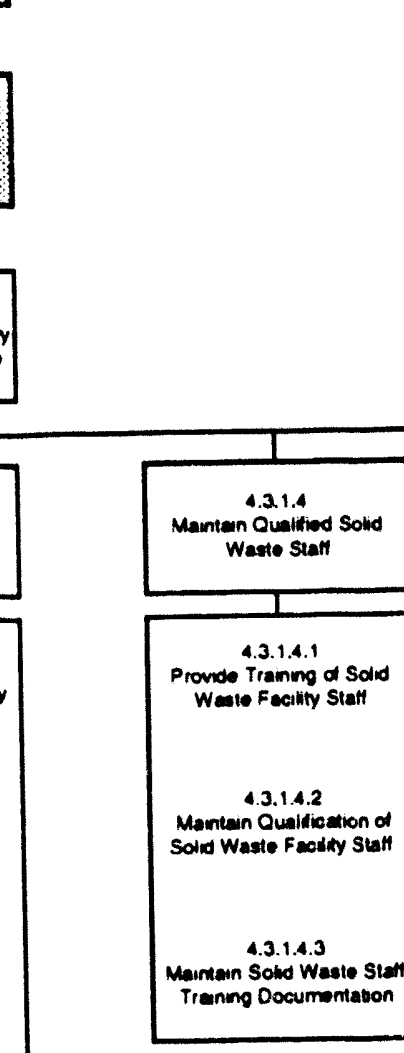




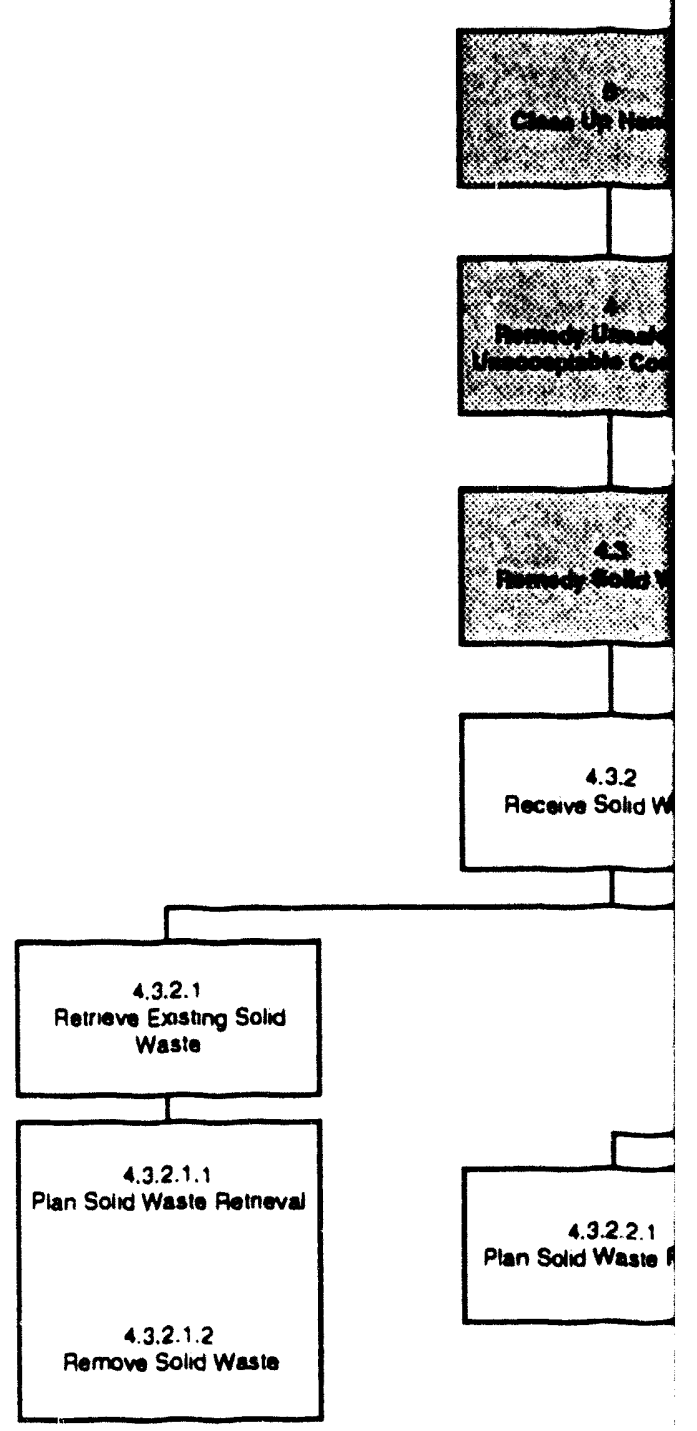




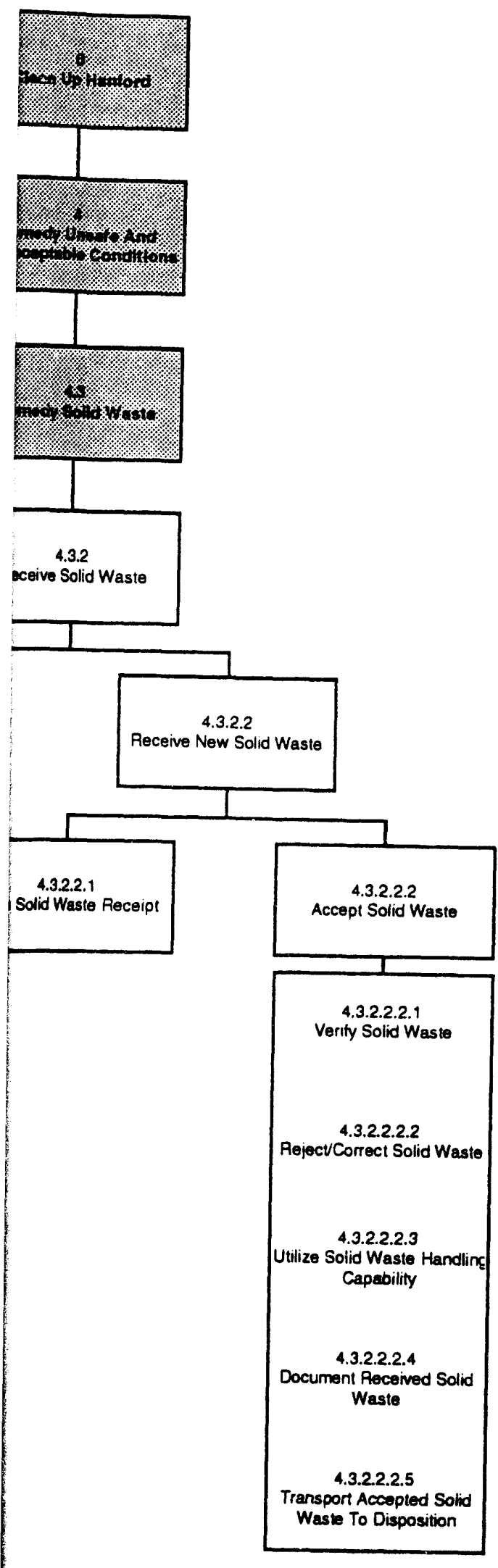




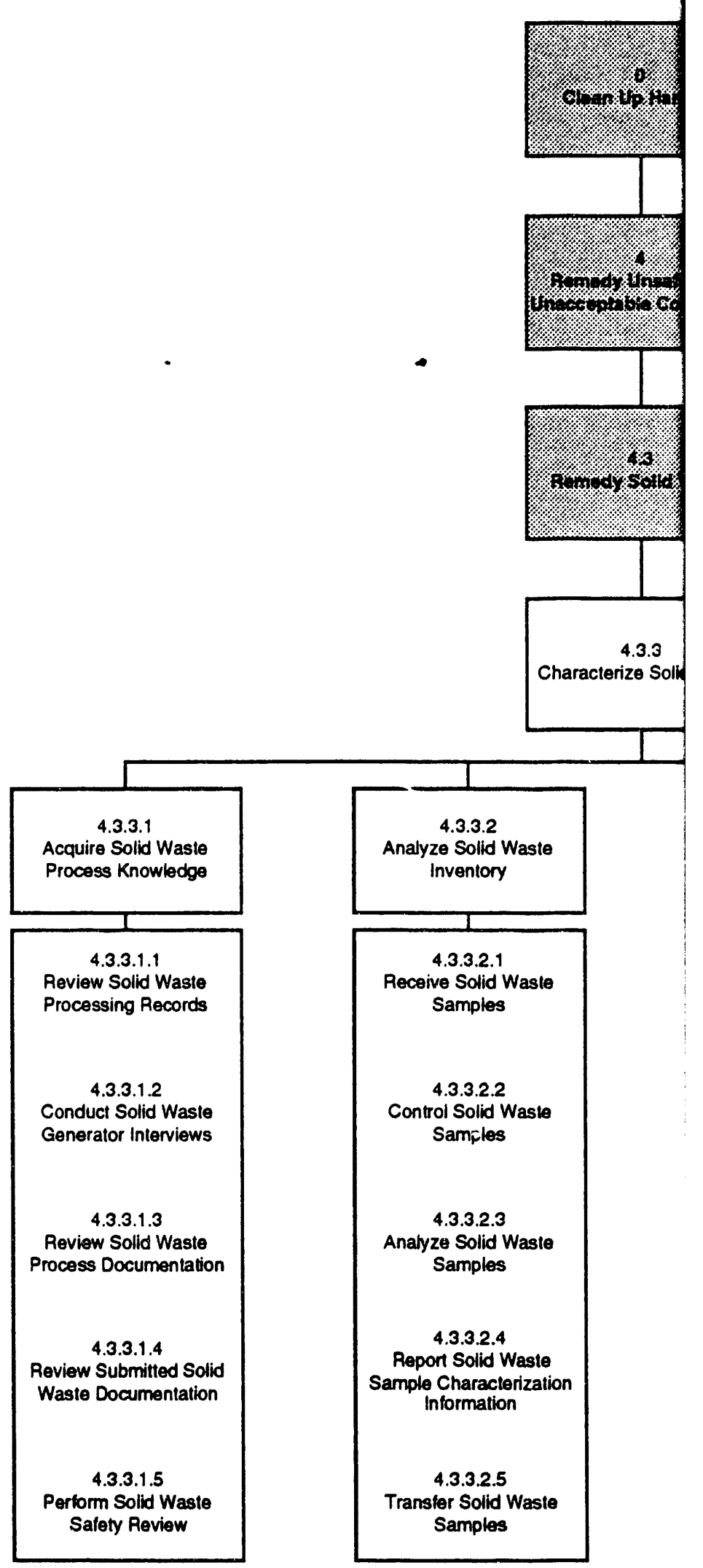




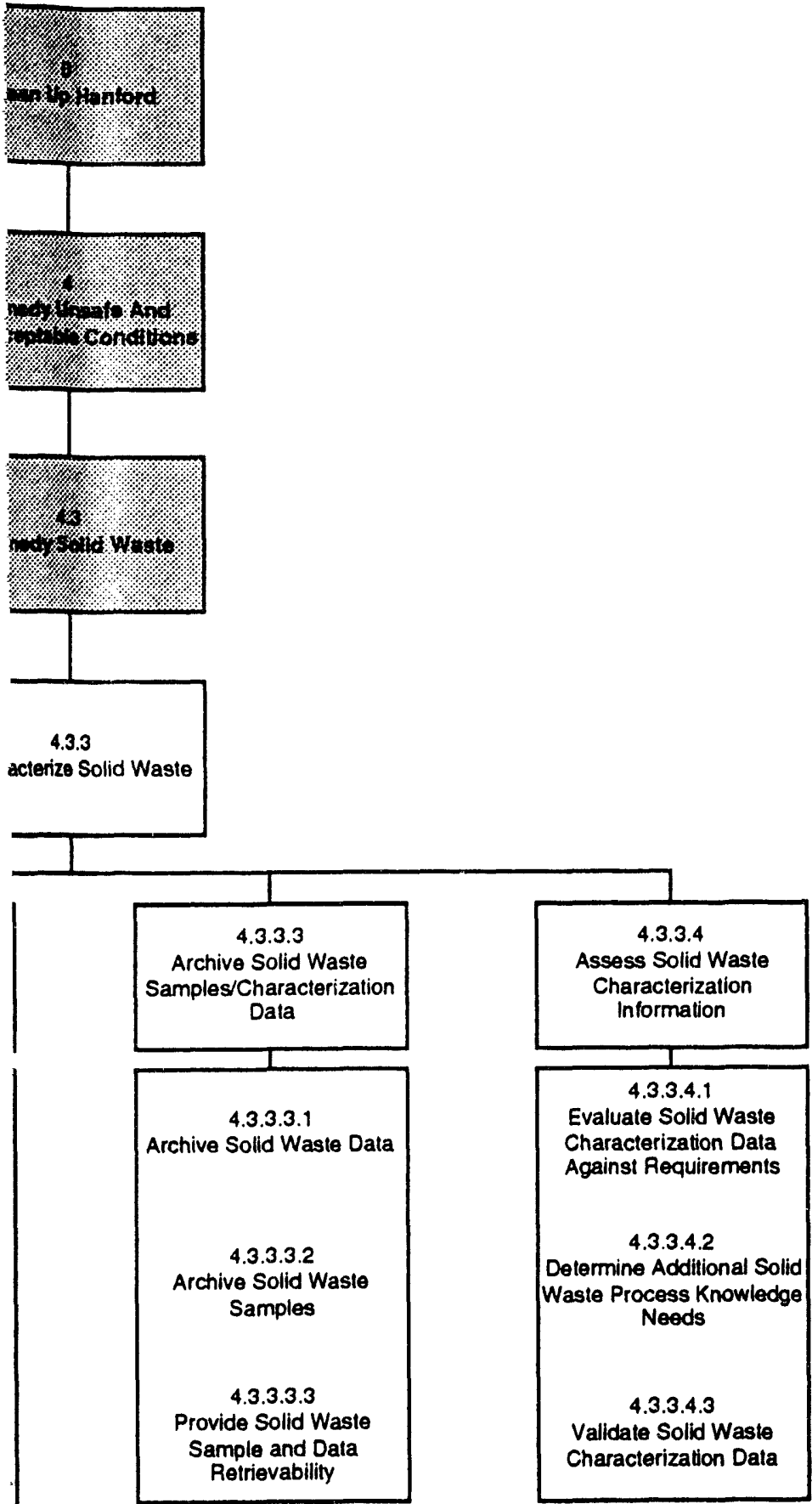




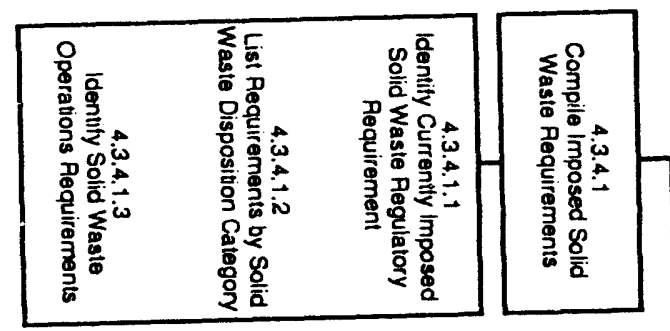




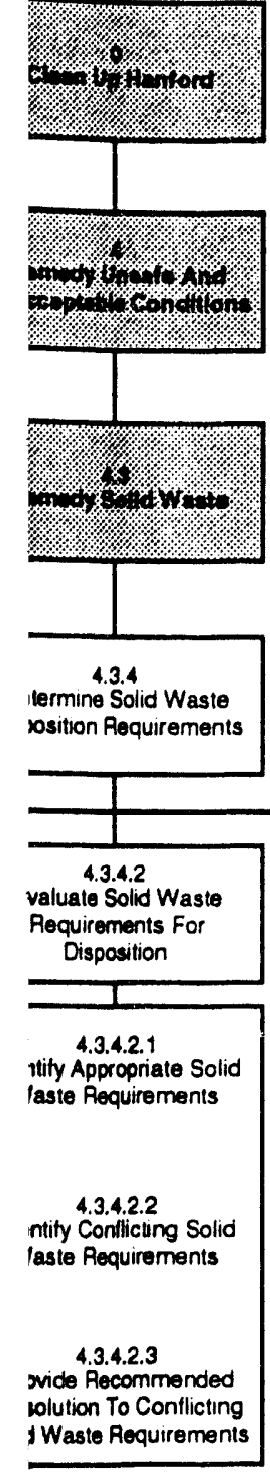

4.3.4.3

Prepare Solid Waste Disposition Specifications

4.3.4.3.1

Develop Solid Waste Specifications for Treatment

4.3.4.3.2

Develop Solid Waste

Specifications for Packaging

$$
\text { 4.3.4.3.3 }
$$

Develop Solid Waste

Specifications for

Certification

4.3.4.3.4

Develop Solid Waste

Specifications for Storage

4.3.4.3.5

Develop Solid Waste

Specifications for Shipping

4.3.4.3.6

Develop Solid Wasto

Specilications lor

Disposition

4.3.4.3.7

Develop Solid Wasto Specifications for Archiving Samples and Records 


\section{Function Hierarchy}

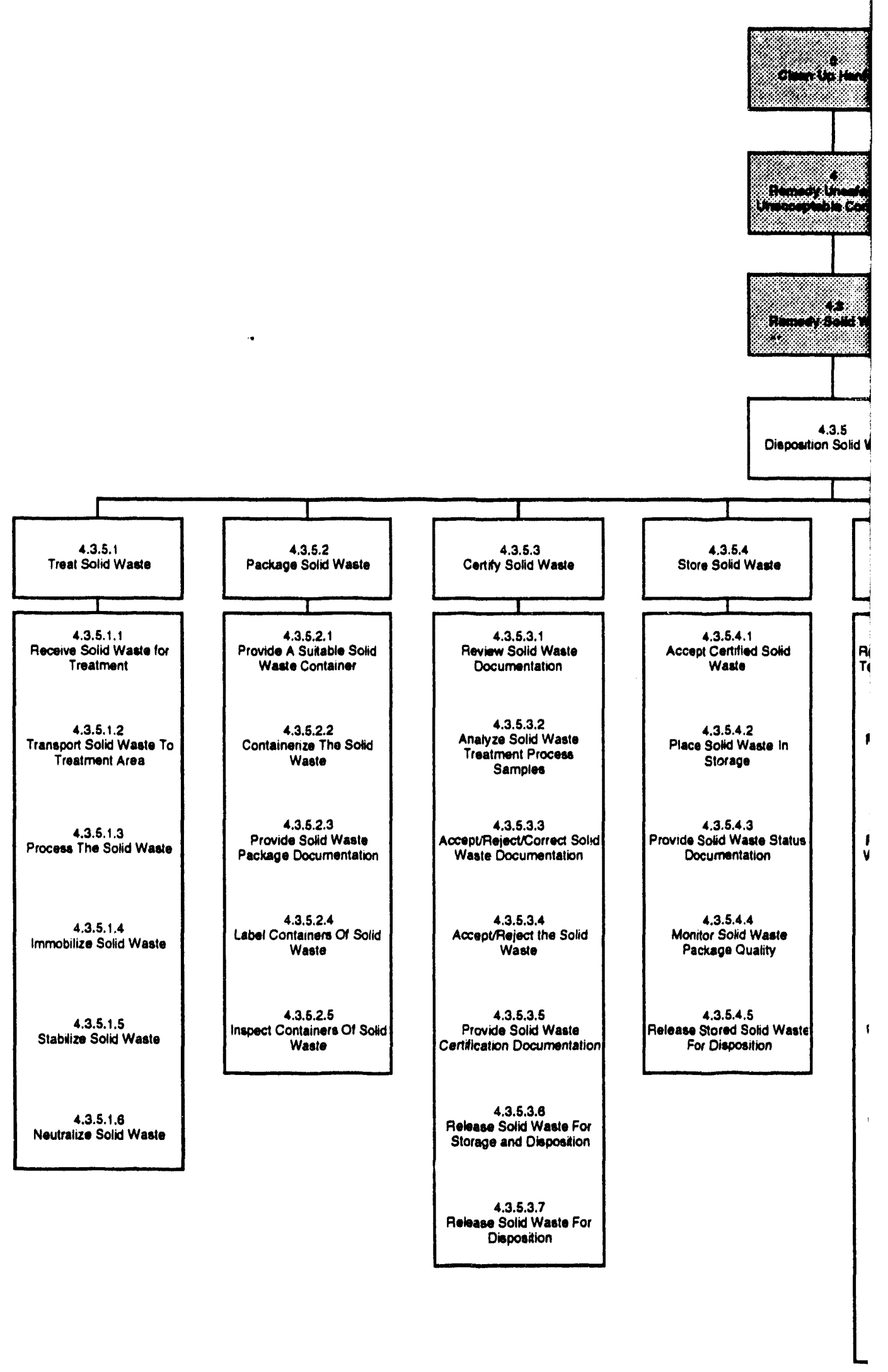




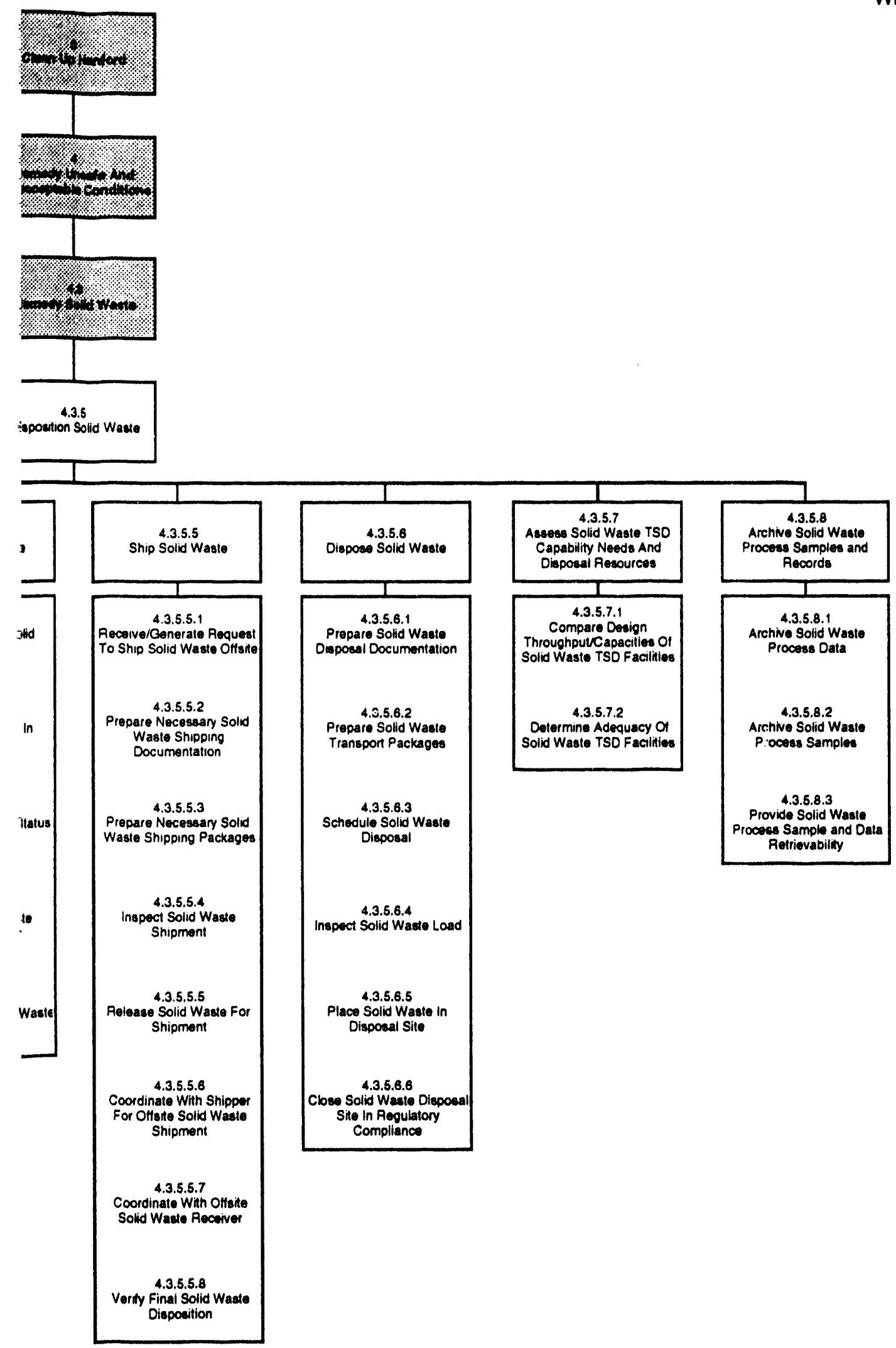




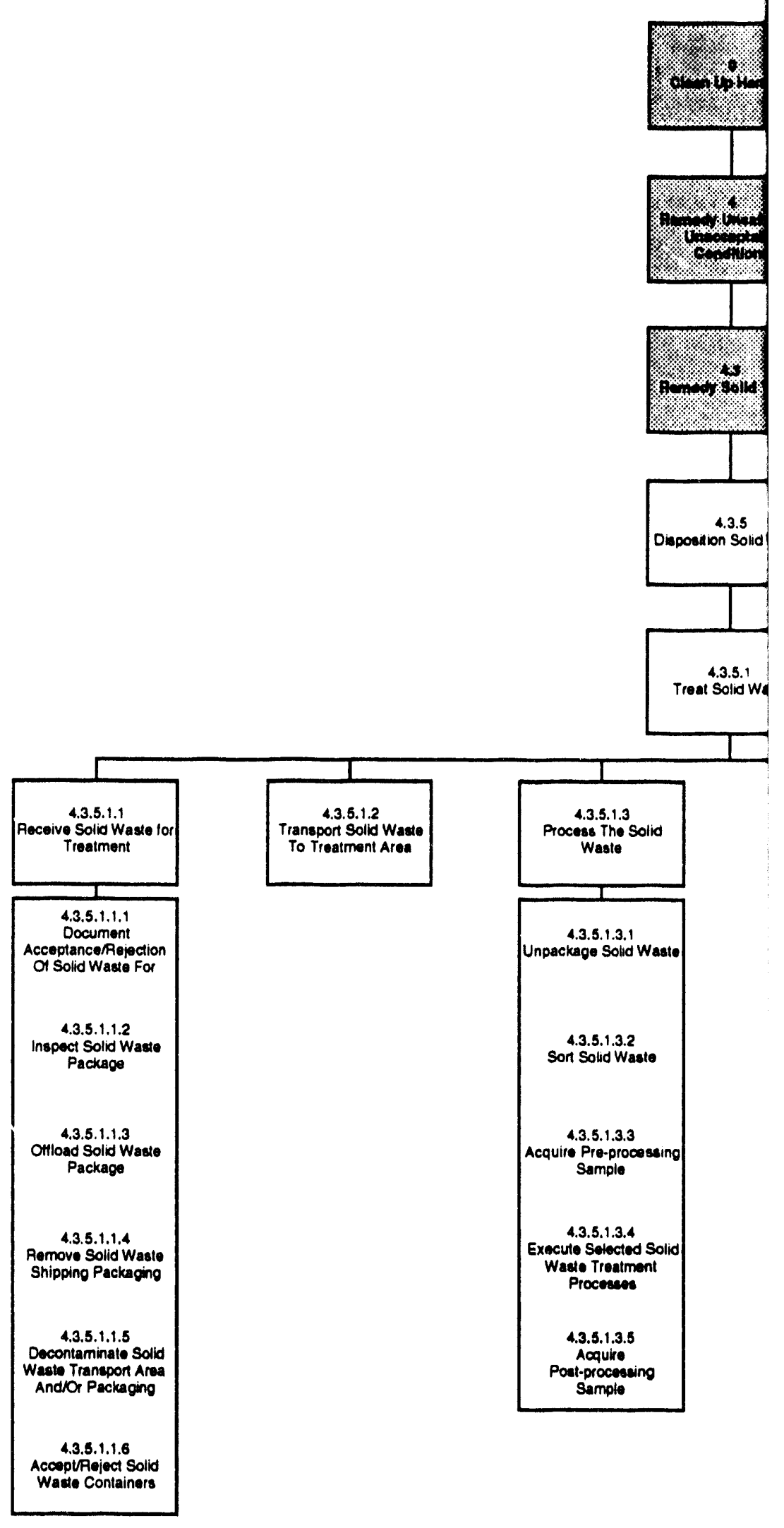




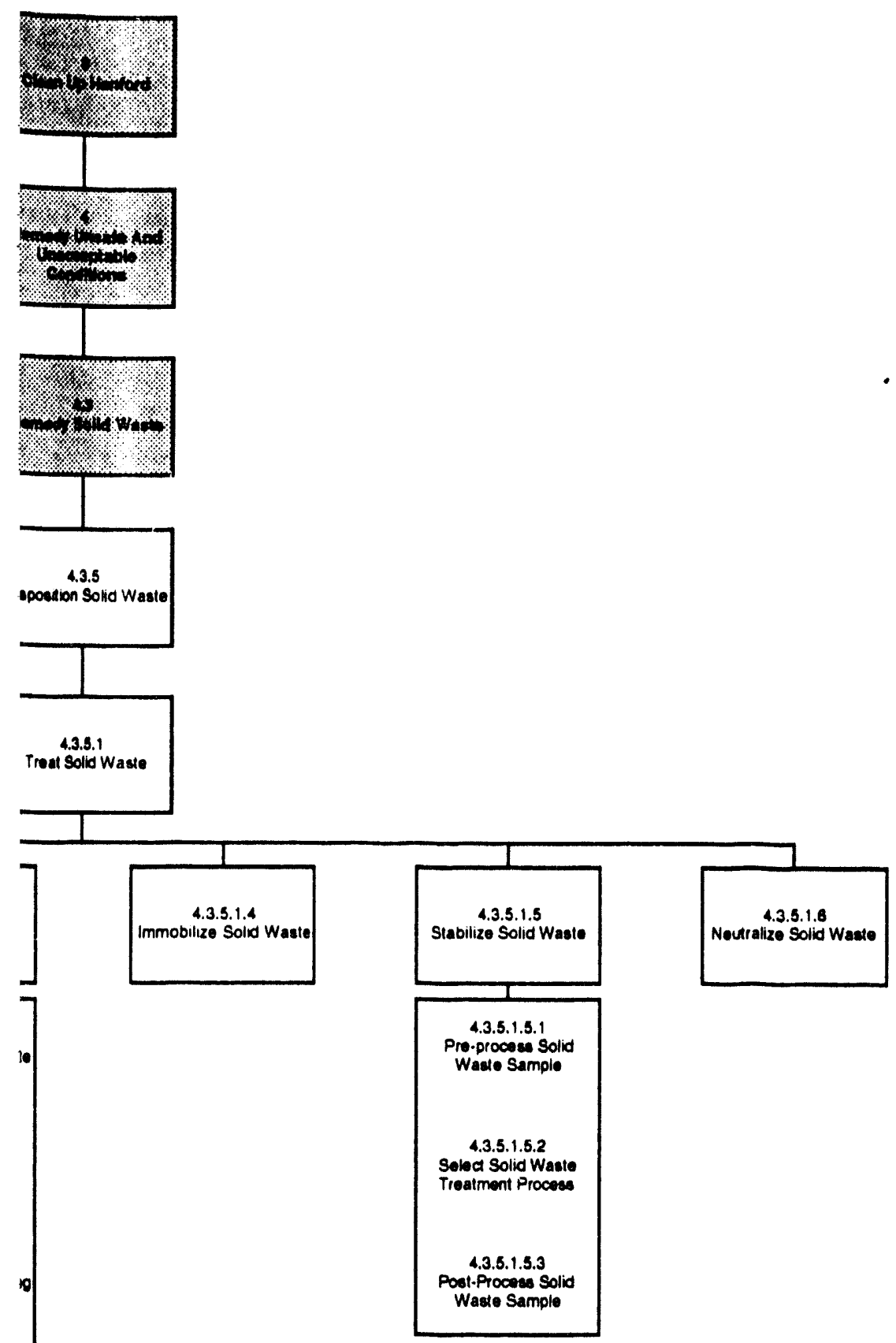


Function Hierarchy

4.3.5.2.1

Provide A Suitable Solid Waste Container

\subsection{1}

Match Container To Treated Solid Waste Type

4.3.5.2.1.2

Match Container To Solid

Waste Acceptance Criteria

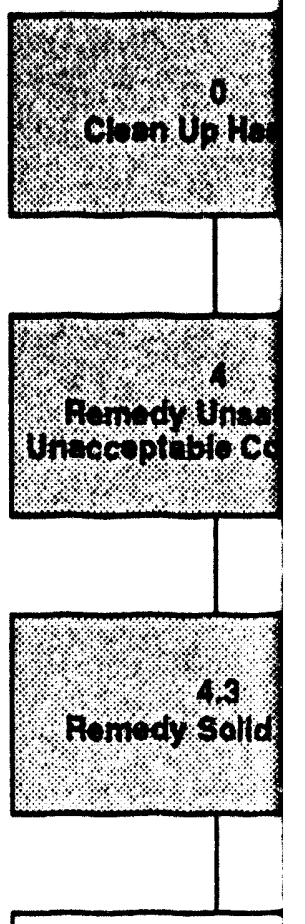

4.3.5

Disposition Solid

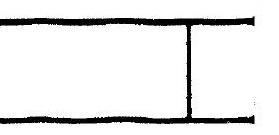

4.3.5.2

Package Solid I

4.3.5.2.2

Containerize The Solid Waste
4.3 .5 .2 .3

Provide Solid V Package Docum

4.3.5.2.3.

Provide Packı

Traceability To li Solid Waste Fer

4.3.5.2.3.:

Develop Solid '

Packaging Certil 


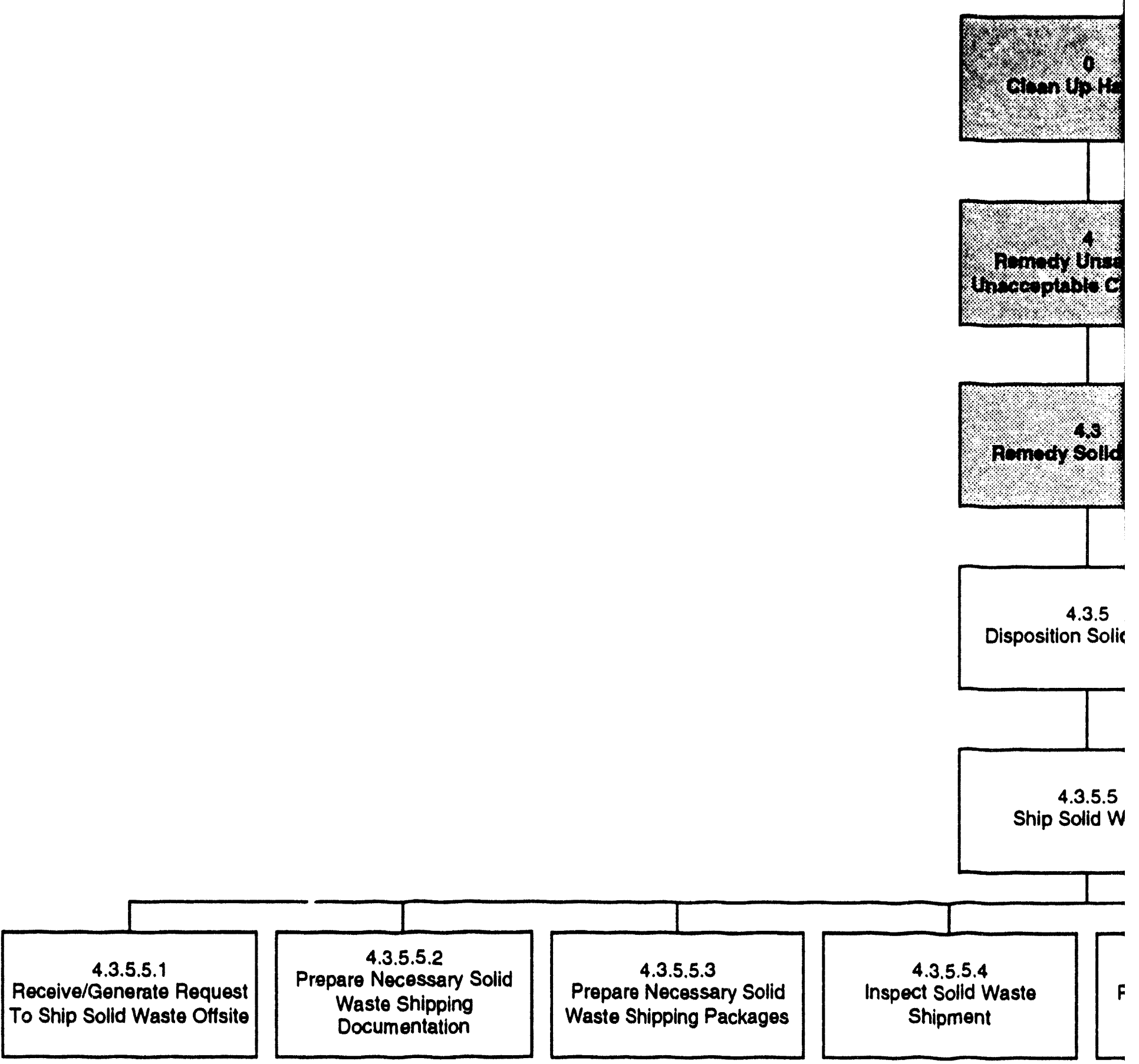



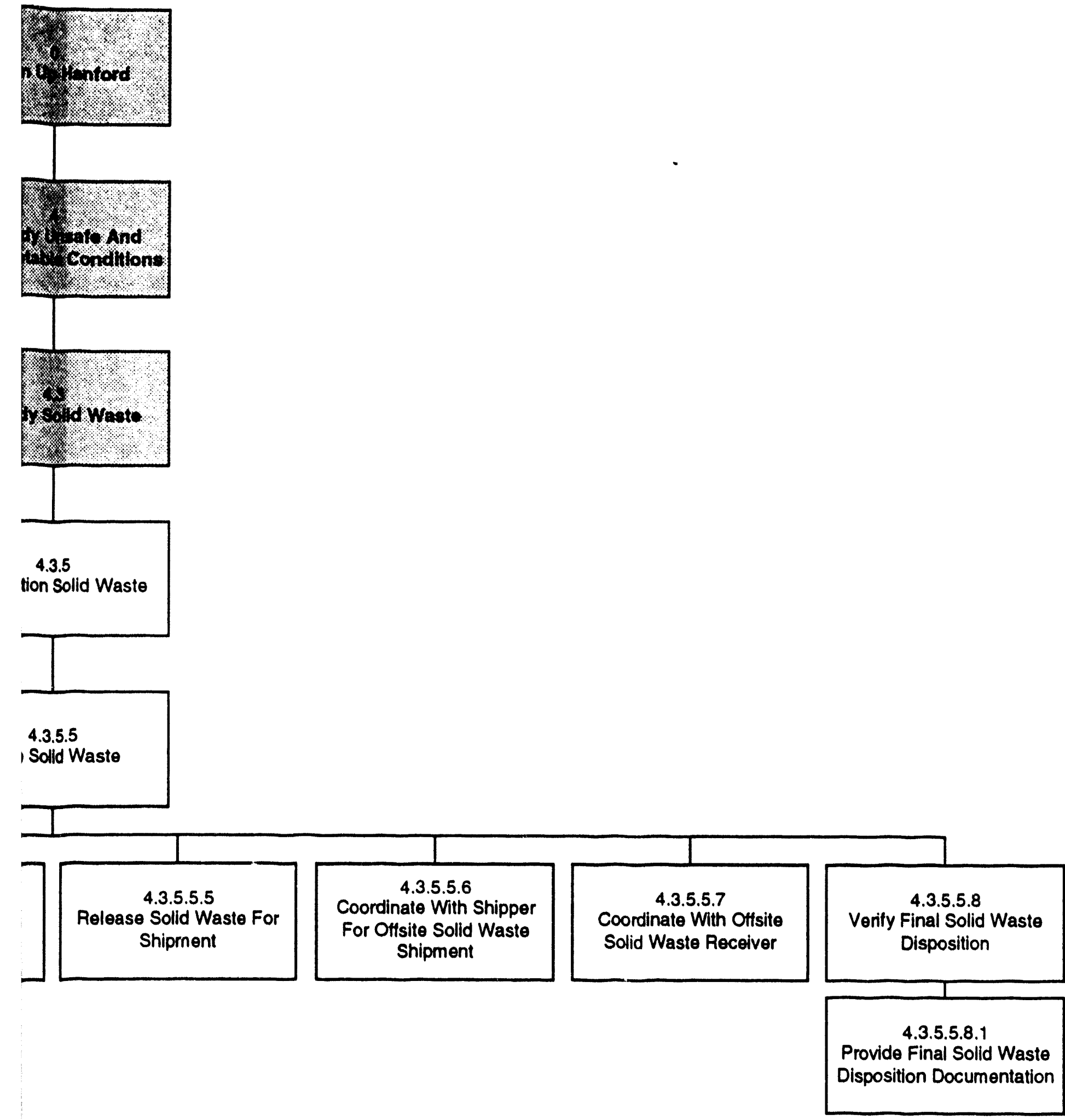


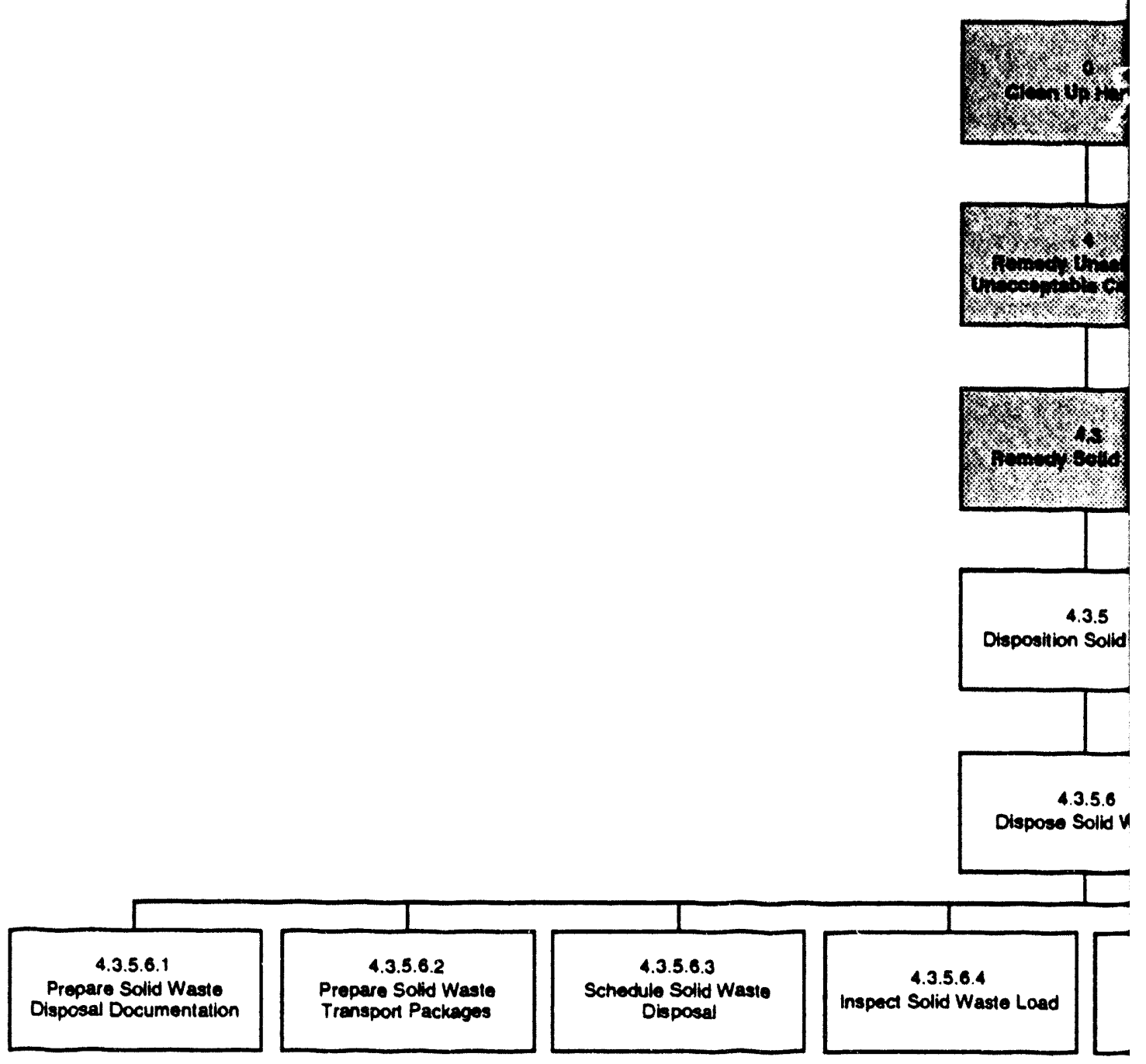



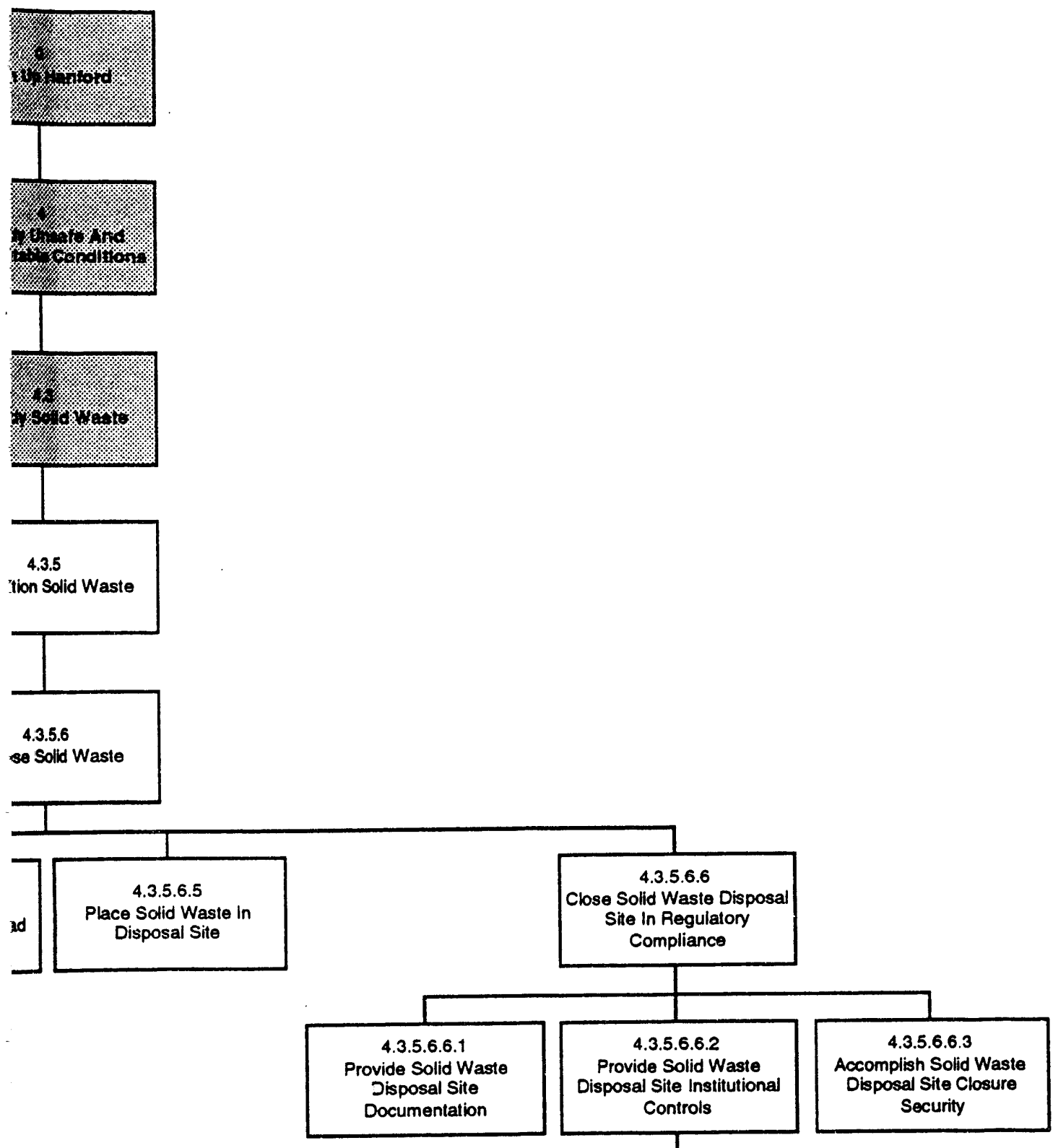

4.5.6.6.2.1

Provide Solid Waste

Disposal Site Fencing

4.3.5.6.6.2.2

Provide Solid Waste

Disposal Site Monitoring

4.3.5.6.6.2.3

Provide Solid Waste

Disposal Site Security 


\section{Function Hierarchy}

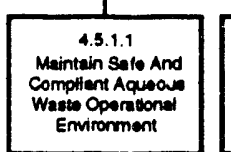

Enviomentent

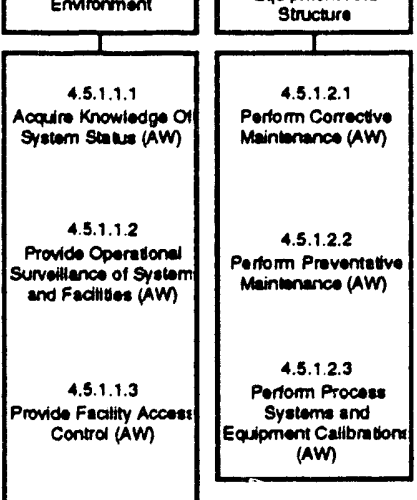

4.5.1.1.4

Provido Work Control

(AW)

4.5.1.1.5

Provide Property

Management (AW)

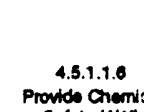

Provido Chemisa

4.5.1.1.7

Provide Mecharica

Saloty (AW)

4.5.1.1.8

rovido Radiation

Seloy (AW)

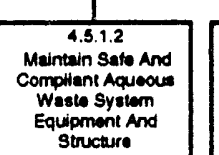

10

$\%$

Meintain Aqueous

Complianco Envolopo

Compliancos Envelope

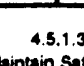

4.5.1.3

Maintain Sate And Compliant Aquous: Wall Documentation

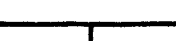

\section{Aquous Wasto Stat}

Manthin Qualifed
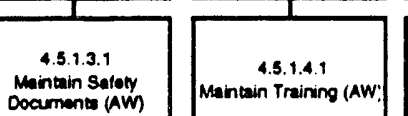
Compliance State

Documentu (AW)

Maintain Training (AW:
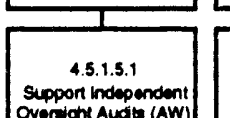

support independent:
Oversight Auditu (AW)

4.5.1.3.2

(a)

Documents (AW)

4.5.1.4.2

Maintain Qualitication

and Contification (AW)

4.5.1.3.2

Asecesmon (AW

4.5.1.3.3

Maintain Maintonenco
Procodures (AW)

4.5.1.5.3

Actoon Plan (AW)

Maintain Complience

4.5.1.3.5

Maintain Inventory

(AW)

4.5.1.3.6

Maintain Enginooring

Documentabion (AW)

4.5.1.3.7

Documentation (AW)
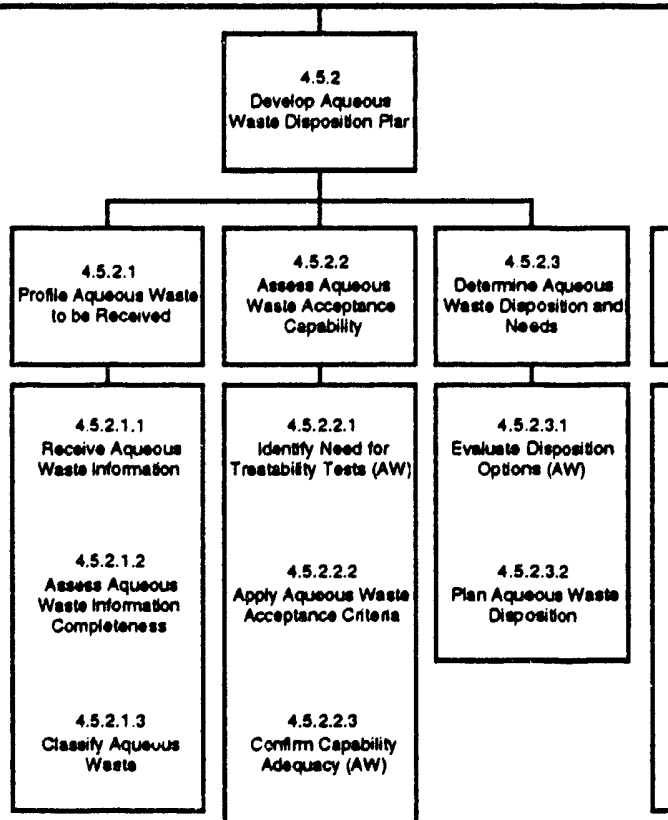

4.5.2.2.

Identily Noed for
Treatabily Toets (AW

4.5.2.2.2 Appty Aquoous Waste

4.5.2.2.3

A.5.2.2.3 Adequancy (AW)

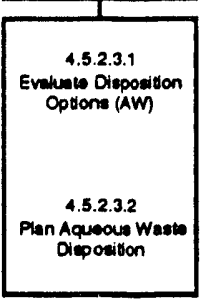

4.5.2.2.4 donotity Need tor Aoditional informaton (AW)

Hanford Site Systems Engineering 

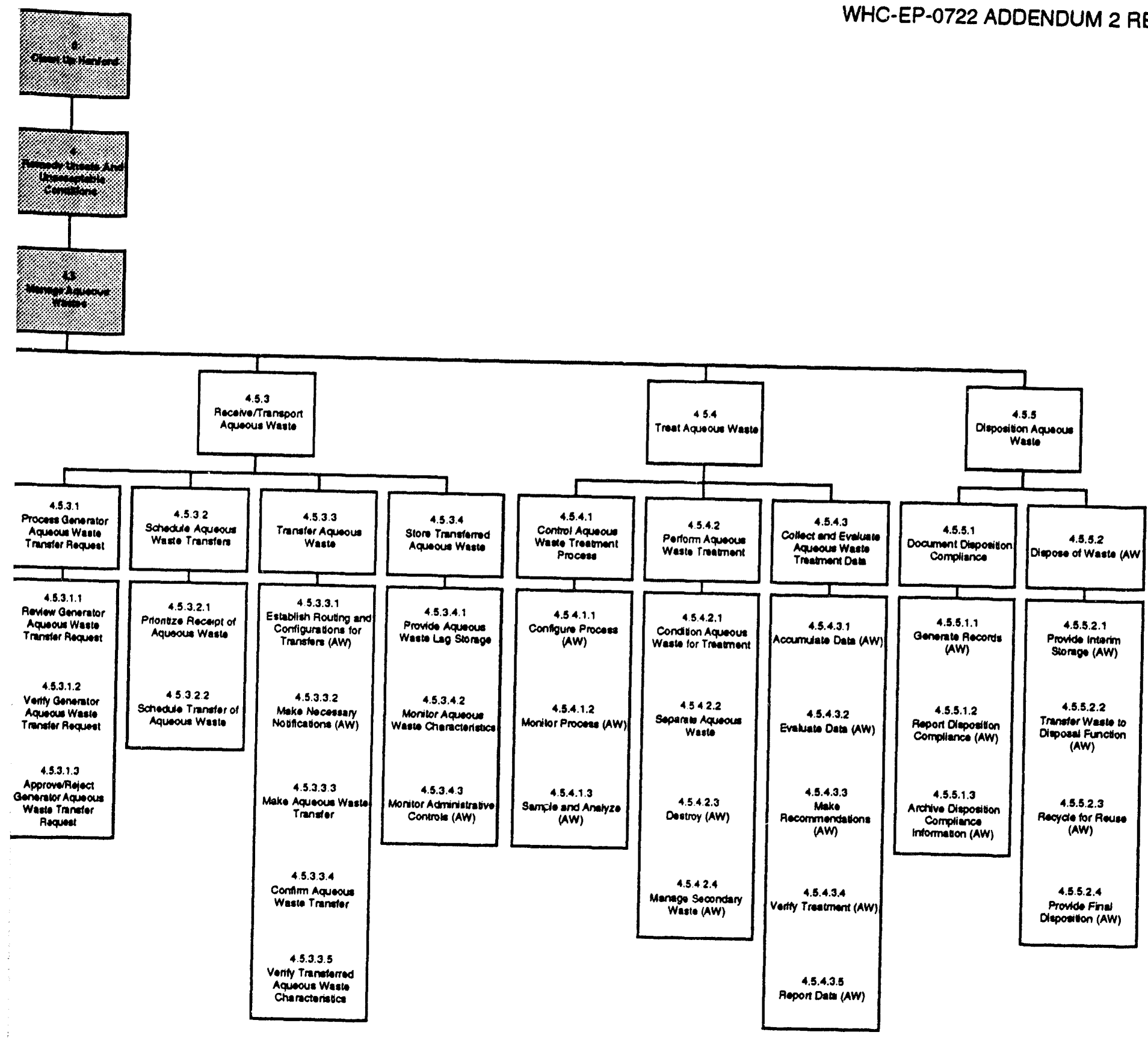


\section{Function Hierarchy}

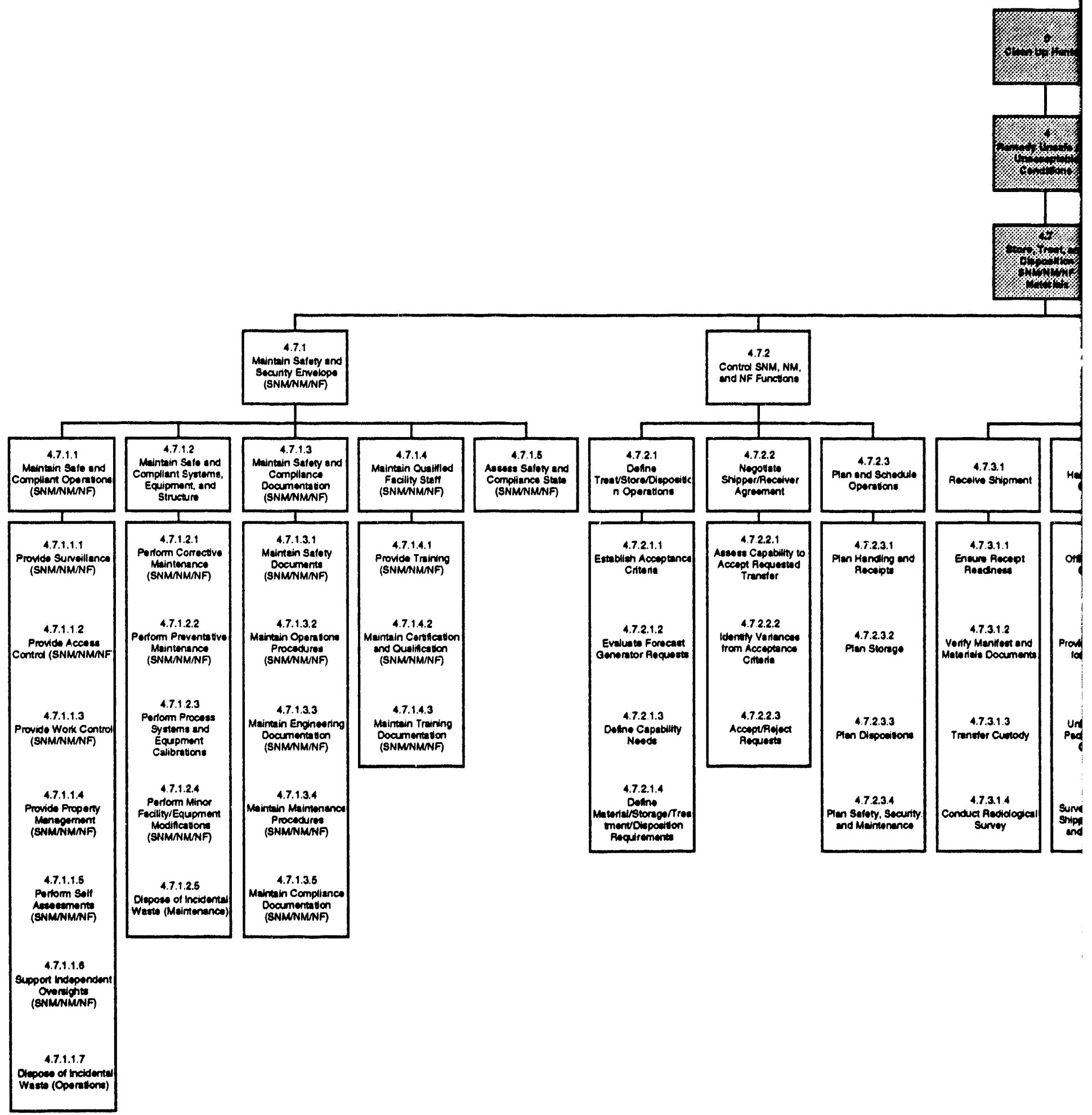

Hanford Site Systems Engineering 


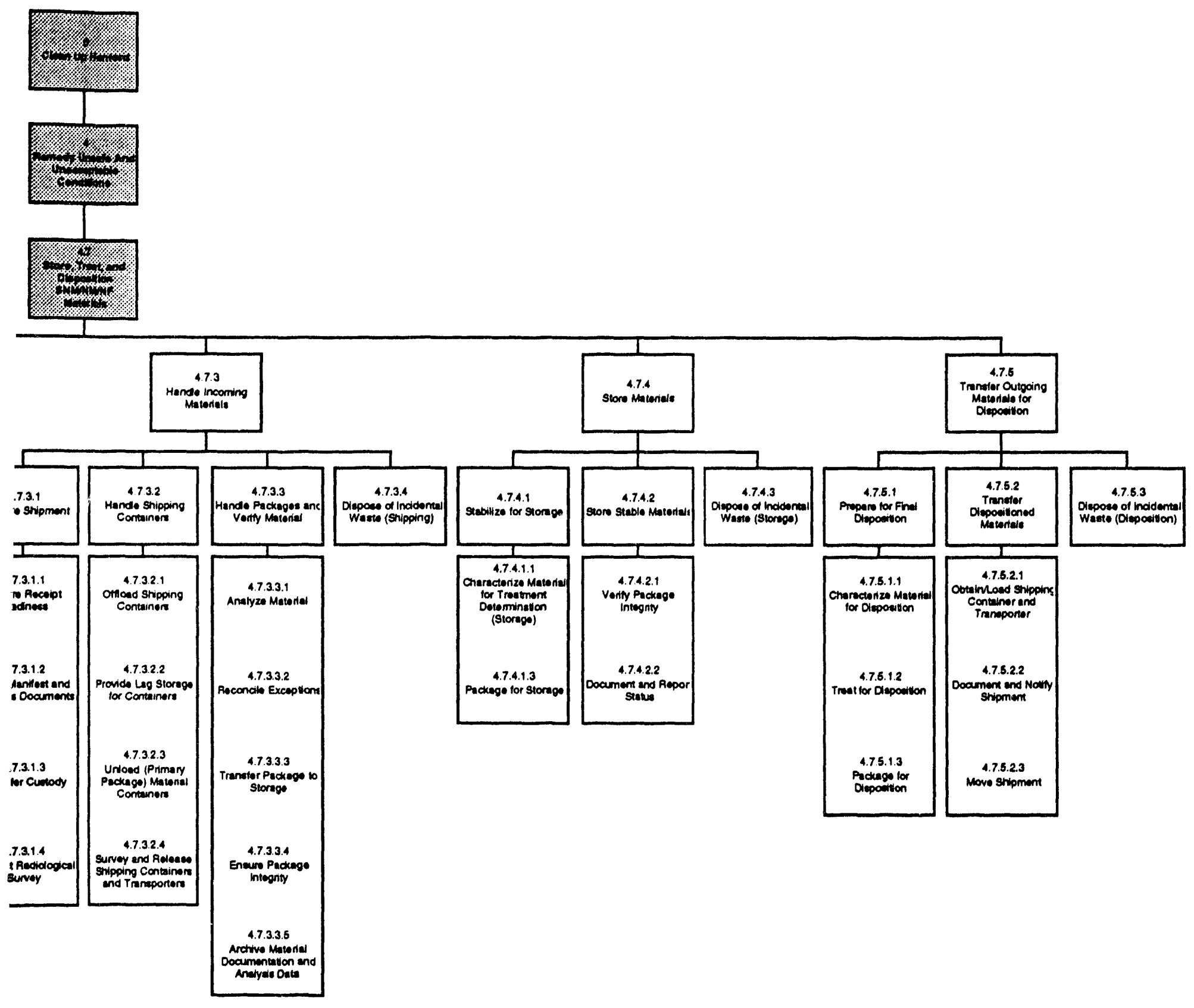




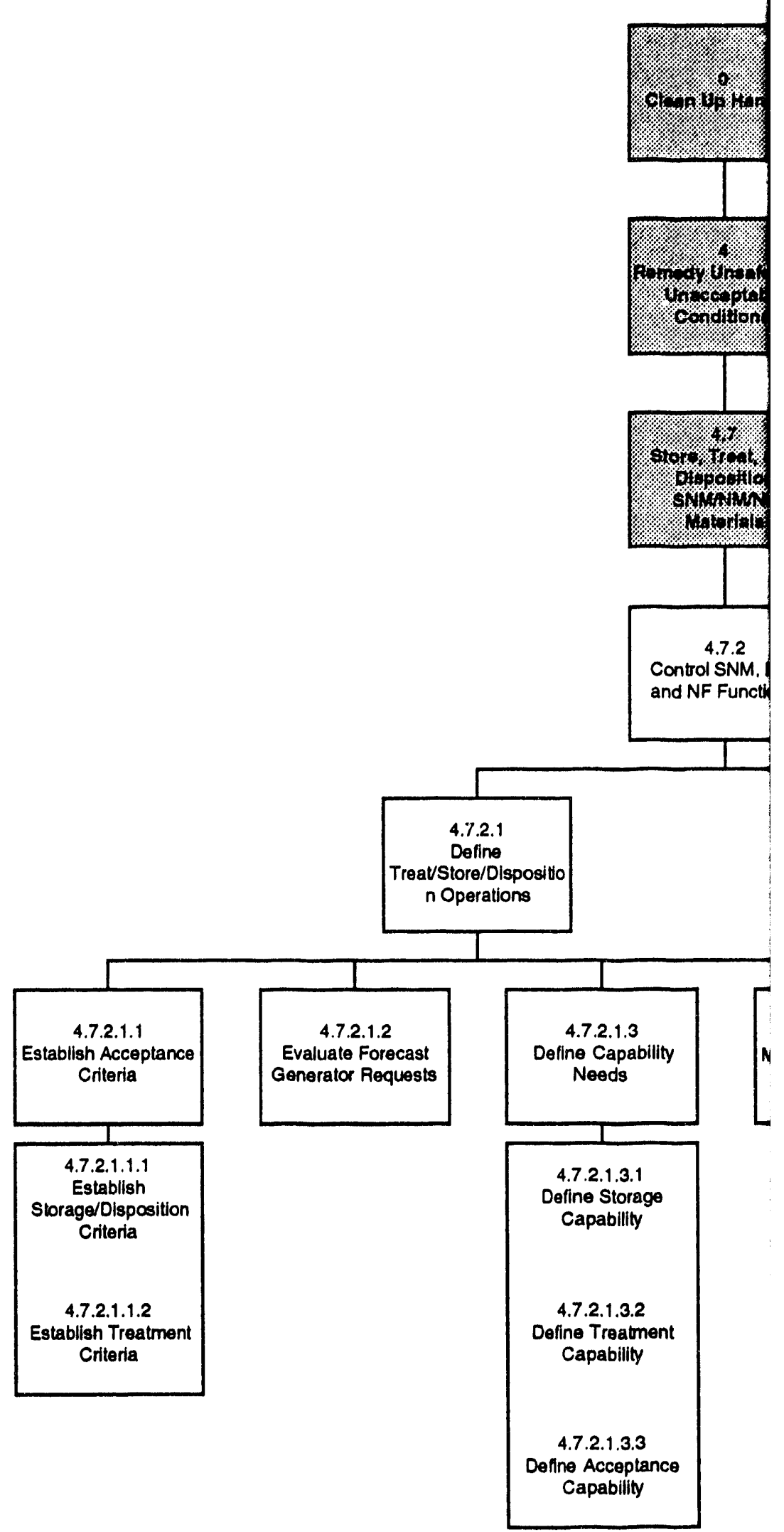


WHC-EP-0722 ADDENDUM 2 REV 0

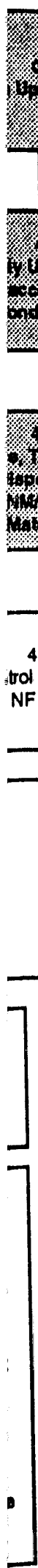

4.7.2.3

Plan and Schedule Operations

Shipper/Receiver Agreement

Or.7. SNM, NM

NF Functions

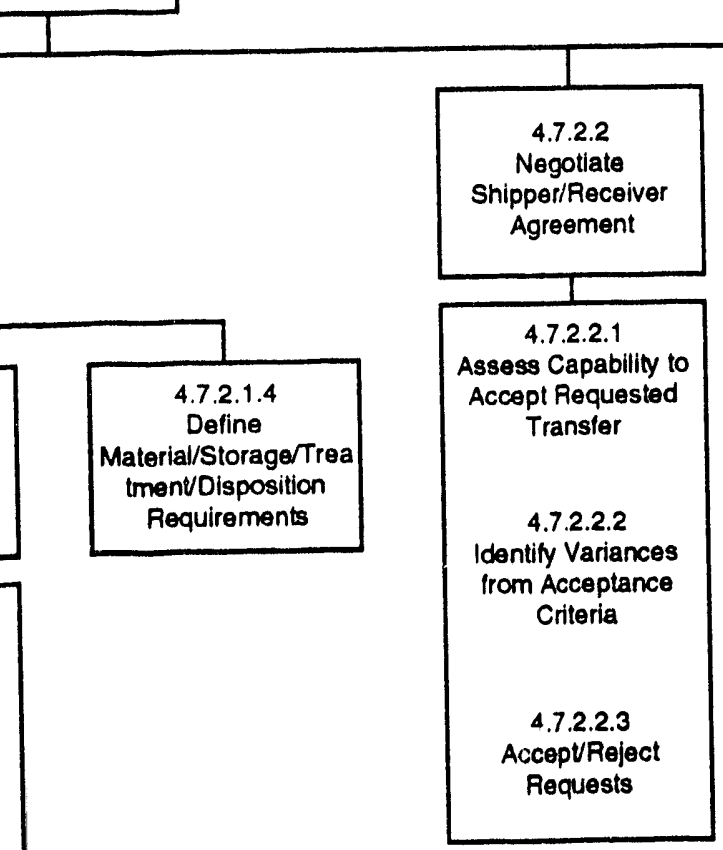

4.7.2.3.1

Plan Handling and

Receipts

4.7.2.3.2

Plan Storage

4.7.2.3.3

Plan Dispositions

4.7.2.3.4.

Plan Safety, Security, and Maintenance 


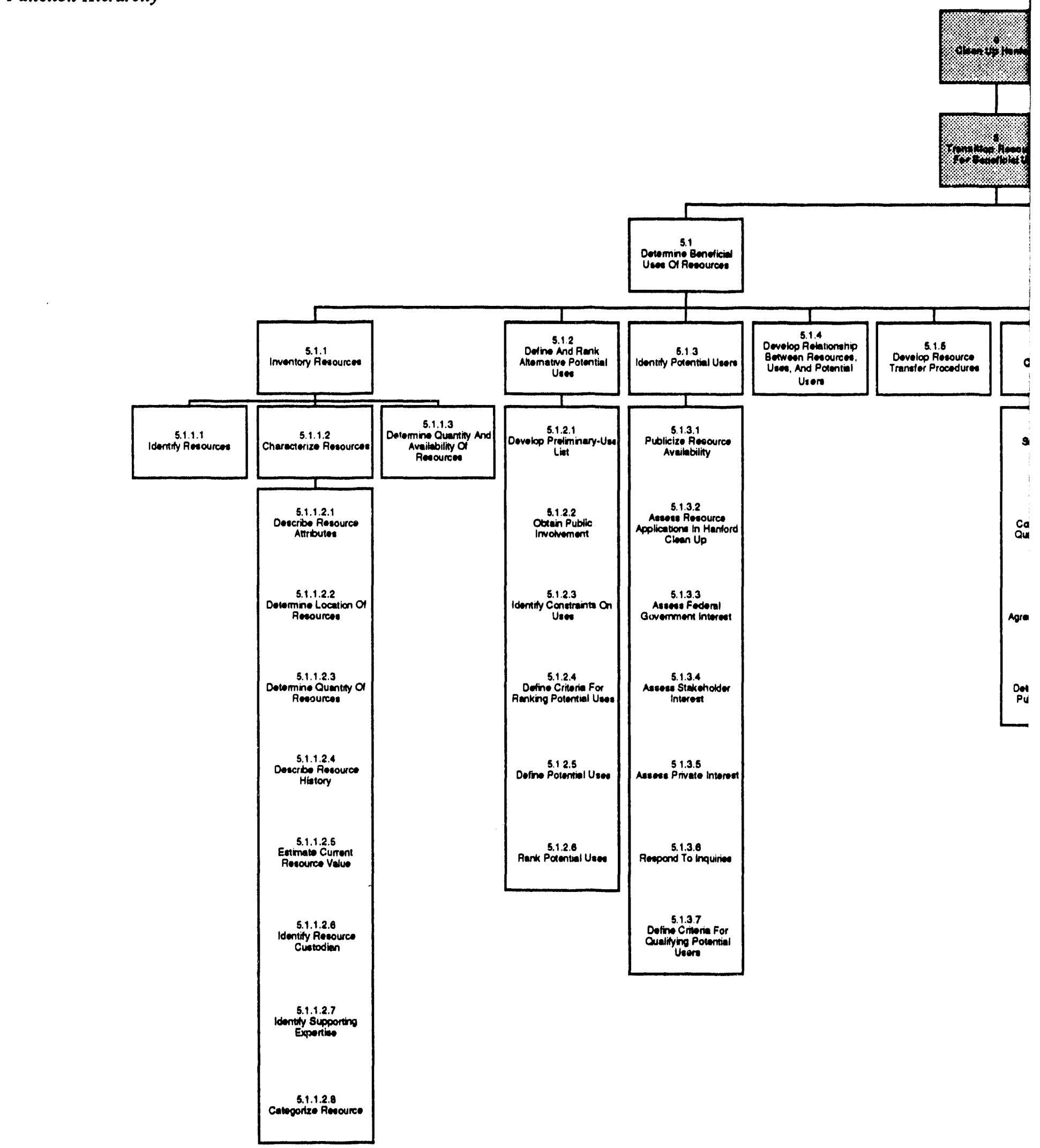


WHC-EP-0722 Addendum 2 Rev 0

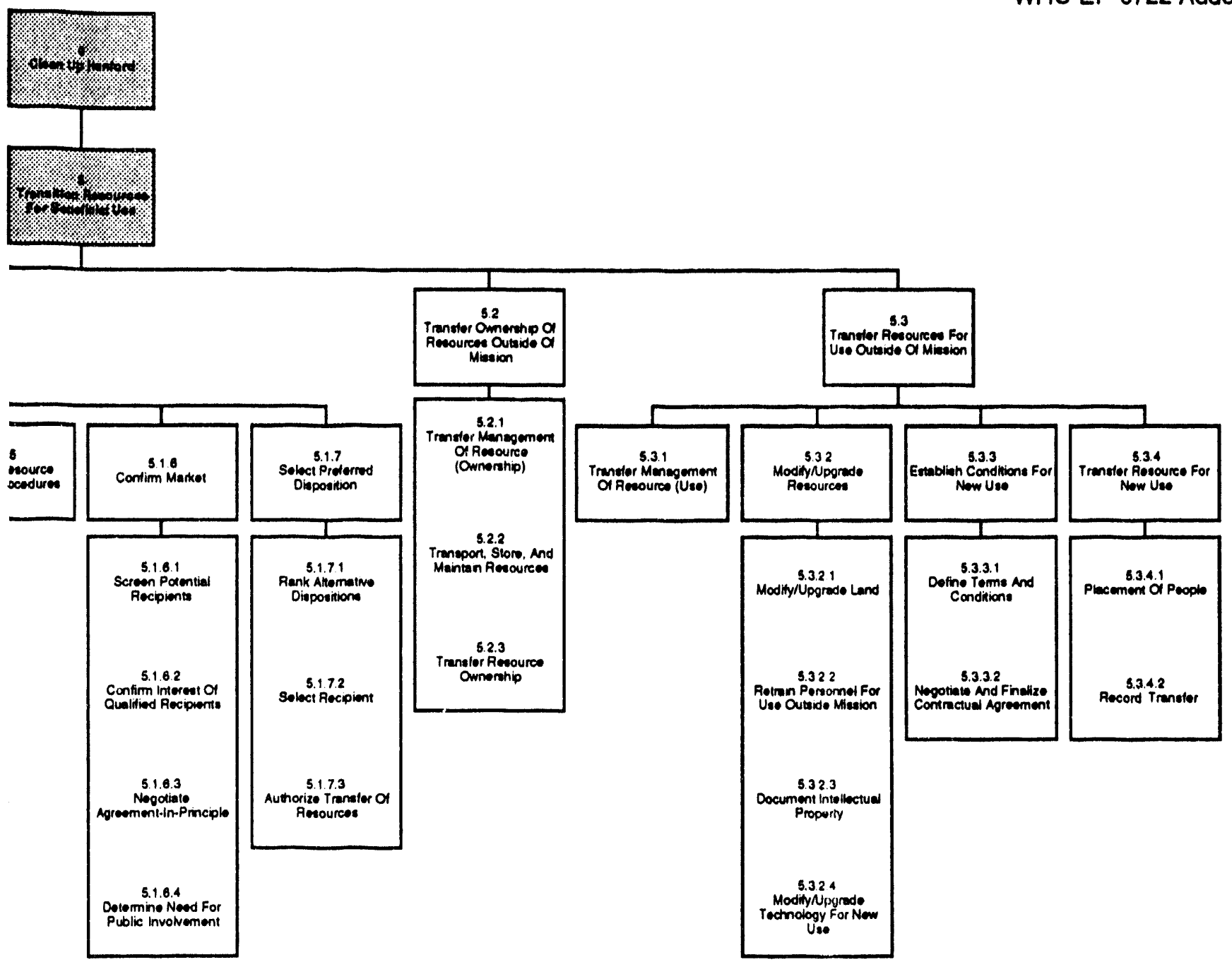


Function Hierarchy

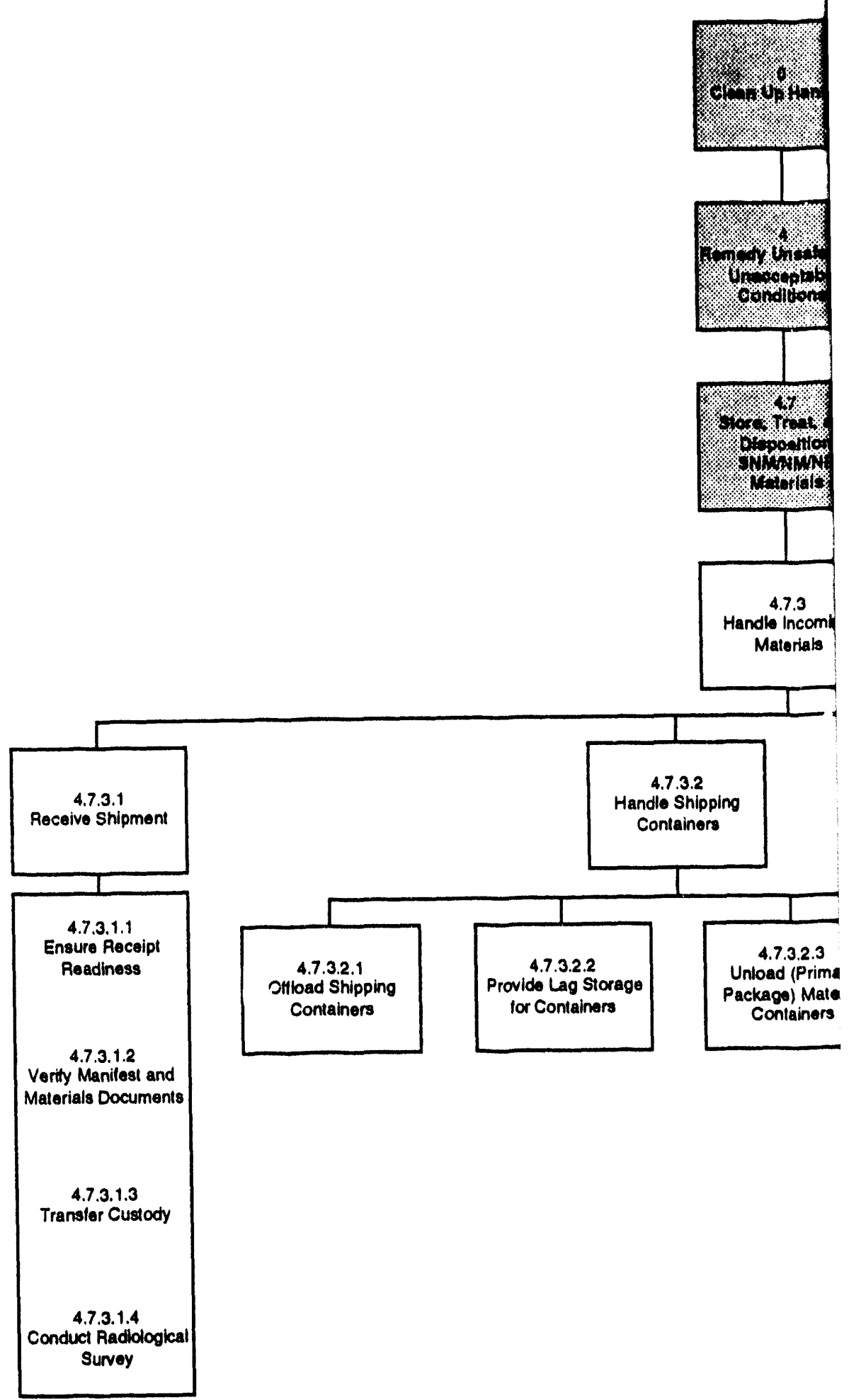




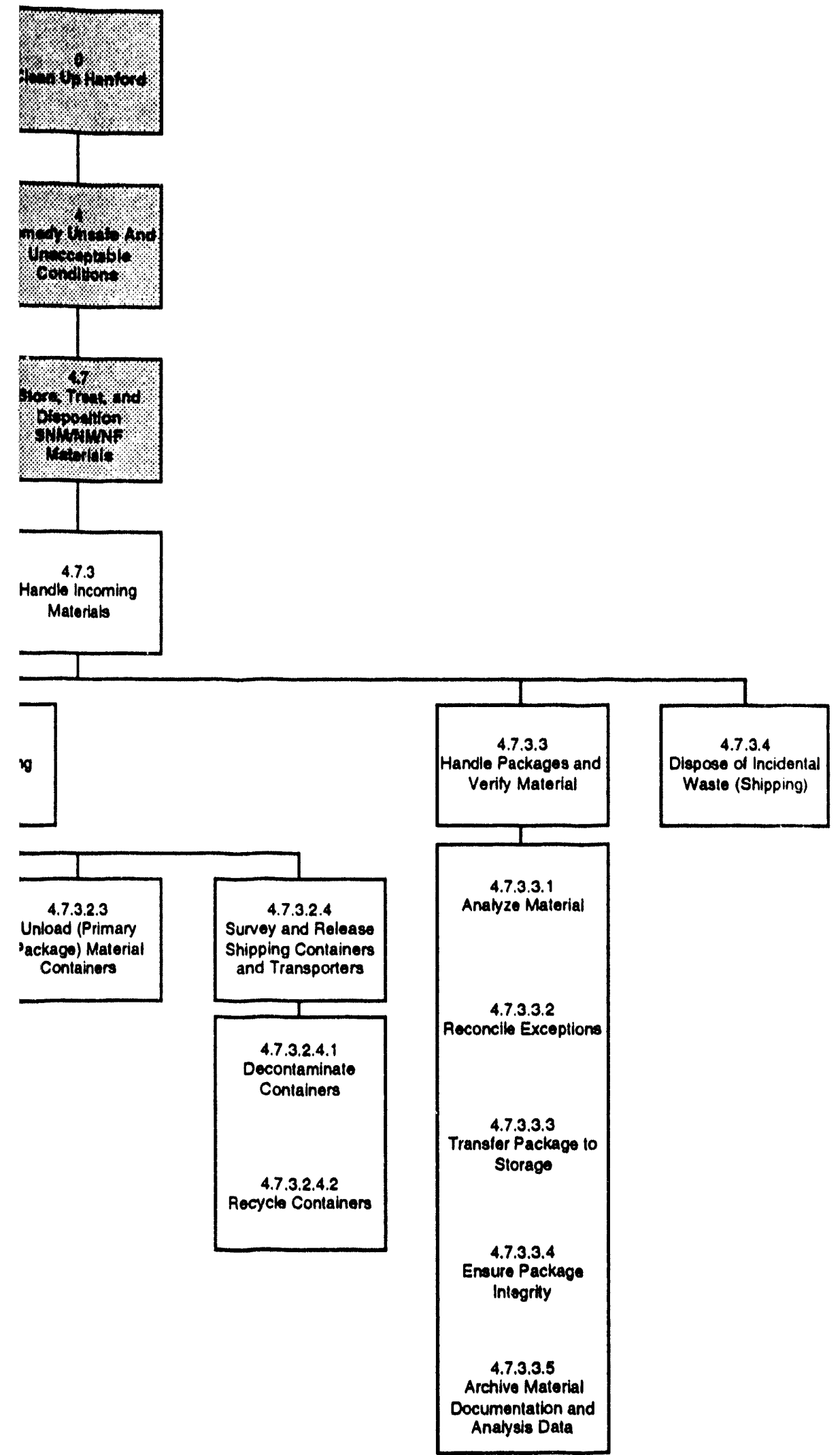


WHC-EP-0722 ADDENDUM 2 REV. 0

SECTION 2

IDEFO DIAGRAMS 
IDEFO DIAGRAMS

LIST OF FIGURES

X Universe Context................................... 2-1

O Clean Up Hanford...................................... 2-2

1 Manage Program.................................... 2-3

1.1 Establish Management System....................... 2-4

1.3 Formulate Program.............................. 2-5

1.4 Direct Program............................... 2-6

1.5 Evaluate Program............................. 2-7

1.6 Help Reinvent Government........................ 2-8

2 Acquire Mission Essential Capabilities...................... 2-9

2.1 Formulate Acquisition Strategy for Mission Essential........ 2-10 Capabilities

2.2 Provide Expertise.............................. 2-11

2.3 Provide Technology.............................. 2-12

2.4 Provide Facilities, Equipment, Infrastructure, \& Supplies.... 2-13

2.5 Provide Essential Information...................... 2-14

3 Obtain Public Acceptance.............................. 2-15

4 Remedy Unsafe and Unacceptable Conditions.................. 2-16

4.1 Deactivate Facilities............................. 2-17

4.1.1 Deactivate Facilities With Special Nuclear Materials \&.... 2-18 Nuclear Materials (Type 1 Fclty)

4.1.1.1 Maintain Safety \& Compliance Envelope (Type 1 Fclty).. 2-19

4.1.1.2 Determine Deactivation Plans \& Negotiate Turnover..... 2-20 Endpoint (Type 1 Fclty)

4.1.1.3 Stabilize \& Reconfigure Facilities for Minimum ...... 2-21 Survelllance (Type 1 Fclty)

4.1.1.4 Disposition Currently Identified Radioactive Materials 2-22 Held As A Potential Product \& Special Nuclear Material (Type 1 Fclty)

4.1.2 Deactivate Facilities With Radioactive \& Hazardous....... 2-23 Material (Type 2 Fclty)

4.1.2.1 Maintain Safety \& Compliance Envelope (Type 2 Fclty).. 2-24

4.1.2.2 Determine Deactivation Plan \& Negotiate Turnover..... 2-25 Endpoint (Type 2 Fclty)

4.1.2.3 Stabilize \& Reconfigure Facilities For Minimum...... 2-26 Surveillance (Type 2 Fclty) 
WHC-EP-0722 ADDENDUM 2 REV. 0

IDEFO DIAGRAMS

LIST OF FIGURES

Page

4.1.3 Deactivate Facilities With Only Hazardous Material, ..... 2-27 Including Asbestos (Type 3 Fclty)

4.1.3.1 Maintain Safety \& Compliance Envelope (Type 3 Fclty).. 2-28

4.1.3.2 Determine Deactivation Plan \& Negotiate Turnover..... 2-29 Endpoint (Type 3 Fclty)

4.1.3.3 Stabilize \& Reconfigure Facilities for Minimum....... 2-30 Survelllance (Type 3 Fclty)

4.1.4 Deactivate Facilities Without Radioactive Or Hazardous.... 2-31 Matertal (Type 4 Fclty)

4.1.4.1 Maintain Safety \& Compliance Envelope (Type 4 Fclty).. 2-32

4.1.4.2 Determine Deactivation Plan \& Negotiate Turnover..... 2-33 Endpoint (Type 4 Fclty)

4.1.4.3 Stabilize \& Reconfigure Facilities For Minimum....... 2-34 Survelllance (Type 4 Fclty)

4.3 Remedy Solid Waste............................... 2-35

4.3.1 Maintain Solid Waste Safety And Compliance Envelope...... 2-36

4.3.2 Receive Solid Waste........................... 2-37

4.3.2.1 Retrieve Existing Solid Waste................. 2-38

4.3.2.1.1 Plan Solid Waste Retrieval.................. 2-39

4.3.2.1.2 Remove Sol id Waste..................... 2-40

4.3.2.2 Receive New Solid Waste..................... 2-41

4.3.2.2.1 Plan Solid Waste Receipt................. 2-42

4.3.2.2.2 Accept Solid Waste..................... 2-43

4.3.3 Characterize Solid Waste........................ 2-44

4.3.4 Determine Solid Waste Disposition Requirements.......... 2-45

4.3.5 Disposition Solid Waste....................... 2-46

4.5 Manage Aqueous Wastes......................... 2-47

4.5.1 Maintain Aquenus Waste Safety and Comp?iance Envelope.... 2-48

4.5.2 Develop Aqueous Waste Disposition Plan............... 2-49

4.5.3 Receive/Transport Aqueous Waste.................. 2-50

4.5.4 Treat Aqueous Waste........................ 2-51

4.5 .5 Disposition Aqueous Waste.................... 2-52

4.6 Correct Unsafe Infrastructure Conditions.............. 2-53 
WHC-EP-0722 ADDENDUM 2 REV. 0

\section{IDEFO DIAGRAMS}

\section{LIST OF FIGURES}

Page

4.7 Store, Treat, and Disposition SNM/NM/NF Materials.......... 2-54

4.7.1 Maintain Safety and Security Envelope............... 2-55

4.7.2 Control SNM, NM, and NF Functions................ 2-56

4.7.3 Handle Incoming Materials..................... 2-57

4.7.4 Store Materials............................ 2-58

4.7.5 Transfer Outgoing Materials for Disposition............ 2-59

5 Transition Resources For Beneficial Use.................. 2-60

5.1 Determine Beneficial Uses of Resources.................. 2-61

5.2 Transfer Ownership of Resources Outside of Mission.......... 2-62

5.3 Transfer Resources For Use Outside of Mission.............. 2-63 
IDEFO Diagrams

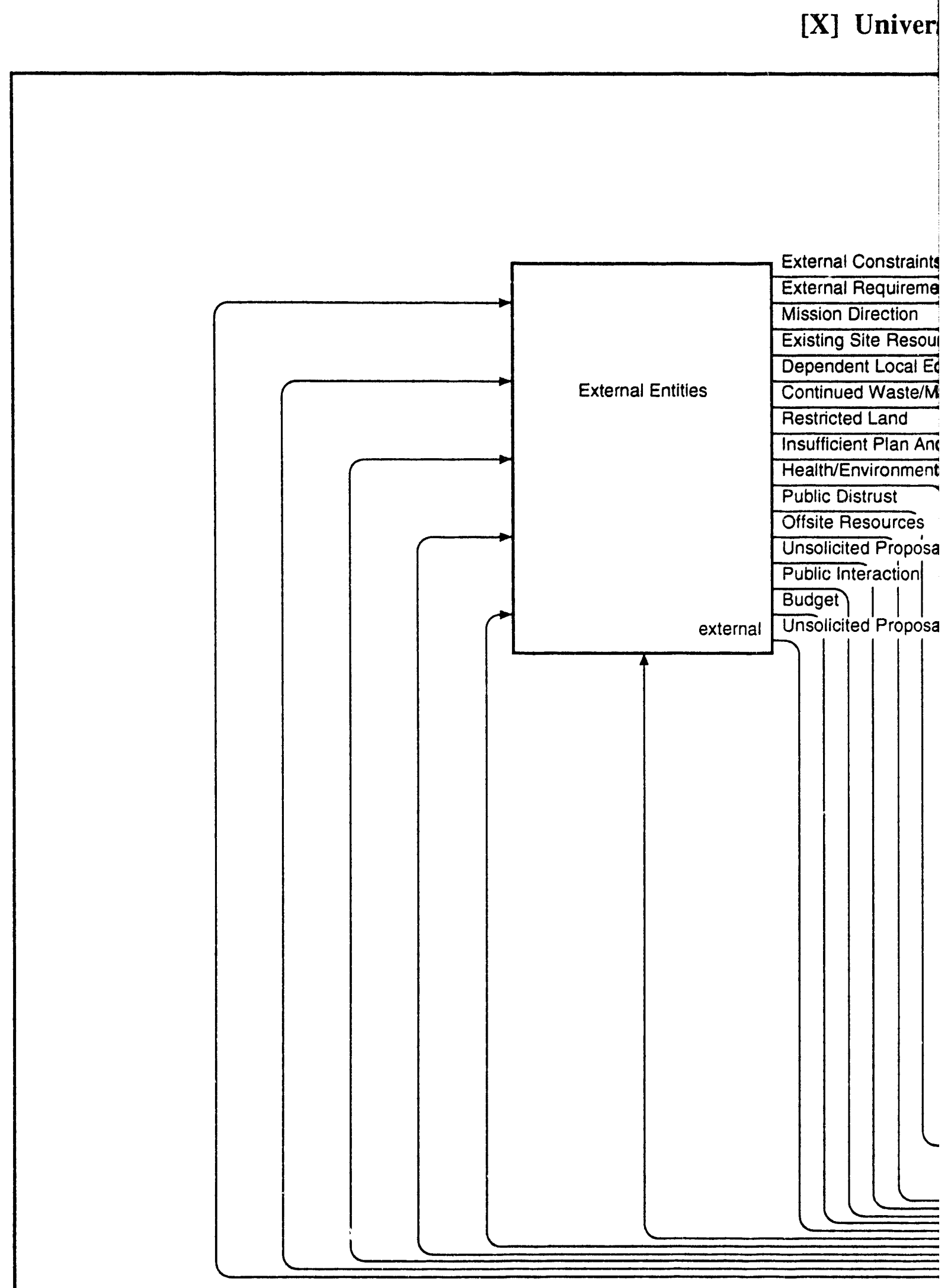

Hanford Site Systems Engineering 


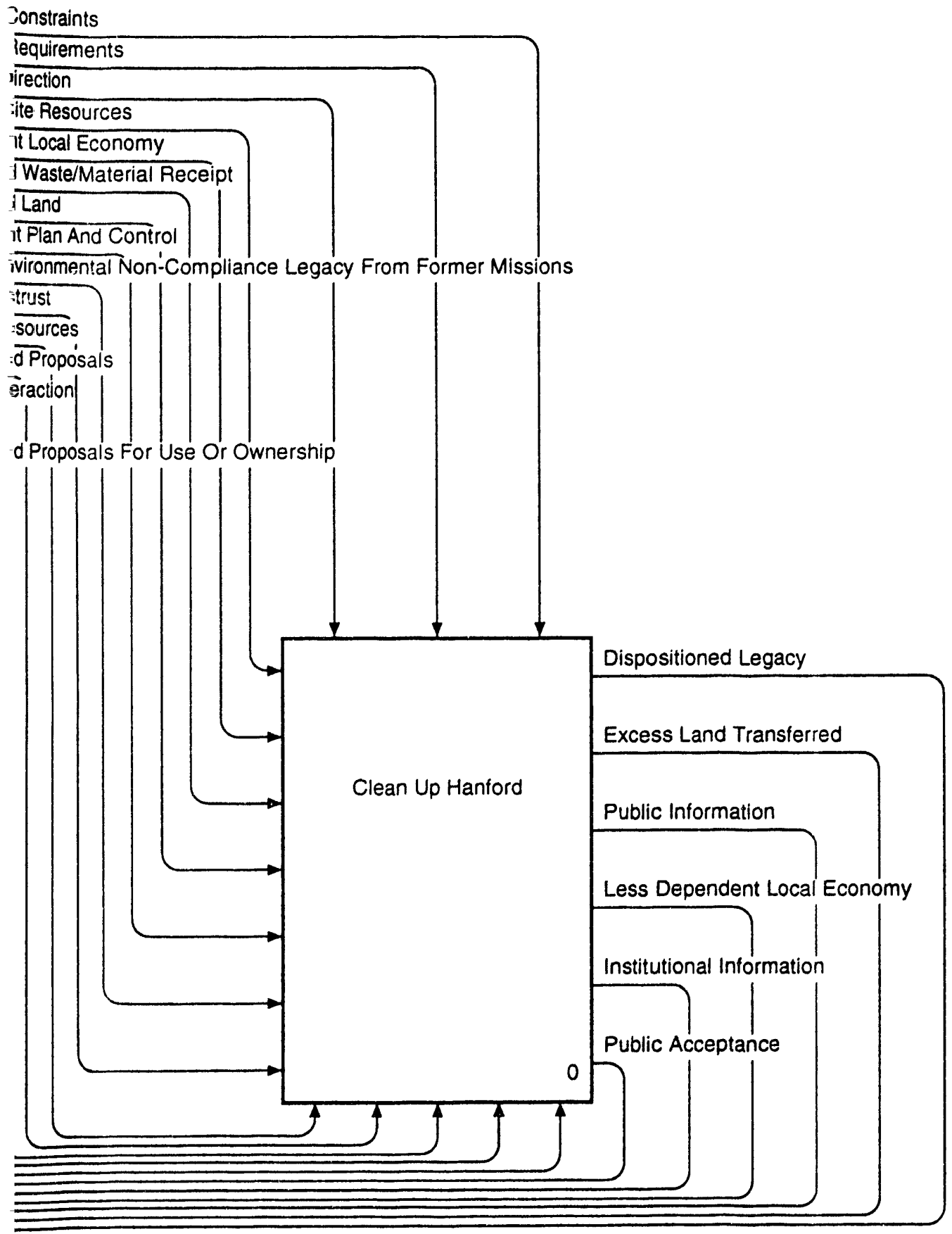




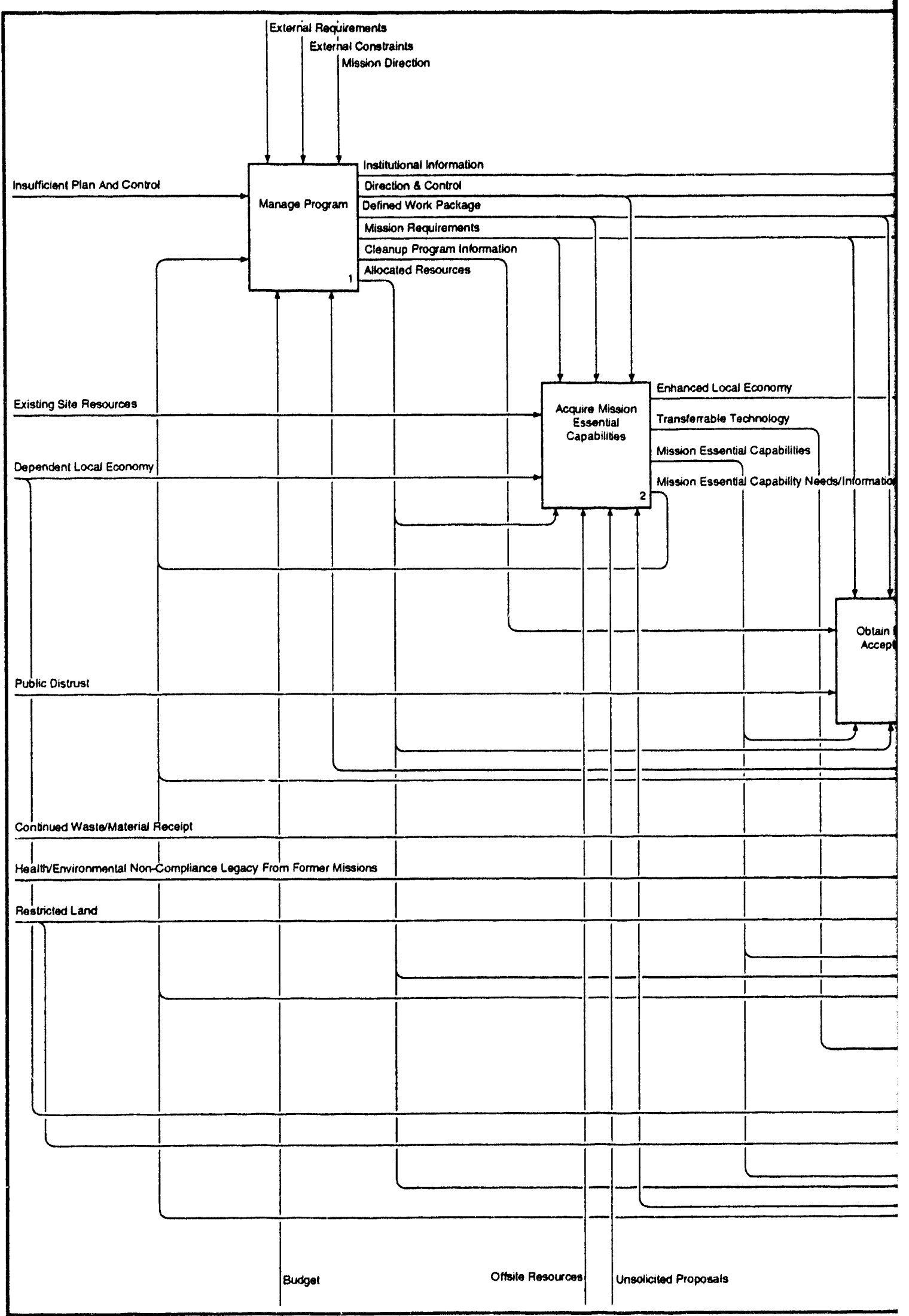




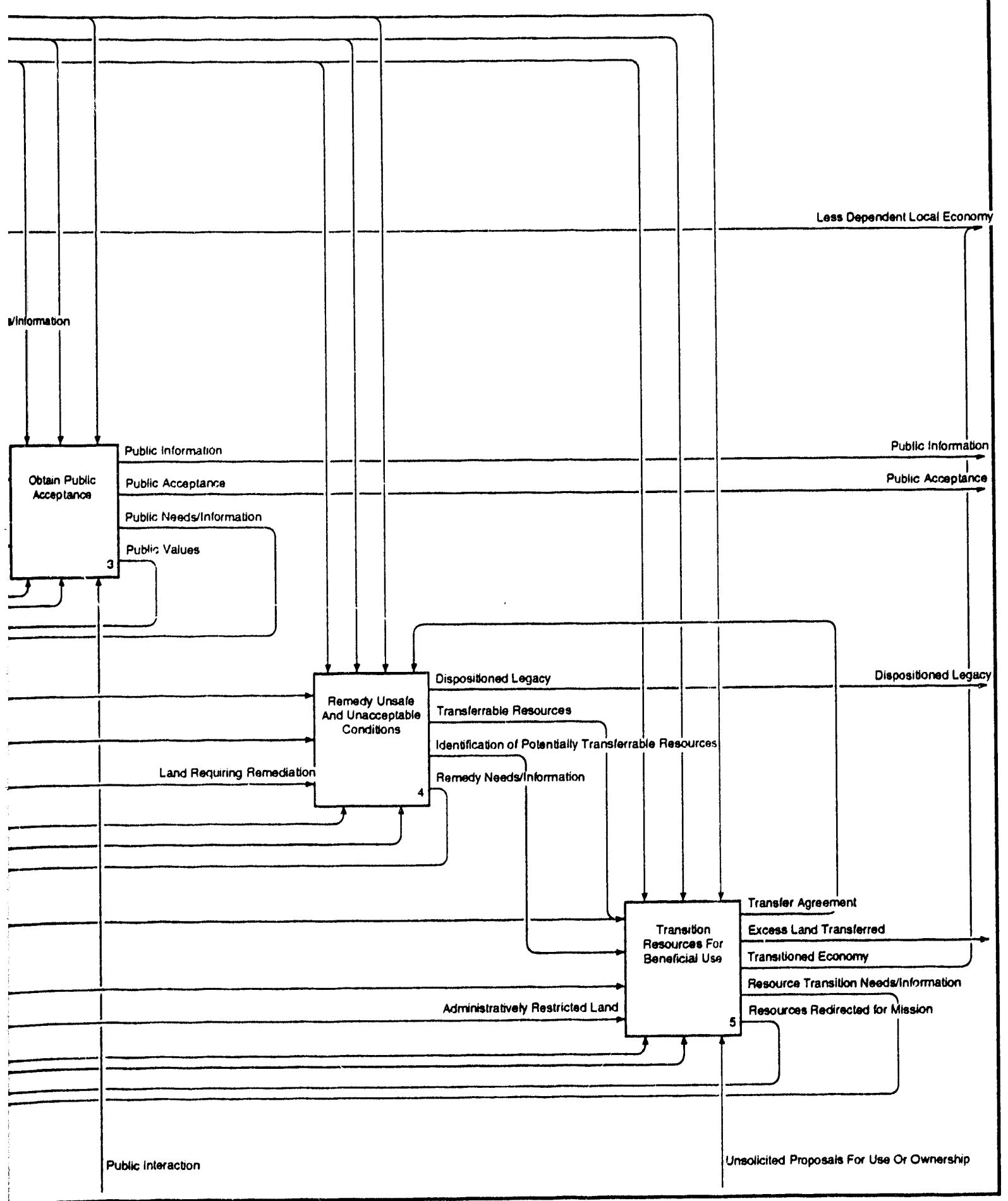




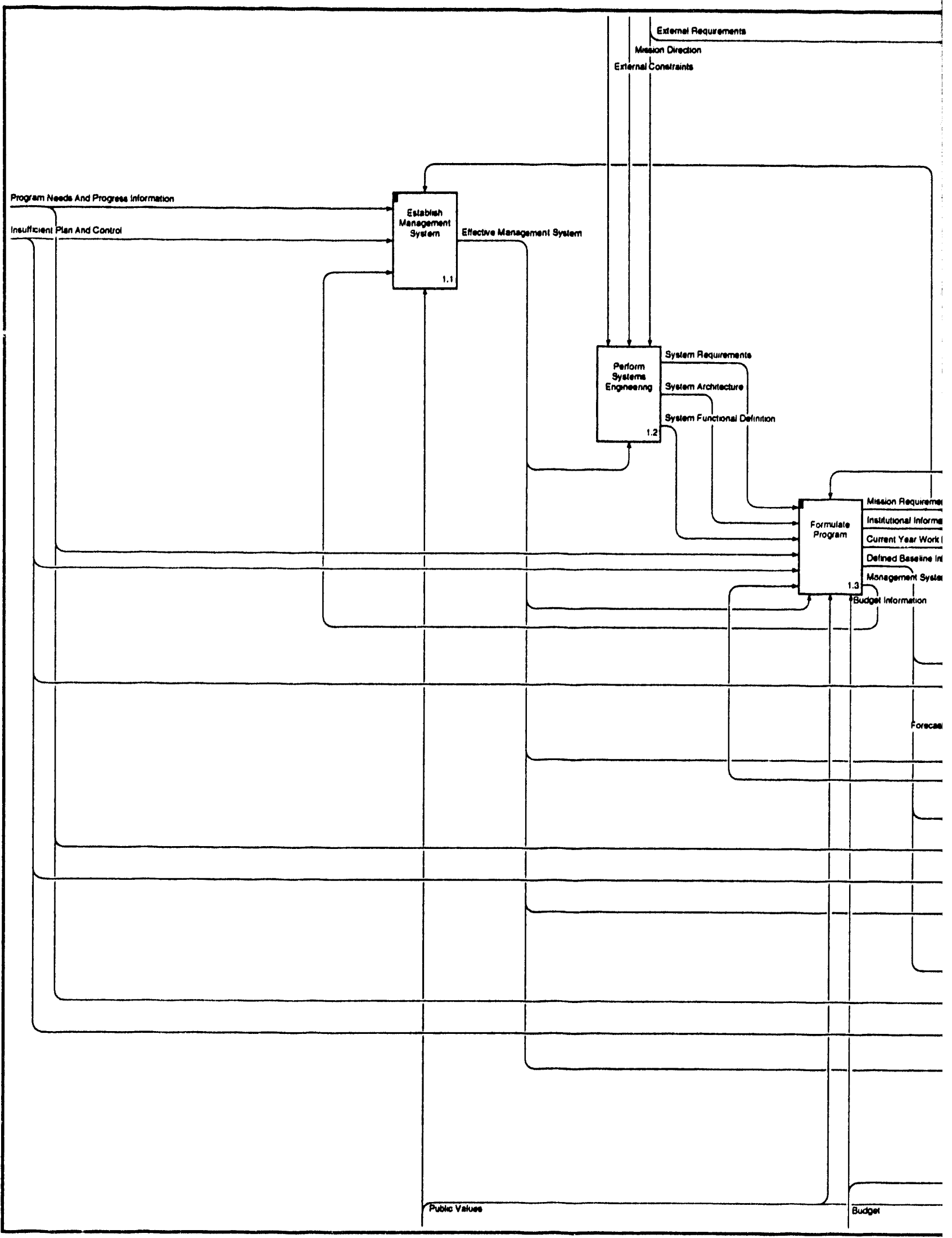

Hanford Site Systems Engineering 


\section{anage Program}

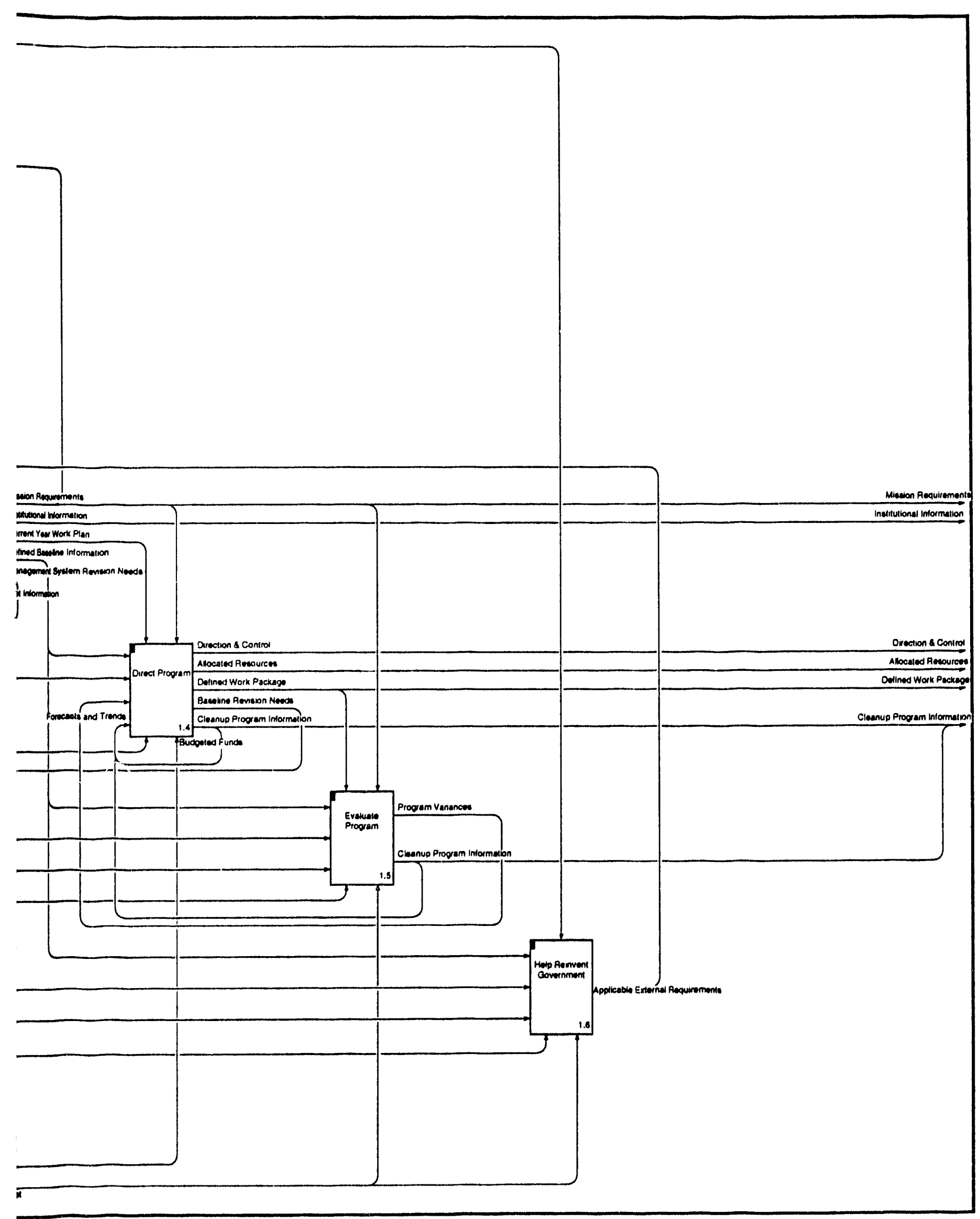




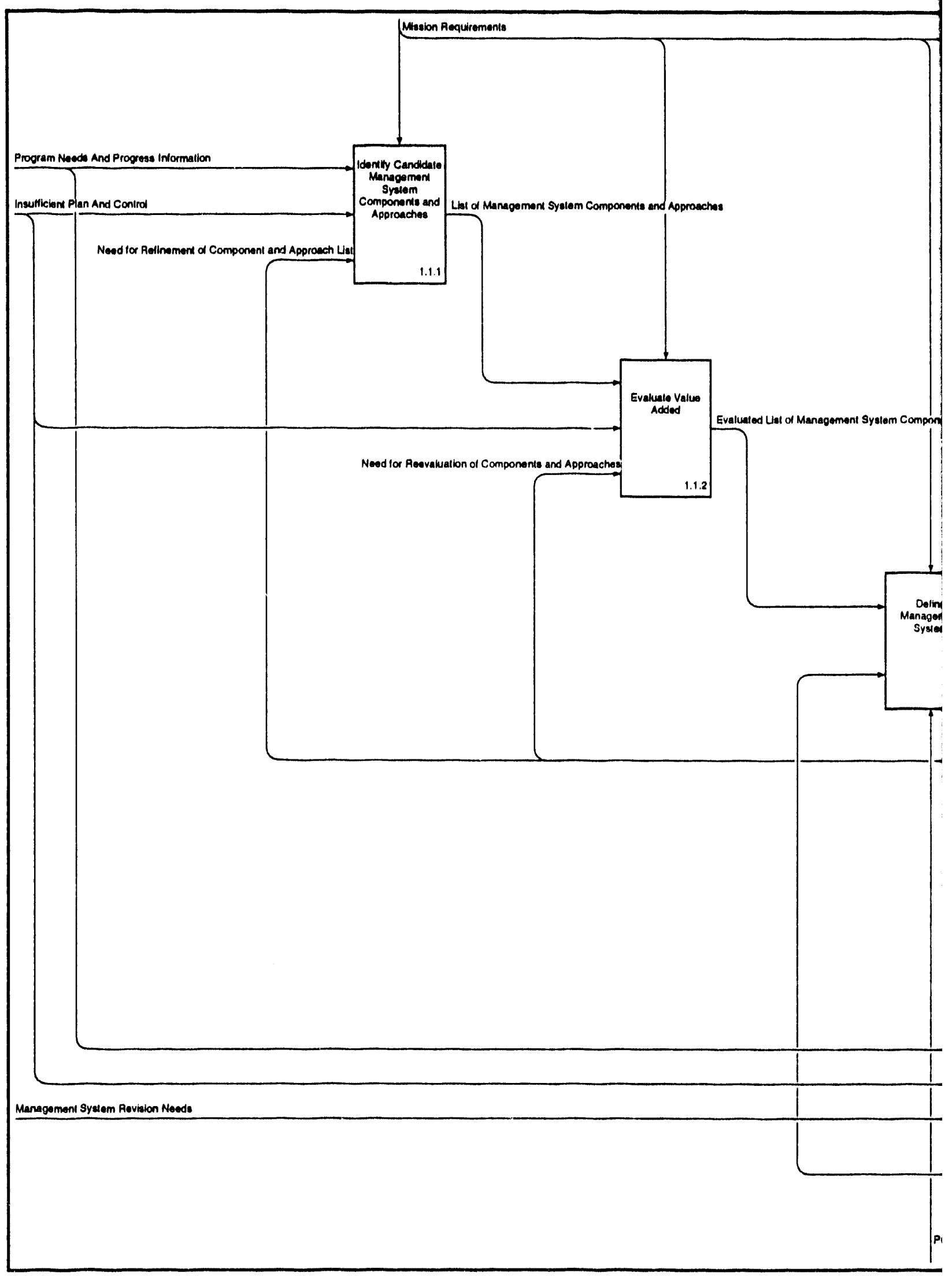

Hanford Site Systems Engineering 


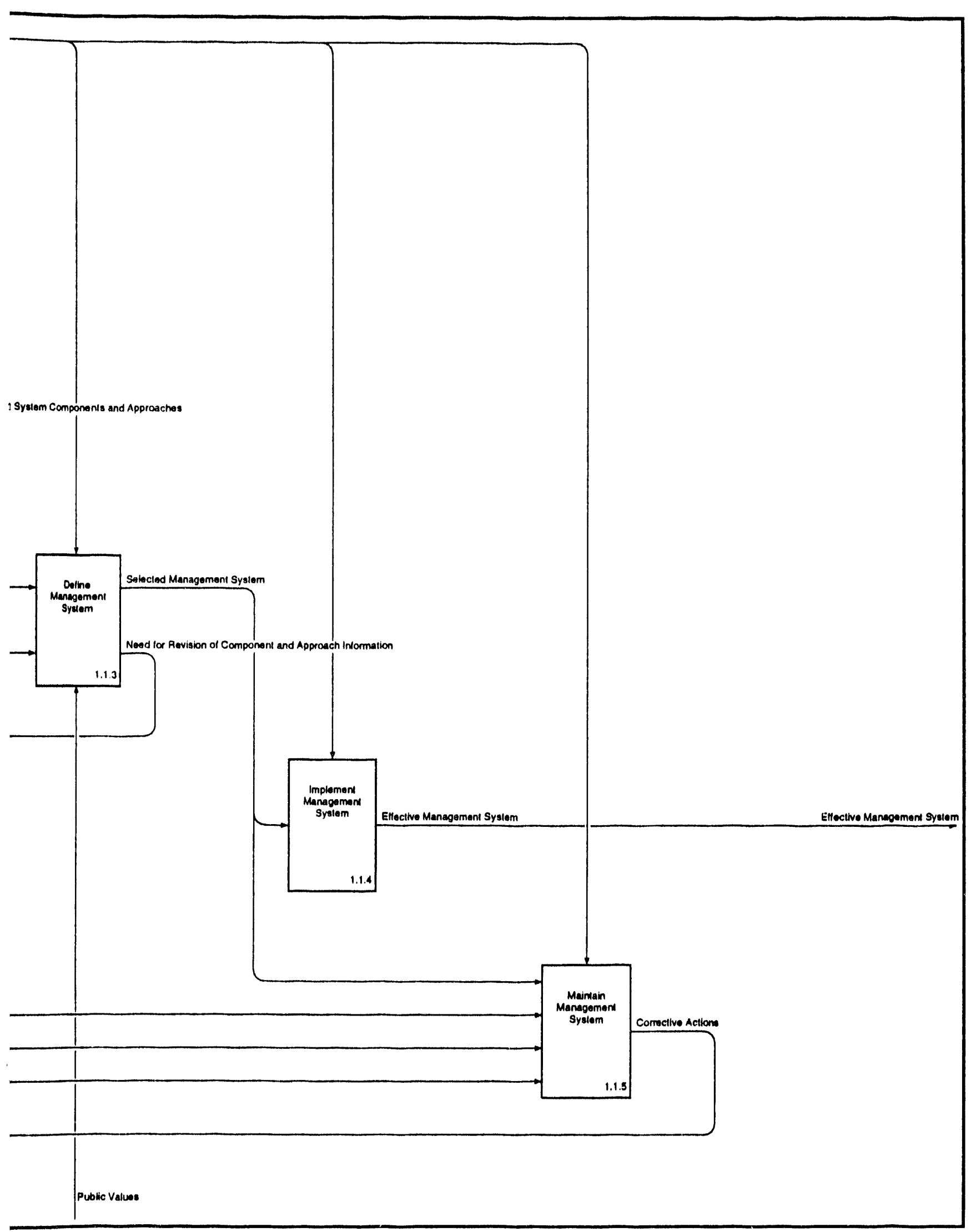




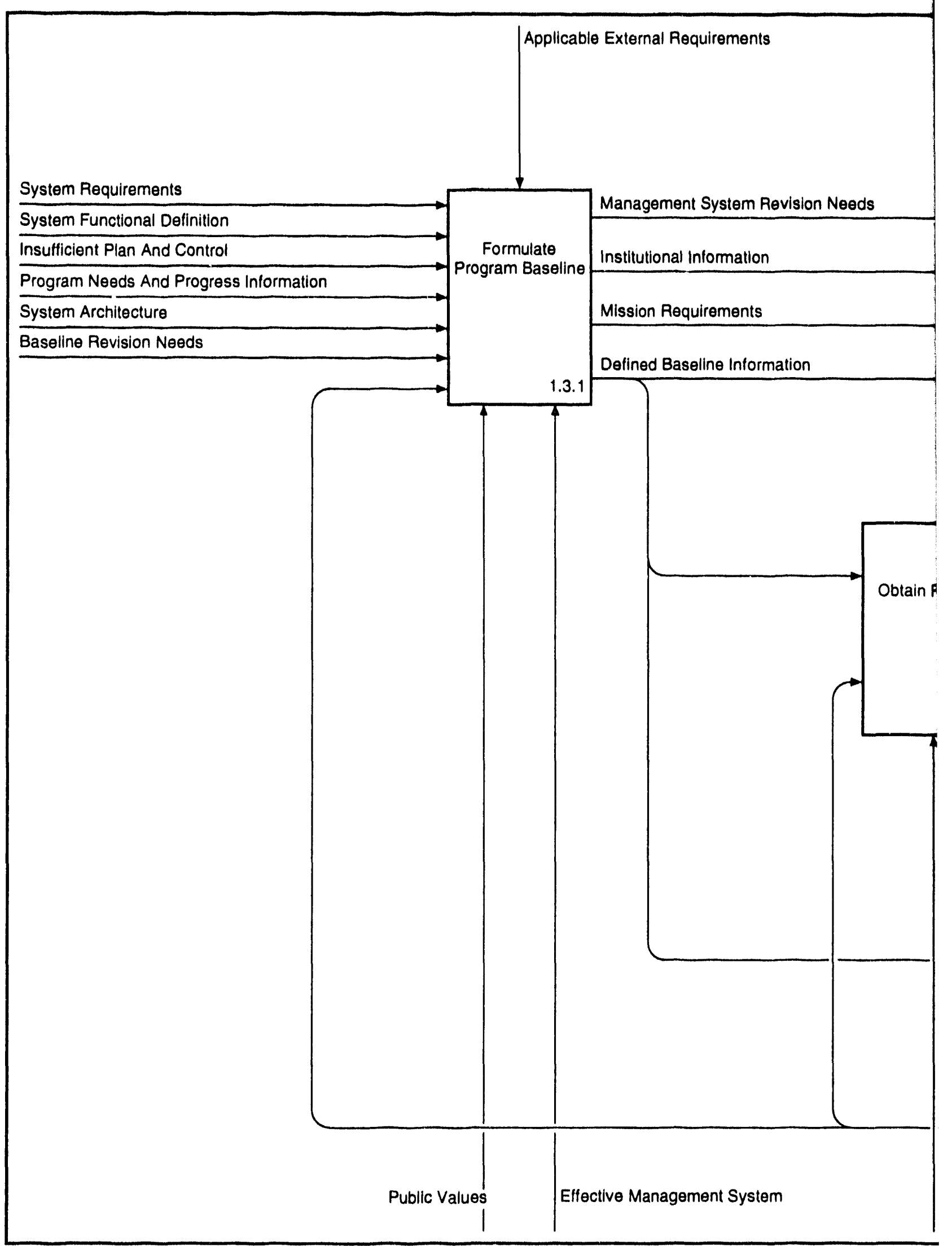




\section{mulate Program}

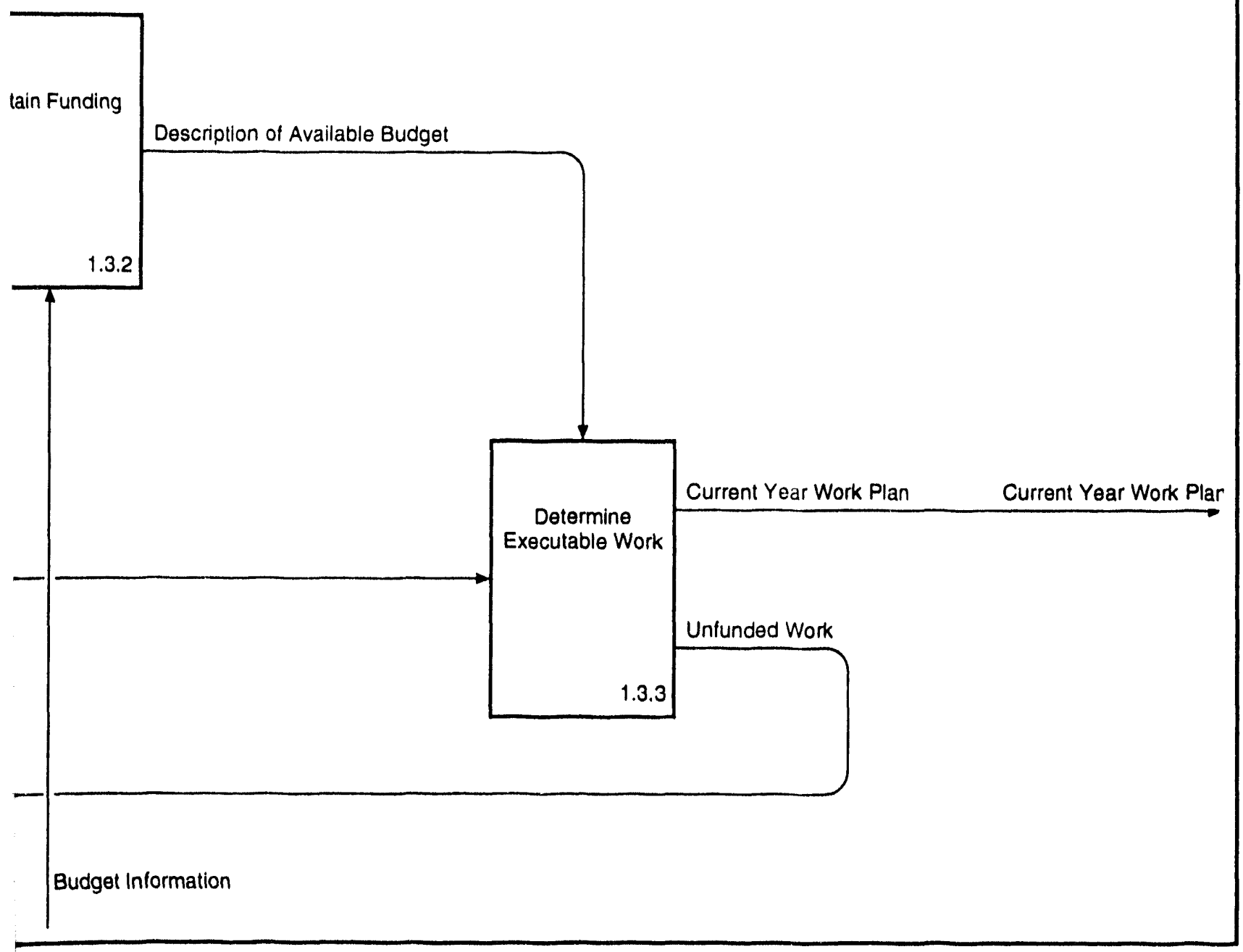




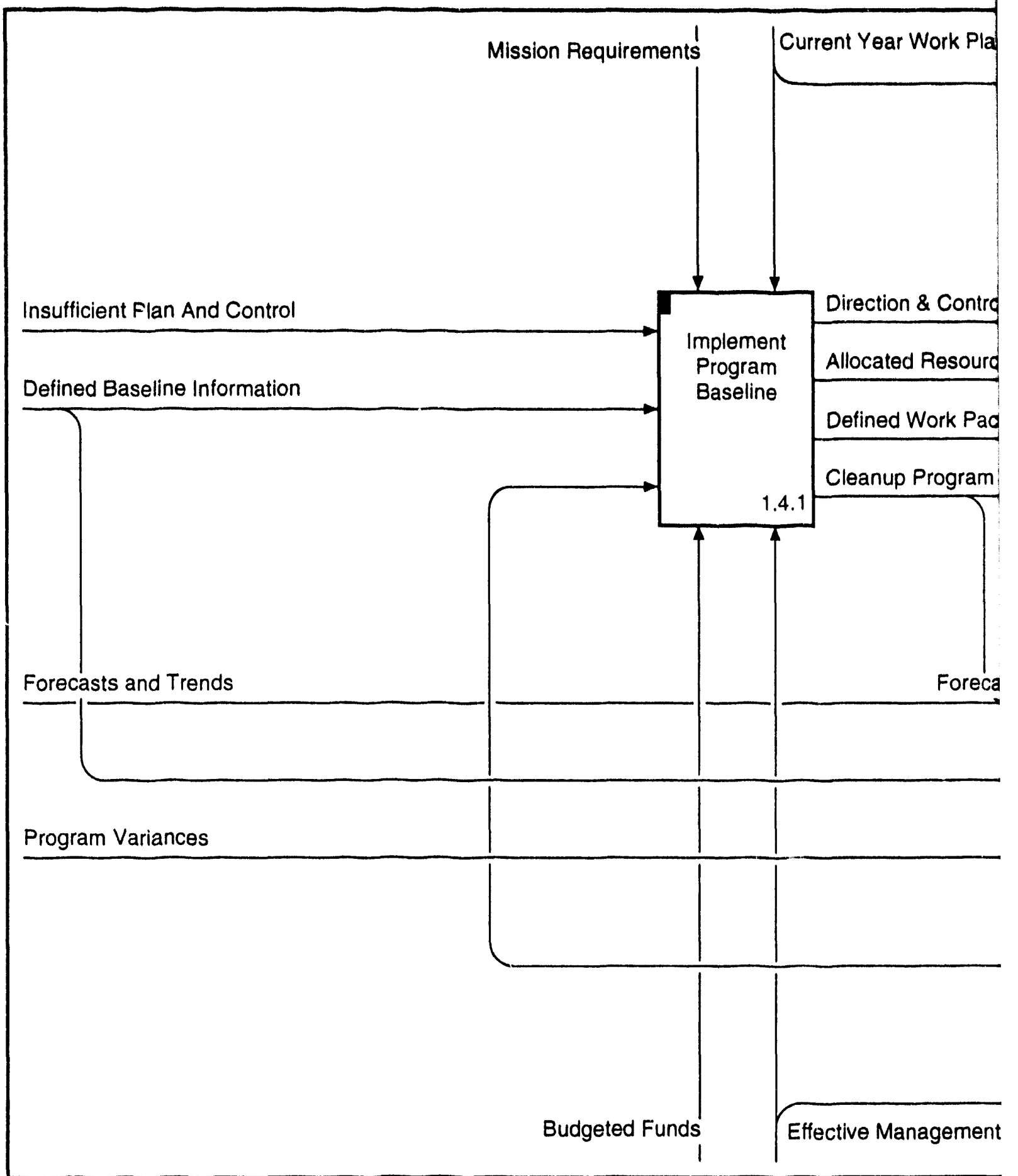




Direction \& Control

gram Information

$\underset{\text { Defined Work Package }}{\stackrel{\text { Allocated Resources }}{.}}$

gram Information

ment System

Baseline Revision Needs

Control

Baseline Revision Needs Baseline Revision Needs Changes
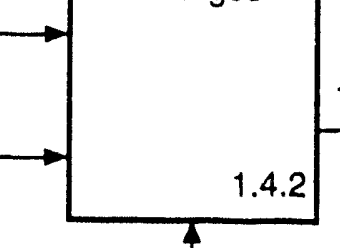

Tactical Changes

Tactical Changes

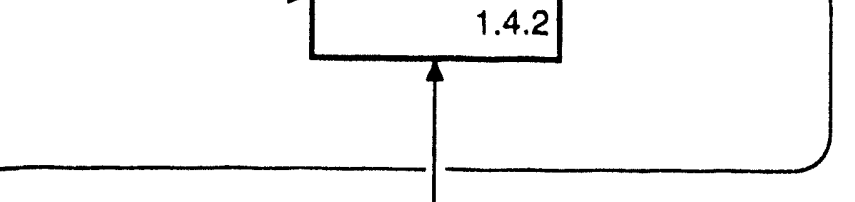




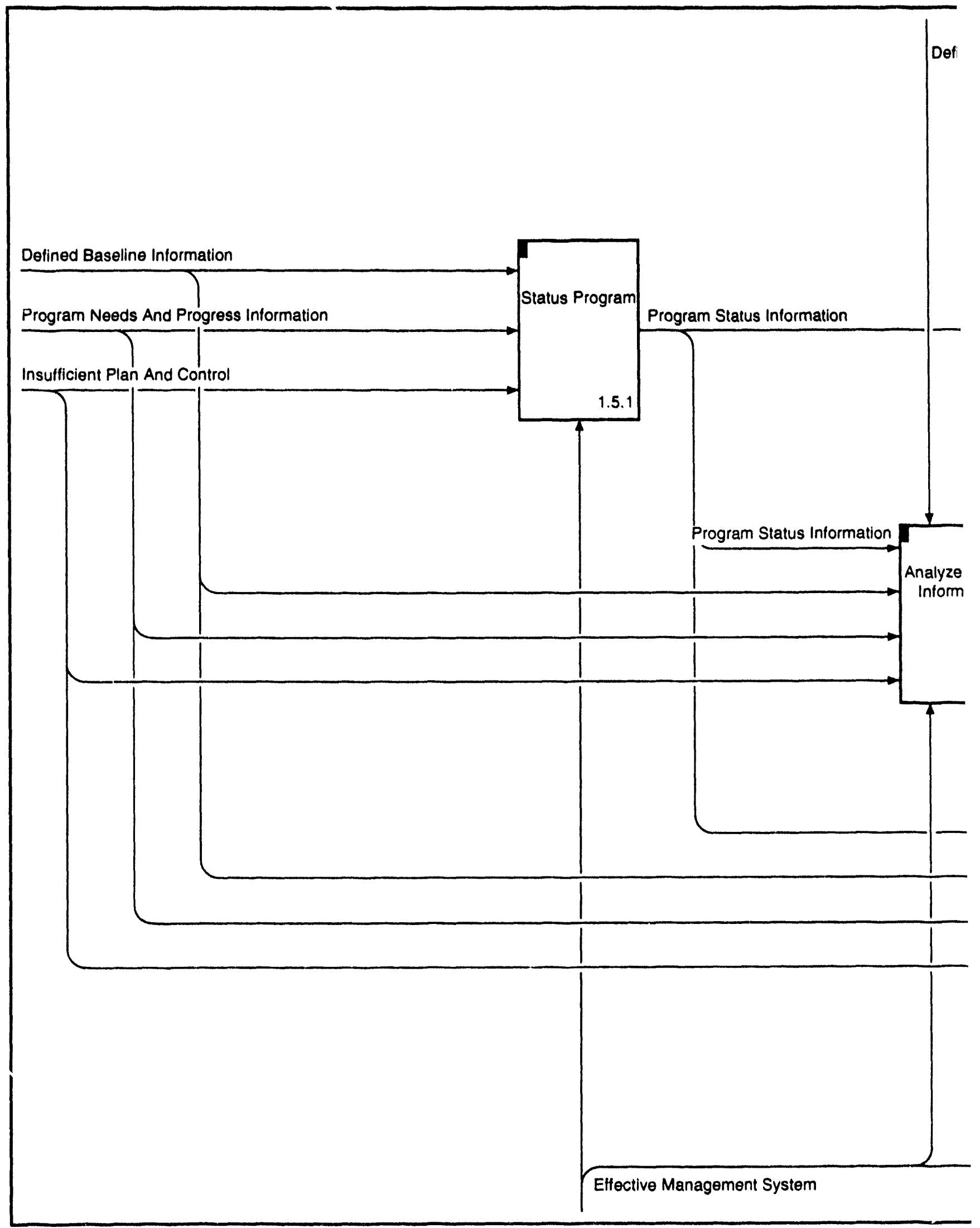




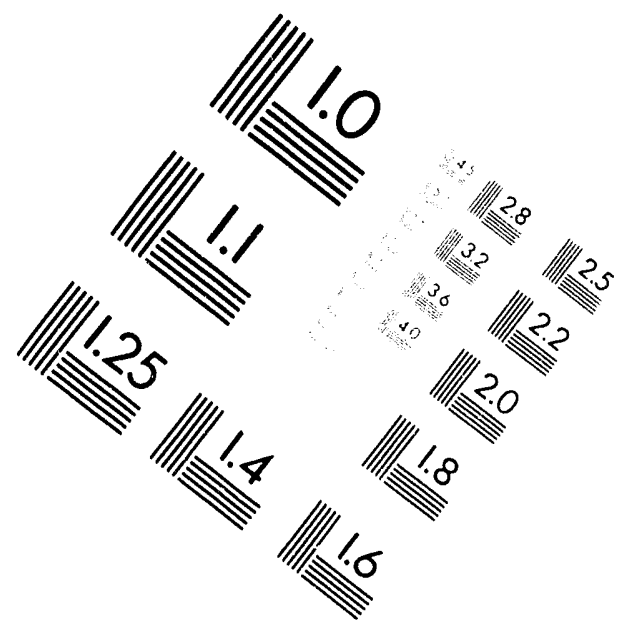

Centimeter

$\begin{array}{llllllllllllllll}1 & 2 & 3 & 4 & 5 & 6 & 7 & 8 & 9 & 10 & 11 & 12 & 13 & 14 & 15 & \mathrm{~mm}\end{array}$

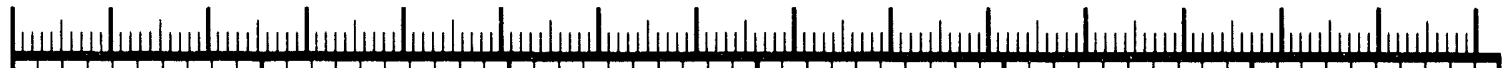
Inches
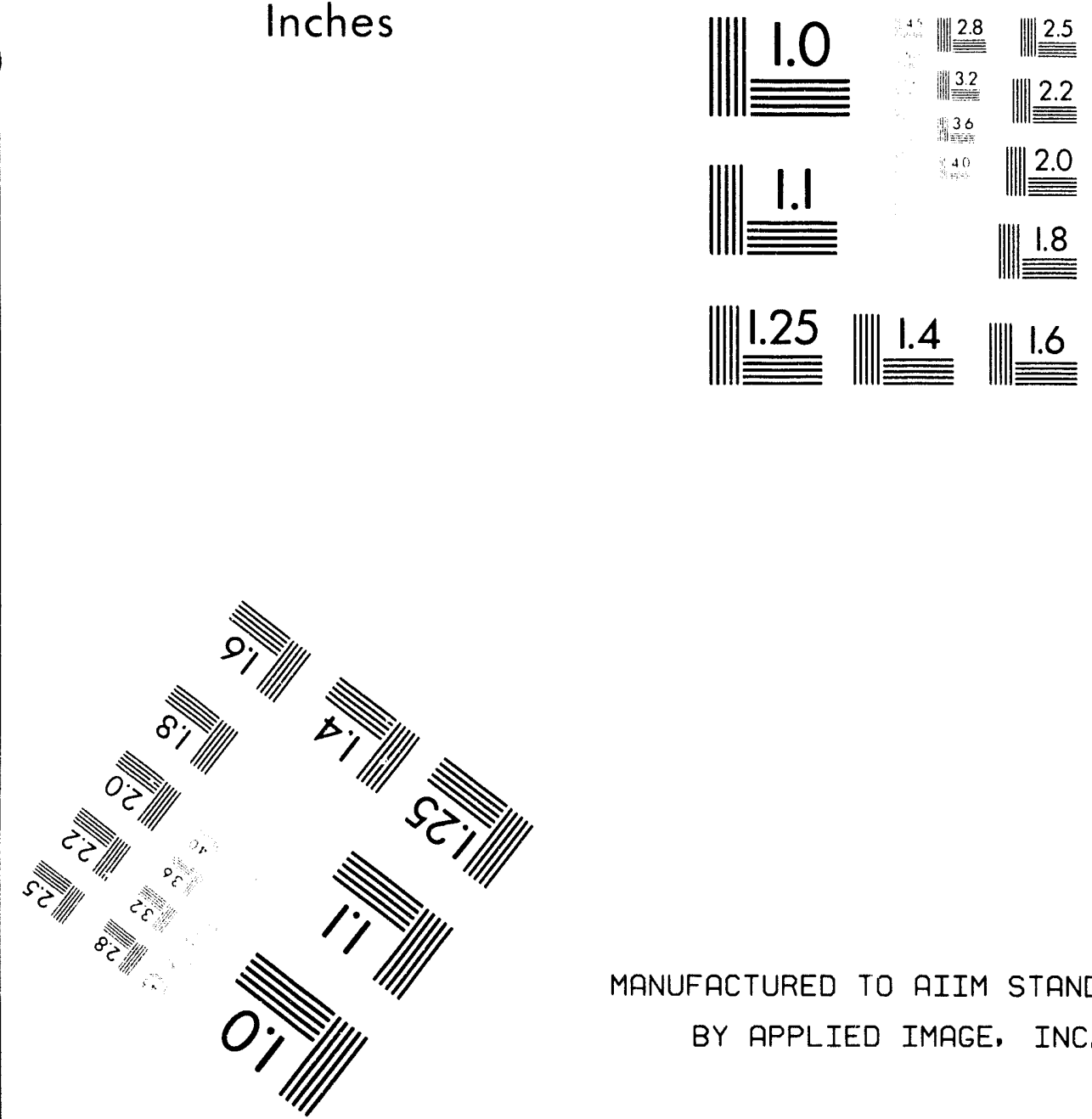

MANUFACTURED TO AIIM STANDARDS BY APPLIED IMAGE, INC.

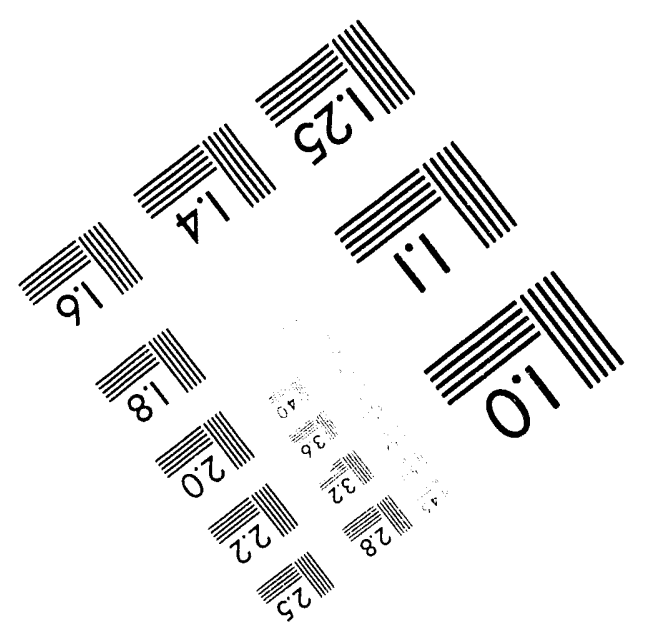



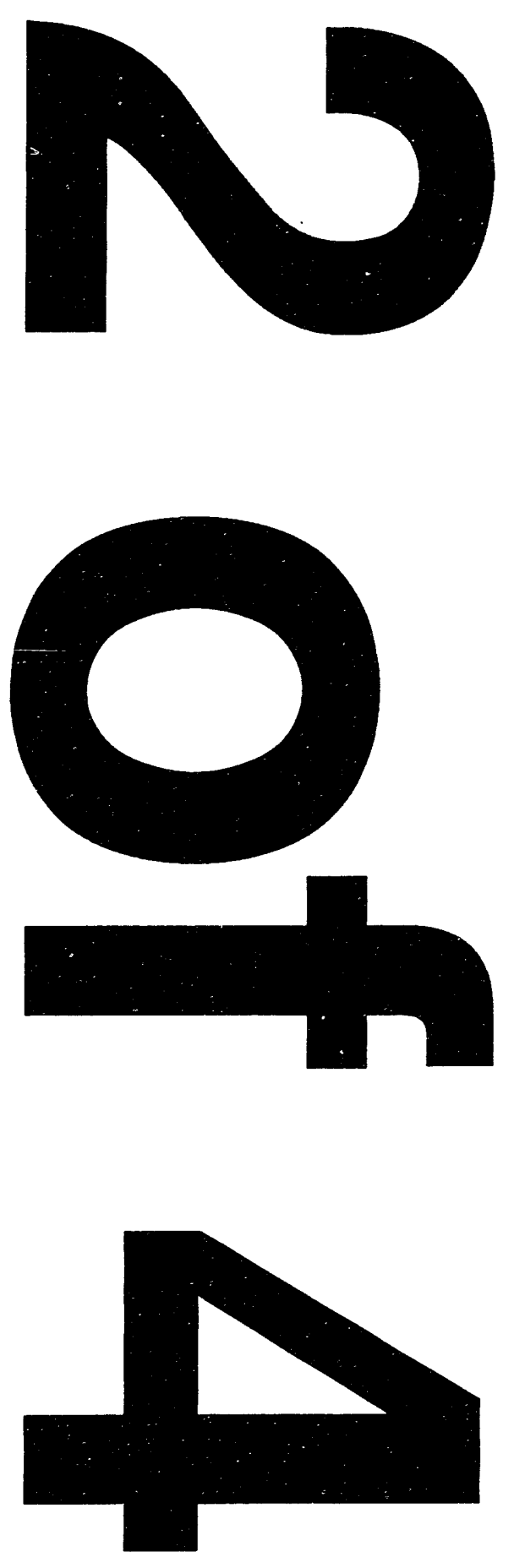


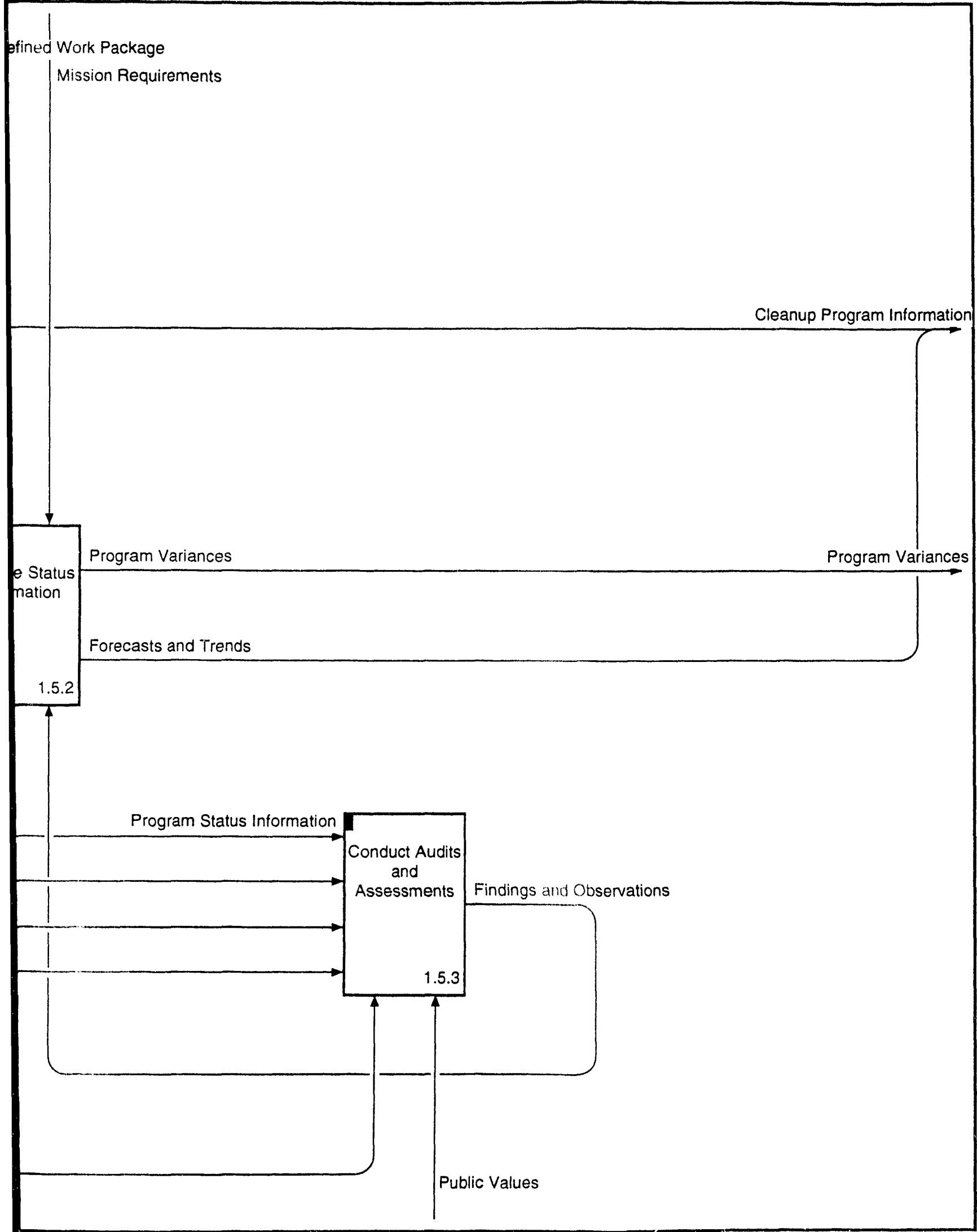




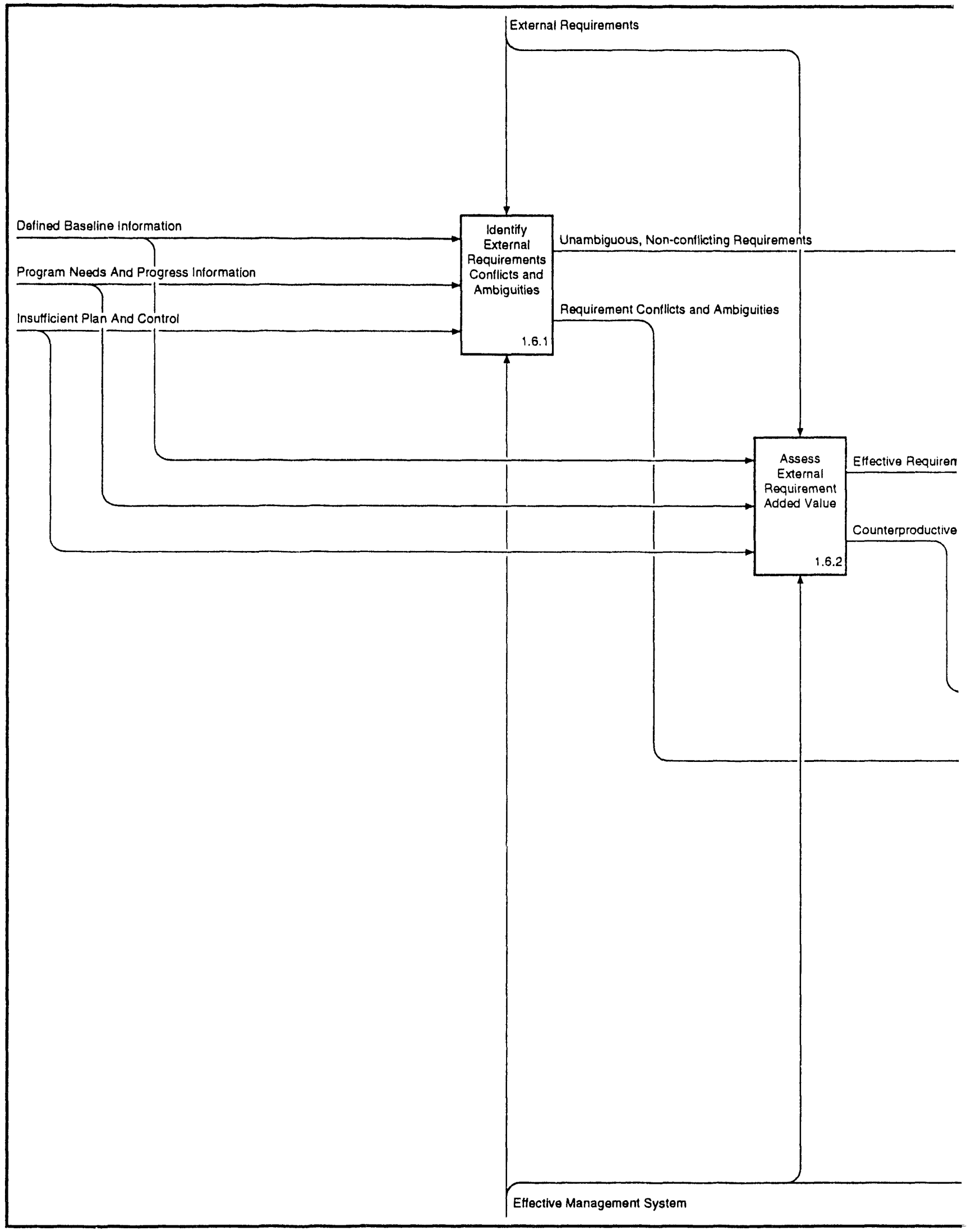




\section{Reinvent Government}

Applicable External Requirements

No Requirements

erproductive Requirements

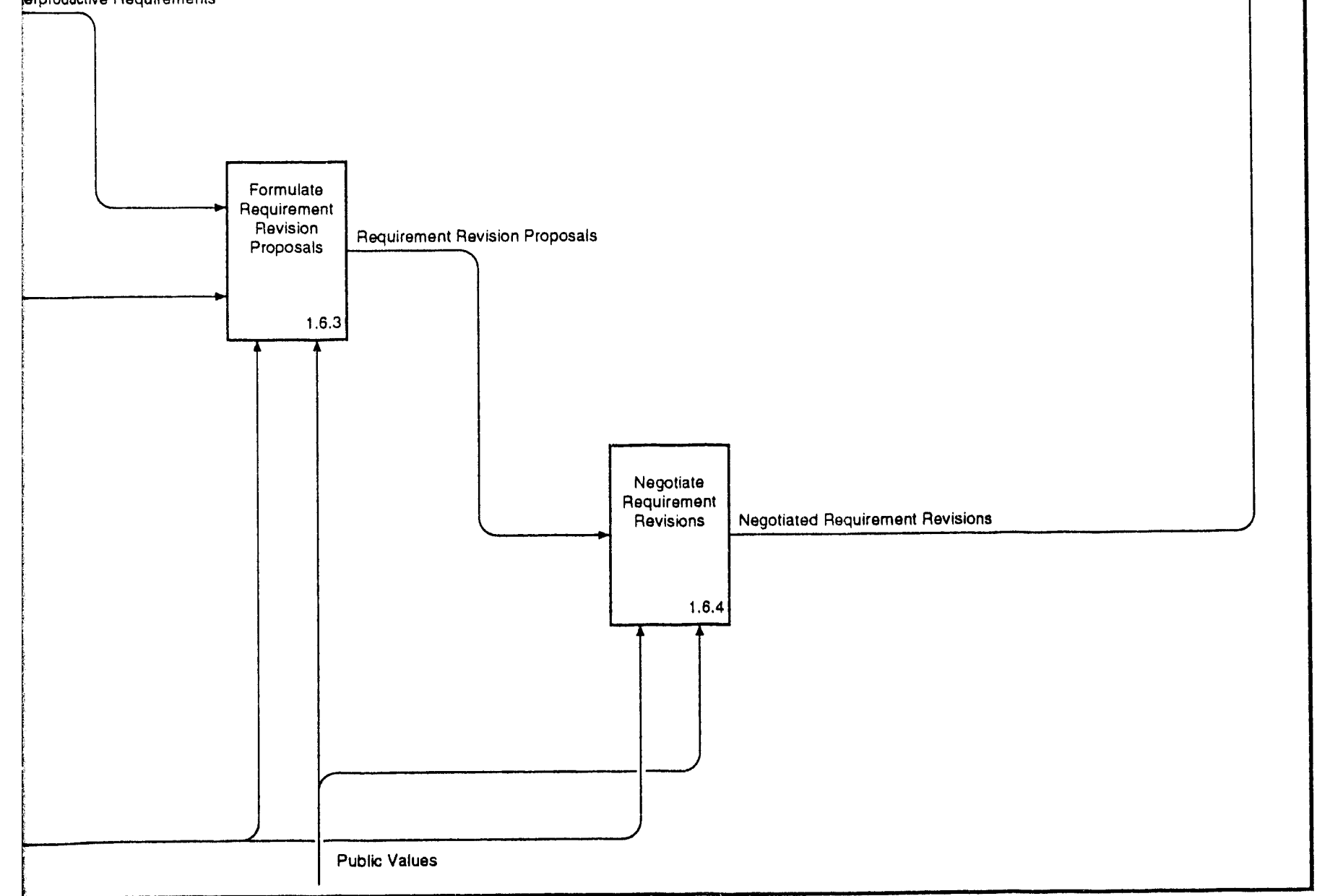




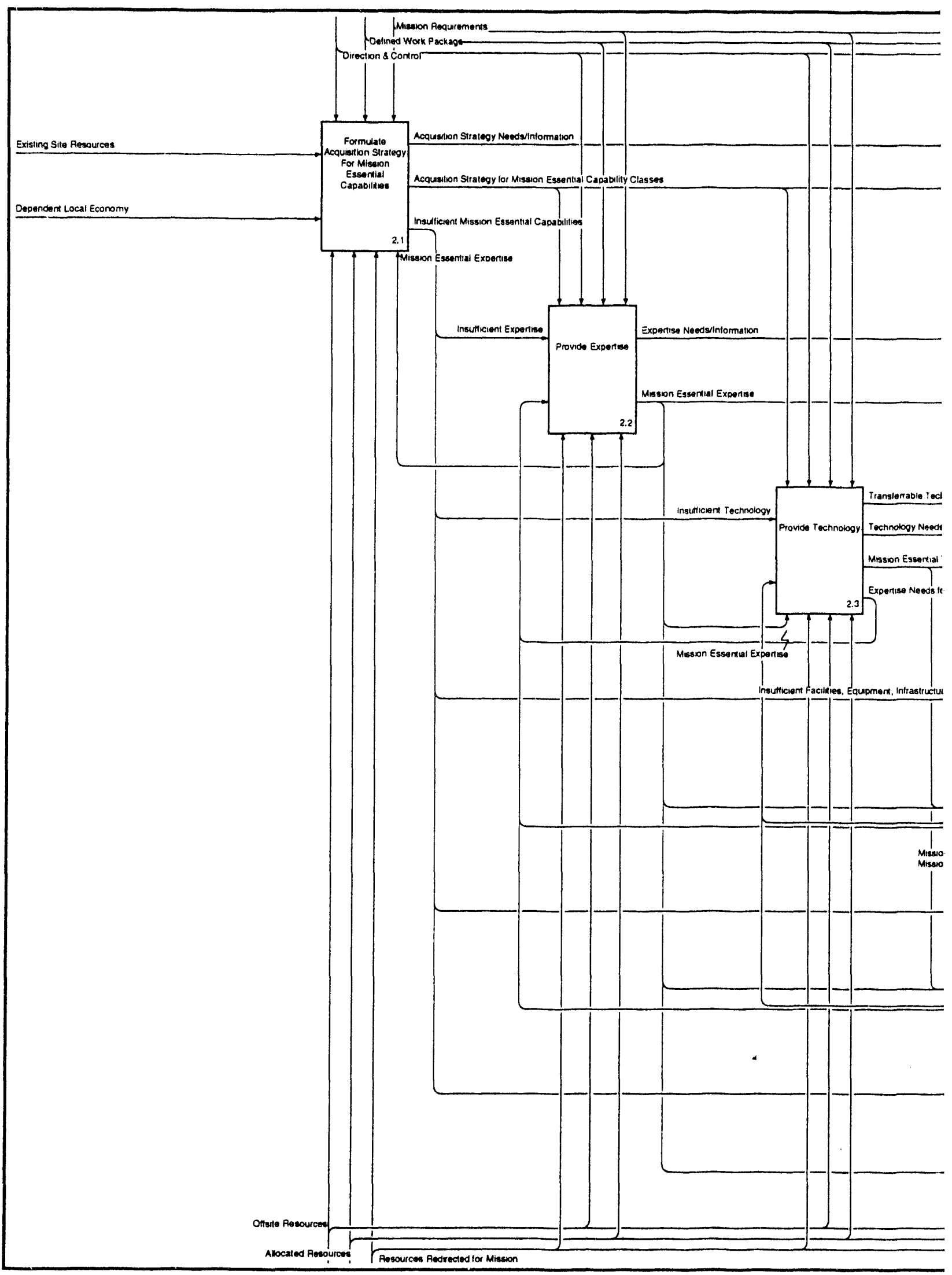




\section{ission Essential Capabilities}

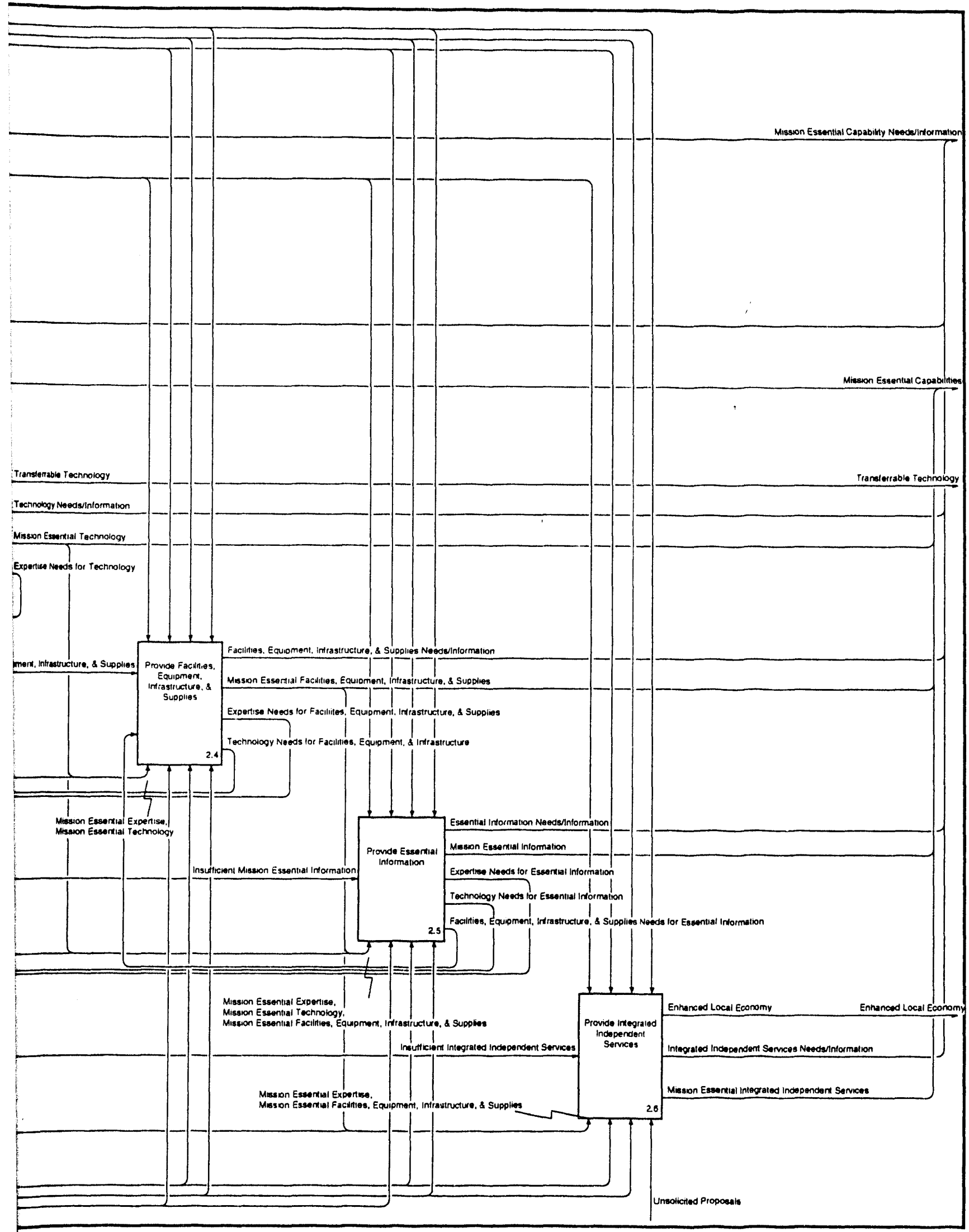

[2/ Acquire Mission Essential Capabilities 


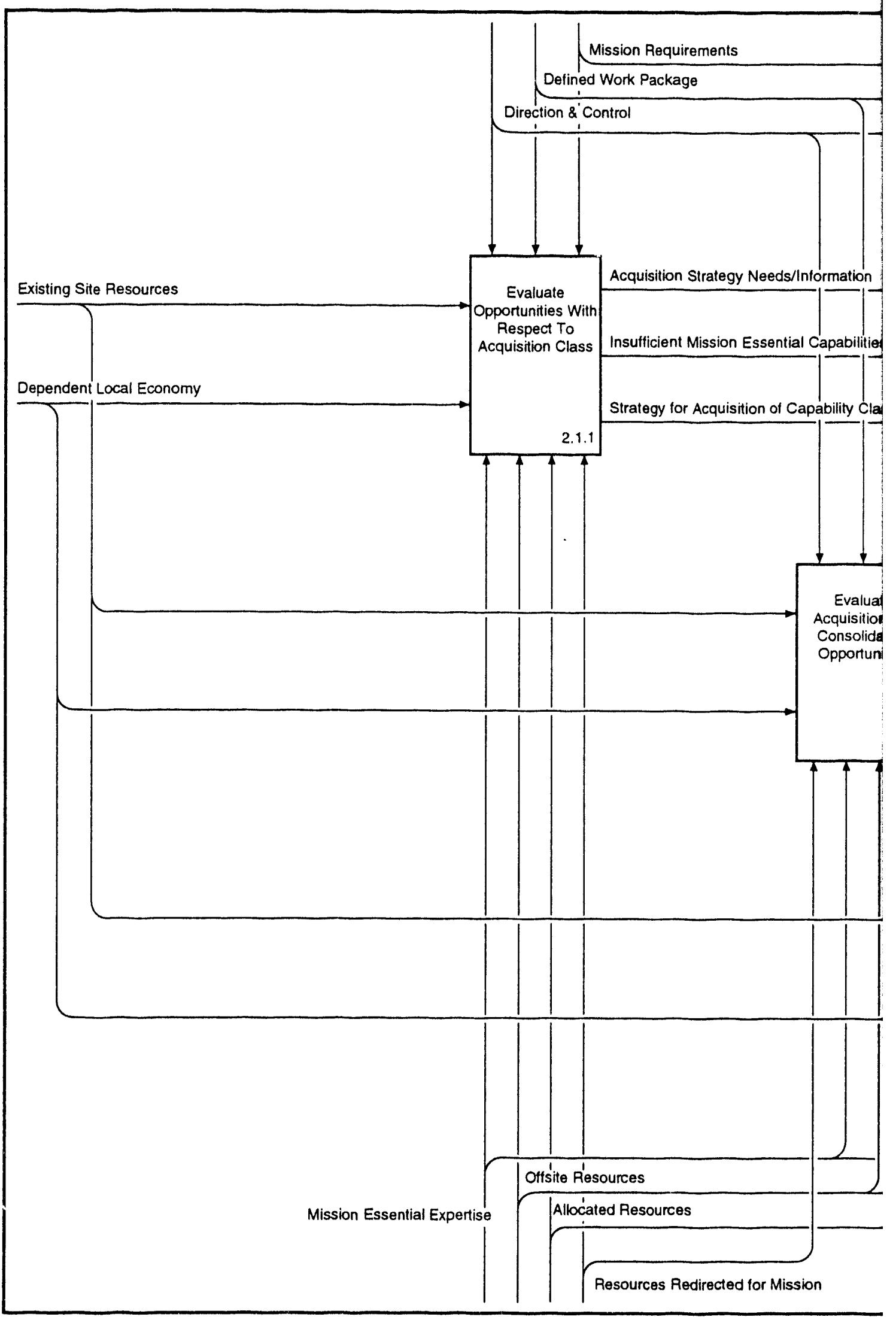




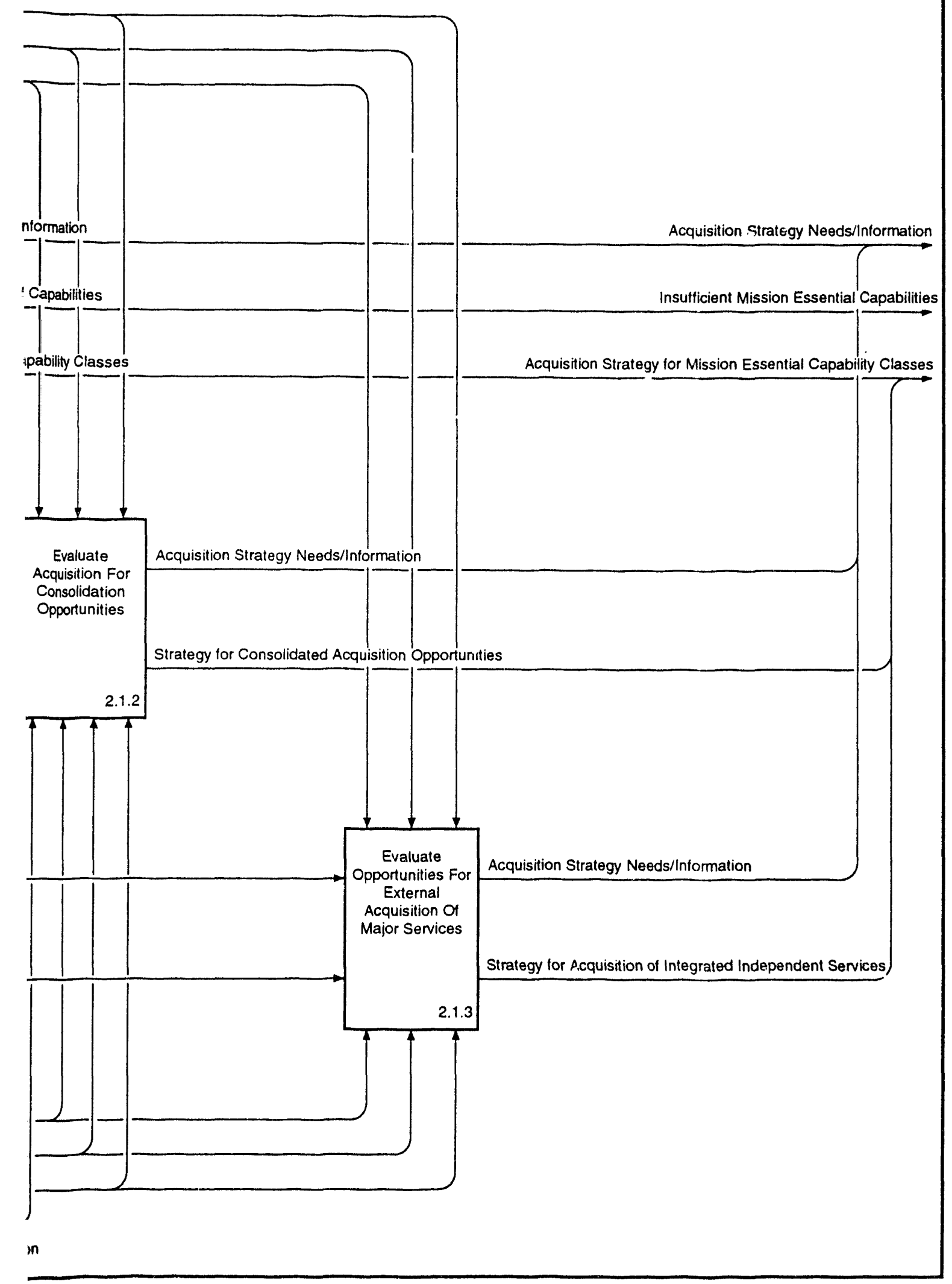




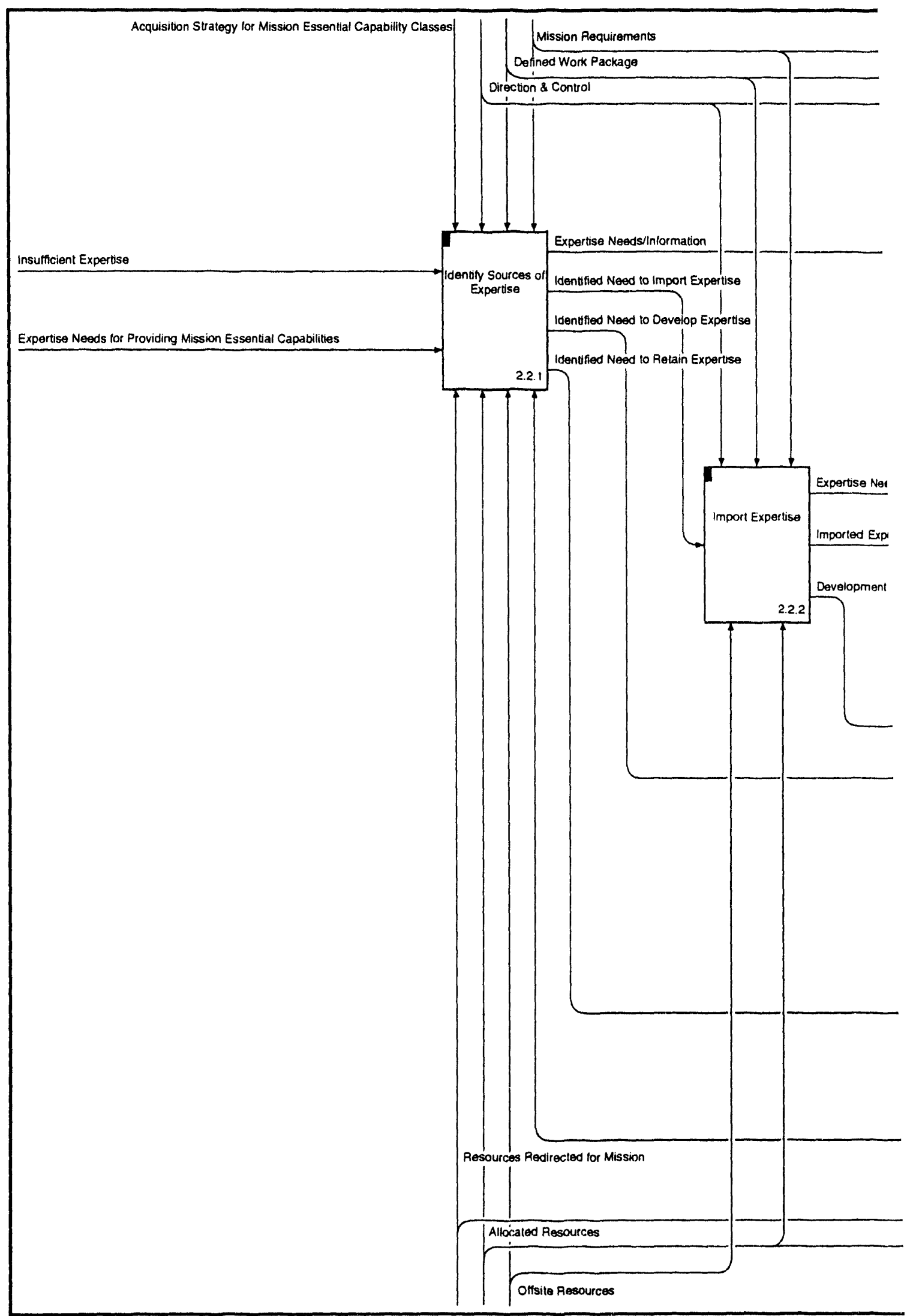




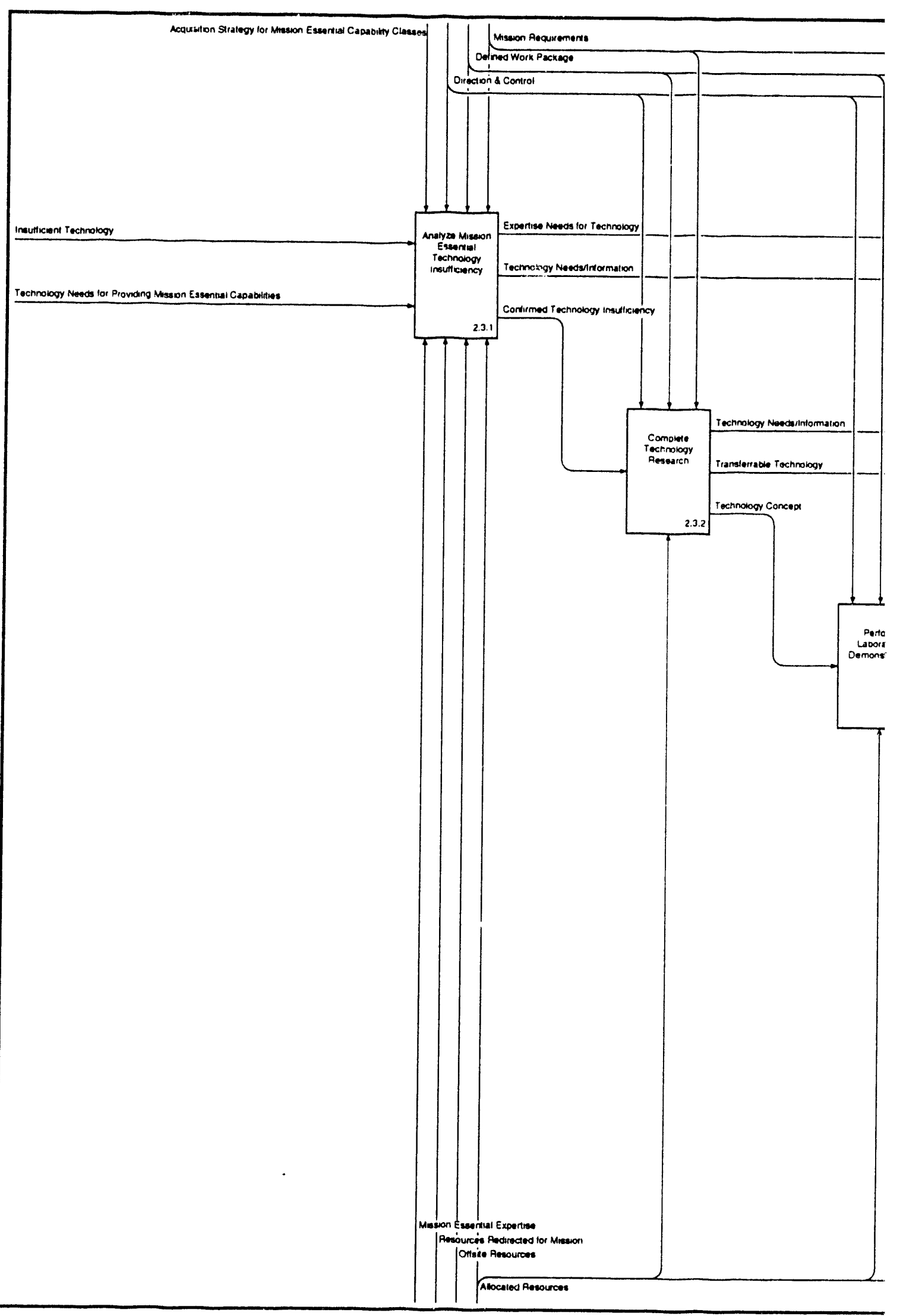




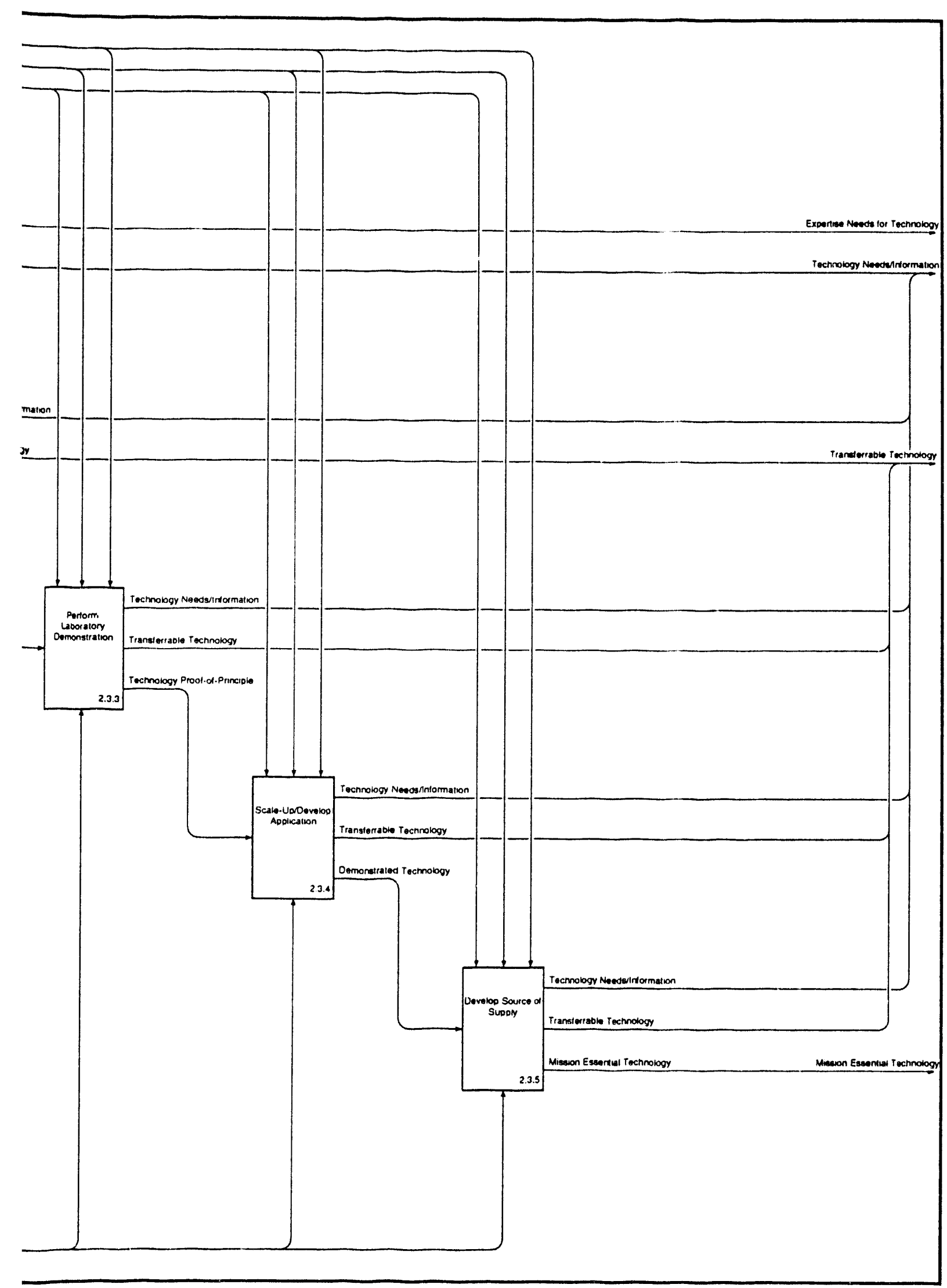


[2.4] Provide Facilities, Equipme

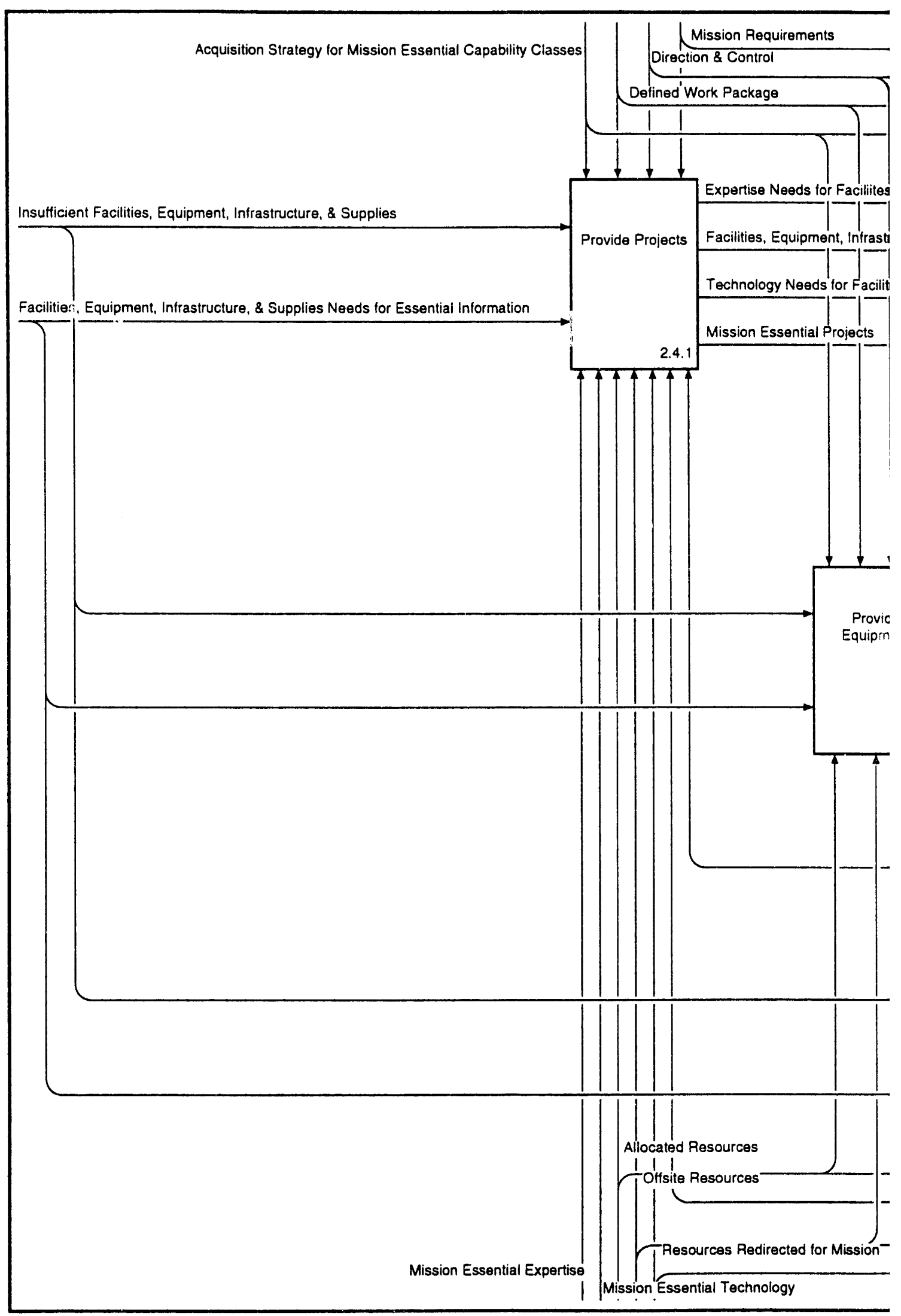




\section{uipment, Infrastructure, \& Supplies}

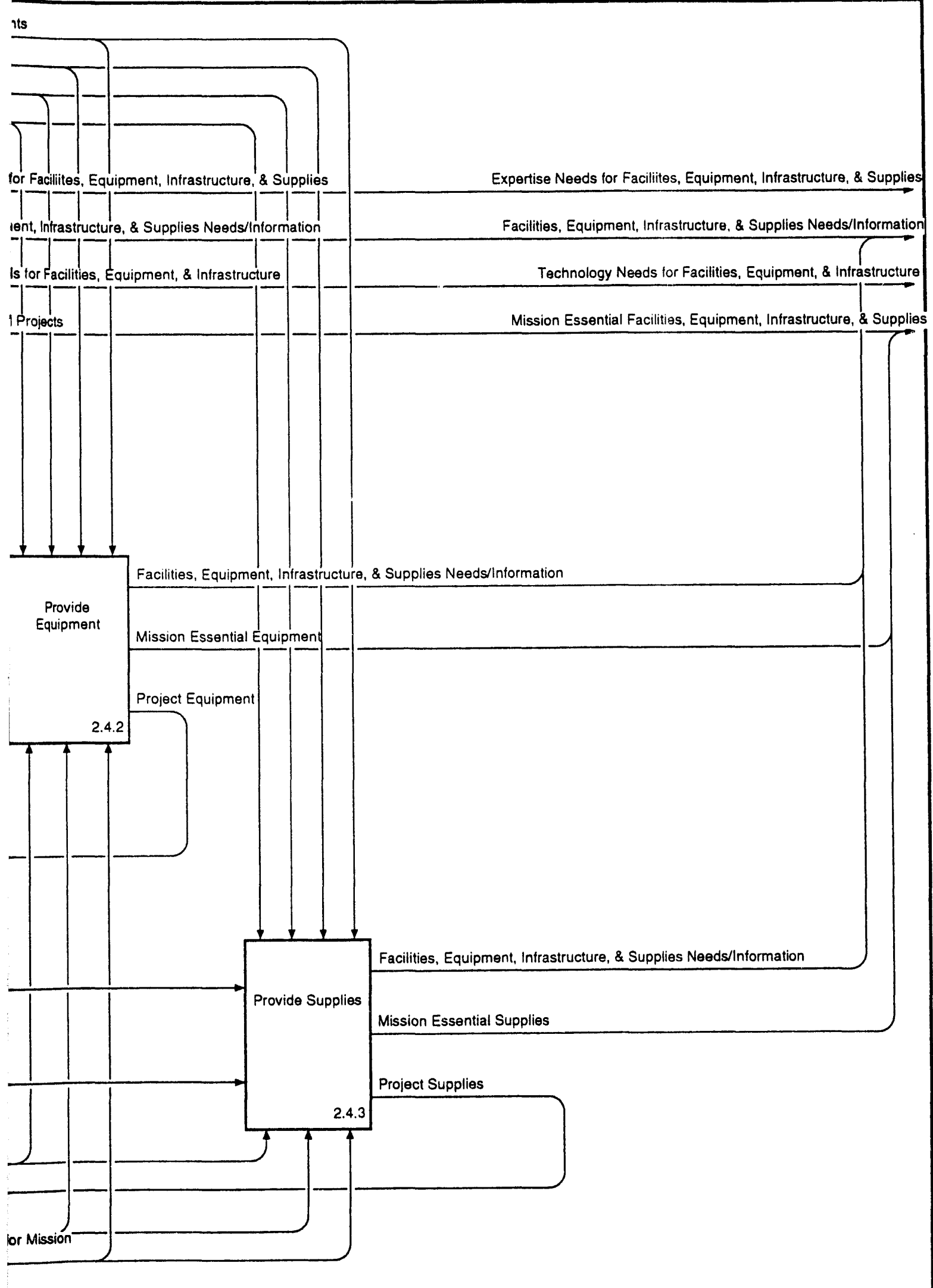




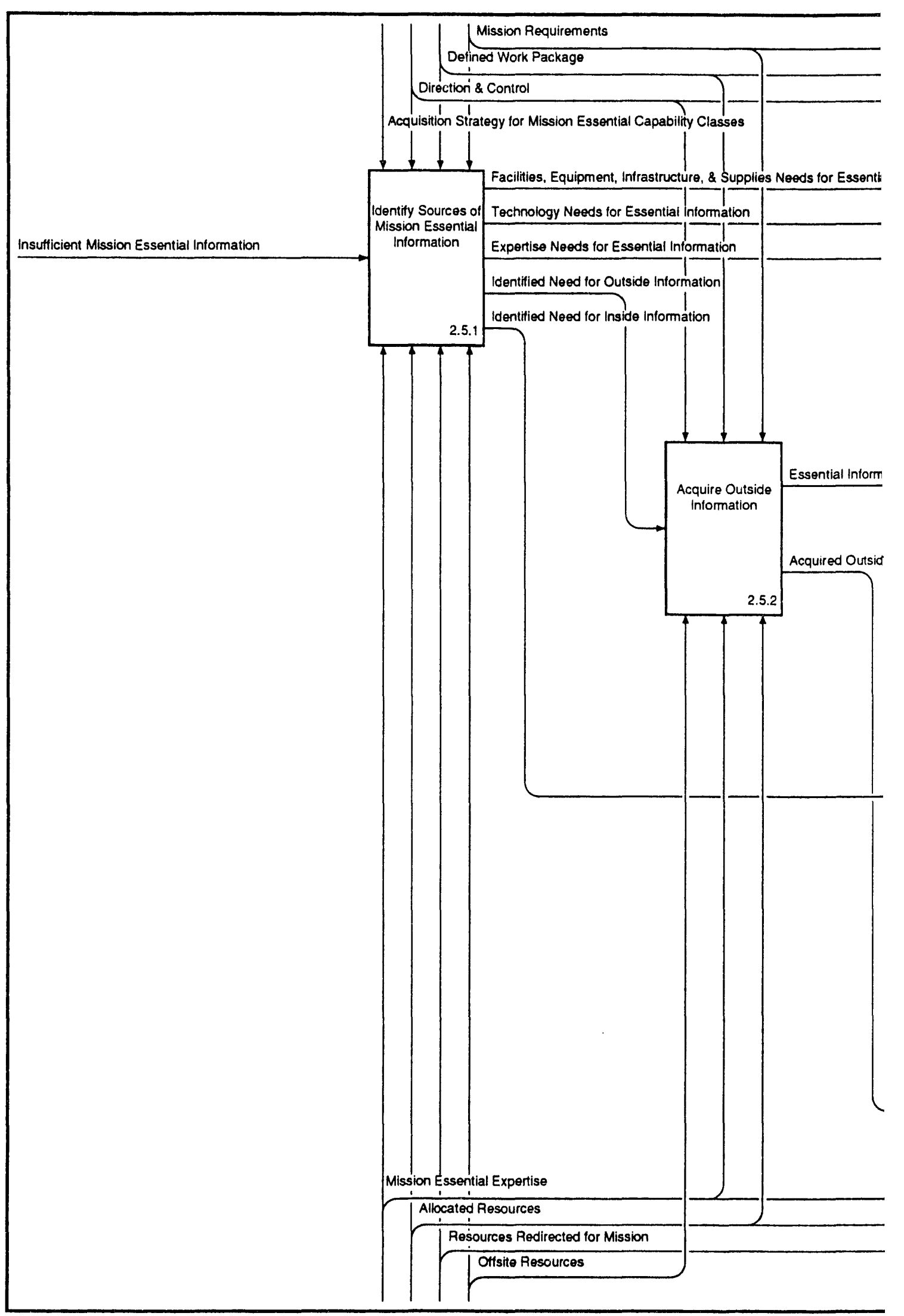




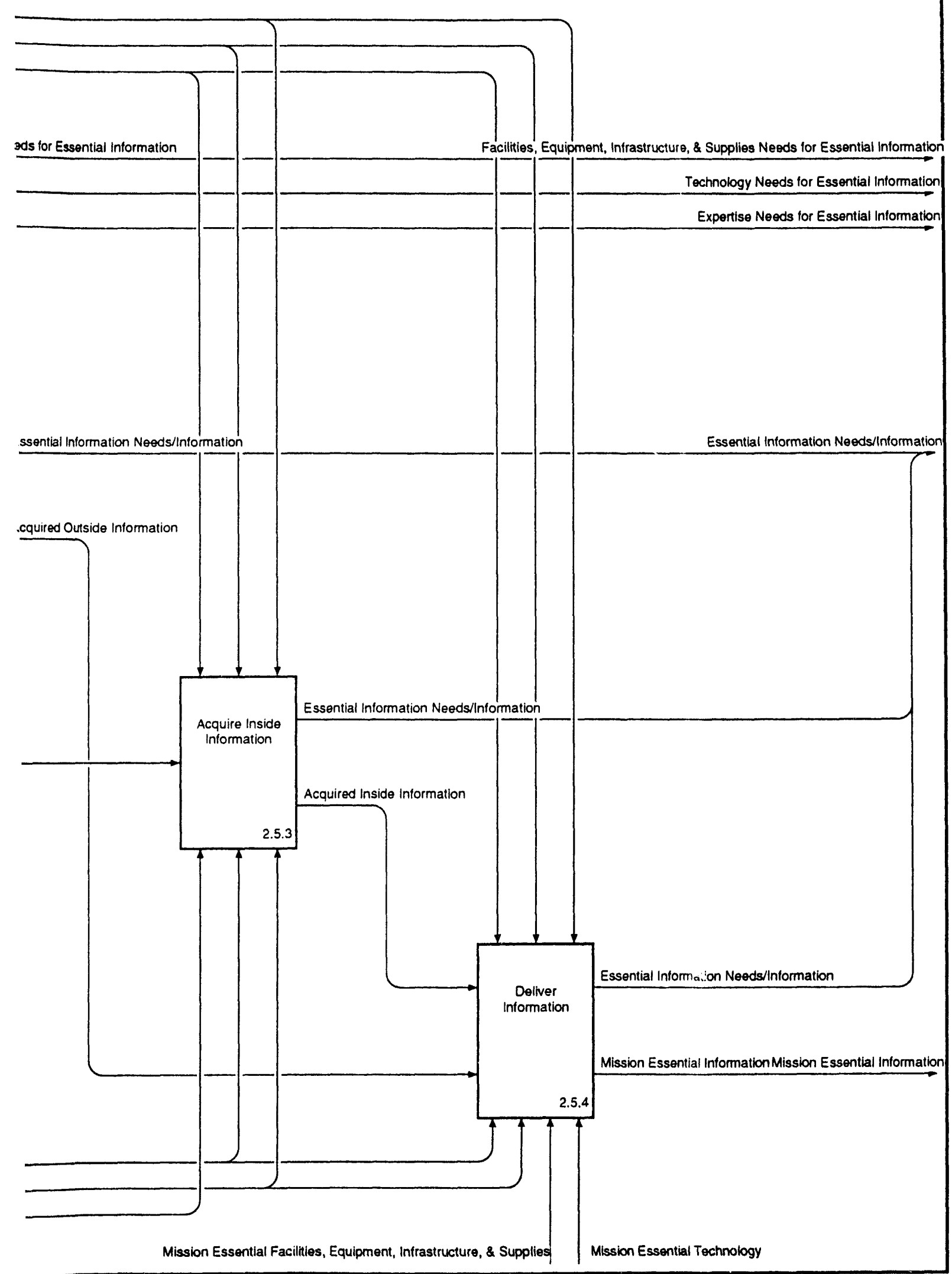




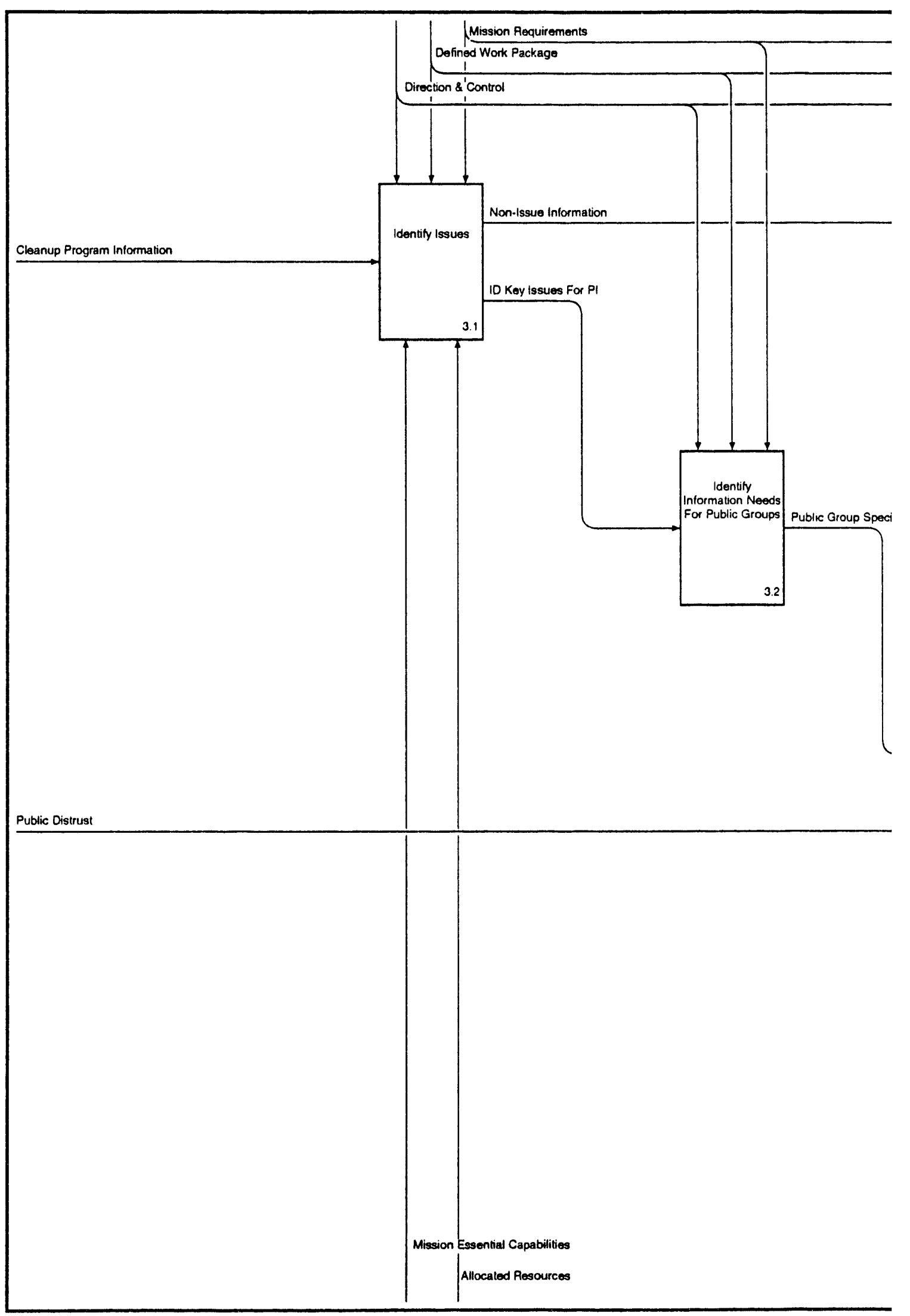




\section{Public Acceptance}

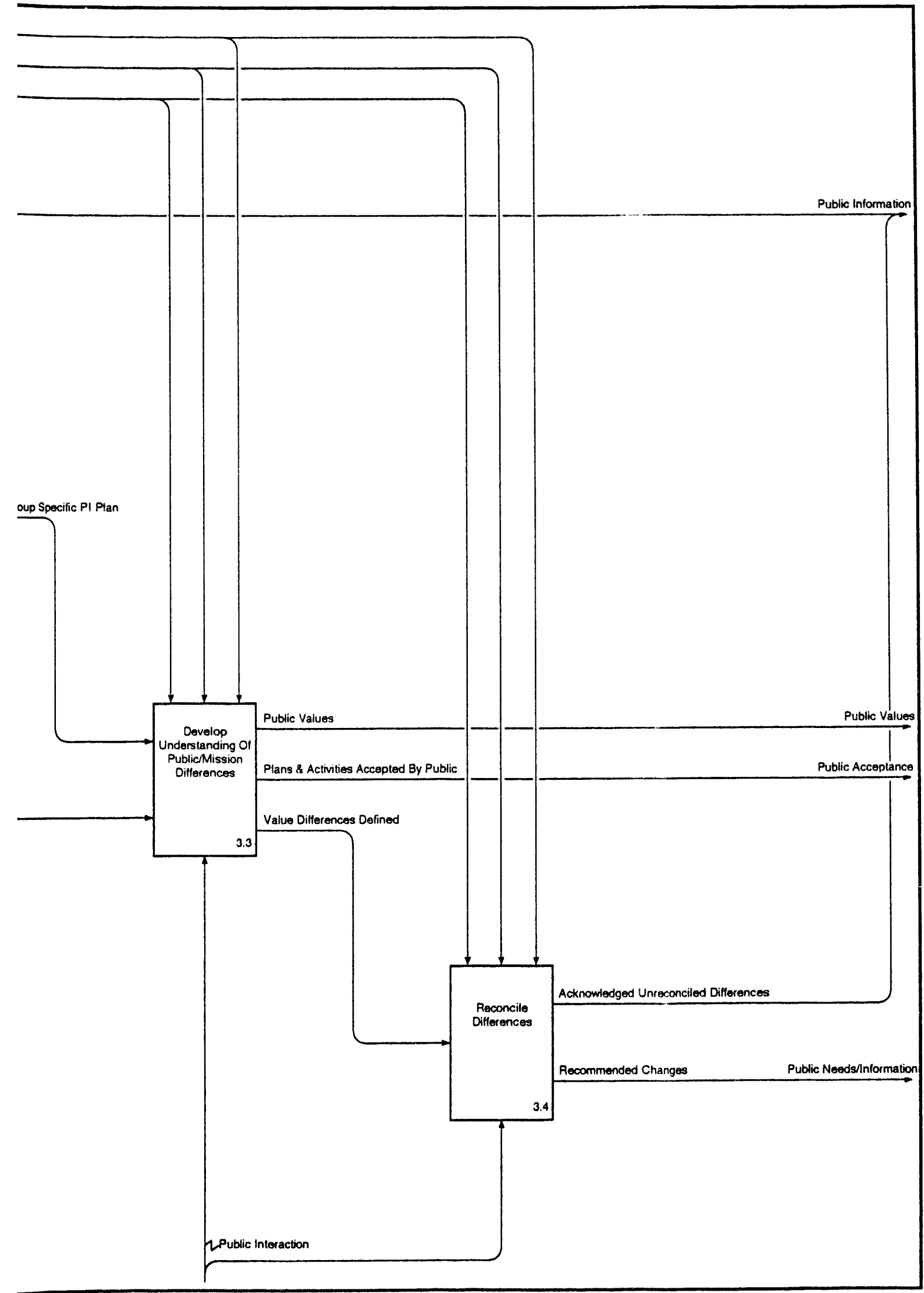




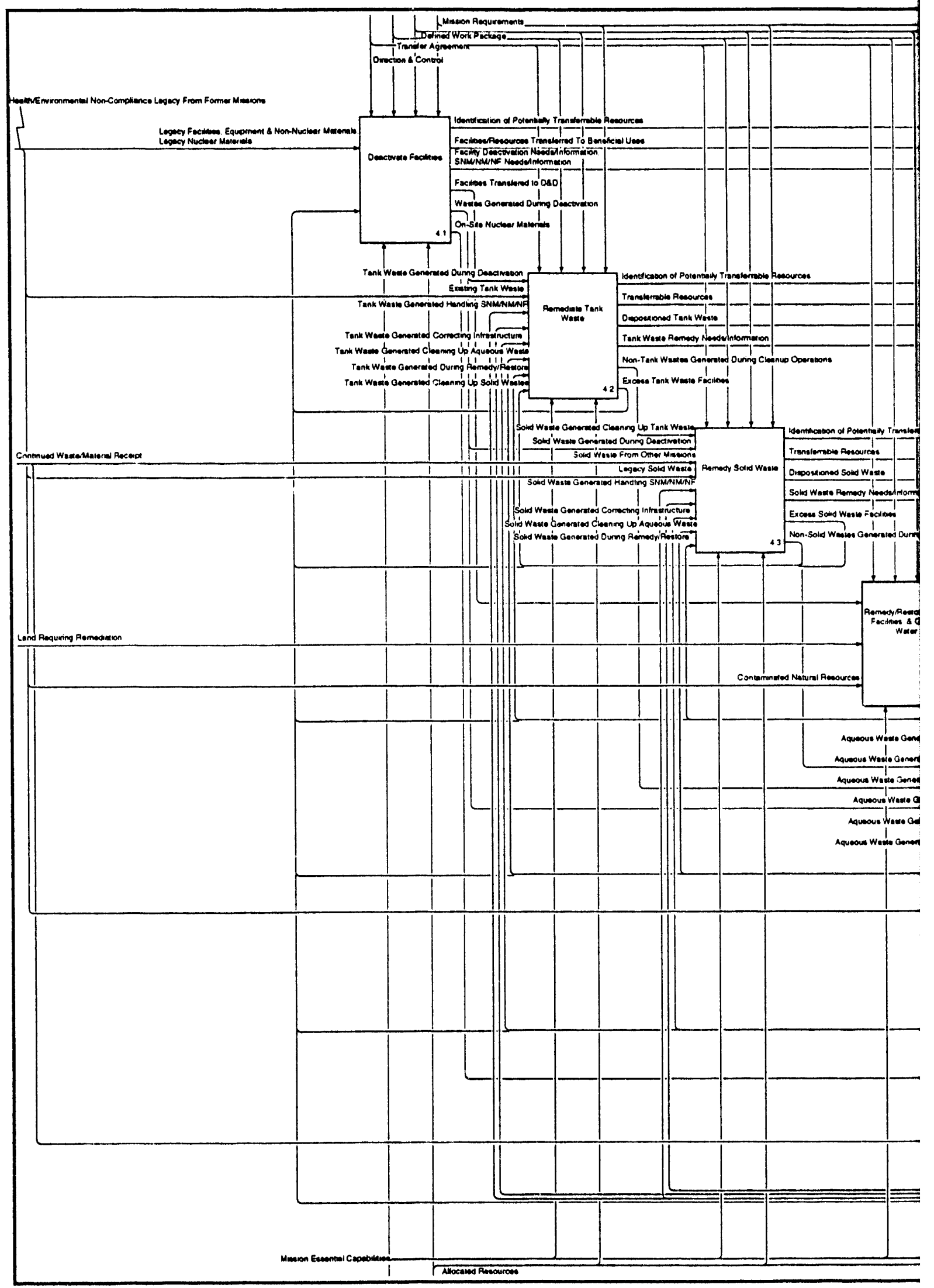

Hanford Site Systems Engineering 


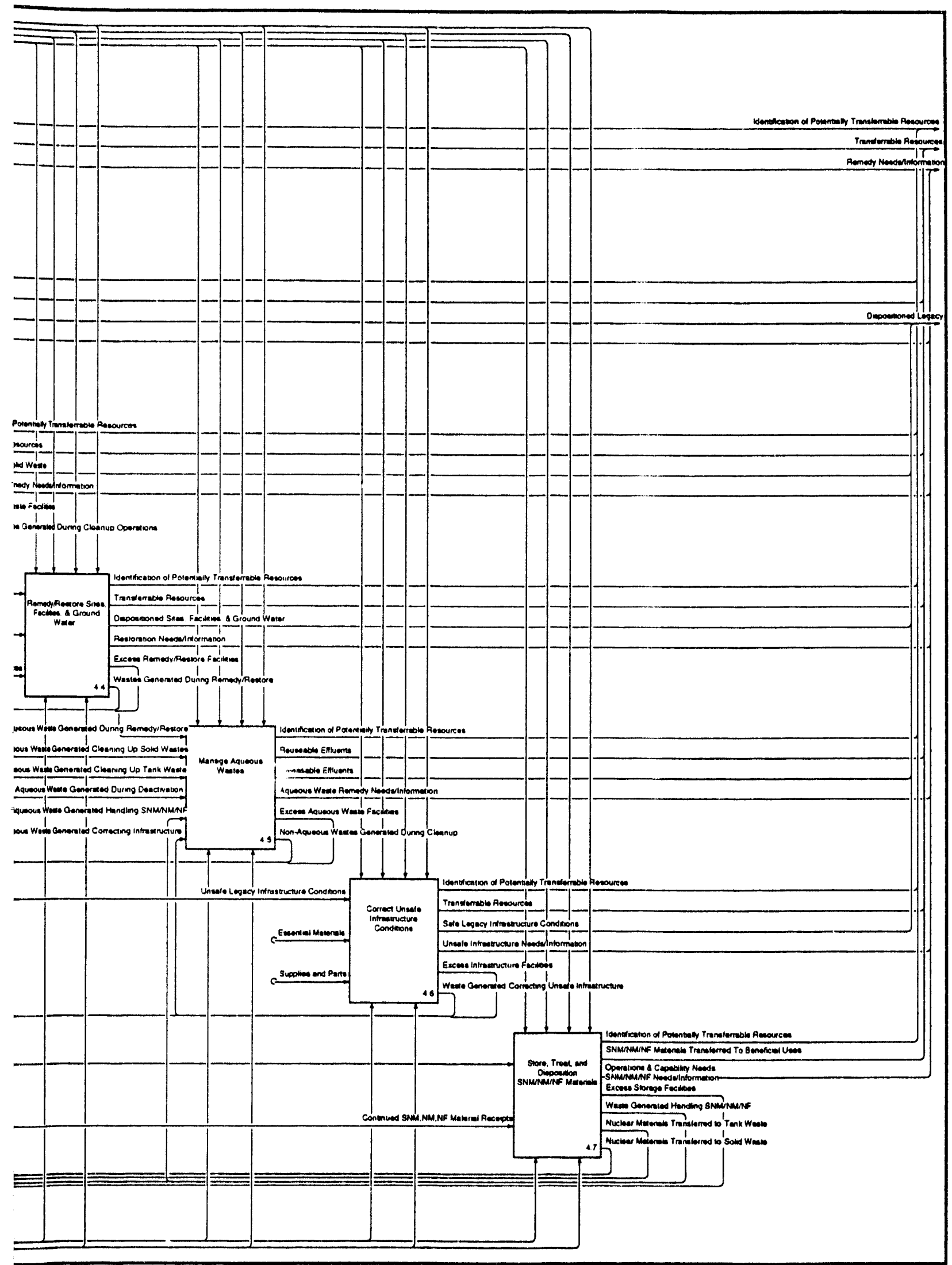


[4.1] Deactiv

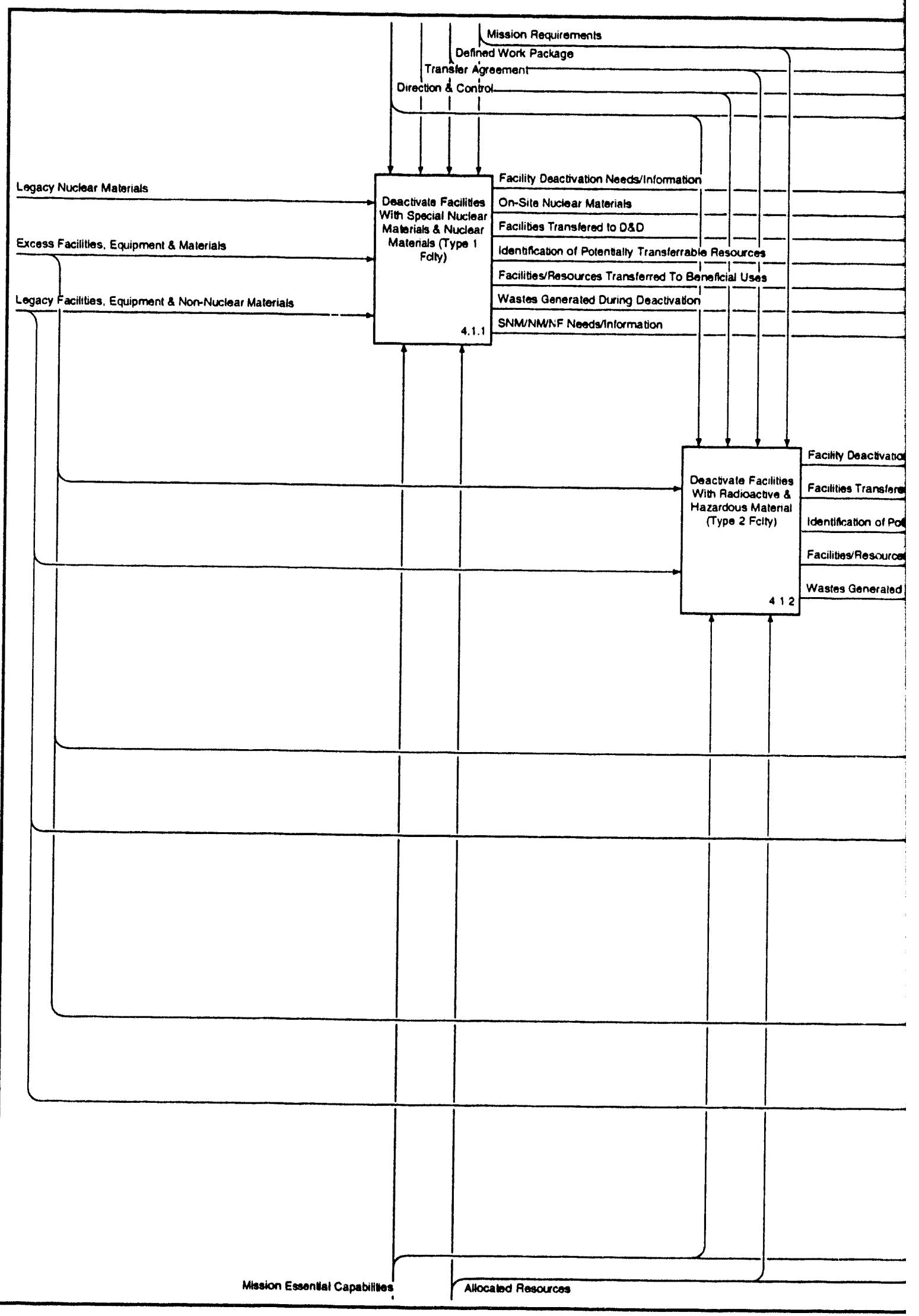

Hanford Site Systems Engineering 


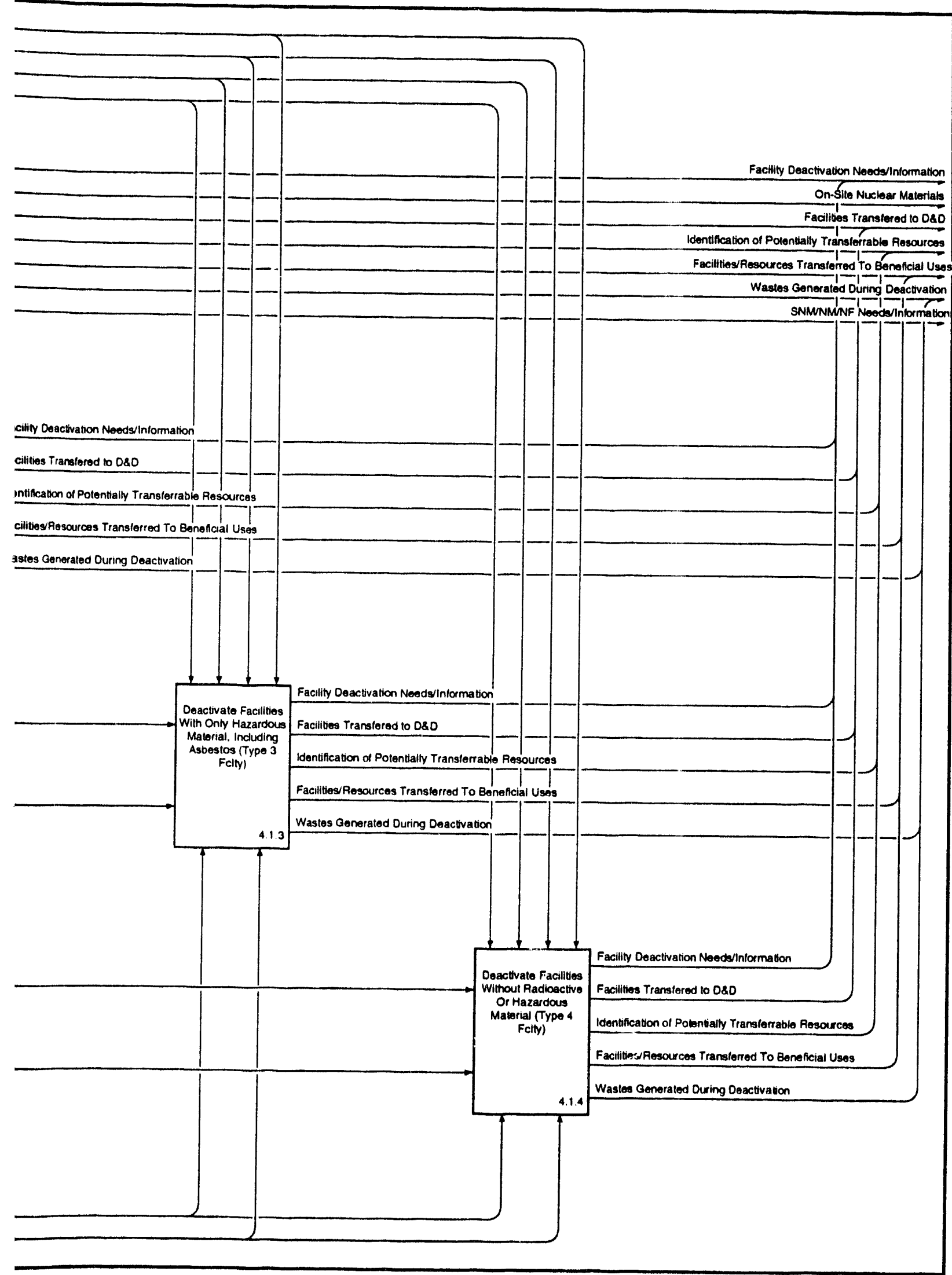




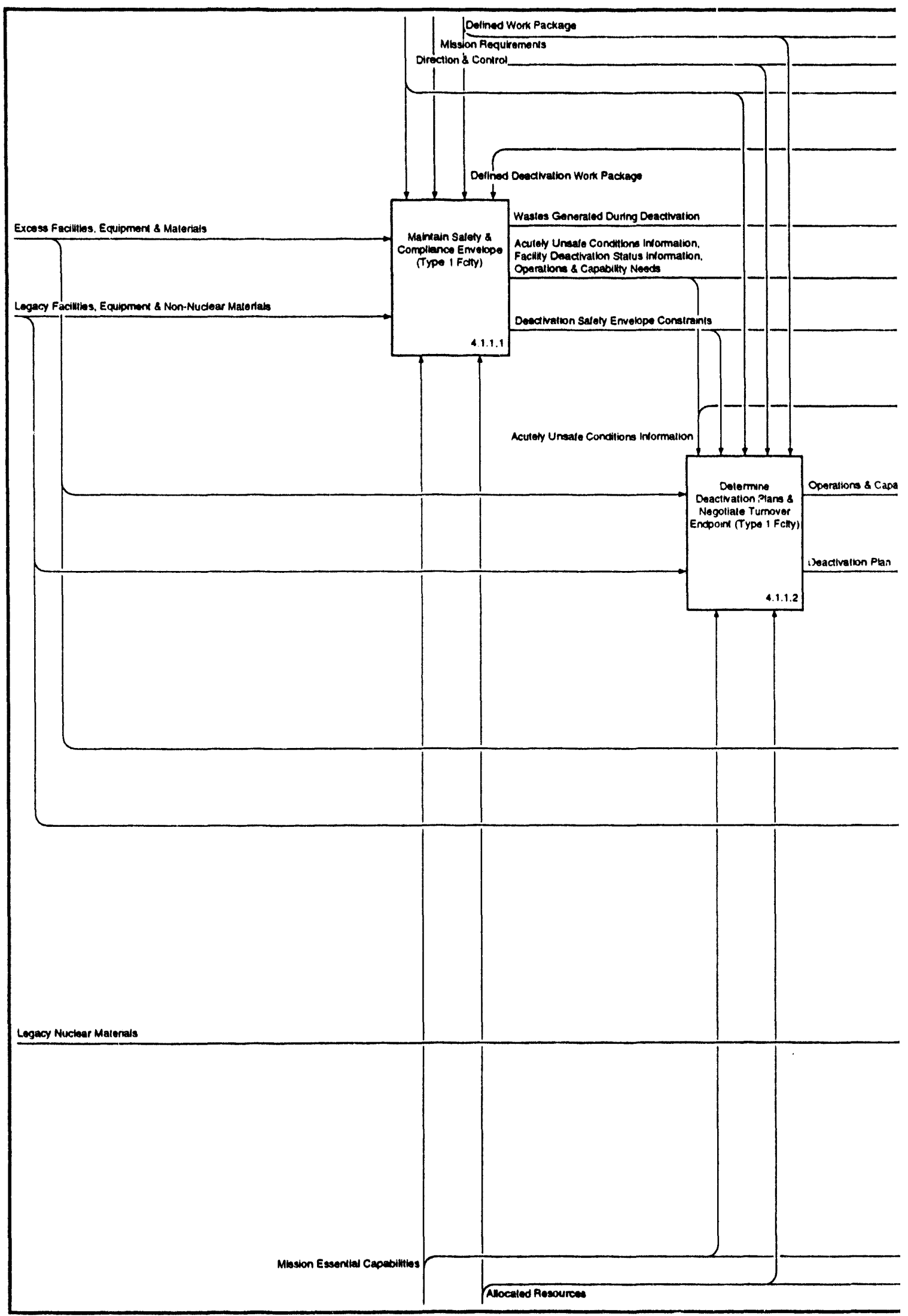




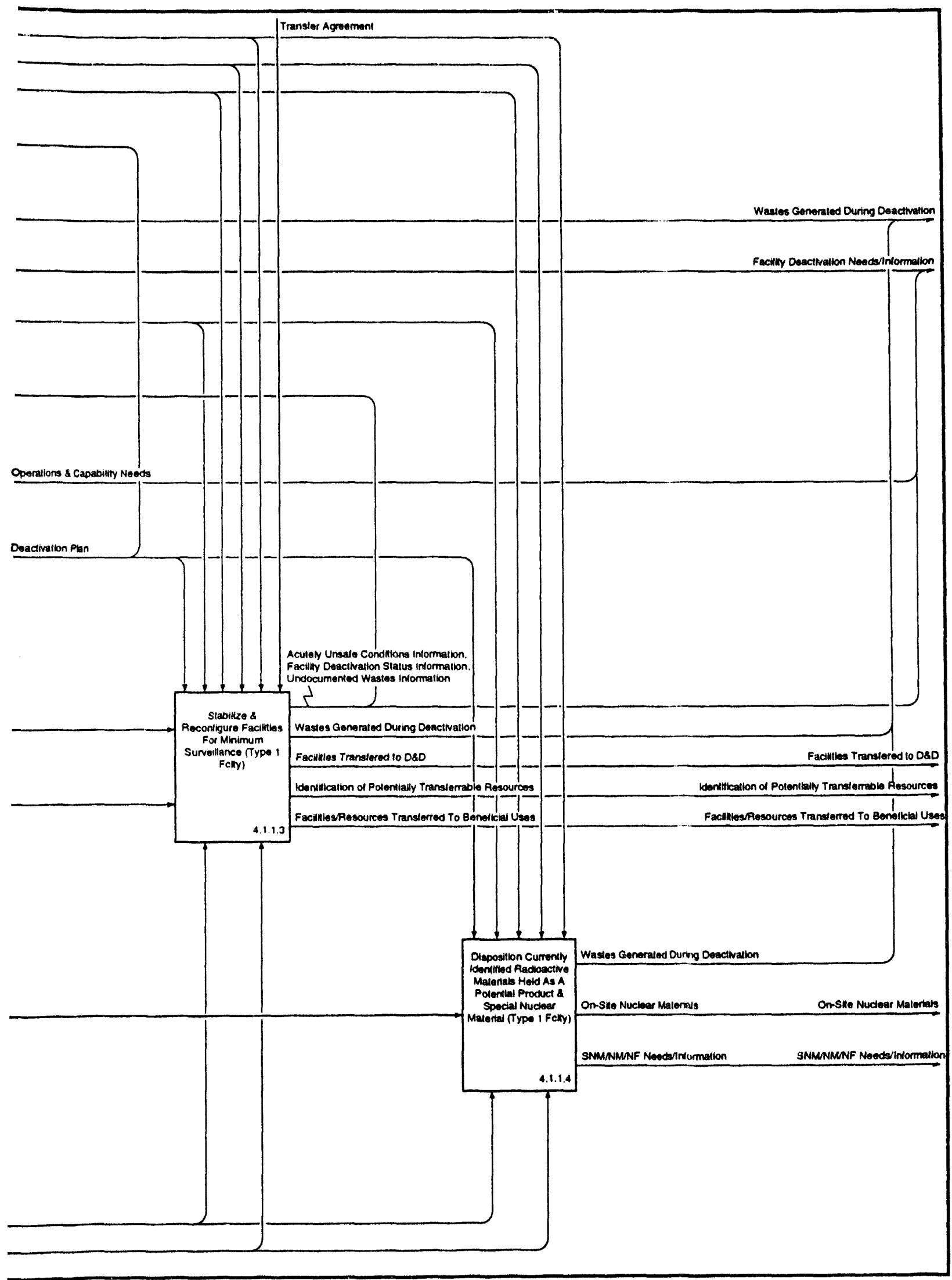




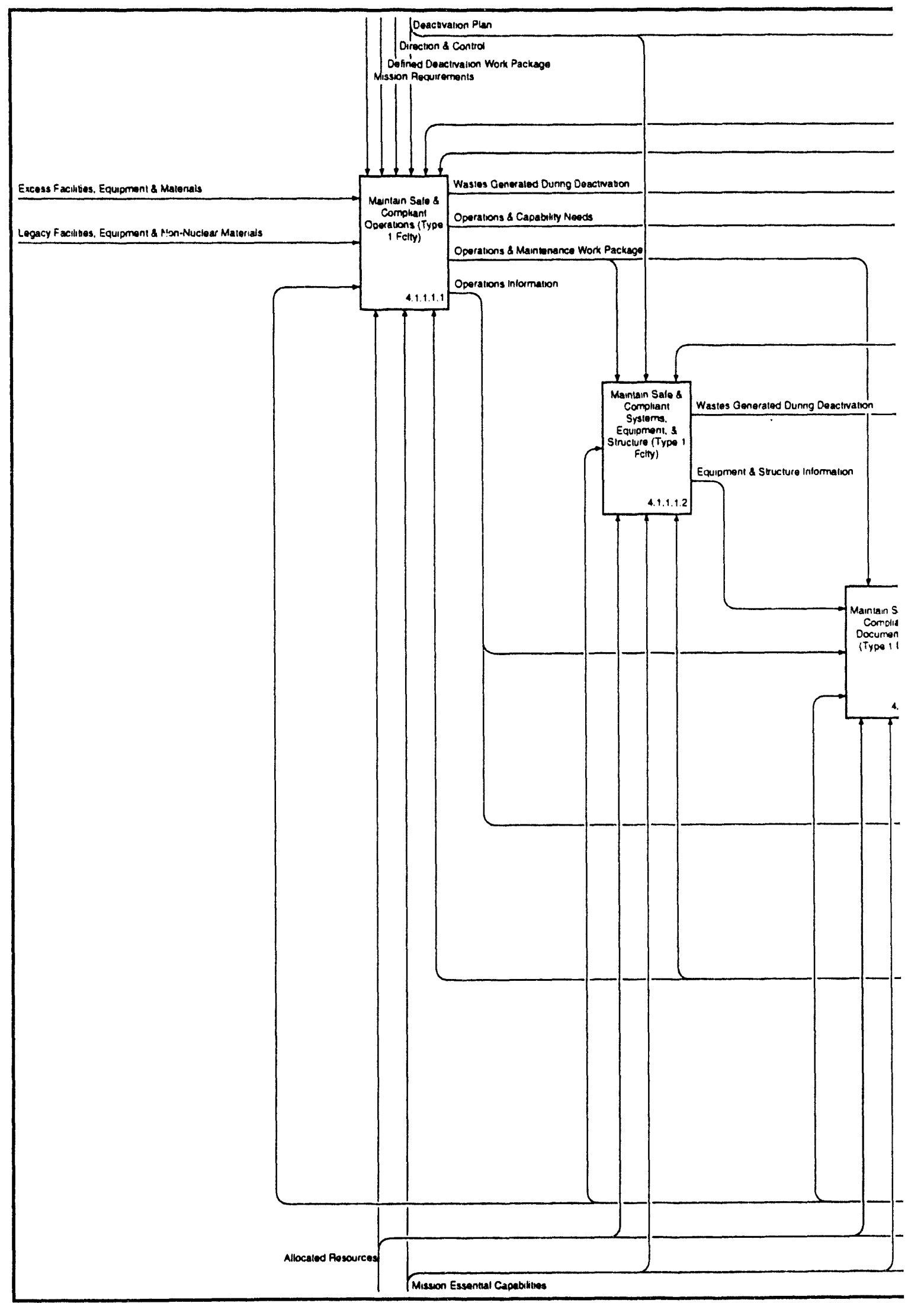




\section{\& Compliance Envelope (Type 1 Fclty)}

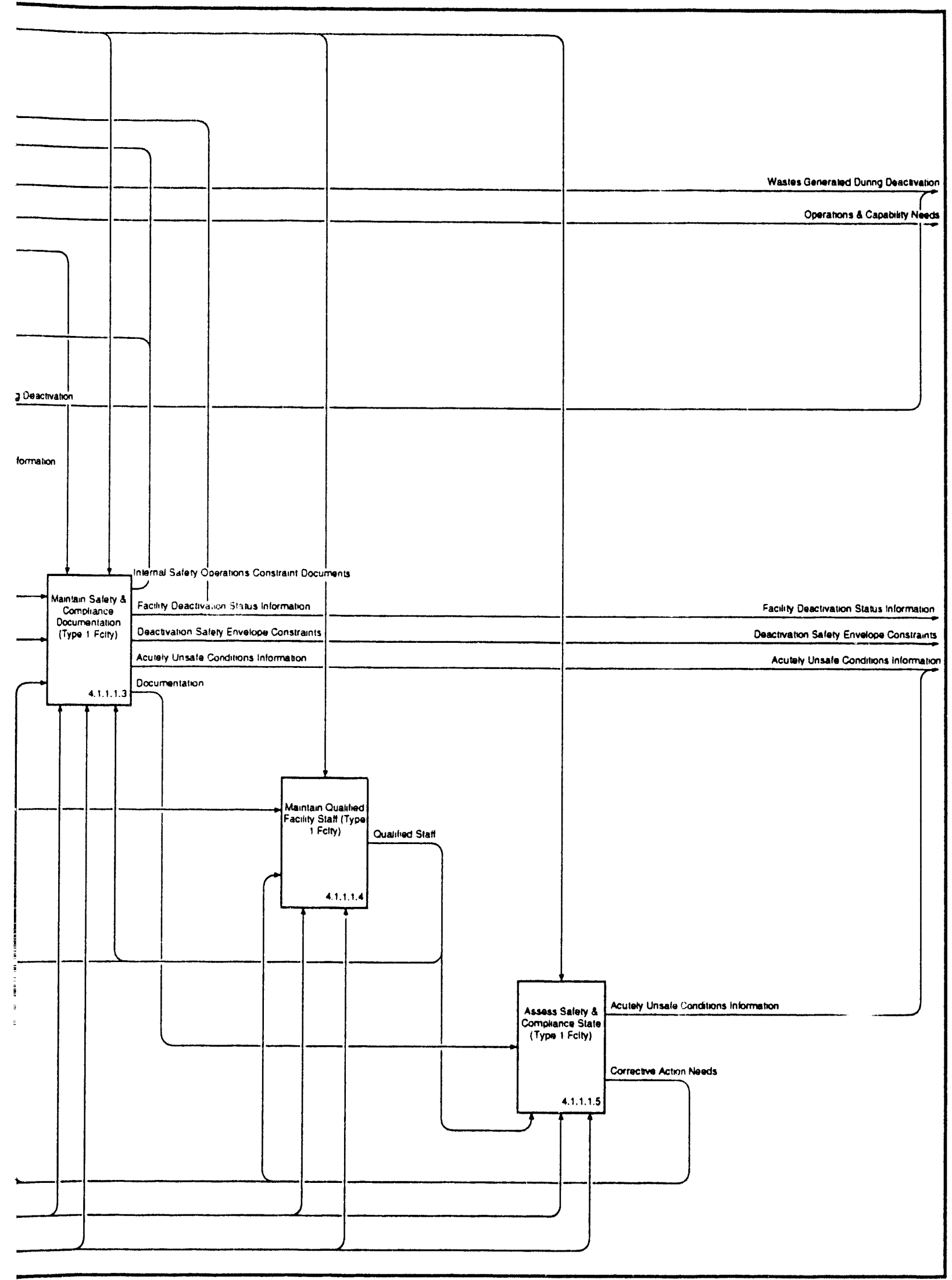


[4.1.1.2] Determine Deactivation Plans \& N

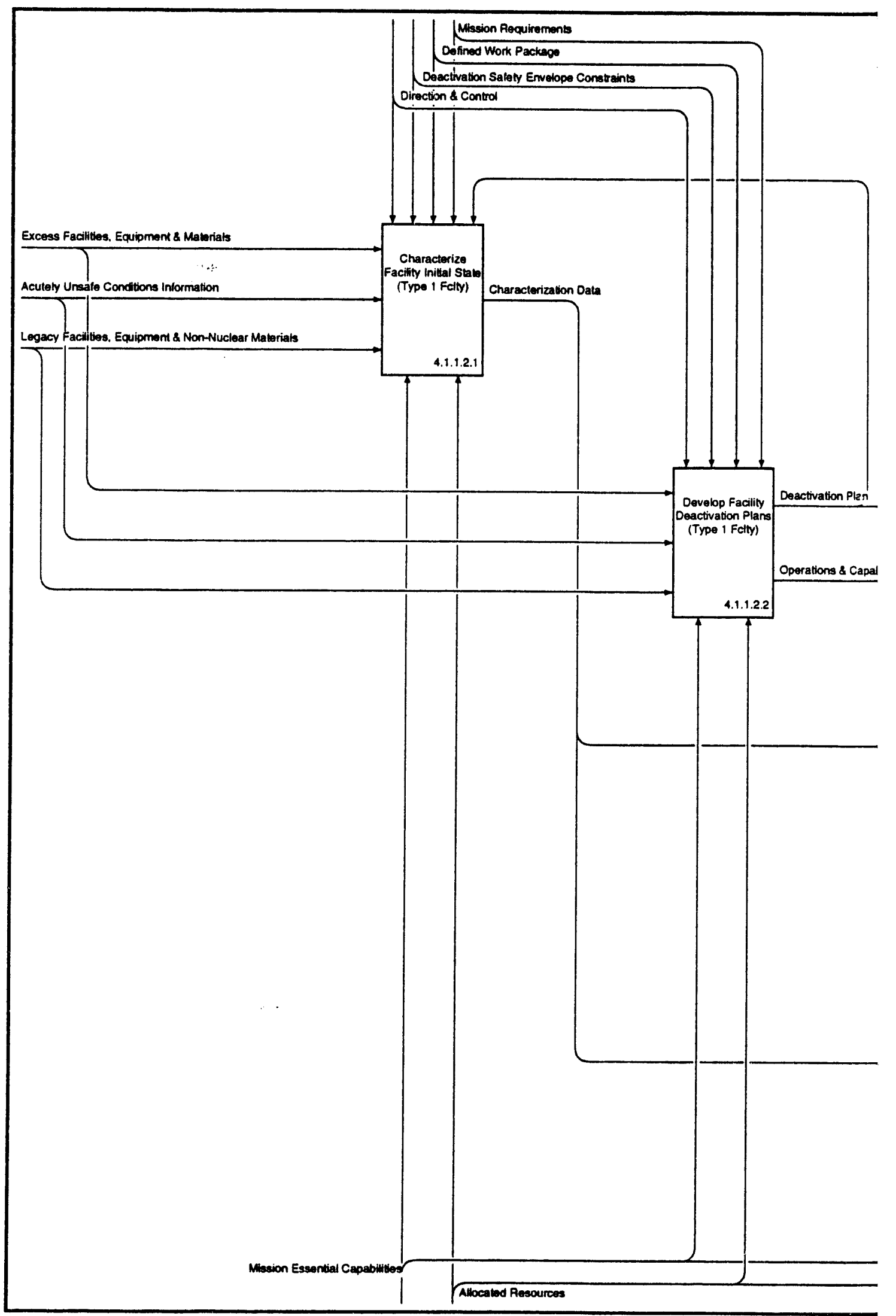




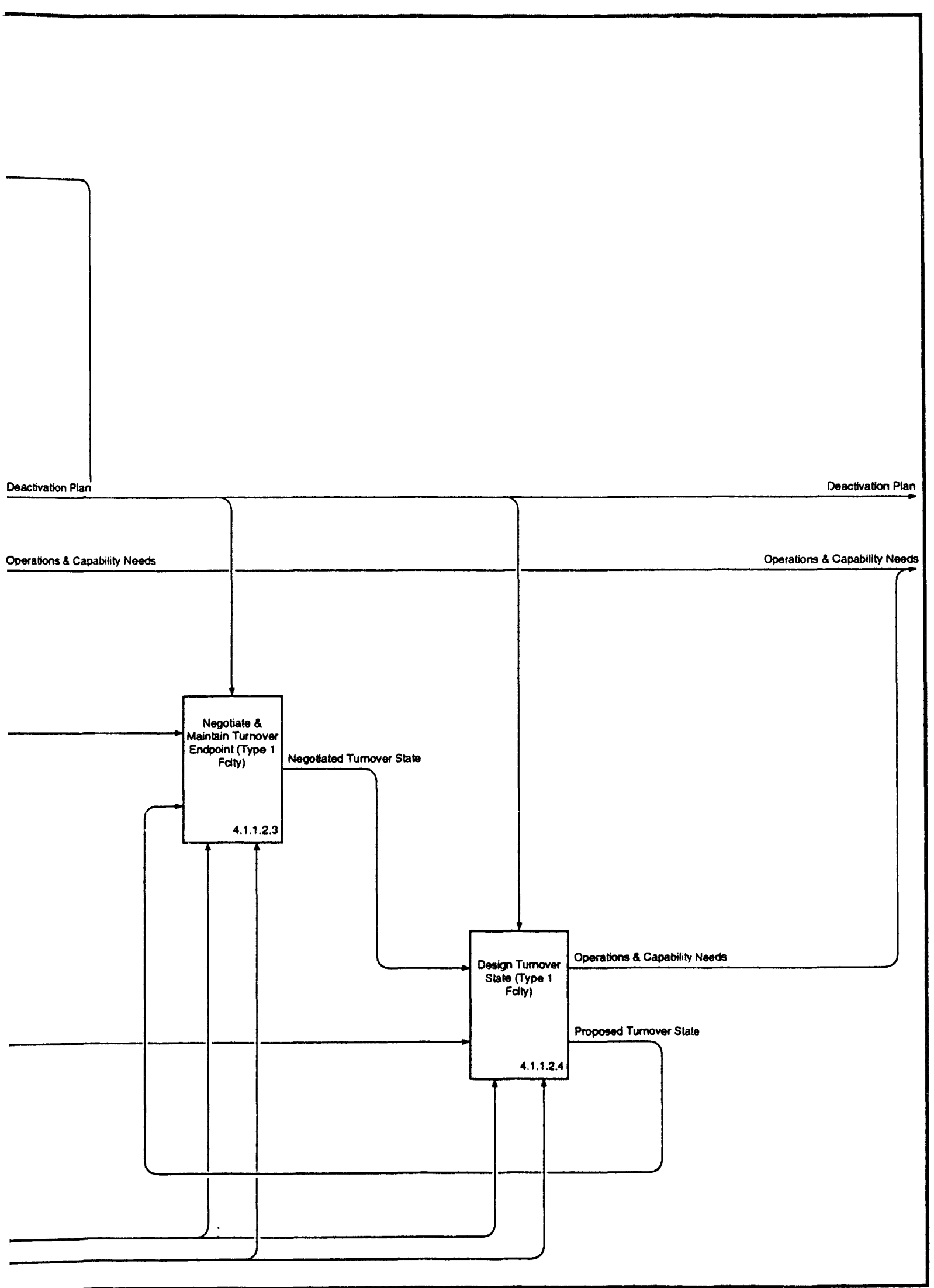

[4.1.1.2] Determine Deactivation Plans \& Negotiate Turnover Endpoint (Type 1 Fclty) 


\section{[4.1.1.3] Stabilize \& Reconfigure Facilities}

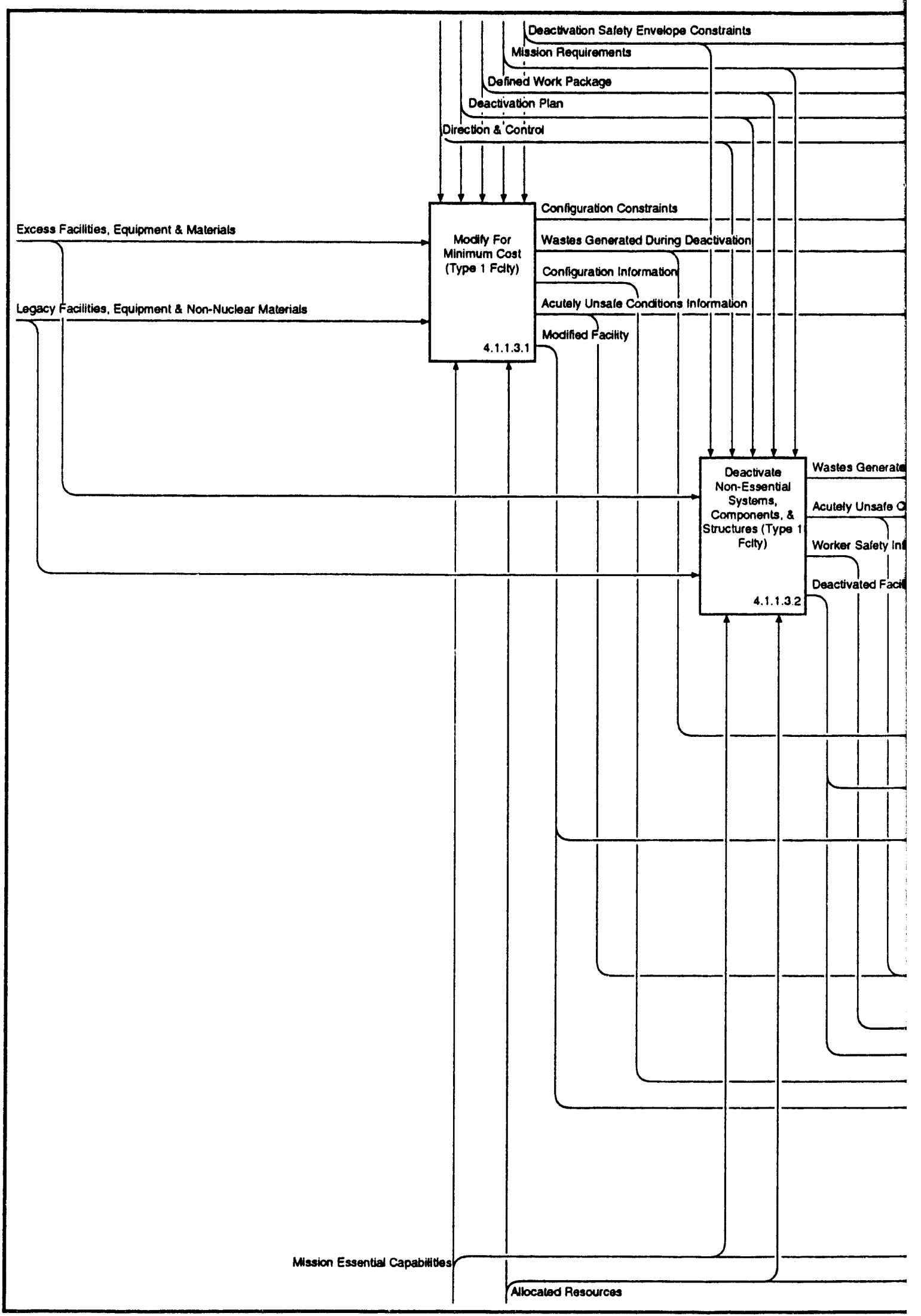




\section{gcilities For Minimum Surveillance (Type 1 Fclty)}

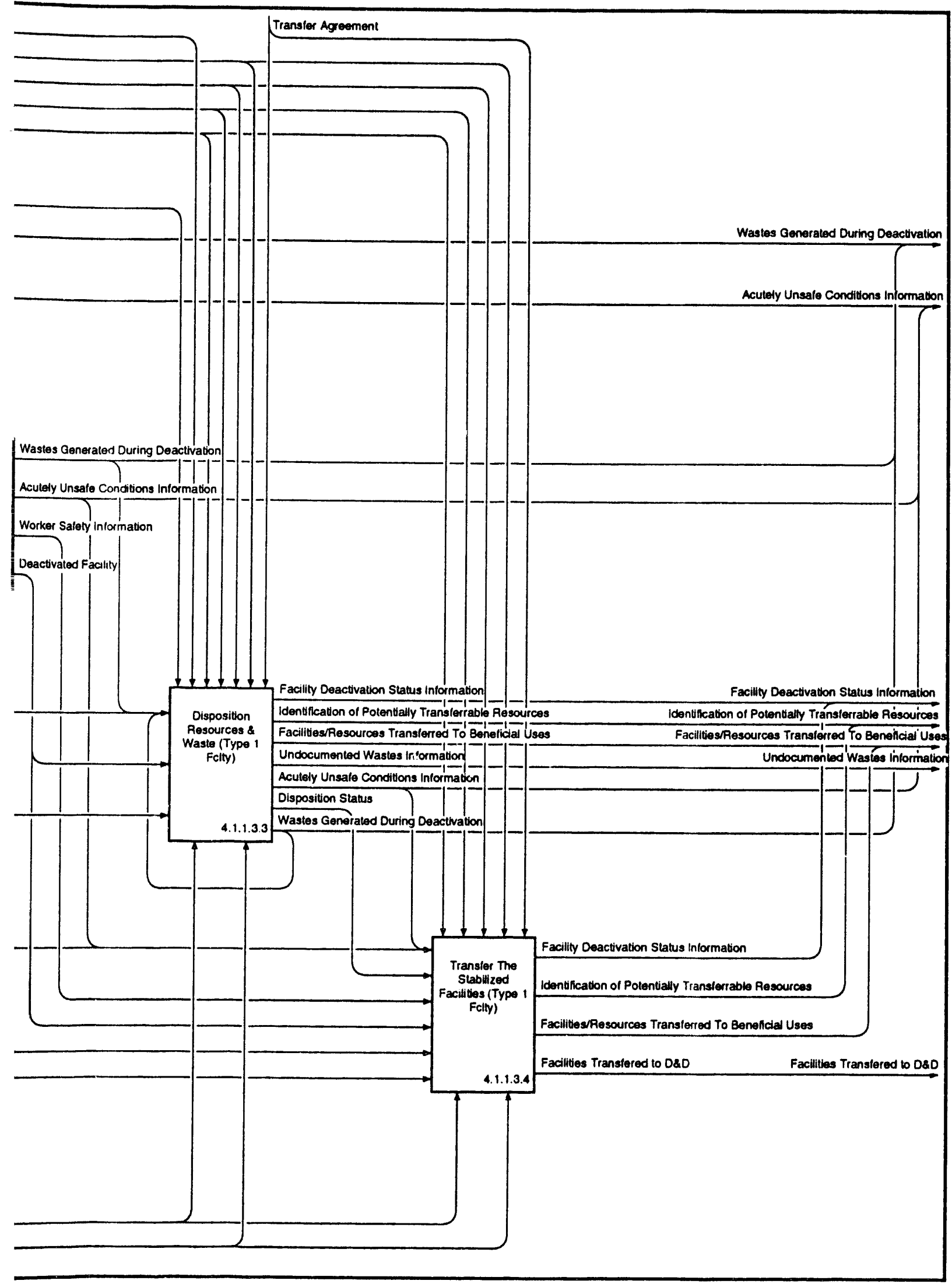

[4.1.1.3] Stabilize \& Reconfigure Facilities For Minimum Surveillance (Type I Fclry) 


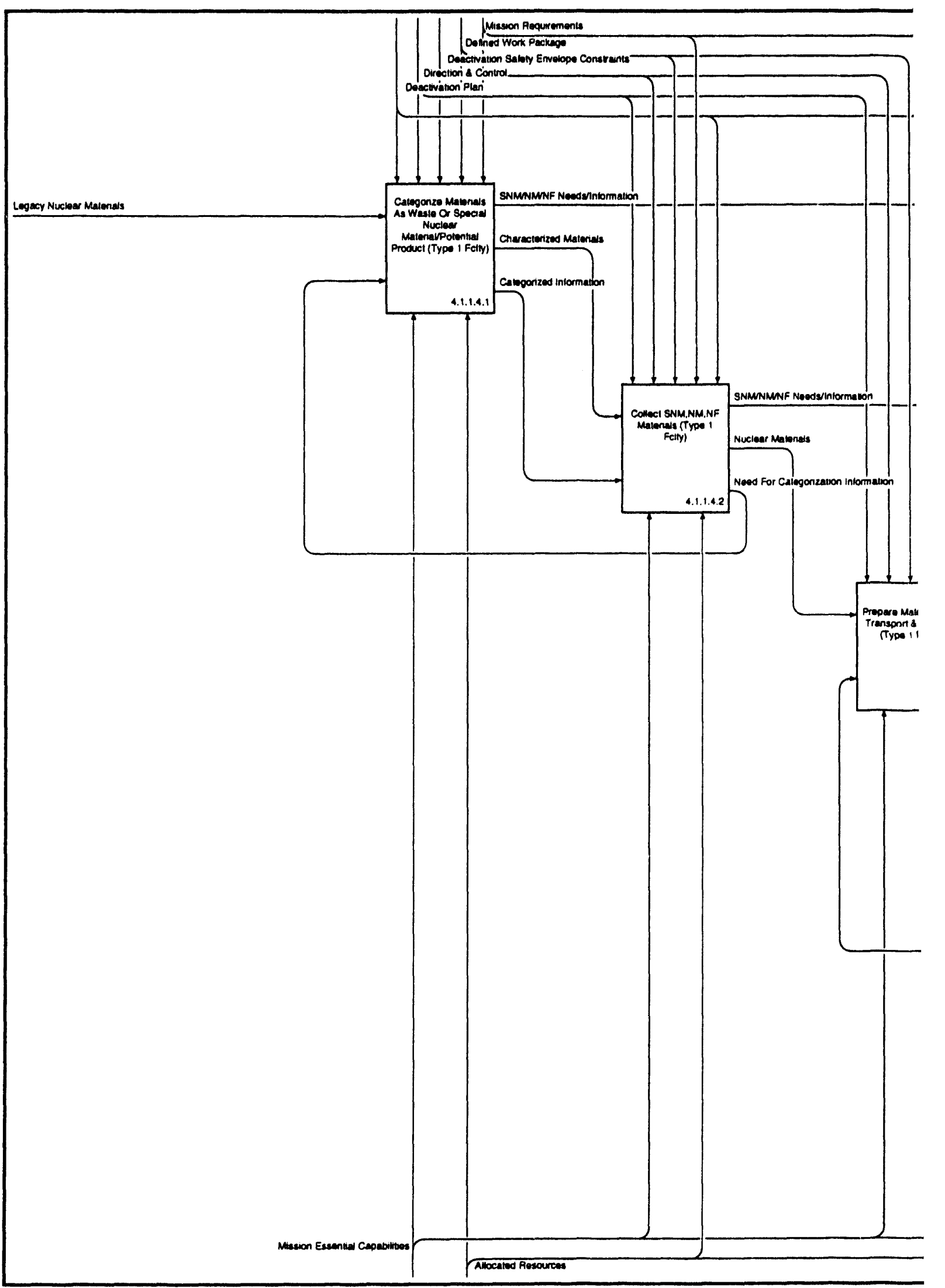




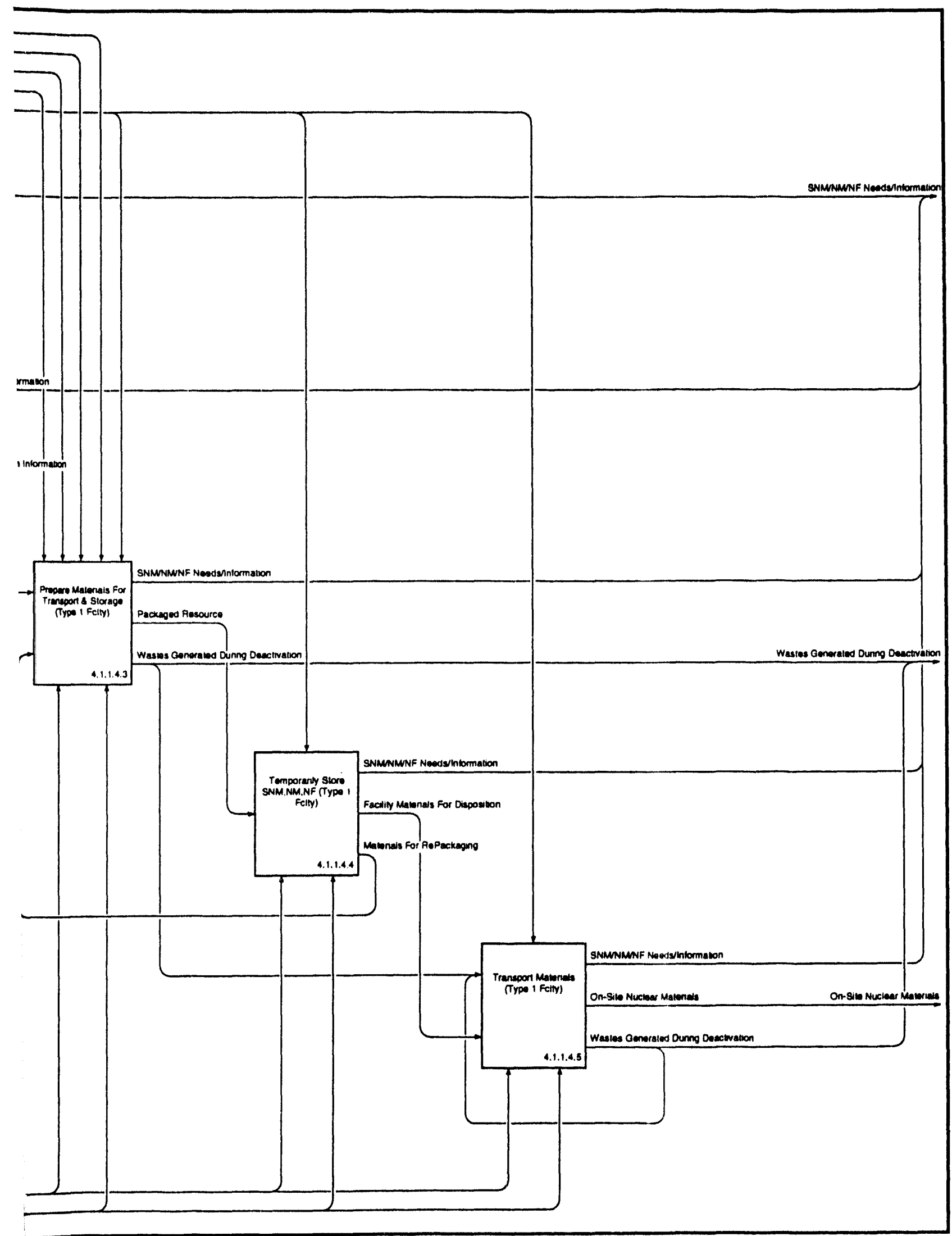

[4.1.1.4] Disposition Currently Identified Radioactive Materials Held As A Potential Product \& Special Nuclear Material (Type 1 Fclty) 


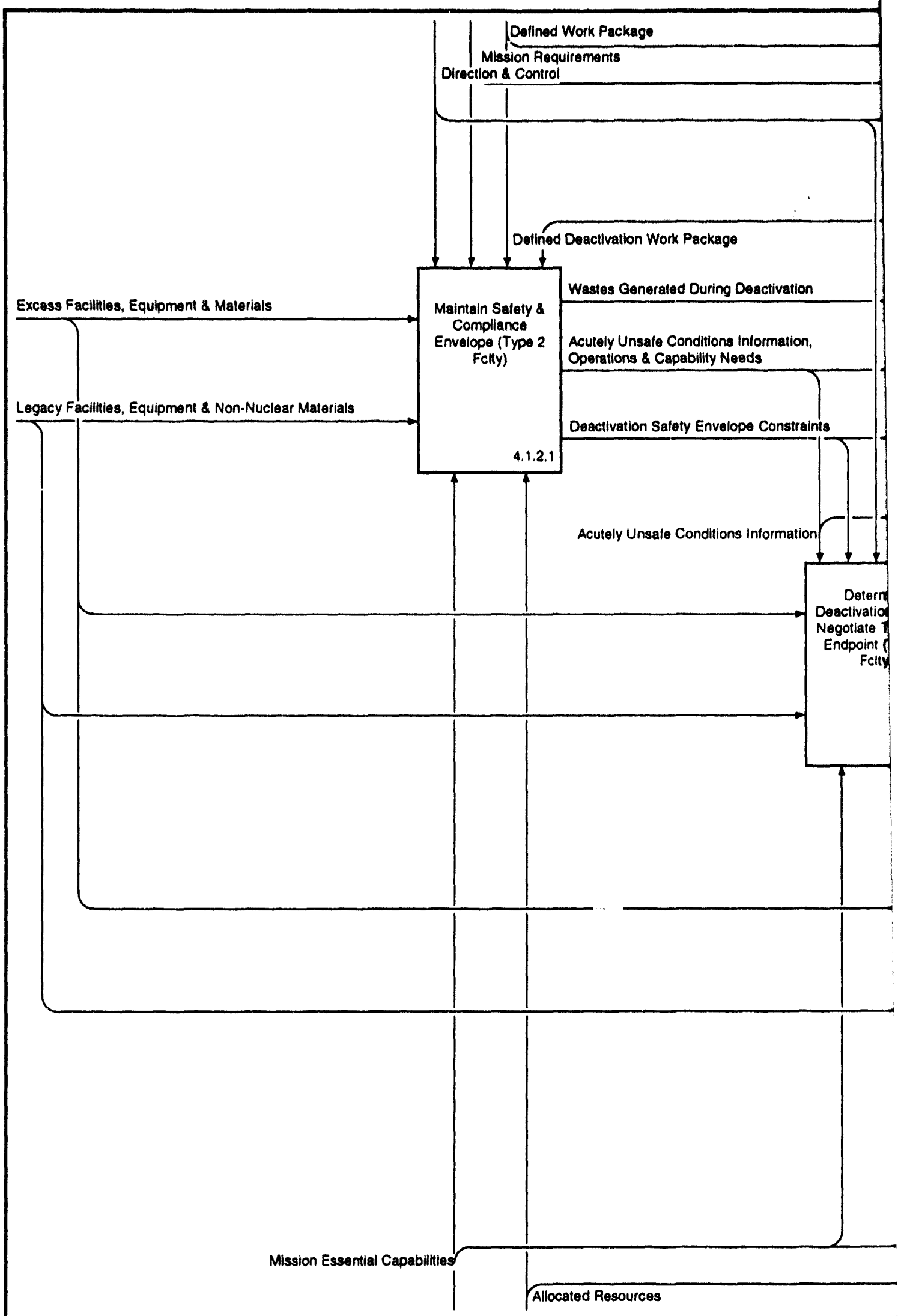




\section{adioactive \& Hazardous Material (Type 2 Fclty)}

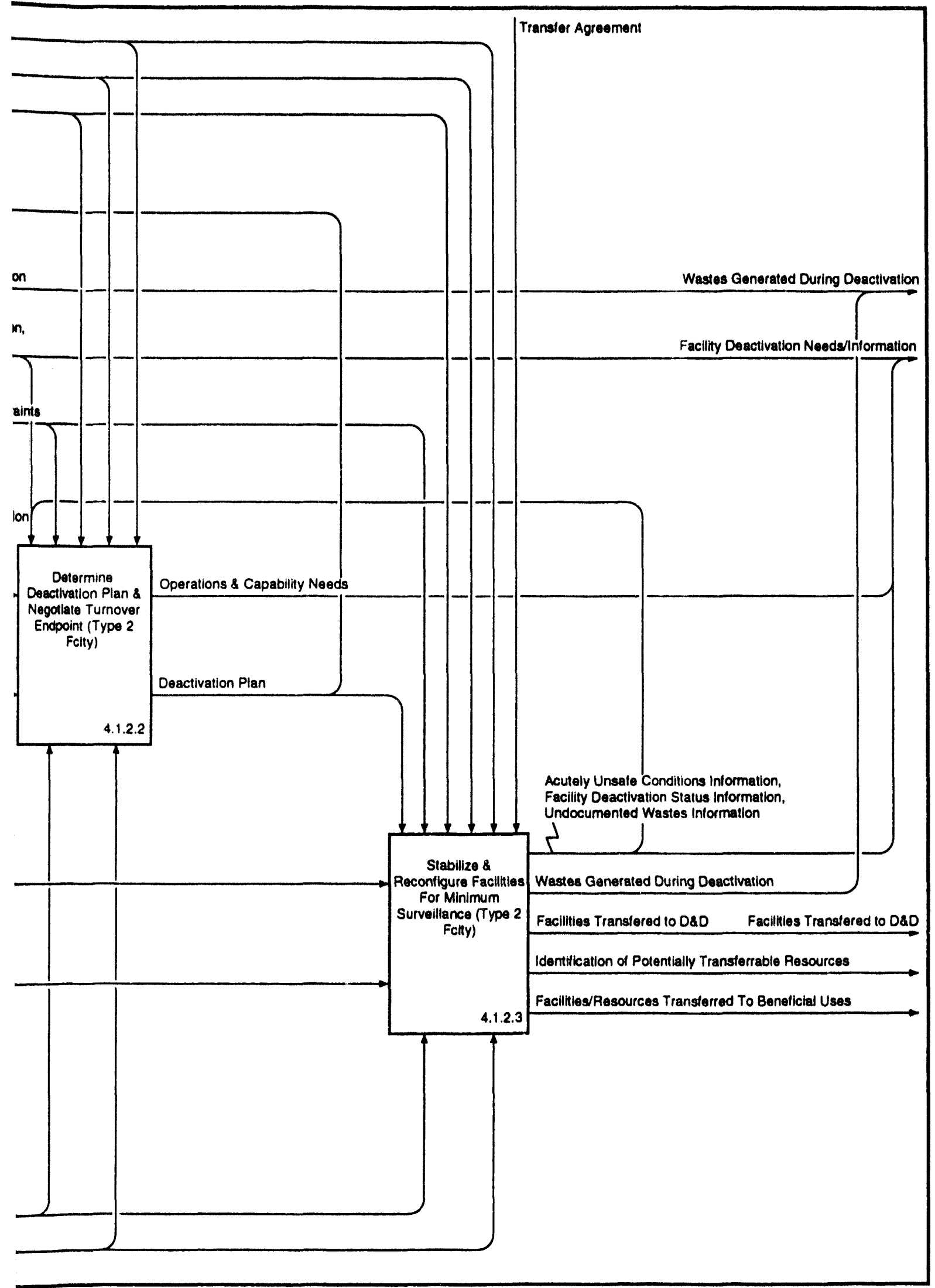




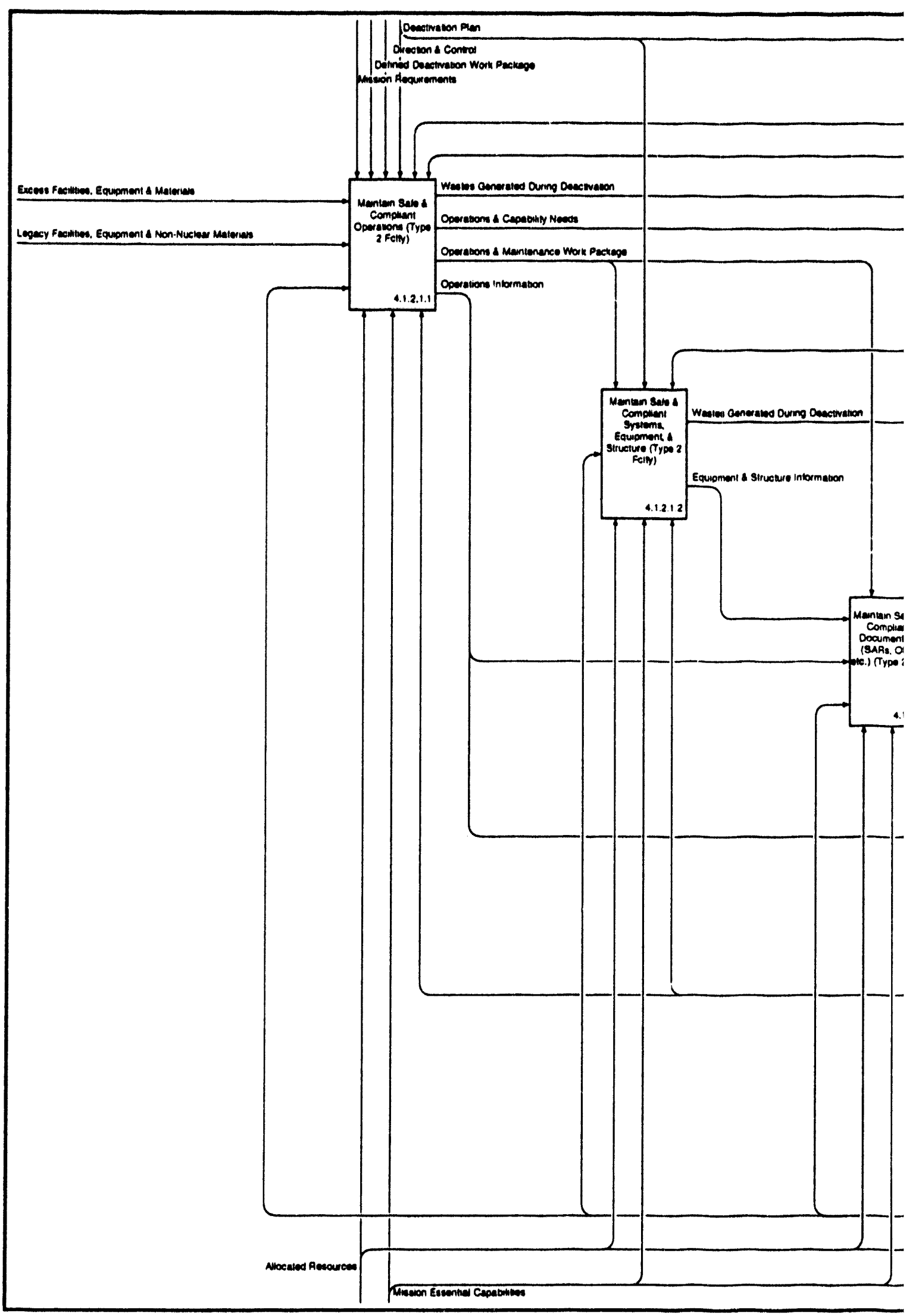




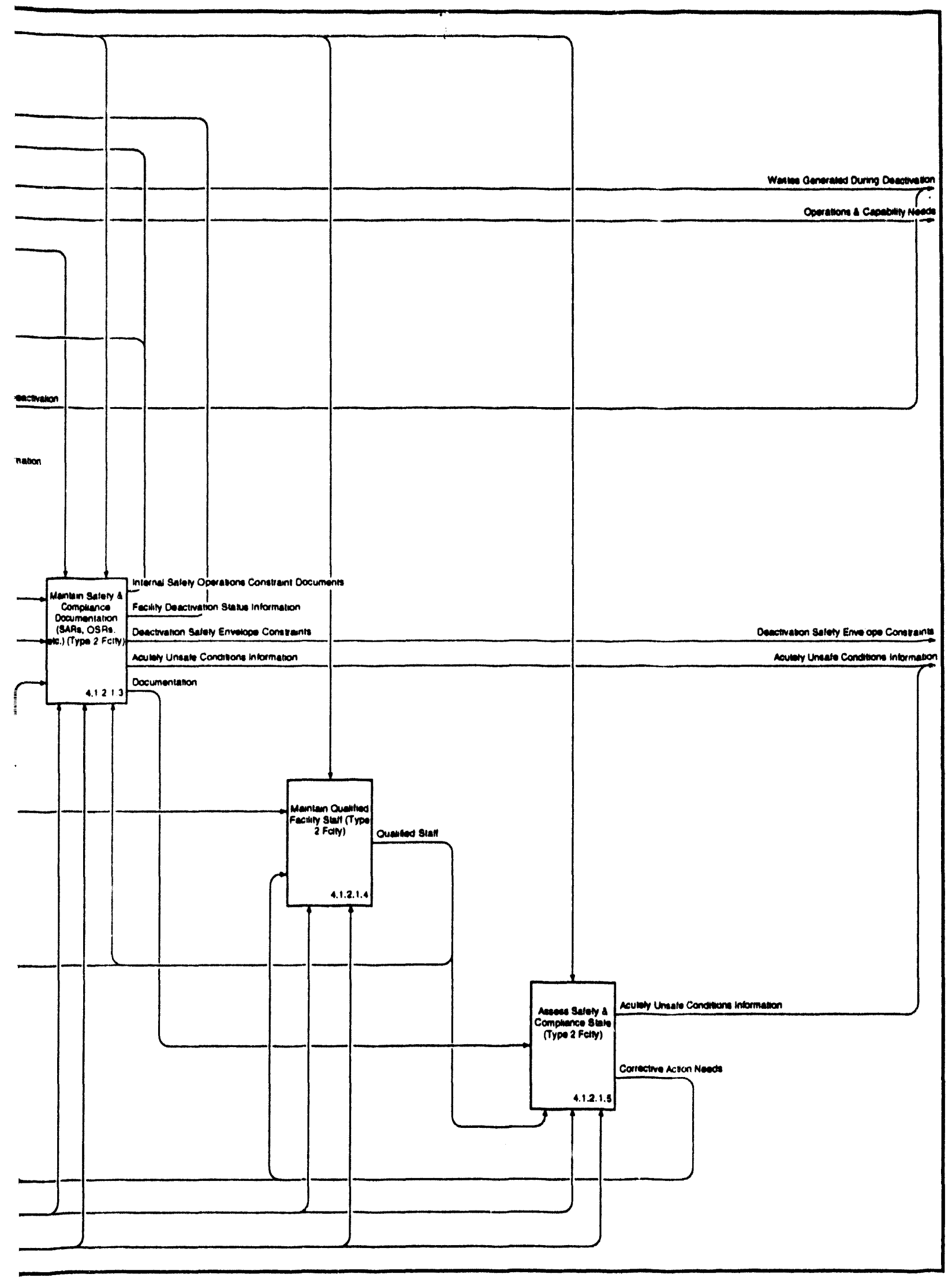




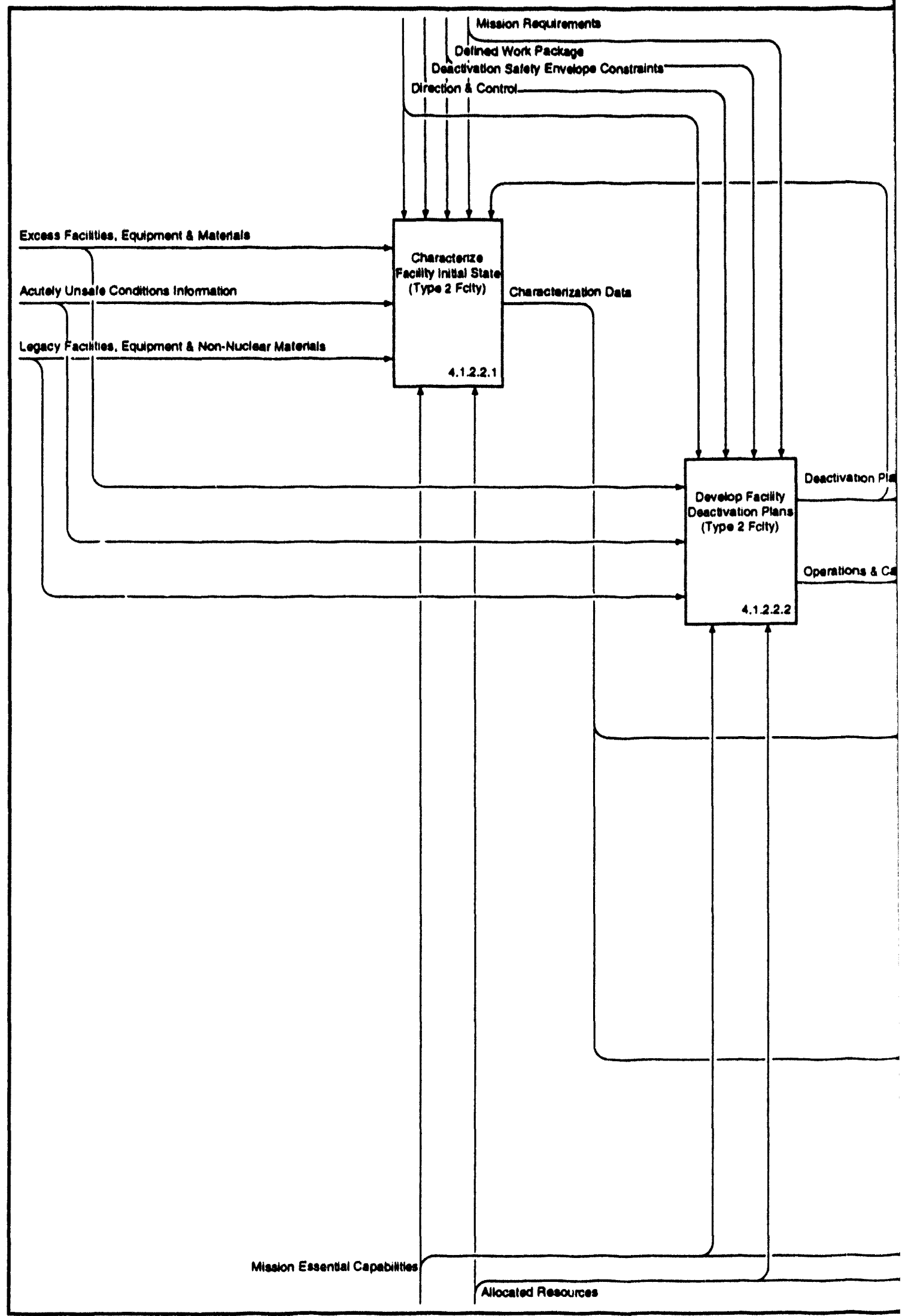




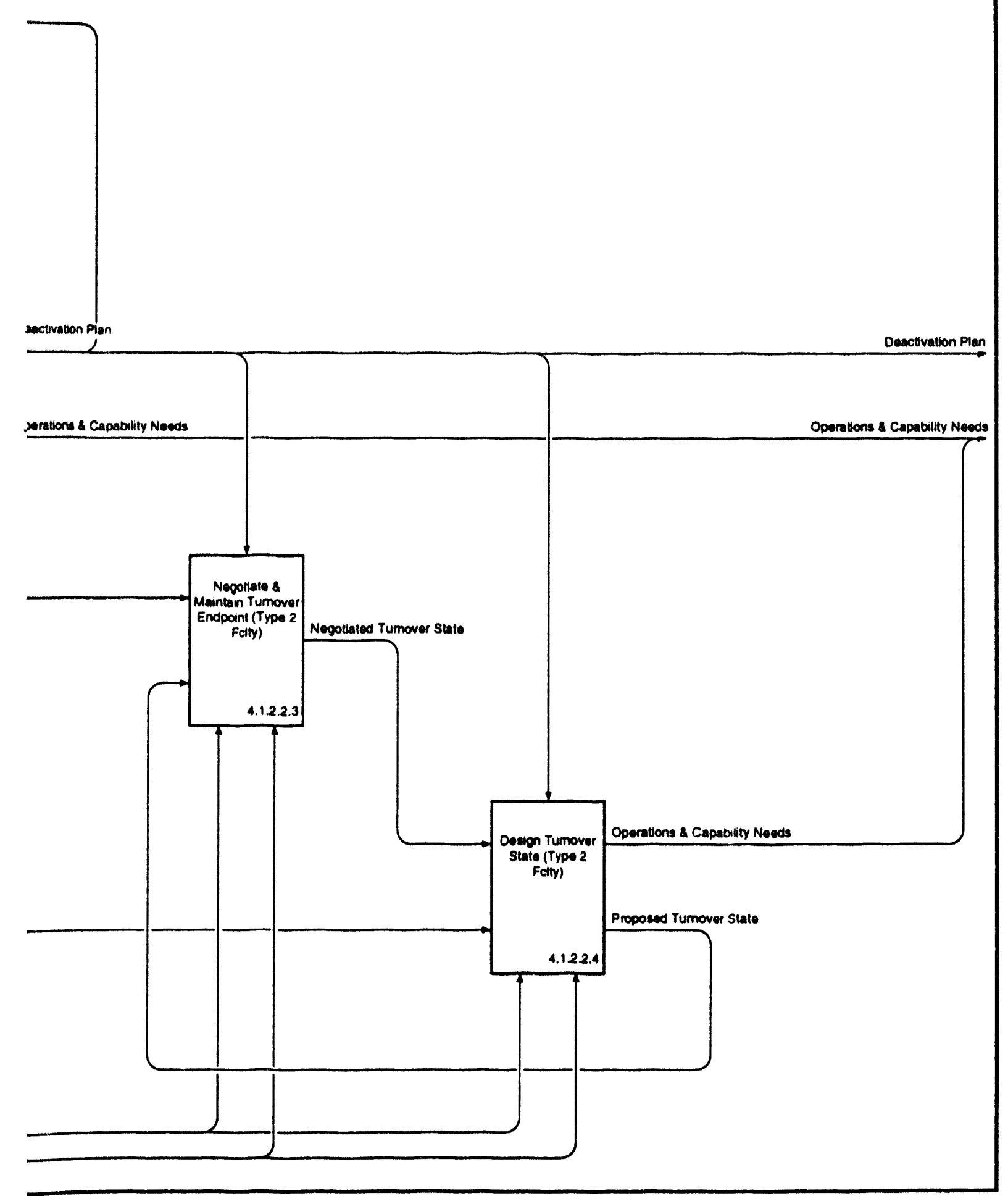

[4.1.2.2] Determine Deactivation Plan \& Negotiate Turnover Endpoint (Type 2 Fclty) 
[4.1.2.3] Stabilize \& Reconfigure Facilities

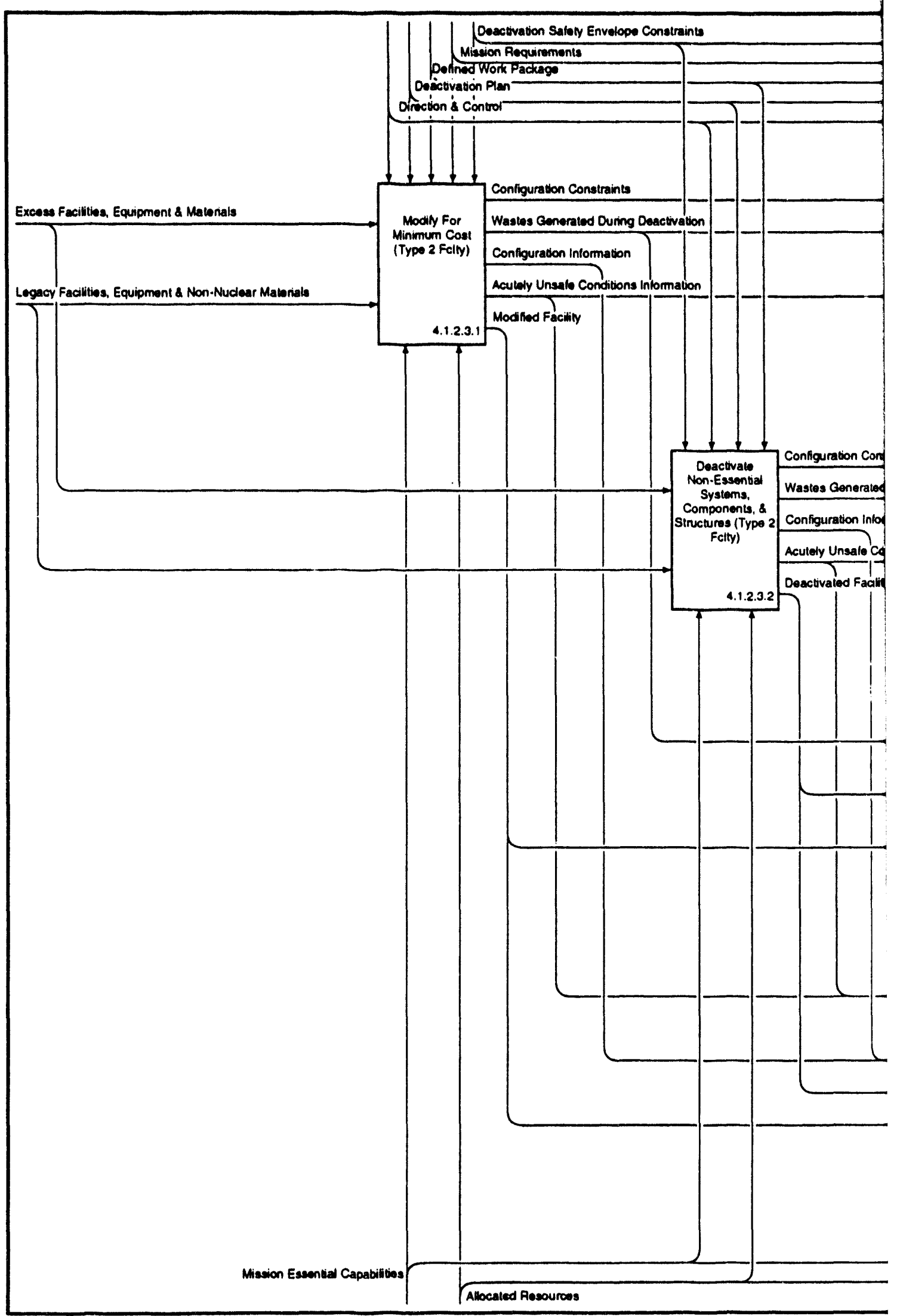




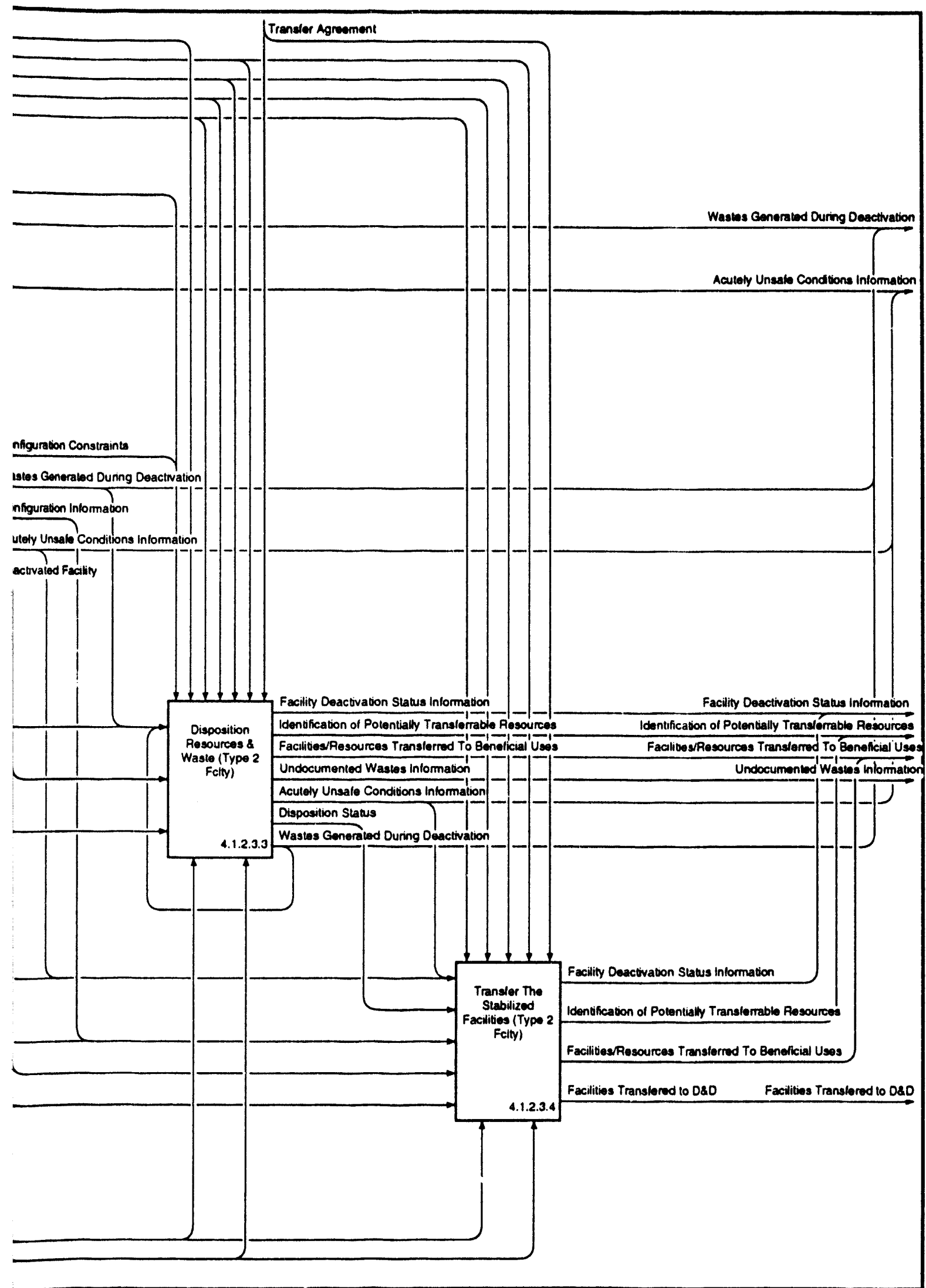

[4.1.2.3) Stabilize \& Reconfigure Facilities For Minimum Surveillance (Type 2 Fclty) 


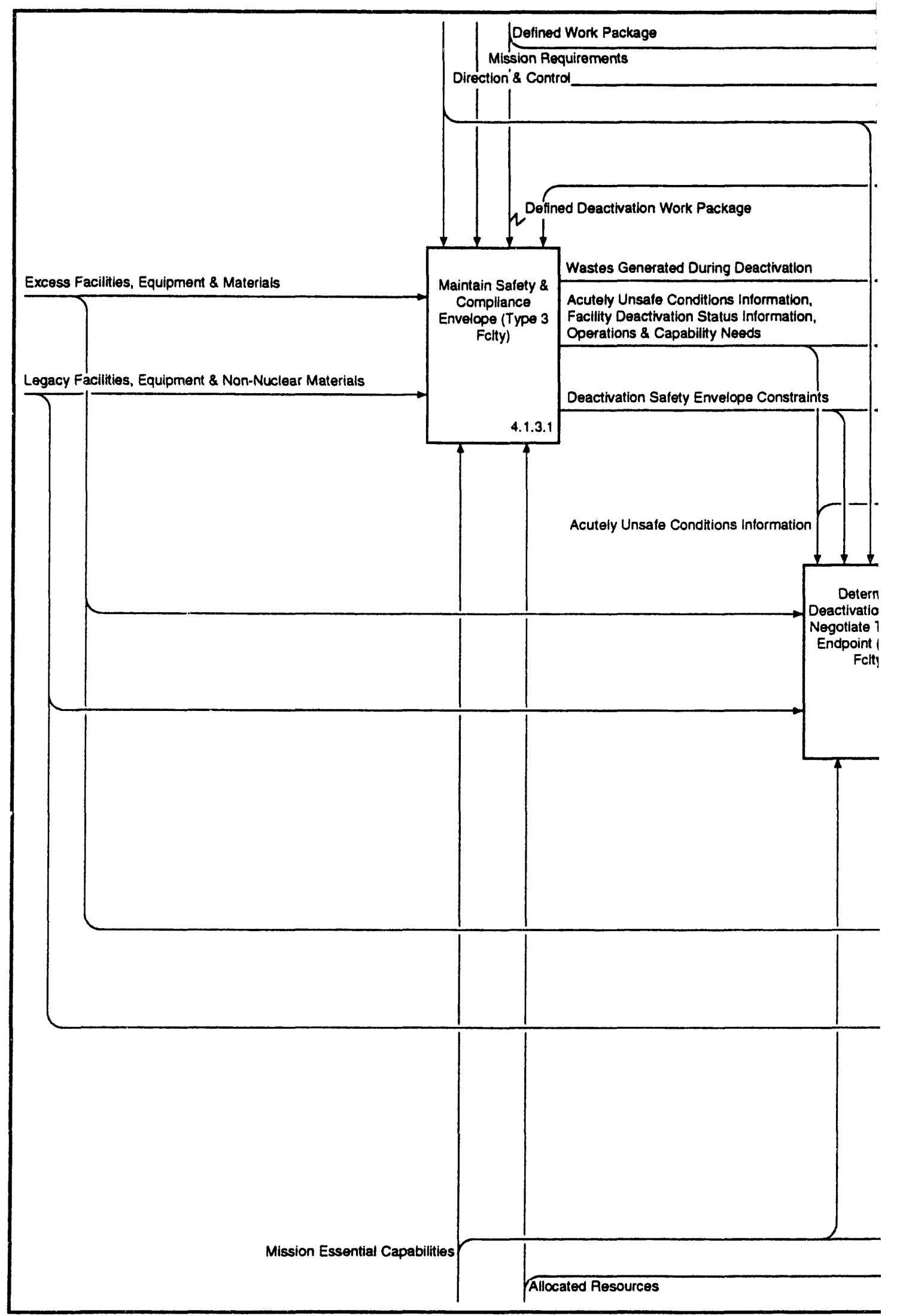




\section{azardous Material, Including Asbestos (Type 3 Fclty)}

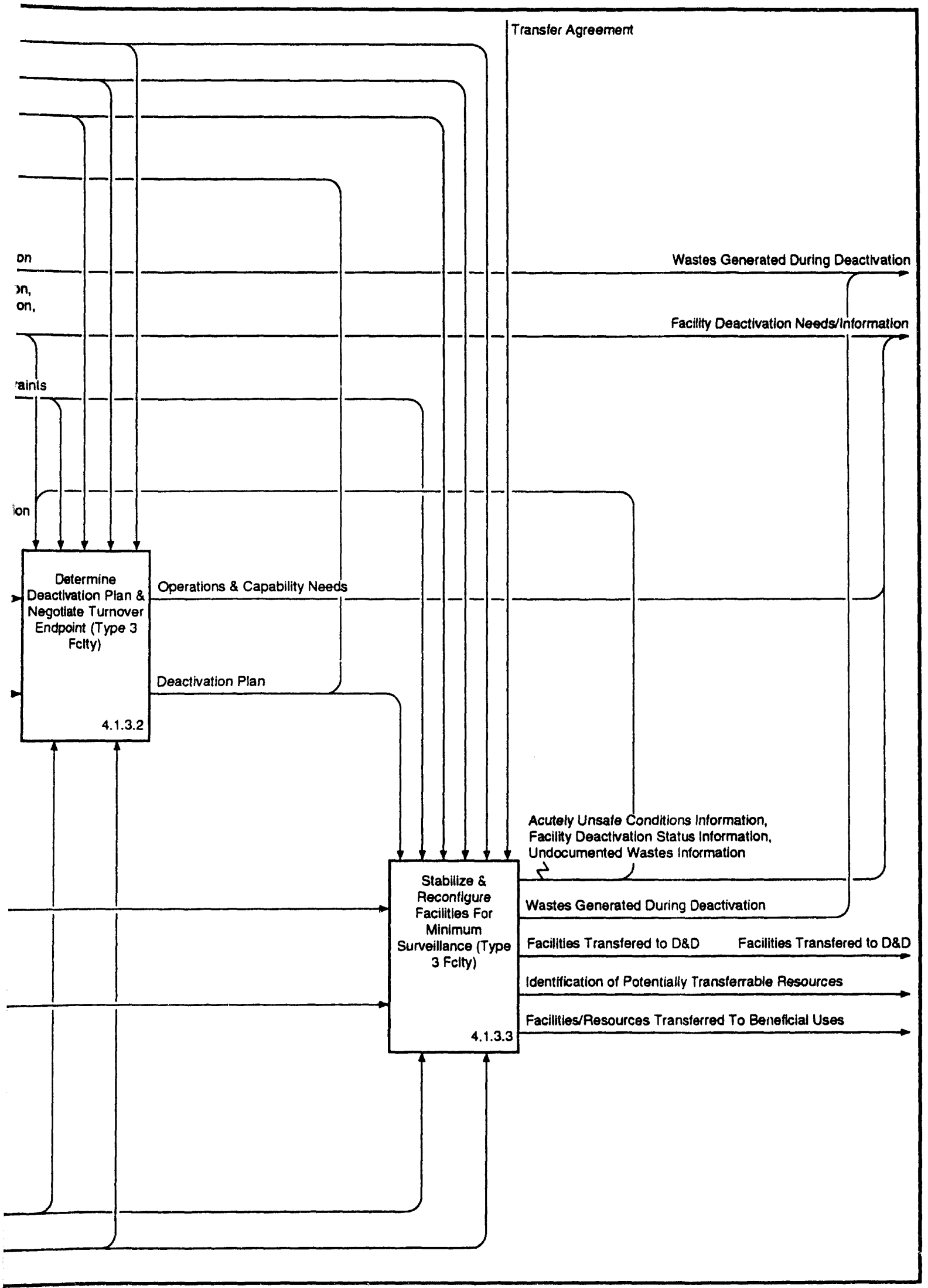


[4.1.3.1] Maintain Safety \& Com]

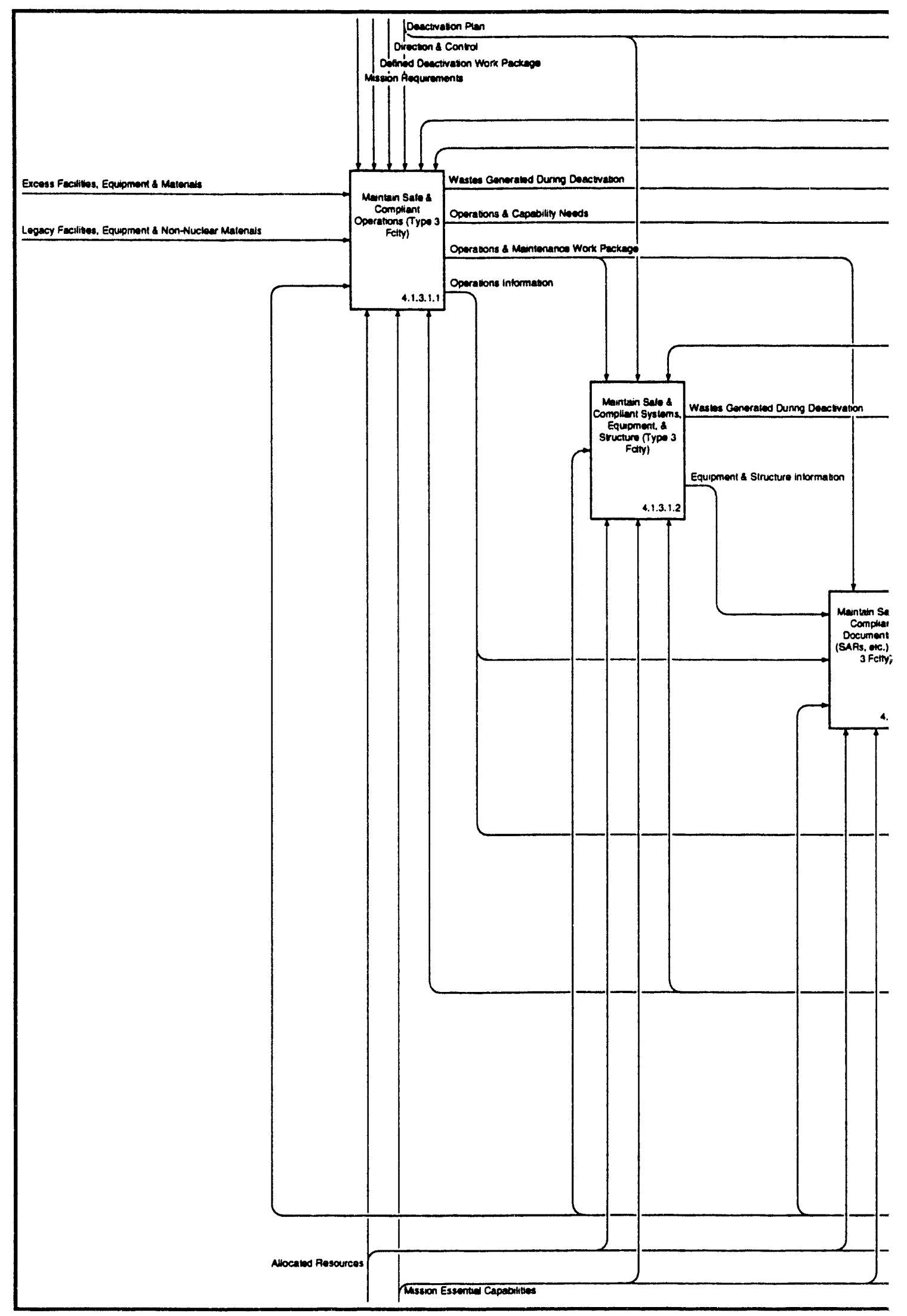




\section{Compliance Envelope (Type 3 Fclty)}

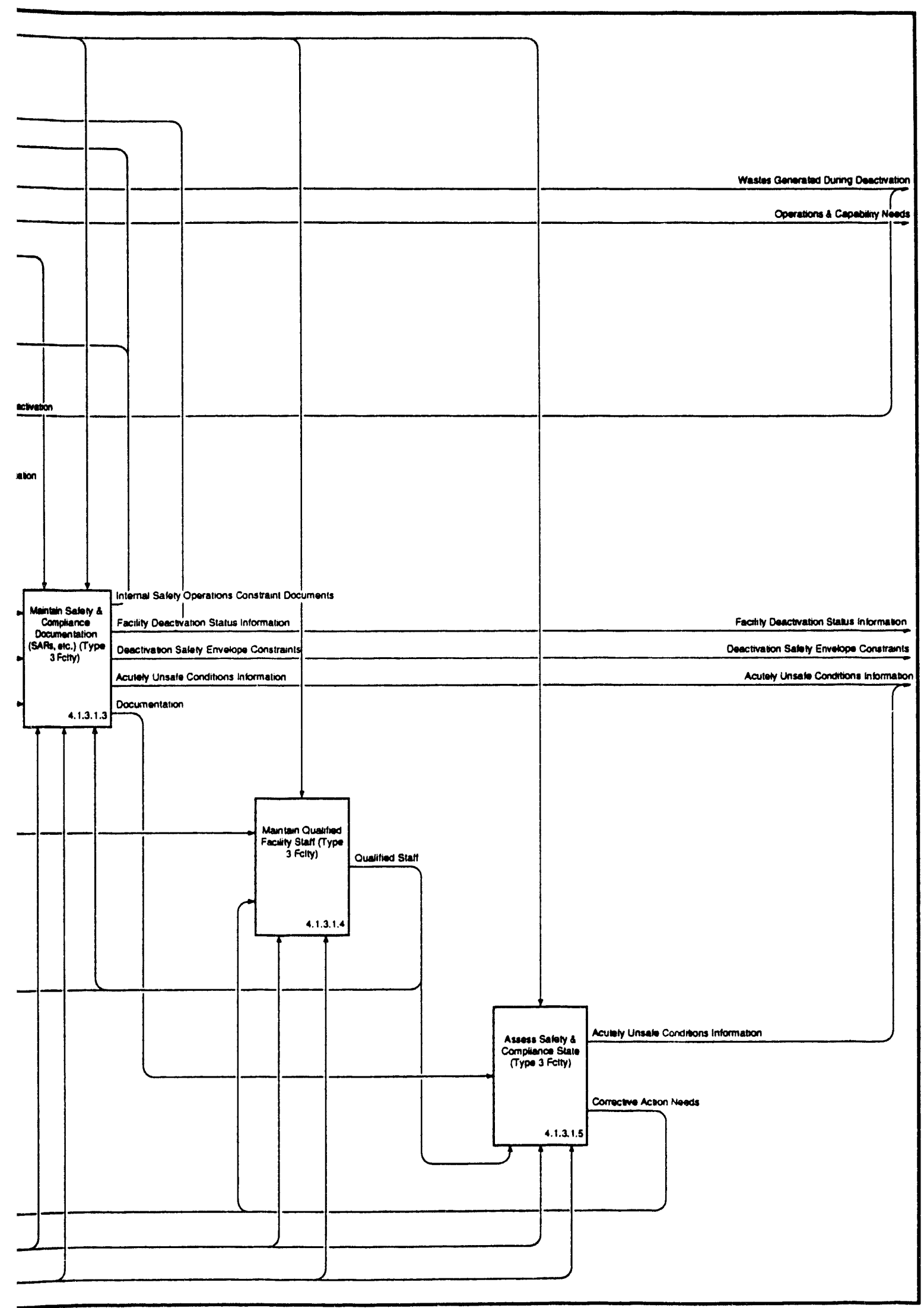


[4.1.3.2] Determine Deactivation Plan \& Ne

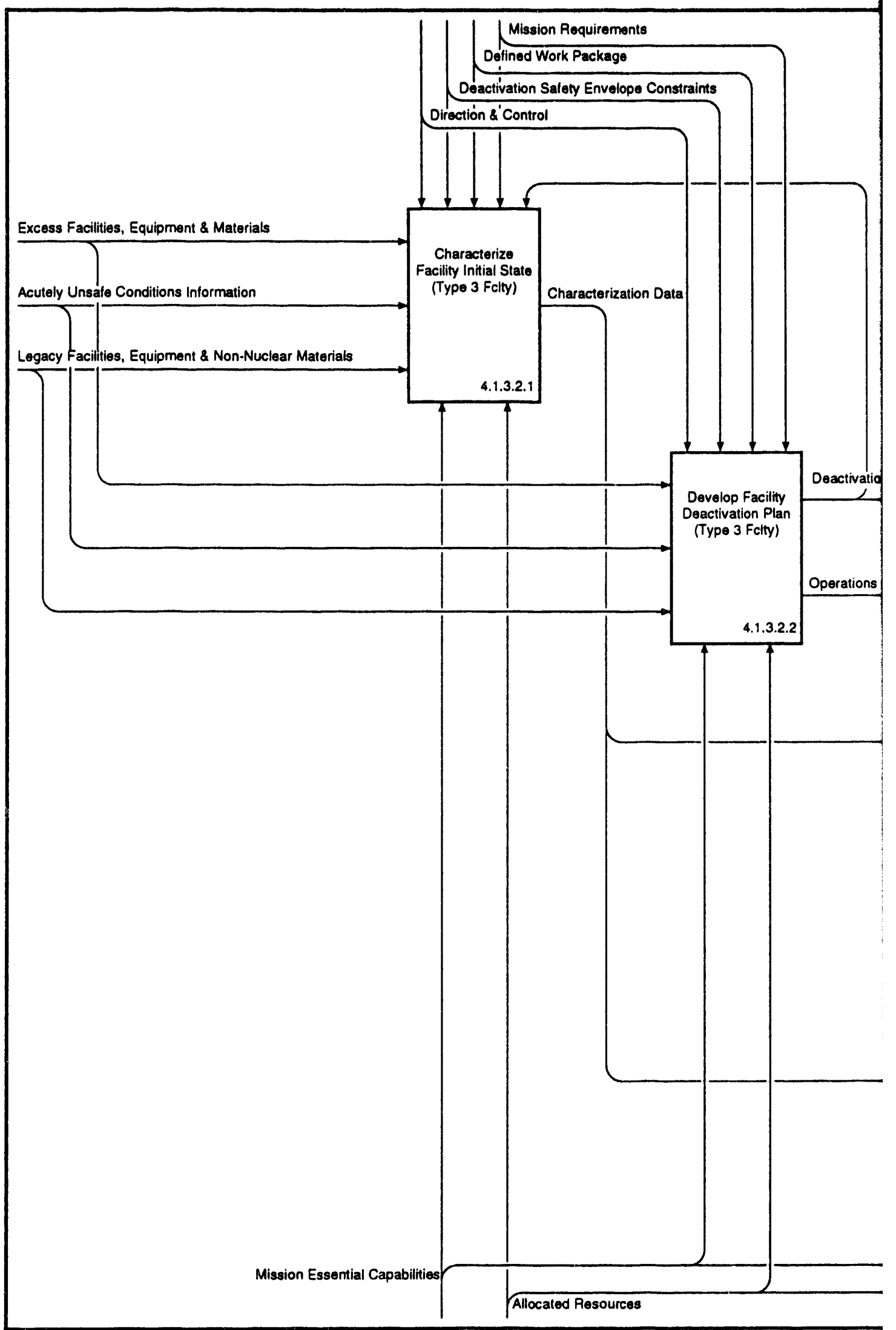




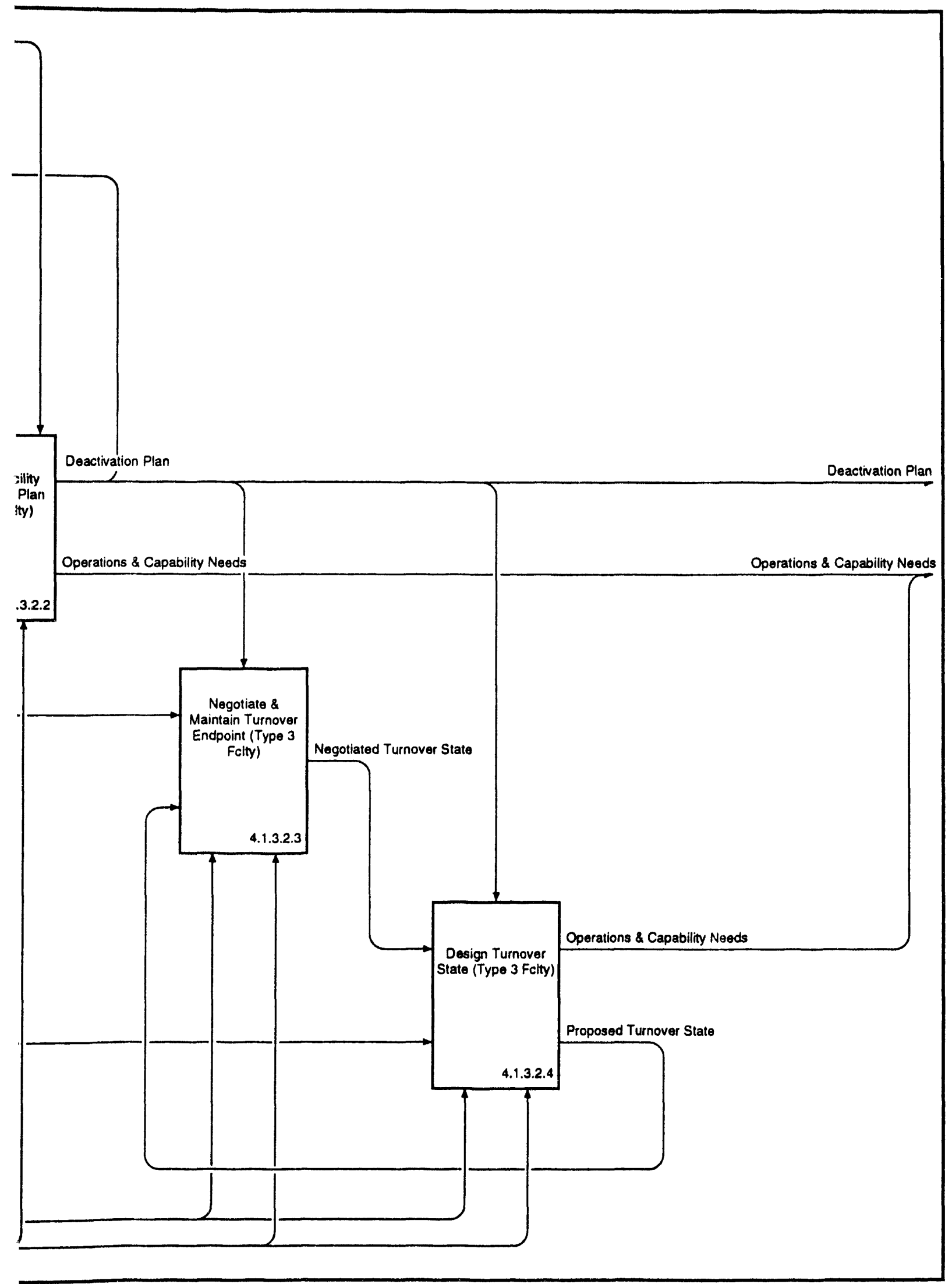




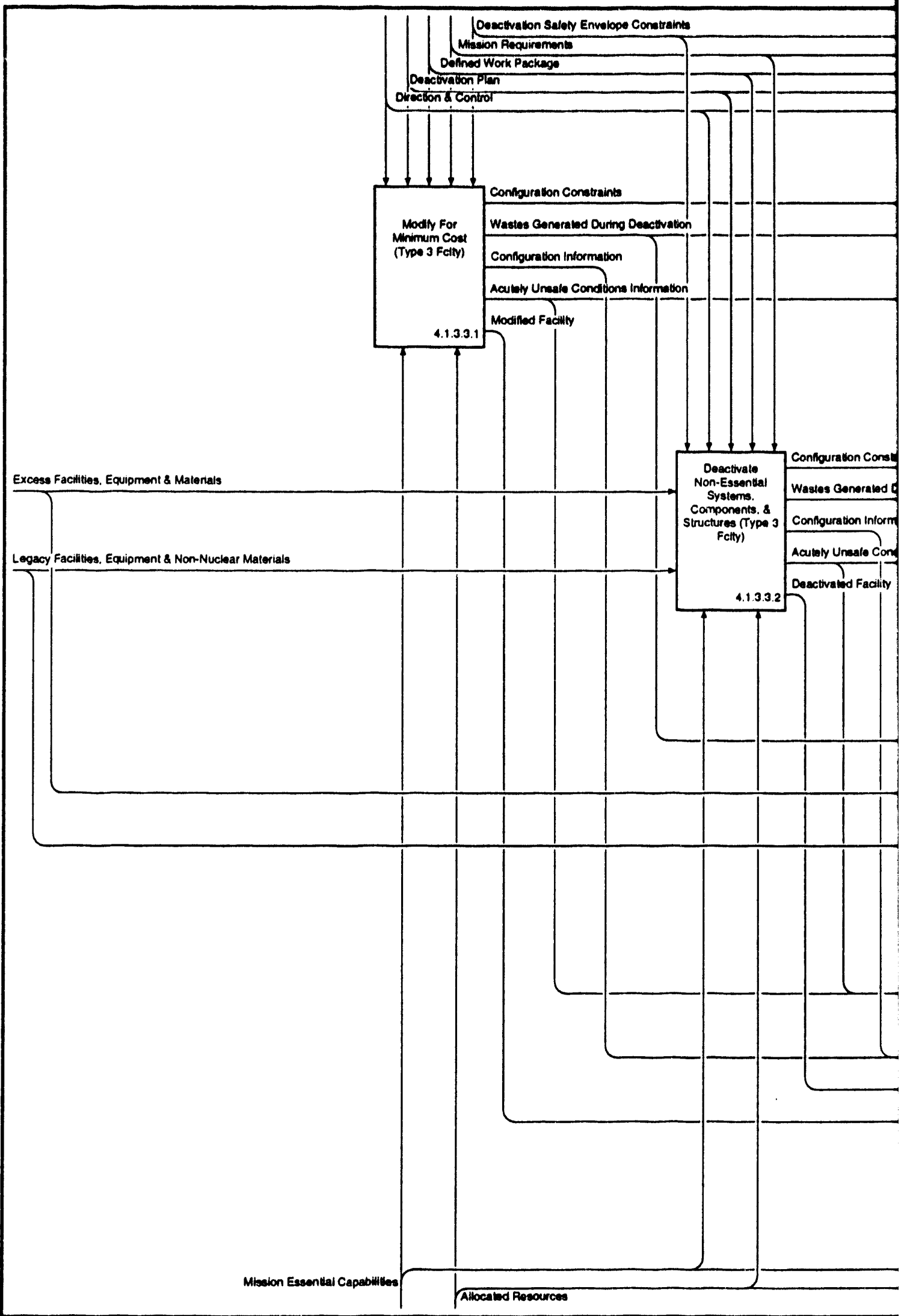




\section{'acilities For Minimum Surveillance (Type 3 Fclty)}

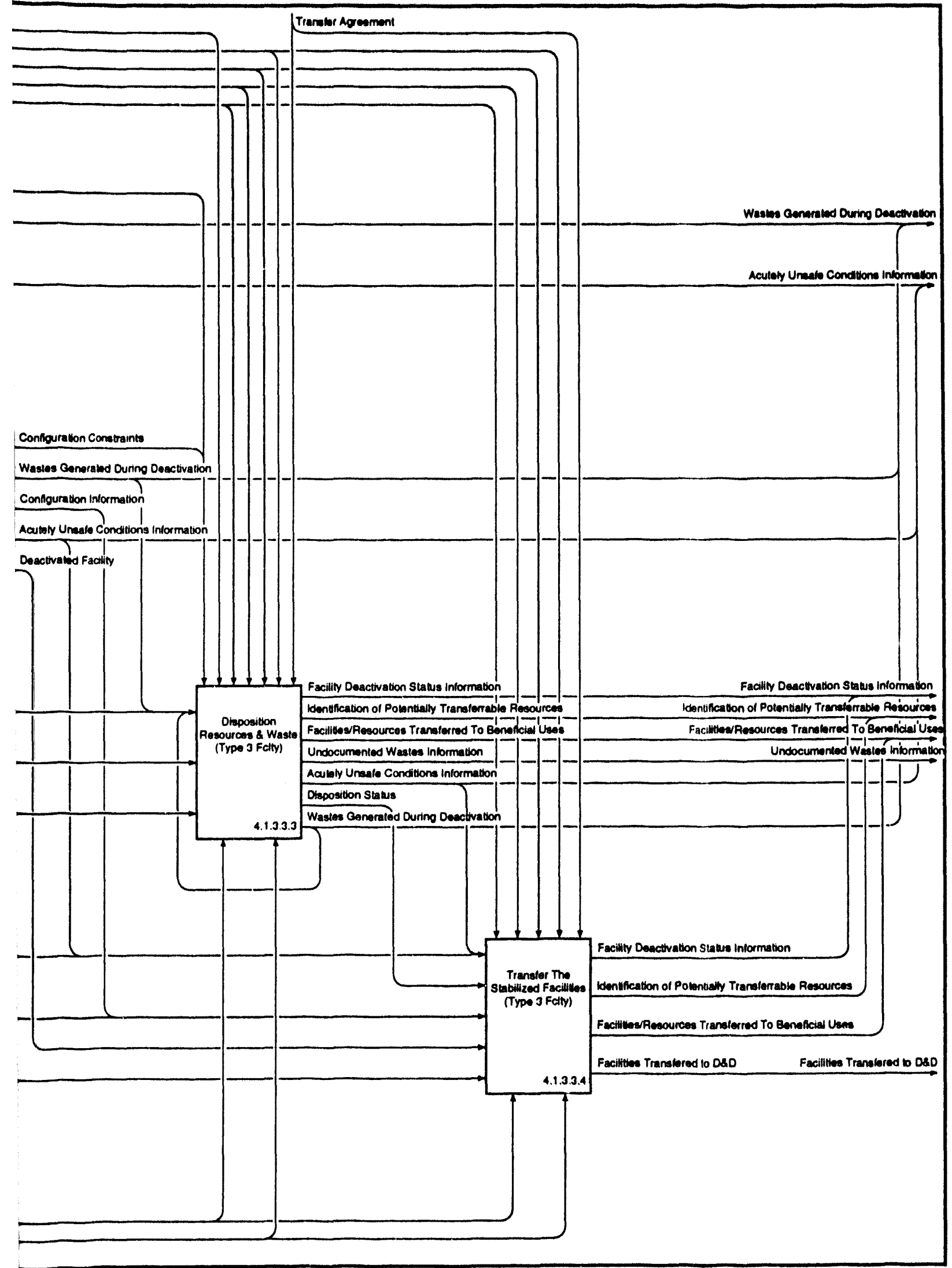

14.1.3.3/ Stabilize \& Reconfigure Facilities For Minimum Surveillance (Type 3 Fcliv) 


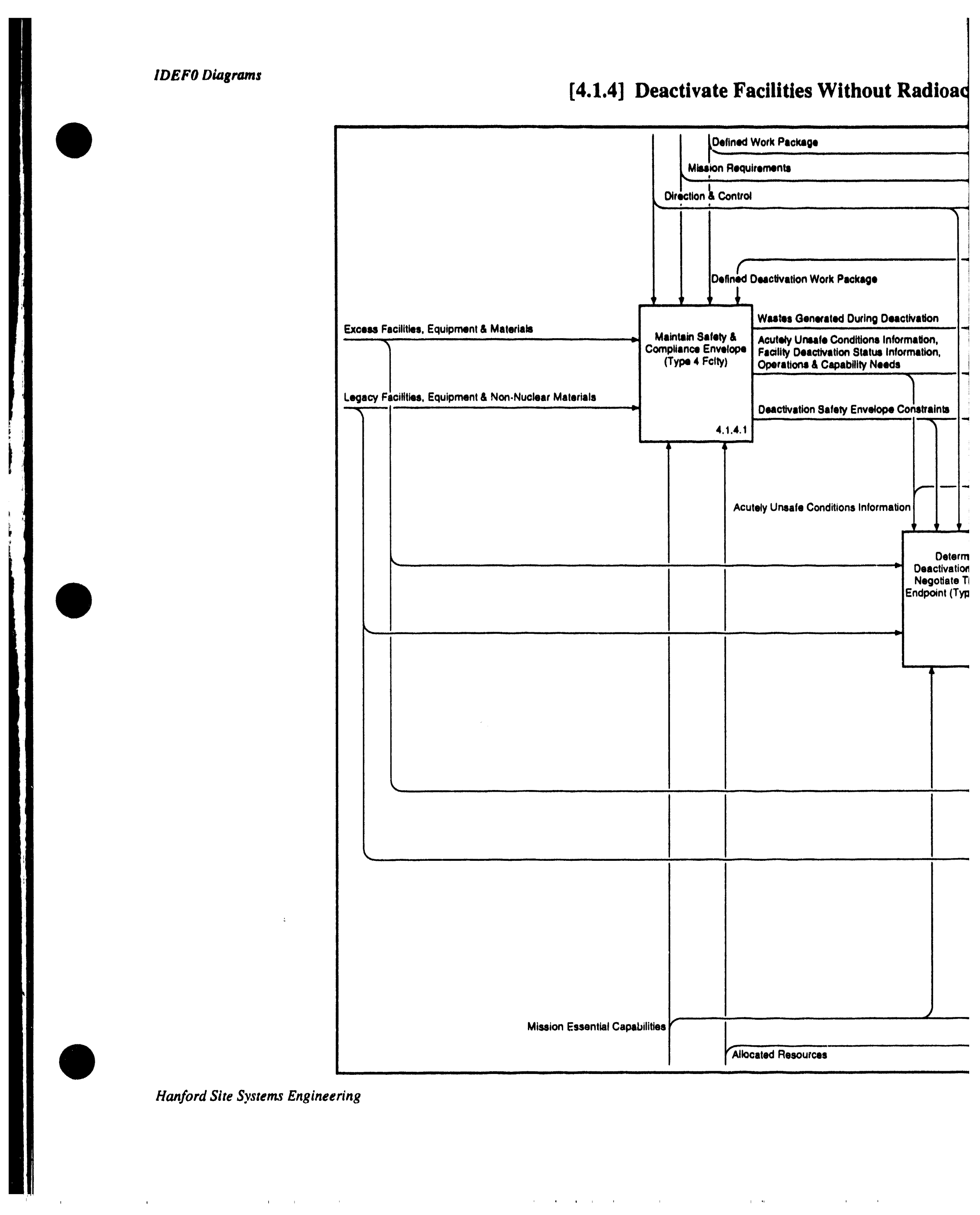




\section{adioactive Or Hazardous Material (Type 4 Fclty)}

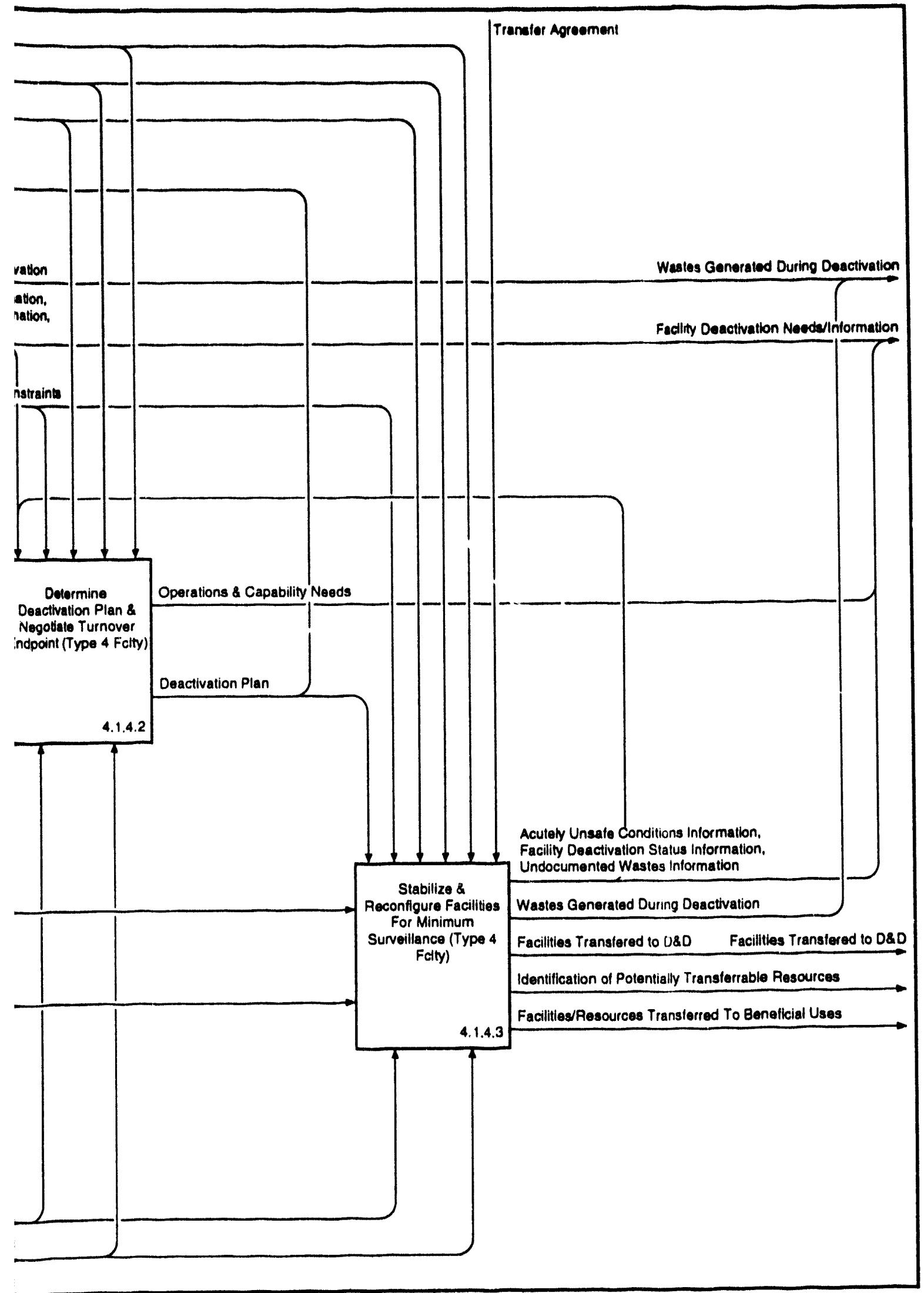

[4.1.4] Deactivate Facilities Without Radioactive Or Hazardous Material (Type 4 Fcly) 


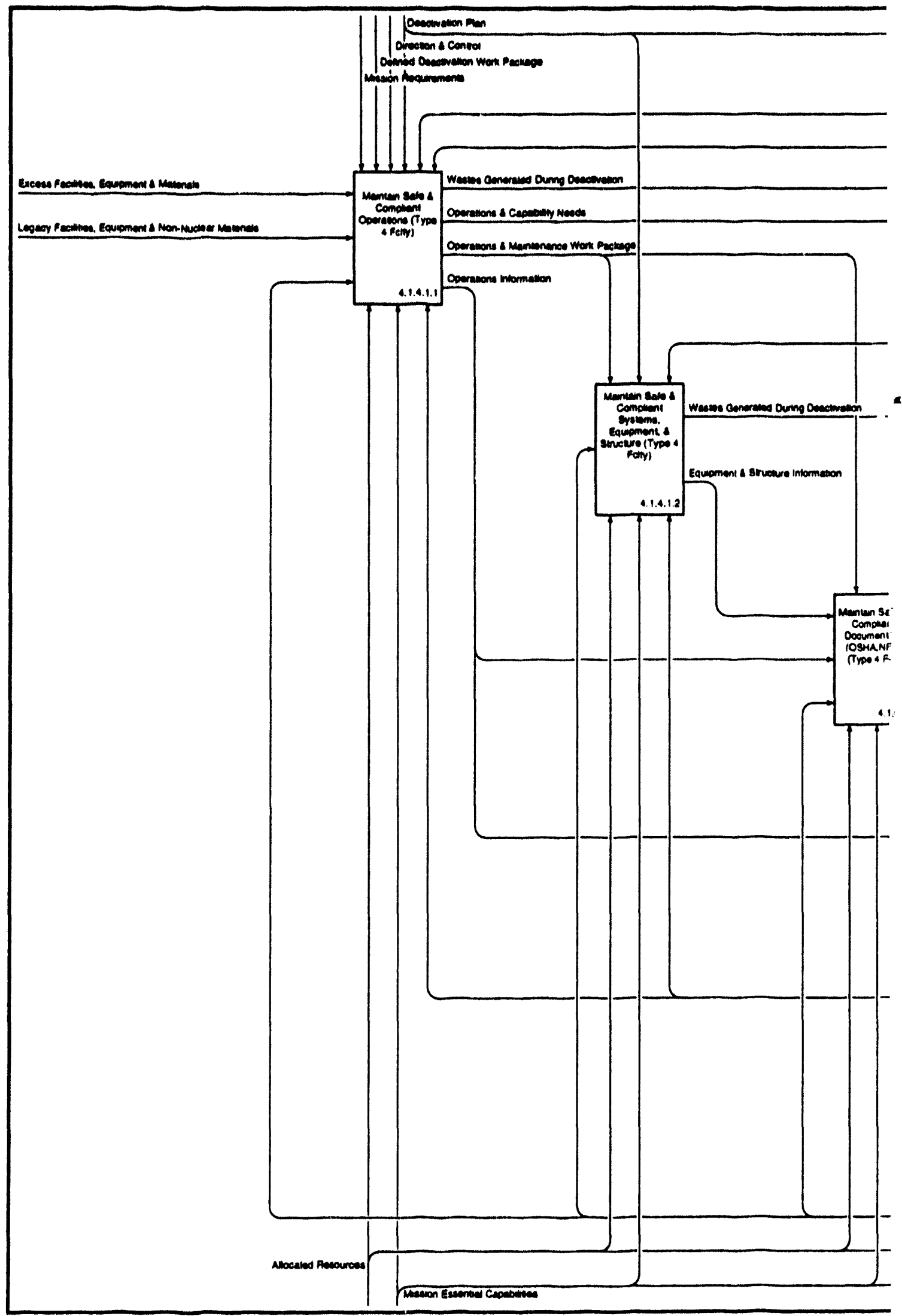

Hanford Site Systems Engineering 
$2 \varepsilon-\tau$

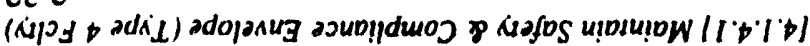

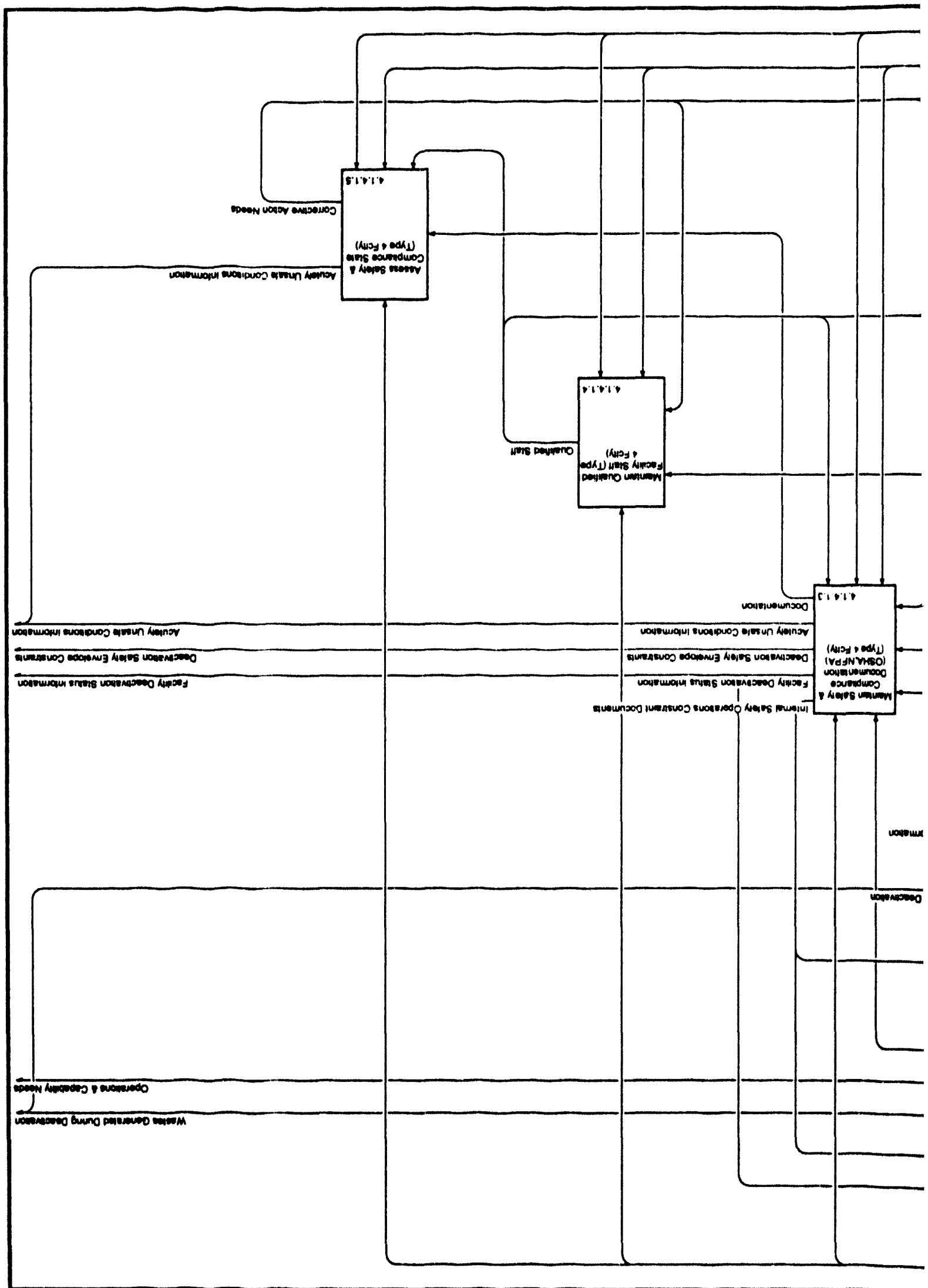




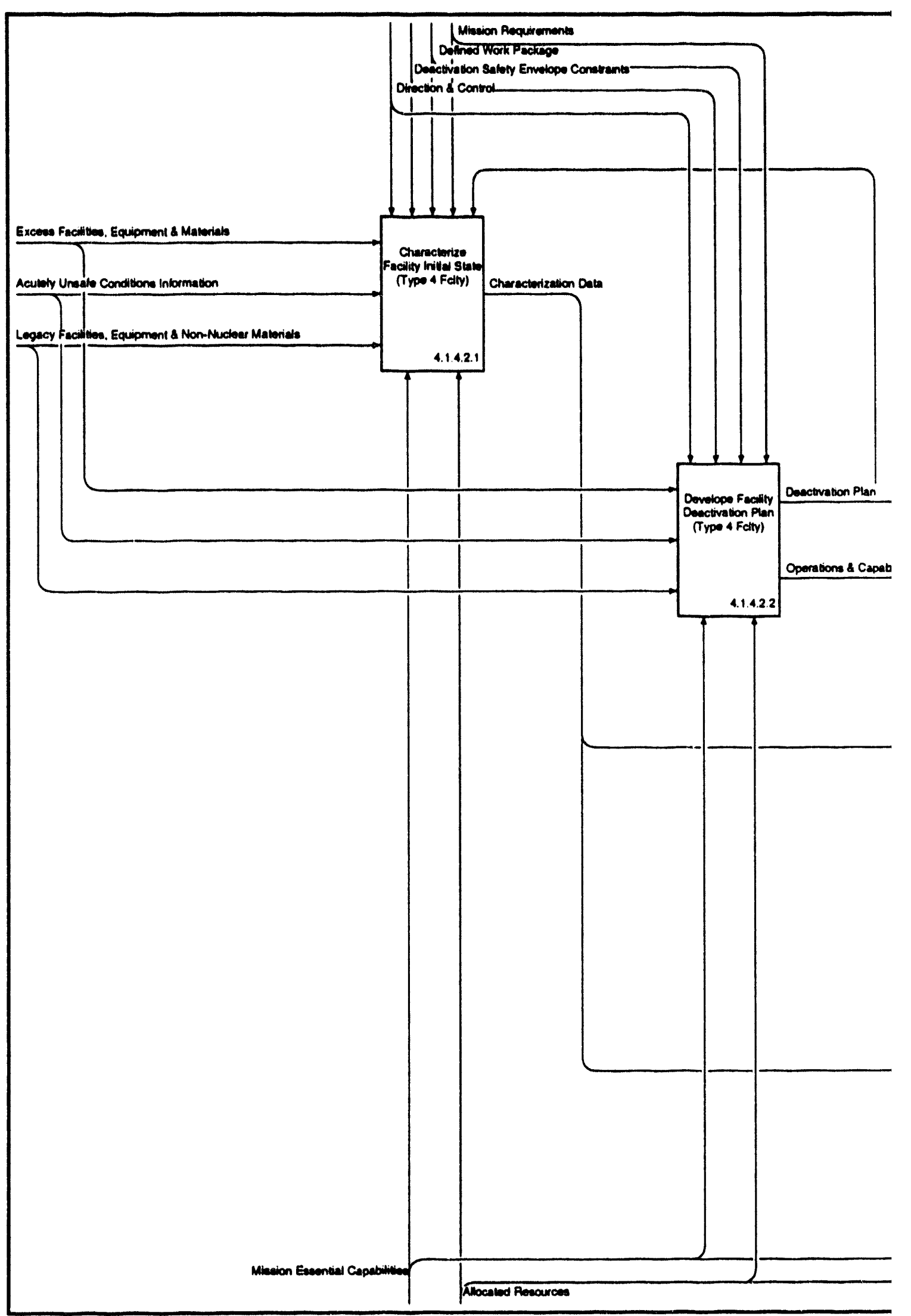




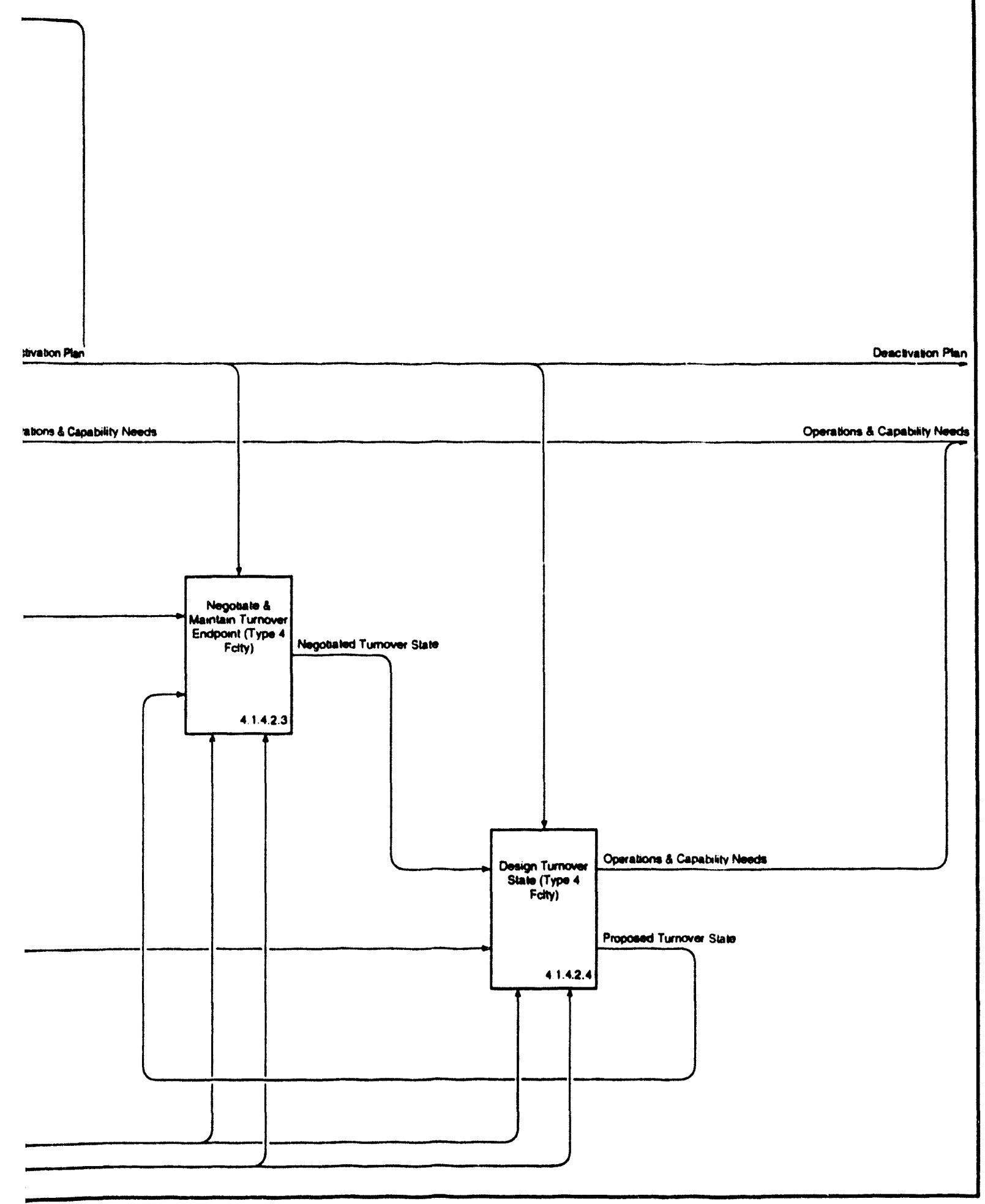

14.1.4.2/ Determine Deactivation Plan \& Negotiate Tumover Endpoint (Type 4 Fclry) 
[4.1.4.3] Stabilize \& Reconfigure Facilities
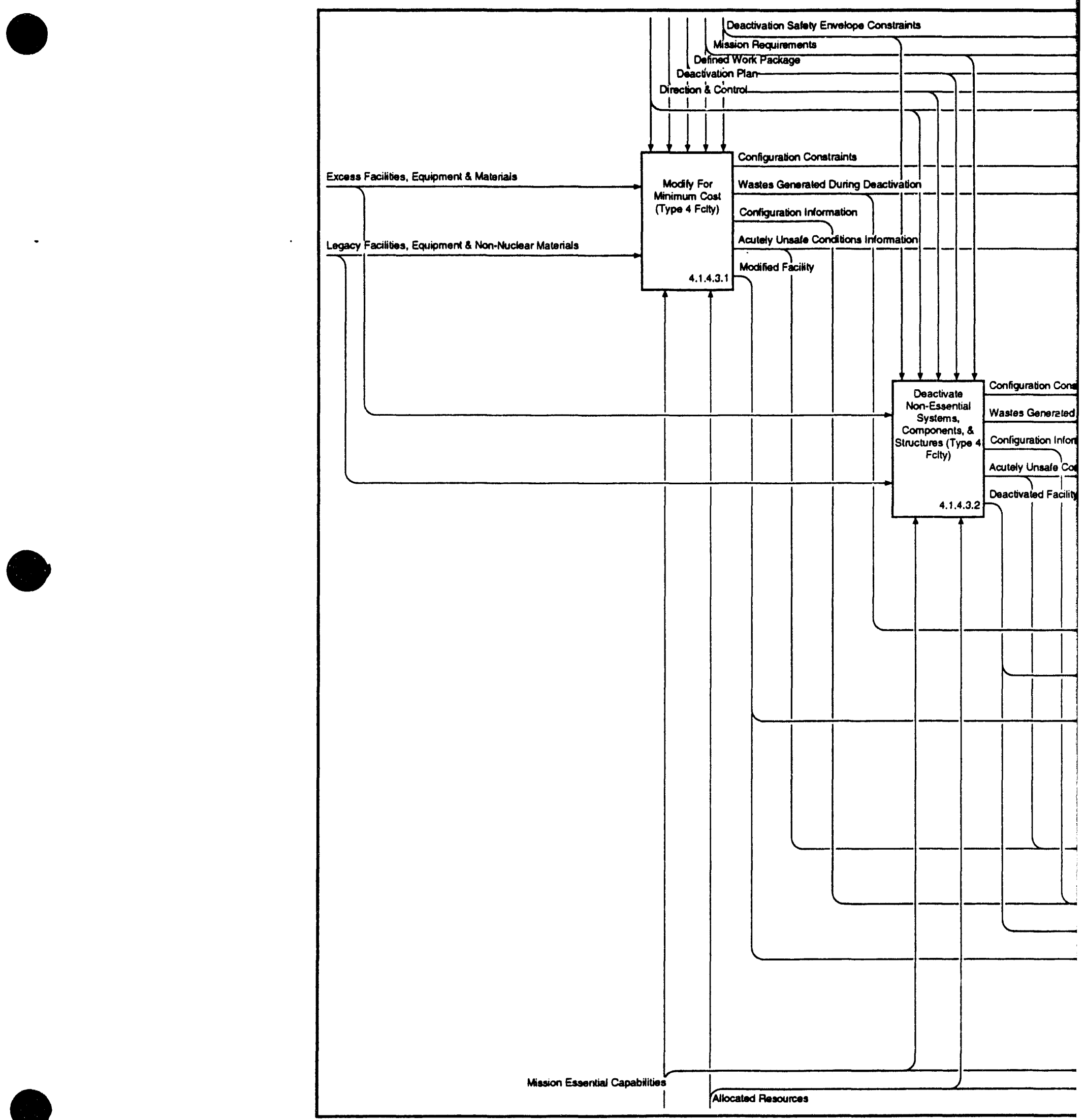


\section{cilities For Minimum Surveillance (Type 4 Fclty)}

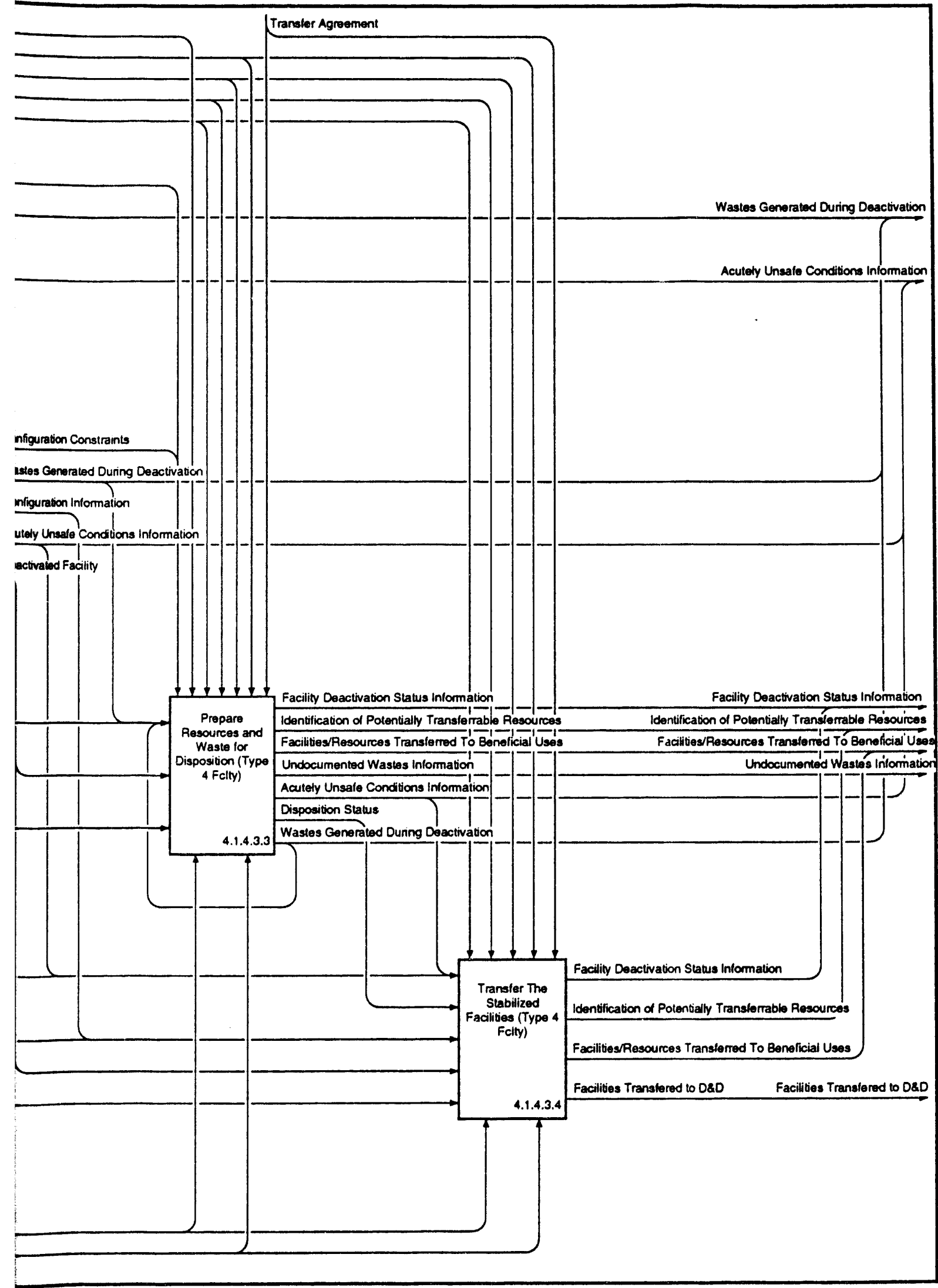


[4.3] Remedy

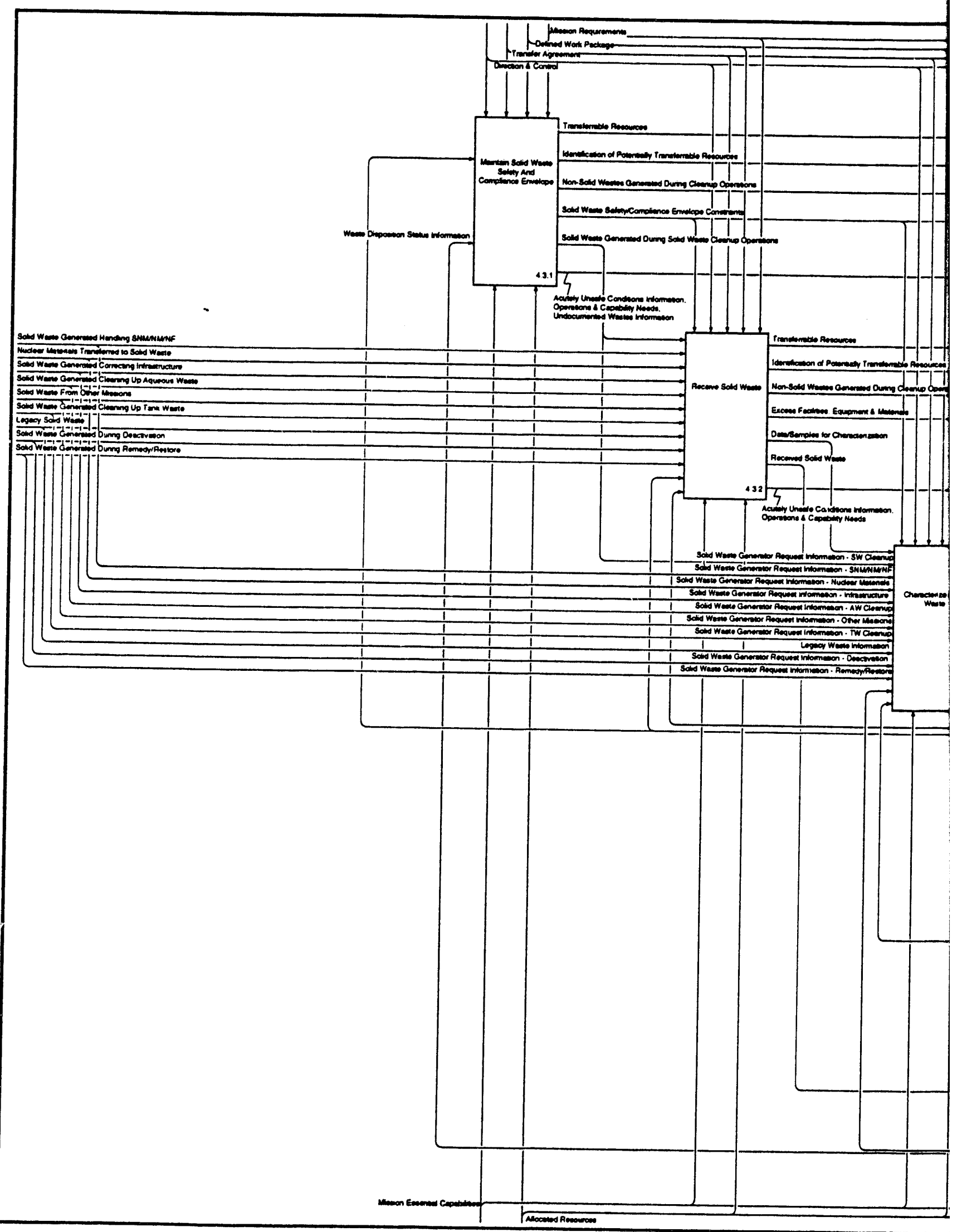

Hanford Site Systems Engineering 


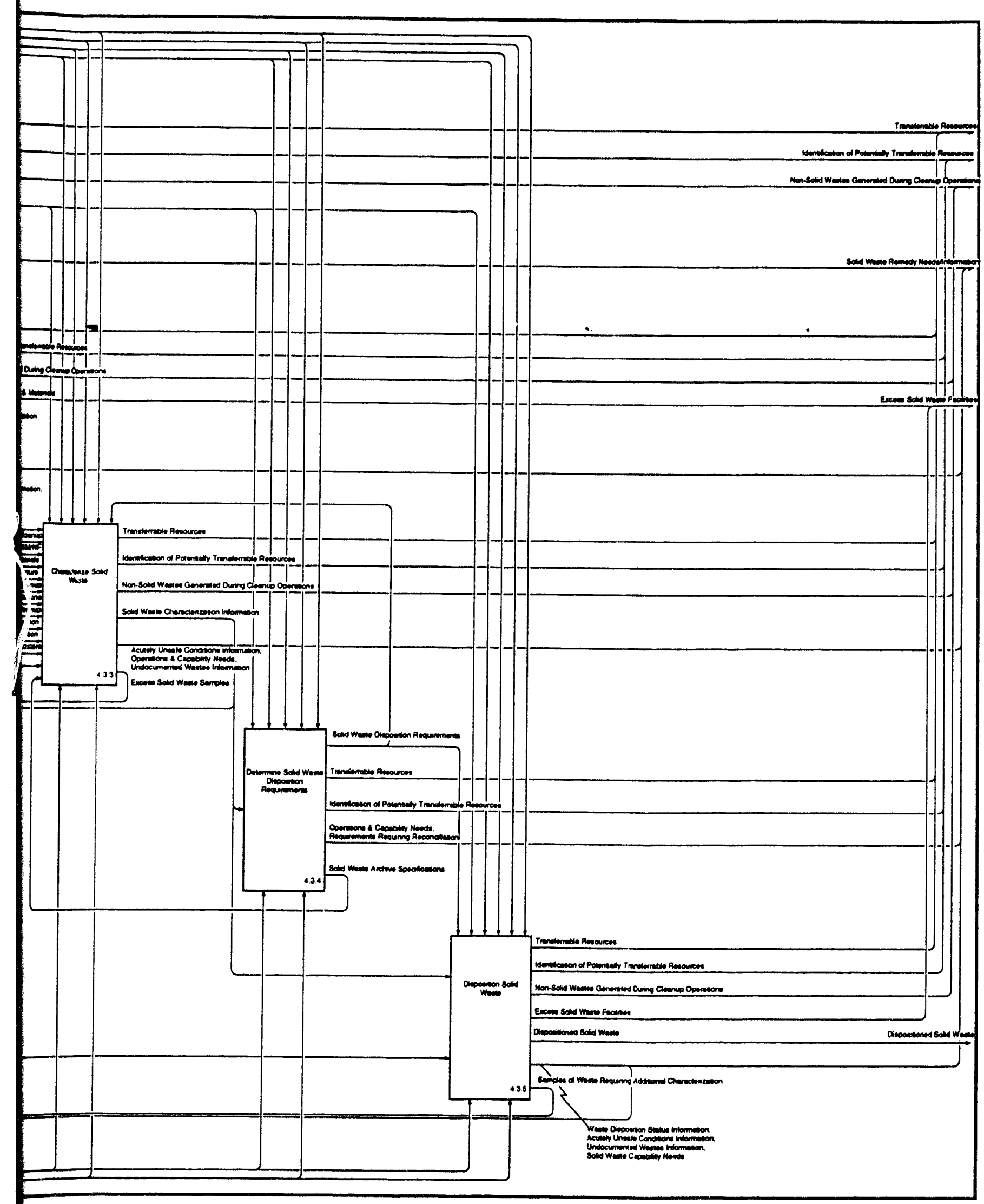




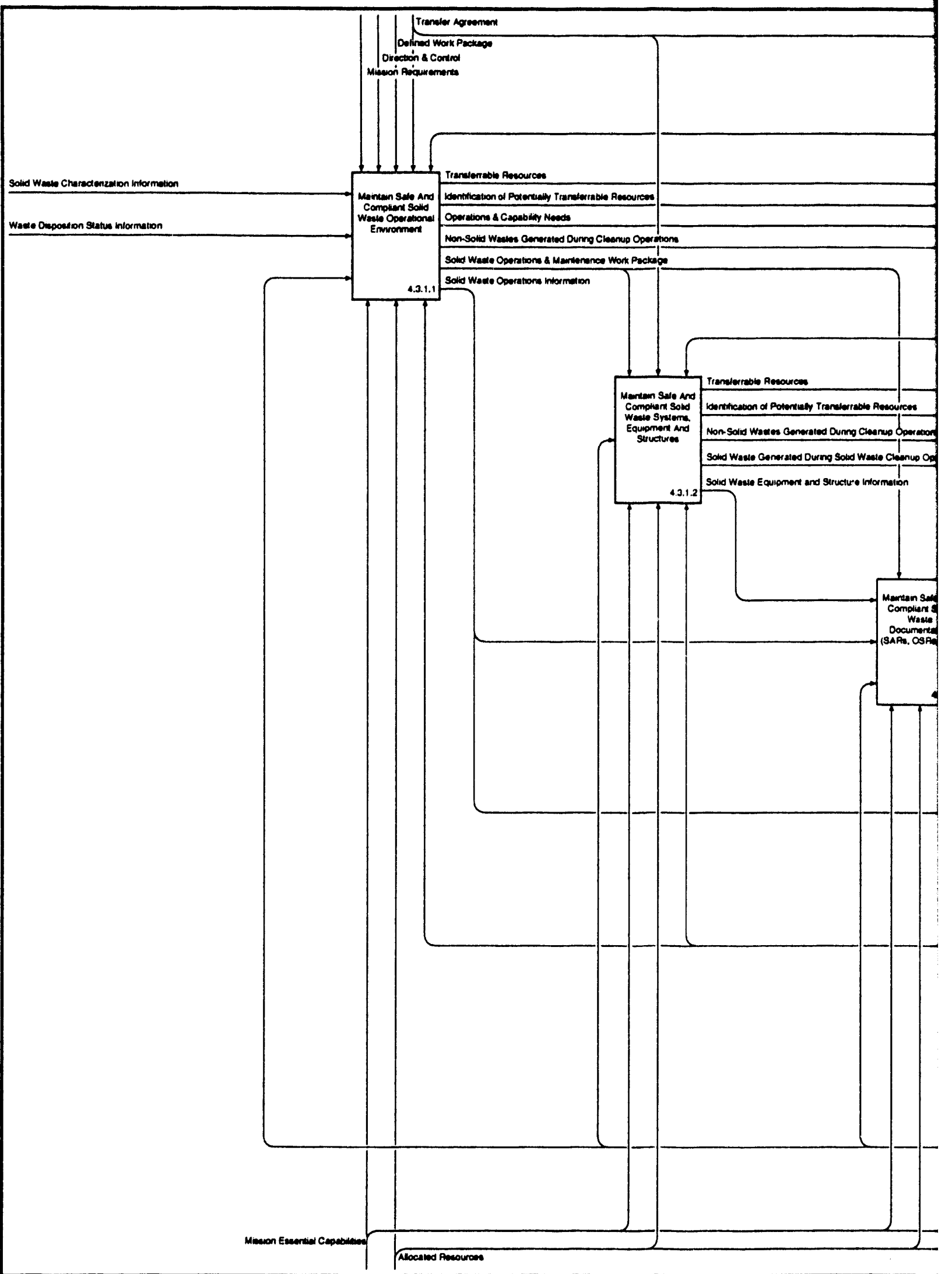




\section{te Safety And Compliance Envelope}

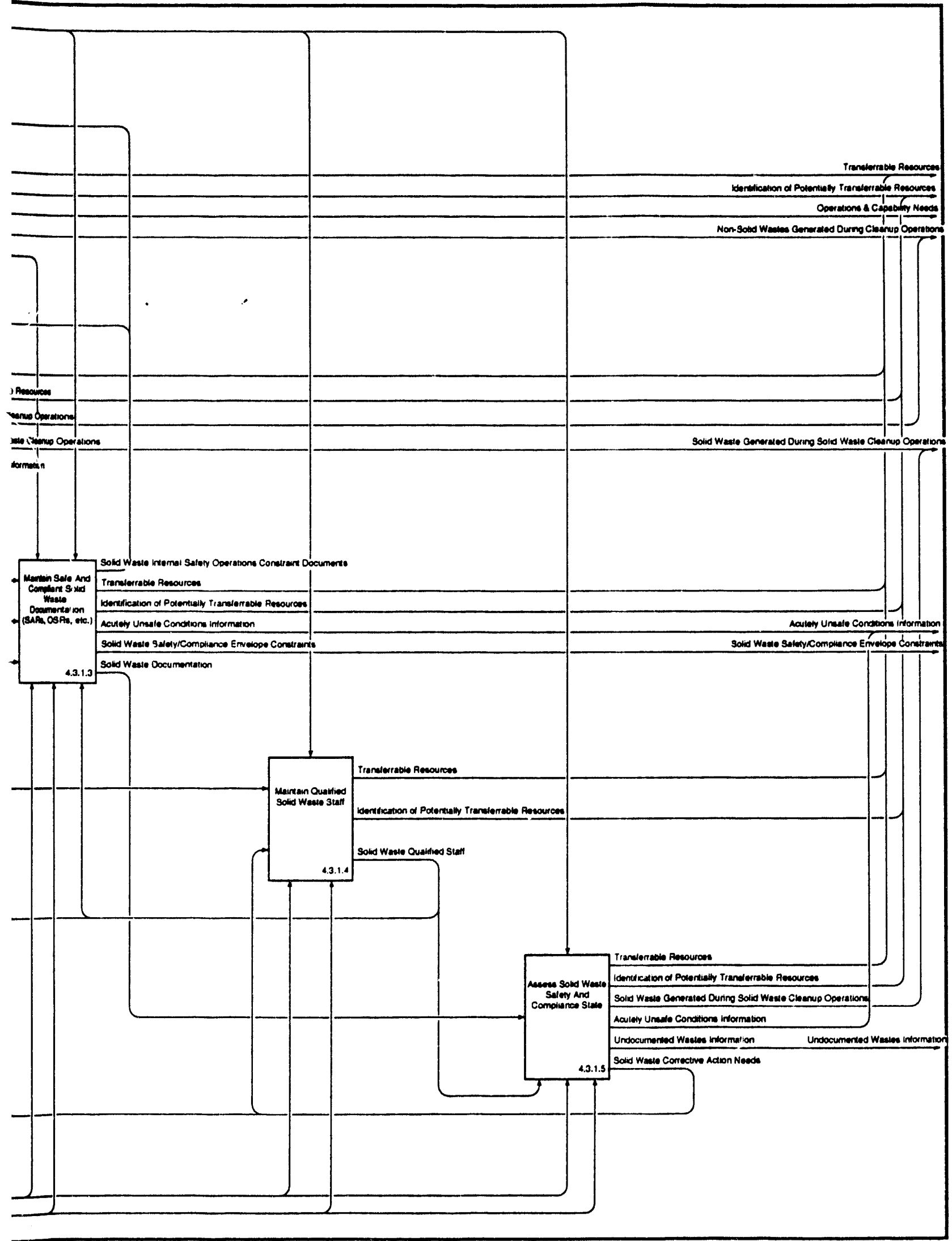

[4.3.1] Maintain Solid Waste Safery And Compliance Envelope 
[4.3.2] Receiv

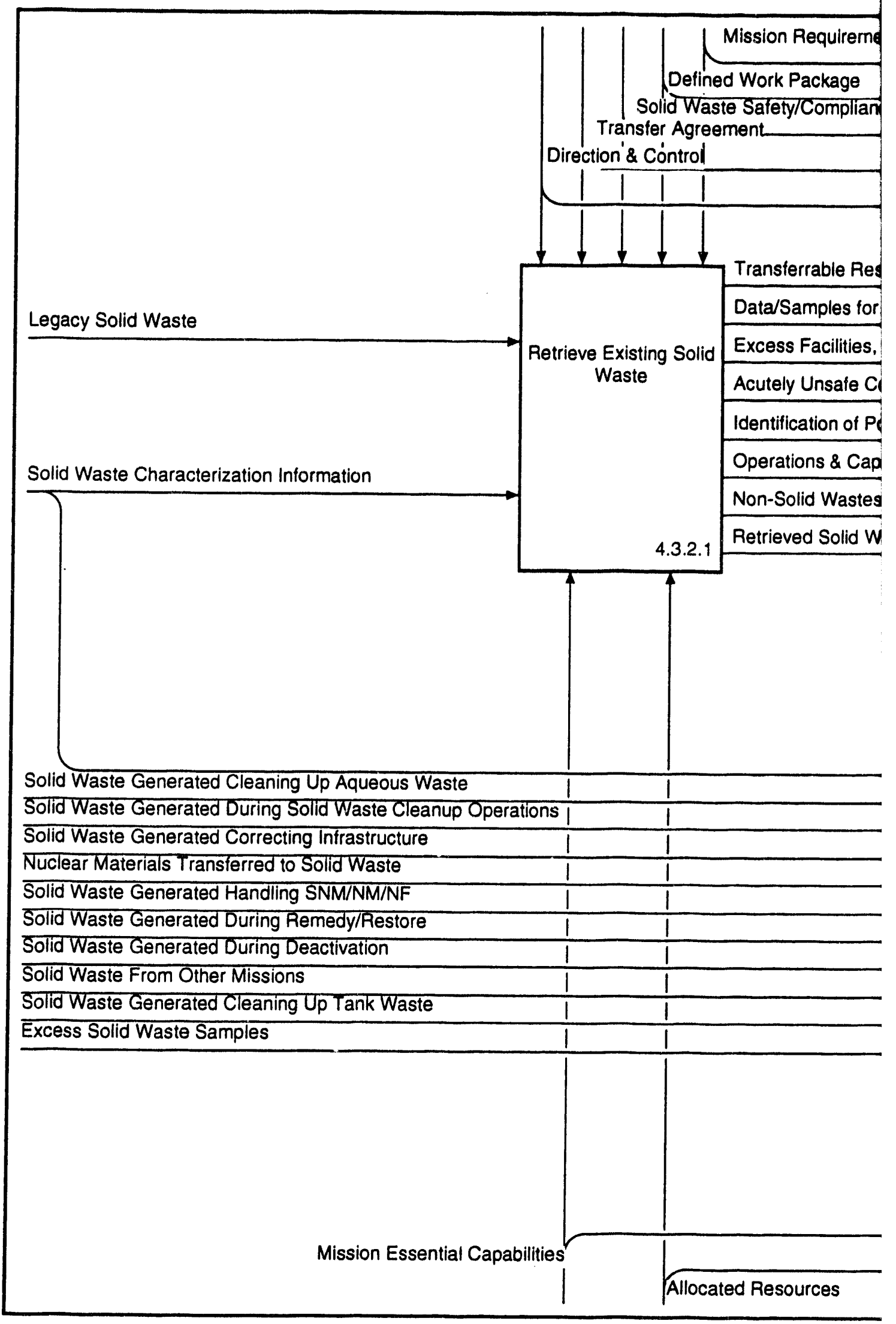




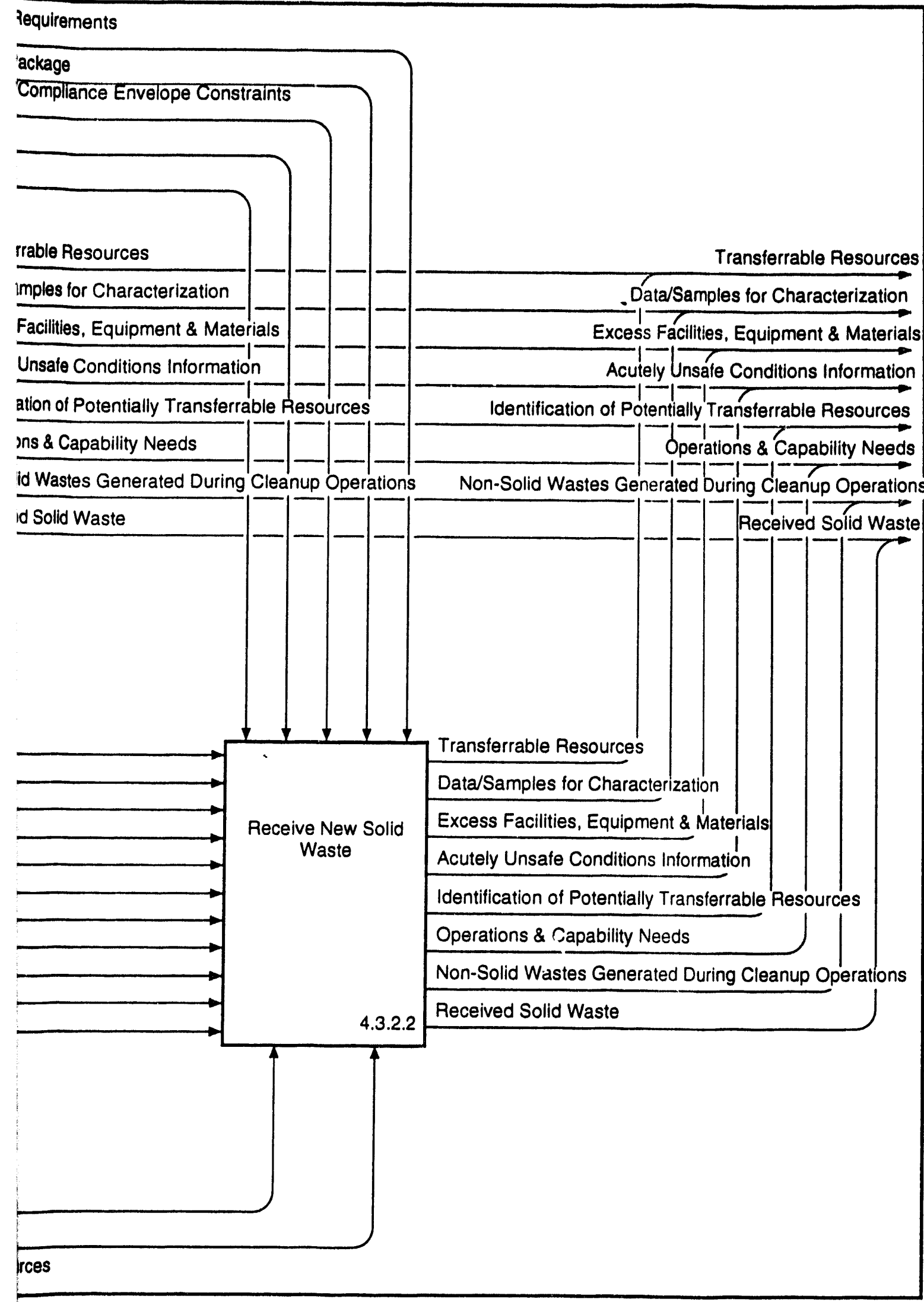


[4.3.2.1] Retrieve Ex

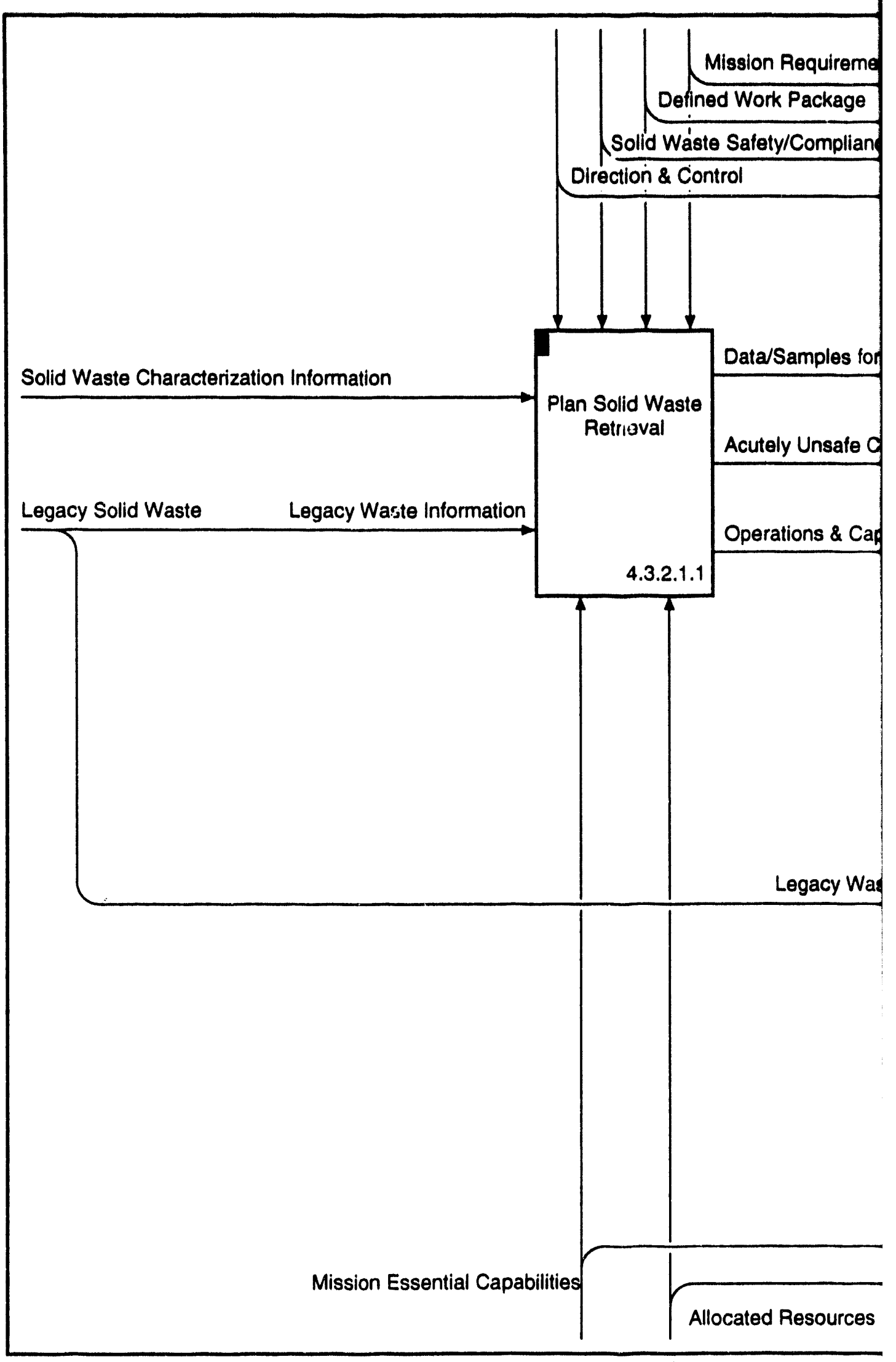




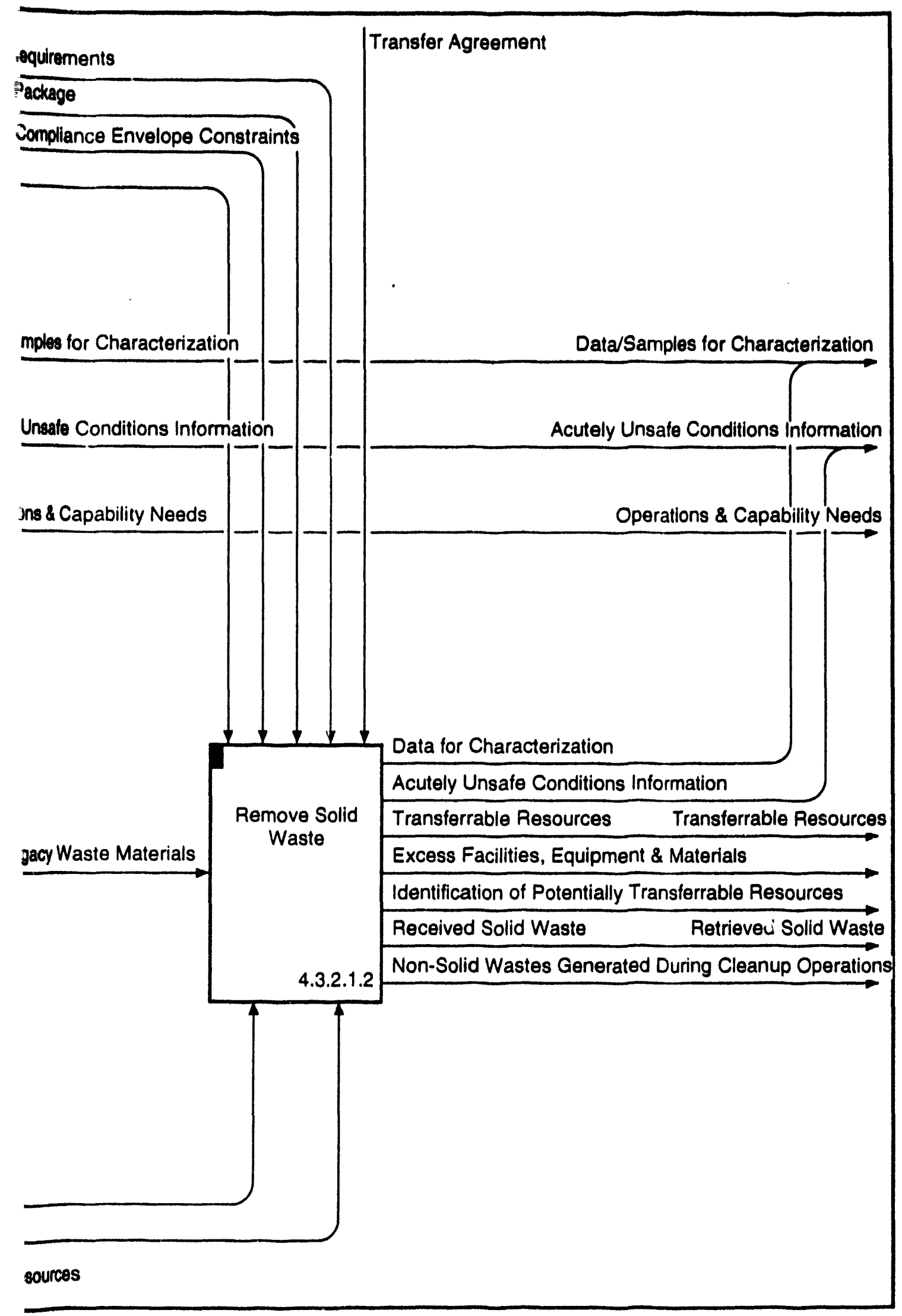


[4.3.2.1.1] Plan Solid

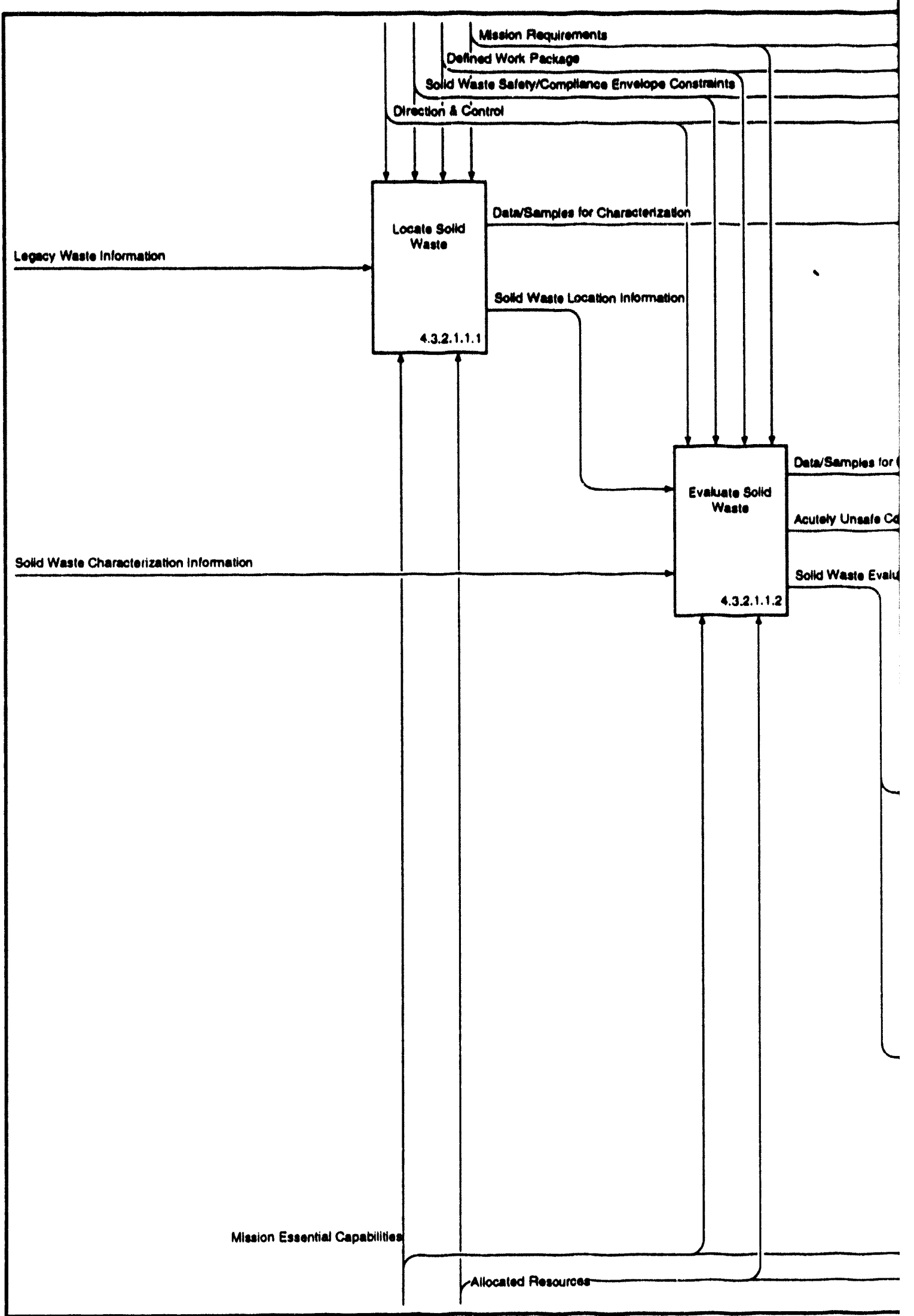




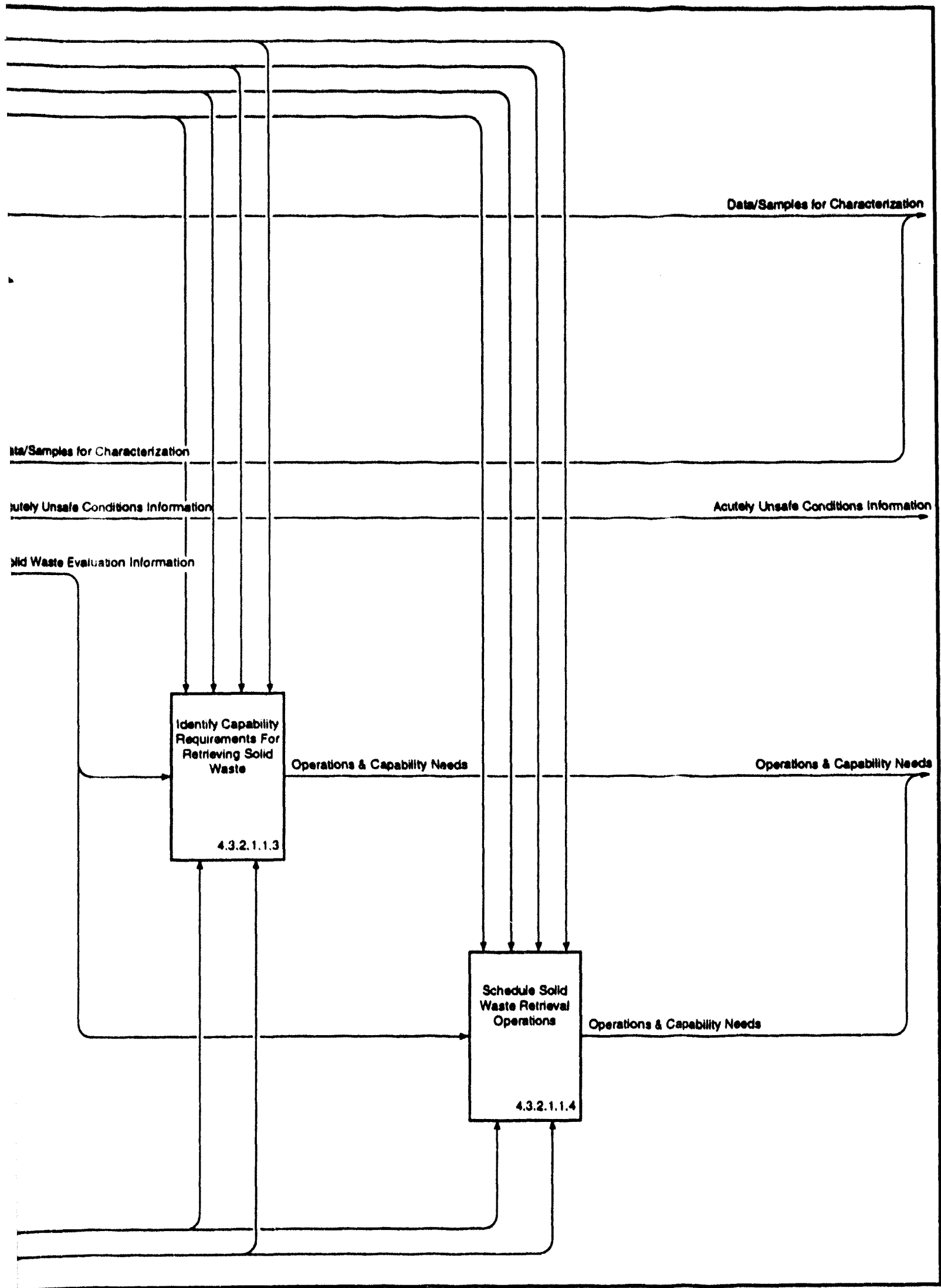



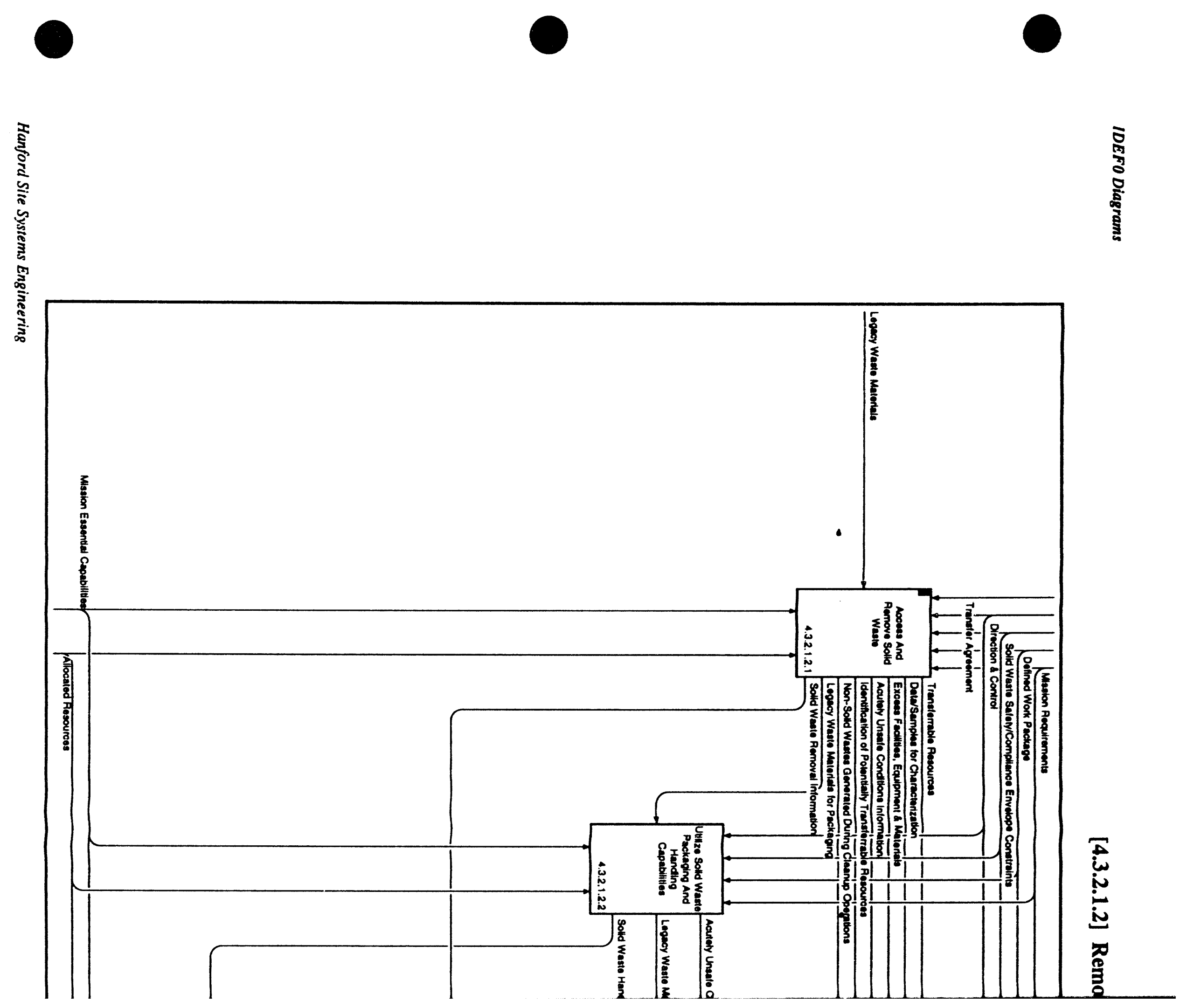


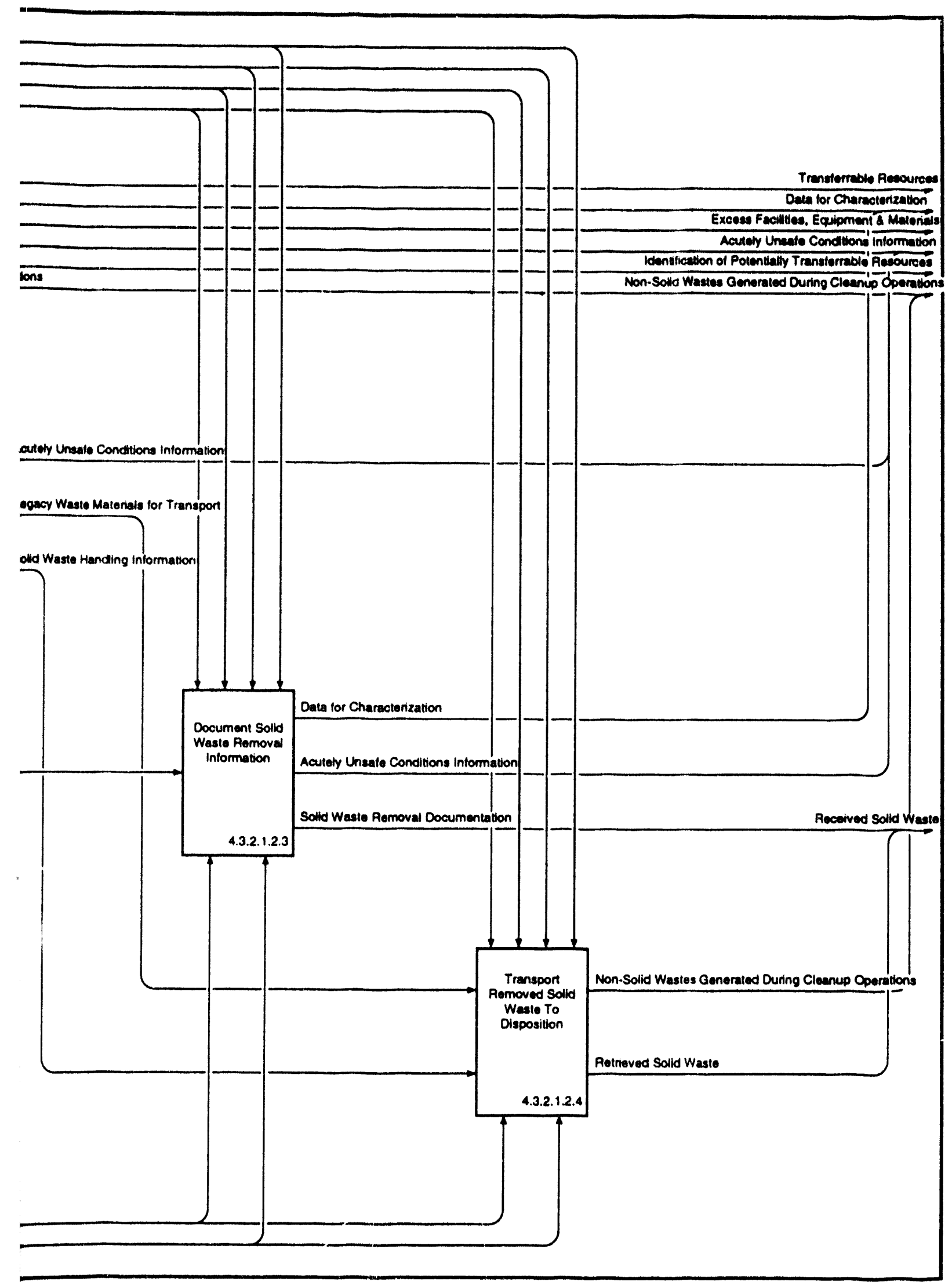




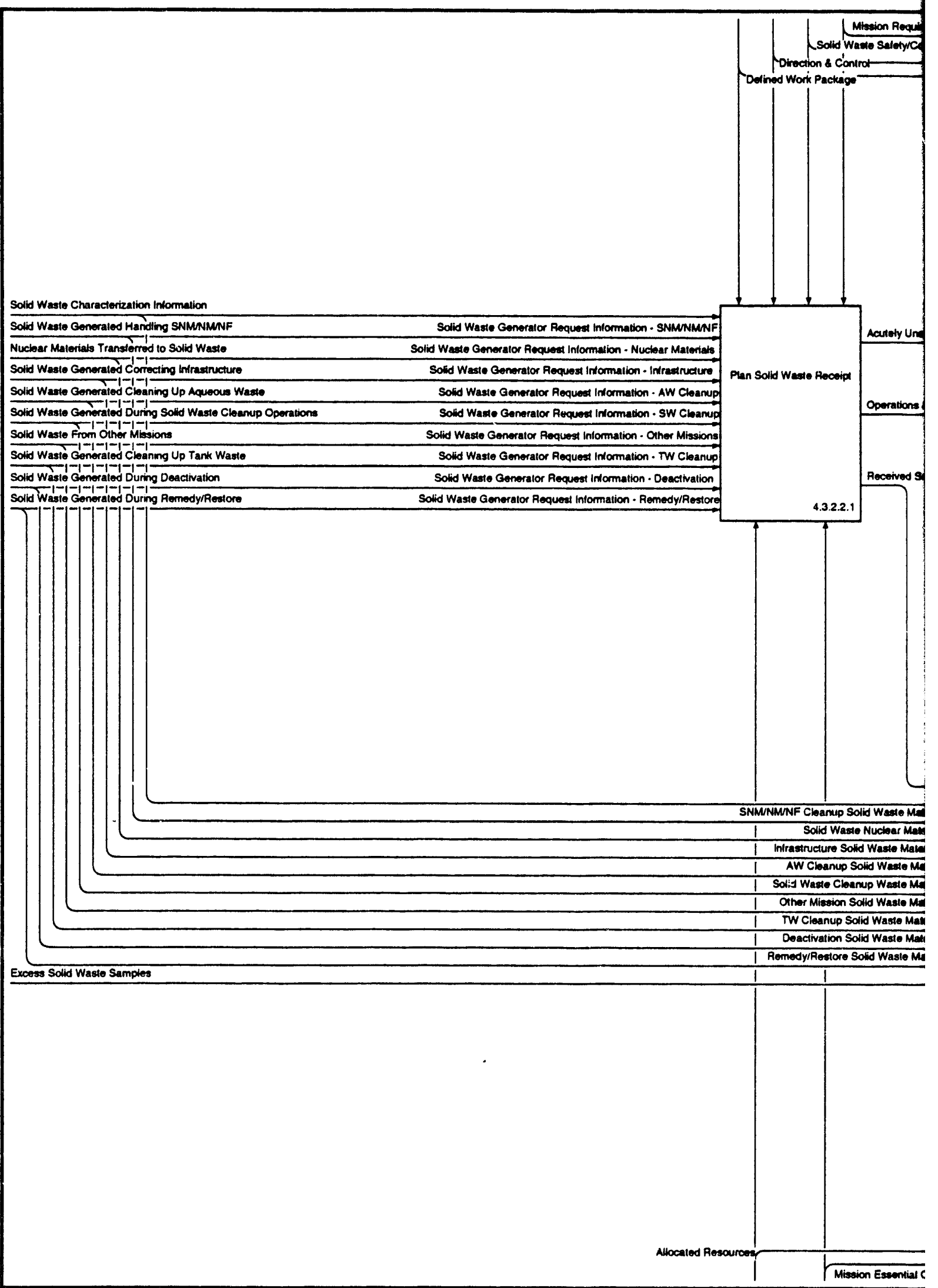




\section{Receive New Solid Waste}

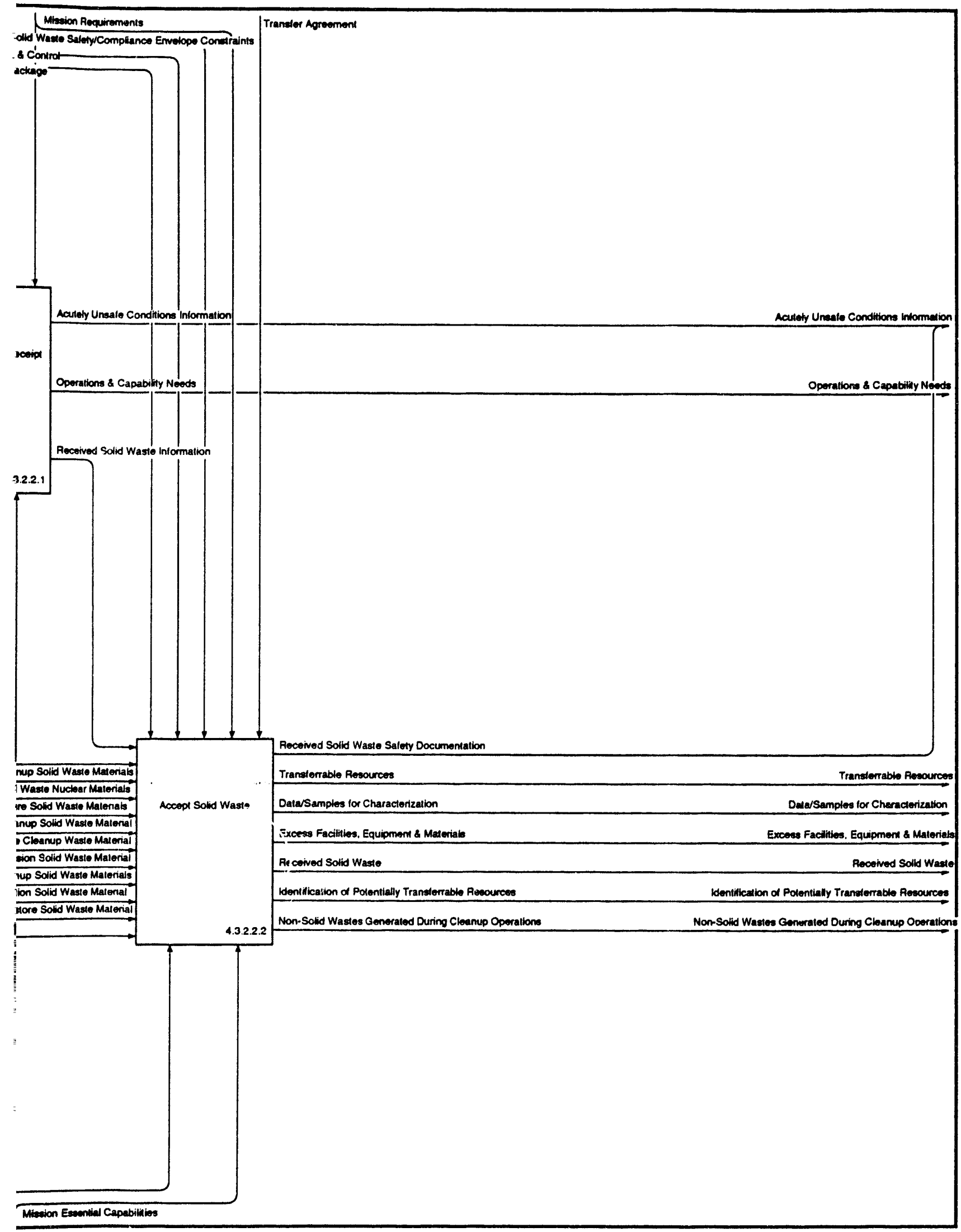




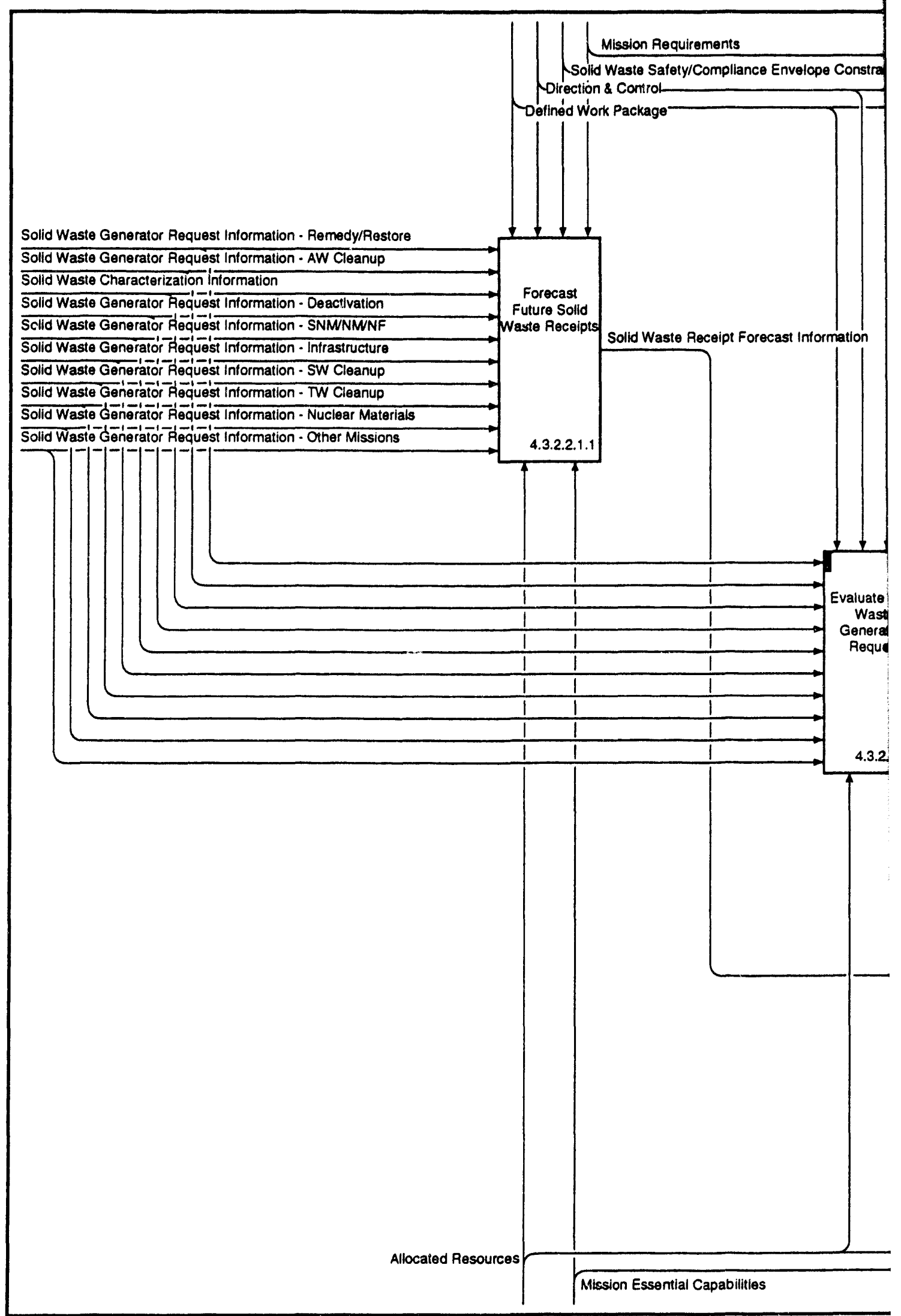




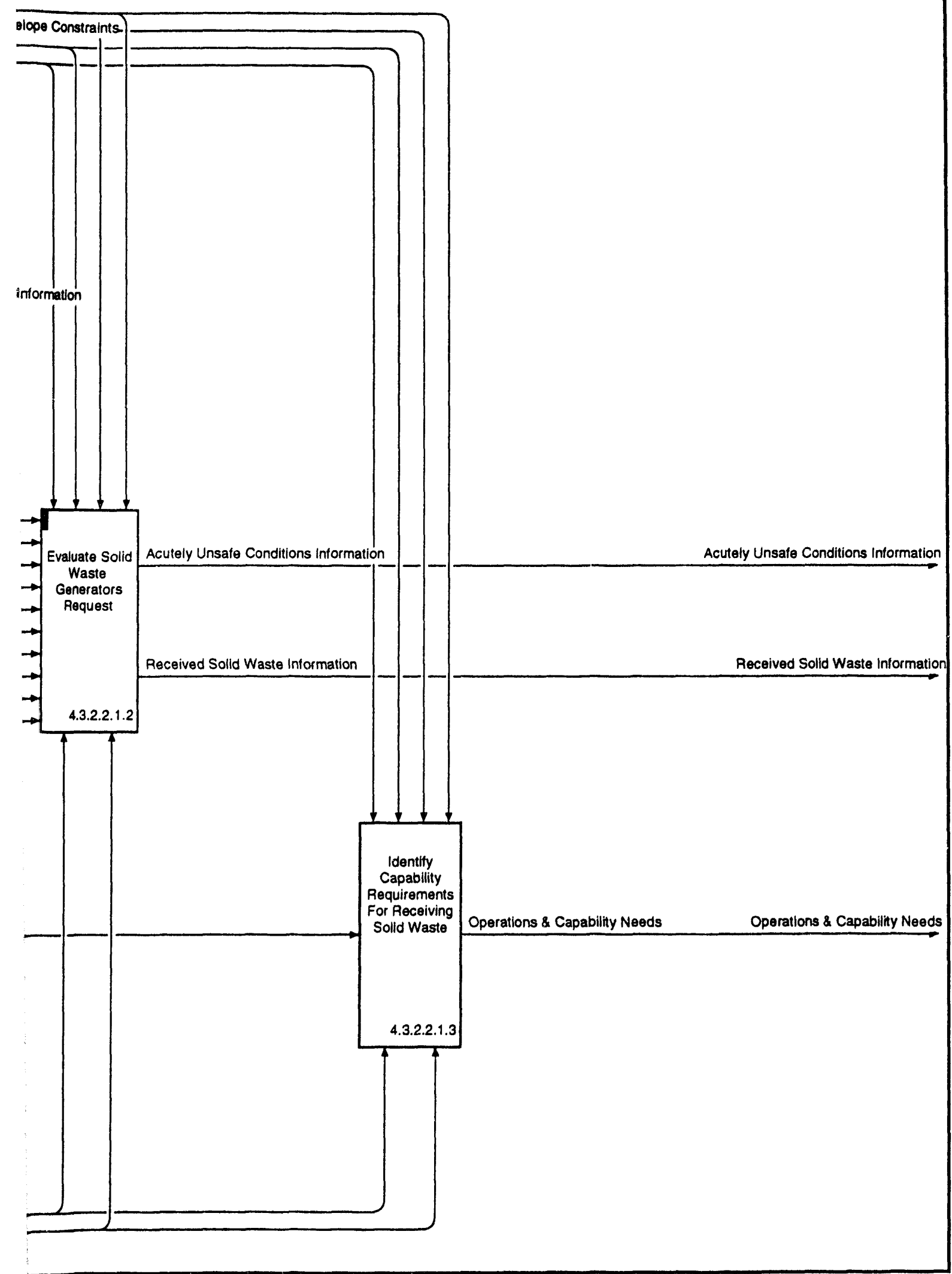




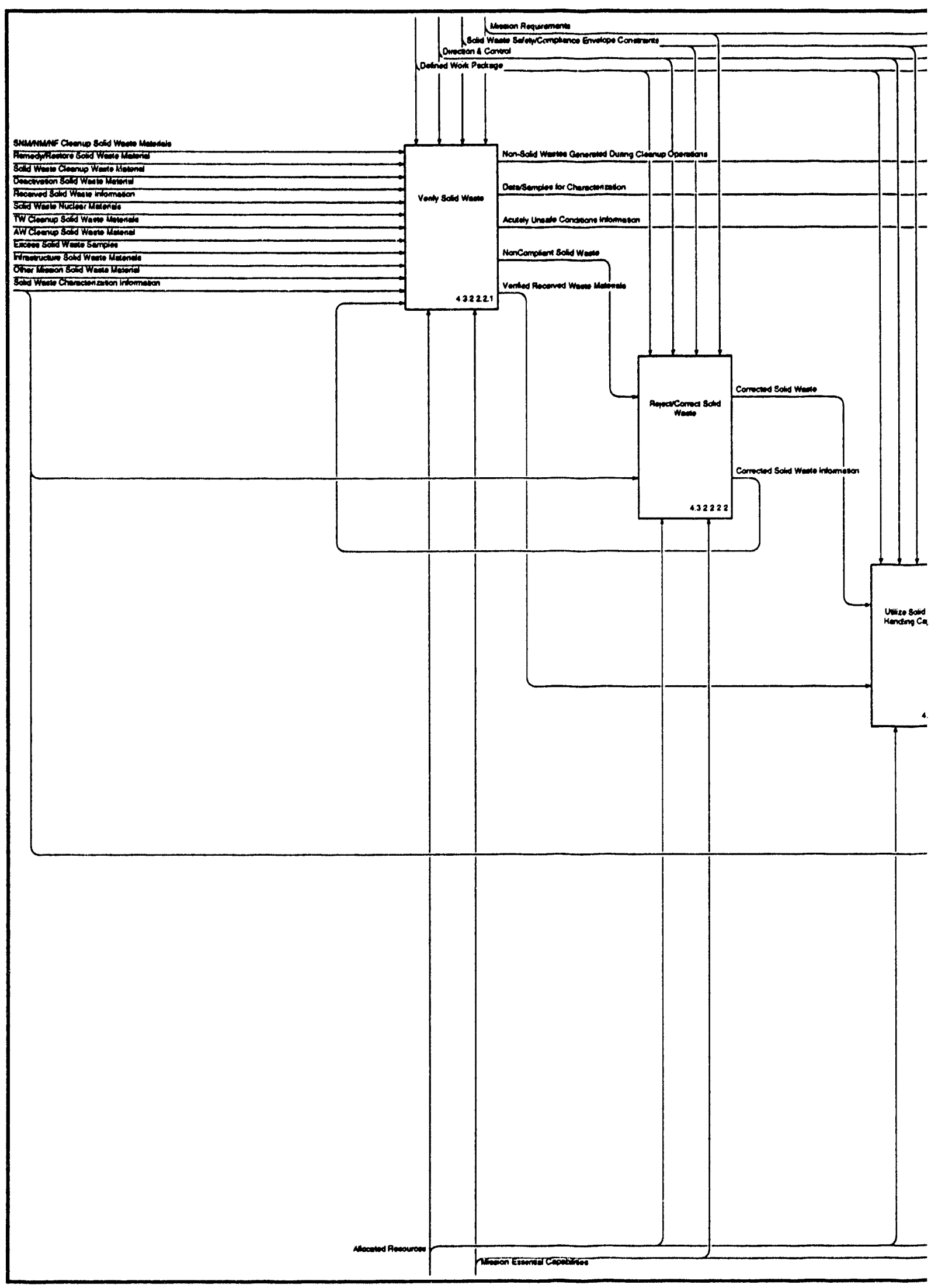




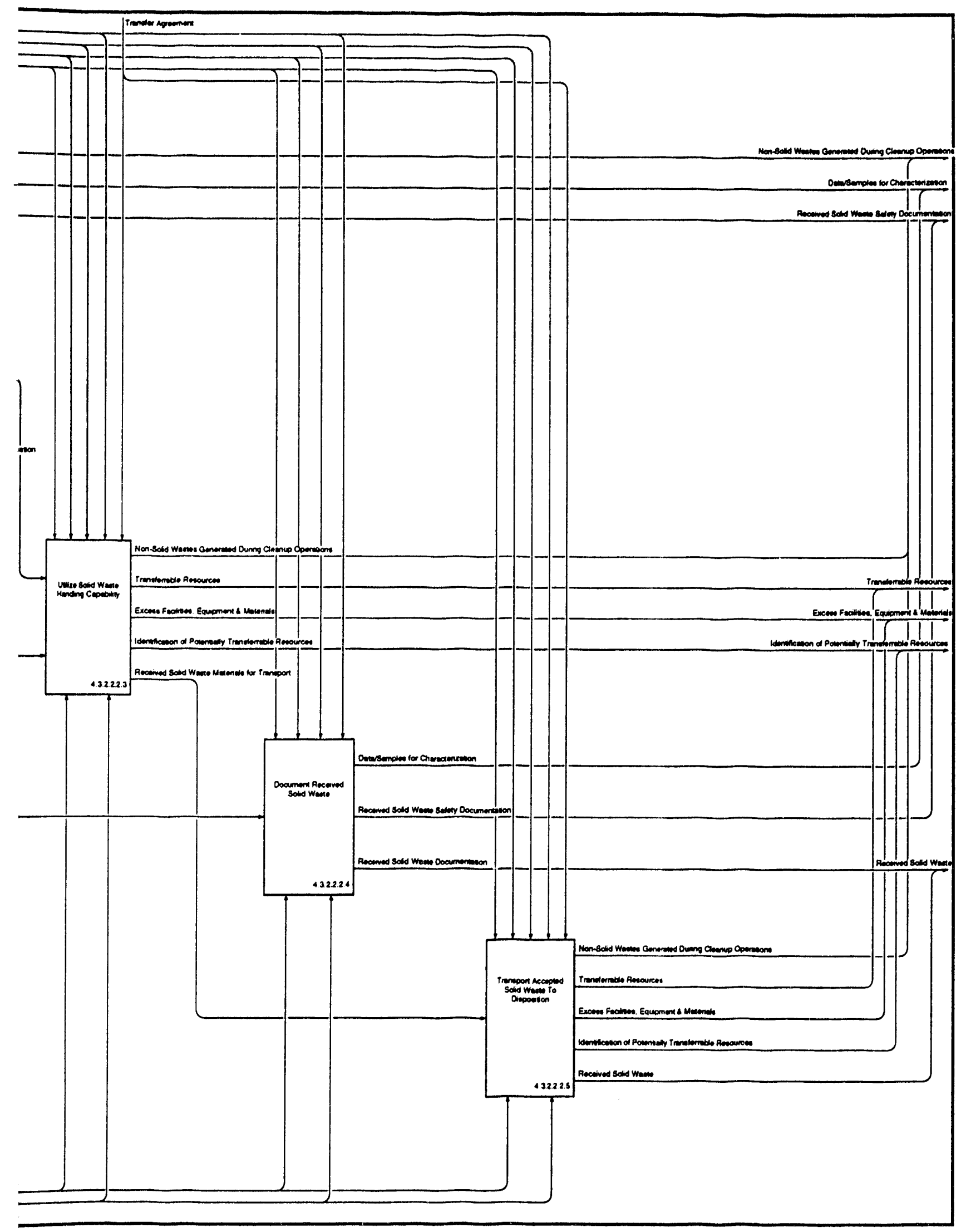




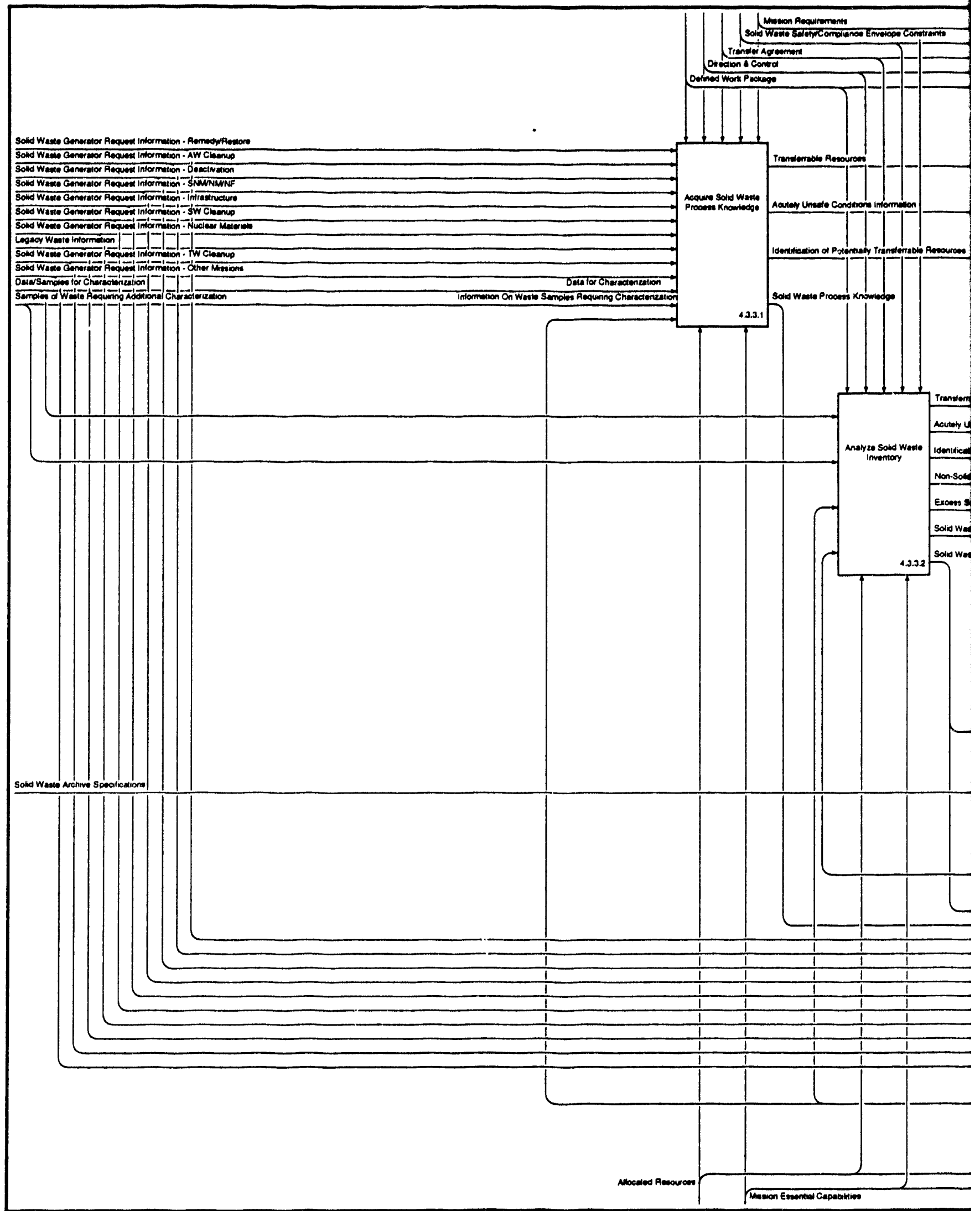

Hanford Site Systems Engineering 


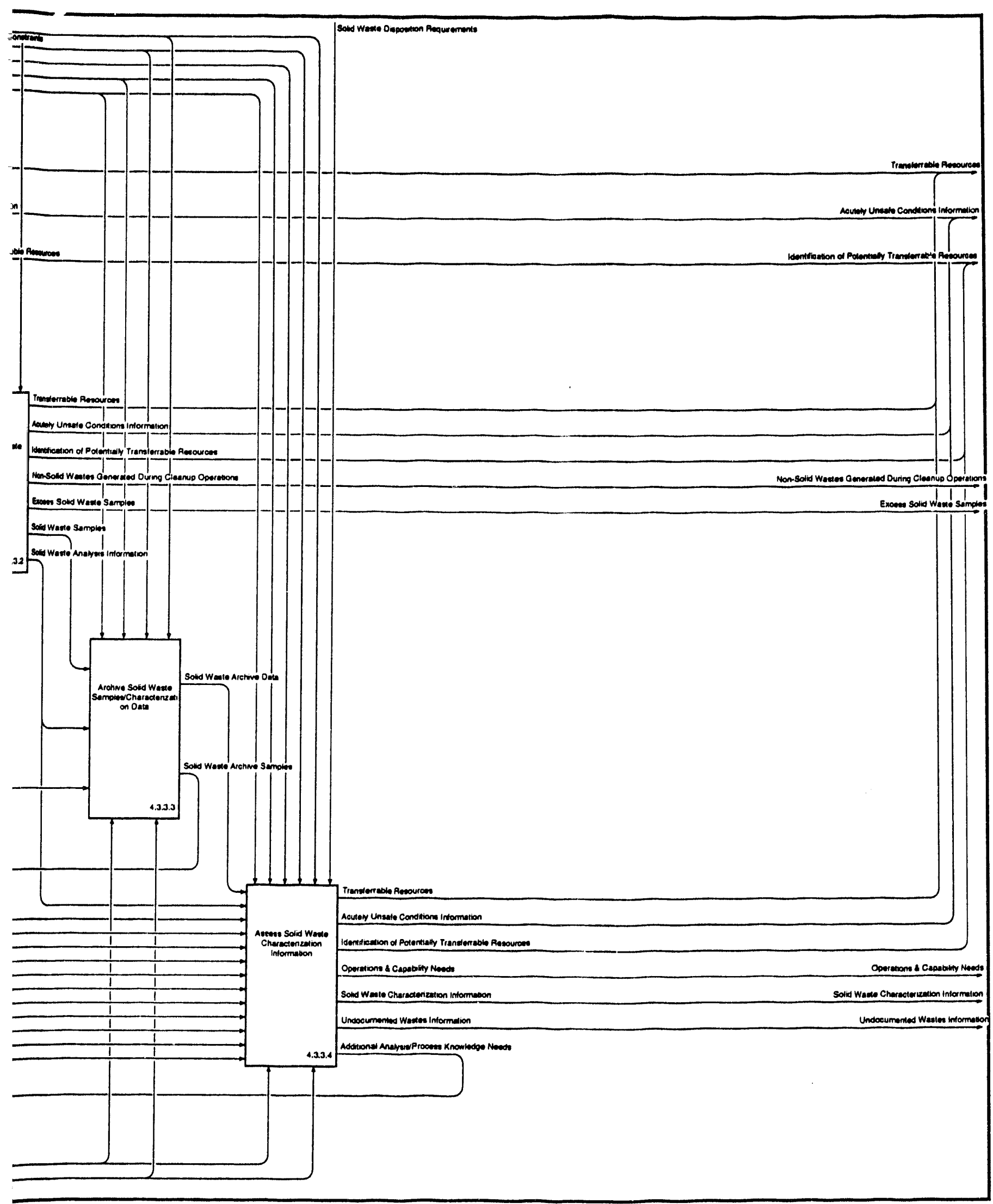


[4.3.4] Determine Solid Wa

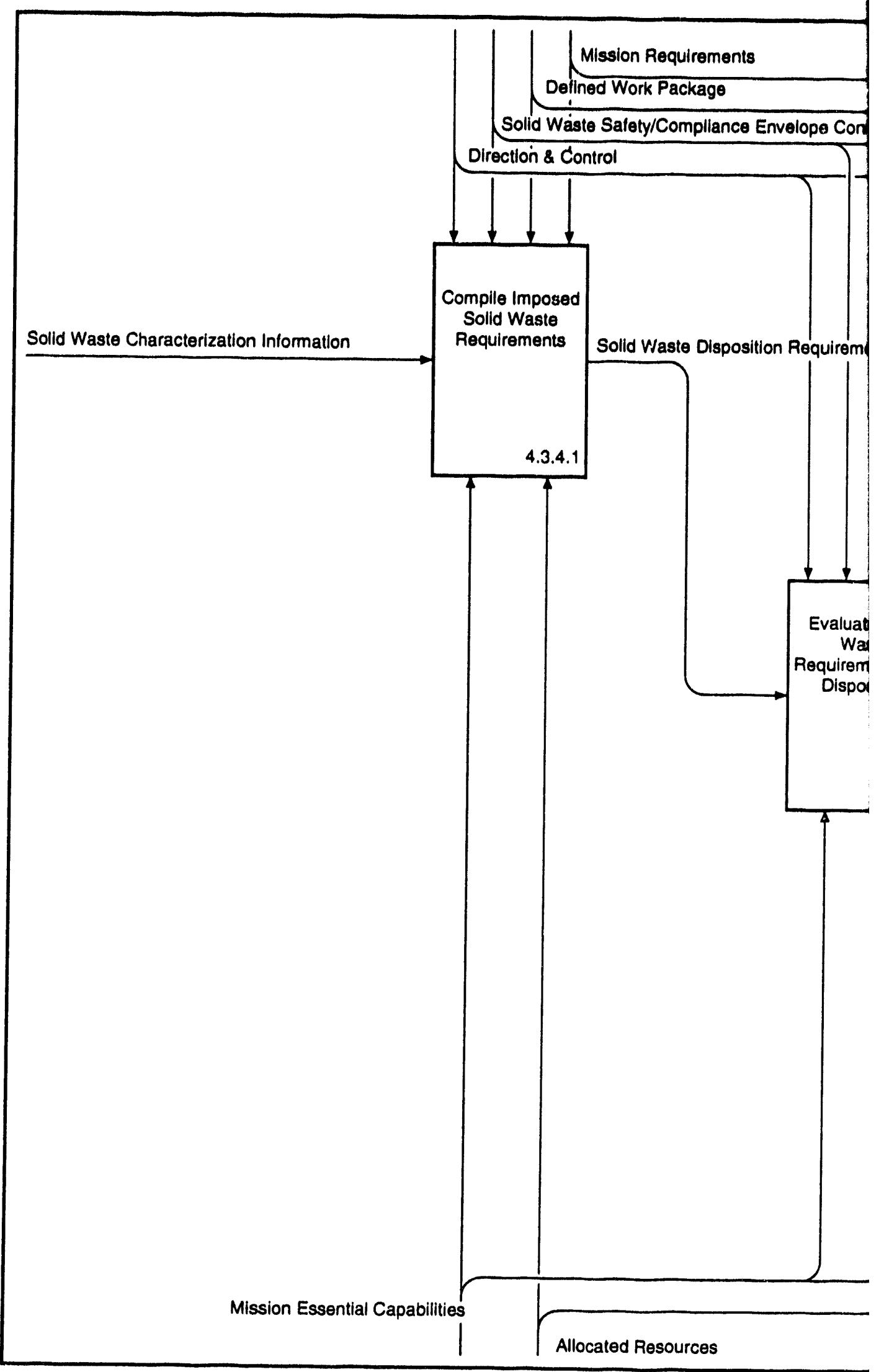




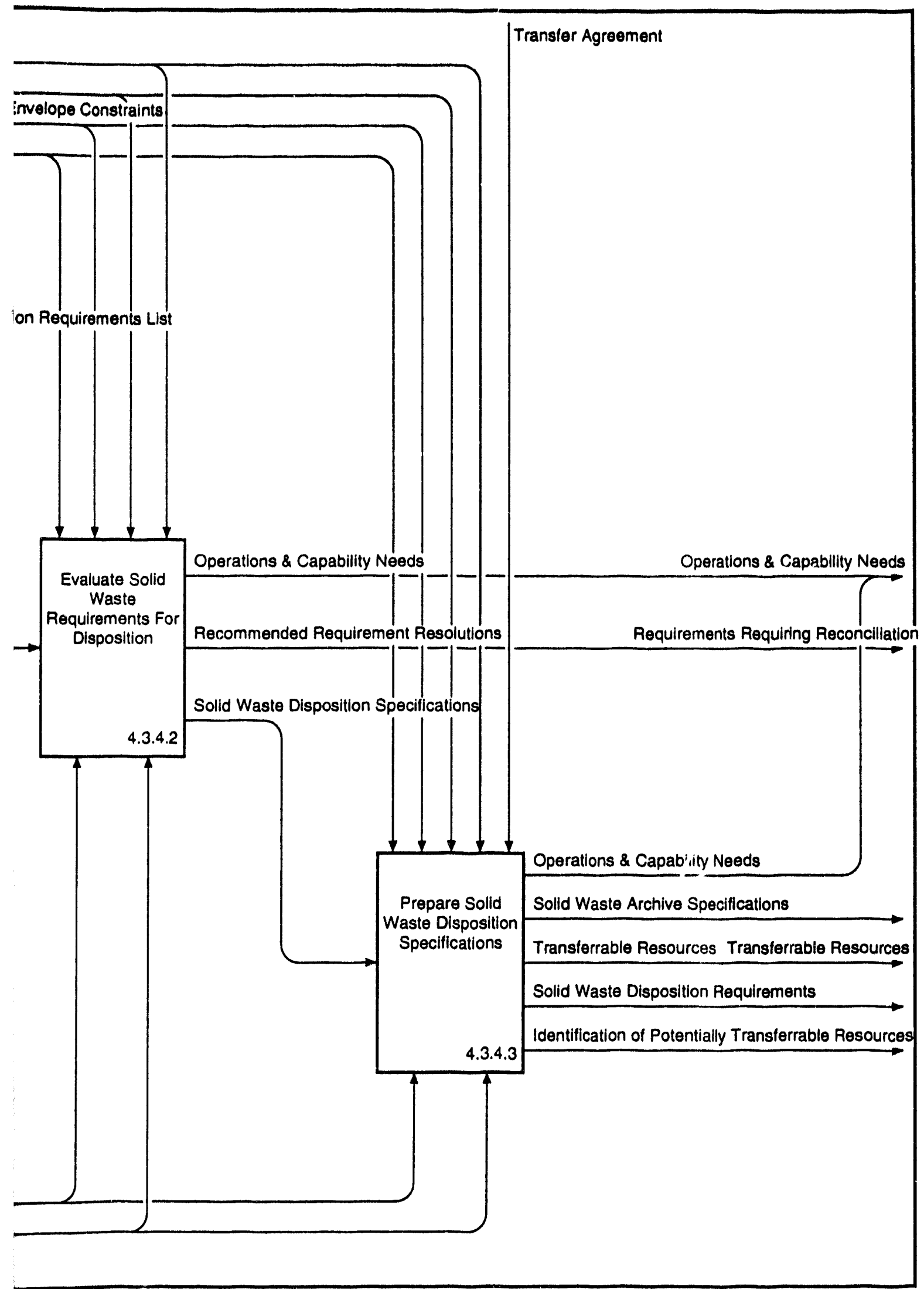


[4.3.5] Dispositi

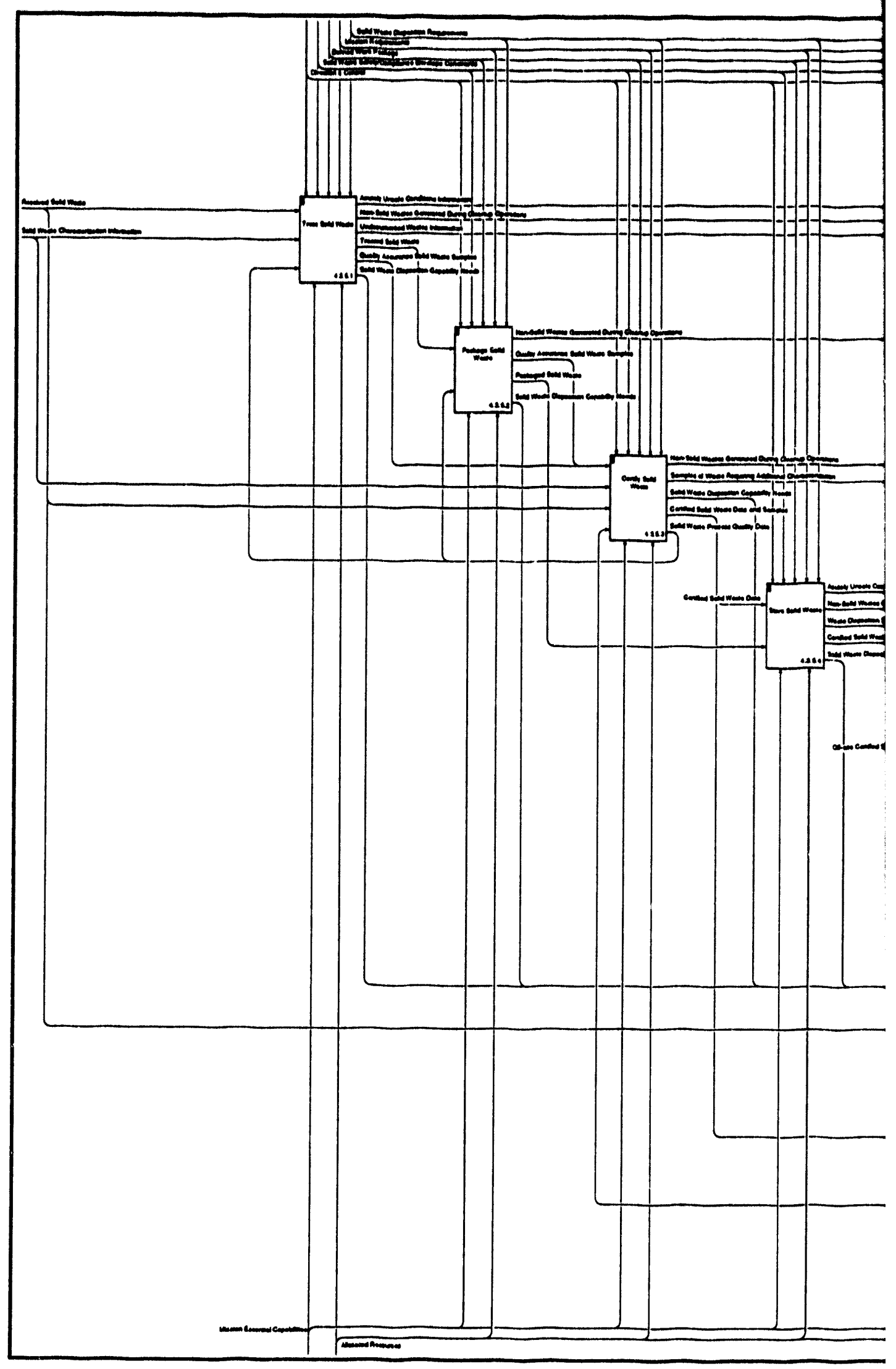




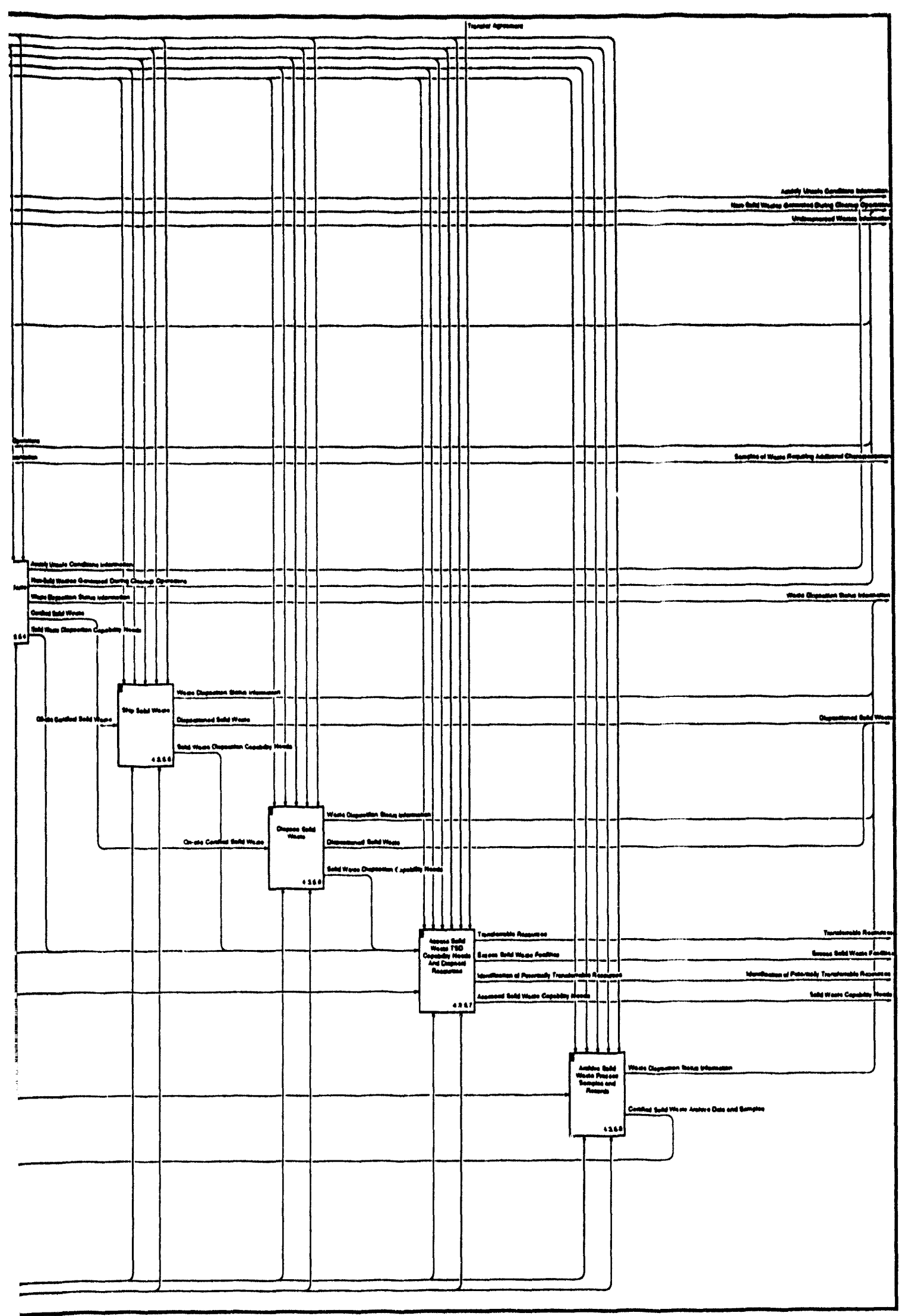




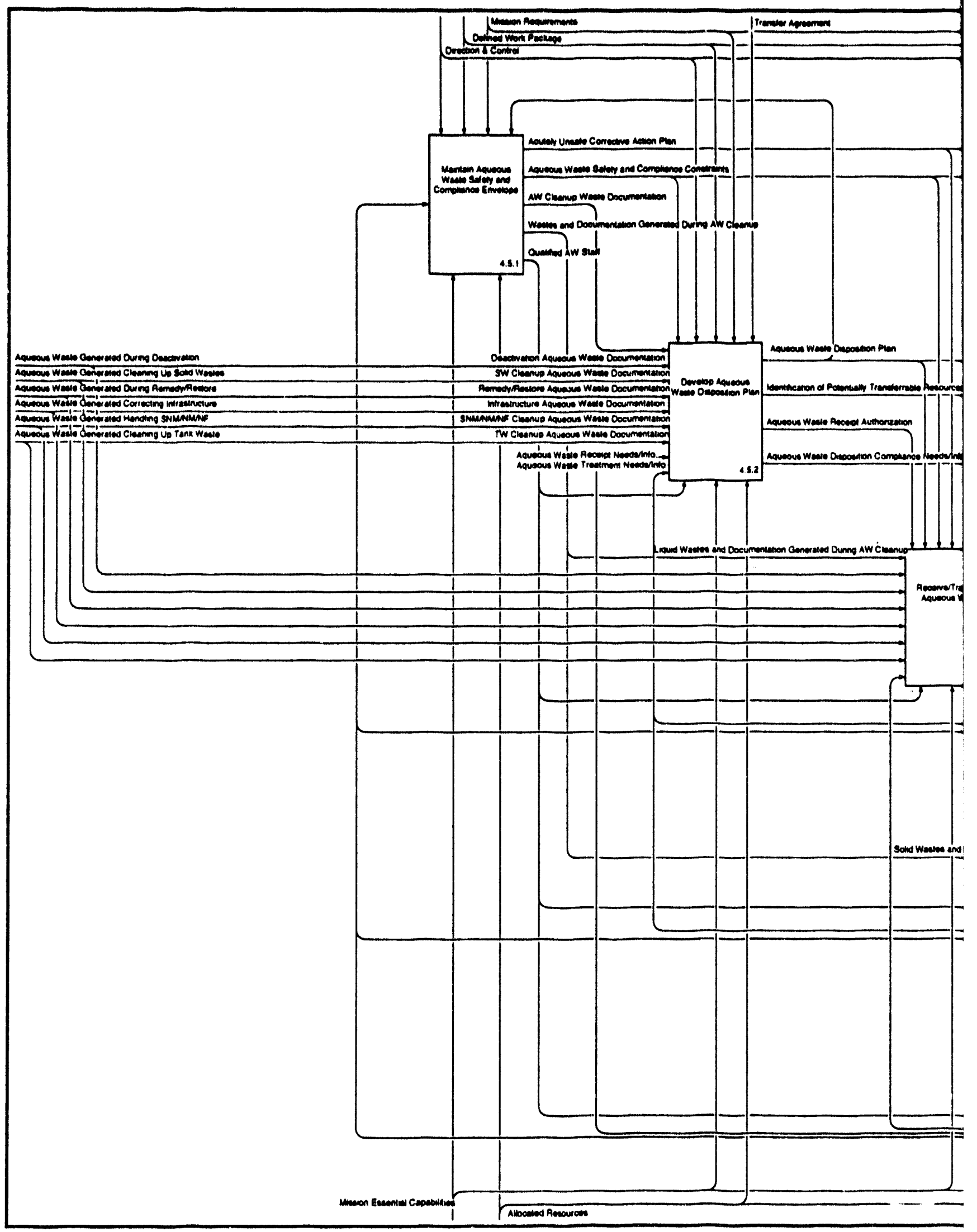




\section{nage Aqueous Wastes}

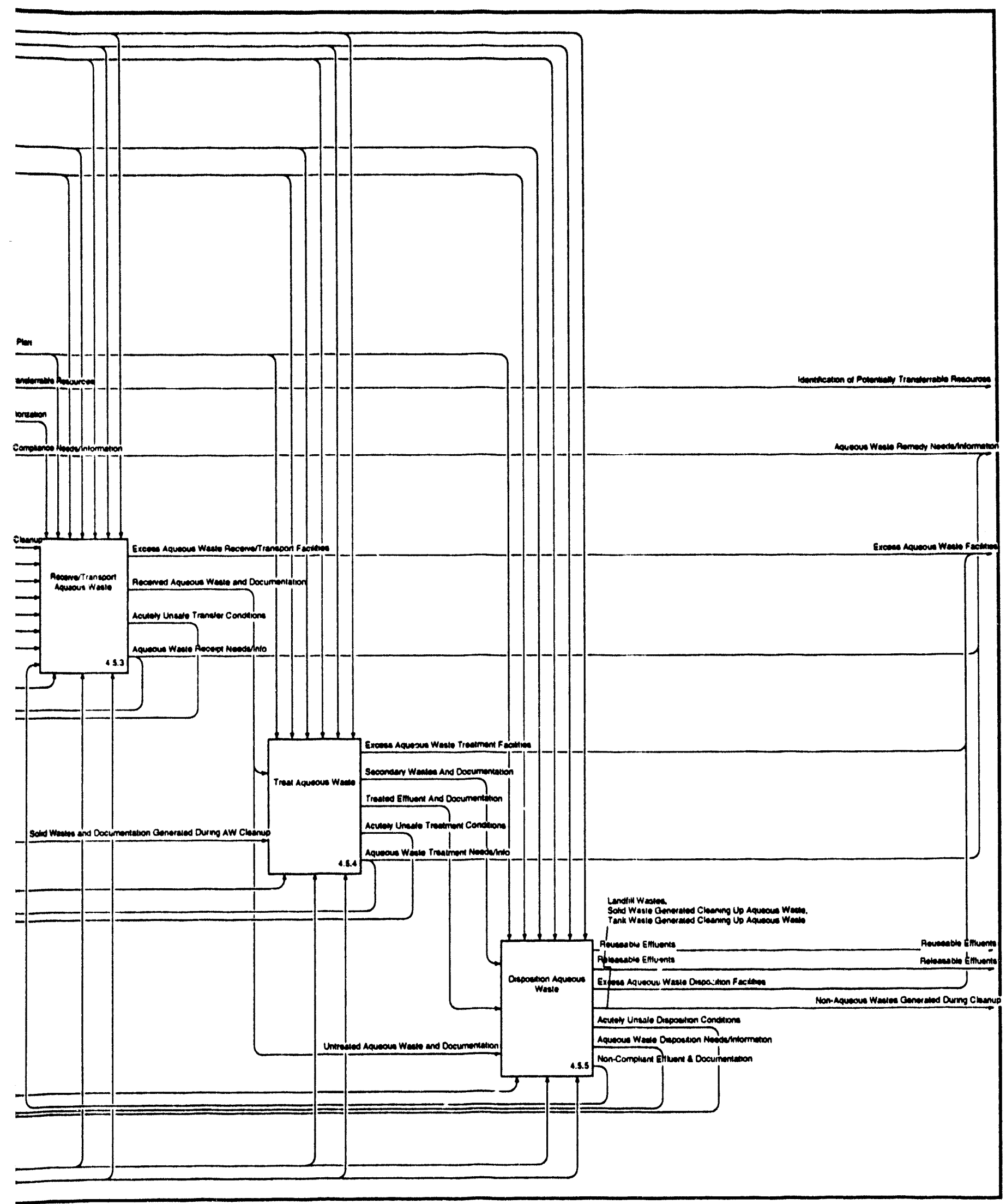




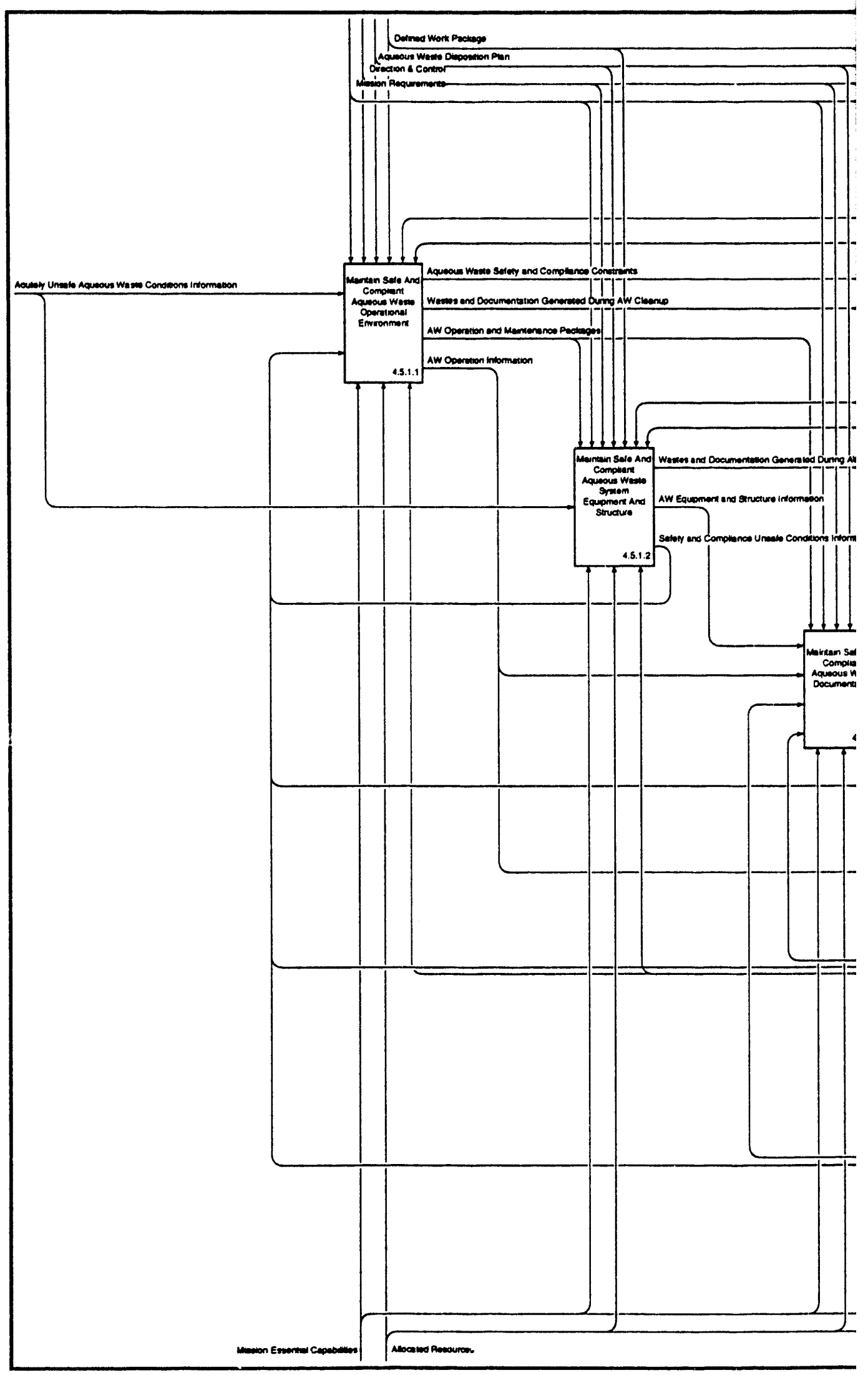




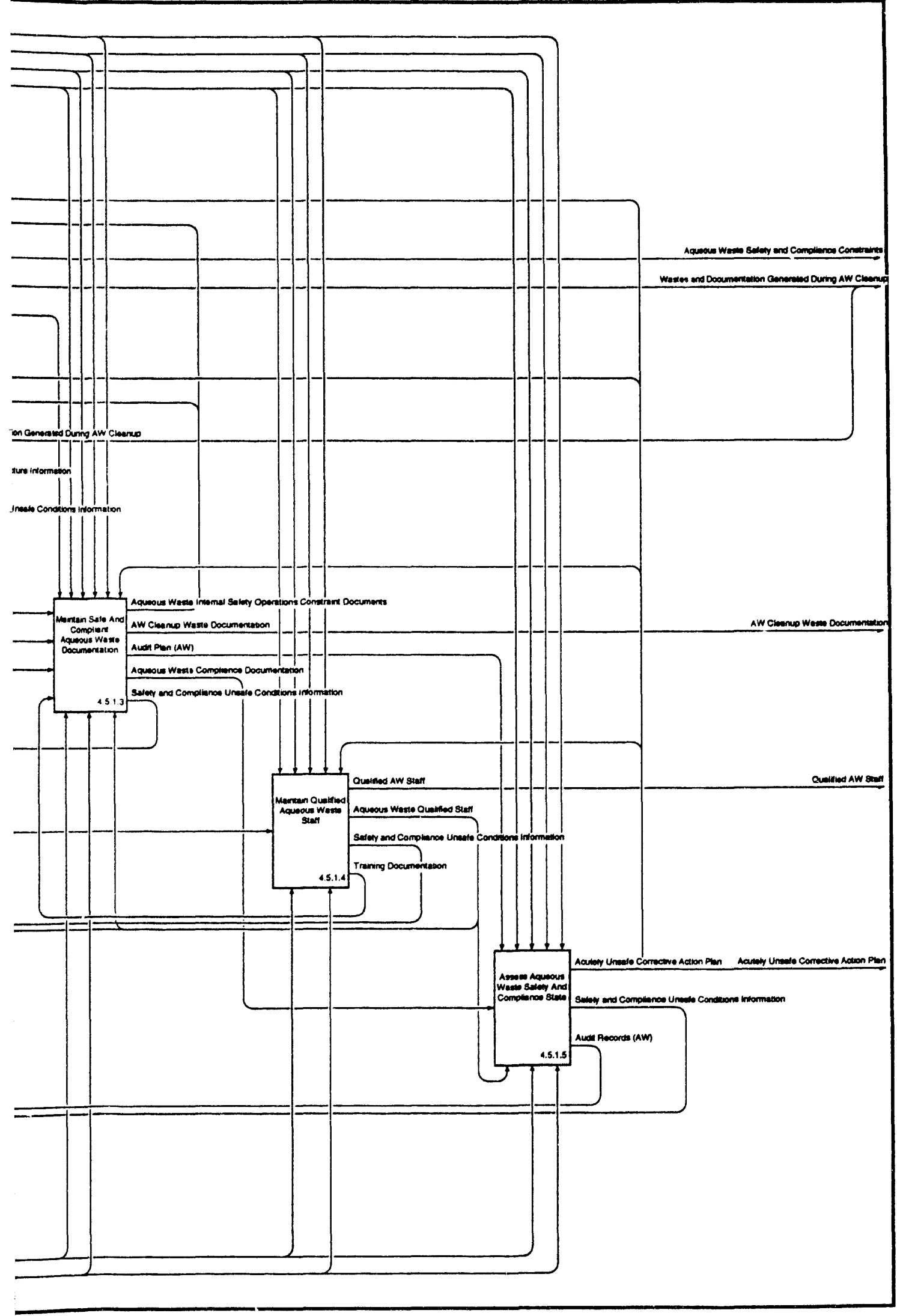




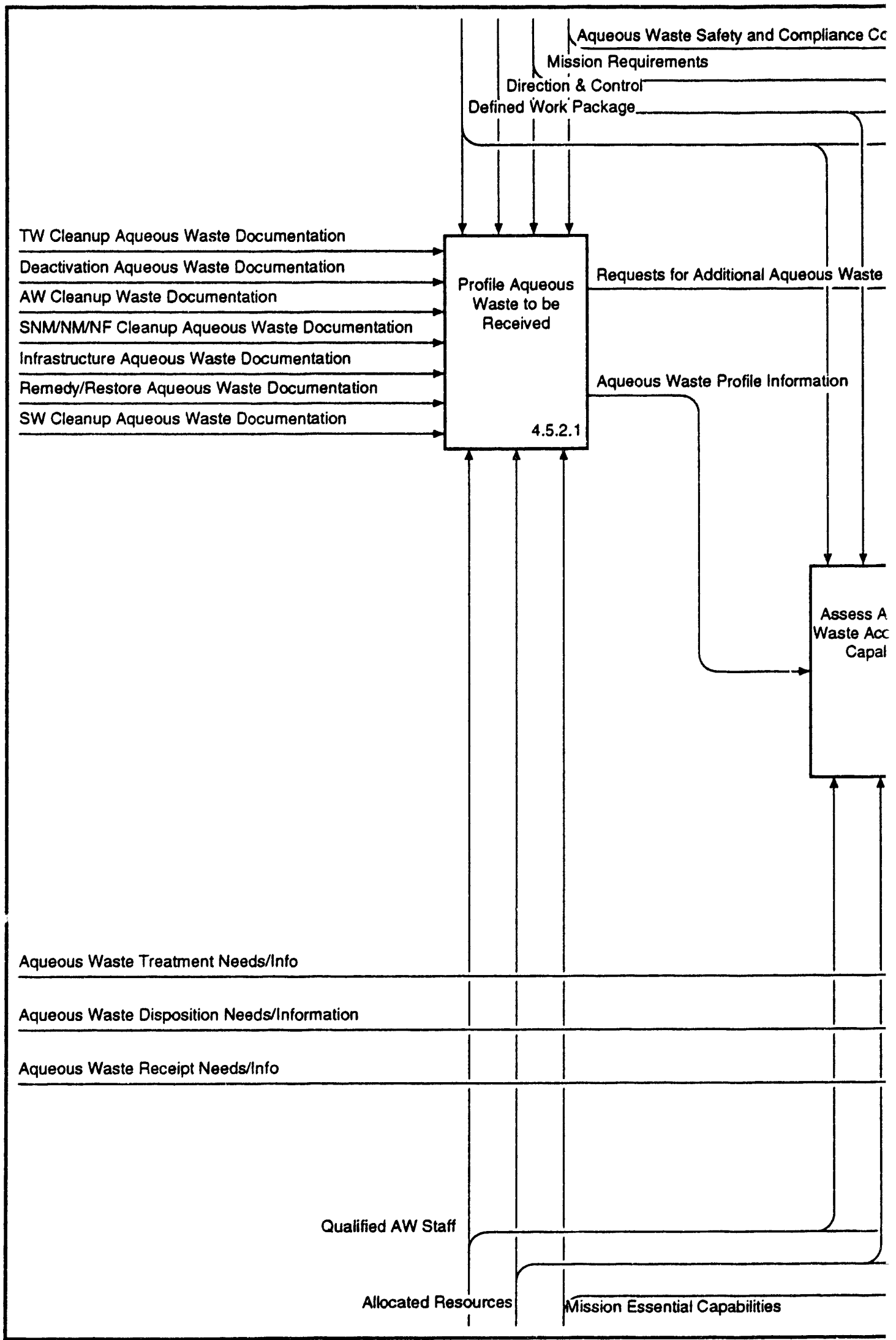

Hanford Site Systems Engineering 


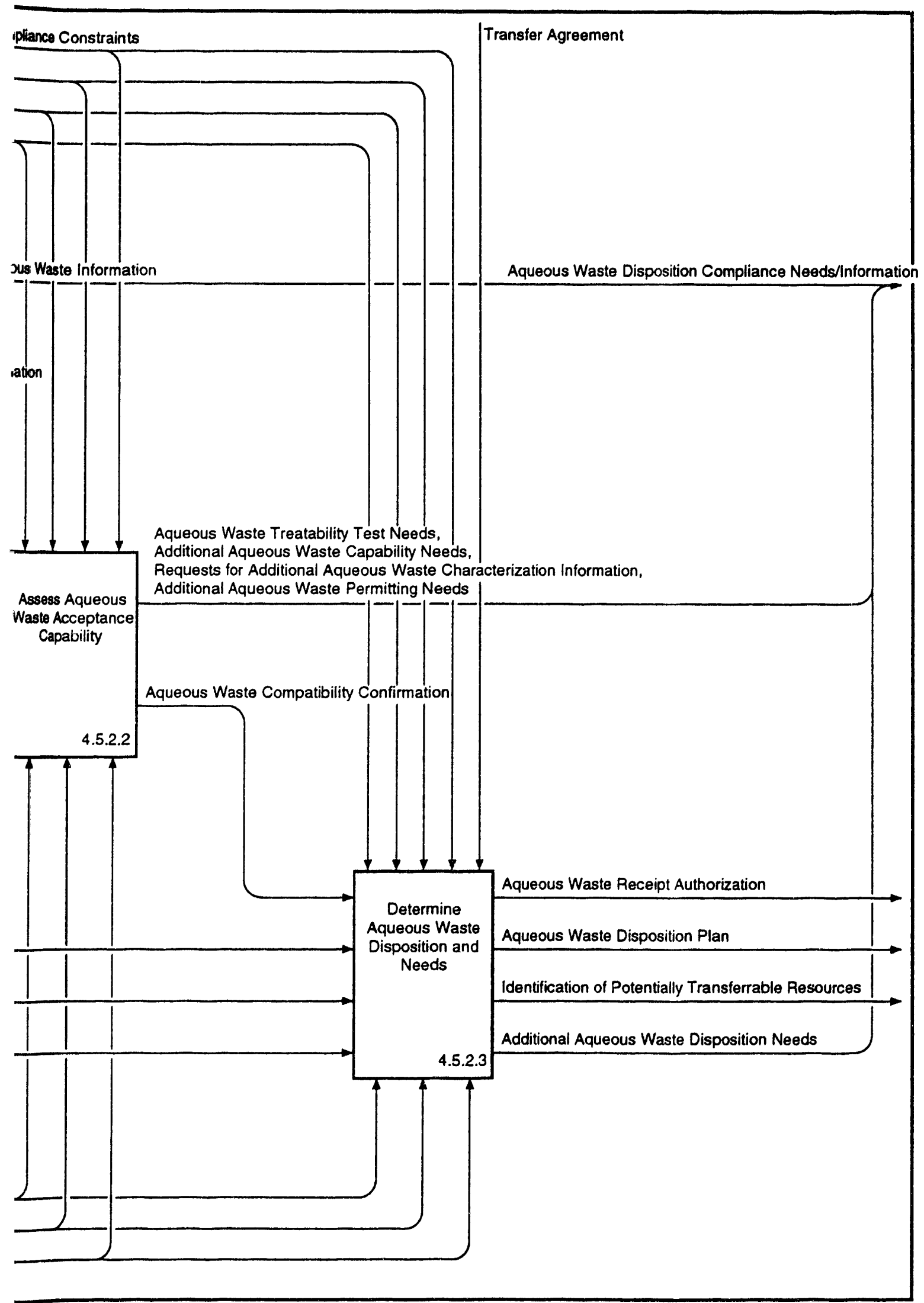


[4.5.3] Receive/Trans
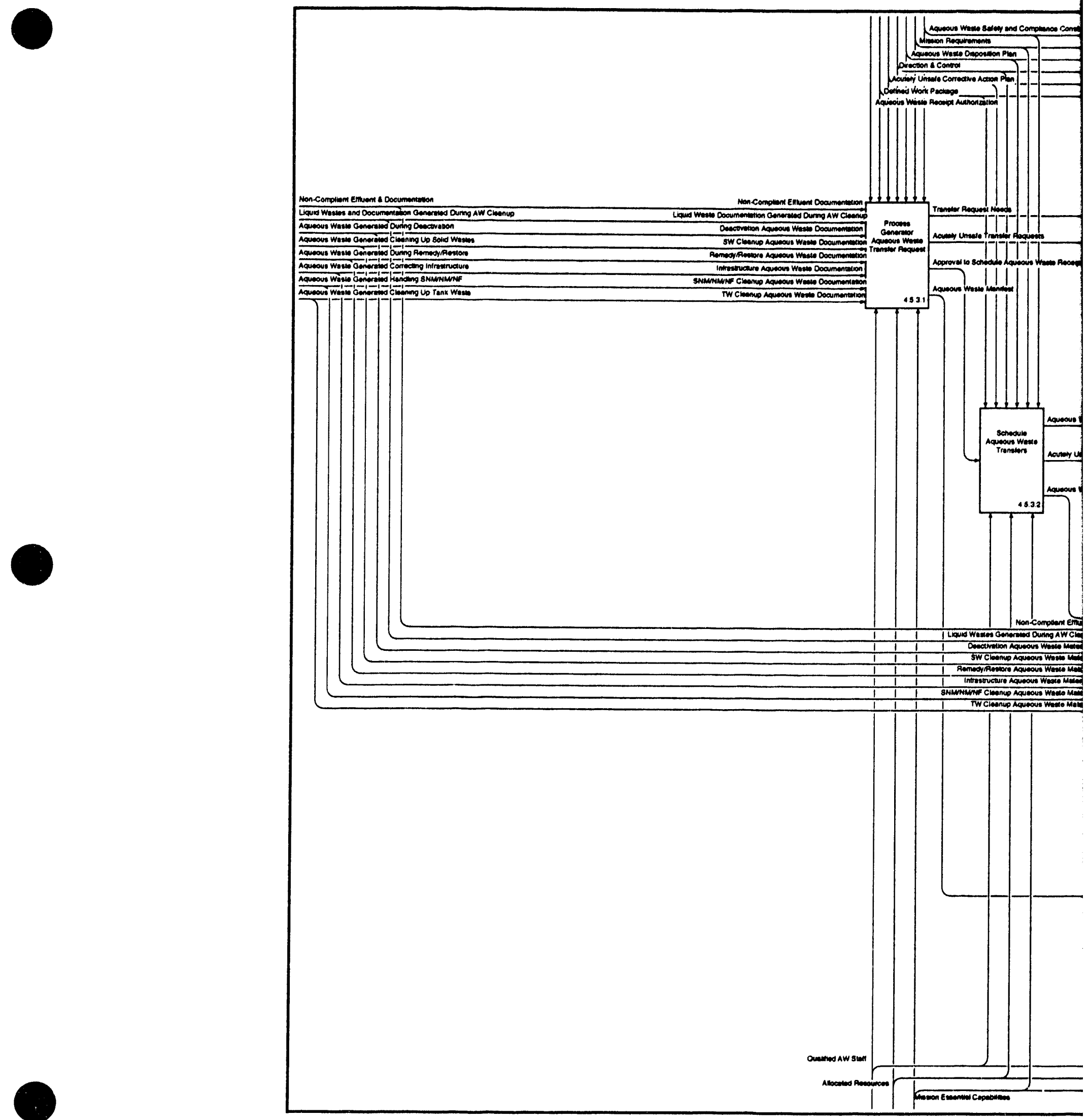


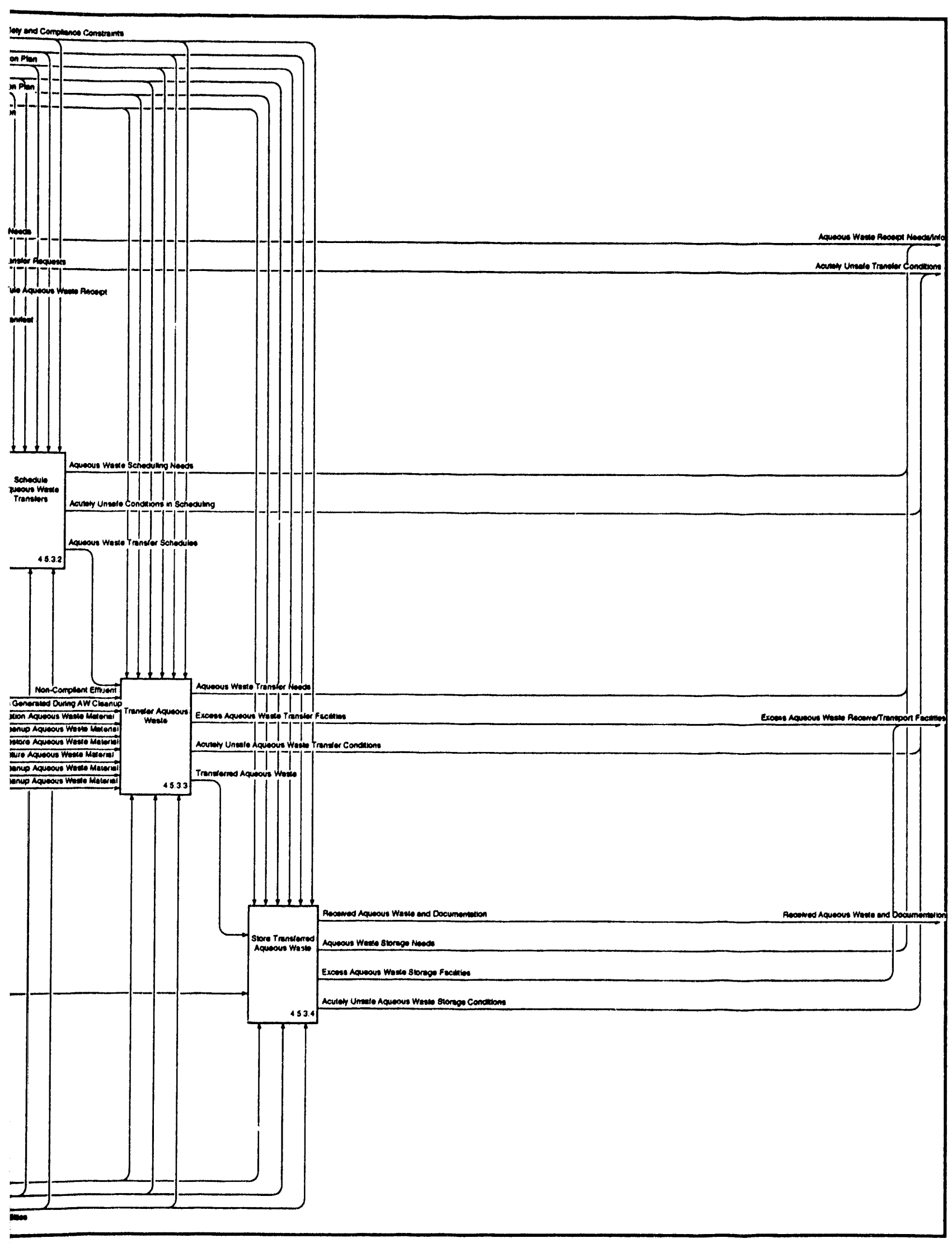




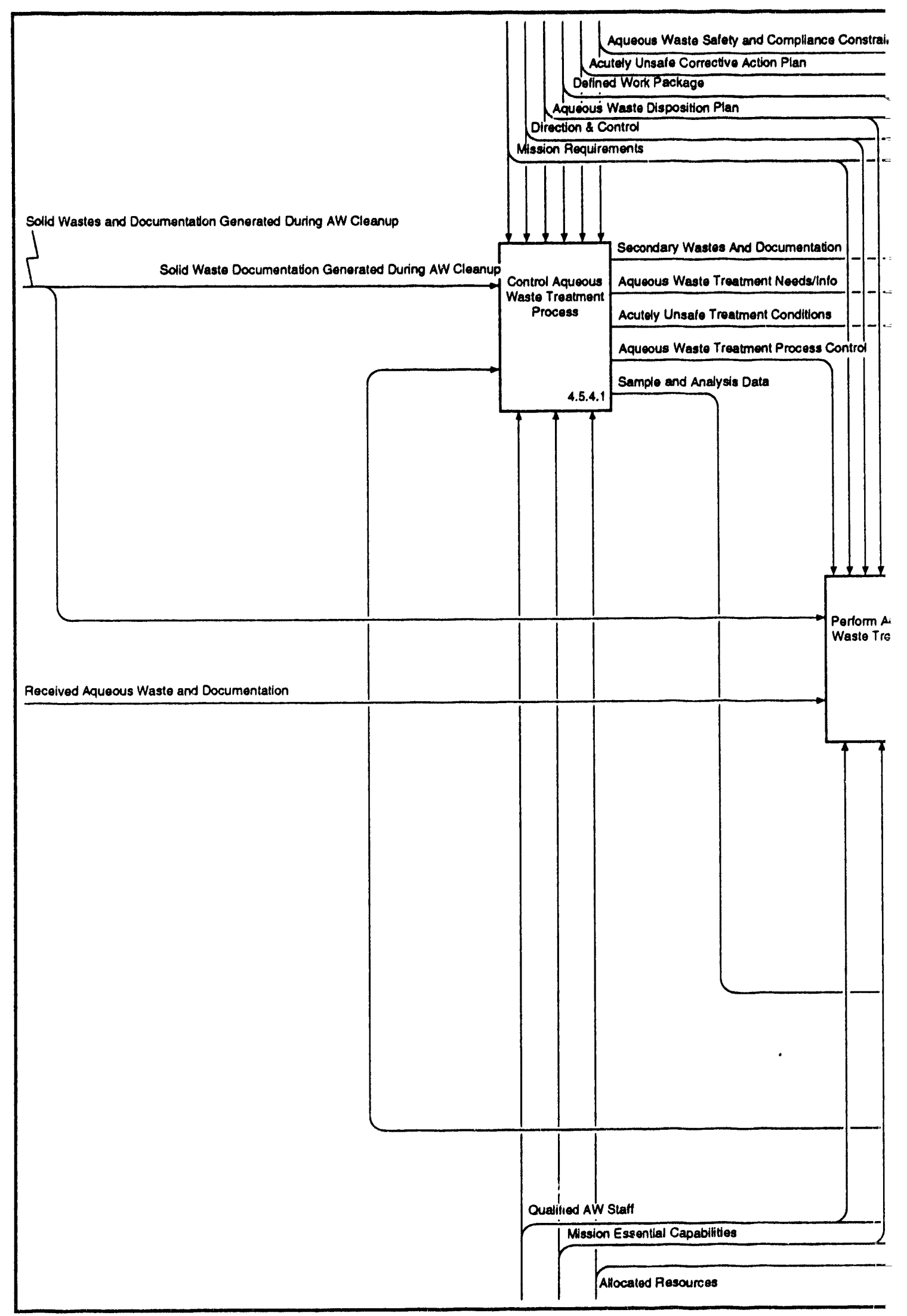

Hanford Site Systems Engineering 


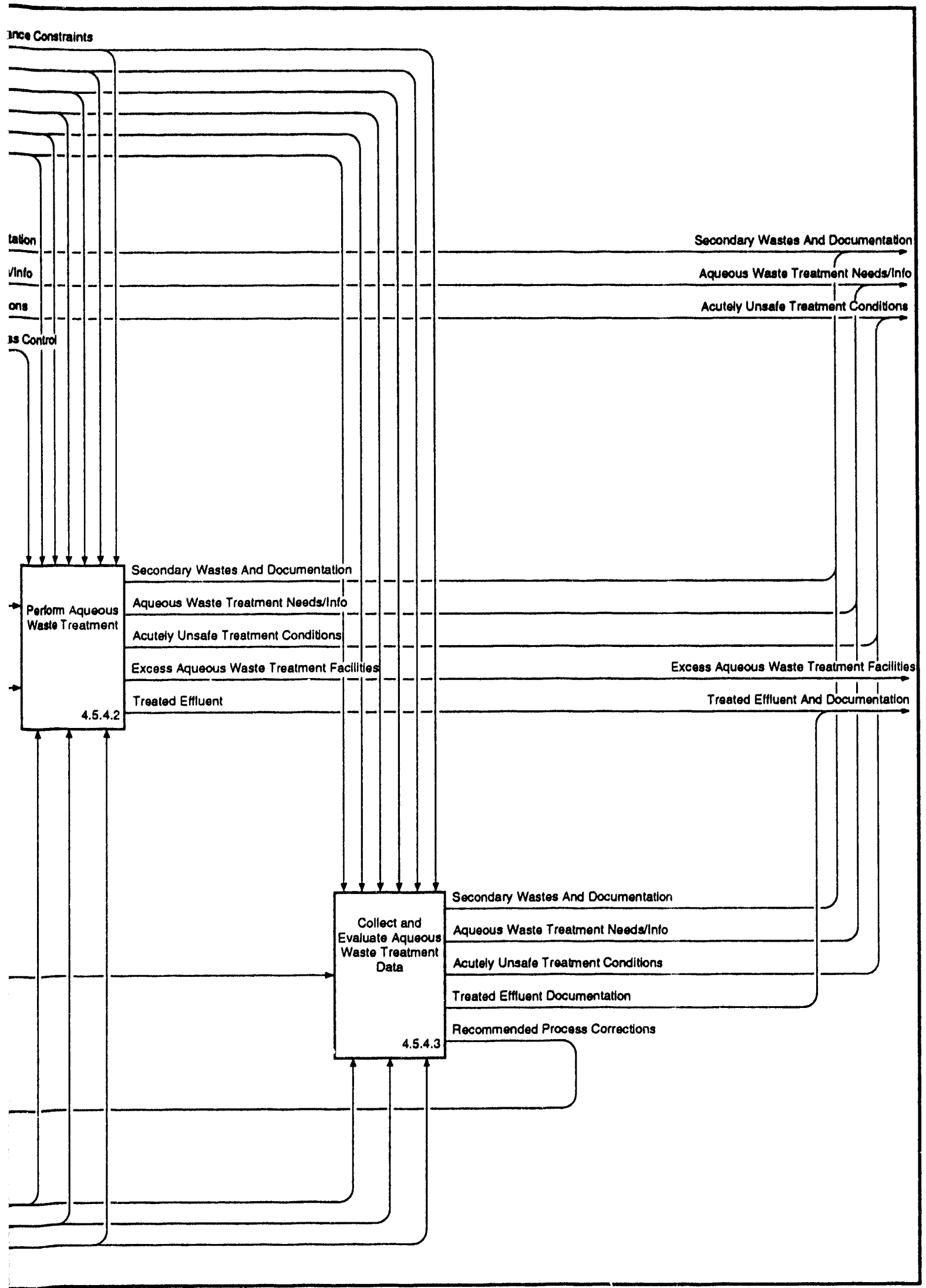




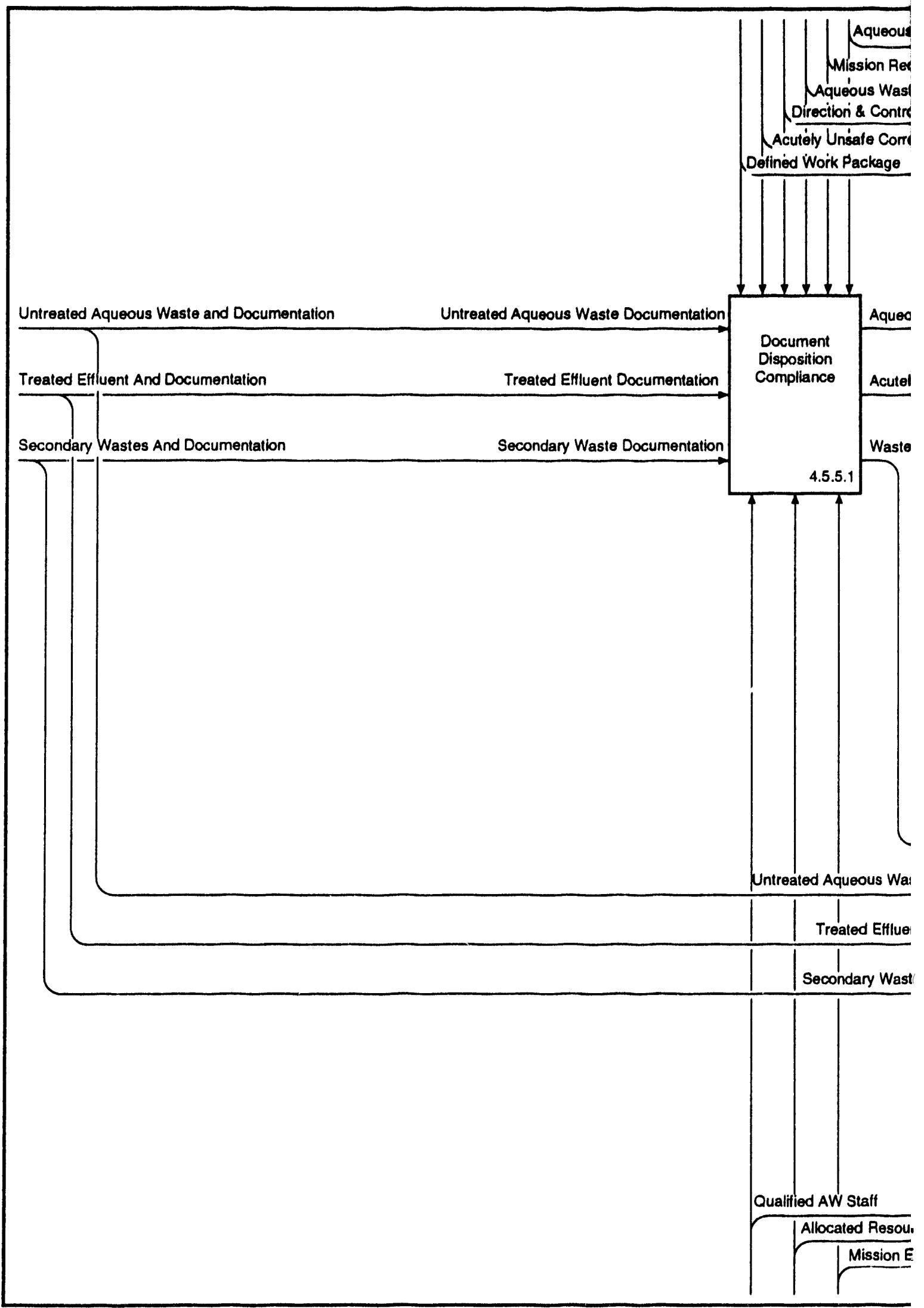




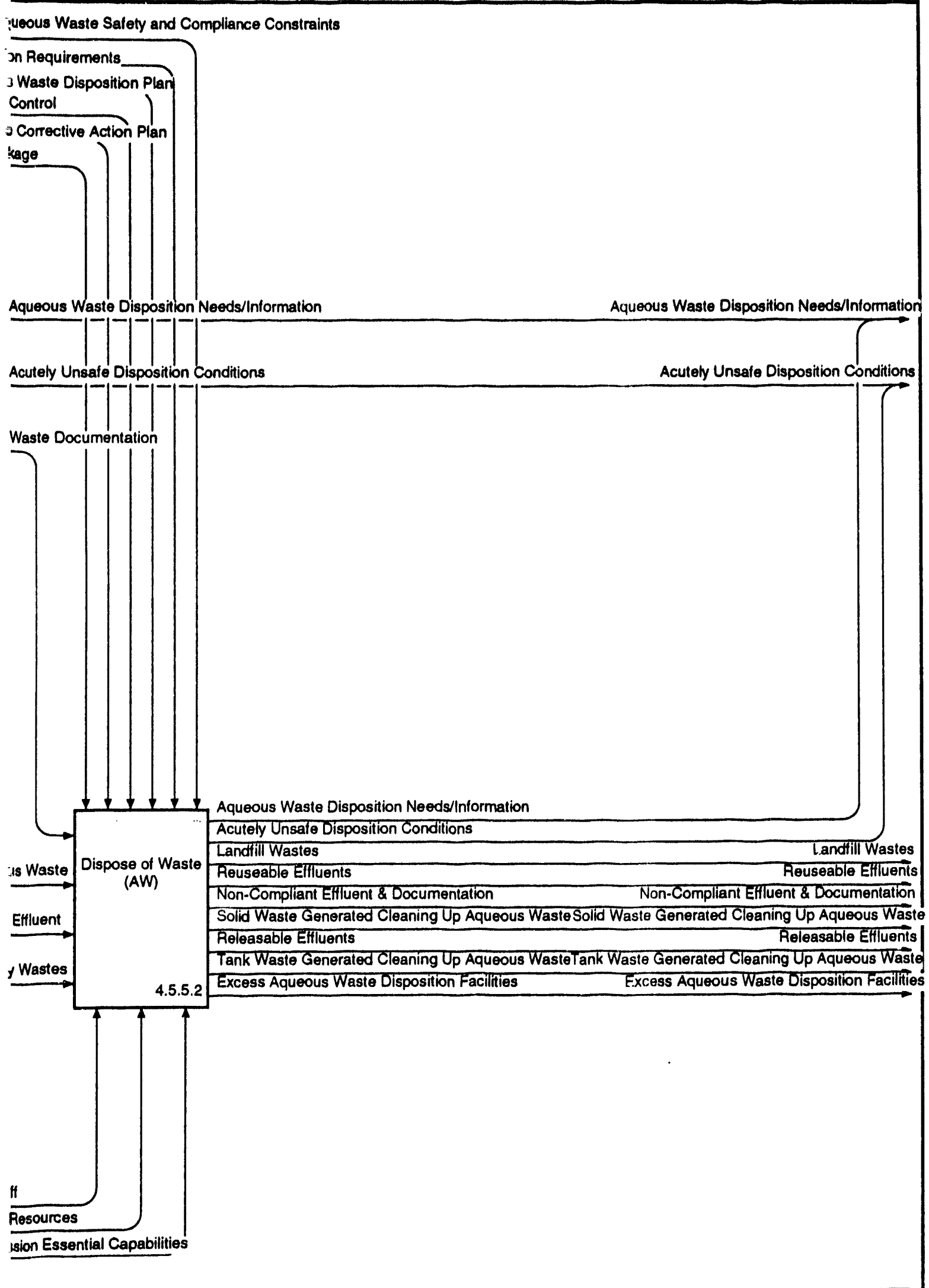




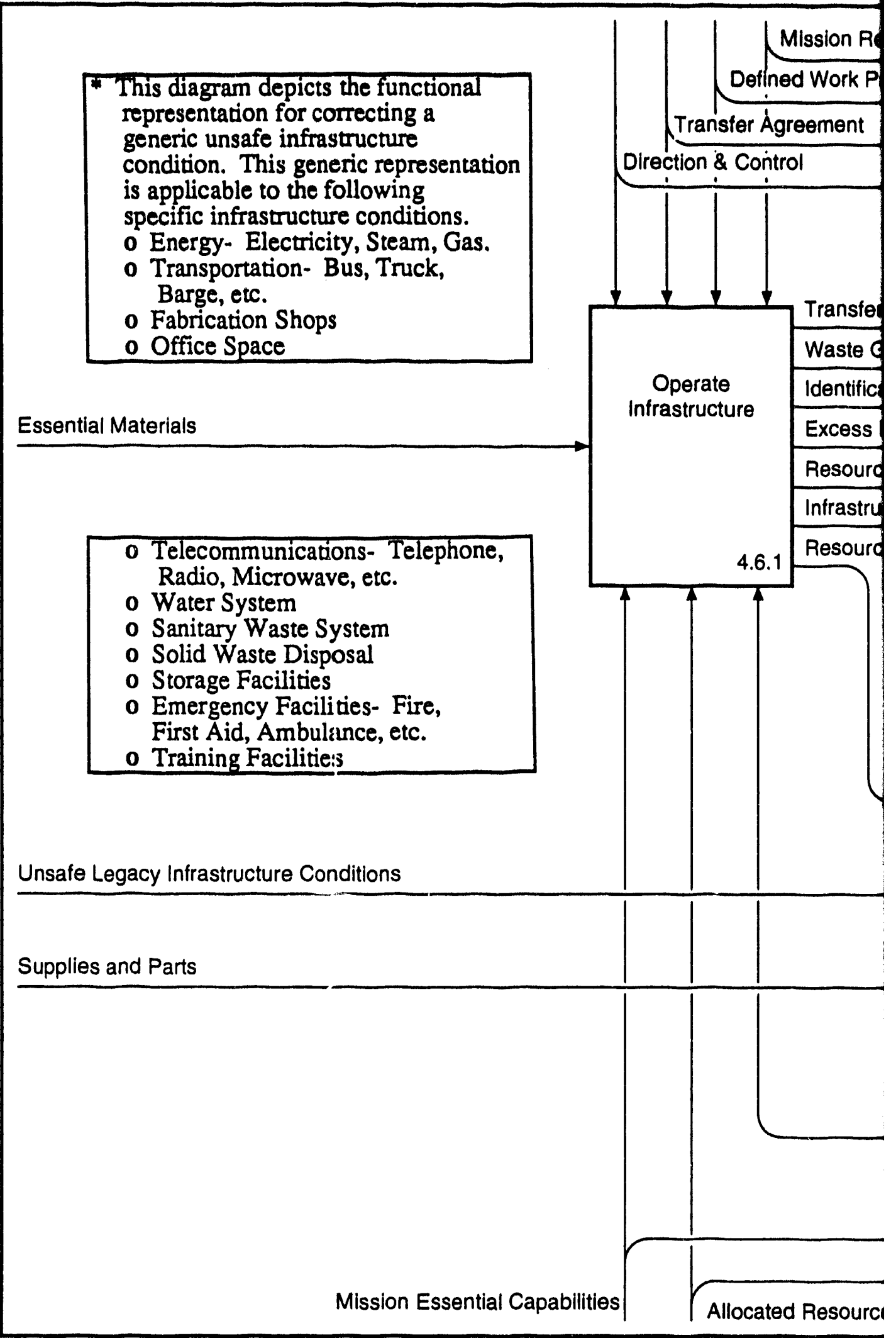




\section{fe Infrastructure Conditions *}

ssion Requirements

\section{; Work Package}

ement

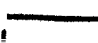

$!$

Transferrable Resources

Transferrable Resources

Waste Generated Correcting Unsafe Infrastructure

Identification of Potentially Transferrable Resources

Excess Infrastructure Facilities

Resource Utilization Information

Infrastructure Status Information

Resource In Need of Maintenance

$\underset{\text { Unsafe Infrastructure Needs/Intormation }}{\longrightarrow \text { Excess Infrastructure Facilities }}$

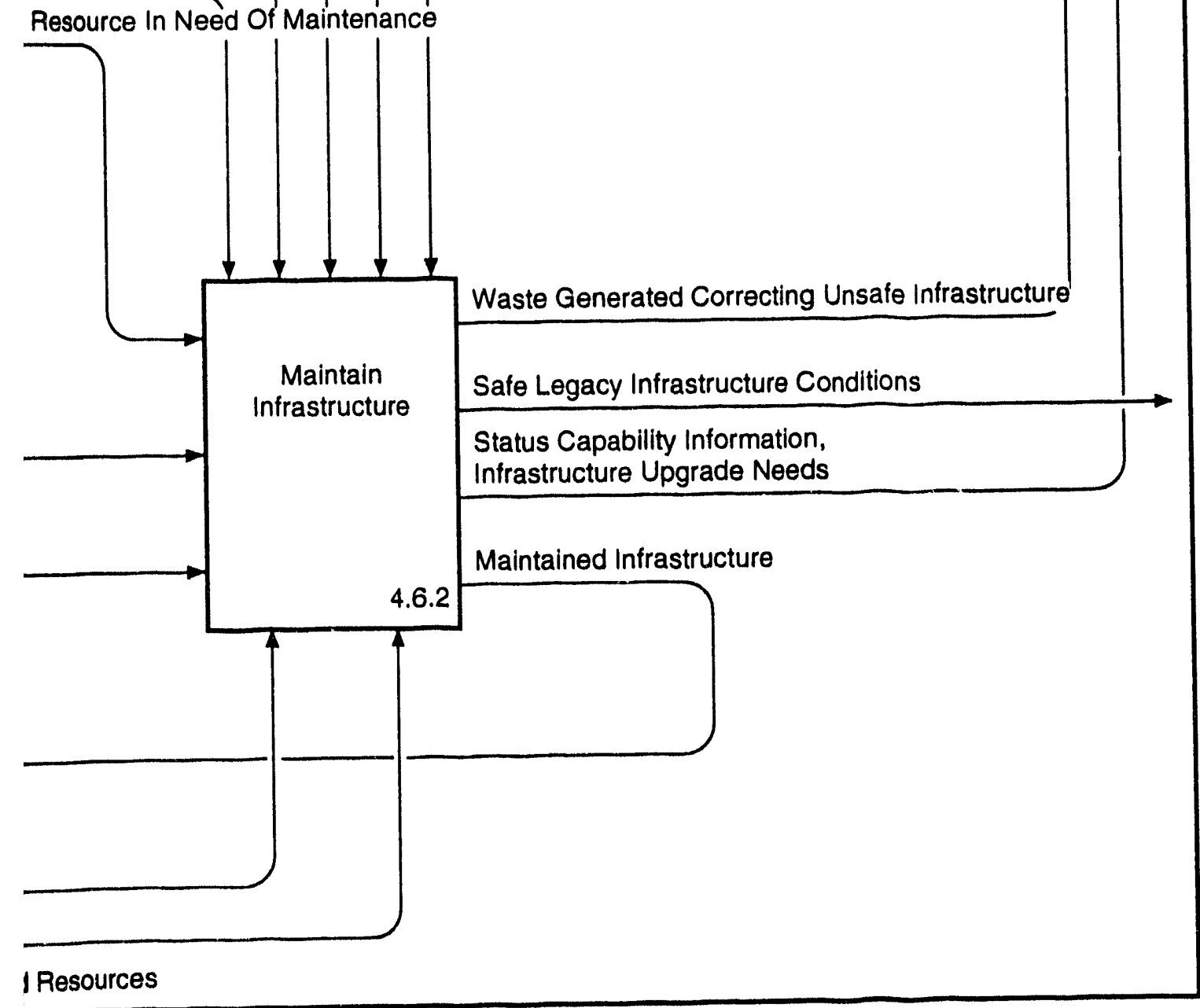




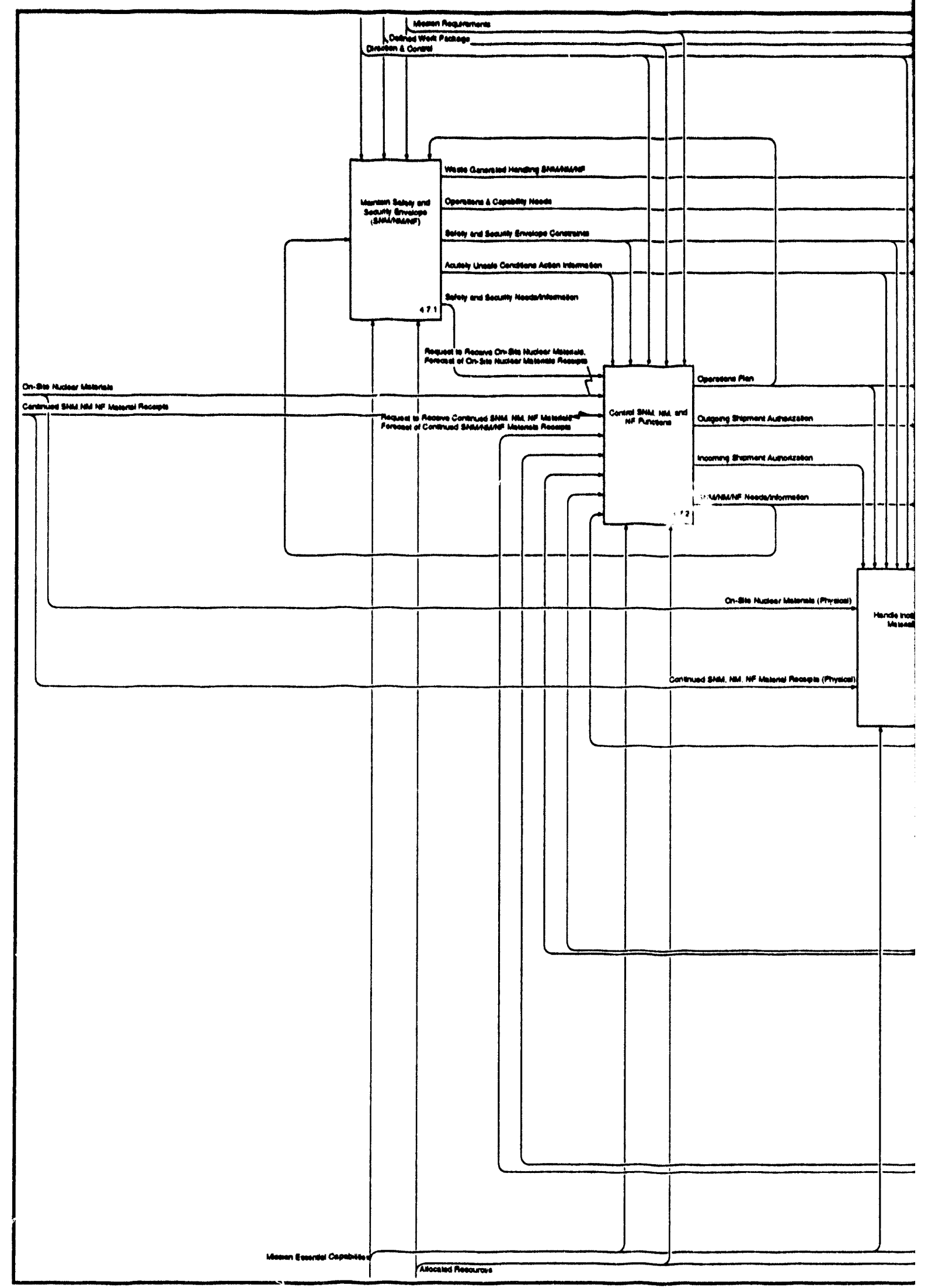




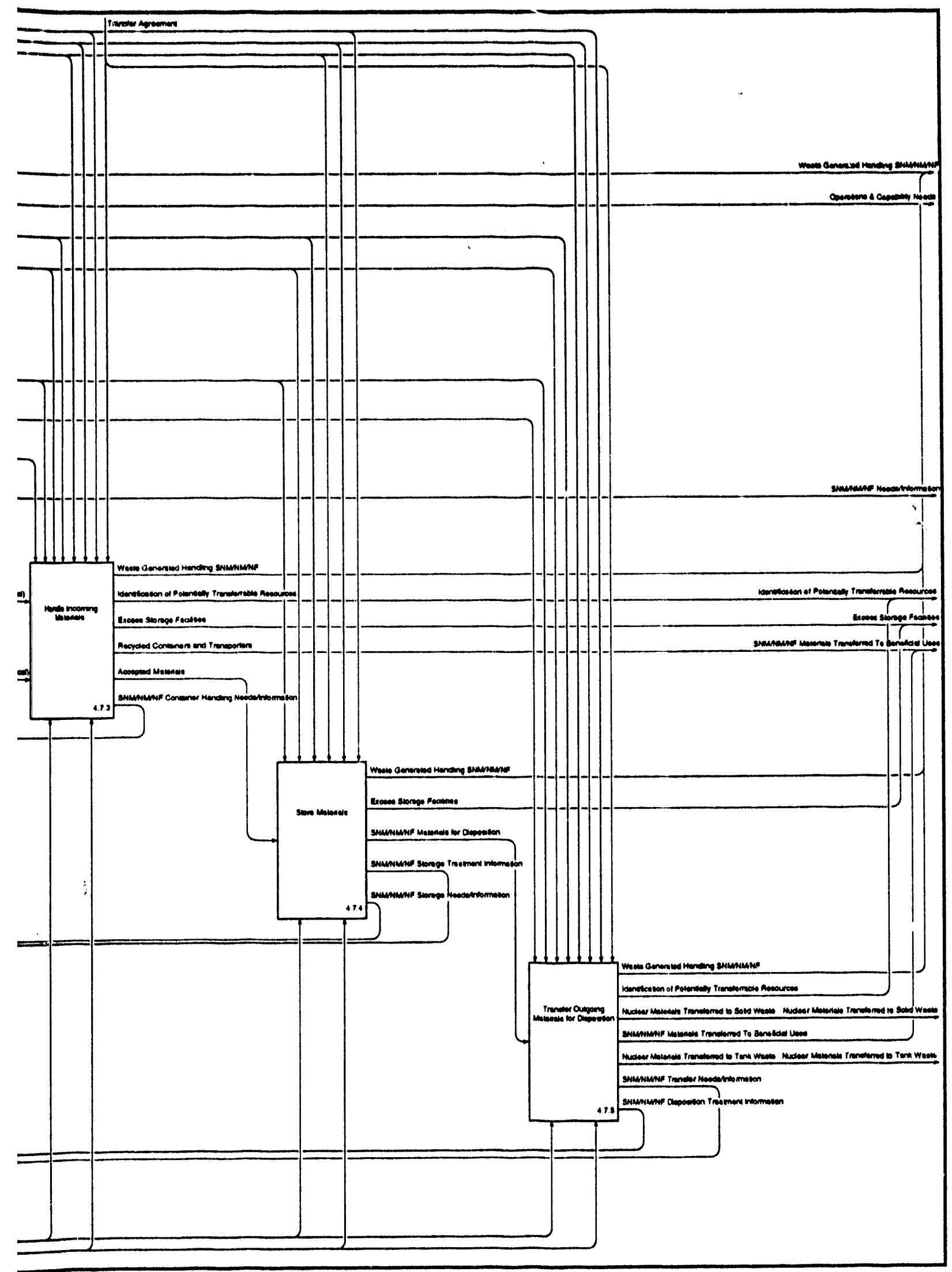

[4.7] Store, Treat, and Disposition SNM/NM/NF Materials 

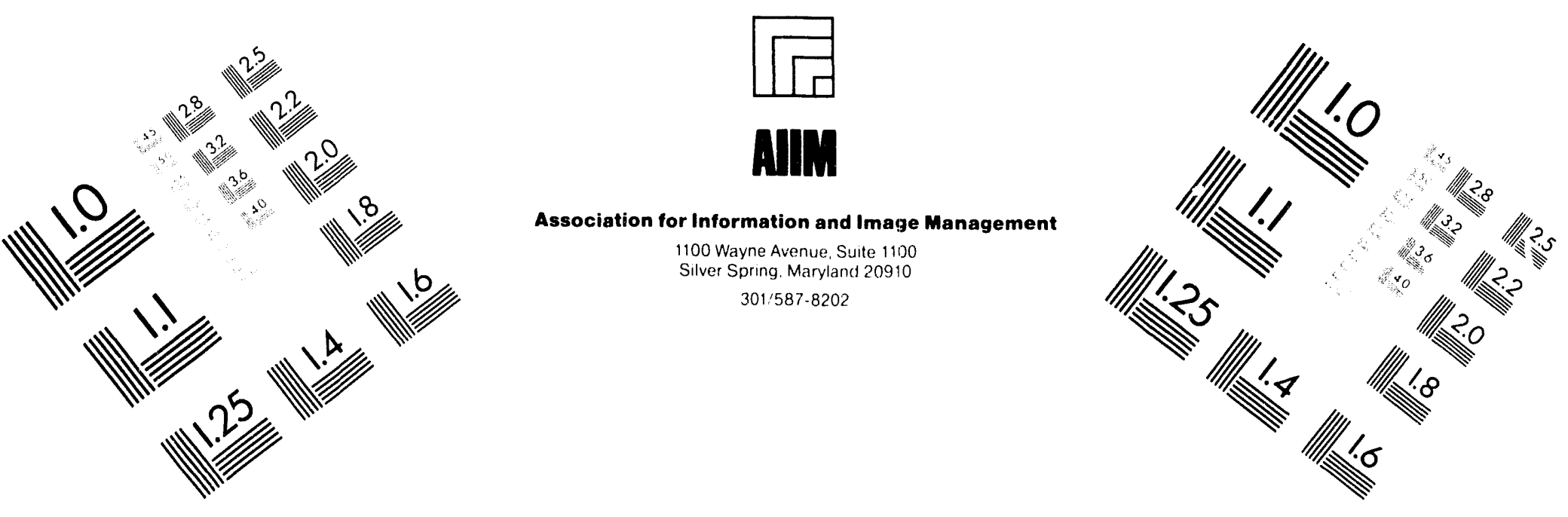

\section{Centimeter}

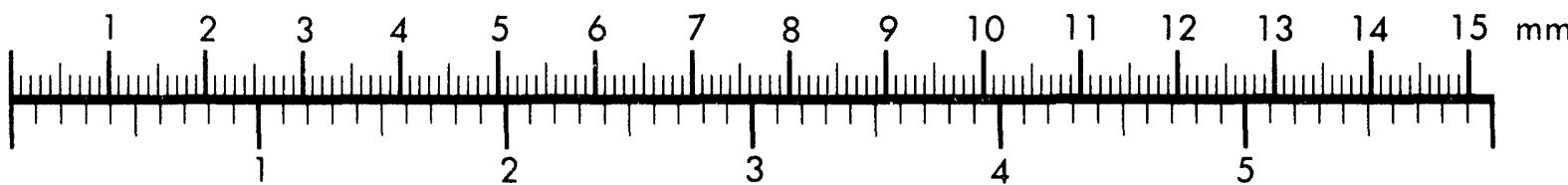
Inches
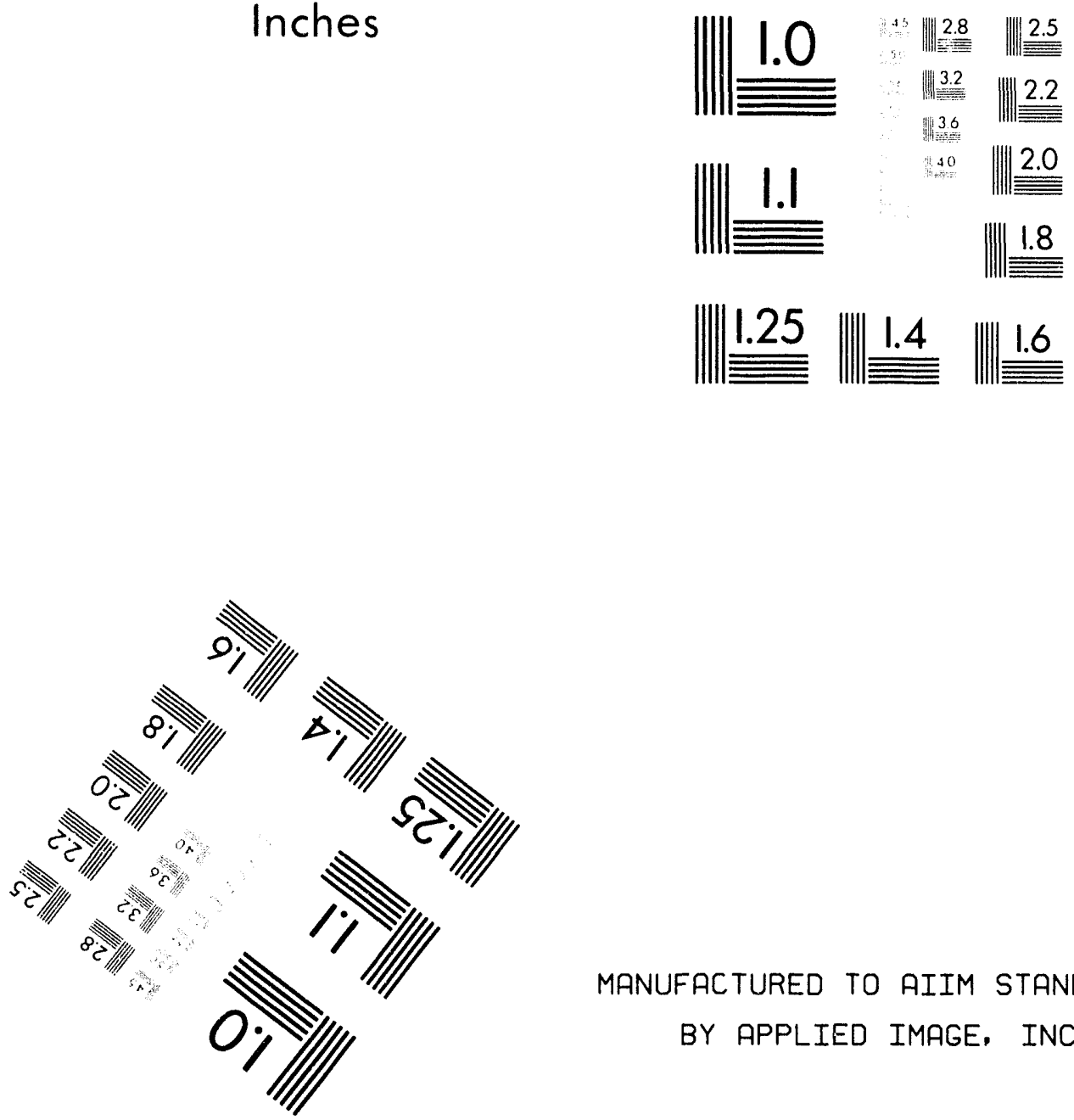

MANUFACTURED TO AIIM STANDARDS BY APPLIED IMAGE, INC.

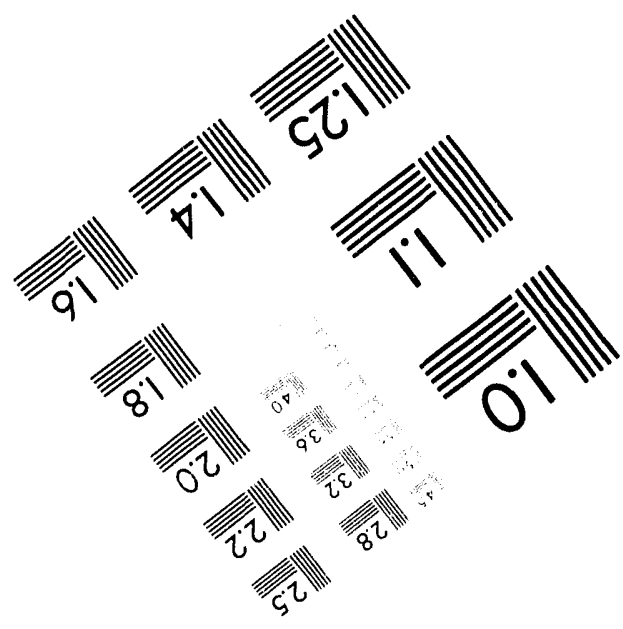



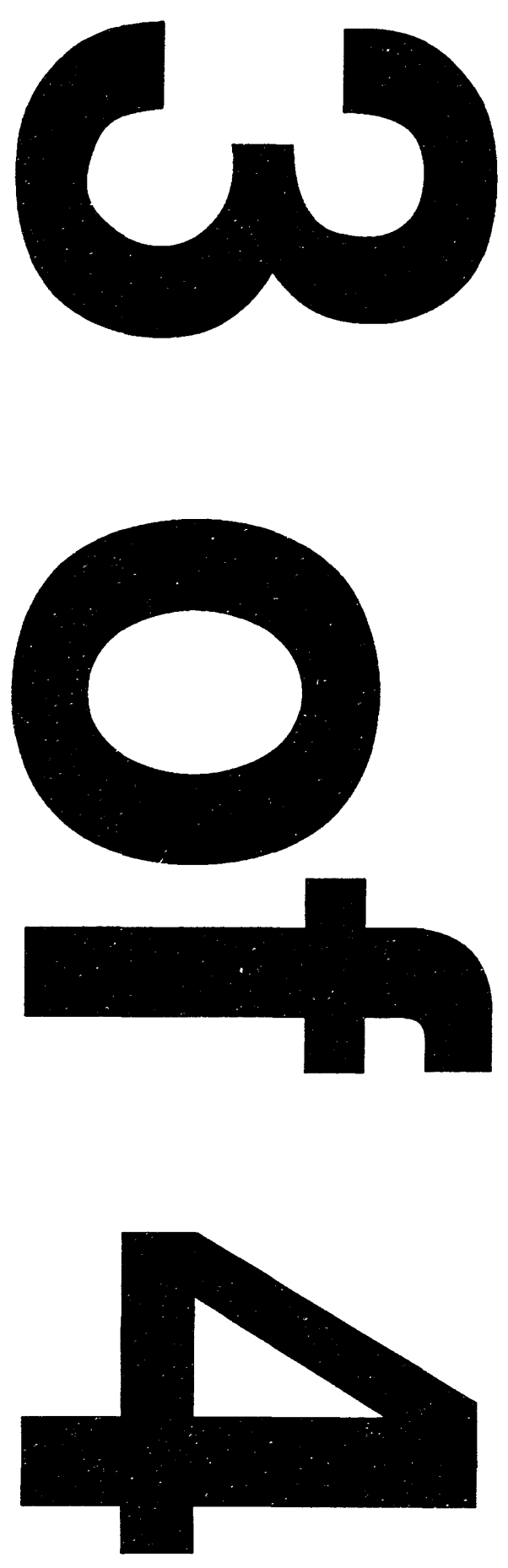


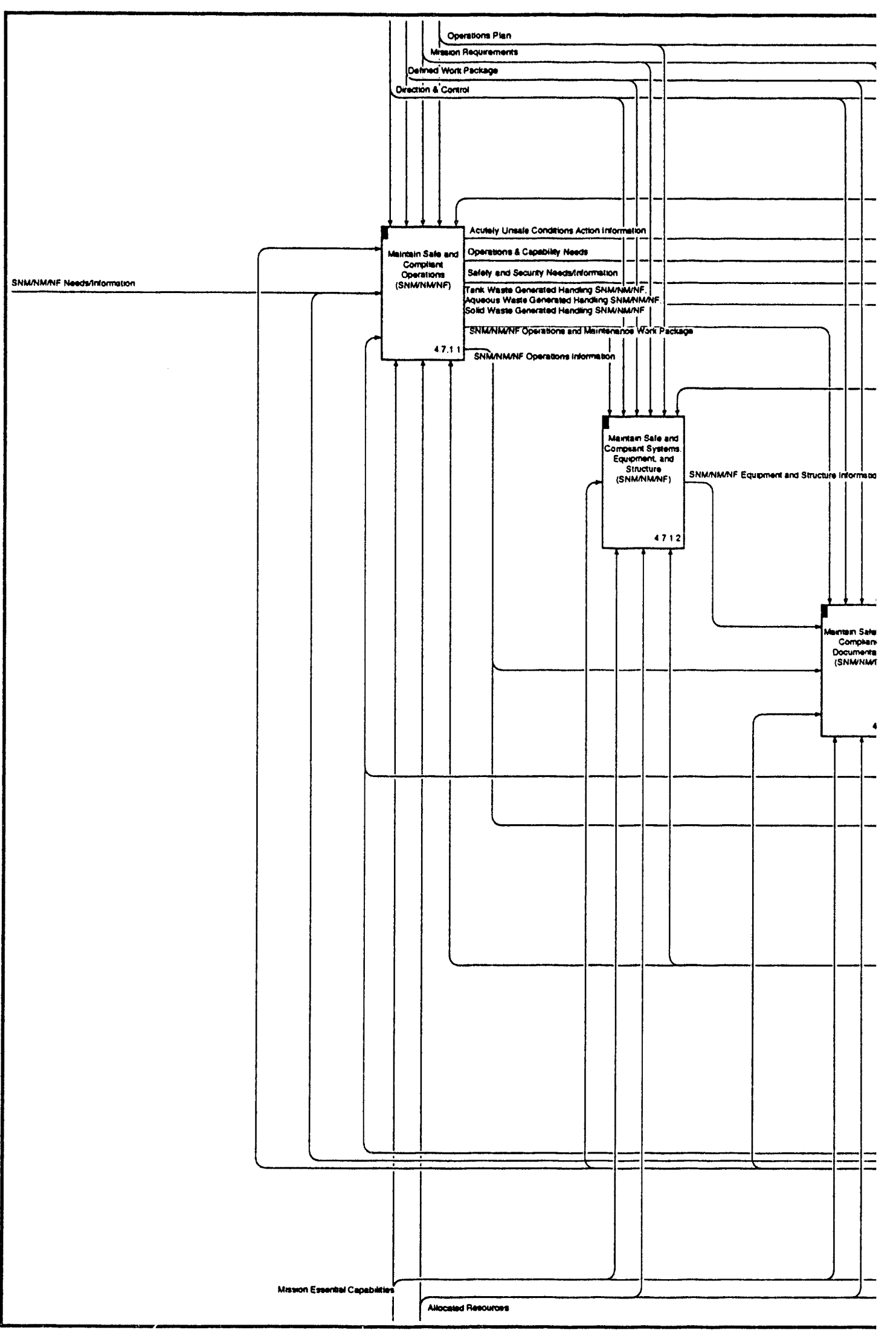




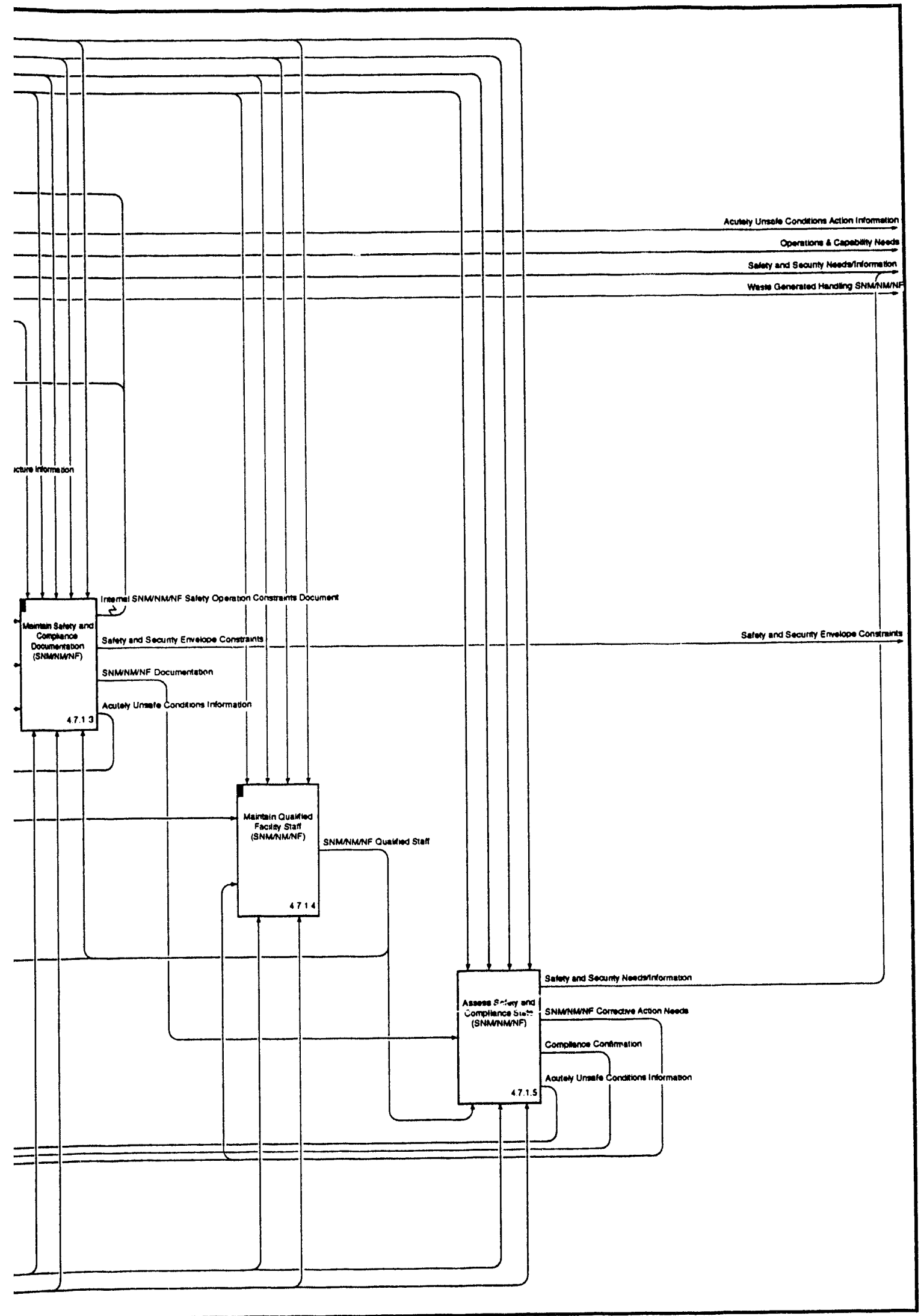




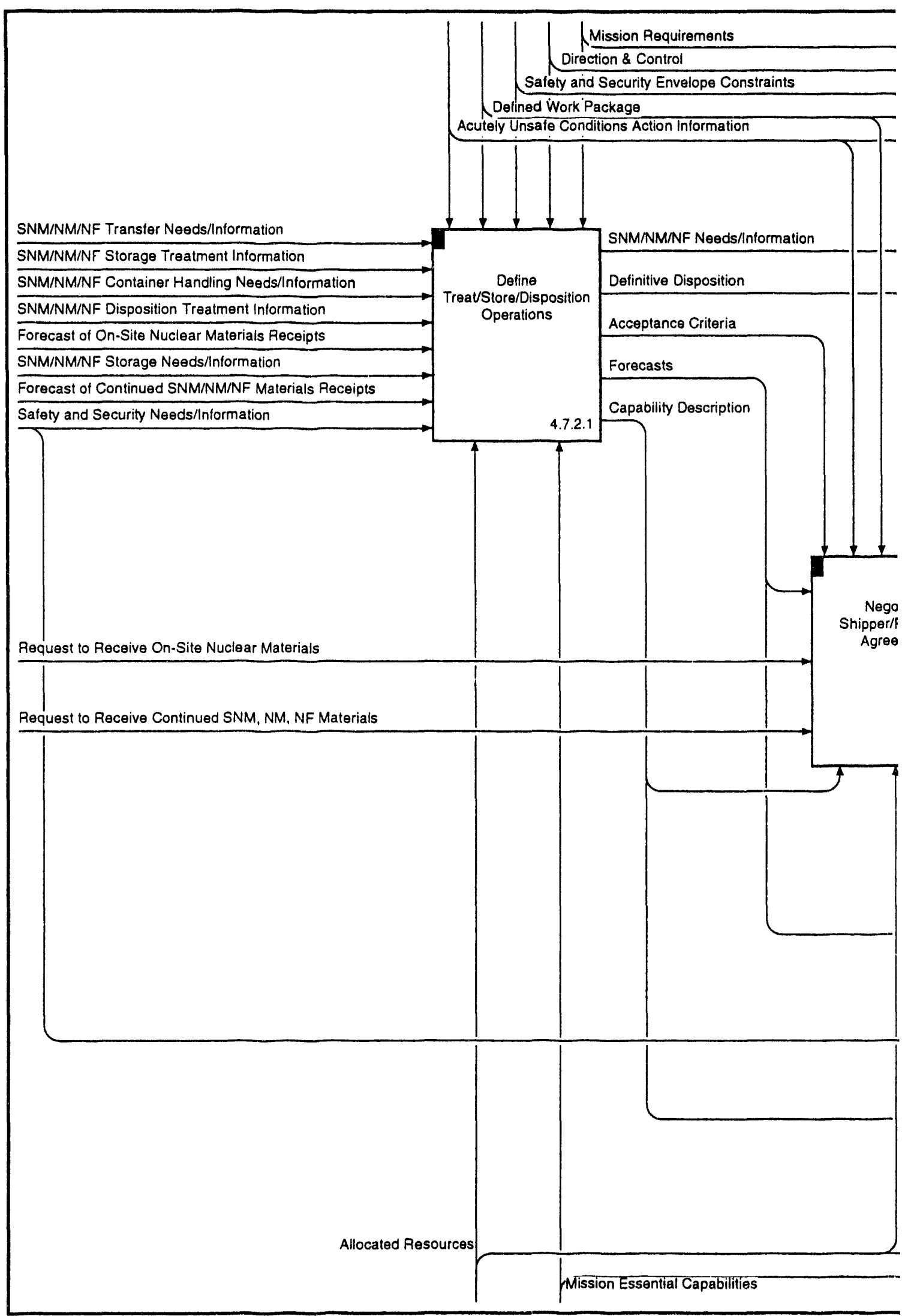




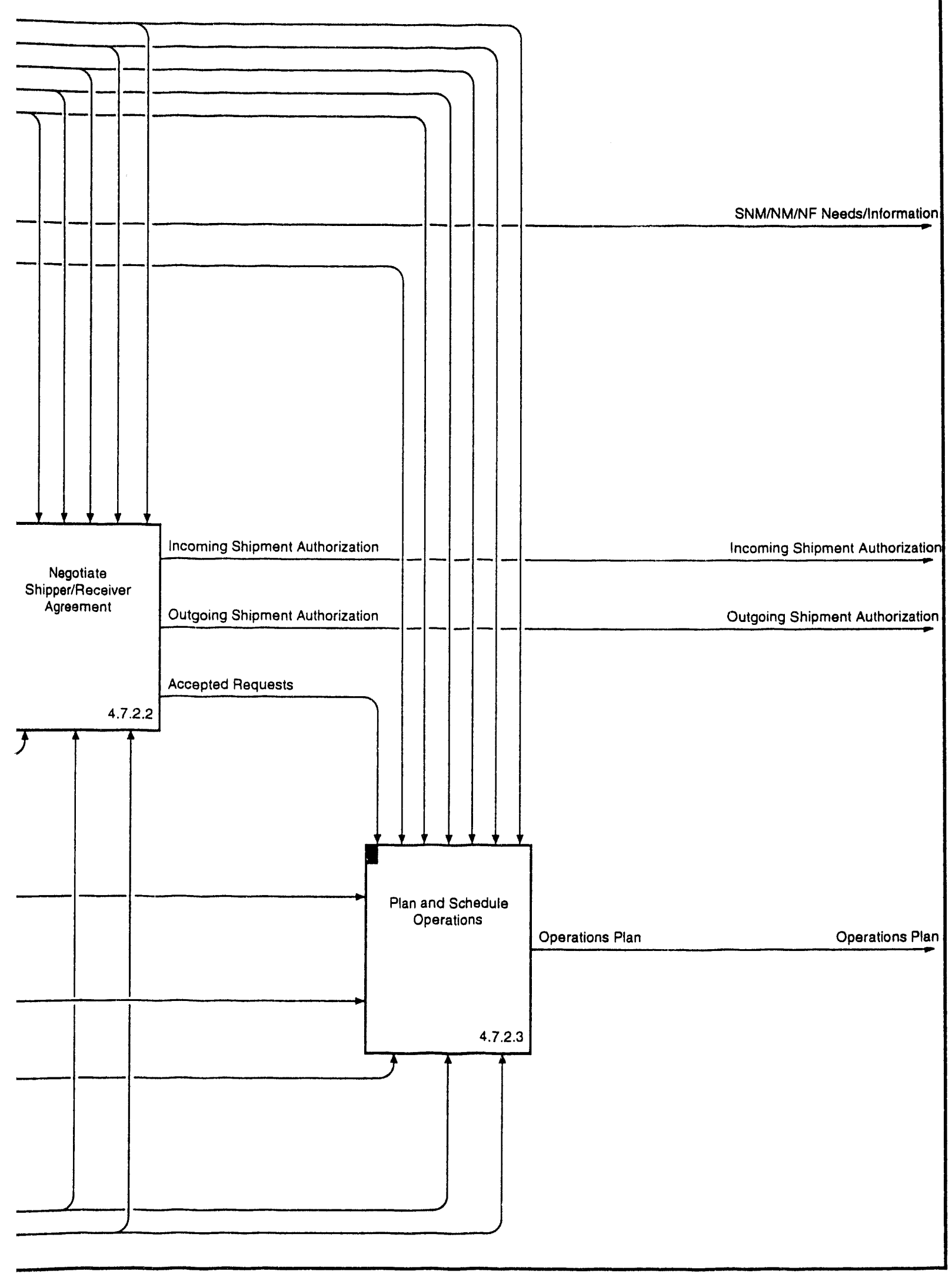




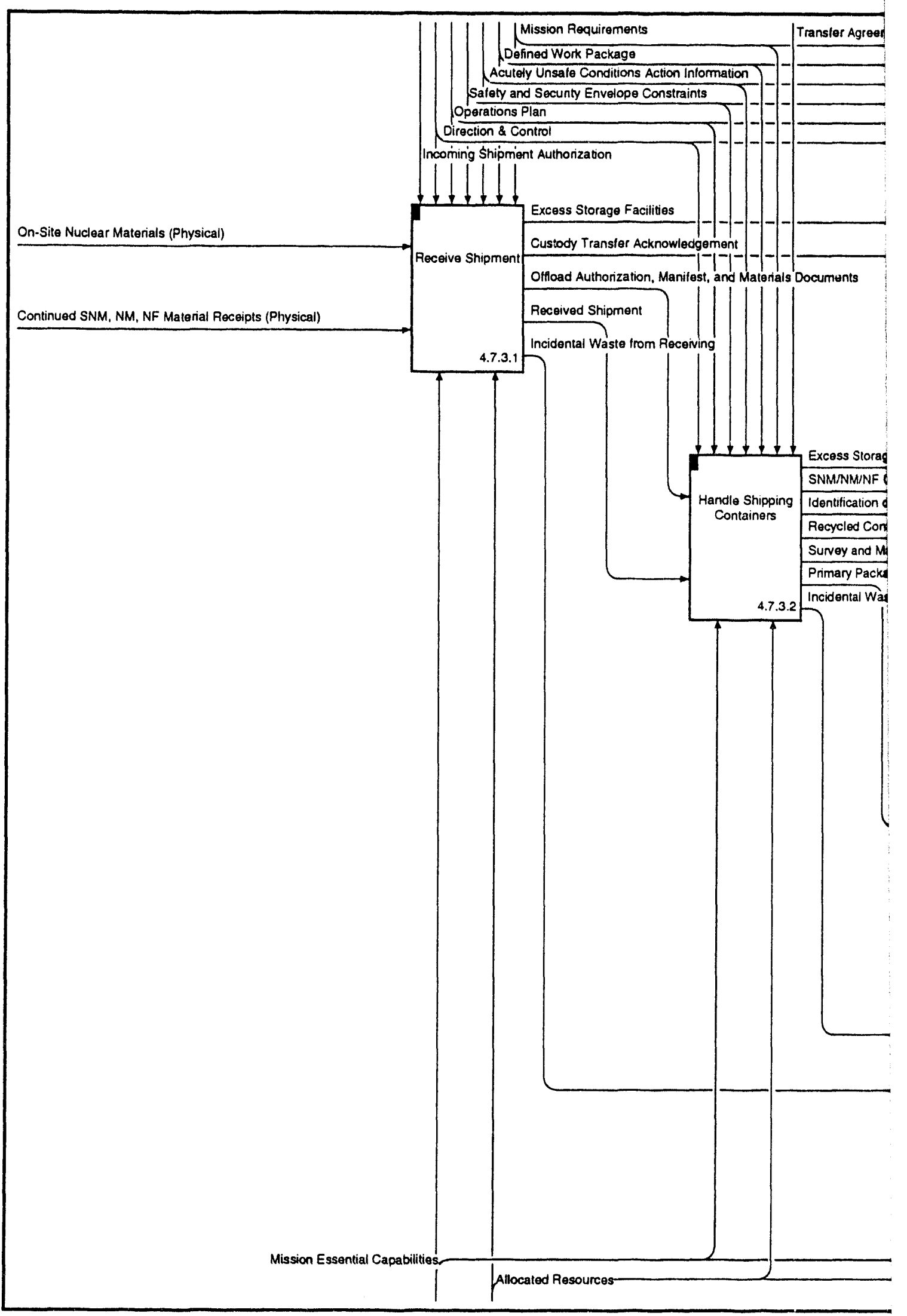




\section{lle Incoming Materials}

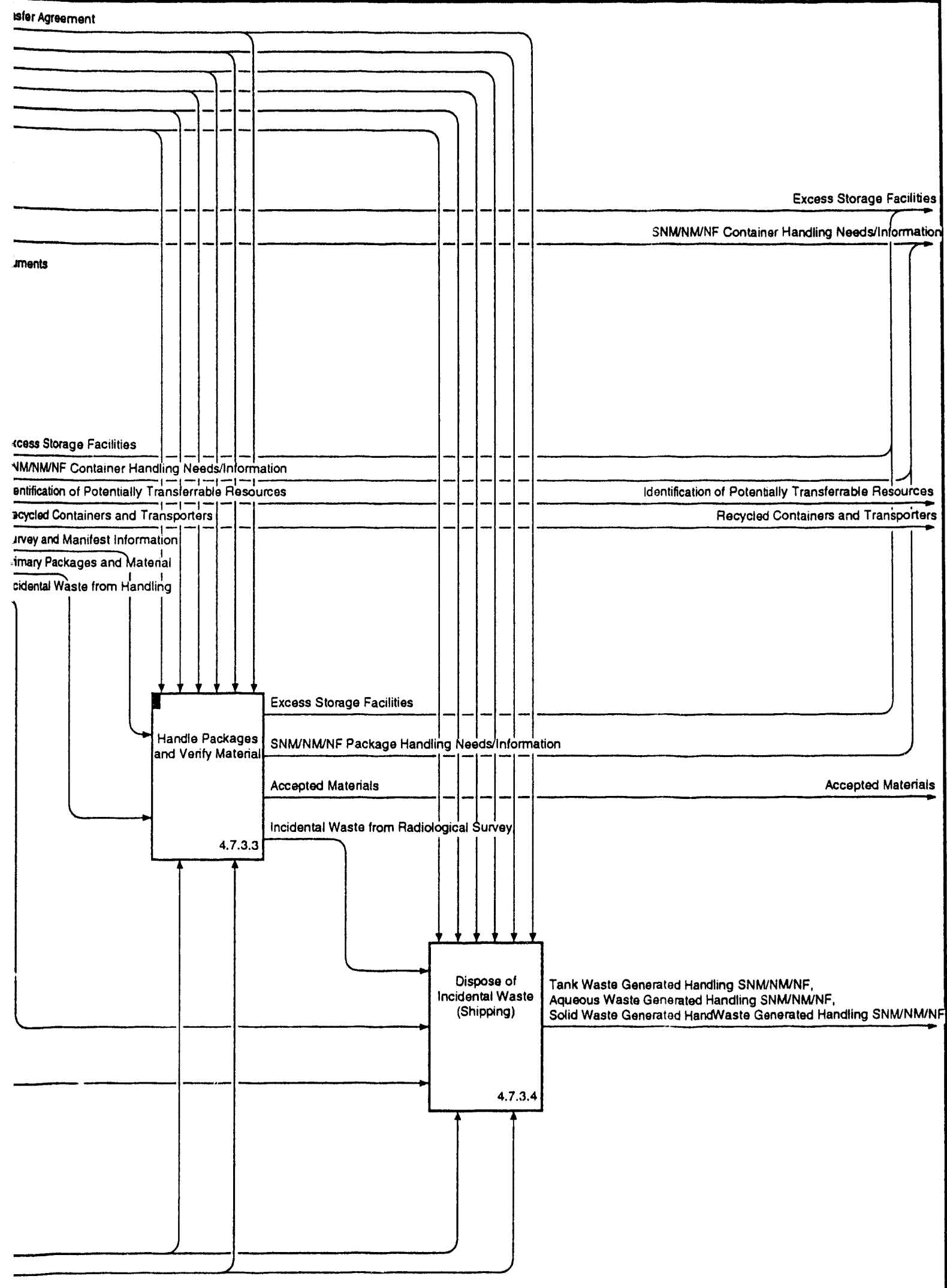




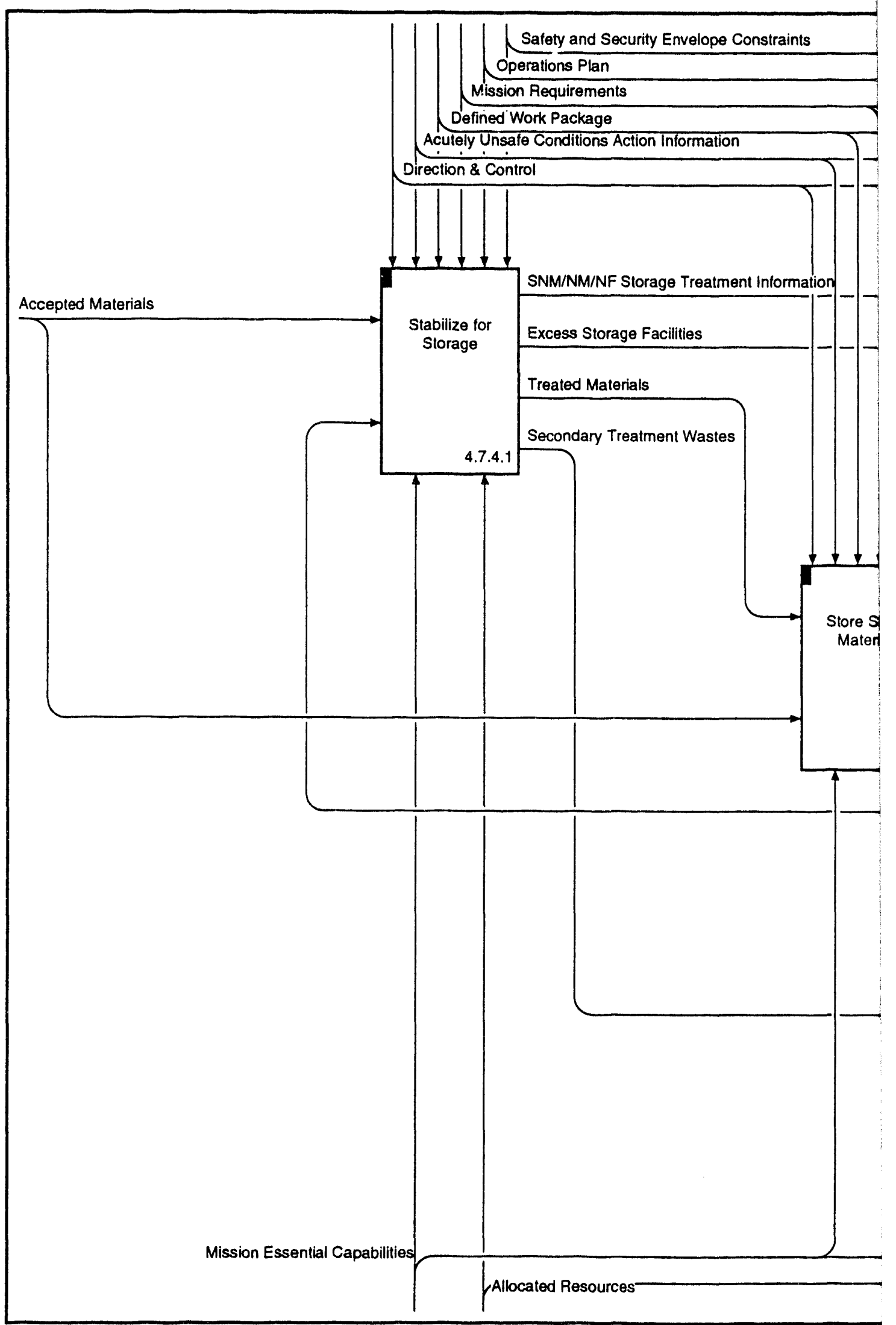


[4.7.5] Transfer Outgoing

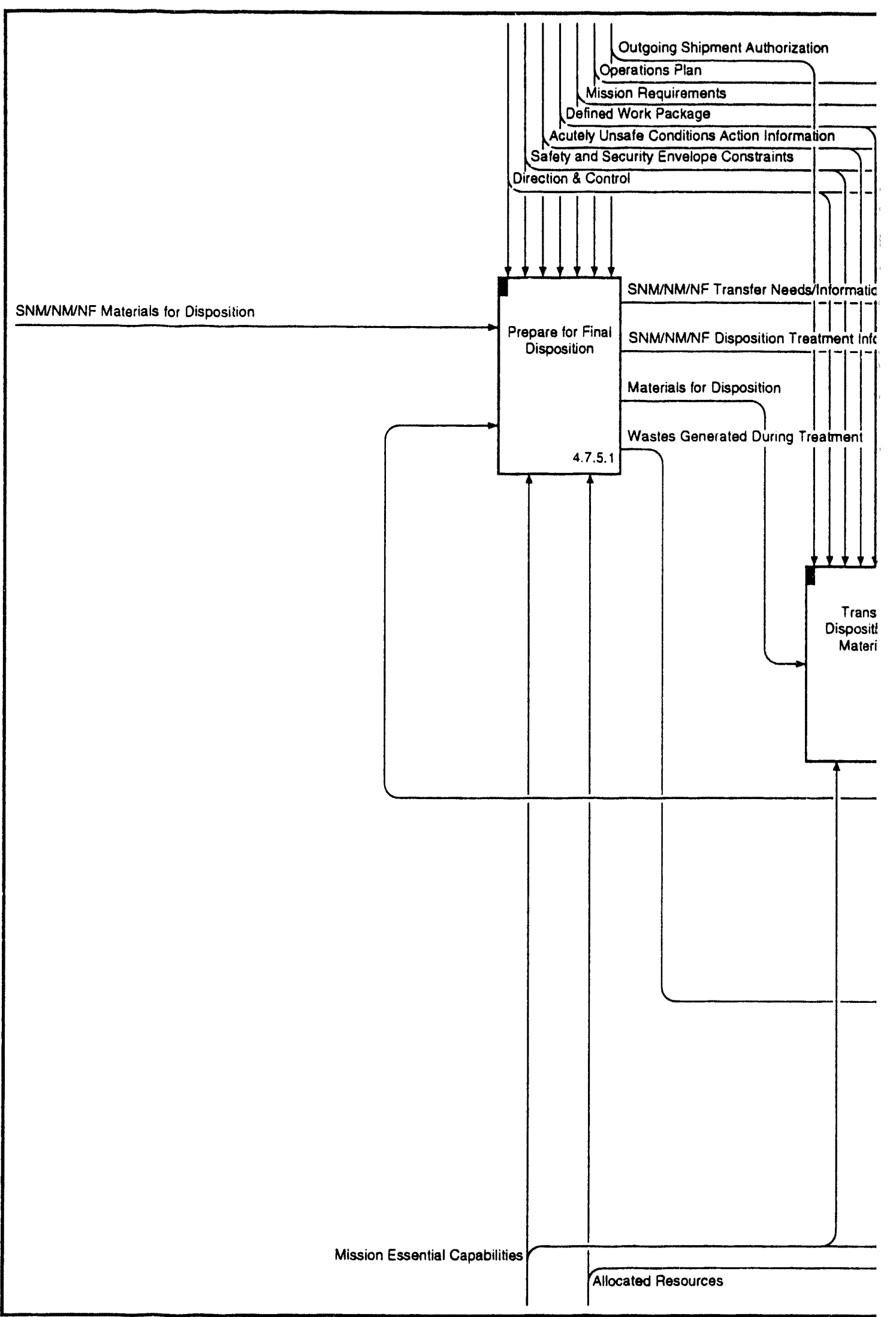

Hanford Site Systems Engineering 


\section{going Materials for Disposition}

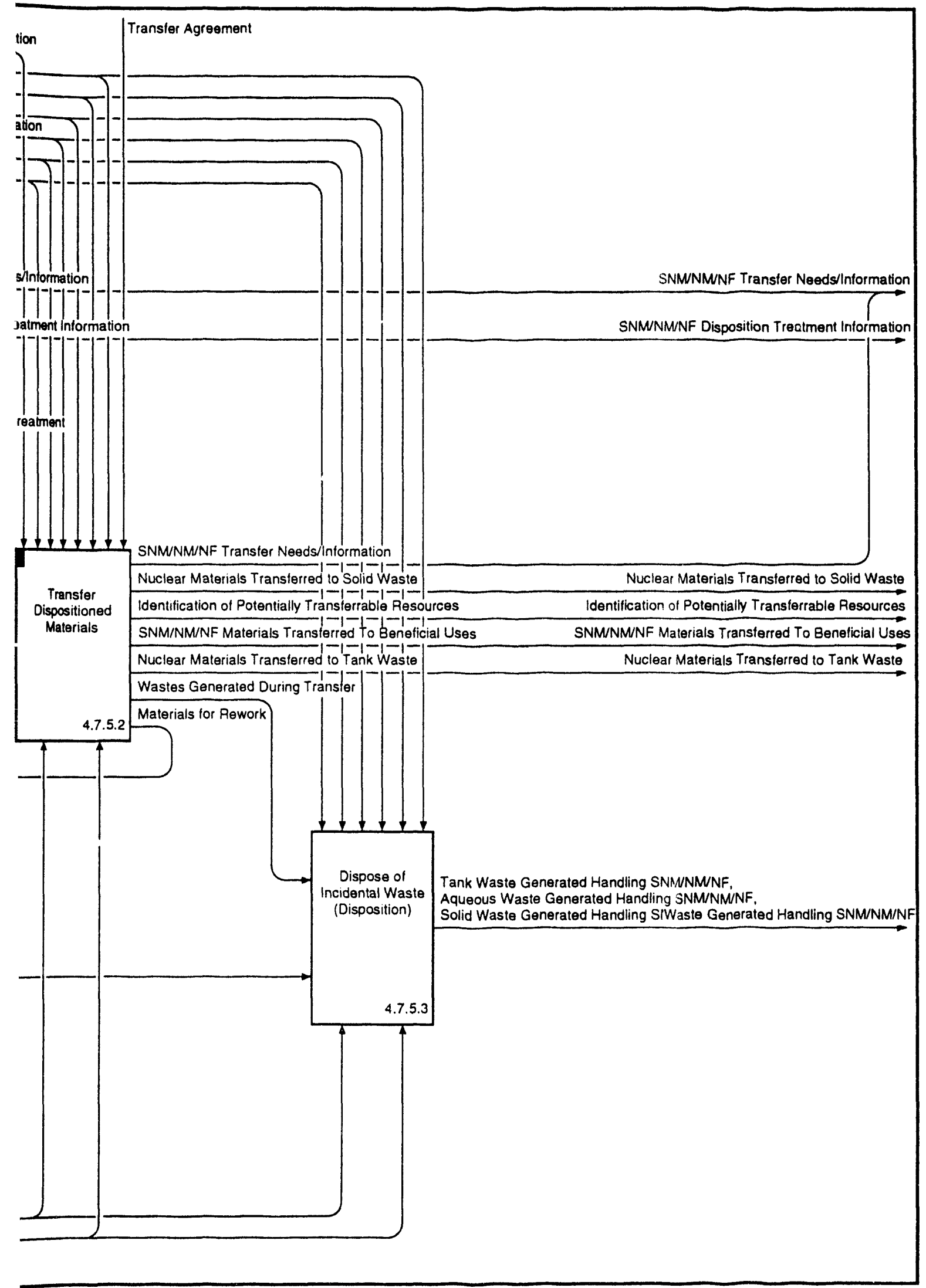




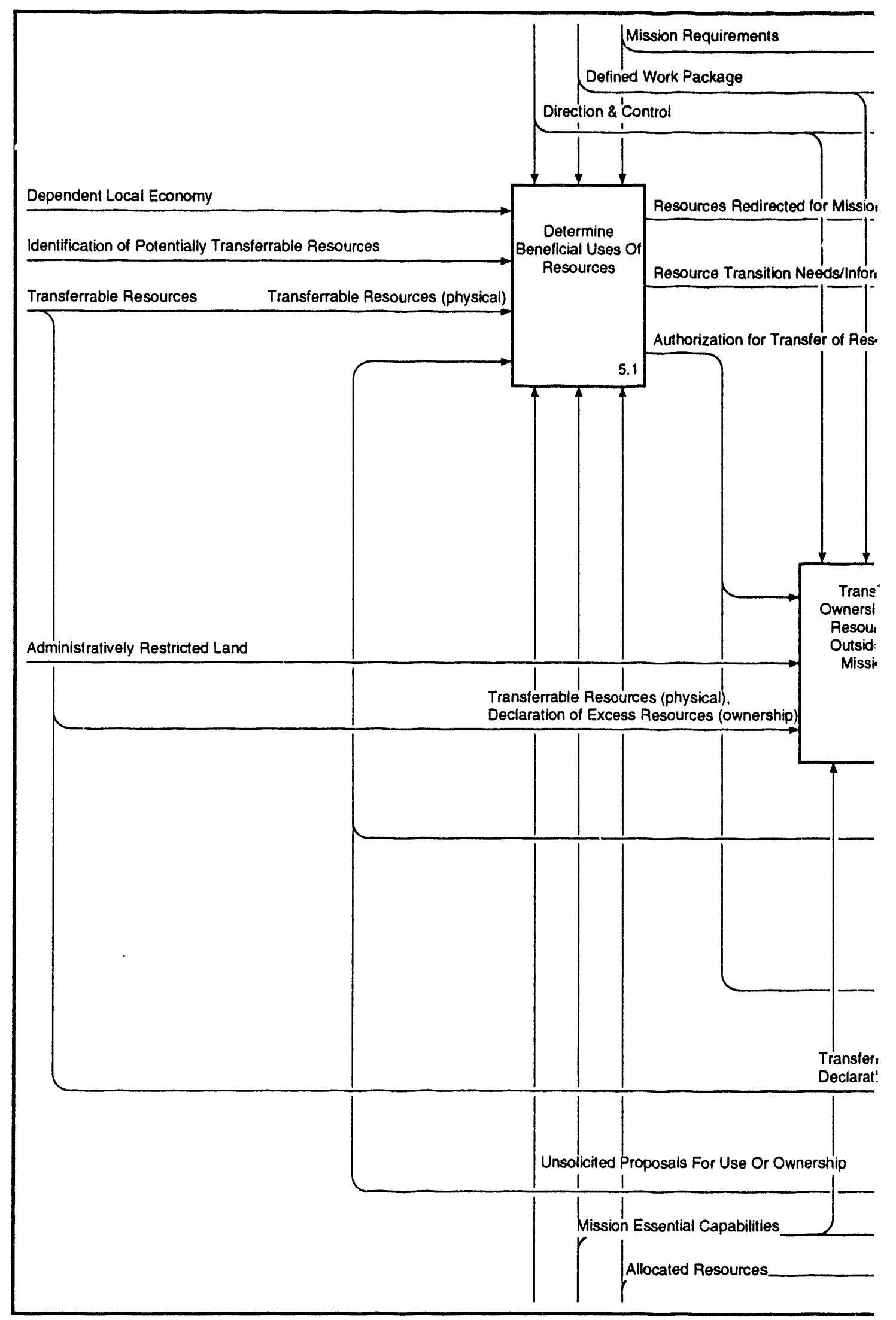




\section{Resources For Beneficial Use}

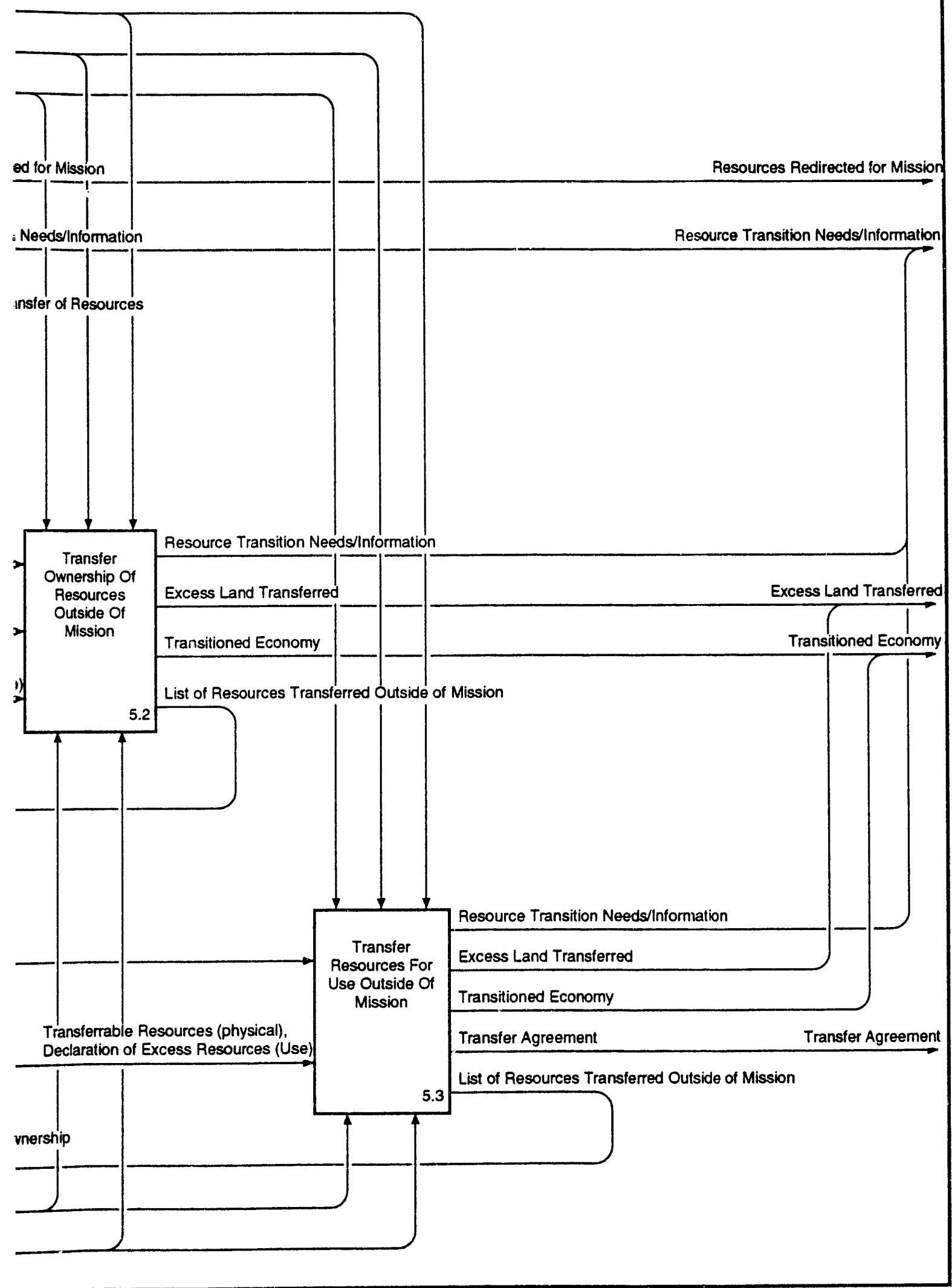

[5] Transition Resources For Beneficial Use 
[5.1] Determine Benefici

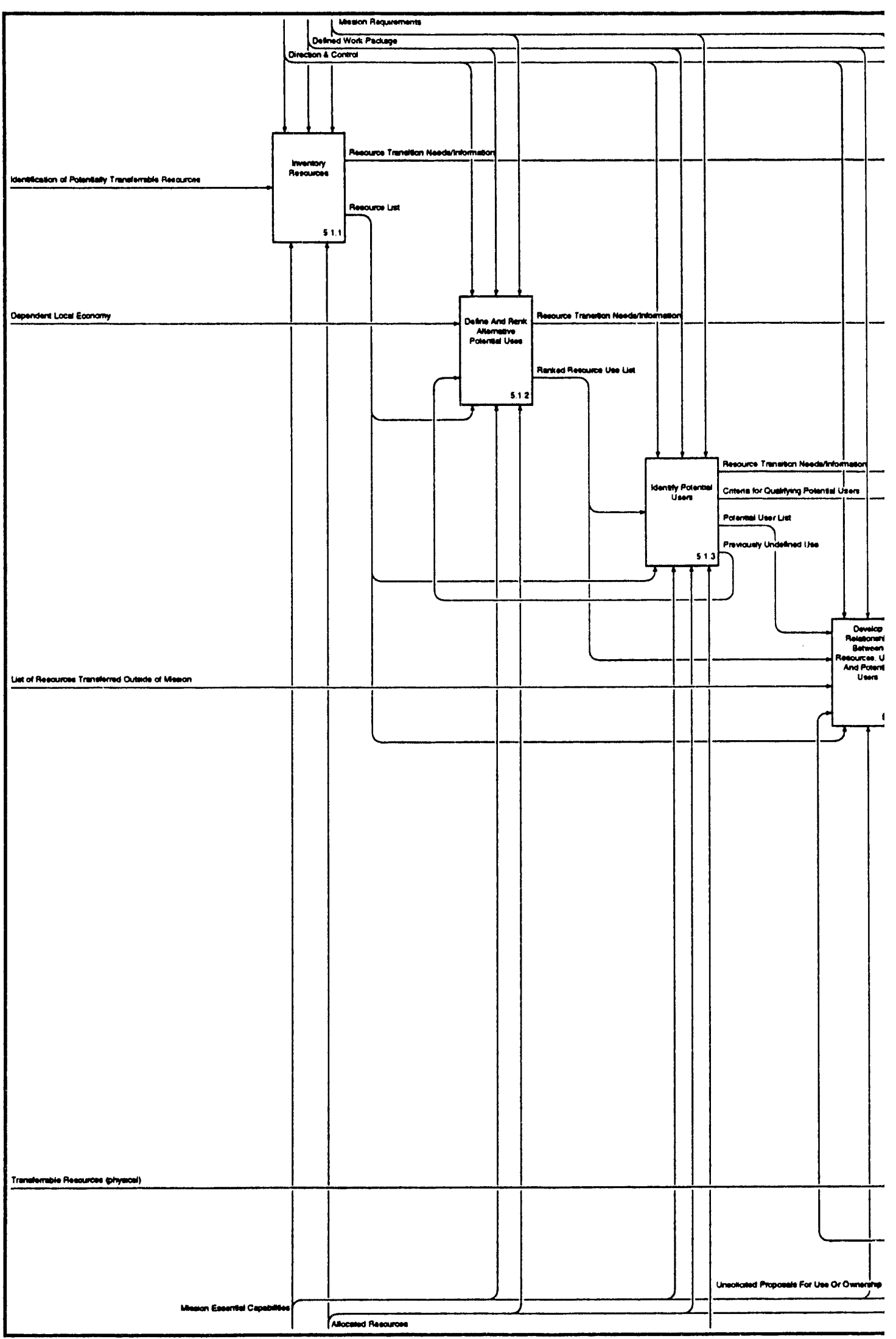




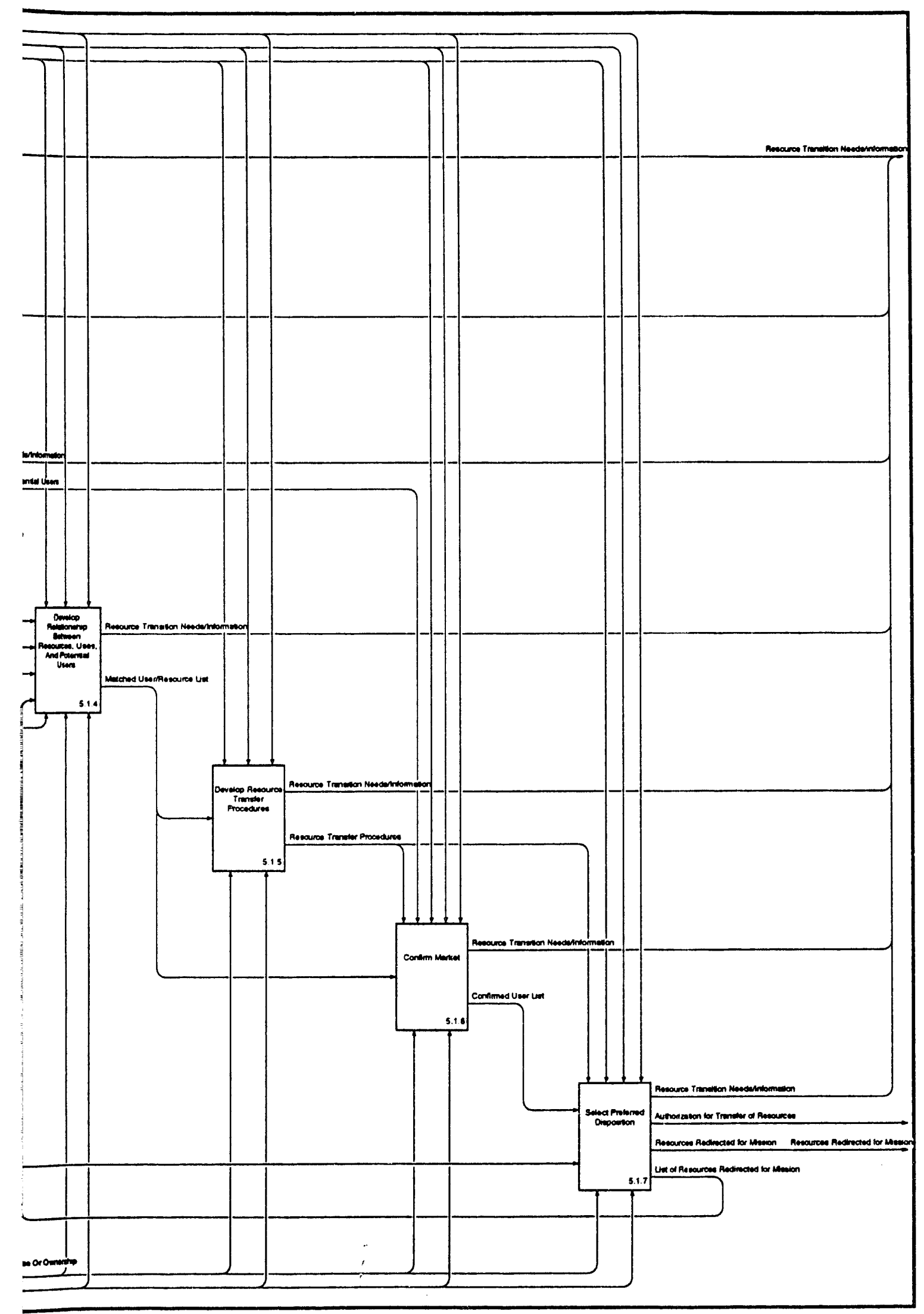




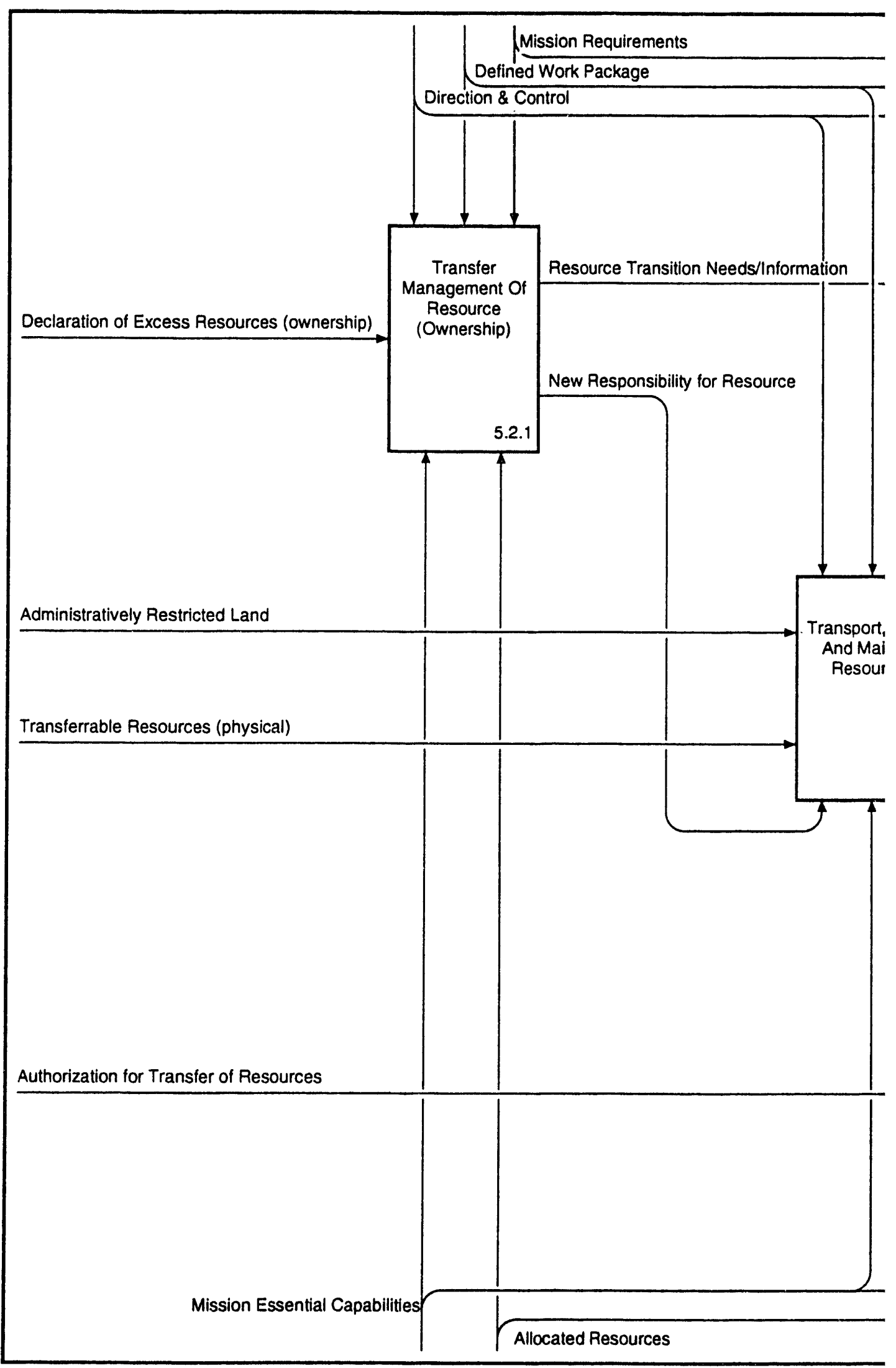




\section{lip Of Resources Outside Of Mission}

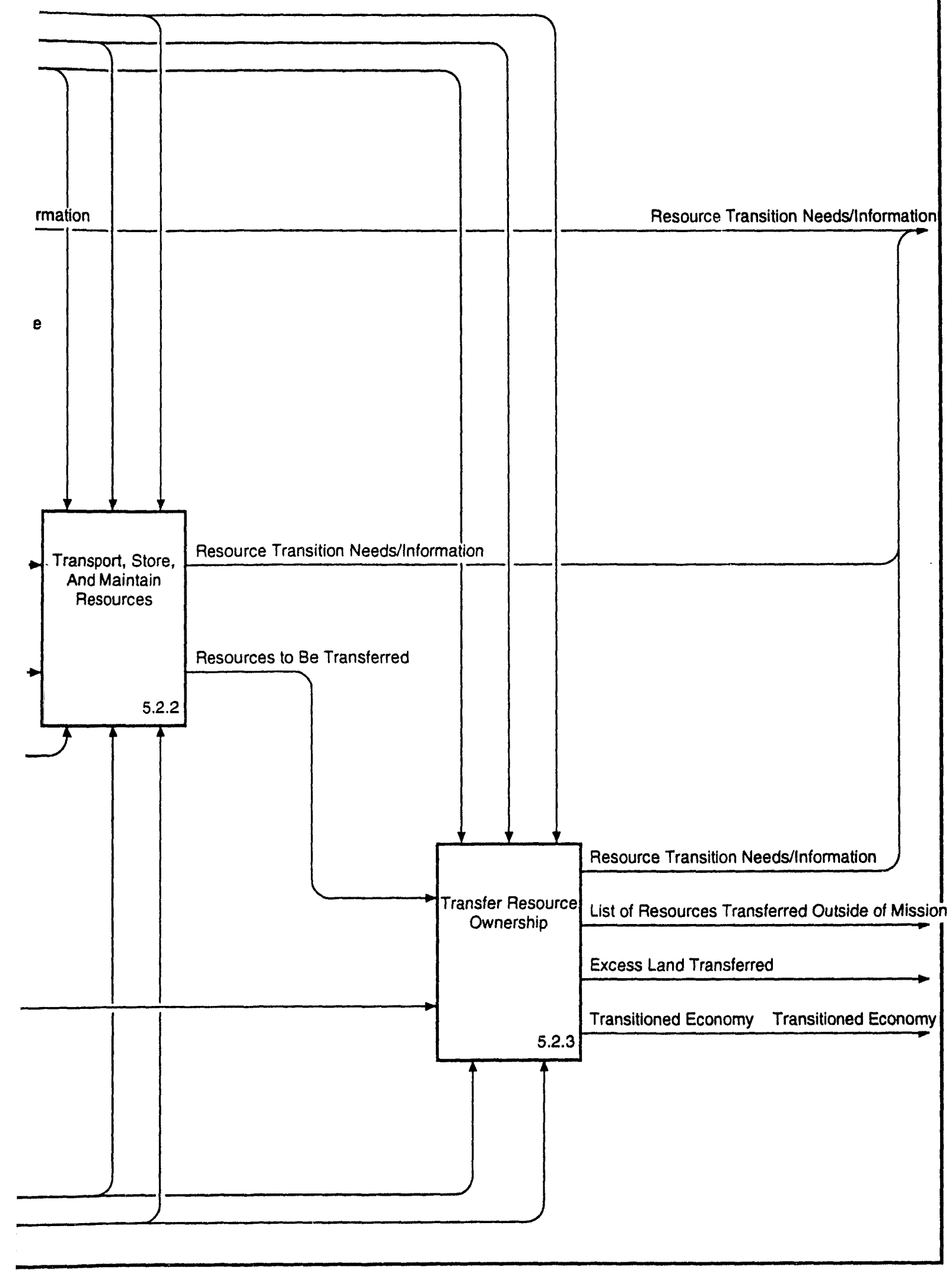

[5.2] Transfer Ownership Of Resources Outside Of Mission 
[5.3] Transfer Resources Fo

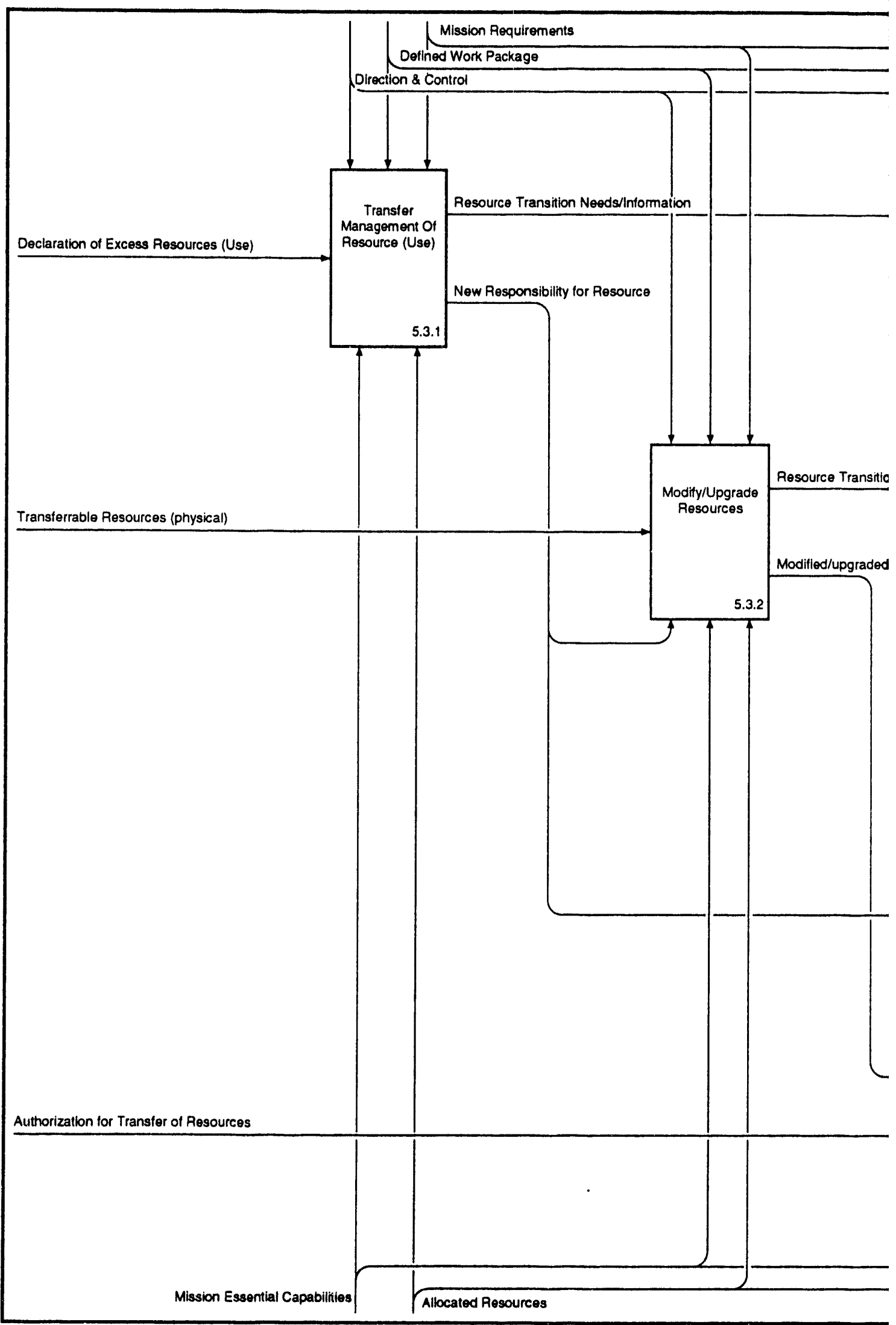




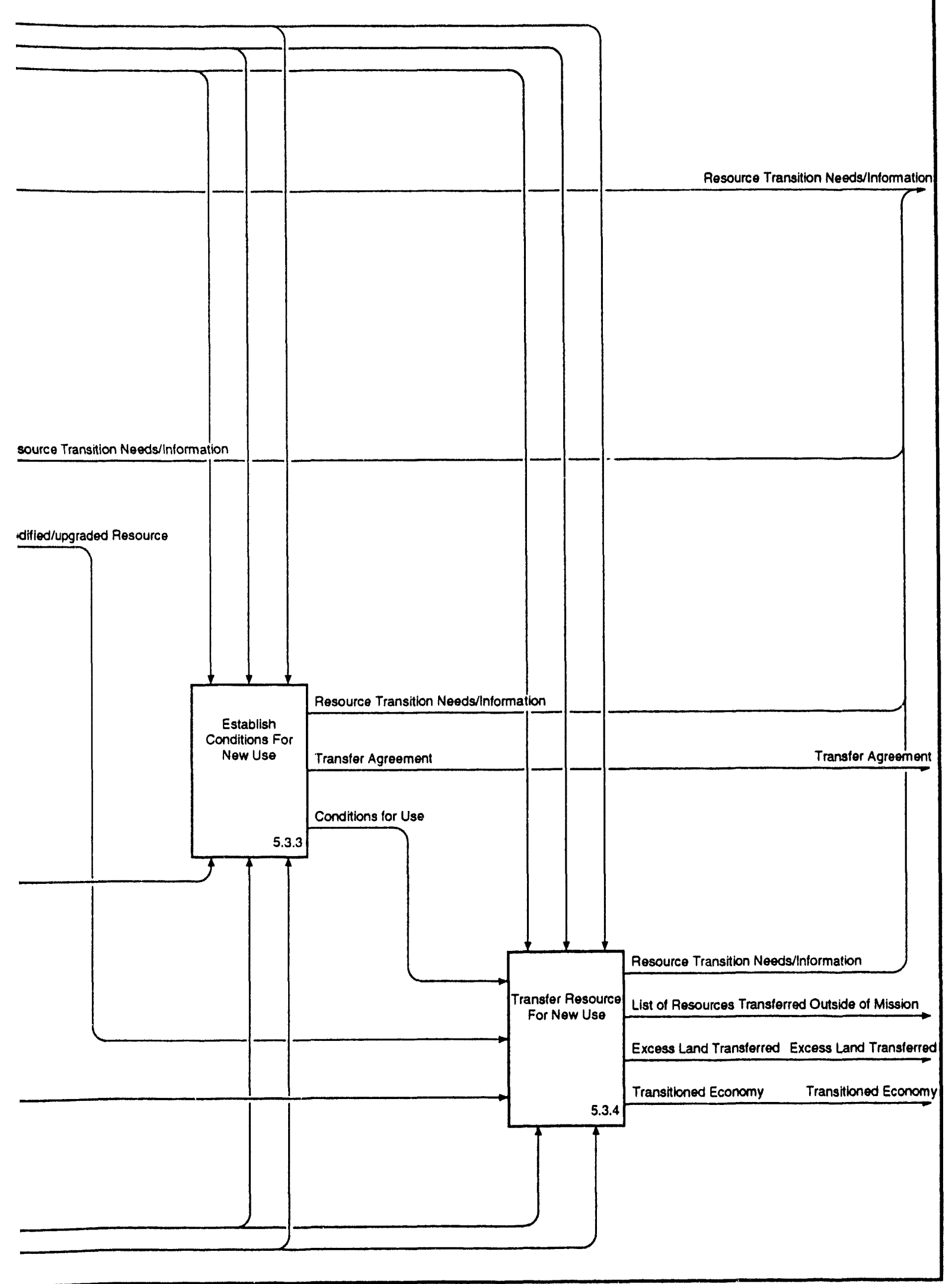


WHC-EP-0722 ADDENDUM 2 REV. 0

SECTION 3

FUNCTION DESCRIPTION TABLE

1 


\begin{tabular}{|c|c|}
\hline Function & Definitions \\
\hline 0 & $\begin{array}{l}\text { Clean Up Hanford } \\
\text { The waste management and environmental restoration mission is to clean up the } \\
\text { Hanford Site, protect the health and safety of the public and our workers, protect } \\
\text { the environment, and provide beneficial use of land and other resources while } \\
\text { serving as the DOE model for environmental restoration. Site cleanup will be in } \\
\text { accordance with the Hanford Federal Facility Agreement and Consent Order } \\
\text { (also known as the Tri-Party Agreement) (Ecology et al. 1990), other } \\
\text { agreements, and will be in compliance with all applicable federal, state, and } \\
\text { local laws. The stakeholders will be active participants in the decision processes } \\
\text { including establishing priorities, determining end states, and developing a } \\
\text { consistent set of rules, regulations, and laws. The work will be leveraged with } \\
\text { providing positive, lasting economic impact in the region. Effectiveness, } \\
\text { efficiency, and discipline in all mission activities will enable us to achieve our } \\
\text { vision and mission in a continuous and substantive manner. }\end{array}$ \\
\hline 1 & $\begin{array}{l}\text { Manage Program } \\
\text { The MANAGE PROGRAM function provides all program planning, management } \\
\text { direction, evaluation, and the management system. This function does not } \\
\text { directly contribute to the final state but provides the management needed to } \\
\text { conduct the mission. The function includes specification of management policies } \\
\text { and procedures, configuration management, scheduling, allocation of all } \\
\text { resources, definition of performance criteria, and resolution of regulatory } \\
\text { problems. }\end{array}$ \\
\hline 1.1 & $\begin{array}{l}\text { Establish Management System } \\
\text { The ESTABLISH MANAGEMENT SYSTEM (1.1) function defines Il procedures, } \\
\text { policies, reporting, configuration, and SE, needed to manage the program. This } \\
\text { function encompasses all routine management areas such as total quality, quality } \\
\text { assurance, personnel management, legal services, fiscal control, prime contracts, } \\
\text { etc. This function also develops the organizatioal structure evaluates the } \\
\text { efficiency of the management work and develops appropriate corrective actions. }\end{array}$ \\
\hline 1.1 .1 & $\begin{array}{l}\text { Identify Candidate Management System Components and Approaches } \\
\text { Define a comprehensive "menu" of organization elements, organization } \\
\text { structures, management principles, and operating policies from which } \\
\text { evaluations and subsequent selections can be made to formulate a management } \\
\text { system. }\end{array}$ \\
\hline 1.1 .2 & $\begin{array}{l}\text { Evaluate Value Added } \\
\text { Identify measures of effectiveness by which the excellence of alternative } \\
\text { management system components can be judged, define acceptability criteria for } \\
\text { each measure of effectiveness, determine the value of each component along the } \\
\text { scale of acceptability, and compile the resulting information for use in } \\
\text { decision-making processes. }\end{array}$ \\
\hline
\end{tabular}




\begin{tabular}{|l|l|}
\hline Function & Definitions \\
\hline 1.1 .2 .1 & Determine Methods for Evaluation \\
\hline 1.1 .2 .2 & Sort Elements with Respect to Evaluation Methodology \\
\hline 1.1 .2 .3 & Perform Evaluation \\
\hline 1.1 .2 .4 & Review with Stakeholders \\
\hline 1.1 .3 & $\begin{array}{l}\text { Define Management System } \\
\text { Assess the management system evaluation data, and select the elements, } \\
\text { structure, principles and policies best suited to management of the Hanford } \\
\text { cleanup mission. }\end{array}$ \\
\hline 1.1 .3 .1 & Select Evaluated Management System Elements \\
\hline 1.1 .3 .2 & Establish Management and Organization Principles \\
\hline 1.1 .3 .3 & Formulate Detailed Management System \\
\hline 1.1 .3 .4 & Obtain Stakeholder Concurrence \\
\hline 1.1 .4 & $\begin{array}{l}\text { Implement Management System } \\
\text { Perform the functions needed to emplace and activate the selected management } \\
\text { system physical and intellectual resources required for initiation of the Hanford } \\
\text { cleanup program. }\end{array}$ \\
\hline 1.1 .4 .1 & Establish Transition Plan \\
\hline 1.1 .4 .2 & Install Management System \\
\hline 1.1 .5 & $\begin{array}{l}\text { Maintain Management System } \\
\text { Assess program evaluation data related to deficiencies in the management system } \\
\text { and prepare recommendations for corrective actions. }\end{array}$ \\
\hline 1.1 .5 .1 & Audit and Surveil Management System \\
\hline 1.1 .5 .2 & Obtain Feedback from Stakeholders \\
\hline 1.1 .5 .3 & Perform Self Assessments \\
\hline 1.1 .5 .4 & Implement Corrective Action \\
\hline 1.2 & $\begin{array}{l}\text { Perform Systems Engineering } \\
\text { The PERFORM SYSTEMS ENGINEERING (1.2) function performs SE activities } \\
\text { for the mission. }\end{array}$ \\
\hline $\begin{array}{l}\text { Formulate Program } \\
\text { The FORMULATE PROGRAM (1.3) function converts the SE product tree into } \\
\text { an executable set of programs consisting of schedules, budgets and priorities and } \\
\text { produces all program descriptive information. }\end{array}$ \\
\hline $\begin{array}{l}\text { Formulate Program Baseline } \\
\text { Define the controlled program cost, schedule, and technical data that describe } 1) \\
\text { the configurations of the physical and management systems and 2) the activities } \\
\text { for the Hanford cleanup program. The baseline information provides the } \\
\text { primary basis for formulating the Work Packages. } \\
\text { Formulate Program Structure }\end{array}$ \\
\hline 1.3 .1 .1 & \\
\hline 1.3 .1 &
\end{tabular}




\begin{tabular}{|l|l|}
\hline Function & Definitions \\
\hline 1.3 .1 .2 & Establish Technical Baseline \\
\hline 1.3 .1 .3 & Establish Schedule Baseline \\
\hline 1.3 .1 .4 & Establish Cost Baseline \\
\hline 1.3 .1 .5 & Validate Planning Baseline \\
\hline 1.3 .2 & $\begin{array}{l}\text { Obtain Funding } \\
\text { Says what we are going to do and how we are going to do it. }\end{array}$ \\
\hline 1.3 .2 .1 & Establish Program Work Priorities \\
\hline 1.3 .2 .2 & Request Funding \\
\hline 1.3 .3 & $\begin{array}{l}\text { Determine Executable Work } \\
\text { Adjust work priorities and resultant fiscal year work scope to reconcile } \\
\text { differences between the requested budget and the budget actually allocated to the } \\
\text { program. }\end{array}$ \\
\hline 1.3 .3 .1 & Reassess Priorities \\
\hline 1.3 .3 .2 & Plan Fiscal Year Work \\
\hline 1.4 & $\begin{array}{l}\text { Direct Program } \\
\text { The DIRECT PROGRAM (1.4) function assigns the work activities defined above } \\
\text { to the performing entity along with budget, work definition and other necessary } \\
\text { resources. }\end{array}$ \\
\hline 1.4 .1 & $\begin{array}{l}\text { Implement Program Baseline } \\
\text { Prepare and issue work packages and supporting directions, and allocate } \\
\text { budgets to support the work defined by the work packages. }\end{array}$ \\
\hline Formulate Detailed Plans \\
\hline 1.4 .1 .1 & Organize Resources \\
\hline 1.4 .1 .2 & Assign Detailed Resources \\
\hline 1.4 .1 .3 & Authorize Work Details \\
\hline 1.4 .1 .5 & Manage Work \\
\hline 1.4 .2 & Control Changes \\
\hline 1.5 & $\begin{array}{l}\text { Evaluate Program } \\
\text { The EVALUATE PROGRAM (1.5) function evaluates progress, quality, and } \\
\text { efficiency of assigned work activities, reports to appropriate authority, and } \\
\text { recommends corrective action. }\end{array}$ \\
\hline $\begin{array}{l}\text { Status Program } \\
\text { Assemble the current status information provided by the physical system } \\
\text { acquisition and operation activities, along with corresponding baseline } \\
\text { information indicating the planned technical, budget and schedule status, for use } \\
\text { in the analysis of cleanup program progress. }\end{array}$ \\
\hline Determine Fiscal Year Status \\
\hline 1.5 .1 .1 & \\
\hline 1.1 &
\end{tabular}




\begin{tabular}{|c|c|}
\hline Function & Definitions \\
\hline 1.5 .1 .2 & Assess Out-Year Status \\
\hline 1.5 .2 & $\begin{array}{l}\text { Analyze Status Information } \\
\text { Analyze the actual and planned progress data to determine the technical, budget } \\
\text { and schedule variances; to forecast future progress based on existing work } \\
\text { packages and direction; and to recommend revised courses of action for } \\
\text { improved program effectiveneness. }\end{array}$ \\
\hline 1.5 .2 .1 & Analyze Baseline Data \\
\hline 1.5 .2 .2 & Develop Forecasts and Trends \\
\hline 1.5 .3 & Conduct Audits and Assessments \\
\hline 1.5 .3 .1 & Conduct Internal Audits, Surveillances, and Assessments \\
\hline 1.5 .3 .2 & Conduct External Audits, Surveillances, and Assessments \\
\hline 1.6 & $\begin{array}{l}\text { Help Reinvent Government } \\
\text { The HELP REINVENT GOVERNMENT (1.6) function reviews requirements, } \\
\text { policies, and directives for mission effectiveness, determines high payoff } \\
\text { candidates for revision, change, or elimination, and actively pursues the desired } \\
\text { change. }\end{array}$ \\
\hline 1.6 .1 & $\begin{array}{l}\text { Identify External Requirements Conflicts and Ambiguities } \\
\text { Review externally-imposed requirements, and identify conflicts between specific } \\
\text { requirements imposed within a single source or in different sources, and } \\
\text { requirements that are not stated with sufficient clarity or specificity to be } \\
\text { translated into specific constraints on system products or processes. }\end{array}$ \\
\hline 1.6 .2 & $\begin{array}{l}\text { Assess External Requirement Added Value } \\
\text { Review externally-imposed requirements to assess the impacts on the cleanup } \\
\text { program and to identify those whose net impacts on system effectiveness; i.e., the } \\
\text { economic, safety or other value of the benefits derived from the requirements } \\
\text { minus the economic, safety or other costs of those regirements; are negative. }\end{array}$ \\
\hline 1.6 .3 & $\begin{array}{l}\text { Formulate Requirement Revision Proposals } \\
\text { Prepare documentation describing the problems related to conflicting, } \\
\text { ambiguous or counterproductive requirements, suggested revisions to those } \\
\text { requirements, and the rationale for those revisions, for use in conducting } \\
\text { interactions with regulatory and policy-making external entities. }\end{array}$ \\
\hline 1.6 .4 & $\begin{array}{l}\text { Negotiate Requirement Revisions } \\
\text { Conduct interactions with regulatory and policy-making external entities to } \\
\text { reach agreements on interpretations, compliance criteria and revisions for } \\
\text { conflicting, ambiguous, and counterproductive requirements. }\end{array}$ \\
\hline 2 & $\begin{array}{l}\text { Acquire Mission Essential Capabilities } \\
\text { The ACQUIRE MISSION ESSENTIAL CAPABILITIES function provides all new } \\
\text { intellectual and physical resources. This includes personnel, consultants, } \\
\text { services, supplies, equipment, construction projects, and subcontracts of all } \\
\text { kinds. }\end{array}$ \\
\hline
\end{tabular}




\begin{tabular}{|c|c|}
\hline Function & Definitions \\
\hline 2.1 & $\begin{array}{l}\text { Formulate Acquisition Strategy For Mission Essential Capabilities } \\
\text { The FORMULATE ACQUISITION STRATEGY FOR MISSION ESSENTIAL } \\
\text { CAPABILITIES (2.1) function screens incoming acquisition needs with respect to } \\
\text { opportunities for consolidation or assignment to a full service, non-Hanford Site } \\
\text { contractor. Opportunities identified are provided to the Manage Program } \\
\text { function for reprogramming. }\end{array}$ \\
\hline 2.1 .1 & $\begin{array}{l}\text { Evaluate Opportunities With Respect To Acquisition Class } \\
\text { Formulate an integrated strategy for the acquisition of mission essential } \\
\text { expertise, technology, facilities, equipment, infrastructure, supplies, and } \\
\text { integrated independent services. Identify acquisition functions and requirements, } \\
\text { evaluate strategic alternatives, and select preferred approaches for acquiring } \\
\text { mission essential capabilities in an integrated and efficiency manner. }\end{array}$ \\
\hline 2.1 .2 & $\begin{array}{l}\text { Evaluate Acquisition For Consolidation Opportunities } \\
\text { Formulate an integrated strategy for the consolidated acquisition of mission } \\
\text { essential capabilities including expertise, technology, facilities, equipment, } \\
\text { infrastructure, supplies, and information. }\end{array}$ \\
\hline 2.1 .3 & $\begin{array}{l}\text { Evaluate Opportunities For External Acquisition Of Major Services } \\
\text { Formulate an integrated strategy for the acquisition of mission essential } \\
\text { integrated independent services. Identify and develop the content and possible } \\
\text { approaches to contracting for a major integrated service such as laundry } \\
\text { services, office space, or other services including contractor-financed investment. }\end{array}$ \\
\hline 2.2 & $\begin{array}{l}\text { Provide Expertise } \\
\text { The PROVIDE EXPERTISE (2.2) function provides new personnel, retrains } \\
\text { existing professional personnel for new assignments outside their usual } \\
\text { discipline, provides expert consultants, and acquires off-site expert services of all } \\
\text { types. }\end{array}$ \\
\hline 2.2 .1 & $\begin{array}{l}\text { Identify Sources of Expertise } \\
\text { Determine sources and methodologies for acquiring qualified personnel to } \\
\text { perform the Hanford clean up mission functions and activities. }\end{array}$ \\
\hline 2.2 .2 & $\begin{array}{l}\text { Import Expertise } \\
\text { Locate, evaluate, select, hire/contract, relocate, train, and deliver qualified } \\
\text { personnel that do not exist within the existing Hanford work force. }\end{array}$ \\
\hline 2.2.2.1 & Find Expertise \\
\hline 2.2 .2 .2 & Evaluate Expertise \\
\hline 2.2 .2 .3 & Select Expertise \\
\hline 2.2 .2 .4 & Hire/Contract Expertise \\
\hline 2.2 .2 .5 & Relocate Expertise \\
\hline 2.2 .2 .6 & Train Expertise \\
\hline 2.2.2.7 & Deliver Expertise \\
\hline
\end{tabular}




\begin{tabular}{|c|c|}
\hline Eunction & Definitions \\
\hline 2.2 .3 & $\begin{array}{l}\text { Develop Expertise } \\
\text { Provide personnel training and certification for the Hanford work force } \\
\text { personnel to perform the Hanford clean up mission functions and activities. }\end{array}$ \\
\hline 2.2 .3 .1 & Screen Candidates \\
\hline 2.2 .3 .2 & Retrain Personnel \\
\hline 2.2 .3 .3 & Certify Personnel \\
\hline 2.2 .4 & $\begin{array}{l}\text { Retain Expertise } \\
\text { Provide personnel salary and benefits, career development opportunities, and a } \\
\text { positive work place to maintain a motivated work force to perform and complete } \\
\text { the Hanford clean up mission. }\end{array}$ \\
\hline 2.2 .4 .1 & Provide Career Pathing \\
\hline 2.2 .4 .2 & Provide Salary and Benefits \\
\hline 2.2 .4 .3 & Provide Career Opportunities \\
\hline 2.2 .4 .4 & Provide Positive Workplace \\
\hline 2.3 & $\begin{array}{l}\text { Provide Technology } \\
\text { The PROVIDE TECHNOLOGIES (2.3) function provides all needed new } \\
\text { technologies not currently available on-site, obtains needed technology from the } \\
\text { Technology Development Program, and provides the technology interface to all } \\
\text { appropriate entities. }\end{array}$ \\
\hline 2.3 .1 & $\begin{array}{l}\text { Analyze Mission Essential Technology Insufficiency } \\
\text { Confirm that a technology insufficiency exists for the Hanford clean up mission. }\end{array}$ \\
\hline 2.3 .2 & $\begin{array}{l}\text { Complete Technology Research } \\
\text { Complete the necessary research to proveldisprove a technological concept. }\end{array}$ \\
\hline 2.3 .3 & $\begin{array}{l}\text { Perform Laboratory Demonstration } \\
\text { Conduct laboratory scale testing of a mission essential technology. }\end{array}$ \\
\hline 2.3 .4 & $\begin{array}{l}\text { Scale-Up/Develop Application } \\
\text { Demonstrate that technology will fulfill its intended function. This may/may not } \\
\text { require full scale demonstration. }\end{array}$ \\
\hline 2.3 .5 & $\begin{array}{l}\text { Develop Source of Supply } \\
\text { Identify existing/potential commercial sources for demonstrated technology. }\end{array}$ \\
\hline 2.4 & $\begin{array}{l}\text { Provide Facilities, Equipment, Infrastructure, \& Supplies } \\
\text { The PROVIDE FACILITIES, EQUIPMEN, INFRASTRUCTURE, \& SUPPLIES } \\
\text { (2.4) function produces all facilities, equipment, software and related items } \\
\text { needed by all work activities. }\end{array}$ \\
\hline 2.4 .1 & $\begin{array}{l}\text { Provide Projects } \\
\text { Define, design, procure, construct, start up and test line item projects, major } \\
\text { systems acquisitions (MSA's), general plant projects, and modifications and } \\
\text { ungrades to facilities and systems. }\end{array}$ \\
\hline
\end{tabular}




\begin{tabular}{|c|c|}
\hline Function & Definitions \\
\hline 2.4.1.1 & $\begin{array}{l}\text { Define Project Scope } \\
\text { Define technical, cost and schedule baselines including engineering studies, } \\
\text { Functional Design Criteria, Conceptual Design Reports, Preliminary Safety } \\
\text { Evaluations, and other preliminary definitions. }\end{array}$ \\
\hline 2.4 .1 .2 & $\begin{array}{l}\text { Define Project Architecture } \\
\text { Define roles and responsibilities and scope of activities to be performed by all } \\
\text { performing organizations. Define management approach. }\end{array}$ \\
\hline 2.4.1.3 & $\begin{array}{l}\text { Obtain Permits and Approvals } \\
\text { Obtain all necessary NEPA, CAA, key decisions, PSAR and FSAR approvals. } \\
\text { Request appropriate authorizations from management. }\end{array}$ \\
\hline 2.4.1.4 & $\begin{array}{l}\text { Design/Analyze Project } \\
\text { Perform Title I, II and III design including preliminary, final design and } \\
\text { engineering inspection. Perform safety analysis, environmental analysis and risk } \\
\text { analysis to support obtaining necessary permits and approvals. Produce } \\
\text { engineering specifications for equipment and component procurement and } \\
\text { construction. }\end{array}$ \\
\hline 2.4.1.5 & $\begin{array}{l}\text { Construct Projects } \\
\text { Procure construction materials and construct/modify facility and systems. }\end{array}$ \\
\hline 2.4 .1 .6 & $\begin{array}{l}\text { Startup Project } \\
\text { Ready project/facility/system for testing. Bump motors, calibrate } \\
\text { instrumentation, groom and align, perform leak and functional test, and correct } \\
\text { deficiencies. }\end{array}$ \\
\hline 2.4.1.7 & $\begin{array}{l}\text { Test Project } \\
\text { Conduct integrated testing of project/facility to verify operability and } \\
\text { performance to requirements. }\end{array}$ \\
\hline 2.4.1.8 & $\begin{array}{l}\text { Prepare Operating Documents } \\
\text { Prepare operating documentation for projectffacility, including operating and } \\
\text { maintenance procedures and technical specifications. }\end{array}$ \\
\hline 2.4 .1 .9 & $\begin{array}{l}\text { Train Operators } \\
\text { Train operations personnel to operate the project/facility. } \\
\end{array}$ \\
\hline 2.4.1.10 & $\begin{array}{l}\text { Accept Project } \\
\text { Accept and turnover project for operation, after testing that has verified } \\
\text { acceptable performance or deficiencies have been are corrected. }\end{array}$ \\
\hline 2.4 .2 & $\begin{array}{l}\text { Provide Equipment } \\
\text { Specify and procure off-the-shelf equipment to support the Hanford clean-up } \\
\text { mission functions and activities. }\end{array}$ \\
\hline 2.4.2.1 & Design Equipment \\
\hline 2.4.2.2 & Procure Equipment \\
\hline 2.4.2.3 & Verify Equipment Operation \\
\hline
\end{tabular}




\begin{tabular}{|c|c|}
\hline Function & Definitions \\
\hline 2.4 .3 & $\begin{array}{l}\text { Provide Supplies } \\
\text { Specify and procure general store items and consumables needed to support the } \\
\text { Hanford clean-up mission functions and activities. }\end{array}$ \\
\hline 2.5 & $\begin{array}{l}\text { Provide Essential Information } \\
\text { The PROVIDES ESSENTIAL INFORMATION FUNCTION (2.5) supplies the } \\
\text { entire effort with access to various offsite databases, proprietary commercial } \\
\text { data, and offsite expert systems and allows wide and facile access to internally } \\
\text { generated information used in Cleanup Mission activities. }\end{array}$ \\
\hline 2.5 .1 & $\begin{array}{l}\text { Identify Sources of Mission Essential Information } \\
\text { Survey and compile capabilities for supplying information classified by topic and } \\
\text { general usage. For internal information, this activity relies on information } \\
\text { products defined by the Manage Program function as resources for identifying } \\
\text { the appropriate sources. Determine whether information should be obtained } \\
\text { internally or externally. }\end{array}$ \\
\hline 2.5 .2 & $\begin{array}{l}\text { Acquire Outside Information } \\
\text { Assess the information functions and requirements, match to the potential } \\
\text { sources of information, investigate the integrity and comprehensiveness of the } \\
\text { information to meet the needs and requirements, and procure the essential } \\
\text { information. }\end{array}$ \\
\hline 2.5.2.1 & $\begin{array}{l}\text { Assess External Functions and Requirements } \\
\text { Evaluate and characterize the information needs inherent in the functions and } \\
\text { requirements for information. }\end{array}$ \\
\hline 2.5.2.2 & $\begin{array}{l}\text { Identify Sources of Outside Information } \\
\text { Match the characterized information needs to potential sources of information } \\
\text { from government, industry, libraries, and information service organizations. }\end{array}$ \\
\hline 2.5.2.3 & $\begin{array}{l}\text { Procure Outside Information } \\
\text { Solicit specific information supply services from those sources that can qualify to } \\
\text { satisfy the characterized information needs and procure the services. }\end{array}$ \\
\hline 2.5 .3 & $\begin{array}{l}\text { Acquire Inside Information } \\
\text { Assess the information functions and requirements, match to potential sources of } \\
\text { information, investigate the integrity and comprehensiveness to meet the } \\
\text { functions and requirements, and arrange access to the information to satisfy } \\
\text { additional uses. }\end{array}$ \\
\hline 2.5.3.1 & $\begin{array}{l}\text { Assess Internal Functions and Requirements } \\
\text { Evaluate and characterize the information needs inherent in the functions and } \\
\text { requirements for the information. }\end{array}$ \\
\hline 2.5.3.2 & $\begin{array}{l}\text { Identify Sources of Inside Information } \\
\text { Match the characterized information needs to potential sources of information } \\
\text { provided by the Manage Program functions. Further refine the specific source of } \\
\text { information based on assessing where best to extract, assess or otherwise secure } \\
\text { the information. }\end{array}$ \\
\hline
\end{tabular}




\begin{tabular}{|c|c|}
\hline Function & Definitions \\
\hline 2.5.3.3 & $\begin{array}{l}\text { Obtain Access to Inside Information } \\
\text { Arrange for the appropriate method of assessing information associated with a } \\
\text { specific function or activity. Secure the approval of the function and activity. } \\
\text { Develop methods that ensure continuity of operations for existing information } \\
\text { processes and for maintaining the accuracy and timeliness of the information. }\end{array}$ \\
\hline 2.5.3.4 & $\begin{array}{l}\text { Standardize Codes, Naming Conventions, and Privileges } \\
\text { Develop common methods of representing information, such as nomenclature, } \\
\text { units of measure, level of detail, and granularity. Develop priviledges for } \\
\text { administering access priviledges and capabilities to change data. }\end{array}$ \\
\hline 2.5 .4 & $\begin{array}{l}\text { Deliver Information } \\
\text { Deploy capabilities that enable staff responsible for specific activities to obtain } \\
\text { the information necessary to perform efforts. }\end{array}$ \\
\hline 2.6 & $\begin{array}{l}\text { Provide Integrated Independent Services } \\
\text { The PROVIDE INTEGRATED INDEPENDENT SERVICES (2.6) function } \\
\text { delivers integrated service contracts of all types including ERMCs, specialty } \\
\text { services like laundry steam supply, etc, to other related integrated efforts. }\end{array}$ \\
\hline 3 & $\begin{array}{l}\text { Obtain Public Acceptance } \\
\text { The OBTAIN PUBLIC ACCEPTANCE function provides all the public } \\
\text { interactions needed to complete the mission including public relations, reviews of } \\
\text { all plans and priorities, as needed to minimize potential conflicts between the } \\
\text { stakeholder interests and mission activities. }\end{array}$ \\
\hline 3.1 & $\begin{array}{l}\text { Identify Issues } \\
\text { The IDENTIFY ACTIVITIES MOST MEANINGFUL TO PUBLIC } \\
\text { INVOLVEMENT (3.1) function defines areas of high interest to the various } \\
\text { public sectors (sometimes called publics). }\end{array}$ \\
\hline 3.1 .1 & $\begin{array}{l}\text { Identify Decisions/Actions/Information Of Interest To The Various Public } \\
\text { Sectors }\end{array}$ \\
\hline 3.1 .2 & $\begin{array}{l}\text { Involve Publics In Identification Of Decisions/Actions/Information Of } \\
\text { Interest To Public Sectors }\end{array}$ \\
\hline 3.1 .3 & Set Public Involvement Baseline For Decisions/Actions/Information \\
\hline 3.2 & $\begin{array}{l}\text { Identify Information Needs For Public Groups } \\
\text { The IDENTIFY NEEDS (3.2) function defines areas where the cleanup mission } \\
\text { needs public involvement. }\end{array}$ \\
\hline 3.2 .1 & Identify Interested Publics For Each Decision/Information Group \\
\hline 3.2 .2 & Define Information Needs For Each Interest Group \\
\hline 3.2.2.1 & Define Information For External Groups \\
\hline 3.2.2.2 & Define Information For Internal Groups \\
\hline 3.2 .3 & $\begin{array}{l}\text { Develop Public Involvement Plan For Each Combination Of Public } \\
\text { Group/(Decision/Information Area) }\end{array}$ \\
\hline
\end{tabular}




\begin{tabular}{|c|c|}
\hline Function & Definitions \\
\hline 3.3 & $\begin{array}{l}\text { Develop Understanding Of Public/Mission Differences } \\
\text { The IDENTIFY PUBLICS (3.3) function classifies the public into associated } \\
\text { groups with common outlooks and needs. }\end{array}$ \\
\hline 3.3 .1 & Define Differences In "Objectives" \\
\hline 3.3.2 & Define Differences In Values \\
\hline 3.3.3 & Define Differences In Concerns \\
\hline 3.3 .4 & Define Differences In Priorities \\
\hline 3.3 .5 & Define Differences On Specific Decisions \\
\hline 3.4 & $\begin{array}{l}\text { Reconcile Differences } \\
\text { The ELIMINATE ADVERSARIAL RELATIONSHIPS (3.4) function conducts } \\
\text { those activities requiring public input and fills the need for public interaction } \\
\text { with respect to public expectations, needs and information. }\end{array}$ \\
\hline 3.4.1 & Narrow Differences Between Mission Plans/Activities \\
\hline 3.4.2 & Develop Compromise/Consensus Positions \\
\hline 4 & $\begin{array}{l}\text { Remedy Unsafe And Unacceptable Conditions } \\
\text { The REMEDY UNSAFE AND UNACCEPTABLE CONDITIONS function } \\
\text { provides the physical changes needed to complete the function and provides the } \\
\text { safe and efficient operation of all facilities. Included within this function are } \\
\text { environmental restoration of buildings, facilities, groundwater, and soils, } \\
\text { treatment of waste materials, interim storage of various materials, disposition of } \\
\text { low-level waste, packaging and shipment of hazardous, transuranic, mixed, and } \\
\text { high-level waste, retrieval of wastes for disposition, regulatory compliance } \\
\text { actions, and disposition of new imported wastes and materials. }\end{array}$ \\
\hline 4.1 & $\begin{array}{l}\text { Deactivate Facilities } \\
\text { The DEACTIVATE FACILITIES (4.1) function contains the safe operation of all } \\
\text { facilities until further operation is no longer mission essential. The primary } \\
\text { output is eventual facility conversion to a state where transfer to the function } 4.4 \\
\text { can take place. }\end{array}$ \\
\hline 4.1 .1 & $\begin{array}{l}\text { Deactivate Facilities With Special Nuclear Materials \& Nuclear Materials } \\
\text { (Type } 1 \text { Fclty) } \\
\text { Deactivates facilities containing the following materials: special nuclear } \\
\text { materials, nuclear materials, nuclear fuels and may include radioactive or } \\
\text { hazardous materials (e.g. Purex, PFP). }\end{array}$ \\
\hline 4.1 .1 .1 & $\begin{array}{l}\text { Maintain Safety \& Compliance Envelope (Type } 1 \text { Fclty) } \\
\text { Maintains the facility structure, qualified staff, safe and compliant equipment, } \\
\text { documentation and provides assessment of safety and compliance states. } \\
\text { Provides all necessary resources for safe and compliant operation in accordance } \\
\text { with governing safety codes and regulations. }\end{array}$ \\
\hline
\end{tabular}




\begin{tabular}{|c|c|}
\hline Function & Definitions \\
\hline 4.1.1.1.1 & $\begin{array}{l}\text { Maintain Safe \& Compliant Operations (Type } 1 \text { Fclty) } \\
\text { Assesses and maintains the facility operations in a safe condition that is } \\
\text { compliant with applicable environmental requirments, DOE orders, and all other } \\
\text { applicable codes, standards, and company procedures. }\end{array}$ \\
\hline 4.1.1.1.1.1 & $\begin{array}{l}\text { Acquire Knowledge Of Facility Status (Type } 1 \text { Fclty) } \\
\text { Acquires knowledge of the facility status, configuration, facility operating } \\
\text { systems, contents, and inventories required to enable deactivation. }\end{array}$ \\
\hline 4.1.1.1.1.2 & $\begin{array}{l}\text { Provide Surveillance (Type } 1 \text { Fclty) } \\
\text { Provides surveillance of the facility operations and operating systems, develop } \\
\text { acutely unsafe condition action plans, perform OSR surveillance procedures, } \\
\text { environmental monitoring, RCRA tracking, and surveillance of safety analysis } \\
\text { compliance. }\end{array}$ \\
\hline 4.1.1.1.1.3 & $\begin{array}{l}\text { Provide Access Control (Type } 1 \text { Fclty) } \\
\text { Provides access control to and internal to the facility for safeguards and security, } \\
\text { safety, and radiological purposes. }\end{array}$ \\
\hline 4.1 .1 .1 .1 .4 & $\begin{array}{l}\text { Provide Work Control (Type } 1 \text { Fclty) } \\
\text { Provides a job control system for the facility activities. }\end{array}$ \\
\hline 4.1.1.1.1.5 & $\begin{array}{l}\text { Provide Property Management (Type } 1 \text { Fclty) } \\
\text { Provides property management for the facility in accordance with DOE orders } \\
\text { and WHC procedures. }\end{array}$ \\
\hline 4.1.1.1.1.6 & $\begin{array}{l}\text { Provide Non-Hazardous Material/Waste Management (Type } 1 \text { Fclty) } \\
\text { Contains, controls, and documents non-hazardous material and waste in } \\
\text { accordance with safe and applicable standards. }\end{array}$ \\
\hline 4.1.1.1.1.7 & $\begin{array}{l}\text { Provide Hazardous Material/Waste Management (Type } 1 \text { Fclty) } \\
\text { Provides containment, control, and documentation of hazardous materials and } \\
\text { wastes in conformance with safety requirements and all applicable hazardous } \\
\text { material/wastes codes and regulations. }\end{array}$ \\
\hline 4.1 .1 .1 .1 .8 & $\begin{array}{l}\text { Provide Radioactive/TRU Material/Waste Management (Type } 1 \text { Fclty) } \\
\text { Provides containment, control, and documentation of radioactive material and } \\
\text { waste in accordance with safety requirements and all applicable codes and } \\
\text { regulations. }\end{array}$ \\
\hline 4.1.1.1.1.9 & $\begin{array}{l}\text { Provide SNM/NM/NF MaterialWaste Management (Type } 1 \text { Fclty) } \\
\text { Provides all special SNM/NM/NF management operations in accordance with } \\
\text { applicable codes and regulations including proper surveillance and security. }\end{array}$ \\
\hline 4.1.1.1.2 & $\begin{array}{l}\text { Maintain Safe \& Compliant Systems, Equipment, \& Structure (Type } 1 \\
\text { Fclty) } \\
\text { Maintains the facility systems and infrastructure in the operational condition } \\
\text { dictated by approved safety and compliance documentation (includes } \\
\text { environmental regulations). }\end{array}$ \\
\hline
\end{tabular}




\begin{tabular}{|c|c|}
\hline Function & Definitions \\
\hline 4.1.1.1.2.1 & $\begin{array}{l}\text { Perform Corrective Maintenance (Type } 1 \text { Fclty) } \\
\text { All maintenance that brings systems and equipment back to their operational } \\
\text { states after failure. }\end{array}$ \\
\hline 4.1.1.1.2.2 & $\begin{array}{l}\text { Perform Preventive Maintenance (Type } 1 \text { Fclty) } \\
\text { Preventive maintenance activities to minimize all unplanned events and } \\
\text { premature equipment failures. }\end{array}$ \\
\hline 4.1.1.1.2.3 & $\begin{array}{l}\text { Perform Process Systems and Equipment Calibrations (Type } 1 \text { Fclty) } \\
\text { Performs facility equipment, instrumentation, and process system calibrations to } \\
\text { ensure accuracy. }\end{array}$ \\
\hline 4.1.1.1.2.4 & $\begin{array}{l}\text { Perform Minor Modifications (Type } 1 \text { Fclty) } \\
\text { Performs minor modifications to facility systems or structure to ensure safe and } \\
\text { compliant operations during the facility deactivation process. }\end{array}$ \\
\hline 4.1 .1 .1 .3 & $\begin{array}{l}\text { Maintain Safety \& Compliance Documentation (Type } 1 \text { Fclty) } \\
\text { Maintains all required facility safety, compliance, engineering, inventory, and } \\
\text { operating documentation during facility deactivation. }\end{array}$ \\
\hline 4.1.1.1.3.1 & $\begin{array}{l}\text { Maintain Safety Documents (Type } 1 \text { Fclty) } \\
\text { Maintains documentation necessary to ensure safe deactivation activities. This } \\
\text { includes FSARs, ISBs, CSERs, ETC. }\end{array}$ \\
\hline 4.1 .1 .1 .3 .2 & $\begin{array}{l}\text { Maintain Operations Procedures (Type } 1 \text { Fclty) } \\
\text { Maintains operations procedures and documentation to support economic, } \\
\text { safety. or environmental compliance requirements. }\end{array}$ \\
\hline 4.1 .1 .1 .3 .3 & $\begin{array}{l}\text { Maintain Engineering Documentation (Type } 1 \text { Fclty) } \\
\text { Maintains configuration drawings and associated engineering documentation } \\
\text { required to operate and maintain the facility in a safe and compliant status. }\end{array}$ \\
\hline 4.1 .1 .1 .3 .4 & $\begin{array}{l}\text { Maintain Maintenance Procedures (Type } 1 \text { Fclty) } \\
\text { Maintains maintenance procedure documentation necessary for safe, efficient, } \\
\text { and compliant operations. }\end{array}$ \\
\hline 4.1 .1 .1 .3 .5 & $\begin{array}{l}\text { Maintain Environmental Compliance Documentation (Type } 1 \text { Fclty) } \\
\text { Maintains appropriate regulatory files and other related environmental } \\
\text { documentation to assure and prove environmental compliance. }\end{array}$ \\
\hline 4.1.1.1.3.6 & $\begin{array}{l}\text { Maintain Inventory Documentation (Type } 1 \text { Fclty) } \\
\text { Maintains nuclear materials documentation in compliance with DOE orders. }\end{array}$ \\
\hline 4.1.1.1.4 & $\begin{array}{l}\text { Maintain Qualified Facility Staff (Type } 1 \text { Fclty) } \\
\text { Provides facility specific training, testing, and training records maintenance to } \\
\text { ensure facility staff remain trained, qualified, and certified (as required) } \\
\text { throughout the facility deactivation process. }\end{array}$ \\
\hline 4.1 .1 .1 .4 .1 & $\begin{array}{l}\text { Provide Training (Type } 1 \text { Fclty) } \\
\text { Provides all training related to the activities necessary to deactivate the facilities } \\
\text { and ensure they remain in a safe and compliant condition. }\end{array}$ \\
\hline
\end{tabular}




\begin{tabular}{|c|c|}
\hline Function & Definitions \\
\hline 4.1.1.1.4.2 & $\begin{array}{l}\text { Maintain Qualification and Certification(Type } 1 \text { Fclty) } \\
\text { Provides periodic personnel skills check, assessment, and testing required to } \\
\text { maintain necessary qualifications and certifications. }\end{array}$ \\
\hline 4.1.1.1.4.3 & $\begin{array}{l}\text { Maintain Training Documentation (Type } 1 \text { Fclty) } \\
\text { Maintains applicable worker training documentation. Documentation includes } \\
\text { worker safetv and competancy qualification and certification. }\end{array}$ \\
\hline 4.1.1.1.5 & $\begin{array}{l}\text { Assess Safety \& Compliance State (Type } 1 \text { Fclty) } \\
\text { Performs/responds to oversight assessments and perform appropriate self } \\
\text { assessments of the facility deactivation activities to evaluate the facility and } \\
\text { operations safety and compliance status. }\end{array}$ \\
\hline 4.1.1.1.5.1 & $\begin{array}{l}\text { Support Independent Oversight Audits (Type } 1 \text { Fclty) } \\
\text { Performs and responds to independant oversight audits. }\end{array}$ \\
\hline 4.1.1.1.5.2 & $\begin{array}{l}\text { Perform Management Assessments (Type } 1 \text { Fclty) } \\
\text { Performs self assessments of facility operations to ensure that safety and } \\
\text { compliance are maintained. }\end{array}$ \\
\hline 4.1 .1 .2 & $\begin{array}{l}\text { Determine Deactivation Plans \& Negotiate Turnover Endpoint (Type } 1 \\
\text { Fclty) } \\
\text { Assesses the current state of the facility, identify and/or negotiate material, and } \\
\text { equipment disposition requirements, develope plans to deactivate facilities, and } \\
\text { negotiate and administrattvely maintain the desired facility turnover endpoint } \\
\text { specifications. Establish and maintain a long-term archive of facility information. }\end{array}$ \\
\hline 4.1.1.2.1 & $\begin{array}{l}\text { Characterize Facility Initial State (Type } 1 \text { Fclty) } \\
\text { Identifies the current state of the facility infrastructure, process systems and } \\
\text { other facility systems. facility contents. equipment. instrumentation, and utilities. }\end{array}$ \\
\hline 4.1.1.2.1.1 & $\begin{array}{l}\text { Perform Walkdowns (Type } 1 \text { Fclty) } \\
\text { Performs walkdowns to compare actual facility state with available } \\
\text { documentation. }\end{array}$ \\
\hline 4.1.1.2.1.2 & $\begin{array}{l}\text { Provide Sampling (Type } 1 \text { Fclty) } \\
\text { Obtains samples to characterize contamination and verify contamination levels, } \\
\text { and to assist with SNM/NM/NF inventory verification. }\end{array}$ \\
\hline 4.1 .1 .2 .1 .3 & $\begin{array}{l}\text { Assess Documentation Completeness (Type } 1 \text { Fclty) } \\
\text { Assesses existing documentation for completeness and verifies with walkdowns, } \\
\text { sampling, and other observations. }\end{array}$ \\
\hline 4.1 .1 .2 .1 .4 & $\begin{array}{l}\text { Define Facility Configuration (Type } 1 \text { Fclty) } \\
\text { Reviews facility drawing packages to determine accuracy and develop additional } \\
\text { configuration control documents as required to document actual configuration of } \\
\text { facility infrastructure, utilities, process systems, equipment, and instrumentation. }\end{array}$ \\
\hline 4.1.1.2.1.5 & $\begin{array}{l}\text { Perform Material/Property Inventory (Type } 1 \text { Fclty) } \\
\text { Performs inventory of all property and materials. }\end{array}$ \\
\hline
\end{tabular}




\begin{tabular}{|c|c|}
\hline Function & Definitions \\
\hline 4.1 .1 .2 .1 .6 & $\begin{array}{l}\text { Summarize \& Document Facility Initial State (Type } 1 \text { Fclty) } \\
\text { Develops and provides a documentation summary or matrix that clearly and } \\
\text { completely defines facility state. }\end{array}$ \\
\hline 4.1 .1 .2 .2 & $\begin{array}{l}\text { Develop Facility Deactivation Plans (Type } 1 \text { Fclty) } \\
\text { Develops strategies to best implement deactivation requirements, plans the } \\
\text { facility deactivation, and identifies and/or negotiates facility equipment } \\
\text { disposition requirements. }\end{array}$ \\
\hline 4.1.1.2.2.1 & $\begin{array}{l}\text { Determine Deactivation Requirements (Type } 1 \text { Fclty) } \\
\text { Determines and documents all facility specific admininstrative, safery, } \\
\text { environmental, regulatory, DOE orders, codes, standards, and other } \\
\text { requirements. }\end{array}$ \\
\hline 4.1.1.2.2.2 & $\begin{array}{l}\text { Assess Capability Needs (Type } 1 \text { Fclty) } \\
\text { Assesses personnel, material, equipment, facility, and technology needs } \\
\text { necessary to support facility deactivation activities. }\end{array}$ \\
\hline 4.1 .1 .2 .2 .3 & $\begin{array}{l}\text { Plan Deactivation (Type } 1 \text { Fclty) } \\
\text { Develops deactivation strategy. plans, and schedules. }\end{array}$ \\
\hline 4.1 .1 .2 .3 & $\begin{array}{l}\text { Negotiate \& Maintain Turnover Endpoint (Type } 1 \text { Fclty) } \\
\text { Negotiates and maintains the desired facility turnover endpoint specifications. }\end{array}$ \\
\hline 4.1 .1 .2 .3 .1 & $\begin{array}{l}\text { Negotiate Turnover Criteria (Type } 1 \text { Fclty) } \\
\text { Negotiates turnover criteria with } D \& D \text { or the transition of resources to } \\
\text { beneficial uses organization. }\end{array}$ \\
\hline 4.1.1.2.3.2 & $\begin{array}{l}\text { Update Turnover Specification (Type } 1 \text { Fclty) } \\
\text { Maintains the negotiated facility deactivation turnover specification. }\end{array}$ \\
\hline 4.1 .1 .2 .4 & $\begin{array}{l}\text { Design Turnover State (Type } 1 \text { Fclty) } \\
\text { Specifies turnover facility, equipment, and material status; develops facility } \\
\text { deactivation turnover specification; develops deactivation engineering } \\
\text { documentation and other supporting analyses. }\end{array}$ \\
\hline 4.1 .1 .2 .4 .1 & $\begin{array}{l}\text { Define Turnover State (Type } 1 \text { Fclty) } \\
\text { Defines configuration specifics, negotiates turnover facility state, and develops } \\
\text { negotiated facility deactivation turnover specification. }\end{array}$ \\
\hline 4.1 .1 .2 .4 .2 & $\begin{array}{l}\text { Develop Engineering and Supporting Analyses (Type } 1 \text { Fclty) } \\
\text { Provides engineering and supporting analyses to support engineered } \\
\text { deactivation package development. Examples include safety analyses, } \\
\text { transportation analyses, and development of NEPA documentation. }\end{array}$ \\
\hline 4.1 .1 .3 & $\begin{array}{l}\text { Stabilize \& Reconfigure Facilities For Minimum Surveillance (Type } 1 \text { Fclty) } \\
\text { Deactivates nonessential systems, system components, and physical structures, } \\
\text { and takes other actions as required to minimize environmental, public, and } \\
\text { personnel hazards. Takes these actions consistent with minimizing continuing } \\
\text { facility costs. }\end{array}$ \\
\hline
\end{tabular}




\begin{tabular}{|c|c|}
\hline Eunction & Definitions \\
\hline 4.1 .1 .3 .1 & $\begin{array}{l}\text { Modify For Minimum Cost (Type } 1 \text { Fclty) } \\
\text { Reconfigures plant systems and structure to minimize cost of maintenance and } \\
\text { operation during deactivation phase and while waiting for } D \& D \text { while retaining } \\
\text { minimum acceptable compliance with safetr and environmental requirements. }\end{array}$ \\
\hline 4.1.1.3.1.1 & $\begin{array}{l}\text { Modify Safety Systems (Type } 1 \text { Fclty) } \\
\text { Makes any appropriate modifications to safety systems such as elimination or } \\
\text { conversion (wet to dry) ef fire systems. }\end{array}$ \\
\hline 4.1.1.3.1.2 & $\begin{array}{l}\text { Modify HVAC (Type } 1 \text { Fclty) } \\
\text { Modifies HVAC for minimum acceptable heating, ventilation and radiological } \\
\text { containment requirements. }\end{array}$ \\
\hline 4.1 .1 .3 .1 .3 & $\begin{array}{l}\text { Modify Security System (Type } 1 \text { Fclty) } \\
\text { Reconfigures security systems consistent with SNM inventory and security } \\
\text { require?zents. }\end{array}$ \\
\hline 4.1 .1 .3 .1 .4 & $\begin{array}{l}\text { Modify Utilities (Type } 1 \text { Fclty) } \\
\text { Modifies or downsizes utility systems to satisfy reduced needs. }\end{array}$ \\
\hline 4.1.1.3.1.5 & $\begin{array}{l}\text { Modify Structure (Type } 1 \text { Fclty) } \\
\text { Modifies structural aspects if savings can be obtained (e.g. adding a firewall } \\
\text { rather than maintainins a fire suppression system). }\end{array}$ \\
\hline 4.1 .1 .3 .2 & $\begin{array}{l}\text { Deactivate Non-Essential Systems, Components, \& Structures (Type } 1 \text { Fclty) } \\
\text { Deactivates non-essential systems, system components, and physical structures } \\
\text { while maintainine safetv and environmental compliance. }\end{array}$ \\
\hline 4.1.1.3.2.1 & $\begin{array}{l}\text { Deactivate Utilities (Type } 1 \text { Fclty) } \\
\text { Deactivates water, sewer, electrical, HLAN, steam, telephone, power, fire system } \\
\text { as appropriate to still maintain minimum safety and environmental compliance. }\end{array}$ \\
\hline 4.1 .1 .3 .2 .2 & $\begin{array}{l}\text { Deactivate Facility Systems (Type } 1 \text { Fclty) } \\
\text { Deactivates facility systems as much as possible to minimize operating and } \\
\text { maintenance costs and stili maintain minimum safety and environmental } \\
\text { compliance. }\end{array}$ \\
\hline 4.1.1.3.2.3 & $\begin{array}{l}\text { Deactivate Process System (Type } 1 \text { Fclty) } \\
\text { Deactivates, drains, flushes, removes, etc., process systems in accordance with } \\
\text { negotiated turnover specifications. }\end{array}$ \\
\hline 4.1 .1 .3 .3 & $\begin{array}{l}\text { Disposition Resources \& Waste (Type } 1 \text { Fclty) } \\
\text { Accumulates, packages, and dispositions resources and waste for facilities } \\
\text { containine SNMINM/NE. }\end{array}$ \\
\hline 4.1 .1 .3 .3 .1 & $\begin{array}{l}\text { Prepare Resources and Waste for Disposition (Type } 1 \text { Fclty) } \\
\text { Accumulates, drains, collects, and dispositions material, equipment, } \\
\text { consumables, etc. and waste or resources for disposal or reuse. }\end{array}$ \\
\hline 4.1.1.3.3.2 & $\begin{array}{l}\text { Package Dispositioned Resources \& Waste (Type } 1 \text { Fclty) } \\
\text { Packases materials for disposal or reuse. }\end{array}$ \\
\hline 4.1 .1 .3 .3 .3 & $\begin{array}{l}\text { Transport Dispositioned Resources \& Waste (Type } 1 \text { Fclty) } \\
\text { Transports materials to disposal or reallocation sites. }\end{array}$ \\
\hline
\end{tabular}




\begin{tabular}{|c|c|}
\hline Eunction & efinitions \\
\hline 4.1 .1 .3 .3 .4 & $\begin{array}{l}\text { Prepare Resource/Waste Documentation (Type } 1 \text { Fclty) } \\
\text { Prepares any documentation required for disposal or certifcation for reuse or } \\
\text { excess. }\end{array}$ \\
\hline 4.1.1.3.3.5 & $\begin{array}{l}\text { Validate Characterization Information (Type } 1 \text { Fclty) } \\
\text { Validates waste or reusable material characterization information. }\end{array}$ \\
\hline 4.1.1.3.4 & $\begin{array}{l}\text { Transfer The Stabilized Facilities (Type } 1 \text { Fclty) } \\
\text { Maintains, and effects transfer of facility structure and surrounding area to D\&D } \\
\text { organizations for remediation or to transition organization for reuse. } \\
\text { privatization. etc. }\end{array}$ \\
\hline 4.1.1.3.4.1 & $\begin{array}{l}\text { Turnover Facilities (Type } 1 \text { Fclty) } \\
\text { After deactivation is complete, maintains and manages the facility until transfer } \\
\text { for beneficial use or } D \& D \text { is accomplished. Performs actual tran.sfer of facility. }\end{array}$ \\
\hline 4.1.1.3.4.2 & $\begin{array}{l}\text { Turnover Facility Information (Type } 1 \text { Fclty) } \\
\text { Transfers actual information on facility status and characterization to receiving } \\
\text { organization. }\end{array}$ \\
\hline 4.1.1.3.4.3 & $\begin{array}{l}\text { Prepare Turnover Documentation (Type } 1 \text { Fclty) } \\
\text { Prepares appropriate documentation on the facility status and supporting } \\
\text { information in accordance with turnover specification. }\end{array}$ \\
\hline 4.1 .1 .4 & $\begin{array}{l}\text { Disposition Currently Identified Radioactive Materials Held As A Potential } \\
\text { Product \& Special Nuclear Material (Type } 1 \text { Fclty) } \\
\text { Collects and prepares materials for temporary storage and transfer, and } \\
\text { transports materials out of the facilit. }\end{array}$ \\
\hline 4.1.1.4.1 & $\begin{array}{l}\text { Categorize Materials As Waste Or Special Nuclear Material/Potential } \\
\text { Product (Type } 1 \text { Fclty) } \\
\text { Categorizes materials as waste or Nuclear Material with potential value (e.g. } \\
\text { cesium cansules. SNM.NF.ETC.). }\end{array}$ \\
\hline 4.1.1.4.1.1 & $\begin{array}{l}\text { Verify Form, Characterization, Physical State \& Inventory } \\
\text { Verifies form. characterization, physical state, and inventory of SNM/NM/NF } \\
\text { materials in the facility. }\end{array}$ \\
\hline 4.1.1.4.1.2 & $\begin{array}{l}\text { Obtain Additional Characterization Information } \\
\text { Obtains additional characterization information to support certification or } \\
\text { SNM NM } / N F \text { disposition decision making. }\end{array}$ \\
\hline 4.1.1.4.1.3 & $\begin{array}{l}\text { Obtain Resource/Waste Decision } \\
\text { Determines if the material is a waste or a product. This may include solicitation } \\
\text { of a decision policy from DOE. }\end{array}$ \\
\hline 4.1 .1 .4 .2 & $\begin{array}{l}\text { Collect SNM,NM,NF Materials (Type } 1 \text { Fclty) } \\
\text { Accumulates materials in logical configuration to minimize facility cost and } \\
\text { maximize subsequent disposition oppurtunities. }\end{array}$ \\
\hline 4.1.1.4.2.1 & $\begin{array}{l}\text { Receive New Offsite SNM,NM,NF Materials } \\
\text { Receives incoming materials in facilities being deactivated if cost effective and it } \\
\text { is the most logical temporary storage facility. }\end{array}$ \\
\hline
\end{tabular}




\begin{tabular}{|c|c|}
\hline Eunction & Definitions \\
\hline 4.1.1.4.2.2 & $\begin{array}{l}\text { Receive Materials From Deactivation Work } \\
\text { Receives material from other facilities being deactivated if designated storage is } \\
\text { cost effective and the most legical temporan storage option. }\end{array}$ \\
\hline 4.1 .1 .4 .3 & $\begin{array}{l}\text { Prepare Materials For Transport \& Storage (Type } 1 \text { Felty) } \\
\text { Stabilizes and packages reactive materials for temporary storage for } \\
\text { transportation to an alternate companver permanent storage or for other use. }\end{array}$ \\
\hline 4.1.1.4.3.1 & $\begin{array}{l}\text { Stabilize Reactive Materials } \\
\text { Treats material as required to meet storage, shipment. and disposition criteria. }\end{array}$ \\
\hline 4.1 .1 .4 .3 .2 & $\begin{array}{l}\text { Package Materials } \\
\text { Packages material for temporary storage or shipment. }\end{array}$ \\
\hline 4.1.1.4.3.3 & $\begin{array}{l}\text { Prepare Documentation } \\
\text { Prepares all documentation for storase, shippins, or alternate use. }\end{array}$ \\
\hline 4.1.1.4.4 & $\begin{array}{l}\text { Temporarily Store SNM,NM,NF (Type } 1 \text { Fclty) } \\
\text { Stores materials until alternate, temporary or permanent storage or beneficial } \\
\text { use is identified. }\end{array}$ \\
\hline 4.1.1.4.4.1 & $\begin{array}{l}\text { Provide Storage Reports } \\
\text { Prepares ald maintains required storage reports. }\end{array}$ \\
\hline 4.1.1.4.4.2 & $\begin{array}{l}\text { Maintain Compliant Storage Facility } \\
\text { Maintains facility and equipment qualifications and operating requirements for } \\
\text { compliant storage. }\end{array}$ \\
\hline 4.1 .1 .4 .5 & $\begin{array}{l}\text { Transport Materials (Type } 1 \text { Fclty) } \\
\text { Transports materials to alternate storage or a beneficial use. }\end{array}$ \\
\hline 4.1 .2 & $\begin{array}{l}\text { Deactivate Facilities With Radioactive \& Hazardous Material (Type } 2 \text { Fclty) } \\
\text { Deactivates facilities containing radioactive or hazardous materials, but which } \\
\text { do not contain SNM/NM/NF. }\end{array}$ \\
\hline 4.1 .2 .1 & $\begin{array}{l}\text { Maintain Safety \& Compliance Envelope (Type } 2 \text { Fclty) } \\
\text { Assesses and maintains the facility structure and its operations in a safe } \\
\text { condition. Maintains a qualified facility staff, and maintain required facility and } \\
\text { oneratine documentation. }\end{array}$ \\
\hline 4.1 .2 .1 .1 & $\begin{array}{l}\text { Maintain Safe \& Compliant Operations (Type } 2 \text { Fclty) } \\
\text { Assesses and maintains the facility operations in a safe condition that is } \\
\text { compliant with applicable environmental requirments, DOE orders, and all other } \\
\text { applicable codes, standards, and company procedures. }\end{array}$ \\
\hline 4.1.2.1.1.1 & $\begin{array}{l}\text { Acquire Knowledge Of Facility Status (Type } 2 \text { Fclty) } \\
\text { Acquires knowledge of the facility status, configuration, facility operating } \\
\text { systems. and contents required to enable deactivation. }\end{array}$ \\
\hline
\end{tabular}




\begin{tabular}{|c|c|}
\hline Eunction & Definitions \\
\hline 4.1.2.1.1.2 & $\begin{array}{l}\text { Provide Surveillance (Type } 2 \text { Fclty) } \\
\text { Provides surveillance of the facility operations and operating systems, develops } \\
\text { acutely unsafe condition action plans, performs OSR surveillance procedures, } \\
\text { environmental monitoring, RCRA tracking, and surveillance of safety analysis } \\
\text { compliance. }\end{array}$ \\
\hline 4.1 .2 .1 .1 .3 & $\begin{array}{l}\text { Provide Access Control (Type } 2 \text { Fclty) } \\
\text { Provides access control to and internal to the facility for safety, security, and } \\
\text { radiological purposes. }\end{array}$ \\
\hline 4.1 .2 .1 .1 .4 & $\begin{array}{l}\text { Provide Work Control (Type } 2 \text { Fclty) } \\
\text { Provides a job control system for the facility activities. }\end{array}$ \\
\hline 4.1 .2 .1 .1 .5 & $\begin{array}{l}\text { Provide Property Management (Type } 2 \text { Fclty) } \\
\text { Provides property management for the facility in accordance with DOE orders } \\
\text { and } W H C \text { procedures. }\end{array}$ \\
\hline 4.1.2.1.1.6 & $\begin{array}{l}\text { Provide Non-Hazardous Material/Waste Management (Type } 2 \text { Fclty) } \\
\text { Contains, controls, and documents non-hazardous material and waste in } \\
\text { accordance with safe and applicable standards. }\end{array}$ \\
\hline 4.1 .2 .1 .1 .7 & $\begin{array}{l}\text { Provide Hazardous MaterialWaste Management (Type } 2 \text { Fclty) } \\
\text { Provides containment, control, and documentation of hazardous materials and } \\
\text { wastes in conformance } n \text { ith safety requirements and all applicable hazardous } \\
\text { material/wastes codes and regulations. }\end{array}$ \\
\hline 4.1 .2 .1 .1 .8 & $\begin{array}{l}\text { Provide Radioactive/Tru Material/Waste Management (Type } 2 \text { Fclty) } \\
\text { Provides containment, control, and documentation of radioactive material and } \\
\text { waste in accordance with safety requirements and all applicable codes and } \\
\text { resulations. }\end{array}$ \\
\hline 4.1.2.1.2 & $\begin{array}{l}\text { Maintain Safe \& Compliant Systems, Equipment, \& Structure (Type } 2 \\
\text { Fclty) } \\
\text { Maintains the facility systems and infrastructure in the operational condition } \\
\text { dictated by approved safety and compliance documentation (includes } \\
\text { environmental regulations). }\end{array}$ \\
\hline 4.1 .2 .1 .2 .1 & $\begin{array}{l}\text { Perform Corrective Maintenance (Type } 2 \text { Fclty) } \\
\text { All maintenance that brings systems and equipment back to their operational } \\
\text { states after failure. }\end{array}$ \\
\hline 4.1.2.1.2.2 & $\begin{array}{l}\text { Perform Preventive Maintenance (Type } 2 \text { Fclty) } \\
\text { Preventive maintenance activities to minimize all unplanned events and } \\
\text { premature equipment failures. }\end{array}$ \\
\hline 4.1 .2 .1 .2 .3 & $\begin{array}{l}\text { Perform Process Systems and Equipment Calibrations (Type } 2 \text { Fclty) } \\
\text { Performs facility equipment, instrumentation, and process system calibrations to } \\
\text { ensure accuracy. }\end{array}$ \\
\hline 4.1 .2 .1 .2 .4 & $\begin{array}{l}\text { Perform Minor Modifications (Type } 2 \text { Fclty) } \\
\text { Performs minor modifications to facility systems or structure to ensure safe and } \\
\text { compliant operations during the facility deactivation process. }\end{array}$ \\
\hline
\end{tabular}




\begin{tabular}{|c|c|}
\hline Function & Definitions \\
\hline 4.1 .2 .1 .3 & $\begin{array}{l}\text { Maintain Safety \& Compliance Documentation (SARs, OSRs, etc.) (Type } 2 \\
\text { Fclty) } \\
\text { Maintains all required facility safety, compliance, engineering, and operating } \\
\text { documentation during facility deactivation. }\end{array}$ \\
\hline 4.1.2.1.3.1 & $\begin{array}{l}\text { Maintain Safety Documents (Type } 2 \text { Fclty) } \\
\text { Maintains documentation necessary to ensure safe deactivation activities. This } \\
\text { includes FSARS. ISBS. CSERS. ETC.. }\end{array}$ \\
\hline 4.1.2.1.3.2 & $\begin{array}{l}\text { Maintain Operations Procedures (Type } 2 \text { Fclty) } \\
\text { Maintains operations procedures and documentation to support economic, } \\
\text { safety, or environmental compliance requirements. }\end{array}$ \\
\hline 4.1 .2 .1 .3 .3 & $\begin{array}{l}\text { Maintain Engineering Documentation (Type } 2 \text { Fclty) } \\
\text { Maintains configuration drawings and associated engineering documentation } \\
\text { required to operate and maintain the facility in a safe and compliant status. }\end{array}$ \\
\hline 4.1 .2 .1 .3 .4 & $\begin{array}{l}\text { Maintain Environmental Compliance Documentation (Type } 2 \text { Fclty) } \\
\text { Maintains appropriate regulatory files and other related environmental } \\
\text { documentation to assure and prove environmental compliance. }\end{array}$ \\
\hline 4.1 .2 .1 .3 .5 & $\begin{array}{l}\text { Maintain Maintenance Procedures (Type } 2 \text { Fclty) } \\
\text { Maintains maintenance procedure documentation necessary for safe, efficient, } \\
\text { and compliant operations. }\end{array}$ \\
\hline 4.1.2.1.4 & $\begin{array}{l}\text { Maintain Qualified Facility Staff (Type } 2 \text { Fclty) } \\
\text { Provides facility specific training, testing, and training records maintenance to } \\
\text { ensure facility staff remain trained, qualified, and certified (as required) } \\
\text { throughout the facility deactivation process. }\end{array}$ \\
\hline 4.1 .2 .1 .4 .1 & $\begin{array}{l}\text { Provide Training (Type } 2 \text { Fclty) } \\
\text { Provides all training related to the activities necessary to deactivate the facilities } \\
\text { and ensure they remain in a safe and compliant condition. }\end{array}$ \\
\hline 4.1 .2 .1 .4 .2 & $\begin{array}{l}\text { Maintain Qualification and Certification (Type } 2 \text { Fclty) } \\
\text { Provides periodic personnel skills check, assessment, and testing. }\end{array}$ \\
\hline 4.1 .2 .1 .4 .3 & $\begin{array}{l}\text { Maintain Training Documentation (Type } 2 \text { Fclty) } \\
\text { Maintains applicable worker training documentation. Documentation includes } \\
\text { worker safety and competancy qualification and certification. }\end{array}$ \\
\hline 4.1 .2 .1 .5 & $\begin{array}{l}\text { Assess Safety \& Compliance State (Type } 2 \text { Fclty) } \\
\text { Performs and responds to oversight assessments and performs appropriate self } \\
\text { assessments of the facility deactivation activities to evaluate the facility and } \\
\text { eperations safety and compliance status. }\end{array}$ \\
\hline 4.1 .2 .1 .5 .1 & $\begin{array}{l}\text { Support Independent Oversight Audits (Type } 2 \text { Fclty) } \\
\text { Performs and responds to independant oversight audits. }\end{array}$ \\
\hline 4.1 .2 .1 .5 .2 & $\begin{array}{l}\text { Perform Management Assessments (Type } 2 \text { Fclty) } \\
\text { Performs self assessments of facility operations to ensure that safety and } \\
\text { compliance are maintained. }\end{array}$ \\
\hline
\end{tabular}




\begin{tabular}{|c|c|}
\hline Function & Definitions \\
\hline 4.1.2.2 & $\begin{array}{l}\text { Determine Deactivation Plan \& Negotiate Turnover Endpoint (Type } 2 \text { Fclty) } \\
\text { Assesses the current state of the facility, identifies and/or negotiates material, } \\
\text { and equipment disposition requirements, develops plans to deactivate facilities, } \\
\text { and negotiates and maintains the desired facility turnover endpoint } \\
\text { specifications. Establishes and maintains a long-term archive of facility } \\
\text { information. }\end{array}$ \\
\hline 4.1.2.2.1 & $\begin{array}{l}\text { Characterize Facility Initial State (Type } 2 \text { Fclty) } \\
\text { Identifies the current state of the facility infrastructure, process systems and } \\
\text { other facility systems, facility contents, equipment, instrumentation, and utilities. }\end{array}$ \\
\hline 4.1.2.2.1.1 & $\begin{array}{l}\text { Perform Walkdowns (Type } 2 \text { Fclty) } \\
\text { Performs walkdowns to compare actual facility state with available } \\
\text { documentation. }\end{array}$ \\
\hline 4.1 .2 .2 .1 .2 & $\begin{array}{l}\text { Provide Sampling (Type } 2 \text { Fclty) } \\
\text { Obtains samples to characterize radioactive and hazardous contamination and } \\
\text { verify contamination levels. }\end{array}$ \\
\hline 4.1 .2 .2 .1 .3 & $\begin{array}{l}\text { Assess Documentation Completeness (Type } 2 \text { Fclty) } \\
\text { Assesses existing documentation for completeness and verifies with walkdowns, } \\
\text { sampling, and other observations. }\end{array}$ \\
\hline 4.1 .2 .2 .1 .4 & $\begin{array}{l}\text { Define Facility Configuration (Type } 2 \text { Fclty) } \\
\text { Reviews facility drawing packages to determine accuracy and develop additional } \\
\text { configuration control documents as required to document actual configuration of } \\
\text { facility infrastructure, utilities, process systems, equipment, and instrumentation. }\end{array}$ \\
\hline 4.1 .2 .2 .1 .5 & $\begin{array}{l}\text { Perform Material/Property Inventory (Type } 2 \text { Fclty) } \\
\text { Performs inventory of all property and materials. }\end{array}$ \\
\hline 4.1 .2 .2 .1 .6 & $\begin{array}{l}\text { Summarize \& Document Facility Initial State (Type } 2 \text { Fclty) } \\
\text { Develops and provides a documentation summary or matrix that clearly and } \\
\text { completely defines facility state. }\end{array}$ \\
\hline 4.1.2.2.2 & $\begin{array}{l}\text { Develop Facility Deactivation Plans (Type } 2 \text { Fclty) } \\
\text { Develops strategies to best implement deactivation requirements, plans the } \\
\text { facility deactivation, and identifies and/or negotiates facility equipment } \\
\text { disposition requirements. }\end{array}$ \\
\hline 4.1.2.2.2.1 & $\begin{array}{l}\text { Determine Deactivation Requirements (Type } 2 \text { Fclty) } \\
\text { Determines and documents all facility specific admininstrative, safety, } \\
\text { environmental, regulatory, DOE orders, codes, standards, and other } \\
\text { requirements. }\end{array}$ \\
\hline 4.1.2.2.2.2 & $\begin{array}{l}\text { Assess Capability Needs (Type } 2 \text { Fclty) } \\
\text { Assesses personnel, material, equipment, facility, and technology needs } \\
\text { necessary to support facility deactivation activities. }\end{array}$ \\
\hline 4.1.2.2.2.3 & $\begin{array}{l}\text { Plan Deactivation (Type } 2 \text { Fclty) } \\
\text { Develops deactivation strategy, plans, and schedules. }\end{array}$ \\
\hline
\end{tabular}




\begin{tabular}{|c|c|}
\hline Function & Definitions \\
\hline 4.1 .2 .2 .3 & $\begin{array}{l}\text { Negotiate \& Maintain Turnover Endpoint (Type } 2 \text { Fclty) } \\
\text { Negotiates and maintains the desired facility turnover endpoint specifications. }\end{array}$ \\
\hline 4.1 .2 .2 .3 .1 & $\begin{array}{l}\text { Negotiate Turnover Criteria (Type } 2 \text { Fclty) } \\
\text { Negotiates turnover criteria with D\&D or the transition of resources to } \\
\text { beneficial uses organization. }\end{array}$ \\
\hline 4.1.2.2.3.2 & $\begin{array}{l}\text { Update Turnover Specification (Type } 2 \text { Fclty) } \\
\text { Maintains the negotiated facility deactivation turnover specification. }\end{array}$ \\
\hline 4.1.2.2.4 & $\begin{array}{l}\text { Design Turnover State (Type } 2 \text { Fclty) } \\
\text { Specifies turnover facility, equipment, and material status; develops facility } \\
\text { deactivation turnover specification; develops deactivation engineering } \\
\text { documentation and other supporting analyses. }\end{array}$ \\
\hline 4.1.2.2.4.1 & $\begin{array}{l}\text { Define Turnover State (Type } 2 \text { Fclty) } \\
\text { Defines configuration specifics, negotiates turnover facility state, and develops } \\
\text { negotiated facility deactivation turnover specification. }\end{array}$ \\
\hline 4.1.2.2.4.2 & $\begin{array}{l}\text { Develop Engineering and Supporting Analyses (Type } 2 \text { Fclty) } \\
\text { Provides engineering and supporting analyses to support engineered } \\
\text { deactivation package development. Examples include safety analyses, } \\
\text { transportation analyses, and development of NEPA documentation. }\end{array}$ \\
\hline 4.1 .2 .3 & $\begin{array}{l}\text { Stabilize \& Reconfigure Facilities For Minimum Surveillance (Type } 2 \text { Fclty) } \\
\text { Deactivates nonessential systems, system components, and physical structures, } \\
\text { and takes other actions as required to minimize environmental, public, and } \\
\text { personnel hazards. Takes these actions consistent with minimizing continuing } \\
\text { facility costs. }\end{array}$ \\
\hline 4.1.2.3.1 & $\begin{array}{l}\text { Modify For Minimum Cost (Type } 2 \text { Fclty) } \\
\text { Reconfigures plant systems and structure to minimize cost of maintenance and } \\
\text { cperation during deactivation phase and while waiting for } D \& D \text { while retaining } \\
\text { minimum acceptable compliance with safetv and environmental requirements. }\end{array}$ \\
\hline 4.1 .2 .3 .1 .1 & $\begin{array}{l}\text { Modify Safety System (Type } 2 \text { Fclty) } \\
\text { Makes any appropriate modifications to safety systems such as elimination or } \\
\text { conversion (wet to dry) of fire systems. }\end{array}$ \\
\hline $4 \cdot 1.2 .3 .1 .2$ & $\begin{array}{l}\text { Modify HVAC (Type } 2 \text { Fclty) } \\
\text { Modifies HVAC for minimum acceptable heating, ventilation and radiological } \\
\text { containment requirements. }\end{array}$ \\
\hline 4.1.2.3.1.3 & $\begin{array}{l}\text { Modify Security System (Type } 2 \text { Fclty) } \\
\text { Reconfigures security systems. }\end{array}$ \\
\hline 4.1.2.3.1.4 & $\begin{array}{l}\text { Modify Utilities (Type } 2 \text { Fclty) } \\
\text { Modifies or downsizes utility systems to satisfy reduced needs. }\end{array}$ \\
\hline 4.1 .2 .3 .1 .5 & $\begin{array}{l}\text { Modify Structure (Type } 2 \text { Fclty) } \\
\text { Modifies structural aspects if savings can be obtained (e.g. adding a firewall } \\
\text { rather than maintaining a fire suppression system). }\end{array}$ \\
\hline
\end{tabular}




\begin{tabular}{|c|c|}
\hline Function & finitions \\
\hline 4.1.2.3.2 & $\begin{array}{l}\text { Deactivate Non-Essential Systems, Components, \& Structures (Type } 2 \text { Fclty) } \\
\text { Deactivates non-essential systems, system components, and physical structures } \\
\text { while maintaining safety and environmental compliance. }\end{array}$ \\
\hline 4.1.2.3.2.1 & $\begin{array}{l}\text { Deactivate Utilities (TyF= } 2 \text { Fclty) } \\
\text { Deactivates water, sewer, electrical, HLAN, steam, telephone, power, fire system } \\
\text { as appropriate to still maintain minimum safety and environmental compliance. }\end{array}$ \\
\hline 4.1.2.3.2.2 & $\begin{array}{l}\text { Deactivate Facility Systems (Type } 2 \text { Fclty) } \\
\text { Deactivates facility systems as much as possible to minimize operating and } \\
\text { maintenance costs and still maintain minimum safety and environmental } \\
\text { compliance. }\end{array}$ \\
\hline 4.1.2.3.2.3 & $\begin{array}{l}\text { Deactivate Process System (Type } 2 \text { Fclty) } \\
\text { Deactivates, drains, flushes, removes, etc., process systems in accordance with } \\
\text { negotiated turnover specifications. }\end{array}$ \\
\hline 4.1.2.3.3 & $\begin{array}{l}\text { Disposition Resources \& Waste (Type } 2 \text { Fclty) } \\
\text { Accumulates, packages, and dispositions resources and waste. }\end{array}$ \\
\hline 4.1.2.3.3.1 & $\begin{array}{l}\text { Accumulate, Remove, and Disposition Resources and Waste (Type } 2 \text { Fclty) } \\
\text { Accumulates, drains, collects, and dispositions material, equipment, } \\
\text { consumables, etc., and waste or resources for disposal or reuse. }\end{array}$ \\
\hline 4.1.2.3.3.2 & $\begin{array}{l}\text { Package Dispositioned Resources \& Waste (Type } 2 \text { Fclty) } \\
\text { Packages materials for disposal or reuse. }\end{array}$ \\
\hline 4.1.2.3.3.3 & $\begin{array}{l}\text { Transport Dispositioned Resources \& Waste (Type } 2 \text { Fclty) } \\
\text { Transports materials to disposal or reallocation sites. }\end{array}$ \\
\hline 4.1.2.3.3.4 & $\begin{array}{l}\text { Prepare Resource/Waste Documentation (Type } 2 \text { Fclty) } \\
\text { Prepares documentation required for disposal or certifcation for reuse or exc }\end{array}$ \\
\hline 4.1.2.3.3.5 & $\begin{array}{l}\text { Validate Characterization Information (Type } 2 \text { Fclty) } \\
\text { Validates waste or reusable material characterization information. }\end{array}$ \\
\hline 4.1.2.3.4 & $\begin{array}{l}\text { Transfer The Stabilized Facilities (Type } 2 \text { Fclty) } \\
\text { Maintains and effects transfer of facility structure and surrounding area to D\&D } \\
\text { organizations for remediation or to transition organization for reuse, } \\
\text { privatization. etc. }\end{array}$ \\
\hline 4.1 .2 .3 .4 .1 & $\begin{array}{l}\text { Turnover Facilities (Type } 2 \text { Fclty) } \\
\text { After deactivation is complete, maintains and manages the facility until transfer } \\
\text { for beneficial use or } D \& D \text { is accomplished. Performs actual transfer of facility. }\end{array}$ \\
\hline 4.1.2.3.4.2 & $\begin{array}{l}\text { Turnover Facility Information (Type } 2 \text { Fclty) } \\
\text { Transfers actual information on facility status and characterization to receiving } \\
\text { organization. }\end{array}$ \\
\hline 4.1.2.3.4.3 & $\begin{array}{l}\text { Prepare Turnover Documentation (Type } 2 \text { Fclty) } \\
\text { Prepares appropriate documentation on the facility status and supporting } \\
\text { information in accordance with turnover specification. }\end{array}$ \\
\hline
\end{tabular}




\begin{tabular}{|c|c|}
\hline Function & Definitions \\
\hline 4.1 .3 & $\begin{array}{l}\text { Deactivate Facilities With Only Hazardous Material, Including Asbestos } \\
\text { (Type } 3 \text { Fclty) } \\
\text { Deactivates facilities containing hazardous materials, including asbestos. }\end{array}$ \\
\hline 4.1.3.1 & $\begin{array}{l}\text { Maintain Safety \& Compliance Envelope (Type } 3 \text { Fclty) } \\
\text { Assessess and maintain the facility structure and its operations in a safe } \\
\text { condition. Maintains a qualified facility staff, and maintains required facility and } \\
\text { operating documentation. }\end{array}$ \\
\hline 4.1.3.1.1 & $\begin{array}{l}\text { Maintain Safe \& Compliant Operations (Type } 3 \text { Fclty) } \\
\text { Acquires knowledge of the facility status, configuration, facility operating } \\
\text { systems, } \\
\text { and contents required to enable deactivation. }\end{array}$ \\
\hline 4.1.3.1.1.1 & $\begin{array}{l}\text { Acquire Knowledge Of Facility Status (Type } 3 \text { Fclty) } \\
\text { Acquires knowledge of the facility status, configuration, facility operating } \\
\text { systems, } \\
\text { and contents required to enable deactivation. }\end{array}$ \\
\hline 4.1.3.1.1.2 & $\begin{array}{l}\text { Provide Surveillance (Type } 3 \text { Fclty) } \\
\text { Provides surveillance of the facility operations and operating systems, develops } \\
\text { acutely unsafe condition action plans, performs OSR surveillance procedures, } \\
\text { environmental monitoring, RCRA tracking, and surveillance of safety analysis } \\
\text { compliance. }\end{array}$ \\
\hline 4.1.3.1.1.3 & $\begin{array}{l}\text { Provide Access Control (Type } 3 \text { Fclty) } \\
\text { Provides access control to and internal to the facility for safety and security } \\
\text { purposes. }\end{array}$ \\
\hline 4.1.3.1.1.4 & $\begin{array}{l}\text { Provide Work Control (Type } 3 \text { Fclty) } \\
\text { Provides a job control system for the facility activities. }\end{array}$ \\
\hline 4.1.3.1.1.5 & $\begin{array}{l}\text { Provide Property Management (Type } 3 \text { Fclty) } \\
\text { Provides property management for the facility in accordance with DOE orders } \\
\text { and WHC procedures. }\end{array}$ \\
\hline 4.1.3.1.1.6 & $\begin{array}{l}\text { Provide Non-Hazardous Material/Waste Management (Type } 3 \text { Fclty) } \\
\text { Contains, controls, and documents non-hazardous material and waste in } \\
\text { accordance with safe and applicable standards. }\end{array}$ \\
\hline 4.1 .3 .1 .1 .7 & $\begin{array}{l}\text { Provide Hazardous MaterialWaste Management (Type } 3 \text { Fclty) } \\
\text { Contains, controls, and documents hazardous materials and wastes in } \\
\text { conformance with safety requirements and all applicable hazardous } \\
\text { materialswastes codes and regulations. }\end{array}$ \\
\hline 4.1 .3 .1 .2 & $\begin{array}{l}\text { Maintain Safe \& Compliant Systems, Equipment, \& Structure (Type } 3 \\
\text { Fclty) } \\
\text { Maintains the facility systems and infrastructure in the operational } \\
\text { condition dictated by approved safety and compliance documentation (includes } \\
\text { environmental regulations). }\end{array}$ \\
\hline
\end{tabular}




\begin{tabular}{|c|c|}
\hline Function & Definitions \\
\hline 4.1 .3 .1 .2 .1 & $\begin{array}{l}\text { Perform Corrective Maintenance (Type } 3 \text { Fclty) } \\
\text { All maintenance that brings systems and equipment back to their operational } \\
\text { states after failure. }\end{array}$ \\
\hline 4.1.3.1.2.2 & $\begin{array}{l}\text { Perform Preventive Maintenance (Type } 3 \text { Fclty) } \\
\text { Preventive maintenance activities to minimize all unplanned events and } \\
\text { premature equipment failures. }\end{array}$ \\
\hline 4.1 .3 .1 .2 .3 & $\begin{array}{l}\text { Perform Process Systems and Equipment Calibrations (Type } 3 \text { Fclty) } \\
\text { Performs facility equipment, instrumentation, and process system calibrations to } \\
\text { ensure accuracy. }\end{array}$ \\
\hline 4.1.3.1.2.4 & $\begin{array}{l}\text { Perform Minor Modifications (Type } 3 \text { Fclty) } \\
\text { Performs minor modifications to facility systems or structure to ensure safe and } \\
\text { compliant operations during the facility deactivation process. }\end{array}$ \\
\hline 4.1 .3 .1 .3 & $\begin{array}{l}\text { Maintain Safety \& Compliance Documentation (SARs, etc.) (Type } 3 \text { Fclty) } \\
\text { Maintains all required facility safety, compliance, engineering, and } \\
\text { operating documentation during facility deactivation. }\end{array}$ \\
\hline 4.1.3.1.3.1 & $\begin{array}{l}\text { Maintain Safety Documents (Type } 3 \text { Fclty) } \\
\text { Maintains documentation necessary to ensure safe deactivation activities. }\end{array}$ \\
\hline 4.1.3.1.3.2 & $\begin{array}{l}\text { Maintain Operations Procedures (Type } 3 \text { Fclty) } \\
\text { Maintains operations procedures and documentation to support economic, } \\
\text { safety, or environmental compliance requirements. }\end{array}$ \\
\hline 4.1 .3 .1 .3 .3 & $\begin{array}{l}\text { Maintain Engineering Docurnentation (Type } 3 \text { Fclty) } \\
\text { Maintains configuration drawings and associated engineering documentation } \\
\text { required to operate and maintain the facility in a safe and compliant status. }\end{array}$ \\
\hline 4.1 .3 .1 .3 .4 & $\begin{array}{l}\text { Maintain Maintenance Procedures (Type } 3 \text { Fclty) } \\
\text { Maintains maintenance procedure documentation necessary for safe, } \\
\text { efficient, and compliant operations. }\end{array}$ \\
\hline 4.1.3.1.3.5 & $\begin{array}{l}\text { Maintain Environmental Compliance Documentation (Type } 3 \text { Fclty) } \\
\text { Maintains appropriate regulatory files and other related environmental } \\
\text { documentation to assure and prove environmental compliance. }\end{array}$ \\
\hline 4.1.3.1.4 & $\begin{array}{l}\text { Maintain Qualified Facility Staff (Type } 3 \text { Fclty) } \\
\text { Provides facility specific training, testing, and training records maintenance to } \\
\text { ensure facility staff remain trained, qualified, and certified (as required) } \\
\text { throughout the facility deactivation process. }\end{array}$ \\
\hline 4.1 .3 .1 .4 .1 & $\begin{array}{l}\text { Provide Training (Type } 3 \text { Fcity) } \\
\text { Provides all training related to the activities necessary to deactivate the } \\
\text { facilities and ensure they remain in a safe and compliant condition. }\end{array}$ \\
\hline 4.1 .3 .1 .4 .2 & $\begin{array}{l}\text { Maintain Qualification and Certification (Type } 3 \text { Fclty) } \\
\text { Provides periodic personnel skills check, assessment, and testing. }\end{array}$ \\
\hline 4.1 .3 .1 .4 .3 & $\begin{array}{l}\text { Maintain Training Documentation (Type } 3 \text { Fclty) } \\
\text { Maintains applicable worker training documentation. Documentation includes } \\
\text { worker safety and competancy qualification and certification. }\end{array}$ \\
\hline
\end{tabular}




\begin{tabular}{|c|c|}
\hline Function & Definitions \\
\hline 4.1 .3 .1 .5 & $\begin{array}{l}\text { Assess Safety \& Compliance State (Type } 3 \text { Fclty) } \\
\text { Performs and responds to oversight assessments and perform appropriate self } \\
\text { assessments of the facility deactivation activities to evaluate the facility and } \\
\text { operations safety and compliance status. }\end{array}$ \\
\hline 4.1.3.1.5.1 & $\begin{array}{l}\text { Support Independent Oversight Audits (Type } 3 \text { Fclty) } \\
\text { Performs and responds to independant oversight audits. }\end{array}$ \\
\hline 4.1.3.1.5.2 & $\begin{array}{l}\text { Perform Management Assessments (Type } 3 \text { Fclty) } \\
\text { Performs self assessments of facility operations to ensure that safety and } \\
\text { compliance are maintained. }\end{array}$ \\
\hline 4.1.3.2 & $\begin{array}{l}\text { Determine Deactivation Plan \& Negotiate Turnover Endpoint (Type } 3 \text { Fclty) } \\
\text { Assesses the current state of the facility; identifies and/or negotiates material, } \\
\text { and } \\
\text { equipment disposition requirements; develops plans to deactivate facilities; and } \\
\text { negotiates and maintains the desired facility turnover endpoint specifications. } \\
\text { Establishes and maintain a long-term archive of facility information. }\end{array}$ \\
\hline 4.1.3.2.1 & $\begin{array}{l}\text { Characterize Facility Initial State (Type } 3 \text { Fclty) } \\
\text { Identifies the current state of the facility infrastructure, process systems and } \\
\text { other } \\
\text { facility systems, facility contents, equipment, instrumentation, and utilities. }\end{array}$ \\
\hline 4.1.3.2.1.1 & $\begin{array}{l}\text { Perform Walkdowns (Type } 3 \text { Fclty) } \\
\text { Performs walkdowns to compare actual facility state with available } \\
\text { documentation. }\end{array}$ \\
\hline 4.1.3.2.1.2 & $\begin{array}{l}\text { Provide Sampling (Type } 3 \text { Fclty) } \\
\text { Obtains samples to characterize hazardous contamination and verifies } \\
\text { contamination levels. }\end{array}$ \\
\hline 4.1.3.2.1.3 & $\begin{array}{l}\text { Assess Documentation Completeness (Type } 3 \text { Fclty) } \\
\text { Assesses existing documentation for completeness and verifies with walkdowns, } \\
\text { sampling, and other observations. }\end{array}$ \\
\hline 4.1.3.2.1.4 & $\begin{array}{l}\text { Define Facility Configuration (Type } 3 \text { Fclty) } \\
\text { Reviews facility drawing packages to determine accuracy and develops } \\
\text { additional } \\
\text { configuration control documents as required to document actual configuration of } \\
\text { facility infrastructure, utilities, process systems, equipment, and instrumentation. }\end{array}$ \\
\hline 4.1.3.2.1.5 & $\begin{array}{l}\text { Perform Material/Property Inventory (Type } 3 \text { Fclty) } \\
\text { Performs inventory of all property and materials. }\end{array}$ \\
\hline 4.1.3.2.1.6 & $\begin{array}{l}\text { Summarize \& Document Facility Initial State (Type } 3 \text { Fclty) } \\
\text { Develops and provides a documentation summary or matrix that clearly and } \\
\text { completely defines facility state. }\end{array}$ \\
\hline
\end{tabular}




\begin{tabular}{|c|c|}
\hline Function & Definitions \\
\hline 4.1.3.2.2 & $\begin{array}{l}\text { Develop Facility Deactivation Plan (Type } 3 \text { Fclty) } \\
\text { Develops strategies to best implement deactivation requirements, plans the } \\
\text { facility } \\
\text { deactivation, and identifies and/or negotiates facility equipment disposition } \\
\text { requirements. }\end{array}$ \\
\hline 4.1.3.2.2.1 & $\begin{array}{l}\text { Determine Deactivation Requirements (Type } 3 \text { Fcity) } \\
\text { Determines and documents all facility specific admininstrative, safert, } \\
\text { environmental, } \\
\text { regulatory, DOE orders, codes, standards, and other requirements. }\end{array}$ \\
\hline 4.1.3.2.2.2 & $\begin{array}{l}\text { Assess Capability Needs (Type } 3 \text { Fclty) } \\
\text { Assesses personnel, material, equipment, facility, and technology needs } \\
\text { necessary to support facility deactivation activities. }\end{array}$ \\
\hline 4.1.3.2.2.3 & $\begin{array}{l}\text { Plan Deactivation (Type } 3 \text { Fclty) } \\
\text { Develops deactivation strategy, plans, and schedules. }\end{array}$ \\
\hline 4.1.3.2.3 & $\begin{array}{l}\text { Negotiate \& Maintain Turnover Endpoint (Type } 3 \text { Fclty) } \\
\text { Negotiates and maintains the desired facility turnover endpoint specifications. }\end{array}$ \\
\hline 4.1.3.2.3.1 & $\begin{array}{l}\text { Negotiate Turnover Criteria (Type } 3 \text { Fclty) } \\
\text { Negotiates turnover criteria with } D \& D \text { or the transition of resources to } \\
\text { beneficial uses organization. }\end{array}$ \\
\hline 4.1 .3 .2 .3 .2 & $\begin{array}{l}\text { Update Turnover Specification (Type } 3 \text { Fclty) } \\
\text { Maintains the negotiated facility deactivation turnover specification. }\end{array}$ \\
\hline 4.1.3.2.4 & $\begin{array}{l}\text { Design Turnover State (Type } 3 \text { Fclty) } \\
\text { Specifies turnover facility, equipment, and material status; develops facility } \\
\text { deactivation turnover specification; develops deactivation engineering } \\
\text { documentation and other supporting analyses. }\end{array}$ \\
\hline 4.1 .3 .2 .4 .1 & $\begin{array}{l}\text { Define Turnover State (Type } 3 \text { Fclty) } \\
\text { Defines configuration specifics, negotiates turnover facility state, and develops } \\
\text { negotiated facility deactivation turnover specification. }\end{array}$ \\
\hline 4.1 .3 .2 .4 .2 & $\begin{array}{l}\text { Develop Engineering and Supporting Analyses (Type } 3 \text { Fclty) } \\
\text { Provides engineering and supporting analyses to support engineered } \\
\text { deactivation package development. Examples include safety analyses, } \\
\text { transportation analyses, and development of NEPA documentation. }\end{array}$ \\
\hline 4.1 .3 .3 & $\begin{array}{l}\text { Stabilize \& Reconfigure Facilities For Minimum Surveillance (Type } 3 \text { Fclty) } \\
\text { Deactivates nonessential systems, system components, and physical structures, } \\
\text { and takes other actions as required to minimize environmental, public, and } \\
\text { personnel hazards. Takes these actions consistent with minimizing continuing } \\
\text { facility costs. }\end{array}$ \\
\hline 4.1 .3 .3 .1 & $\begin{array}{l}\text { Modify For Minimum Cost (Type } 3 \text { Fclty) } \\
\text { Reconfigures plant systems and structure to minimize cost of maintenance and } \\
\text { operation during deactivation phase and while waiting for } D \& D \text { while retaining } \\
\text { minimum acceptable compliance with safety and environmental requirements. }\end{array}$ \\
\hline
\end{tabular}




\begin{tabular}{|c|c|}
\hline Function & Definitions \\
\hline 4.1 .3 .3 .1 .1 & $\begin{array}{l}\text { Modify Safety System (Type } 3 \text { Fclty) } \\
\text { Makes any appropriate modifications to safety systems such as elimination or } \\
\text { conversion (wet to dry) of fire systems. }\end{array}$ \\
\hline 4.1 .3 .3 .1 .2 & $\begin{array}{l}\text { Modify HVAC (Type } 3 \text { Fclty) } \\
\text { Modifies HVAC for minimum acceptable heating, ventilation requirements. }\end{array}$ \\
\hline 4.1.3.3.1.3 & $\begin{array}{l}\text { Modify Security System (Type 3 Fclty) } \\
\text { Reconfigures security systems. }\end{array}$ \\
\hline 4.1 .3 .3 .1 .4 & $\begin{array}{l}\text { Modify Utilities (Type } 3 \text { Fclty) } \\
\text { Modifies or downsizes utility systems to satisfy reduced needs. }\end{array}$ \\
\hline 4.1 .3 .3 .1 .5 & $\begin{array}{l}\text { Modify Structure (Type } 3 \text { Fclty) } \\
\text { Modifies structural aspects if savings can be obtained (e.g. adding a firewall } \\
\text { rather than maintaining a fire suppression system). }\end{array}$ \\
\hline 4.1.3.3.2 & $\begin{array}{l}\text { Deactivate Non-Essential Systems, Components, \& Structures (Type } 3 \text { Fclty) } \\
\text { Deactivates non-essential systems, system components, and physical structures } \\
\text { while maintaining safety and environmental compliance. }\end{array}$ \\
\hline 4.1.3.3.2.1 & $\begin{array}{l}\text { Deactivate Utilities (Type } 3 \text { Fclty) } \\
\text { Deactivates water, sewer, electrical, HLAN, steam, telephone, power, fire system } \\
\text { as appropriate to still maintain minimum safety and environmental compliance. }\end{array}$ \\
\hline 4.1 .3 .3 .2 .2 & $\begin{array}{l}\text { Deactivate Facility Systems (Type } 3 \text { Fclty) } \\
\text { Deactivates facility systems as much as possible to minimize operating and } \\
\text { maintenance costs and still maintain minimum safery and environmental } \\
\text { compliance. }\end{array}$ \\
\hline 4.1.3.3.2.3 & $\begin{array}{l}\text { Deactivate Process System (Type } 3 \text { Fclty) } \\
\text { Deactivates, drains, flushs, removes, etc., process systems in accordance with } \\
\text { negotiated turnover specifications. }\end{array}$ \\
\hline 4.1 .3 .3 .3 & $\begin{array}{l}\text { Disposition Resources \& Waste (Type } 3 \text { Fclty) } \\
\text { Accumulates, packages, and dispositions resources and waste. }\end{array}$ \\
\hline 4.1 .3 .3 .3 .1 & $\begin{array}{l}\text { Accumulate, Remove, and Disposition Resources and Waste (Type } 3 \text { Fclty) } \\
\text { Accumulates, drains, collects, and dispositions material, equipment, } \\
\text { consumables, etc. and waste or resources for disposal or reuse. }\end{array}$ \\
\hline 4.1.3.3.3.2 & $\begin{array}{l}\text { Package Dispositioned Resources \& Waste (Type } 3 \text { Fclty) } \\
\text { Packages materials for disposal or reuse. }\end{array}$ \\
\hline 4.1 .3 .3 .3 .3 & $\begin{array}{l}\text { Transport Dispositioned Resources \& Waste (Type } 3 \text { Fclty) } \\
\text { Transports materials to disposal or reallocation sites. }\end{array}$ \\
\hline 4.1 .3 .3 .3 .4 & $\begin{array}{l}\text { Prepare Resource/Waste Documentation (Type } 3 \text { Fclty) } \\
\text { Prepares documentation required for disposal or certifcation for reuse or excess. }\end{array}$ \\
\hline 4.1.3.3.3.5 & $\begin{array}{l}\text { Validate Characterization Information (Type } 3 \text { Fclty) } \\
\text { Validates waste or reusable material characterization information. }\end{array}$ \\
\hline
\end{tabular}




\begin{tabular}{|c|c|}
\hline Eunction & Definitions \\
\hline 4.1.3.3.4 & $\begin{array}{l}\text { Transfer The Stabilized Facilities (Type } 3 \text { Fclty) } \\
\text { Maintains and effects transfer of facility structure and surrounding area to } \\
D \& D \text { organizations for remediation or to transition organization for reuse, } \\
\text { privatization, etc. }\end{array}$ \\
\hline 4.1.3.3.4.1 & $\begin{array}{l}\text { Turnover Facilities (Type } 3 \text { Fclty) } \\
\text { After deactivation is complete, maintains and manages the facility until } \\
\text { transfer for beneficial use or D\&D is accomplished. Performs actual transfer of } \\
\text { facility. }\end{array}$ \\
\hline 4.1.3.3.4.2 & $\begin{array}{l}\text { Turnover Facility Information (Type } 3 \text { Fclty) } \\
\text { Transfers actual information on facility status and characterization to receiving } \\
\text { organization. }\end{array}$ \\
\hline 4.1.3.3.4.3 & $\begin{array}{l}\text { Prepare Turnover Documentation (Type } \mathbf{3} \text { Fclty) } \\
\text { Prepares appropriate documentation on the facility status and supporting } \\
\text { information in accordance with turnover specification. }\end{array}$ \\
\hline 4.1.4 & $\begin{array}{l}\text { Deactivate Facilities Without Radioactive Or Hazardous Material (Type } 4 \\
\text { Fclty) } \\
\text { Deactivates facilities without radioactive or hazardous materials. Examples } \\
\text { include office buildings, etc. }\end{array}$ \\
\hline 4.1.4.1 & $\begin{array}{l}\text { Maintain Safety \& Compliance Envelope (Type } 4 \text { Fclty) } \\
\text { Assesses and maintains the facility structure and its operations in a safe } \\
\text { condition. Maintains a qualified facility staff, and maintains required facility and } \\
\text { operating documentation. }\end{array}$ \\
\hline 4.1.4.1.1 & $\begin{array}{l}\text { Maintain Safe \& Compliant Operations (Type } 4 \text { Fclty) } \\
\text { Acquires knowledge of the facility status, configuration, facility operating } \\
\text { systems, } \\
\text { and contents required to enable deactivation. }\end{array}$ \\
\hline 4.1.4.1.1.1 & $\begin{array}{l}\text { Acquire Knowledge Of Facility Status (Type } 4 \text { Fclty) } \\
\text { Acquires knowledge of the facility status, configuration, facility operating } \\
\text { systems, } \\
\text { and contents required to enable deactivation. }\end{array}$ \\
\hline 4.1.4.1.1.2 & $\begin{array}{l}\text { Provide Surveillance (Type } 4 \text { Fclty) } \\
\text { Provides surveillance of the facility operations and operating systems, develops } \\
\text { acutely unsafe condition action plans, environmental monitoring, and } \\
\text { surveillance of safetv analvsis compliance. }\end{array}$ \\
\hline 4.1 .4 .1 .1 .3 & $\begin{array}{l}\text { Provide Access Control (Type } 4 \text { Fclty) } \\
\text { Provides access control to and internal to the facility for safety and security } \\
\text { purposes. }\end{array}$ \\
\hline 4.1 .4 .1 .1 .4 & $\begin{array}{l}\text { Provide Work Control (Type } 4 \text { Fclty) } \\
\text { Provides a job control system for the facility activities. }\end{array}$ \\
\hline
\end{tabular}




\begin{tabular}{|c|c|}
\hline Function & Definitions \\
\hline 4.1.4.1.1.5 & $\begin{array}{l}\text { Provide Property Management (Type } 4 \text { Fclty) } \\
\text { Provides property management for the facility in accordance with DOE orders } \\
\text { and WHC procedures. }\end{array}$ \\
\hline 4.1 .4 .1 .1 .6 & $\begin{array}{l}\text { Provide Non-Hazardous Material/Waste Management (Type } 4 \text { Fclty) } \\
\text { Contains, controls, and documents non-hazardous material and waste in } \\
\text { accordance with safe and applicable standards. }\end{array}$ \\
\hline 4.1.4.1.2 & $\begin{array}{l}\text { Maintain Safe \& Compliant Systems, Equipment, \& Structure (Type } 4 \\
\text { Fclty) } \\
\text { Maintains the facility systems and infrastructure in the operational } \\
\text { condition dictated by approved safety and compliance documentation (includes } \\
\text { environmental resulations). }\end{array}$ \\
\hline 4.1 .4 .1 .2 .1 & $\begin{array}{l}\text { Perform Corrective Maintenance (Type } 4 \text { Fclty) } \\
\text { All maintenance that brings systems and equipment back to their operational } \\
\text { states after failure. }\end{array}$ \\
\hline 4.1.4.1.2.2 & $\begin{array}{l}\text { Perform Preventive Maintenance (Type } 4 \text { Fclty) } \\
\text { Preventive maintenance activities to minimize all unplanned events and } \\
\text { premature equinment failures. }\end{array}$ \\
\hline 4.1 .4 .1 .2 .3 & $\begin{array}{l}\text { Perform Process Systems and Equipment Calibration (Type } 4 \text { Fclty) } \\
\text { Performs facility equipment, instrumentation, and process system calibrations to } \\
\text { ensure accuracy. }\end{array}$ \\
\hline 4.1.4.1.2.4 & $\begin{array}{l}\text { Perform Minor Modifications (Type } 4 \text { Fcity) } \\
\text { Performs minor modifications to facility systems or structure to ensure safe and } \\
\text { compliant operations during the facility deactivation process. }\end{array}$ \\
\hline 4.1.4.1.3 & $\begin{array}{l}\text { Maintain Safety \& Compliance Documentation (OSHA,NFPA) (Type } 4 \\
\text { Fclty) } \\
\text { Maintains all required facility safery, compliance, engineering, and operating } \\
\text { documentation during facility deactivation. }\end{array}$ \\
\hline 4.1 .4 .1 .3 .1 & $\begin{array}{l}\text { Maintain Safety Documents (Type } 4 \text { Fclty) } \\
\text { Maintains documentation necessary to ensure safe deactivation activities. }\end{array}$ \\
\hline 4.1 .4 .1 .3 .2 & $\begin{array}{l}\text { Maintain Engineering Documentation (Type } 4 \text { Fclty) } \\
\text { Maintains configuration drawings and associated engineering documentation } \\
\text { required to operate and maintain the facility in a safe and compliant status. }\end{array}$ \\
\hline 4.1 .4 .1 .3 .3 & $\begin{array}{l}\text { Maintain Operations Procedures (Type } 4 \text { Fclty) } \\
\text { Maintains operations procedures and documentation to support economic. } \\
\text { safetw. or environmental compliance requirements. }\end{array}$ \\
\hline 4.1 .4 .1 .3 .4 & $\begin{array}{l}\text { Maintain Maintenance Procedures (Type } 4 \text { Fclty) } \\
\text { Maintains maintenance procedure documentation necessary for safe, } \\
\text { efficient. and compliant operations. }\end{array}$ \\
\hline
\end{tabular}




\begin{tabular}{|c|c|}
\hline Eunction & Definitions \\
\hline 4.1.4.1.4 & $\begin{array}{l}\text { Maintain Qualified Facillty Staff (Type } 4 \text { Fclty) } \\
\text { Provides facility specific training, testing, and training records } \\
\text { maintenance to ensure facility staff remain trained, qualified, and certified (as } \\
\text { required) } \\
\text { throughout the facility deactivation process. }\end{array}$ \\
\hline 4.1.4.1.4.1 & $\begin{array}{l}\text { Provide Training (Type } 4 \text { Fclty) } \\
\text { Provides all training related to the activities necessary to deactivate the } \\
\text { facilities and ensure they remain in a safe and compliant condition. }\end{array}$ \\
\hline 4.1 .4 .1 .4 .2 & $\begin{array}{l}\text { Maintain Qualification and Certification(Type } 4 \text { Fclty) } \\
\text { Provides periodic personnel skills check, assessment. and testing. }\end{array}$ \\
\hline 4.1.4.1.4.3 & $\begin{array}{l}\text { Maintain Training Documentation (Type } 4 \text { Fclty) } \\
\text { Maintains applicable worker training documentation. Documentation includes } \\
\text { worker safety and competancy qualification and certification. }\end{array}$ \\
\hline 4.1 .4 .1 .5 & $\begin{array}{l}\text { Assess Safety \& Compliance State (Type } 4 \text { Fclty) } \\
\text { Performs and responds to oversight assessments and perform appropriate self } \\
\text { assessments of the facility deactivation activities to evaluate the facility and } \\
\text { eperations safetr and compliance status. }\end{array}$ \\
\hline 4.1.4.1.5.1 & $\begin{array}{l}\text { Support Independent Oversight Audits (Type } 4 \text { Folty) } \\
\text { Performs and reinonds to independant oversight audits. }\end{array}$ \\
\hline 4.1.4.1.5.2 & $\begin{array}{l}\text { Perform Management Assessments (Type } 4 \text { Fclty) } \\
\text { Performs self assessments of facility operations to ensure that safety and } \\
\text { compliance are maintained. }\end{array}$ \\
\hline 4.1.4.2 & $\begin{array}{l}\text { Determine Deactivation Plan \& Negotiate Turnover Endpoint (Type } 4 \text { Fclty) } \\
\text { Assesses the current state of the facility, identifies and/or negotiates material, } \\
\text { and } \\
\text { equipment disposition requirements, develops plans to deactivate facilities, and } \\
\text { negotiates and maintains the desired facility turnover endpoint } \\
\text { specifications. Establishes and maintain a long-term archive of facility } \\
\text { information. }\end{array}$ \\
\hline 4.1.4.2.1 & $\begin{array}{l}\text { Characterize Facility Initial State (Type } 4 \text { Fclty) } \\
\text { Identifies the current state of the facility infrastructure, process systems and } \\
\text { other } \\
\text { facility sustems, facility contents, equipment, instrumentation, and utilities. }\end{array}$ \\
\hline 4.1.4.2.1.1 & $\begin{array}{l}\text { Perform Walkdowns (Type } 4 \text { Fclty) } \\
\text { Performs walkdowns to compare actual facility state with available } \\
\text { documentation. }\end{array}$ \\
\hline 4.1 .4 .2 .1 .2 & $\begin{array}{l}\text { Provide Sampling (Type } 4 \text { Fclty) } \\
\text { Obtains samples to characterize and verify contamination levels. }\end{array}$ \\
\hline 4.1.4.2.1.3 & $\begin{array}{l}\text { Assess Documentation Completeness (Type } 4 \text { Fclty) } \\
\text { Assesses existing documentation for completeness and verifies with walkdowns, } \\
\text { sampling, and other observations. }\end{array}$ \\
\hline
\end{tabular}




\begin{tabular}{|c|c|}
\hline Eunction & Definitions \\
\hline 4.1 .4 .2 .1 .4 & $\begin{array}{l}\text { Define Facility Configuration (Type } 4 \text { Fclty) } \\
\text { Reviews facility drawing packages to determine accuracy and develop additional } \\
\text { configuration control documents as required to document actual configuration of } \\
\text { facilitin infrastructure. utilities. process systems, equipment. and instrumentation. }\end{array}$ \\
\hline 4.1 .4 .2 .1 .5 & $\begin{array}{l}\text { Perform Material/Property Inventory (Type } 4 \text { Fclty) } \\
\text { Performs inventory of all property and materials. }\end{array}$ \\
\hline 4.1.4.2.1.6 & $\begin{array}{l}\text { Summarize \& Document Facility Initial State (Type } 4 \text { Fclty) } \\
\text { Develops and provides a documentation summary or matrix that clearly and } \\
\text { completely defines facility state. }\end{array}$ \\
\hline 4.1 .4 .2 .2 & $\begin{array}{l}\text { Develope Facility Deactivation Plan (Type } 4 \text { Fclty) } \\
\text { Develops strategies to best implement deactivation requirements, plans the } \\
\text { facility deactivation, and identifies and/or negotiates facility equipment } \\
\text { disposition requirements. }\end{array}$ \\
\hline 4.1.4.2.2.1 & $\begin{array}{l}\text { Determine Deactivation Requirements (Type } 4 \text { Fclty) } \\
\text { Determines and documents all facility specific admininstrative, safety, } \\
\text { environmental, regulatory, DOE orders, codes, standards, and other } \\
\text { requirements. }\end{array}$ \\
\hline 4.1.4.2.2.2 & $\begin{array}{l}\text { Assess Capability Needs (Type } 4 \text { Fclty) } \\
\text { Assesses personnel, material, equipment, facility, and technology needs } \\
\text { necessary to support facility deactivation activities. }\end{array}$ \\
\hline 4.1.4.2.2.3 & $\begin{array}{l}\text { Plan Deactivation (Type } 4 \text { Fclty) } \\
\text { Develops deactivation strategy. plans, and schedules. }\end{array}$ \\
\hline 4.1.4.2.3 & $\begin{array}{l}\text { Negotiate \& Maintain Turnover Endpoint (Type } 4 \text { Fclty) } \\
\text { Negotiates and maintains the desired facility turnover endpoint specifications. }\end{array}$ \\
\hline 4.1 .4 .2 .3 .1 & $\begin{array}{l}\text { Negotiate Turnover Criteria (Type } 4 \text { Fclty) } \\
\text { Negotiates turnover criteria with D\&D or the transition of resources to } \\
\text { beneficial } \\
\text { uses organization. }\end{array}$ \\
\hline 4.1 .4 .2 .3 .2 & $\begin{array}{l}\text { Develop Turnover Requirements (Type } 4 \text { Fclty) } \\
\text { Identifies facilitu specific turnover requirements. }\end{array}$ \\
\hline 4.1.4.2.3.3 & $\begin{array}{l}\text { Negotiate Turnover Requirements (Type } 4 \text { Fclty) } \\
\text { Negotiates turnover criteria with D\&D or the transition of resources to } \\
\text { beneficial } \\
\text { uses orsanization. }\end{array}$ \\
\hline 4.1.4.2.3.4 & $\begin{array}{l}\text { Document Turnover Requirements (Type } 4 \text { Fclty) } \\
\text { Prepares turnover specification. }\end{array}$ \\
\hline 4.1 .4 .2 .3 .5 & $\begin{array}{l}\text { Update Turnover Specification (Type } 4 \text { Fclty) } \\
\text { Maintains the negotiated facility deactivation turnover specification. }\end{array}$ \\
\hline
\end{tabular}




\begin{tabular}{|c|c|}
\hline Eunction & Definitions \\
\hline 4.1.4.2.4 & $\begin{array}{l}\text { Design Turnover State (Type } 4 \text { Fclty) } \\
\text { Specifies turnover facility, equipment, and material status; develops facility } \\
\text { deactivation turnover specification; develops deactivation engineering } \\
\text { decumentation and other supportine analyses. }\end{array}$ \\
\hline 4.1.4.2.4.1 & $\begin{array}{l}\text { Define Turnover State (Type } 4 \text { Fclty) } \\
\text { Defines configuration specifics, negotiates turnover facility state, and develops } \\
\text { nefotiated facility deactivation turnover specification. }\end{array}$ \\
\hline 4.1 .4 .2 .4 .2 & $\begin{array}{l}\text { Develop Engineering and Supporting Analyses (Type } 4 \text { Fclty) } \\
\text { Provides engineering and supporting analyses to support engineered } \\
\text { deactivation package development. Examples include safety analyses, } \\
\text { transportation analuses, and develonment of NEPA decumentation. }\end{array}$ \\
\hline 4.1.4.3 & $\begin{array}{l}\text { Stabilize \& Reconfigure Facilities For Minimum Surveillance (Type } 4 \text { Fclty) } \\
\text { Deactivates nonessential systems, system components, and physical structures, } \\
\text { and takes other actions as required to minimize environmental, public, and } \\
\text { personnel hazards. Takes these actions consistent with minimizing continuing } \\
\text { facilioy costs. }\end{array}$ \\
\hline 4.1.4.3.1 & $\begin{array}{l}\text { Modify For Minimum Cost (Type } 4 \text { Fclty) } \\
\text { Reconfigures plant systems and structure to minimize cost of maintenance and } \\
\text { operation during deactivation phase and while waiting for } D \& D \text { while retaining } \\
\text { minimum accentable compliance with safetrand environmental requirements. }\end{array}$ \\
\hline 4.1 .4 .3 .1 .1 & $\begin{array}{l}\text { Modify Safety System (Type } 4 \text { Fclty) } \\
\text { Makes any appropriate modifications to safety systems such as elimination or } \\
\text { conversion (wet to dry) of fire systems. }\end{array}$ \\
\hline 4.1.4.3.1.2 & $\begin{array}{l}\text { Modify HVAC (Type } 4 \text { Fclty) } \\
\text { Modifies HVAC for minimum acceptable heating, ventilation requirements. }\end{array}$ \\
\hline 4.1.4.3.1.3 & $\begin{array}{l}\text { Modify Security System (Type 4 Fclty) } \\
\text { Reconfigures securify systems. }\end{array}$ \\
\hline 4.1 .4 .3 .1 .4 & $\begin{array}{l}\text { Modify Utilities (Type } 4 \text { Fclty) } \\
\text { Modifies or downsizes utility systems to satisfy reduced needs. }\end{array}$ \\
\hline 4.1 .4 .3 .1 .5 & $\begin{array}{l}\text { Modify Structure (Type } 4 \text { Fclty) } \\
\text { Modifies structural aspects if savings can be obtained (e.g. adding a firewall } \\
\text { rather than maintainine a fire suppression system). }\end{array}$ \\
\hline 4.1.4.3.2 & $\begin{array}{l}\text { Deactivate Non-Essential Systems, Components, \& Structures (Type } 4 \text { Fclty) } \\
\text { Deactivates non-essential systems, system components, and physical structures } \\
\text { while maintainine safety and environmental compliance. }\end{array}$ \\
\hline 4.1 .4 .3 .2 .1 & $\begin{array}{l}\text { Deactivate Utilities (Type } 4 \text { Fclty) } \\
\text { Deactivates water, sewer, electrical, HLAN, steam, telephone, power, fire system } \\
\text { as appropriate to still maintain minimum safen and environmental compliance. }\end{array}$ \\
\hline
\end{tabular}




\begin{tabular}{|c|c|}
\hline Function & Definitions \\
\hline 4.1.4.3.2.2 & $\begin{array}{l}\text { Deactivate Facility Systems (Type } 4 \text { Fclty) } \\
\text { Deactivates facility systems as much as possible to minimize operating and } \\
\text { maintenance costs and still maintain minimum safety and environmental } \\
\text { compliance. }\end{array}$ \\
\hline 4.1.4.3.3 & $\begin{array}{l}\text { Prepare Resources and Waste for Disposition (Type } 4 \text { Fclty) } \\
\text { Accumulules. Dackages, and dispositions resources and waste. }\end{array}$ \\
\hline 4.1.4.3.3.1 & $\begin{array}{l}\text { Accumulate, Remove, and Disposition Resources \& Waste (Type } 4 \text { Fclty) } \\
\text { Accumulates, drains, collects, and dispositions material, equipment. } \\
\text { consumables, etc., and waste or resources for disposal or reuse. }\end{array}$ \\
\hline 4.1 .4 .3 .3 .2 & $\begin{array}{l}\text { Package Dispositioned Resources \& Waste (Type } 4 \text { Fclty) } \\
\text { Packages materials for disposal or reuse. }\end{array}$ \\
\hline 4.1.4.3.3.3 & $\begin{array}{l}\text { Transport Dispositioned Resources \& Waste (Type } 4 \text { Fclty) } \\
\text { Transports materials to disposal or reallocation sites. }\end{array}$ \\
\hline 4.1.4.3.3.4 & $\begin{array}{l}\text { Prepare Resource/Waste Documentation (Type } 4 \text { Fclty) } \\
\text { Prepares documentation required for disposal or certifcation for reuse or excess. }\end{array}$ \\
\hline 4.1.4.3.4 & $\begin{array}{l}\text { Transfer The Stabilized Facilities (Type } 4 \text { Fclty) } \\
\text { Maintains and effects transfer of facility structure and surrounding area to } \\
D \& D \text { organizations for remediation or to transition organization for reuse. } \\
\text { privatization, etc. }\end{array}$ \\
\hline 4.1.4.3.4.1 & $\begin{array}{l}\text { Turnover Facilities (Type } 4 \text { Fclty) } \\
\text { After deactivation is complete, maintains and manages the facility until } \\
\text { transfer for beneficial use or } D \& D \text { is accomplished. Performs actual transfer of } \\
\text { facility. }\end{array}$ \\
\hline 4.1.4.3.4.2 & $\begin{array}{l}\text { Turnover Facility Information (Type } 4 \text { Fclty) } \\
\text { Transfers actual information on facility status and characterization to receiving } \\
\text { organization. }\end{array}$ \\
\hline 4.1 .4 .3 .4 .3 & $\begin{array}{l}\text { Prepare Turnover Documentation (Type } 4 \text { Fclty) } \\
\text { Prepares appropriate documentation on the facility status and supporting } \\
\text { information } \\
\text { in accordance with turnover specification. }\end{array}$ \\
\hline 4.2 & $\begin{array}{l}\text { Remediate Tank Waste } \\
\text { The REMEDY TANK WASTE (4.2) function is directed to removal, treatment, } \\
\text { and disposition of highly radioactive wastes located in tanks around the Hanford } \\
\text { Site. Emphasis is on the } 177 \text { waste storage tanks located on the } 200 \text { Area } \\
\text { plateau. This function also contains newly generated liquid/slurry wastes from } \\
\text { operating facilities and other Hanford Site sources as well as all High Level } \\
\text { waste in tanks other than the } 177 \text { waste tanks. }\end{array}$ \\
\hline 4.3 & $\begin{array}{l}\text { Remedy Solid Waste } \\
\text { The REMEDY SOLID WASTE (4.3) function is charged with disposition of } \\
\text { buried wastes and solid waste materials generated during past missions as well } \\
\text { as solid wastes generated during the cleanup mission. }\end{array}$ \\
\hline
\end{tabular}




\begin{tabular}{|c|c|}
\hline Eunction & Definitions \\
\hline 4.3 .1 & $\begin{array}{l}\text { Maintain Solid Waste Safety And Compliance Envelope } \\
\text { Continually assess and maintain the waste form and the facility structures and } \\
\text { operations in a safe and compliant condition. Includes maintaining a qualified } \\
\text { facility staff and maintaining required safert/compliance documentation. }\end{array}$ \\
\hline 4.3.1.1 & $\begin{array}{l}\text { Maintain Safe And Compliant Solid Waste Operational Environment } \\
\text { Continually assess and maintain the solid waste operational environment in a } \\
\text { safe and compliant condition, with respect to applicable environmental } \\
\text { requirements, DOE orders, and all other applicable codes, standards, and } \\
\text { company procedures. }\end{array}$ \\
\hline 4.3.1.1.1 & $\begin{array}{l}\text { Acquire Knowledge Of Solid Waste Status } \\
\text { Acquire knowledge of the solid waste status, including solid waste facility status, } \\
\text { configuration, operating, systems, and inventories. }\end{array}$ \\
\hline 4.3 .1 .1 .2 & $\begin{array}{l}\text { Provide Surveillance of Solid Waste Facility Operations } \\
\text { Provide surveillance of the solid waste facility operations and operating systems. } \\
\text { develop acutely unsafe condition action plans, perform OSR surveillance } \\
\text { procedures, environmental monitoring, RCRA tracking, and surveillance of } \\
\text { safety analysis compliance. }\end{array}$ \\
\hline 4.3.1.1.3 & $\begin{array}{l}\text { Provide Access Control to Solid Waste Facilities } \\
\text { Provide access control to and internal to the solid waste facility for safeguards } \\
\text { and security safetw and radiological purposes. }\end{array}$ \\
\hline 4.3.1.1.4 & $\begin{array}{l}\text { Provide Work Control for Solid Waste Facilities } \\
\text { Provide work control for the solid waste facilitu activities. }\end{array}$ \\
\hline 4.3.1.1.5 & $\begin{array}{l}\text { Provide Property Management for Solid Waste Facilities } \\
\text { Provide propertu management for the solid waste facilities. }\end{array}$ \\
\hline 4.3 .1 .1 .6 & $\begin{array}{l}\text { Provide Non-Hazardous Solid Waste Material Management } \\
\text { Contain, control, and document non-hazardous solid waste material in } \\
\text { accordance with safe and applicable standards. }\end{array}$ \\
\hline 4.3.1.1.7 & $\begin{array}{l}\text { Provide Hazardous Solid Waste Material Management } \\
\text { Contain, control, and document hazardous solid waste material in accordance } \\
\text { with safe and applicable codes and regulations. }\end{array}$ \\
\hline 4.3.1.1.8 & $\begin{array}{l}\text { Provide Radioactive/TRU Solid Waste Material Management } \\
\text { Contain, control, and document radioactive solid waste material in accordance } \\
\text { with safe and applicable codes and regulations. }\end{array}$ \\
\hline 4.3.1.2 & $\begin{array}{l}\text { Maintain Safe And Compliant Solid Waste Systems, Equipment And } \\
\text { Structures } \\
\text { Continually assess and maintain the solid waste facility systems, equipment, and } \\
\text { structures, and their operations in a safe condition. Maintain a qualified solid } \\
\text { waste staff, and maintain required solid waste facility and operating } \\
\text { documentation. }\end{array}$ \\
\hline
\end{tabular}




\begin{tabular}{|c|c|}
\hline Function & Definitions \\
\hline 4.3.1.2.1 & $\begin{array}{l}\text { Perform Routine Maintenance for Solid Waste Systems, Equipment, and } \\
\text { Structures } \\
\text { Maintenance that includes all normal scheduled, routine, and emergent } \\
\text { corrective maintenance that is not covered in preventative maintenance or other } \\
\text { calibration procedures. This function performs solid waste facility systems and } \\
\text { infrastructure maintenance. }\end{array}$ \\
\hline 4.3.1.2.2 & $\begin{array}{l}\text { Perform Preventative Maintenance on Solid Waste Systems, Equipment, } \\
\text { and Structures } \\
\text { Includes maintenance above and beyond all routine maintenance to prevent } \\
\text { premature equipment failures. }\end{array}$ \\
\hline 4.3.1.2.3 & $\begin{array}{l}\text { Perform Solid Waste Equipment Calibrations } \\
\text { Perform solid waste process equipment/instrument calibrations. }\end{array}$ \\
\hline 4.3.1.2.4 & $\begin{array}{l}\text { Perform Minor Modifications to Solid Waste Facility Systems } \\
\text { Perform minor modifications to solid waste facility systems, equipment, and } \\
\text { structures necessary to ensure safe and compliant operations. }\end{array}$ \\
\hline 4.3.1.3 & $\begin{array}{l}\text { Maintain Safe And Compliant Solid Waste Documentation (SARs, OSRs, } \\
\text { etc.) } \\
\text { Maintain required solid waste facility and operating documentation related to } \\
\text { safe and compliant operations. }\end{array}$ \\
\hline 4.3.1.3.1 & $\begin{array}{l}\text { Maintain Solid Waste Facility Operations Safety Documents } \\
\text { Maintain solid waste facility operations safety documents necessary to ensure } \\
\text { safe and compliant operations. This documentation includes FSARs, ISBs, } \\
\text { CSERs, etc. }\end{array}$ \\
\hline 4.3.1.3.2 & $\begin{array}{l}\text { Maintain Solid Waste Facility Operations Procedures } \\
\text { Continually maintain solid waste facility operations procedures and } \\
\text { documentation to support economic, safety, or environmental compliance } \\
\text { requirements. }\end{array}$ \\
\hline 4.3.1.3.3 & $\begin{array}{l}\text { Maintain Solid Waste Facility Configuration Drawings } \\
\text { Continually maintain configuration drawings and associated engineering } \\
\text { documentation required to operate and maintain the solid waste facility in a safe } \\
\text { and compliant status. }\end{array}$ \\
\hline 4.3.1.3.4 & $\begin{array}{l}\text { Maintain Maintenance Procedures for Solid Waste Systems, Equipment, } \\
\text { and Structure } \\
\text { Continually maintain maintenance procedures documentation necessary for safe, } \\
\text { efficient, and compliant operation of solid waste systems, equipment, and } \\
\text { structures. }\end{array}$ \\
\hline 4.3.1.3.5 & $\begin{array}{l}\text { Maintain Solid Waste Environmental Compliance Documentation } \\
\text { Continually maintain appropriate regulatory files and other related } \\
\text { documentation to assure and prove environmental compliance of the solid waste } \\
\text { operations. }\end{array}$ \\
\hline
\end{tabular}




\begin{tabular}{|c|c|}
\hline Function & Definitions \\
\hline 4.3.1.3.6 & $\begin{array}{l}\text { Maintain Solid Waste Inventory Documentation } \\
\text { Maintain solid waste inventory documentation in compliance with DOE orders. }\end{array}$ \\
\hline 4.3.1.4 & $\begin{array}{l}\text { Maintain Qualified Solid Waste Staff } \\
\text { Provide solid waste facility specific training, testing, and training records } \\
\text { maintenance to ensure solid waste fucility staff remain trained, qualified, and } \\
\text { certified throughout the solid waste facility operation. }\end{array}$ \\
\hline 4.3 .1 .4 .1 & $\begin{array}{l}\text { Provide Training of Solid Waste Facility Staff } \\
\text { Provide all staff training related to the solid waste facility activities to ensure the } \\
\text { facilities remain in a safe and compliant condition. }\end{array}$ \\
\hline 4.3 .1 .4 .2 & $\begin{array}{l}\text { Maintain Qualification of Solid Waste Facility Staff } \\
\text { Provides periodic solid waste facility personnel skills check, assessment and } \\
\text { testing required to maintain necessary qualifications and certifications. }\end{array}$ \\
\hline 4.3.1.4.3 & $\begin{array}{l}\text { Maintain Solid Waste Staff Training Documentation } \\
\text { Continuous maintenance of applicable solid waste worker training } \\
\text { documentation. Documentation includes worker safety and competency } \\
\text { certification. }\end{array}$ \\
\hline 4.3.1.5 & $\begin{array}{l}\text { Assess Solid Waste Safety And Compliance State } \\
\text { Perform/respond to oversight assessments and perform appropriate self } \\
\text { assessments of the solid waste facility activities to evaluate the facility and } \\
\text { operations safety and compliance status. }\end{array}$ \\
\hline 4.3 .1 .5 .1 & $\begin{array}{l}\text { Support Independent Oversight Solid Waste Audits } \\
\text { Perform and respond to independent oversight audits of the solid waste to ensure } \\
\text { safe and compliant state. }\end{array}$ \\
\hline 4.3.1.5.2 & $\begin{array}{l}\text { Perform Solid Waste Management Assessments } \\
\text { Perform self assessments of solid waste facility operations to ensure that safety } \\
\text { and compliance are maintained. }\end{array}$ \\
\hline 4.3 .2 & $\begin{array}{l}\text { Receive Solid Waste } \\
\text { Retrieve legacy solid waste and receive solid waste generated during cleanup } \\
\text { operations and solid waste from other missions. }\end{array}$ \\
\hline 4.3.2.1 & $\begin{array}{l}\text { Retrieve Existing Solid Waste } \\
\text { Retrieve legacy solid waste; includes retrieval planning and solid waste access } \\
\text { and removal, obtains waste characterization samples, generates waste } \\
\text { information, establishes retrieval capability requirements, and forecasts future } \\
\text { retrieval operations. }\end{array}$ \\
\hline 4.3.2.1.1 & $\begin{array}{l}\text { Plan Solid Waste Retrieval } \\
\text { Receive solid waste and legacy solid waste characterization information and } \\
\text { information on non-compliant conditions of solid waste. Based on input } \\
\text { information, forecast future retrieval operations, evaluate solid waste status, } \\
\text { identify needed capacity requirements and schedule retrieval operations. }\end{array}$ \\
\hline
\end{tabular}




\begin{tabular}{|c|c|}
\hline Function & Definitions \\
\hline 4.3 .2 .1 .1 .1 & $\begin{array}{l}\text { Locate Solid Waste } \\
\text { Based upon available information, verify solid waste burial locations for } \\
\text { retrieval operations and identify non-complaint conditions. }\end{array}$ \\
\hline 4.3.2.1.1.2 & $\begin{array}{l}\text { Evaluate Solid Waste } \\
\text { Review solid waste characterization information and compare with actual solid } \\
\text { waste labeling for accuracy and completeness. Includes determination of } \\
\text { container condition and recognition of non-compliant conditions. }\end{array}$ \\
\hline 4.3.2.1.1.3 & $\begin{array}{l}\text { Identify Capability Requirements For Retrieving Solid Waste } \\
\text { Based upon solid waste characterization information and evaluation of solid } \\
\text { waste, specify needed capability for solid waste retrieval operations. }\end{array}$ \\
\hline 4.3.2.1.1.4 & $\begin{array}{l}\text { Schedule Solid Waste Retrieval Operations } \\
\text { Develop solid waste retrieval operation schedules based upon resource } \\
\text { capabilities, and program requirements. }\end{array}$ \\
\hline 4.3.2.1.2 & $\begin{array}{l}\text { Remove Solid Waste } \\
\text { Operations in which solid waste is accessed and removed from storage locations, } \\
\text { sorted. packaged, documented and transported for disposition. }\end{array}$ \\
\hline 4.3.2.1.2.1 & $\begin{array}{l}\text { Access And Remove Solid Waste } \\
\text { Physical access to solid waste storage locations and removal of solid waste. }\end{array}$ \\
\hline 4.3.2.1.2.2 & $\begin{array}{l}\text { Utilize Solid Waste Packaging And Handling Capabilities } \\
\text { Following sorting of solid waste, re-package (if necessary), and prepare for } \\
\text { disposition. }\end{array}$ \\
\hline 4.3.2.1.2.3 & $\begin{array}{l}\text { Document Solid Waste Removal Information } \\
\text { This is information related to solid waste removal that is needed for handling and } \\
\text { storage thereof. }\end{array}$ \\
\hline 4.3.2.1.2.4 & $\begin{array}{l}\text { Transport Removed Solid Waste To Disposition } \\
\text { Transport of retrieved solid waste for treatment, storage, and disposal. }\end{array}$ \\
\hline 4.3.2.2 & $\begin{array}{l}\text { Receive New Solid Waste } \\
\text { Receives solid waste generated during Hanford Cleanup mission operations and } \\
\text { from other missions; includes solid waste receipt planning, characterization } \\
\text { information verification for acceptance, establishes receipt capability } \\
\text { requirements, and forecasts future receipt operations. }\end{array}$ \\
\hline 4.3.2.2.1 & $\begin{array}{l}\text { Plan Solid Waste Receipt } \\
\text { Establish schedules for receipt of solid waste based upon solid waste } \\
\text { characterization information, generator requests, and information related to } \\
\text { receipt capability. }\end{array}$ \\
\hline 4.3.2.2.1.1 & $\begin{array}{l}\text { Forecast Future Solid Waste Receipts } \\
\text { Evaluate solid waste storage and characterization information and planned solid } \\
\text { waste removal operations and project solid waste receipt schedules. }\end{array}$ \\
\hline 4.3 .2 .2 .1 .2 & $\begin{array}{l}\text { Evaluate Solid Waste Generators Request } \\
\text { Review solid waste generator requests for completeness and accuracy prior to } \\
\text { providing authorization for receipt of solid waste. }\end{array}$ \\
\hline
\end{tabular}




\begin{tabular}{|c|c|}
\hline Function & Definitions \\
\hline 4.3 .2 .2 .1 .3 & $\begin{array}{l}\text { Identify Capability Requirements For Receiving Solid Waste } \\
\text { Compare solid waste removal information and transport plans to establish } \\
\text { personnel. equipment and facility requirements for planned solid waste receipt. }\end{array}$ \\
\hline 4.3.2.2.2 & $\begin{array}{l}\text { Accept Solid Waste } \\
\text { Authorize receipt of solid waste from Hanford cleanup and other missions by } \\
\text { verifying characterization information for correctness, off-loading and transport } \\
\text { capability and maintaining solid waste information. }\end{array}$ \\
\hline 4.3.2.2.2.1 & $\begin{array}{l}\text { Verify Solid Waste } \\
\text { Review solid waste characterization information and generator requests to } \\
\text { ensure waste materials comply with accepted solid waste descriptions. }\end{array}$ \\
\hline 4.3.2.2.2.2 & $\begin{array}{l}\text { Reject/Correct Solid Waste } \\
\text { Based upon evaluation of generator requests and current or proposed solid waste } \\
\text { packaging, correct generator requests as necessary or deny authorization for } \\
\text { receipt of solid waste. }\end{array}$ \\
\hline 4.3.2.2.2.3 & $\begin{array}{l}\text { Utilize Solid Waste Handling Capability } \\
\text { Remove solid waste from transport vehicle and store in appropriate facilities. }\end{array}$ \\
\hline 4.3.2.2.2.4 & $\begin{array}{l}\text { Document Received Solid Waste } \\
\text { Maintain solid waste characterization information records during temporary } \\
\text { storage and generate information required for transport to disposition. }\end{array}$ \\
\hline 4.3.2.2.2.5 & $\begin{array}{l}\text { Transport Accepted Solid Waste To Disposition } \\
\text { Shipment of received solid waste for treatment. storage, and disposal. }\end{array}$ \\
\hline 4.3 .3 & $\begin{array}{l}\text { Characterize Solid Waste } \\
\text { Identify, inventory, and characterize the waste by reviewing the process } \\
\text { operations (current and historical), by reviewing new waste information and by } \\
\text { sampling and analyzing the waste inventory. Assess the current condition of the } \\
\text { waste. Archive the results of these investigations. }\end{array}$ \\
\hline 4.3 .3 .1 & $\begin{array}{l}\text { Acquire Solid Waste Process Knowledge } \\
\text { Review the solid waste processing records, process documentation, and } \\
\text { submitted documentation to acquire knowledge about the solid waste generation } \\
\text { process. Conduct interviews with solid waste generators as needed to obtain } \\
\text { such knowledge and perform a safety review of the solid waste. Provide this } \\
\text { process knowledge to enable the solid waste characterization assessment. }\end{array}$ \\
\hline 4.3.3.1.1 & $\begin{array}{l}\text { Review Solid Waste Processing Records } \\
\text { Review the solid waste processing records to acquire knowledge of the process } \\
\text { that generated the solid waste to support its characterization and disposition. }\end{array}$ \\
\hline 4.3.3.1.2 & $\begin{array}{l}\text { Conduct Solid Waste Generator Interviews } \\
\text { Conduct interviews as needed with the solid waste generators to acquire } \\
\text { knowledge of the process that generated the solid waste to support its } \\
\text { characterization and disposition. }\end{array}$ \\
\hline
\end{tabular}




\begin{tabular}{|c|c|}
\hline Function & Definitions \\
\hline 4.3 .3 .1 .3 & $\begin{array}{l}\text { Review Solid Waste Process Documentation } \\
\text { Review the solid waste process documentation to acquire knowledge of the } \\
\text { process that generated the solid waste to support its characterization and } \\
\text { disposition. }\end{array}$ \\
\hline 4.3.3.1.4 & $\begin{array}{l}\text { Review Submitted Solid Waste Documentation } \\
\text { Review the solid waste generator requests to acquire knowledge of the process } \\
\text { that generated the solid waste to support its characterization and disposition. }\end{array}$ \\
\hline 4.3.3.1.5 & $\begin{array}{l}\text { Perform Solid Waste Safety Review } \\
\text { Perform a safety review of the solid waste process knowledge documentation to } \\
\text { identify any unsafe conditions associated with the solid waste to ensure that } \\
\text { safety requirements are met and that further solid waste characterization can } \\
\text { continue. }\end{array}$ \\
\hline 4.3.3.2 & $\begin{array}{l}\text { Analyze Solid Waste Inventory } \\
\text { Receive the solid waste samples and perform analysis thereof to obtain required } \\
\text { characterization data. Provide this analysis information to enable the solid } \\
\text { waste characterization assessment. Transfer the solid waste samples and } \\
\text { accompanying characterization data to solid waste archives. Send any excess } \\
\text { solid waste samples on to the Receive Solid Waste function to facilitate } \\
\text { disposition. }\end{array}$ \\
\hline 4.3.3.2.1 & $\begin{array}{l}\text { Receive Solid Waste Samples } \\
\text { Receive the solid waste samples that require characterization analysis. }\end{array}$ \\
\hline 4.3.3.2.2 & $\begin{array}{l}\text { Control Solid Waste Samples } \\
\text { Provide control of the solid waste samples throughout the characterization } \\
\text { analysis process. }\end{array}$ \\
\hline 4.3.3.2.3 & $\begin{array}{l}\text { Analyze Solid Waste Samples } \\
\text { Analyze the solid waste samples to provide characterization data to support its } \\
\text { disposition. }\end{array}$ \\
\hline 4.3.3.2.4 & $\begin{array}{l}\text { Report Solid Waste Sample Characterization Information } \\
\text { Report the solid waste sample characterization analysis information to enable } \\
\text { assessment against its disposition requirements. }\end{array}$ \\
\hline 4.3.3.2.5 & $\begin{array}{l}\text { Transfer Solid Waste Samples } \\
\text { Transfer the solid waste samples and accompanying characterization data to } \\
\text { solid waste archives. Send any excess solid waste samples on to the Receive } \\
\text { Solid Waste function to facilitate disposition. }\end{array}$ \\
\hline 4.3 .3 .3 & $\begin{array}{l}\text { Archive Solid Waste Samples/Characterization Data } \\
\text { Provide archival storage and retrieval operations for the analyzed solid waste } \\
\text { samples and the accompanving characterization data. }\end{array}$ \\
\hline 4.3.3.3.1 & $\begin{array}{l}\text { Archive Solid Waste Data } \\
\text { Provide archival storage of the solid waste sample characterization data. }\end{array}$ \\
\hline 4.3.3.3.2 & $\begin{array}{l}\text { Archive Solid Waste Samples } \\
\text { Provide archival storage of the solid waste samples. }\end{array}$ \\
\hline
\end{tabular}




\begin{tabular}{|c|c|}
\hline Function & Definitions \\
\hline 4.3 .3 .3 .3 & $\begin{array}{l}\text { Provide Solid Waste Sample and Data Retrievability } \\
\text { Provide archival retrieval operations for the analyzed solid waste samples and } \\
\text { the accompanving characterization data. }\end{array}$ \\
\hline 4.3.3.4 & $\begin{array}{l}\text { Assess Solid Waste Characterization Information } \\
\text { Evaluate the solid waste characterization information, consisting of generator } \\
\text { request information, process knowledge information, and waste sample } \\
\text { characterization data, against the solid waste disposition requirements and } \\
\text { provide validation of meeting the disposition requirements. Determine any } \\
\text { additional solid waste process knowledge needs to support disposition. }\end{array}$ \\
\hline 4.3.3.4.1 & $\begin{array}{l}\text { Evaluate Solid Waste Characterization Data Against Requirements } \\
\text { Evaluate the solid waste characterization information, consisting of generator } \\
\text { request information, process knowledge information, and waste sample } \\
\text { characterization data, against the solid waste disposition requirements. }\end{array}$ \\
\hline 4.3 .3 .4 .2 & $\begin{array}{l}\text { Determine Additional Solid Waste Process Knowledge Needs } \\
\text { Determine any additional solid waste process knowledge needs to support } \\
\text { disposition. }\end{array}$ \\
\hline 4.3.3.4.3 & $\begin{array}{l}\text { Validate Solid Waste Characterization Data } \\
\text { Validate the solid waste characterization information, consisting of generator } \\
\text { request information, process knowledge information, and waste sample } \\
\text { characterization data meets the disposition requirements. }\end{array}$ \\
\hline 4.3 .4 & $\begin{array}{l}\text { Determine Solid Waste Disposition Requirements } \\
\text { Compile and sort imposed requirements, define requirements that result from } \\
\text { proposed solutions and required capabilities, and assess constraints under which } \\
\text { the function must operate. }\end{array}$ \\
\hline 4.3 .4 .1 & $\begin{array}{l}\text { Compile Imposed Solid Waste Requirements } \\
\text { Collect state, federal, DOE and any other regulatory documents which pertain to } \\
\text { transport, handling, packaging and disposition of solid waste. }\end{array}$ \\
\hline 4.3.4.1.1 & $\begin{array}{l}\text { Identify Currently Imposed Solid Waste Regulatory Requirement } \\
\text { Review regulatory documents to identify requirements which address transport, } \\
\text { packaging, handling, and disposition of solid waste. }\end{array}$ \\
\hline 4.3.4.1.2 & $\begin{array}{l}\text { List Requirements by Solid Waste Disposition Category } \\
\text { From regulatory documents which pertain to solid waste, generate a summary } \\
\text { list of requirements by disposition category. }\end{array}$ \\
\hline 4.3 .4 .1 .3 & $\begin{array}{l}\text { Identify Solid Waste Operations Requirements } \\
\text { Generate a summary of solid waste operating requirements from all regulations } \\
\text { which apply to transport, handling, packaging and disposition for solid waste. }\end{array}$ \\
\hline 4.3.4.2 & $\begin{array}{l}\text { Evaluate Solid Waste Requirements For Disposition } \\
\text { Review and evaluate list of solid waste disposition requirements to verify } \\
\text { applicability and completeness for disposition categories. }\end{array}$ \\
\hline
\end{tabular}




\begin{tabular}{|c|c|}
\hline Function & Definitions \\
\hline 4.3 .4 .2 .1 & $\begin{array}{l}\text { Identify Appropriate Solid Waste Requirements } \\
\text { Correlate solid waste regulatory requirements with appropriate tasks in solid } \\
\text { waste operations. }\end{array}$ \\
\hline 4.3 .4 .2 .2 & $\begin{array}{l}\text { Identify Conflicting Solid Waste Require } 7 \text { ents } \\
\text { Review regulatory requirements for regulations which are contradictory and } \\
\text { propose resolutions thereof. }\end{array}$ \\
\hline 4.3.4.2.3 & $\begin{array}{l}\text { Provide Recommended Resolution To Conflicting Solid Waste Requirements } \\
\text { Provide a recommended resolution to conflicting solid waste regulatory } \\
\text { requirements for review and acceptance by the Manage Program function and } \\
\text { the appropriate regulatory agencies. }\end{array}$ \\
\hline 4.3.4.3 & $\begin{array}{l}\text { Prepare Solid Waste Disposition Specifications } \\
\text { Generate specifications for solid waste operations includingtreatment, } \\
\text { packaging, certification, storage, shipping. disposition, and archiving samples } \\
\text { and records. }\end{array}$ \\
\hline 4.3 .4 .3 .1 & $\begin{array}{l}\text { Develop Solid Waste Specifications for Treatment } \\
\text { Develop and write specifications for solid waste treatment. }\end{array}$ \\
\hline 4.3.4.3.2 & $\begin{array}{l}\text { Develop Solid Waste Specifications for Packaging } \\
\text { Develop and write specifications for packaging solid waste for disposition. }\end{array}$ \\
\hline 4.3.4.3.3 & $\begin{array}{l}\text { Develop Solid Waste Specifications for Certification } \\
\text { Develop and write specifications for certification of solid waste. }\end{array}$ \\
\hline 4.3.4.3.4 & $\begin{array}{l}\text { Develop Solid Waste Specifications for Storage } \\
\text { Develop and write specifications for safe storage of solid waste. }\end{array}$ \\
\hline 4.3 .4 .3 .5 & $\begin{array}{l}\text { Develop Solid Waste Specifications for Shipping } \\
\text { Develop and write specifications for shipment of solid waste. }\end{array}$ \\
\hline 4.3.4.3.6 & $\begin{array}{l}\text { Develop Solid Waste Specifications for Disposition } \\
\text { Develop and write specifications for disposition of solid waste. }\end{array}$ \\
\hline 4.3.4.3.7 & $\begin{array}{l}\text { Develop Solid Waste Specifications for Archiving Samples and Records } \\
\text { Develop and write specifications for storage of archive solid waste samples and } \\
\text { for characterization information records. }\end{array}$ \\
\hline 4.3 .5 & $\begin{array}{l}\text { Disposition Solid Waste } \\
\text { Package and certify the solid waste, store or ship it as needed, treat and/or } \\
\text { immobilize it, and accomplish final disposition. }\end{array}$ \\
\hline 4.3 .5 .1 & $\begin{array}{l}\text { Treat Solid Waste } \\
\text { Provide treatment of solid waste to neutralize, immobilize, and stabilize it for } \\
\text { storage and final disposition. }\end{array}$ \\
\hline 4.3 .5 .1 .1 & $\begin{array}{l}\text { Receive Solid Waste for Treatment } \\
\text { Receive and document acceptance/rejection of solid waste for treatment. Provide } \\
\text { inspection and offloading of the solid waste package. Provide removal of the } \\
\text { solid waste shipping package and acceptance or rejection of the solid waste } \\
\text { containers. Provide decontamination of solid waste transport areas and/or } \\
\text { packaging as needed. }\end{array}$ \\
\hline
\end{tabular}




\begin{tabular}{|c|c|}
\hline Function & Definitions \\
\hline 4.3 .5 .1 .1 .1 & $\begin{array}{l}\text { Document Acceptance/Rejection Of Solid Waste For Treatment } \\
\text { Provide documentation of receipt and acceptance/rejection of solid waste for } \\
\text { treatment. }\end{array}$ \\
\hline 4.3.5.1.1.2 & $\begin{array}{l}\text { Inspect Solid Waste Package } \\
\text { Provide inspection of the solid waste package. }\end{array}$ \\
\hline 4.3.5.1.1.3 & $\begin{array}{l}\text { Offload Solid Waste Package } \\
\text { Provide offloading of the solid waste package. }\end{array}$ \\
\hline 4.3 .5 .1 .1 .4 & $\begin{array}{l}\text { Remove Solid Waste Shipping Packaging } \\
\text { Provide removal of the solid waste shipping packaging. }\end{array}$ \\
\hline 4.3 .5 .1 .1 .5 & $\begin{array}{l}\text { Decontaminate Solid Waste Transport Area And/Or Packaging } \\
\text { Provide any needed decontamination of the solid waste transport area and/or } \\
\text { packagins. }\end{array}$ \\
\hline 4.3 .5 .1 .1 .6 & $\begin{array}{l}\text { Accept/Reject Solid Waste Containers } \\
\text { Provide acceptance or rejection of the solid waste containers. }\end{array}$ \\
\hline 4.3.5.1.2 & $\begin{array}{l}\text { Transport Solid Waste To Treatment Area } \\
\text { Transport the solid waste to the selected treatment process area. }\end{array}$ \\
\hline 4.3 .5 .1 .3 & $\begin{array}{l}\text { Process The Solid Waste } \\
\text { Process the solid waste using the selected treatment process. Provide samples of } \\
\text { the solid waste before and after processing for quality assurance certification. }\end{array}$ \\
\hline 4.3.5.1.3.1 & $\begin{array}{l}\text { Unpackage Solid Waste } \\
\text { Unpackage the solid waste. }\end{array}$ \\
\hline 4.3 .5 .1 .3 .2 & $\begin{array}{l}\text { Sort Solid Waste } \\
\text { Sort the solid waste according to the selected treatment process. }\end{array}$ \\
\hline 4.3.5.1.3.3 & $\begin{array}{l}\text { Acquire Pre-processing Sample } \\
\text { Acquire a pre-processing solid waste sample for quality assurance certification } \\
\text { of the treatment process. }\end{array}$ \\
\hline 4.3 .5 .1 .3 .4 & $\begin{array}{l}\text { Execute Selected Solid Waste Treatment Processes } \\
\text { Execute the selected solid waste treatment processes. }\end{array}$ \\
\hline 4.3 .5 .1 .3 .5 & $\begin{array}{l}\text { Acquire Post-processing Sample } \\
\text { Acquire a post-piccessing solid waste sample for quality assurance certification } \\
\text { of the treatment process. }\end{array}$ \\
\hline 4.3.5.1.4 & $\begin{array}{l}\text { Immobilize Solid Waste } \\
\text { Provide immobilization of the treated solid waste. }\end{array}$ \\
\hline 4.3.5.1.5 & $\begin{array}{l}\text { Stabilize Solid Waste } \\
\text { Provide stabilization of the treated solid waste. }\end{array}$ \\
\hline 4.3.5.1.5.1 & $\begin{array}{l}\text { Pre-process Solid Waste Sample } \\
\text { Acquire a pre-processing solid waste sample to be used in treatment quality } \\
\text { assurance and effectiveness evaluations. }\end{array}$ \\
\hline
\end{tabular}




\begin{tabular}{|c|c|}
\hline Function & Definitions \\
\hline 4.3 .5 .1 .5 .2 & $\begin{array}{l}\text { Select Solid Waste Treatment Process } \\
\text { Select an appropriate solid waste treatment process that will stabilize the wastes } \\
\text { and allow the treated waste product to meet its final disposition reauirements. }\end{array}$ \\
\hline 4.3.5.1.5.3 & $\begin{array}{l}\text { Post-Process Solid Waste Sample } \\
\text { Acquire a post-processing solid waste sample to be used in treatment quality } \\
\text { assurance and effectiveness evaluations. }\end{array}$ \\
\hline 4.3.5.1.6 & $\begin{array}{l}\text { Neutralize Solid Waste } \\
\text { Provide neutralization of the treated solid waste, if needed. }\end{array}$ \\
\hline 4.3.5.2 & $\begin{array}{l}\text { Package Solid Waste } \\
\text { Containerize the treated solid waste in a container suitable for the waste type. } \\
\text { Provide solid waste packaging documentation including packaging certifications } \\
\text { and solid waste feedstock traceability. Provide appropriate labeling and } \\
\text { inspection of the containers of solid waste. }\end{array}$ \\
\hline 4.3.5.2.1 & $\begin{array}{l}\text { Provide A Suitable Solid Waste Container } \\
\text { Provide a container for the treated solid waste suicable for the waste type that } \\
\text { meets the solid waste disposition requirements. }\end{array}$ \\
\hline 4.3.5.2.1.1 & $\begin{array}{l}\text { Match Container To Treated Solid Waste Type } \\
\text { Match container to the treated solid waste suitable for the waste type. }\end{array}$ \\
\hline 4.3 .5 .2 .1 .2 & $\begin{array}{l}\text { Match Container To Solid Waste Acceptance Criteria } \\
\text { Match container to the treated solid waste that meets the solid waste disposition } \\
\text { requirements. }\end{array}$ \\
\hline 4.3.5.2.2 & $\begin{array}{l}\text { Containerize The Solid Waste } \\
\text { Place the solid waste in a suitable container and package with other similar } \\
\text { waste containers derived from the same waste feedstock. }\end{array}$ \\
\hline 4.3 .5 .2 .3 & $\begin{array}{l}\text { Provide Solid Waste Package Documentation } \\
\text { Provide documentation of the solid waste package including traceability to the } \\
\text { incoming solid waste feedstock and the certifications developed for the waste } \\
\text { package. }\end{array}$ \\
\hline 4.3 .5 .2 .3 .1 & $\begin{array}{l}\text { Provide Packaging Traceability To Incoming Solid Waste Feedstock } \\
\text { Provide documentation of the solid waste package traceability to the incoming } \\
\text { solid waste feedstock. }\end{array}$ \\
\hline 4.3 .5 .2 .3 .2 & $\begin{array}{l}\text { Develop Solid Waste Packaging Certifications } \\
\text { Develop solid waste packaging certifications that show that the solid waste } \\
\text { packaging was appropriate and suitable for the solid waste. }\end{array}$ \\
\hline 4.3.5.2.4 & $\begin{array}{l}\text { Label Containers Of Solid Waste } \\
\text { Provide appropriatc labeling of the solid waste containers. }\end{array}$ \\
\hline 4.3 .5 .2 .4 .1 & $\begin{array}{l}\text { Provide Traceability To Incoming Solid Waste Feedstock } \\
\text { Provide solid waste container labeling information that gives traceability of the } \\
\text { containerized solid waste to its former incoming or before treatment solid waste } \\
\text { feedstock. }\end{array}$ \\
\hline
\end{tabular}




\begin{tabular}{|c|c|}
\hline Eunction & Definitions \\
\hline 4.3.5.2.4.2 & $\begin{array}{l}\text { Develop Solld Waste Package Certifications } \\
\text { Develop solid waste package certifications that will be included as part of the } \\
\text { solid waste container labeling. These certifications, when done, will ensure that } \\
\text { appropriate waste container packaging is accomplished, allowing waste package } \\
\text { transfer to storage and final disposition. }\end{array}$ \\
\hline 4.3.5.2.5 & $\begin{array}{l}\text { Inspect Containers Of Solld Waste } \\
\text { Inspect the solid waste containers to verify the correct labeling and the container } \\
\text { integrity. }\end{array}$ \\
\hline 4.3.5.3 & $\begin{array}{l}\text { Certify Solid Waste } \\
\text { Certify that the solid waste was treated according to the disposition requirements } \\
\text { and that the documentation is in order to allow release of the solid waste } \\
\text { package for storage and disposition. This function will also send samples back } \\
\text { to the characterization function if needed. }\end{array}$ \\
\hline 4.3 .5 .3 .1 & $\begin{array}{l}\text { Review Solid Waste Documentation } \\
\text { Review the solid waste documentation to verify that the solid waste treatment was } \\
\text { appropriate and that the waste packase meets the disposition requirements. }\end{array}$ \\
\hline 4.3 .5 .3 .2 & $\begin{array}{l}\text { Analyze Solid Waste Treatment Process Samples } \\
\text { Analyze the solid waste treatment process samples to verify that the treatment } \\
\text { process produced a treated solid waste product that meets the disposition } \\
\text { requirements. Transfer these samples and analysis data to archival storage } \\
\text { when analysis is finished. }\end{array}$ \\
\hline 4.3 .5 .3 .3 & $\begin{array}{l}\text { Accept/Reject/Correct Solld Waste Documentation } \\
\text { Determine the state of the solid waste documentation and either accept, reject, or } \\
\text { correct the documentation to allow release of the solid waste package for storage } \\
\text { and disposition. }\end{array}$ \\
\hline 4.3 .5 .3 .4 & $\begin{array}{l}\text { Accept/Reject the Solid Waste } \\
\text { Determine from the treatment analysis results whether the treatment process was } \\
\text { appropriate and effective at producing a product that meets the disposition } \\
\text { requirements and either accept or reject the treated solid waste. }\end{array}$ \\
\hline 4.3 .5 .3 .5 & $\begin{array}{l}\text { Provide Solid Waste Certification Documentation } \\
\text { Document the certification of the solid waste documentation that allows release } \\
\text { of the solid waste package for storage and disposition. }\end{array}$ \\
\hline 4.3.5.3.6 & $\begin{array}{l}\text { Release Solid Waste For Storage and Disposition } \\
\text { Release the certified solid waste package for storage and disposition. }\end{array}$ \\
\hline 4.3.5.3.7 & $\begin{array}{l}\text { Release Solid Waste For Disposition } \\
\text { Release the certified solid waste package for final disposition. }\end{array}$ \\
\hline 4.3.5.4 & $\begin{array}{l}\text { Store Solid Waste } \\
\text { Store the solid waste before shipment to the disposal site. }\end{array}$ \\
\hline 4.3.5.4.1 & $\begin{array}{l}\text { Accept Certified Solid Waste } \\
\text { Accept the certified solid waste for storage before shipment to the disposal site. }\end{array}$ \\
\hline
\end{tabular}




\begin{tabular}{|c|c|}
\hline Eunction & Definitions \\
\hline 4.3 .5 .4 .2 & $\begin{array}{l}\text { Place Solld Waste In Storage } \\
\text { Place the certified solid waste in storage before shipment } 10 \text { the disposal site. }\end{array}$ \\
\hline 4.3.5.4.3 & $\begin{array}{l}\text { Provide Solid Waste Status Documentation } \\
\text { Provide certified solid waste storase status documentation. }\end{array}$ \\
\hline 4.3.5.4.4 & $\begin{array}{l}\text { Monitor Solid Waste Package Quality } \\
\text { Monitor the certified solid waste package to verify its integrity and ouality. }\end{array}$ \\
\hline 4.3.5.4.5 & $\begin{array}{l}\text { Release Stored Solid Waste For Disposition } \\
\text { Release the certified solid waste package for shipment to offsite and onsite } \\
\text { disnosal sites. }\end{array}$ \\
\hline 4.3 .5 .5 & $\begin{array}{l}\text { Ship Solid Waste } \\
\text { Receive the certified solid waste package and prepare the necessary shipment } \\
\text { requests and documentation. Coordinate the solid waste package shipment with } \\
\text { shippers and receivers to verify that the final waste disposition was } \\
\text { accomplished. Document the final solid waste disposition status. }\end{array}$ \\
\hline 4.3.5.5.1 & $\begin{array}{l}\text { Receive/Generate Request To Ship Solid Waste OfIsite } \\
\text { Receive the certified solid waste package and prepare the necessary offsite } \\
\text { shipment requests and associated documentation. }\end{array}$ \\
\hline 4.3.5.5.2 & $\begin{array}{l}\text { Prepare Necessary Solid Waste Shipping Documentation } \\
\text { Prepare the necessary solid waste package shipment documentation. }\end{array}$ \\
\hline 4.3.5.5.3 & $\begin{array}{l}\text { Prepare Necessary Solid Waste Shipping Packages } \\
\text { Prepare the necessary solid waste shipnine packages. }\end{array}$ \\
\hline 4.3.5.5.4 & $\begin{array}{l}\text { Inspect Solid Waste Shipment } \\
\text { Inspect the solid waste package before shipment to verify that the shipment meets } \\
\text { its release requirements. }\end{array}$ \\
\hline 4.3 .5 .5 .5 & $\begin{array}{l}\text { Release Solid Waste For Shipment } \\
\text { Release the solid waste packase for shipment to the waste disposal site. }\end{array}$ \\
\hline 4.3 .5 .5 .6 & $\begin{array}{l}\text { Coordinate With Shipper For Offsite Solid Waste Shipment } \\
\text { Coordinate the solid waste package offsite shipment with the shipper to verify } \\
\text { that waste package shipment to the disposal site is accomplished. }\end{array}$ \\
\hline 4.3.5.5.7 & $\begin{array}{l}\text { Coordinate With Offsite Solid Waste Receiver } \\
\text { Coordinate the solid waste package offsite shipment with the waste package } \\
\text { receiver to verify that waste package shipment to the disposal site is } \\
\text { accomplished. }\end{array}$ \\
\hline 4.3 .5 .5 .8 & $\begin{array}{l}\text { Verify Final Solid Waste Disposition } \\
\text { Verify that waste package shipment to the disposal site was accomplished and } \\
\text { that final disposition was completed. }\end{array}$ \\
\hline 4.3 .5 .5 .8 .1 & $\begin{array}{l}\text { Provide Final Solid Waste Disposition Documentation } \\
\text { Provide documentation that final solid waste package disposition was comf }\end{array}$ \\
\hline
\end{tabular}




\begin{tabular}{|c|c|}
\hline Eunction & Definitions \\
\hline 4.3 .5 .6 & $\begin{array}{l}\text { Dispose Solid Waste } \\
\text { Schedule the solid waste package disposal and prepare the waste transport } \\
\text { package and disposal documentation. Provide final inspection of the solid waste } \\
\text { package load and place in disposal site. Close the disposal site in regulatory } \\
\text { compliance and provide institutional controls and closure security to maintain } \\
\text { the disposal site security and integritu. }\end{array}$ \\
\hline 4.3 .5 .6 .1 & $\begin{array}{l}\text { Prepare Solld Waste Disposal Documentation } \\
\text { Prepare she solid waste package disposaldocumentation. }\end{array}$ \\
\hline 4.3.5.6.2 & $\begin{array}{l}\text { Prepare Solid Waste Transport Packages } \\
\text { Prepare the solid waste transport packages. }\end{array}$ \\
\hline 4.3.5.6.3 & $\begin{array}{l}\text { Schedule Solid Waste Disposal } \\
\text { Schedule the solid waste packare disposal. }\end{array}$ \\
\hline 4.3.5.6.4 & $\begin{array}{l}\text { Inspect Solid Waste Load } \\
\text { Provide final inspection of the solid waste package load to verify package } \\
\text { integritu and consistency of the documentation. }\end{array}$ \\
\hline 4.3 .5 .6 .5 & $\begin{array}{l}\text { Place Solid Waste In Disposal Site } \\
\text { Place the solid waste package in the disposal site. }\end{array}$ \\
\hline 4.3.5.6.6 & $\begin{array}{l}\text { Close Solid Waste Disposal Site In Regulatory Compliance } \\
\text { Close the solid waste disposal site in regulatory compliance. }\end{array}$ \\
\hline 4.3 .5 .6 .6 .1 & $\begin{array}{l}\text { Provide Solid Waste Disposal Site Documentation } \\
\text { Provide solid waste disposal site documentation to verify completion of solid } \\
\text { waste package disposal. }\end{array}$ \\
\hline 4.3 .5 .6 .6 .2 & $\begin{array}{l}\text { Provide Solid Waste Disposal Site Institutional Controls } \\
\text { Provide solid waste disposal site institutional controls to maintain the integrity of } \\
\text { the site. }\end{array}$ \\
\hline 4.3 .5 .6 .6 .2 .1 & $\begin{array}{l}\text { Provide Solid Waste Disposal Site Fencing } \\
\text { Provide solid waste disposal site fencing to maintain the integrity of the site. }\end{array}$ \\
\hline 4.3.5.6.6.2.2 & $\begin{array}{l}\text { Provide Solid Waste Disposal Site Monitoring } \\
\text { Provide solid waste disposal site monitoring to assure that the site integrity is } \\
\text { maintained. }\end{array}$ \\
\hline 4.3 .5 .6 .6 .2 .3 & $\begin{array}{l}\text { Provide Solid Waste Disposal Site Security } \\
\text { Provide solid waste disposal site security to assure that the site integrity is } \\
\text { maintained. }\end{array}$ \\
\hline 4.3.5.6.6.3 & $\begin{array}{l}\text { Accomplish Solid Waste Disposal Site Closure Security } \\
\text { Provide solid waste disposal site closure security to maintain the security } \\
\text { integrity of the site. }\end{array}$ \\
\hline 4.3 .5 .7 & $\begin{array}{l}\text { Assess Solid Waste TSD Capability Needs And Disposal Resources } \\
\text { Assess the solid waste treatment, storage, and disposal (TSD) capability needs } \\
\text { and resources based on required versus design throughput and capacities. }\end{array}$ \\
\hline
\end{tabular}




\begin{tabular}{|c|c|}
\hline Function & Definitions \\
\hline 4.3 .5 .7 .1 & $\begin{array}{l}\text { Compare Design Throughput/Capacities Of Solid Waste TSD Facilities With } \\
\text { Needs } \\
\text { Compare required versus design throughput and capacities of solid waste TSD } \\
\text { facilities to identify additional capability needs. }\end{array}$ \\
\hline 4.3.5.7.2 & $\begin{array}{l}\text { Determine Adequacy Of Solid Waste TSD Facilities } \\
\text { Determine inadequacies or excess capacities of solid waste TSD facilities to } \\
\text { identify additional capability needs or resources that could be used by other } \\
\text { Cleanun functions. }\end{array}$ \\
\hline 4.3.5.8 & $\begin{array}{l}\text { Archive Solid Waste Process Samples and Records } \\
\text { Provide archival storage and retrieval operations for the analyzed solid waste } \\
\text { process samples and the accompanvine analvsis data. }\end{array}$ \\
\hline 4.3.5.8.1 & $\begin{array}{l}\text { Archive Solid Waste Process Data } \\
\text { Provide archival storage of the solid waste process, sample analysis data. }\end{array}$ \\
\hline 4.3.5.8.2 & $\begin{array}{l}\text { Archive Solid Waste Process Samples } \\
\text { Provide archival storase of the solid waste process samples. }\end{array}$ \\
\hline 4.3.5.8.3 & $\begin{array}{l}\text { Provide Solid Waste Process Sample and Data Retrievability } \\
\text { Provide archival retrieval operations for the analyzed solid waste process } \\
\text { samples and the accompanying analysis data. }\end{array}$ \\
\hline 4.4 & $\begin{array}{l}\text { Remedy/Restore Sites, Facilities, \& Ground Water } \\
\text { The REMEDYIRESTORE SITES, FACILITIES, AND GROUND WATER (4.4) } \\
\text { function restores sites, deactivated facilities, ground water, and related items to } \\
\text { an acceptable state. This function includes restoration of chemically } \\
\text { contaminated lands, and removal or disposition of buildings, fission reactors, } \\
\text { chemical processing plants, infrastructure, etc. This function includes new as } \\
\text { well as lepacy items. }\end{array}$ \\
\hline 4.5 & $\begin{array}{l}\text { Manage Aqueous Wastes } \\
\text { Manages and treats aqueous wastes which are generated by the other functions } \\
\text { under [4.0] and ongoing operations (PNL, sanitary wastes). Develops an } \\
\text { Aqueous Waste Disposition Plan, transports and receives aqueous waste, treats } \\
\text { the aqueous waste (if required), and dispositions the waste. Aqueous wastes } \\
\text { which would result in the treatment facility operating outside of the safety and } \\
\text { regulatory envelope will not be accepted. Trans-Uranic (TRU) wastes will not be } \\
\text { processed and the concentration of TRU's in aqueous waste processed must be } \\
\text { below established limits. Appropriate and required documentation must } \\
\text { accompany all aqueous waste. }\end{array}$ \\
\hline 4.5 .1 & $\begin{array}{l}\text { Maintain Aqueous Waste Safety and Compliance Envelope } \\
\text { Maintains the facility structure. qualified staff, safe and compliant equipment. } \\
\text { documentation and provides assessment of safety and compliance states. } \\
\text { Provides all necessary resources for safe and compliant operation in accordance } \\
\text { with soverning safety codes and regulations. }\end{array}$ \\
\hline
\end{tabular}




\begin{tabular}{|c|c|}
\hline Eunction & Definitions \\
\hline 4.5 .1 .1 & $\begin{array}{l}\text { Maintain Safe And Compliant Aqueous Waste Operational Environment } \\
\text { Assesses and maintains facility operations in a safe condition compliant with } \\
\text { applicable codes, standards, and company procedures. ALARA is achieved in/by } \\
\text { 4.5.1.1. Operational safety and compliance aspects of the system are } \\
\text { maintained, including work control, property m management, and safety for } \\
\text { materials. Information on unsafe conditions enter this function and, with } \\
\text { acquired system knowledge the information is passed to } 4.5 .1 .3 \text { and then to } \\
\text { 4.5.1.5 to develop a corrective action plan. Some wastes may be generated by } \\
\text { this function, the solid wastes are passed to } 4.5 .5 \text { for disposition and the liquid } \\
\text { wastes are passed to } 4.5 .2 \text { for plannine and treatment. }\end{array}$ \\
\hline 4.5.1.1.1 & $\begin{array}{l}\text { Acquire Knowledge Of System Status (AW) } \\
\text { Identifies the initial state of the operation activities, including the people, facility. } \\
\text { and the aqueous waste. }\end{array}$ \\
\hline 4.5 .1 .1 .2 & $\begin{array}{l}\text { Provide Operational Surveillance of System and Facilities (AW) } \\
\text { Provides the surveillance of the aqueous waste system for safety and compliance. }\end{array}$ \\
\hline 4.5 .1 .1 .3 & $\begin{array}{l}\text { Provide Facility Access Control (AW) } \\
\text { Controls access to hazardous areas. It provides barriers, controls, security, and } \\
\text { controls access to chemical, mechanical, and radiological hazards. }\end{array}$ \\
\hline 4.5 .1 .1 .4 & $\begin{array}{l}\text { Provide Work Control (AW) } \\
\text { Provides the job control system (JCS) which develops the details associated with } \\
\text { doing a job, usually specific to a facility. (The JCS provides more detail than the } \\
\text { work package which comes down from management.) }\end{array}$ \\
\hline 4.5.1.1.5 & $\begin{array}{l}\text { Provide Property Management (AW) } \\
\text { Provides property management for the facility in accordance with DOE orders } \\
\text { and contractor procedures. }\end{array}$ \\
\hline 4.5 .1 .1 .6 & $\begin{array}{l}\text { Provide Chemical Safety (AW) } \\
\text { Provides containment, control, and documentation for hazardous and } \\
\text { non-hazardous chemicals. This may include barriers, storage, and other } \\
\text { enerations. }\end{array}$ \\
\hline 4.5 .1 .1 .7 & $\begin{array}{l}\text { Provide Mechanical Safety (AW) } \\
\text { Provide Mechanical Safety (AW) Provides for safety for mechanical items, } \\
\text { which includes things like belt guards, warning labels, and other items used for } \\
\text { equipment safety. }\end{array}$ \\
\hline 4.5 .1 .1 .8 & $\begin{array}{l}\text { Provide Radiation Safety (AW) } \\
\text { Provides radiological safety, including shielding, containment, control, and } \\
\text { documentation of radioactive material and waste, etc. }\end{array}$ \\
\hline 4.5 .1 .2 & $\begin{array}{l}\text { Maintain Safe And Compliant Aqueous Waste System Equipment And } \\
\text { Structure } \\
\text { Maintains the facility systems and infrastructure in the operational condition } \\
\text { dictated by approved safety and compliance documentation (includes } \\
\text { environmental resulations). }\end{array}$ \\
\hline
\end{tabular}




\begin{tabular}{|c|c|}
\hline Function & Definitions \\
\hline 4.5 .1 .2 .1 & $\begin{array}{l}\text { Perform Corrective Maintenance (AW) } \\
\text { Performs all maintenance that brings systems and equipment back to their } \\
\text { operational states after failure. }\end{array}$ \\
\hline 4.5.1.2.2 & $\begin{array}{l}\text { Perform Preventative Maintenance (AW) } \\
\text { Performs preventative maintenance activities to minimize all unplanned events } \\
\text { and premature equipment failures. }\end{array}$ \\
\hline 4.5.1.2.3 & $\begin{array}{l}\text { Perform Process Systems and Equipment Calibrations (AW) } \\
\text { Performs facility and equipment calibration maintenance. }\end{array}$ \\
\hline 4.5 .1 .3 & $\begin{array}{l}\text { Maintain Safe And Compliant Aqueous Waste Documentation } \\
\text { Prepares, updates, and stores the documentation associated with the aqueous } \\
\text { waste facilities. This includes safety, training, operations, compliance, inventory. } \\
\text { and engineering documentation, and maintenance procedures. }\end{array}$ \\
\hline 4.5.1.3.1 & $\begin{array}{l}\text { Maintain Safety Documents (AW) } \\
\text { Maintains the safety documentation associated with the aqueous waste facilities. }\end{array}$ \\
\hline 4.5.1.3.2 & $\begin{array}{l}\text { Maintain Operations Documents (AW) } \\
\text { Maintains the operations documentation for the aqueous waste facilities. }\end{array}$ \\
\hline 4.5.1.3.3 & $\begin{array}{l}\text { Maintain Maintenance Procedures (A W) } \\
\text { Maintains the maintenance procedures associated with the aqueous waste } \\
\text { facilities. }\end{array}$ \\
\hline 4.5.1.3.4 & $\begin{array}{l}\text { Maintain Compliance Documentation (A W) } \\
\text { Maintains compliance documentation for the aqueous waste facilities, which } \\
\text { includes regulatory, company, OSHA, and other documentation required by any } \\
\text { source, to assure and prove compliance. (A "catch-all" for any required } \\
\text { documentation which does not fit into any of the other categories under 4.5.1.3.) }\end{array}$ \\
\hline 4.5 .1 .3 .5 & $\begin{array}{l}\text { Maintain Inventory Documentation (AW) } \\
\text { Maintains the documentation which may be required for inventories of chemicals } \\
\text { or radionuclides. }\end{array}$ \\
\hline 4.5 .1 .3 .6 & $\begin{array}{l}\text { Maintain Engineering Documentation (AW) } \\
\text { Maintains the engineering documentation for the aqueous waste facilities, } \\
\text { including drawings/diagrams. }\end{array}$ \\
\hline 4.5.1.3.7 & $\begin{array}{l}\text { Maintain Training Documentation (AW) } \\
\text { Maintains the training documentation for qualification and certification of the } \\
\text { aqueous waste facility staff. }\end{array}$ \\
\hline 4.5.1.4 & $\begin{array}{l}\text { Maintain Qualified Aqueous Waste Staff } \\
\text { Maintains the qualification and certification of the staff of the aqueous waste } \\
\text { facilities through training and/or testing. }\end{array}$ \\
\hline 4.5 .1 .4 .1 & $\begin{array}{l}\text { Maintain Training (AW) } \\
\text { Provides the training for operators, staff, and maintenance personnel for the } \\
\text { aqueous waste facilities. }\end{array}$ \\
\hline
\end{tabular}




\begin{tabular}{|c|c|}
\hline Function & Definitions \\
\hline 4.5 .1 .4 .2 & $\begin{array}{l}\text { Maintain Qualification and Certification (AW) } \\
\text { Provides periodic personnel skills check, assessment, and testing required to } \\
\text { maintain the qualification and certification (as required) of employees of the } \\
\text { aqueous waste facilities. }\end{array}$ \\
\hline 4.5.1.5 & $\begin{array}{l}\text { Assess Aqueous Waste Safety And Compliance State } \\
\text { Performs self assessments independent oversight of the aqueous waste facilities. } \\
\text { Also develops the corrective action plans for unsafe conditions in the aqueous } \\
\text { waste facilities. }\end{array}$ \\
\hline 4.5.1.5.1 & $\begin{array}{l}\text { Support Independent Oversight Audits (AW) } \\
\text { Provides support for and responds to external, independent oversight audits, } \\
\text { without being directly involved in those audits. }\end{array}$ \\
\hline 4.5.1.5.2 & $\begin{array}{l}\text { Perform Self Assessment (AW) } \\
\text { Periodically self assesses the safety and compliance of the aqueous waste } \\
\text { facilities. }\end{array}$ \\
\hline 4.5.1.5.3 & $\begin{array}{l}\text { Develop Corrective Action Plan (AW) } \\
\text { Develops the plan for correcting unsafe conditions in the aqueous waste } \\
\text { facilities. }\end{array}$ \\
\hline 4.5 .2 & $\begin{array}{l}\text { Develop Aqueous Waste Disposition Plan } \\
\text { Receives and determines the characteristics of the aqueous waste to be } \\
\text { transferred, assesses the acceptability of the aqueous waste, and develops a plan } \\
\text { for the disposition of the aqueous waste, identifies needs for improvements and } \\
\text { additions. Scheduling of the waste transfer, treatment, and disposition is done } \\
\text { later (in function 4.5.3). Information and intelligence function for managing } \\
\text { aqueous waste. Input documentation will be associated with the waste which will } \\
\text { provide compositions or a list of constituents of the waste being received, or } \\
\text { appropriate waste codes. }\end{array}$ \\
\hline 4.5 .2 .1 & $\begin{array}{l}\text { Profile Aqueous Waste to be Received } \\
\text { Receives the aqueous waste documentation from the generators for each } \\
\text { requested transfer, assesses the completeness of the information received, and } \\
\text { develops a profile for the waste. Additional information may be required from } \\
\text { the waste generator to further classify the waste and assess it traceability. The } \\
\text { documentation will include concentrations of constituents in the waste and will } \\
\text { determine any waste codes. }\end{array}$ \\
\hline 4.5.2.1.1 & $\begin{array}{l}\text { Receive Aqueous Waste Information } \\
\text { Receives the information associated with waste that is being requested to be } \\
\text { transferred to the aqueous waste facilities. }\end{array}$ \\
\hline 4.5.2.1.2 & $\begin{array}{l}\text { Assess Aqueous Waste Information Completeness } \\
\text { Assesses the completeness of the information received. If the information is } \\
\text { incomplete, requests for additional information are returned to the generator. }\end{array}$ \\
\hline
\end{tabular}




\begin{tabular}{|c|c|}
\hline Function & Definitions \\
\hline 4.5 .2 .1 .3 & $\begin{array}{l}\text { Classify Aqueous Waste } \\
\text { Classifies the aqueous waste according to the information received. The waste } \\
\text { codes generated are later used to determine the final disposition of the aqueous } \\
\text { waste, and the actual acceptability of the waste to be transferred. }\end{array}$ \\
\hline 4.5 .2 .2 & $\begin{array}{l}\text { Assess Aqueous Waste Acceptance Capability } \\
\text { Determines if the current treatment facilities can accept the aqueous waste based } \\
\text { on the acceptance criteria and permits for the treatment, storage and disposal } \\
\text { facilities, depending on the waste codes and other characteristics of the aqueous } \\
\text { waste. Will identify the need for additional treatability tests or waste } \\
\text { characterization information. The output is a confirmation of the compatibility } \\
\text { of the aqueous waste. }\end{array}$ \\
\hline 4.5 .2 .2 .1 & $\begin{array}{l}\text { Identify Need for Treatability Tests (AW) } \\
\text { Identifies the need for treatability tests in cases where the treatability of an } \\
\text { aqueous waste is in question. }\end{array}$ \\
\hline 4.5 .2 .2 .2 & $\begin{array}{l}\text { Apply Aqueous Waste Acceptance Criteria } \\
\text { Determines if the aqueous waste meets the facility criteria for acceptance. This } \\
\text { is essentially a checklist of regulatory and other constraints. }\end{array}$ \\
\hline 4.5 .2 .2 .3 & $\begin{array}{l}\text { Confirm Capability Adequacy (AW) } \\
\text { Confirms that the aqueous waste can be processed in the aqueous waste } \\
\text { facilities. This is above and beyond the criteria in } 4.5 .2 .2 .2 \text { in that the capability } \\
\text { may be based on technical, economic, and safety considerations. }\end{array}$ \\
\hline 4.5.2.2.4 & $\begin{array}{l}\text { Identify Need for Additional Characterization Information (AW) } \\
\text { Identifies the need for additional aqueous waste characterization information. } \\
\text { Additional characterization tests may be necessary to determine the } \\
\text { concentration and constituents of the aqueous waste. }\end{array}$ \\
\hline 4.5.2.3 & $\begin{array}{l}\text { Determine Aqueous Waste Disposition and Needs } \\
\text { Determines the needs for disposition of the aqueous waste, evaluates the } \\
\text { disposition options, selects the disposition option, plans the disposition of the } \\
\text { waste (note that scheduling is done in 4.5.3), and authorizes the transfer of the } \\
\text { waste. Uses the input and paperwork generated by functions 4.5.2.1 and 4.5.2.2. }\end{array}$ \\
\hline 4.5 .2 .3 .1 & $\begin{array}{l}\text { Evaluate Disposition Options (AW) } \\
\text { Options for disposition of the waste are determined and evaluated based on } \\
\text { information associated with the waste. }\end{array}$ \\
\hline 4.5.2.3.2 & $\begin{array}{l}\text { Plan Aqueous Waste Disposition } \\
\text { Plans for the disposition of the aqueous waste are developed, including the } \\
\text { transfer of the waste, the facility configuration required, and plans all other } \\
\text { aspects for the processing of the waste. }\end{array}$ \\
\hline
\end{tabular}




\begin{tabular}{|c|c|}
\hline Function & Definitions \\
\hline 4.5 .3 & $\begin{array}{l}\text { Receive/Transport Aqueous Waste } \\
\text { Incoming aqueous waste is received, stored and/or transported to the aqueous } \\
\text { waste treatment or disposal facilities including approval of the transfer request, } \\
\text { scheduling and prioritization, transfer of the waste, and storage of the waste until } \\
\text { it is treated. Associated with the waste will be all of the required documentation, } \\
\text { including the manifest for each waste transfer. Verification of the characteristics } \\
\text { of the waste received, as well as monitoring the waste while in storage is also } \\
\text { performed. }\end{array}$ \\
\hline 4.5.3.1 & $\begin{array}{l}\text { Process Generator Aqueous Waste Transfer Request } \\
\text { Performs final review and approves or rejects of the transfer request for the } \\
\text { aqueous waste. Information determined in } 4.5 .2 \text { will be included, as well as } \\
\text { waste packaging information. Additional information needed to verify the } \\
\text { transfer request will be requested by this function from the aqueous waste } \\
\text { geneiator. Rejected paperwork is returned to the generator for further work. }\end{array}$ \\
\hline 4.5.3.1.1 & $\begin{array}{l}\text { Review and Approve Generator Aqueous Waste Transfer Request } \\
\text { Reviews the aqueous waste transfer request submitted by the aqueous waste } \\
\text { generator. }\end{array}$ \\
\hline 4.5.3.1.2 & $\begin{array}{l}\text { Verify Generator Aqueous Waste Transfer Request } \\
\text { Verifies the aqueous waste transfer request submitted by the aqueous waste } \\
\text { generator, may also verify the waste to be transferred. }\end{array}$ \\
\hline 4.5.3.1.3 & $\begin{array}{l}\text { Approve/Reject Generator Aqueous Waste Transfer Request } \\
\text { Gives the final approval or a rejection to transfer the aqueous waste from the } \\
\text { generator. }\end{array}$ \\
\hline 4.5 .3 .2 & $\begin{array}{l}\text { Schedule Aqueous Waste Transfers } \\
\text { Schedules waste transfers based on priorities, other transfers, and facility } \\
\text { receiving capabilities. }\end{array}$ \\
\hline 4.5.3.2.1 & $\begin{array}{l}\text { Prioritize Receipt of Aqueous Waste } \\
\text { Develops the priorities of the wastes transfers to be received. }\end{array}$ \\
\hline 4.5.3.2.2 & $\begin{array}{l}\text { Schedule Transfer of Aqueous Waste } \\
\text { Schedules the transfer of aqueous waste based on the prioritization from } \\
\text { 4.5.3.2.I and on the availability of the necessarv aqueous waste facilities. }\end{array}$ \\
\hline 4.5 .3 .3 & $\begin{array}{l}\text { Transfer Aqueous Waste } \\
\text { Executes the actual transfer/receiving of the aqueous waste. This will include } \\
\text { establishing the routing of the waste, making the necessary notifications, } \\
\text { transferring the waste, confirming transfer of the waste, and verification that the } \\
\text { waste characteristics match those on the manifest and supporting documents. } \\
\text { Transfer needs will be requested, and the transferred waste will be transferred. }\end{array}$ \\
\hline 4.5 .3 .3 .1 & $\begin{array}{l}\text { Establish Routing and Configurations for Transfers (AW) } \\
\text { Establishes, configures, and verifies the transfer route for the aqueous waste. } \\
\text { (For example switching the valves on pipe transfers and barricading roads). }\end{array}$ \\
\hline
\end{tabular}




\begin{tabular}{|c|c|}
\hline Function & Definitions \\
\hline 4.5 .3 .3 .2 & $\begin{array}{l}\text { Make Necessary Notifications (AW) } \\
\text { Notifies the appropriate authorities or personnel when waste is going to be } \\
\text { transferred. (For example, the state patrol or department of transportation may } \\
\text { need to know when radioactive or hazardous waste is transferred overland by } \\
\text { truck.) }\end{array}$ \\
\hline 4.5.3.3.3 & $\begin{array}{l}\text { Make Aqueous Waste Transfer } \\
\text { Physically transfers the aqueous waste by whatever means is planned (truck, } \\
\text { pipe. etc.) to the aqueous waste facilities. }\end{array}$ \\
\hline 4.5.3.3.4 & $\begin{array}{l}\text { Confirm Aqueous Waste Transfer } \\
\text { May return a confirmation to the aqueous waste generator that the waste was } \\
\text { received. Determines if the waste is successfully transferred. Checks for pipe } \\
\text { leaks or routing errors. }\end{array}$ \\
\hline 4.5 .3 .3 .5 & $\begin{array}{l}\text { Verify Transferred Aqueous Waste Characteristics } \\
\text { The characteristics of the waste (composition and constituents) are verified, as } \\
\text { necessary, to see that they meet what was described in the transfer } \\
\text { documentation and/or manifest. If the aqueous waste is not what was described, } \\
\text { or is outside the ability for the aqueous waste facilities to process, it may be } \\
\text { rejected. }\end{array}$ \\
\hline 4.5.3.4 & $\begin{array}{l}\text { Store Transferred Aqueous Waste } \\
\text { When necessary, aqueous waste will be stored after it has been transferred. The } \\
\text { stored waste will be monitored and evaluated as necessary against establish } \\
\text { criteria. Monitoring of waste characteristics may involve sampling analysis or } \\
\text { instrumentation. }\end{array}$ \\
\hline 4.5.3.4.1 & $\begin{array}{l}\text { Provide Aqueous Waste Lag Storage } \\
\text { Provides Interim storage for the aqueous waste before treatment or disposition, if } \\
\text { the waste does not require treatment. (If the waste is received directly into the } \\
\text { treatment facilities, this function will be by-passed.) }\end{array}$ \\
\hline 4.5.3.4.2 & $\begin{array}{l}\text { Monitor Aqueous Waste Characteristics } \\
\text { While the aqueous waste is in storage awaiting treatment or disposition, some } \\
\text { characteristics may have to be monitored, depending on the type of aqueous } \\
\text { waste. }\end{array}$ \\
\hline 4.5 .3 .4 .3 & Monitor Administrative Controls (AW) \\
\hline 4.5 .4 & $\begin{array}{l}\text { Treat Aqueous Waste } \\
\text { Treats the aqueous waste by whatever method has been developed in the Aqueous } \\
\text { Waste Disposition Plan. During treatment, required stack, effluent, and process } \\
\text { data are collected. The output streams from the treatment process may be stored, } \\
\text { and during storage some waste verification may be performed, if necessary. }\end{array}$ \\
\hline 4.5.4.1 & $\begin{array}{l}\text { Control Aqueous Waste Treatment Process } \\
\text { Controls the treatment of the aqueous waste, including facility configuration, } \\
\text { process monitoring, and sampling and analysis the input and output streams. }\end{array}$ \\
\hline
\end{tabular}




\begin{tabular}{|c|c|}
\hline Function & Definitions \\
\hline 4.5 .4 .1 .1 & $\begin{array}{l}\text { Configure Process (AW) } \\
\text { Sets the initial facility process configuration/ and controls the process set points. }\end{array}$ \\
\hline 4.5 .4 .1 .2 & $\begin{array}{l}\text { Monitor Process (AW) } \\
\text { Monitors the aqueous waste treatment process, and passes the data to the data } \\
\text { collection and evaluation function, 4.5.4.3 and 4.5.4.1.1. (Monitoring may } \\
\text { include sampling and analysis.) }\end{array}$ \\
\hline 4.5 .4 .1 .3 & $\begin{array}{l}\text { Sample and Analyze (AW) } \\
\text { Samples and analyzes the input streams and effluents as necessary during the } \\
\text { treatment of the aqueous waste. }\end{array}$ \\
\hline 4.5.4.2 & $\begin{array}{l}\text { Perform Aqueous Waste Treatment } \\
\text { Performs the actual aqueous waste treatment, which could be some combination } \\
\text { of conditioning, separation, and destruction. Secondary waste is managed by } \\
\text { this function. }\end{array}$ \\
\hline 4.5.4.2.1 & $\begin{array}{l}\text { Condition Aqueous Waste for Treatment } \\
\text { Conditions the aqueous waste. For some forms of aqueous waste this may be the } \\
\text { only treatment required. (Examples include feed equilization, temperature and } \\
\text { Ph adjustment, etc.) }\end{array}$ \\
\hline 4.5 .4 .2 .2 & $\begin{array}{l}\text { Separate Aqueous Waste } \\
\text { Separates desired constituents from the aqueous waste. }\end{array}$ \\
\hline 4.5 .4 .2 .3 & $\begin{array}{l}\text { Destroy (AW) } \\
\text { Destroys desired constituents of the aqueous waste. }\end{array}$ \\
\hline 4.5.4.2.4 & $\begin{array}{l}\text { Manage Secondary Waste (AW) } \\
\text { Handles the secondary waste generated by the aqueous waste facilities, including } \\
\text { any additional treatment, packaging, storage and development of the required } \\
\text { waste manifest documentation. Includes solid wastes generated during cleanup } \\
\text { of the aqueous waste facilities when correcting non-compliant conditions. }\end{array}$ \\
\hline 4.5.4.3 & $\begin{array}{l}\text { Collect and Evaluate Aqueous Waste Treatment Data } \\
\text { Collects and evaluates the process data associated with the aqueous waste } \\
\text { facilities, including stack offgas, liquid effluent secondary waste data. The data is } \\
\text { evaluated and recommendations are made based on the evaluations. The data is } \\
\text { also used to verify the treatment of the waste is proceeding as planned. The data } \\
\text { is also reported to the regulatory groups as required. }\end{array}$ \\
\hline 4.5.4.3.1 & $\begin{array}{l}\text { Accumulate Data (AW) } \\
\text { Accumulates the data generated in the aqueous waste facilities. Includes } \\
\text { archival of the data. }\end{array}$ \\
\hline 4.5.4.3.2 & $\begin{array}{l}\text { Evaluate Data (AW) } \\
\text { Evaluates the accumulated data to show trending and behavior. }\end{array}$ \\
\hline 4.5.4.3.3 & $\begin{array}{l}\text { Make Recommendations (AW) } \\
\text { Makes recommendations based on the evaluation of the data trends to further } \\
\text { improve process efficiency. }\end{array}$ \\
\hline
\end{tabular}




\begin{tabular}{|c|c|}
\hline Function & Definitions \\
\hline 4.5.4.3.4 & $\begin{array}{l}\text { Verify Treatment (AW) } \\
\text { Verifies that the treatment of the aqueous waste is meeting expectations and that } \\
\text { the facilities are operating correctly. }\end{array}$ \\
\hline 4.5.4.3.5 & $\begin{array}{l}\text { Report Data (AW) } \\
\text { Reports the necessary data to the appropriate regulatory agencies as required. } \\
\text { (For example stack effluent data may be required.) }\end{array}$ \\
\hline 4.5.5 & $\begin{array}{l}\text { Disposition Aqueous Waste } \\
\text { Performs final disposition. Waste may be disposed, reused, or released. } \\
\text { Verification is performed to ensure that the waste meets all regulatory and } \\
\text { permit requirements. }\end{array}$ \\
\hline 4.5.5.1 & $\begin{array}{l}\text { Document Disposition Compliance } \\
\text { Documents the compliant disposition of wastes. }\end{array}$ \\
\hline 4.5 .5 .1 .1 & $\begin{array}{l}\text { Generate Records (AW) } \\
\text { Generates the associated records and manifests for the disposition of the waste. }\end{array}$ \\
\hline 4.5.5.1.2 & $\begin{array}{l}\text { Report Disposition Compliance (AW) } \\
\text { Reports the compliance of the waste disposition. }\end{array}$ \\
\hline 4.5.5.1.3 & $\begin{array}{l}\text { Archive Disposition Compliance Information (AW) } \\
\text { Retains the disposition compliance information generated. }\end{array}$ \\
\hline 4.5.5.2 & $\begin{array}{l}\text { Dispose of Waste (AW) } \\
\text { Disposes of the waste by transferring it to the appropriate disposal functions or } \\
\text { processing facilities determined in the disposition plan. }\end{array}$ \\
\hline 4.5.5.2.1 & $\begin{array}{l}\text { Provide Interim Storage (AW) } \\
\text { Performs the interim storage of the waste before it is disposed or recycled. }\end{array}$ \\
\hline 4.5.5.2.2 & $\begin{array}{l}\text { Transfer Waste to Disposal Function (AW) } \\
\text { Transfers the waste to the appropriate disposal function as determined by the } \\
\text { disposition plan. }\end{array}$ \\
\hline 4.5.5.2.3 & $\begin{array}{l}\text { Recycle for Reuse (AW) } \\
\text { Recycles aqueous effluents within the aqueous waste facility, or makes it } \\
\text { available for reuse in other facilities or applications. }\end{array}$ \\
\hline 4.5.5.2.4 & $\begin{array}{l}\text { Provide Final Disposition (AW) } \\
\text { Provides the final disposition of the wastes which can be disposed of directly by } \\
\text { the aqueous waste facilities. }\end{array}$ \\
\hline 4.6 & $\begin{array}{l}\text { Correct Unsafe Infrastructure Conditions } \\
\text { The CORRECT UNSAFE INFRASTRUCTURE CONDITIONS (4.6) function has } \\
\text { a dual purpose, namely to correct all unsafe or non-compliant conditions as well } \\
\text { as operation of all the services and facilities not identified with the programs or } \\
\text { integrated cleanup activities. Infrastructure includes such items as roads, } \\
\text { utilities, transportation, office buildings }\end{array}$ \\
\hline 4.6 .1 & Operate Infrastructure \\
\hline
\end{tabular}




\begin{tabular}{|c|c|}
\hline Function & Definitions \\
\hline 4.6 .2 & $\begin{array}{l}\text { Maintain Infrastructure } \\
\text { Requires a safe operation to be maintained along with performing the upgrades } \\
\text { necessary to support the safe operation function. }\end{array}$ \\
\hline 4.7 & $\begin{array}{l}\text { Store, Treat, and Disposition SNM/NM/NF Materials } \\
\text { The management of Special Nuclear Materials (SNM), Nuclear Materials }(N M), \\
\text { and Nuclear Fuels (NF) is the receiving, handling, processing, storing, and } \\
\text { transfer for ultimate disposition of the materials in a safe and efficient way. } \\
\text { SNM/NM/NF in this function includes plutonium as inventoried, uranium, } \\
\text { thorium, retrieved special materials, irradiated fuel and other irradiated non } \\
\text { waste materials, cesium and strontium capsules, and miscellaneous actinides } \\
\text { such as neptunium and californium, and nuclear standards/sources. }\end{array}$ \\
\hline 4.7 .1 & $\begin{array}{l}\text { Maintain Safety and Security Envelope (SNM/NM/NF) } \\
\text { Maintains the physical facility, qualified staff, safe and compliant equipment, } \\
\text { documentation and provides assessment of safety and compliant states. Provides } \\
\text { all necessary safety and security resources for compliance with all governing } \\
\text { safety/security codes and regulations. Also included is the periodic verification } \\
\text { of material inventory and sample analysis of the materials. }\end{array}$ \\
\hline 4.7.1.1 & $\begin{array}{l}\text { Maintain Safe and Compliant Operations (SNM/NM/NF) } \\
\text { Assesses and maintains the SNM facility operations in a safe condition that is } \\
\text { compliant with environmental, DOE Orders, Regulatory requirements, and all } \\
\text { other applicable codes, standards, and company procedures. }\end{array}$ \\
\hline 4.7.1.1.1 & $\begin{array}{l}\text { Provide Surveillance (SNM/NM/NF) } \\
\text { Provide surveillance of all facility operations and operating systems, develop } \\
\text { acutely unsafe condition action plans, perform OSR surveillance procedures, } \\
\text { environmental monitoring, RCRA tracking, and surveillance of safety analysis } \\
\text { compliance. }\end{array}$ \\
\hline 4.7 .1 .1 .2 & $\begin{array}{l}\text { Provide Access Control (SNM/NM/NF) } \\
\text { Provide access control to and internal to the facility for safeguards and security, } \\
\text { safety and radiological purposes. }\end{array}$ \\
\hline 4.7.1.1.3 & $\begin{array}{l}\text { Provide Work Control (SNM/NM/NF) } \\
\text { Provides the job control system for the facility activities. }\end{array}$ \\
\hline 4.7.1.1.4 & $\begin{array}{l}\text { Provide Property Management (SNM/NM/NF) } \\
\text { Provides property management for the facilities in accordance with DOE orders } \\
\text { and WHC procedures. }\end{array}$ \\
\hline 4.7 .1 .1 .5 & $\begin{array}{l}\text { Perform Self Assessments (SNM/NM/NF) } \\
\text { Internal assessment of the facility operations to ensure that safety and } \\
\text { compliance are maintained. }\end{array}$ \\
\hline 4.7 .1 .1 .6 & $\begin{array}{l}\text { Support Independent Oversights (SNM/NM/NF) } \\
\text { Provides personnel to assist with independent safety and compliant assessment } \\
\text { audits. }\end{array}$ \\
\hline
\end{tabular}




\begin{tabular}{|c|c|}
\hline Function & Definitions \\
\hline 4.7 .1 .1 .7 & $\begin{array}{l}\text { Dispose of Incidental Waste (Operations) } \\
\text { Arranges for the disposal of solid, liquid, and aqueous wastes generated during } \\
\text { the maintenance of the safetr and compliance envelope. }\end{array}$ \\
\hline 4.7.1.2 & $\begin{array}{l}\text { Maintain Safe and Compliant Systems, Equipment, and Structure } \\
\text { (SNM/NM/NF) } \\
\text { Maintains the facility infrastructure and facility systems in the operational } \\
\text { condition dictated by approved safety and compliance documentation. } \\
(\text { SNM/NM/NF) }\end{array}$ \\
\hline 4.7 .1 .2 .1 & $\begin{array}{l}\text { Perform Corrective Maintenance (SNM/NM/NF) } \\
\text { All maintenance that brings systems and equipment back to its operational state } \\
\text { after failure. }\end{array}$ \\
\hline 4.7.1.2.2 & $\begin{array}{l}\text { Perform Preventative Maintenance (SNM/NM/NF) } \\
\text { Preventative maintenance activities to minimize all unplanned events and } \\
\text { premature equipment failures. }\end{array}$ \\
\hline 4.7 .1 .2 .3 & $\begin{array}{l}\text { Perform Process Systems and Equipment Calibrations (SNM/NM/NF) } \\
\text { Performs facility equipment, instrumentation and process system calibrations to } \\
\text { ensure accuracy. }\end{array}$ \\
\hline 4.7.1.2.4 & $\begin{array}{l}\text { Perform Minor Facility/Equipment Modifications (SNM/NM/NF) } \\
\text { Performs minor modifications to SNM facility systems or structures necessary to } \\
\text { ensure safe and compliant operations which do not require new capabilities from } \\
\text { provide mission essential capabilities. }\end{array}$ \\
\hline 4.7.1.2.5 & $\begin{array}{l}\text { Dispose of Incidental Waste (Maintenance) } \\
\text { Arranges for the disposal of the solid, liquid, and aqueous wastes generated } \\
\text { during the facility maintenance activities. }\end{array}$ \\
\hline 4.7 .1 .3 & $\begin{array}{l}\text { Maintain Safety and Compliance Documentation (SNM/NM/NF) } \\
\text { Maintains all required facility safety, compliance, engineering, inventory and } \\
\text { operating documentation. }\end{array}$ \\
\hline 4.7.1.3.1 & $\begin{array}{l}\text { Maintain Safety Documents (SNM/NM/NF) } \\
\text { Maintains documentation necessary to ensure economical, safe and compliant } \\
\text { handling, storage, treatment, and disposition of SNM/NM/NF materials. }\end{array}$ \\
\hline 4.7.1.3.2 & $\begin{array}{l}\text { Maintain Operations Procedures (SNM/NM/NF) } \\
\text { Maintains operating procedure documentation. }\end{array}$ \\
\hline 4.7 .1 .3 .3 & $\begin{array}{l}\text { Maintain Engineering Documentation (SNM/NM/NF) } \\
\text { Maintains configuration drawings and associated engineering documentation } \\
\text { required to maintain the facility in a safe and compliant status. }\end{array}$ \\
\hline 4.7.1.3.4 & $\begin{array}{l}\text { Maintain Maintenance Procedures (SNM/NM/NF) } \\
\text { Maintains maintenance procedure documentation necessary for efficient, safe, } \\
\text { and compliant operation. }\end{array}$ \\
\hline 4.7 .1 .3 .5 & $\begin{array}{l}\text { Maintain Compliance Documentation (SNM/NM/NF) } \\
\text { Maintains documentation to assure and prove compliance with appropriate } \\
\text { environmental, regulatory and DOE orders. }\end{array}$ \\
\hline
\end{tabular}




\begin{tabular}{|c|c|}
\hline Function & Definitions \\
\hline 4.7 .1 .3 .5 .1 & $\begin{array}{l}\text { Maintain SNM/NM/NF Inventory Documentation } \\
\text { Maintains an inventory of all materials in the SNM/NM/NF category in } \\
\text { accordance with DOE orders. }\end{array}$ \\
\hline 4.7 .1 .3 .5 .2 & $\begin{array}{l}\text { Maintain Criticality Documentation (SNM, NM, NF) } \\
\text { Maintains criticality calculations and other applicable documents. }\end{array}$ \\
\hline 4.7 .1 .4 & $\begin{array}{l}\text { Maintain Qualified Facility Staff (SNM/NM/NF) } \\
\text { Provides facility specific training, testing, and training records maintenance to } \\
\text { ensure the facility staff remain trained, qualified, and certified as required. }\end{array}$ \\
\hline 4.7 .1 .4 .1 & $\begin{array}{l}\text { Provide Training (SNM/NM/NF) } \\
\text { Provides all training related to the handling, storage, treatment, and disposition } \\
\text { of SNM/NM/NF materials and training necessary to safely operate and maintain } \\
\text { the facility and facility operating systems. }\end{array}$ \\
\hline 4.7 .1 .4 .2 & $\begin{array}{l}\text { Maintain Certification and Qualification (SNM/NM/NF) } \\
\text { Provides periodic personnel skills check, assessment, and testing required to } \\
\text { maintain the necessarv qualification and certification. }\end{array}$ \\
\hline 4.7 .1 .4 .3 & $\begin{array}{l}\text { Maintain Training Documentation (SNM/NM/NF) } \\
\text { Maintains applicable worker training documentation. Documentation includes } \\
\text { worker safety and competency qualification and certification. }\end{array}$ \\
\hline 4.7 .1 .5 & $\begin{array}{l}\text { Assess Safety and Compliance State (SNM/NM/NF) } \\
\text { Assesses the handling, treatment, storage, and disposition of all SNM/NM/NF } \\
\text { materials to ensure that safety and compliance is maintained. }\end{array}$ \\
\hline 4.7 .2 & $\begin{array}{l}\text { Control SNM, NM, and NF Functions } \\
\text { Plans, coordinates, and schedules all the necessary operations within the } 4.7 \\
\text { function. The function defines the treatment, storage, and transfer needs for the } \\
\text { materials; establishes the acceptance criteria; and performs the acceptance or } \\
\text { rejection of the material shipping documentation. The function does not include } \\
\text { the physical work to perform the operations. }\end{array}$ \\
\hline 4.7 .2 .1 & $\begin{array}{l}\text { Define Treat/Store/Disp osition Operations } \\
\text { Performs the planning and assessment of necessary material treatment, storage } \\
\text { and disposition actions necessary for safe and efficient material receipts, } \\
\text { treatment, storage and disposition. }\end{array}$ \\
\hline 4.7 .2 .1 .1 & $\begin{array}{l}\text { Establish Acceptance Criteria } \\
\text { Establishes the criteria necessary to accept the material based on the capibility } \\
\text { to handle, treat. store and disposition the material. }\end{array}$ \\
\hline 4.7 .2 .1 .1 .1 & $\begin{array}{l}\text { Establish Storage/Disposition Criteria } \\
\text { Identifies storage and disposition criteria associated with the proposed receipt of } \\
\text { SNM/NM/NF material. }\end{array}$ \\
\hline 4.7.2.1.1.2 & $\begin{array}{l}\text { Establish Treatment Criteria } \\
\text { Identifies the treatment criteria associated with the proposed receipt of } \\
\text { SNM/NM/NF materials. }\end{array}$ \\
\hline
\end{tabular}




\begin{tabular}{|c|c|}
\hline Eunction & Definitions \\
\hline 4.7 .2 .1 .2 & $\begin{array}{l}\text { Evaluate Forecast Generator Requests } \\
\text { Evaluates generator forecasts to determine storage capacity needs, treatment } \\
\text { eptions and disposition prospects for all forecasted materials receipts. }\end{array}$ \\
\hline 4.7 .2 .1 .3 & $\begin{array}{l}\text { Define Capabillty Needs } \\
\text { Defines existing handlins, treatment, storase and disposition capabilities. }\end{array}$ \\
\hline 4.7 .2 .1 .3 .1 & $\begin{array}{l}\text { Deffne Storage Capability } \\
\text { Defines the remaining facility storase capability. }\end{array}$ \\
\hline 4.7 .2 .1 .3 .2 & $\begin{array}{l}\text { Define Treatment Capability } \\
\text { Defines the existing facility specific treatment canabilities. }\end{array}$ \\
\hline 4.7 .2 .1 .3 .3 & $\begin{array}{l}\text { Define Acceptance Capability } \\
\text { Defines the SNM/NM/NF material form, type, consistency, and packaging } \\
\text { required for acceptance into the facilio. }\end{array}$ \\
\hline 4.7.2.1.4 & $\begin{array}{l}\text { Define Materia/Storage/Treatment/Disposition Requirements } \\
\text { Defines the material handling, treatment. storage and disposition requirements } \\
\text { necessary to process a specific material shipment. }\end{array}$ \\
\hline 4.7 .2 .2 & $\begin{array}{l}\text { Negotiate Shipper/Receiver Agreement } \\
\text { Negotiates acceptance terms and requirements with the material shipper. }\end{array}$ \\
\hline 4.7.2.2.1 & $\begin{array}{l}\text { Assess Capability to Accept Requested Transfer } \\
\text { Examines existing facility handling, treatment, storage, and disposition } \\
\text { capabilities to verify capability exists to accept the proposed transfer. }\end{array}$ \\
\hline 4.7.2.2.2 & $\begin{array}{l}\text { Identify Variances from Acceptance Criteria } \\
\text { Compares the facility SNM/NM/NF acceptance criteria with the proposed } \\
\text { SNM/NM/NF current owner criteria to determine variances. }\end{array}$ \\
\hline 4.7 .2 .2 .3 & $\begin{array}{l}\text { Accept/Reject Requests } \\
\text { Transfer requests are accepted or rejected based upon the facility acceptance } \\
\text { capability. }\end{array}$ \\
\hline 4.7 .2 .3 & $\begin{array}{l}\text { Plan and Schedule Operations } \\
\text { Plans all work operations for specific material shipment transfers. Plans } \\
\text { necessary to receive, treat, store and disposition specific material transfers are } \\
\text { performed. }\end{array}$ \\
\hline 4.7 .2 .3 .1 & $\begin{array}{l}\text { Plan Handling and Receipts } \\
\text { Develops plans to receive and handle SNM/NM/NF materials. }\end{array}$ \\
\hline 4.7.2.3.2 & $\begin{array}{l}\text { Plan Storage } \\
\text { Develops SNM/NM/NF storage plans. }\end{array}$ \\
\hline 4.7 .2 .3 .3 & $\begin{array}{l}\text { Plan Dispositions } \\
\text { Develops the SNM/NM/NF disposition plans. }\end{array}$ \\
\hline 4.7.2.3.4 & $\begin{array}{l}\text { Plan Safety, Security, and Maintenance } \\
\text { Plans all facility safety, security, and maintenance activities. }\end{array}$ \\
\hline
\end{tabular}




\begin{tabular}{|c|c|}
\hline Eunction & Definitions \\
\hline 4.7 .3 & $\begin{array}{l}\text { Handle Incoming Materials } \\
\text { Physically handles the material shipping container and packaged material } \\
\text { receipt. It is responsible for disposal of the wastes generated during the } \\
\text { handline process. }\end{array}$ \\
\hline 4.7 .3 .1 & $\begin{array}{l}\text { Receive Shipment } \\
\text { The process of receiving the actual shipment of materials. This function ensures } \\
\text { readiness to accept material, ensures transfer of custody, verifies manifest and } \\
\text { material documentation, and is responsible for obtaining radiological surveys. }\end{array}$ \\
\hline 4.7.3.1.1 & $\begin{array}{l}\text { Ensure Receipt Readiness } \\
\text { Verifies the readiness to receive a specific SNM/NM/NF shipment. }\end{array}$ \\
\hline 4.7 .3 .1 .2 & $\begin{array}{l}\text { Verify Manifest and Materials Documents } \\
\text { Examines manifest and material documents for accuracy and completeness. }\end{array}$ \\
\hline 4.7 .3 .1 .3 & $\begin{array}{l}\text { Transfer Custody } \\
\text { Performs the SNMINMINF material transfer of custody as defined in } 5630 . \mathrm{Wa} \text {. }\end{array}$ \\
\hline 4.7 .3 .1 .4 & $\begin{array}{l}\text { Conduct Radiological Survey } \\
\text { Performs radiological surveys to ensure the received shipment shipping } \\
\text { containers are not externally radioactively contaminated. }\end{array}$ \\
\hline 4.7.3.2 & $\begin{array}{l}\text { Handle Shipping Containers } \\
\text { Performs lag storage, shipping container unloading, shipping container } \\
\text { decontamination, and recycling of the shipping containers. }\end{array}$ \\
\hline 4.7 .3 .2 .1 & $\begin{array}{l}\text { Offload Shipping Containers } \\
\text { Performs the unloading of shippins containers from the transporter. }\end{array}$ \\
\hline 4.7 .3 .2 .2 & $\begin{array}{l}\text { Provide Lag Storage for Containers } \\
\text { Provides temporary storage for received shipping containers. }\end{array}$ \\
\hline 4.7 .3 .2 .3 & $\begin{array}{l}\text { Unload (Primary Package) Material Containers } \\
\text { Removes the primary package containing SNM/NM/NF materials from the } \\
\text { shippins container. }\end{array}$ \\
\hline 4.7.3.2.4 & $\begin{array}{l}\text { Survey and Release Shipping Containers and Transporters } \\
\text { Releases the shipping containers and transporters after radiological surveys are } \\
\text { performed to ensure they are not externally radiologically contaminated. }\end{array}$ \\
\hline 4.7 .3 .2 .4 .1 & $\begin{array}{l}\text { Decontaminate Containers } \\
\text { Decontaminates shippine containers to levels acceptable for reuse. }\end{array}$ \\
\hline 4.7 .3 .2 .4 .2 & $\begin{array}{l}\text { Recycle Containers } \\
\text { Recycles the shipping containers for reuse. }\end{array}$ \\
\hline 4.7.3.3 & $\begin{array}{l}\text { Handle Packages and Verify Material } \\
\text { Physically handles the packages containing material. It verifies that the material } \\
\text { is of the form, type, consistency and quantity noted in the material documentation } \\
\text { and shipping manifest. }\end{array}$ \\
\hline
\end{tabular}




\begin{tabular}{|c|c|}
\hline Eunction & \\
\hline 4.7 .3 .3 .1 & $\begin{array}{l}\text { Analyze Material } \\
\text { Characterizes samples and/or material to verify the actual SNM/NM/NF material } \\
\text { matches the associated manifest and material documentation. }\end{array}$ \\
\hline 4.7 .3 .3 .2 & $\begin{array}{l}\text { Reconcile Exceptions } \\
\text { Reconciles the differences between the actual material characteristics and those } \\
\text { material characteristics noted in the manifest and materialdocumentation. }\end{array}$ \\
\hline 4.7.3.3.3 & $\begin{array}{l}\text { Transfer Package to Storage } \\
\text { Transfers the SNM/NMINE material package to storage. }\end{array}$ \\
\hline 4.7 .3 .3 .4 & $\begin{array}{l}\text { Ensure Package Integrity } \\
\text { Examines and evaluates the SNM/NM/NF material package condition. }\end{array}$ \\
\hline 4.7 .3 .3 .5 & $\begin{array}{l}\text { Archive Material Documentation and Analysis Data } \\
\text { Ensures the safe, secure storage and retrieval of SNM/NM/NF analysis data and } \\
\text { information. }\end{array}$ \\
\hline 4.7.3.4 & $\begin{array}{l}\text { Dispose of Incidental Waste (Shipping) } \\
\text { Arranges for the disposal of solid, liquid, and effluent wastes generated during } \\
\text { the handling of incomine materials. }\end{array}$ \\
\hline 4.7.4 & $\begin{array}{l}\text { Store Materials } \\
\text { Prepares and stabilizes the materials for storage and stores these materials until } \\
\text { they are transferred to disposition. It is responsible for disposal of wastes } \\
\text { Renerated durine the material storage process. }\end{array}$ \\
\hline 4.7.4.1 & $\begin{array}{l}\text { Stabilize for Storage } \\
\text { Treats the SNM/NMNNF material to permit safe storage. }\end{array}$ \\
\hline 4.7 .4 .1 .1 & $\begin{array}{l}\text { Characterize Material for Treatment Determination (Storage) } \\
\text { Examines the material characterization to determine treatment necessary to store } \\
\text { SNM/NMINE material. }\end{array}$ \\
\hline 4.7 .4 .1 .3 & $\begin{array}{l}\text { Package for Storage } \\
\text { Performs the packaging necessary to store SNM/NMINF materials. }\end{array}$ \\
\hline 4.7.4.2 & $\begin{array}{l}\text { Store Stable Materials } \\
\text { Provides all periodic inspections and tests to ensure the safety and security of the } \\
\text { materials placed in storase. }\end{array}$ \\
\hline 4.7.4.2.1 & $\begin{array}{l}\text { Verify Package Integrity } \\
\text { Examines and evaluates the SNMINM/NF storage packuging condition. }\end{array}$ \\
\hline 4.7.4.2.2 & $\begin{array}{l}\text { Document and Report Status } \\
\text { Provides information on the status of all stored SNM/NM/NF materials and } \\
\text { maintains SNM/NM/NF inventories. }\end{array}$ \\
\hline 4.7.4.3 & $\begin{array}{l}\text { Dispose of Incidental Waste (Storage) } \\
\text { Arranges for the disposal of solid, liquid, and effluent wastes generated during } \\
\text { the treatment of material prior to storage and during the actual storage of the } \\
\text { material. }\end{array}$ \\
\hline
\end{tabular}




\begin{tabular}{|l|l|}
\hline Function & Definitions \\
\hline 4.7 .5 & $\begin{array}{l}\text { Transfer Outgoing Materials for Disposition } \\
\text { Prepares, stabilizes, and transfers materials for dispostion. The disposition of } \\
\text { materials is one of two processes: The transfer of useable material or the transfer } \\
\text { of materials for disposal. It arranges for disposal of incidental wastes generated } \\
\text { during the dispostion process. }\end{array}$ \\
\hline $\begin{array}{l}\text { Prepare for Final Disposition } \\
\text { Characterizes material for disposition treatment, treats the material and } \\
\text { packases itfor disposition. }\end{array}$ \\
\hline $\begin{array}{l}\text { Characterize Material for Disposition } \\
\text { Examines material characterization to determine necessary disposition actions. }\end{array}$ \\
\hline $\begin{array}{l}\text { Treat for Disposition } \\
\text { Performs the necessary operations to comply with disposition criteria. }\end{array}$ \\
\hline $\begin{array}{l}\text { Package for Disposition } \\
\text { Provides the necessary packaging to allow for the SNM/NM/NF material } \\
\text { disposition. }\end{array}$ \\
\hline $\begin{array}{l}\text { Transfer Dispositioned Materials } \\
\text { Obtains and loads the shipping containers and transporter. It prepares the } \\
\text { necessary shipping documentation, makes the necessary notifications, and ships } \\
\text { the material. }\end{array}$ \\
\hline $\begin{array}{l}\text { Obtain/Load Shipping Container and Transporter } \\
\text { Arranges for the necessary transporter and shipping containers, loads the } \\
\text { shipping containers, and places the shipping containers on the transporter to } \\
\text { transfer the materials to disposition. }\end{array}$ \\
\hline $\begin{array}{l}\text { Document and Notify Shipment } \\
\text { Performs the necessary notification prior to the material shipment and prepares } \\
\text { the necessary shipping documentation. }\end{array}$ \\
\hline $\begin{array}{l}\text { Move Shipment } \\
\text { Transfers the SNM/NM/NF materials to disposition. }\end{array}$ \\
\hline $\begin{array}{l}\text { 4.7.7.5pose of Incidental Waste (Disposition) } \\
\text { Arranges for the disposal of solid, liquid and effluent wastes generated during } \\
\text { the treatment and dispositioning of the materials. }\end{array}$ \\
$\begin{array}{l}\text { Transition Resources For Beneficial Use } \\
\text { The TRANSTION RESOURCES FOR BENEFICIAL USE function provides for } \\
\text { tre disposal of excess land, materials and personnel; recycling of material, } \\
\text { facilities, equipment and personnel to uses within the mission; and smooth } \\
\text { economic transition to a viable local economy as the cleanup mission winds } \\
\text { down. }\end{array}$ \\
\hline 5
\end{tabular}




\begin{tabular}{|l|l|}
\hline Function & Definitions \\
\hline 5.1 & $\begin{array}{l}\text { Determine Beneficial Uses Of Resources } \\
\text { The DETERMINE BENEFICIAL USES OF RESOURCES (5.1) function } \\
\text { determines which resources are candidates for use outside the mission, seeks } \\
\text { "customers" to receive the resources, and establishes agreements to actually } \\
\text { transfer specific items, as well as personnel. }\end{array}$ \\
\hline 5.1 .1 & $\begin{array}{l}\text { Inventory Resources } \\
\text { The process of identifying, characterizing, and determining the quantity and } \\
\text { availability ofpotential Hanford transferable resources. }\end{array}$ \\
\hline 5.1 .1 .1 & $\begin{array}{l}\text { Identify Resources } \\
\text { The process of determining which resources would qualify as being potentially } \\
\text { useful or beneficial to government. public and private domain groups. }\end{array}$ \\
\hline 5.1.1.2 & $\begin{array}{l}\text { Characterize Resources } \\
\text { Determine location of (map) resources, describe resource history, estimate } \\
\text { current resource value (cultural and monetary), identify resource custodian, } \\
\text { identify supporting expertise, assess requirements and constraints on transfer of } \\
\text { resource, and prepare resource database. }\end{array}$ \\
\hline $\begin{array}{l}\text { Describe Resource Attributes } \\
\text { Data that describe the current characteristics of Hanford physical and } \\
\text { intellectual resources. }\end{array}$ \\
\hline Determine Location Of Resources \\
Determine Ouantity Of Resources \\
\hline 5.1 .1 .2 .1
\end{tabular}




\begin{tabular}{|c|c|}
\hline Function & Definitions \\
\hline 5.1 .2 & $\begin{array}{l}\text { Define And Rank Alternative Potential Uses } \\
\text { The process of establishing alternative potential uses for Hanford transferable } \\
\text { resources and of ranking these alternative uses. The function includes public } \\
\text { involvement, identification of the constraints on the use and transfer of the } \\
\text { resource, and the criteria for ranking potential uses. }\end{array}$ \\
\hline 5.1 .2 .1 & Develop Preliminary-Use List \\
\hline 5.1.2.2 & $\begin{array}{l}\text { Obtain Public Involvement } \\
\text { Public involement is needed here to provide feedback about proposed possible } \\
\text { uses of resources. } \\
\text { Public involvement is defined as the point where the public needs to be involved } \\
\text { before any further actions or decisions are carried out. The amount of public } \\
\text { involvement generallv depends upon the situation at hand. }\end{array}$ \\
\hline 5.1 .2 .3 & Identify Constraints On Uses \\
\hline 5.1 .2 .4 & Define Criteria For Ranking Potential Uses \\
\hline 5.1 .2 .5 & Define Potential Uses \\
\hline 5.1 .2 .6 & Rank Potential Uses \\
\hline 5.1 .3 & $\begin{array}{l}\text { Identify Potential Users } \\
\text { The process of publicizing Hanford resource availability and of assessing the } \\
\text { potential uses of available resources by the Hanford Cleanup Mission, by other } \\
\text { Government agencies, by stakeholders, and by other private interest. The } \\
\text { process includes responding to unsolicited inquiries by the public about } \\
\text { available resources and including these potential users in the resource transfer } \\
\text { and evaluation process. Criteria for qualifying potential users will also be } \\
\text { established by this function. }\end{array}$ \\
\hline 5.1.3.1 & $\begin{array}{l}\text { Publicize Resource Availability } \\
\text { Communication of transferable/available resource information to the public. }\end{array}$ \\
\hline 5.1 .3 .2 & Assess Resource Applications In Hanford Clean Up \\
\hline 5.1 .3 .3 & Assess Federal Government Interest \\
\hline 5.1.3.4 & $\begin{array}{l}\text { Assess Stakeholder Interest } \\
\text { The assessment of stakeholder interest that includes any public element that has } \\
\text { an interest in the Hanford mission including governments, regulators, and } \\
\text { special interest groups. }\end{array}$ \\
\hline 5.1 .3 .5 & Assess Private Interest \\
\hline 5.1 .3 .6 & Respond To Inquiries \\
\hline 5.1 .3 .7 & Define Criteria For Qualifying Potential Users \\
\hline 5.1 .4 & $\begin{array}{l}\text { Develop Relationship Between Resources, Uses, And Potential Users } \\
\text { The process of matching the available (transferable) resources, with potential } \\
\text { alternative uses, and the potential users. A database management system will be } \\
\text { used to develop and analyze the information relationships. }\end{array}$ \\
\hline
\end{tabular}




\begin{tabular}{|c|c|}
\hline Function & Definitions \\
\hline 5.1 .5 & $\begin{array}{l}\text { Develop Resource Transfer Procedures } \\
\text { Define the process for redirecting resources for mission use, transfer of use } \\
\text { outside of mission, and transfer of ownership including the requirements and } \\
\text { constraints on these transfers. }\end{array}$ \\
\hline 5.1 .6 & $\begin{array}{l}\text { Confirm Market } \\
\text { The process of screening potential recipients against criteria for qualifying } \\
\text { potential users, confirming the interest of qualified recipients, and negotiating an } \\
\text { agreement in principle with the recipient for the resource transfer. The public } \\
\text { will be involved in this process as appropriate. }\end{array}$ \\
\hline 5.1.6.1 & Screen Potential Recipients \\
\hline 5.1.6.2 & Confirm Interest Of Qualified Recipients \\
\hline 5.1 .6 .3 & Negotiate Agreement-In-Principle \\
\hline 5.1 .6 .4 & Determine Need For Public Involvement \\
\hline 5.1 .7 & $\begin{array}{l}\text { Select Preferred Disposition } \\
\text { The process of evaluating the alternative disposition options for the resource, } \\
\text { selecting the preferred recipient and authorizing the resource transfer. The } \\
\text { process also includes temporary storage of resources that are redirected for } \\
\text { mission use. }\end{array}$ \\
\hline 5.1.7.1 & Rank Alternative Dispositions \\
\hline 5.1 .7 .2 & Select Recipient \\
\hline 5.1 .7 .3 & Authorize Transfer Of Resources \\
\hline 5.2 & $\begin{array}{l}\text { Transfer Ownership Of Resources Outside Of Mission } \\
\text { The TRANSFER RESOURCES FOR USE OUTSIDE THE MISSION (5.3) } \\
\text { function provides for use of excess mission resources to an outside user without } \\
\text { actually transferring ownership. }\end{array}$ \\
\hline 5.2 .1 & $\begin{array}{l}\text { Transfer Management Of Resource (Ownership) } \\
\text { The process of transferring the management (custodianship) of resources within } \\
\text { the Hanford internal management system. }\end{array}$ \\
\hline 5.2 .2 & $\begin{array}{l}\text { Transport, Store, And Maintain Resources } \\
\text { The process of transporting, storing, and maintaining resources destined for new } \\
\text { ownership outside of the Hanford mission. }\end{array}$ \\
\hline 5.2 .3 & $\begin{array}{l}\text { Transfer Resource Ownership } \\
\text { The process of finalizing the resource ownership transfer. }\end{array}$ \\
\hline 5.3 & $\begin{array}{l}\text { Transfer Resources For Use Outside Of Mission } \\
\text { The process of transferring management of the resource, modifying/upgrading } \\
\text { the resource, establishing the conditions for use, and finalizing the transfer of } \\
\text { resource use outside of the Hanford mission. }\end{array}$ \\
\hline 5.3 .1 & $\begin{array}{l}\text { Transfer Management Of Resource (Use) } \\
\text { The process of transferring the management (custodianship) of resource use } \\
\text { within the Hanford management system. }\end{array}$ \\
\hline
\end{tabular}




\begin{tabular}{|l|l|}
\hline Function & Definitions \\
\hline 5.3 .2 & $\begin{array}{l}\text { Modify/Upgrade Resources } \\
\text { The process of modifying and upgrading resources destined for new use outside } \\
\text { of the Hanford mission. This includes modifying/upgrading land, retraining } \\
\text { personnel, documenting intellectual property, and modifying/upgrading } \\
\text { technology for new use. }\end{array}$ \\
\hline Modify/Upgrade Land \\
\hline 5.3 .2 .1 & Retrain Personnel For Use Outside Mission \\
\hline 5.3 .2 .2 & Document Intellectual Property \\
\hline 5.3 .2 .3 & Modify/Upgrade Technology For New Use \\
\hline 5.3 .3 & $\begin{array}{l}\text { Establish Conditions For New Use } \\
\text { The process of defining the terms and conditions for new use of the resource, and } \\
\text { negotiating and finalizing contractual agreements. }\end{array}$ \\
\hline Define Terms And Conditions \\
\hline 5.3 .3 .1 & Negotiate And Finalize Contractual Agreement \\
\hline 5.3 .3 .2 & $\begin{array}{l}\text { Transfer Resource For New Use } \\
\text { The process of finalizing the transfer of resource use. This includes the } \\
\text { placement of people and completing resource transfer records. }\end{array}$ \\
\hline 5.3 .4 & Placement Of People \\
\hline 5.3 .4 .1 & Record Transfer \\
\hline 5.3 .4 .2 &
\end{tabular}


WHC-EP-0722 ADDENDUM 2 REV. 0

SECTION 4

INTERFACE DESCRIPTION TABLE

$\mathbf{i}$ 


\begin{tabular}{|l|l|}
\hline Interface & Constituents \\
\hline AW Cleanup Solid Waste Material & \\
\hline $\begin{array}{l}\text { AW Cleanup Waste Documentation } \\
\text { Documentation associated with the aqueous wastes } \\
\text { generated durins cleanup of the aqueous waste facilities. }\end{array}$ & \\
\hline AW Equipment and Structure Information & \\
\hline AW Operation Information & \\
\hline AW Operation and Maintenance Packages & \\
\hline $\begin{array}{l}\text { Acceptance Criteria } \\
\text { Criteria necessary to accept material based on the existing } \\
\text { capability to handle, treat, store, and dispose of the } \\
\text { material. }\end{array}$ & \\
\hline $\begin{array}{l}\text { Accepted Materials } \\
\text { Materials that meet the acceptance criteria and their form. } \\
\text { type, consistency, and quantity have been verified. }\end{array}$ & \\
\hline $\begin{array}{l}\text { Accepted Requests } \\
\text { Shipping requests that have been accepted based upon the } \\
\text { facility acceptance capability. }\end{array}$ & \\
\hline Acknowledged Unreconciled Differences & \\
\hline $\begin{array}{l}\text { Acquired Inside Information } \\
\text { Hanford-internal access arrangements and/or data files } \\
\text { that satisfy the need for mission essential information. }\end{array}$ & \\
\hline $\begin{array}{l}\text { Acquired Outside Information } \\
\text { Hanford-external access arrangements and/or data files } \\
\text { that satisfy the need for mission essential information. }\end{array}$ & \\
\hline $\begin{array}{l}\text { Acquisition Strategy Needs/Information } \\
\text { Feedback to the Manage Program function describing the } \\
\text { status of activities, including problems impeding successful } \\
\text { execution of the work defined in the Work Packages. }\end{array}$ & \\
\hline $\begin{array}{l}\text { Acquisition Strategy for Mission Essential Capability } \\
\text { Classes } \\
\text { Definition of an integrated strategy for the acquisition of } \\
\text { mission essential expertise, technology, facilities, } \\
\text { equipment, infrastructure, supplies, and integrated } \\
\text { independent services. Definition includes identification of } \\
\text { acquisition functions and requirements, and strategic } \\
\text { alternatives and selected approaches for acquiring mission } \\
\text { essential capabilities in an integrated and efficiency } \\
\text { inanner. }\end{array}$ & $\begin{array}{l}\text { Capability Classes } \\
\text { Ctrategy for Acquisition of } \\
\text { Integrated Independent Services } \\
\text { Strategy for Consolidated }\end{array}$ \\
\hline
\end{tabular}




\begin{tabular}{|c|c|}
\hline Interface & Constituents \\
\hline $\begin{array}{l}\text { Acutely Unsafe Aqueous Waste Conditions Information } \\
\text { Aqueous waste unsafe conditions and information, does } \\
\text { not include OSHA, for example. These are conditions } \\
\text { detected during the cleanup operations which need to be } \\
\text { immediately fed back to the program management function } \\
\text { for consideration which may result in new direction \& } \\
\text { control, defined work packages, and/or mission } \\
\text { requirements. }\end{array}$ & $\begin{array}{l}\text { - Acutely Unsafe Disposition } \\
\text { Conditions } \\
\text { - Acutely Unsafe Transfer } \\
\text { Conditions } \\
\text { - Acutely Unsafe Treatment } \\
\text { Conditions }\end{array}$ \\
\hline $\begin{array}{l}\text { Acutely Unsafe Aqueous Waste Storage Conditions } \\
\text { Unsafe or non-compliant conditions which arise during the } \\
\text { storage of aqueous waste. }\end{array}$ & \\
\hline \multicolumn{2}{|l|}{ Acutely Unsafe Aqueous Waste Transfer Conditions } \\
\hline \multicolumn{2}{|l|}{$\begin{array}{l}\text { Acutely Unsafe Conditions Action Information } \\
\text { Information defining the corrective action(s) necessary to } \\
\text { remedy acutely unsafe conditions. }\end{array}$} \\
\hline $\begin{array}{l}\text { Acutely Unsafe Conditions Information } \\
\text { Nuclear or chemically unsafe conditions and information, } \\
\text { does not include OSHA, for example. These are conditions } \\
\text { detected during the cleanup operations which need to be } \\
\text { immediately fed back to the program management function } \\
\text { for consideration which may result in new direction \& } \\
\text { control, defined work packages, and/or mission } \\
\text { requirements. }\end{array}$ & $\begin{array}{l}\text { - Received Solid Waste Safety } \\
\text { Documentation } \\
\end{array}$ \\
\hline \multicolumn{2}{|l|}{ Acutely Unsafe Conditions in Scheduling } \\
\hline \multicolumn{2}{|l|}{$\begin{array}{l}\text { Acutely Unsafe Corrective Action Plan } \\
\text { Plan to correct unsafe conditions in the rqueous waste } \\
\text { facilities. }\end{array}$} \\
\hline \multicolumn{2}{|c|}{$\begin{array}{l}\text { Acutely Unsafe Disposition Conditions } \\
\text { Conditions which are unsafe or non-compliant related to } \\
\text { the disposition of the aqueous waste. }\end{array}$} \\
\hline $\begin{array}{l}\text { Acutely Unsafe Transfer Conditions } \\
\text { Unsafe or non-compliant conditions related to the transfer } \\
\text { of aqueous waste from the waste generator. Parent arrow } \\
\text { of Acutely Unsafe Transfer Requests, Acutely Unsafe } \\
\text { Conditions in Scheduling, Acutely Unsafe Aqueous Waste } \\
\text { Transfer Conditions, and Acutely Unsafe Aqueous Waste } \\
\text { Storage Conditions. }\end{array}$ & $\begin{array}{l}\text { - Acutely Unsafe Aqueous Waste } \\
\text { Storage Conditions } \\
\text { - Acutely Unsafe Aqueous Waste } \\
\text { Transfer Conditions } \\
\text { - Acutely Unsafe Conditions in } \\
\text { Scheduling } \\
\text { - Acutely Unsafe Transfer Requests }\end{array}$ \\
\hline $\begin{array}{l}\text { Acutely Unsafe Transfer Requests } \\
\text { Transfer requests which are unsafe or non-compliant. }\end{array}$ & \\
\hline
\end{tabular}




\begin{tabular}{|l|l|}
\hline Interface & Constituents \\
\hline $\begin{array}{l}\text { Acutely Unsafe Treatment Conditions } \\
\text { The conditions which are identified as unsafe or } \\
\text { non-compliant during the treatment of aqueous waste. }\end{array}$ & \\
\hline $\begin{array}{l}\text { Additional Analysis/Process Knowledge Needs } \\
\text { Needs for additional analysis of the solid waste samples or } \\
\text { solid waste process knowledge to complete assessment of } \\
\text { characterization information. }\end{array}$ & \\
\hline $\begin{array}{l}\text { Additional Aqueous Waste Capability Needs } \\
\text { Additional needs requested to obtain the capability to treat } \\
\text { the aqueous waste. }\end{array}$ & \\
\hline $\begin{array}{l}\text { Additional Aqueous Waste Disposition Needs } \\
\text { Request for additional needs for waste disposition. }\end{array}$ & \\
\hline $\begin{array}{l}\text { Additional Aqueous Waste Permitting Needs } \\
\text { Additional needs due to permit or lack of permits for the } \\
\text { acceptance of the aqueous waste. }\end{array}$ & \\
\hline Administratively Restricted Land & \\
\hline $\begin{array}{l}\text { Allocated Resources } \\
\text { Financial resources authorized to the implementing } \\
\text { organization for completion of the function }\end{array}$ & \\
\hline $\begin{array}{l}\text { Applicable External Requirements } \\
\text { The total set of external requirements that emerges; after } \\
\text { interactions with external entities to resolve conflicts, } \\
\text { ambiguities, and effectiveness issues; to become the basis } \\
\text { for formulation of the Hanford cleanup program. }\end{array}$ & $\begin{array}{l}\text { Requirements } \\
\text { Approval to Schedule Aqueous Waste Receipt }\end{array}$ \\
\hline $\begin{array}{l}\text { Aqueous Waste Compatibility Confirmation } \\
\text { Confirmation that the aqueous waste is compatible with } \\
\text { the aqueous waste treatment facilities. }\end{array}$ & \\
\hline $\begin{array}{l}\text { Aqueous Waste Compliance Documentation } \\
\text { Aqueous waste compliance documentation, which is used } \\
\text { to assess and ensure the safety and compliance state of the } \\
\text { facilities is maintained. }\end{array}$ & \\
\hline
\end{tabular}




\begin{tabular}{|c|c|}
\hline Interface & Constituents \\
\hline $\begin{array}{l}\text { Aqueous Waste Disposition Compliance } \\
\text { Needs/Information } \\
\text { The information or needs required for compliance and } \\
\text { disposition of the waste. Parent arrow of Requests for } \\
\text { Additional Aqueous Waste Information, Requests for } \\
\text { Additional Aqueous Waste Characterization Information, } \\
\text { Additional Aqueous Waste Permitting Needs, Additional } \\
\text { Aqueous Waste Capability Needs, Aqueous Waste } \\
\text { Treatability Test Needs, and Additional Aqueous Waste } \\
\text { Disposition Needs. }\end{array}$ & $\begin{array}{l}\text { - Additional Aqueous Waste } \\
\text { Capability Needs } \\
\text { - Additional Aqueous Waste } \\
\text { Disposition Needs } \\
\text { - Additional Aqueous Waste } \\
\text { Permitting Needs } \\
\text { - Aqueous Waste Treatability Test } \\
\text { Needs } \\
\text { - Requests for Additional Aqueous } \\
\text { Waste Characterization Information } \\
\text { - Requests for Additional Aqueous } \\
\text { Waste Information }\end{array}$ \\
\hline \multicolumn{2}{|l|}{$\begin{array}{l}\text { Aqueous Waste Disposition Needs/Information } \\
\text { The information and needs for the disposition of the } \\
\text { aqueous waste. }\end{array}$} \\
\hline \multicolumn{2}{|l|}{$\begin{array}{l}\text { Aqueous Waste Disposition Plan } \\
\text { Plan developed which specifies the transfer, storage, } \\
\text { treatment, and final disposition plan for the aqueous waste } \\
\text { to be received. }\end{array}$} \\
\hline Aqueous Waste Generated Cleaning Up Solid Wastes & $\begin{array}{l}\text { - SW Cleanup Aqueous Waste } \\
\text { Documentation } \\
\text { - SW Cleanup Aqueous Waste } \\
\text { Material }\end{array}$ \\
\hline Aqueous Waste Generated Cleaning Up Tank Waste & $\begin{array}{l}\text { - TW Cleanup Aqueous Waste } \\
\text { Documentation } \\
\text { - TW Cleanup Aqueous Waste } \\
\text { Material }\end{array}$ \\
\hline Aqueous Waste Generated Correcting Infrastructure & $\begin{array}{l}\text { - Infrastructure Aqueous Waste } \\
\text { Documentation } \\
\text { - Infrastructure Aqueous Waste } \\
\text { Material }\end{array}$ \\
\hline $\begin{array}{l}\text { Aqueous Waste Generated During Deactivation } \\
\text { Any thing that is not tank or solid waste including liquid } \\
\text { effluence, hazardous liquid waste, and mixed hadardous } \\
\text { waste. TBD. }\end{array}$ & $\begin{array}{l}\text { - Deactivation Aqueous Waste } \\
\text { Documentation } \\
\text { - Deactivation Aqueous Waste } \\
\text { Material }\end{array}$ \\
\hline Aqueous Waste Generated During Remedy/Restore & $\begin{array}{l}\text { - Remedy/Restore Aqueous Waste } \\
\text { Documentation } \\
\text { - Remedy/Restore Aqueous Waste } \\
\text { Material }\end{array}$ \\
\hline
\end{tabular}




\begin{tabular}{|c|c|}
\hline Interface & Constituents \\
\hline $\begin{array}{l}\text { Aqueous Waste Generated Handling SNM/NM/NF } \\
\text { Aqueous wastes is liquid effluent, process condensate, } \\
\text { purge water, steam condensates, cooling water, storm } \\
\text { water, floor drain (cleaning wastes), } D \& D \text { generated } \\
\text { wastes, janitorial wastes, and laboratory wastes. }\end{array}$ & $\begin{array}{l}\text { - SNM/NM/NF Cleanup Aqueous } \\
\text { Waste Documentation } \\
\text { - SNM/NM/NF Cleanup Aqueous } \\
\text { Waste Material }\end{array}$ \\
\hline $\begin{array}{l}\text { Aqueous Waste Internal Safety Operations Constraint } \\
\text { Documents } \\
\text { These are documents that relate the internal safety } \\
\text { operational constraints for the aqueous waste systems, } \\
\text { equipment, and structures. }\end{array}$ & \\
\hline $\begin{array}{l}\text { Aqueous Waste Manifest } \\
\text { The manifest, required by environmental regulations, to be } \\
\text { associated and transmitted with the aqueous waste. }\end{array}$ & \\
\hline $\begin{array}{l}\text { Aqueous Waste Profile Information } \\
\text { Aqueous waste profile information provided by the } \\
\text { aqueous waste generator. }\end{array}$ & \\
\hline $\begin{array}{l}\text { Aqueous Waste Qualified Staff } \\
\text { These are personnel that have been trained and qualified } \\
\text { to perform the aqueous waste facility operations. }\end{array}$ & \\
\hline $\begin{array}{l}\text { Aqueous Waste Receipt Authorization } \\
\text { Authorization passed to the Receive/Transport function } \\
\text { which allows the receiving of the aqueous waste. }\end{array}$ & \\
\hline $\begin{array}{l}\text { Aqueous Waste Receipt Needs/Info } \\
\text { The information and needs for receiving the aqueous } \\
\text { waste. Parent arrow is Aqueous Waste Processing Needs } \\
\text { and Information. }\end{array}$ & $\begin{array}{l}\text { - Aqueous Waste Scheduling Needs } \\
\text { - Aqueous Waste Storage Needs } \\
\text { - Aqueous Waste Transfer Needs } \\
\text { - Transfer Request Needs }\end{array}$ \\
\hline $\begin{array}{l}\text { Aqueous Waste Remedy Needs/Information } \\
\text { In the course of managing waste generated during } \\
\text { operations and clean up, needs and information may be } \\
\text { discovered which may impact direction \& control, mission } \\
\text { requirements, and defined work packages. This } \\
\text { information must be fed back to the MANAGE PROGRAM } \\
\text { function for evaluation. }\end{array}$ & $\begin{array}{l}\text { - Aqueous Waste Disposition } \\
\text { Compliance Needs/Information } \\
\text { - Aqueous Waste Needs/Info } \\
\text { - Aqueous Waste Receipt } \\
\text { Needs/Info } \\
\text { - Aqueous Waste Treatment } \\
\text { Needs/Info } \\
\end{array}$ \\
\hline $\begin{array}{l}\text { Aqueous Waste Safety and Compliance Constraints } \\
\text { Control mechanism to maintain the safety and compliance } \\
\text { envelope. }\end{array}$ & \\
\hline $\begin{array}{l}\text { Aqueous Waste Scheduling Needs } \\
\text { The needs derived from scheduling the aqueous waste } \\
\text { transfer. Parent arrow is Aqueous Waste Receipt Needs } \\
\text { and Information. }\end{array}$ & \\
\hline
\end{tabular}




\begin{tabular}{|l|l}
\hline Interface & Constituents \\
\hline
\end{tabular}

Aqueous Waste Storage Needs

The needs and requirements for aqueous waste storage.

Parent arrow is Aqueous Waste Receipt Needs and Information.

Aqueous Waste Transfer Needs

The needs and requirements for aqueous waste transfer.

Parent arrow is Aqueous Waste Receipt Needs and

Information.

Aqueous Waste Transfer Schedules

The schedules of aqueous waste transfers and the schedules of the required facilities.

Aqueous Waste Treatability Test Needs

Request for treatability tests to determine the treatability

of the aqueous waste by the facilities.

Aqueous Waste Treatment Needs/Info

The needs and information associated with the treatment

of the aqueous waste.

Aqueous Waste Treatment Process Control

The direction and control generated to control the aqueous waste treatment.

Assessed Solid Waste Capability Needs

These are assessed capability needs to support retrieval, treatment storage and disposal of the solid waste.

Audit Plan (AW)

The plan for auditing the safety and compliance state of the aqueous waste facilities.

Audit Records (AW)

Records generated during audits of the safety and compliance state of the aqueous waste facilities.

Authorization for Transfer of Resources

Approval of the recommended disposition of resources that satisfy the transfer requirements and constraints for transfer outside of mission.

Baseline Revision Needs

Budget

Budget (funding) to conduct the mission from the DOE customer.

Budget Information

Budgeted Funds 


\begin{tabular}{|l|l|}
\hline Interface & Constituents \\
\hline $\begin{array}{l}\text { Capability Description } \\
\text { Information on the existing facility treat, store, handling, } \\
\text { and disposition capabilities and personnel readiness. }\end{array}$ & \\
\hline $\begin{array}{l}\text { Career Development Information } \\
\text { Feedback to the Develop Expertise function from the } \\
\text { Retain Expertise function describing the career history, } \\
\text { pathing, and opportunities for personnel supporting the } \\
\text { Hanford clean-up mission. }\end{array}$ & \\
\hline $\begin{array}{l}\text { Categorized Information } \\
\text { Information on what type or category material fits in. (ie. } \\
\text { waste. SNM. NM. NE. potential product. etc) }\end{array}$ & \\
\hline $\begin{array}{l}\text { Certified Solid Waste } \\
\text { This is solid waste that meets the certification } \\
\text { requirements that allow either on-site or off-site } \\
\text { disposition. }\end{array}$ & $\cdot$ Off-site Certified Solid Waste \\
\hline $\begin{array}{l}\text { Certified Solid Waste Archive Data and Samples } \\
\text { This is archive data and samples that were part of the solid } \\
\text { waste certification process that were requested to support } \\
\text { further certification work. }\end{array}$ & \\
\hline $\begin{array}{l}\text { Certified Solid Waste Data } \\
\text { This is data related to the solid waste certification process } \\
\text { that will allow storage and disposition of the solid waste } \\
\text { and will be included in the waste disposition status } \\
\text { information. }\end{array}$ & \\
\hline $\begin{array}{l}\text { Certified Solid Waste Data and Samples } \\
\text { This is data and samples that were part of the solid waste } \\
\text { certification process that require archival storage. }\end{array}$ & \\
\hline $\begin{array}{l}\text { Characterization Data } \\
\text { Information collected from facility walkdowns and } \\
\text { documentation review about the actual physical } \\
\text { configuration of the facility, status of process and other } \\
\text { facility systems, material contents, and contamination } \\
\text { areas and levels. }\end{array}$ & \\
\hline $\begin{array}{l}\text { Characterized Materials } \\
\text { Material in the facility that has been categorized. }\end{array}$ & \\
\hline $\begin{array}{l}\text { Cleanup Program Information } \\
\text { Cleanup Program Information consisting of program } \\
\text { plans for review, comment and information, progress } \\
\text { information, and resource allocation information to the } \\
\text { OBTAIN PUBLIC TRUST function for public review and } \\
\text { information. }\end{array}$ & $\cdot$ Program Status Information \\
\hline
\end{tabular}




\begin{tabular}{|c|c|}
\hline Interface & Constituents \\
\hline \multicolumn{2}{|l|}{$\begin{array}{l}\text { Compliance Confirmation } \\
\text { Acknowledgement that current facility status and } \\
\text { operations meet all safety and compliance criteria. }\end{array}$} \\
\hline \multicolumn{2}{|l|}{$\begin{array}{l}\text { Conditions for Use } \\
\text { Limitations on the use of transferable resources } \\
\text { (redirected resources within the mission and resources } \\
\text { with uses outside of the mission). }\end{array}$} \\
\hline \multicolumn{2}{|l|}{$\begin{array}{l}\text { Configuration Constraints } \\
\text { Constraints placed on the disposition of resources and } \\
\text { waste by modifications to the facility to minimize cost. } \\
\text { maintenance, surveillance, and security. }\end{array}$} \\
\hline \multicolumn{2}{|l|}{$\begin{array}{l}\text { Configuration Information } \\
\text { Status on how the facility should be modified to minimize } \\
\text { cost. maintenance, surveillance, and security. }\end{array}$} \\
\hline \multicolumn{2}{|l|}{$\begin{array}{l}\text { Confirmed Technology Insufficiency } \\
\text { Confirmed need for the development of a new technology } \\
\text { to support the Hanford clean-up mission. }\end{array}$} \\
\hline \multicolumn{2}{|l|}{$\begin{array}{l}\text { Confirmed User List } \\
\text { A list of qualified individuals and groups committed to the } \\
\text { acquisition or use of a specific resource. }\end{array}$} \\
\hline \multicolumn{2}{|l|}{ Contaminated Natural Resources } \\
\hline \multicolumn{2}{|l|}{$\begin{array}{l}\text { Continued SNM, NM, NF Material Receipts (Physical) } \\
\text { The actual material involved in the continued offsite } \\
\text { material receipts. }\end{array}$} \\
\hline $\begin{array}{l}\text { Continued SNM,NM,NF Material Receipts } \\
\text { SNM/NM/NF materials and associated documentation and } \\
\text { forecasts information that are coming from offsite. }\end{array}$ & $\begin{array}{l}\text { - Continued SNM, NM, NF Material } \\
\text { Receipts (Physical) } \\
\text { - Forecast of Continued } \\
\text { SNM/NM/NF Materials Receipts } \\
\text { - Request to Receive Continued } \\
\text { SNM,NM, NF Materials } \\
\end{array}$ \\
\hline $\begin{array}{l}\text { Continued Waste/Material Receipt } \\
\text { The Hanford Site continues to receive waste and other } \\
\text { materials from offsita sources and provides waste } \\
\text { management servic. including disposal, for those } \\
\text { muterials. } \\
\text { Until national policy chany dictate otherwise, the } \\
\text { Hanford Site will provide waste management services for } \\
\text { the rest of the DOE complex and related activities (e.g., } \\
\text { nuclear submarine compartments). The Hanford Site's } \\
\text { expected role in a new national policy is yet to be } \\
\text { articulated. }\end{array}$ & $\begin{array}{l}\text { - Continued SNM,NM,NF Material } \\
\text { Receipts } \\
\text { - Solid Waste From Other Missions }\end{array}$ \\
\hline
\end{tabular}




\section{Interface \\ Constituents}

Corrected Solid Waste

Noncompliant solid waste that has had its noncompliance issues resolved.

Corrected Solid Waste Information

Information assoicated with noncompliant solid waste that has had its noncompliance issues resolved.

Corrective Action Needs

Actions that must be taken to ensure the safety and compliance envelop of the facility is maintained.

\section{Corrective Actions}

Recommended revisions to the structure, principles or policies comprising the management system, formulated in response to the findings of audits, surveillances or other assessments of program effectiveness.

\section{Counterproductive Requirements}

Requirements for which the economic, safety or other value of the benefits derived from compliance with the requirement are less than the economic, safety or other costs of compliance.

\section{Criteria for Qualifying Potential Users}

The basis for determining whether or not a potential user should receive ownership or use of a specific resource. Consideration must be given to factors such as: financial responsibility, impact on local economy, social benefits, etc.

\section{Current Year Work Plan}

Custody Transfer Acknowledgement

An acknowledgement of custody transfer.

Data for Characterization

Data assoicated with the solid waste that will be used in its characterization.

Data/Samples for Characterization

This is new waste information and samples obtained during solid waste access and removal operations and through the verification process associated with accentance.

Deactivated Facility A facility who has all non-essential components and structures deactivated.

\section{Deactivation Aqueous Waste Documentation}




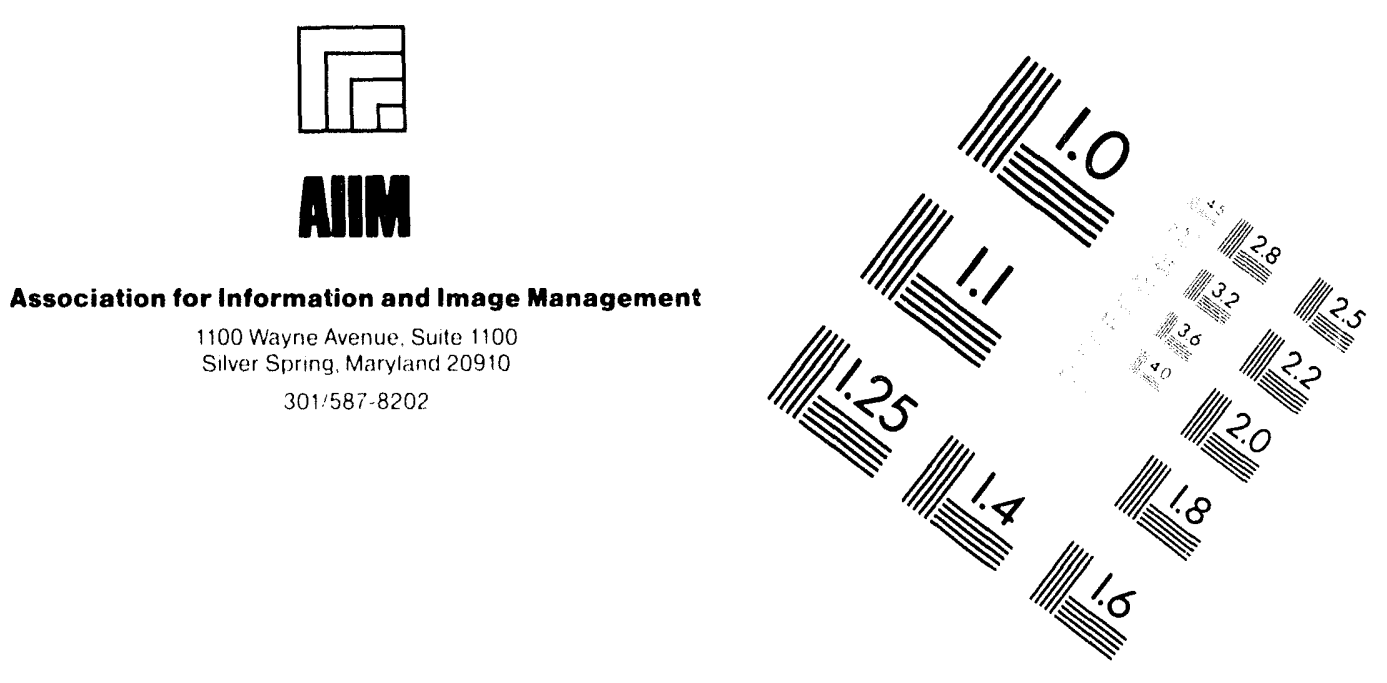

\section{Centimeter}

$\begin{array}{llllllllllllllll}1 & 2 & 3 & 4 & 5 & 6 & 7 & 8 & 9 & 10 & 11 & 12 & 13 & 14 & 15 & \mathrm{~mm}\end{array}$

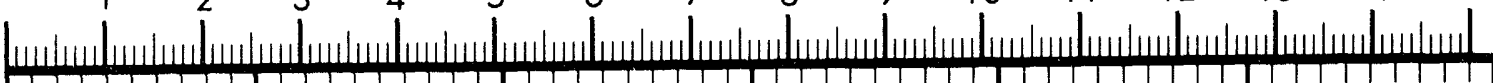

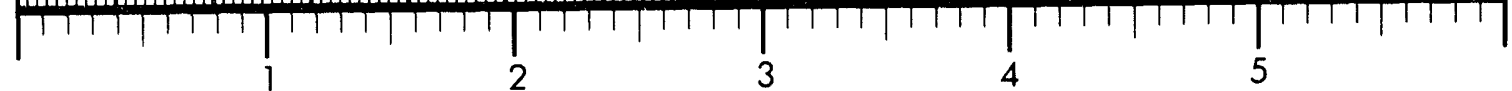
Inches
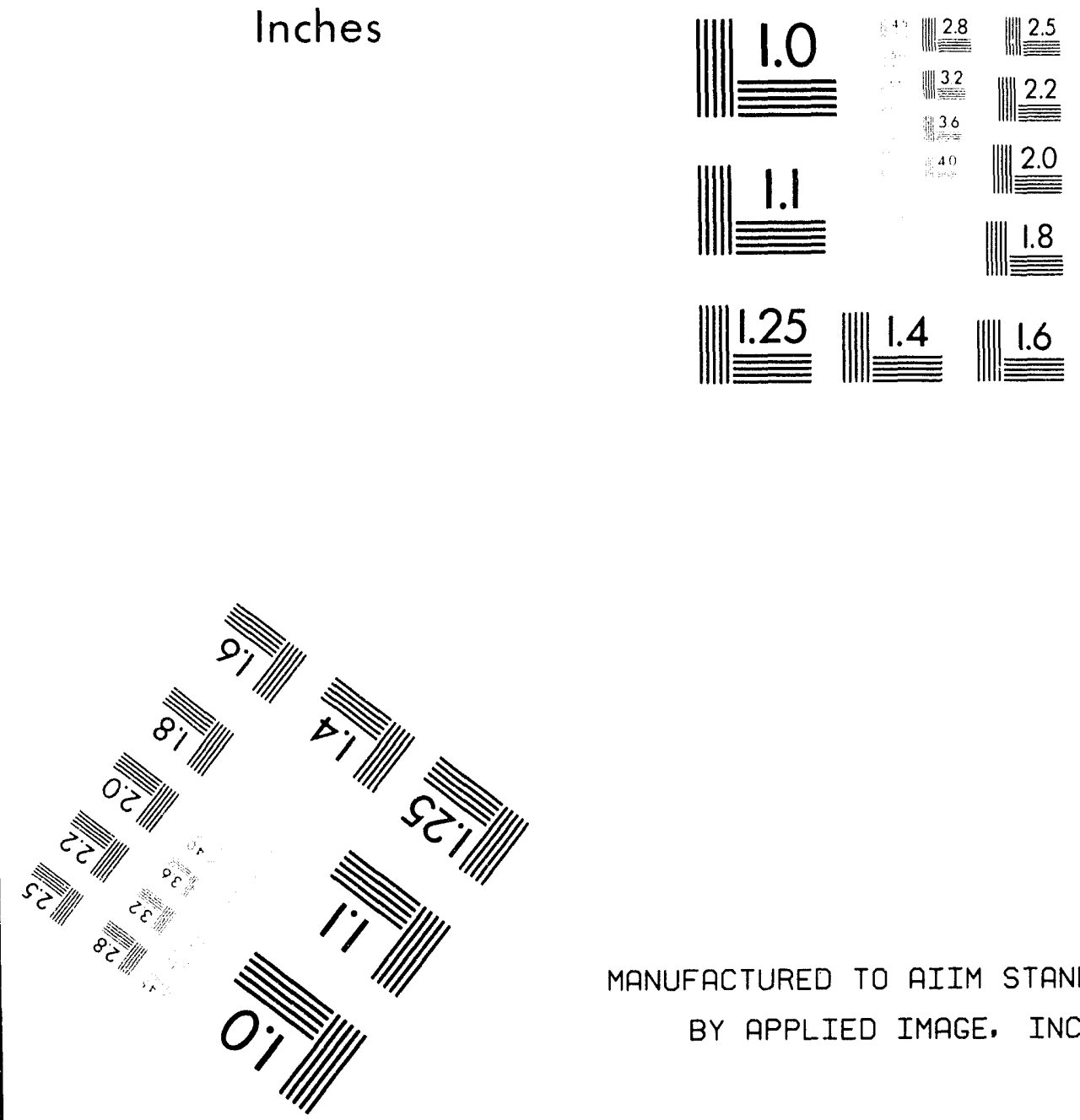
MANUFACTURED TO AIIM STANDARDS BY APPLIED IMAGE, INC.

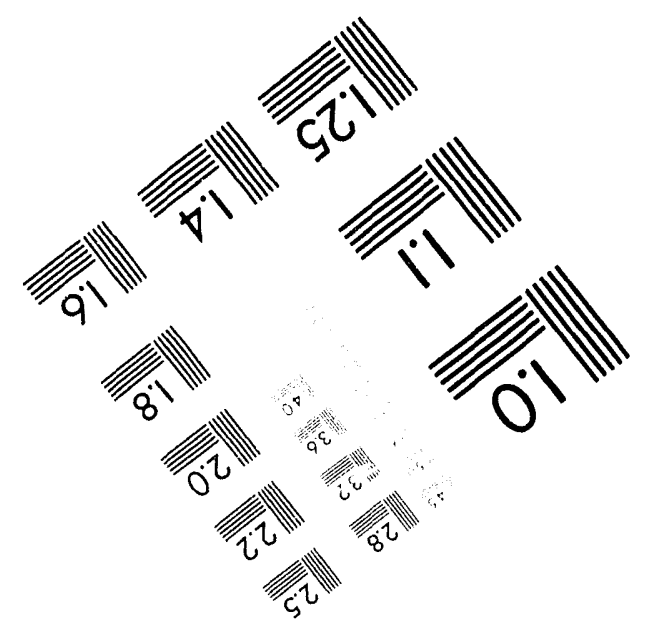



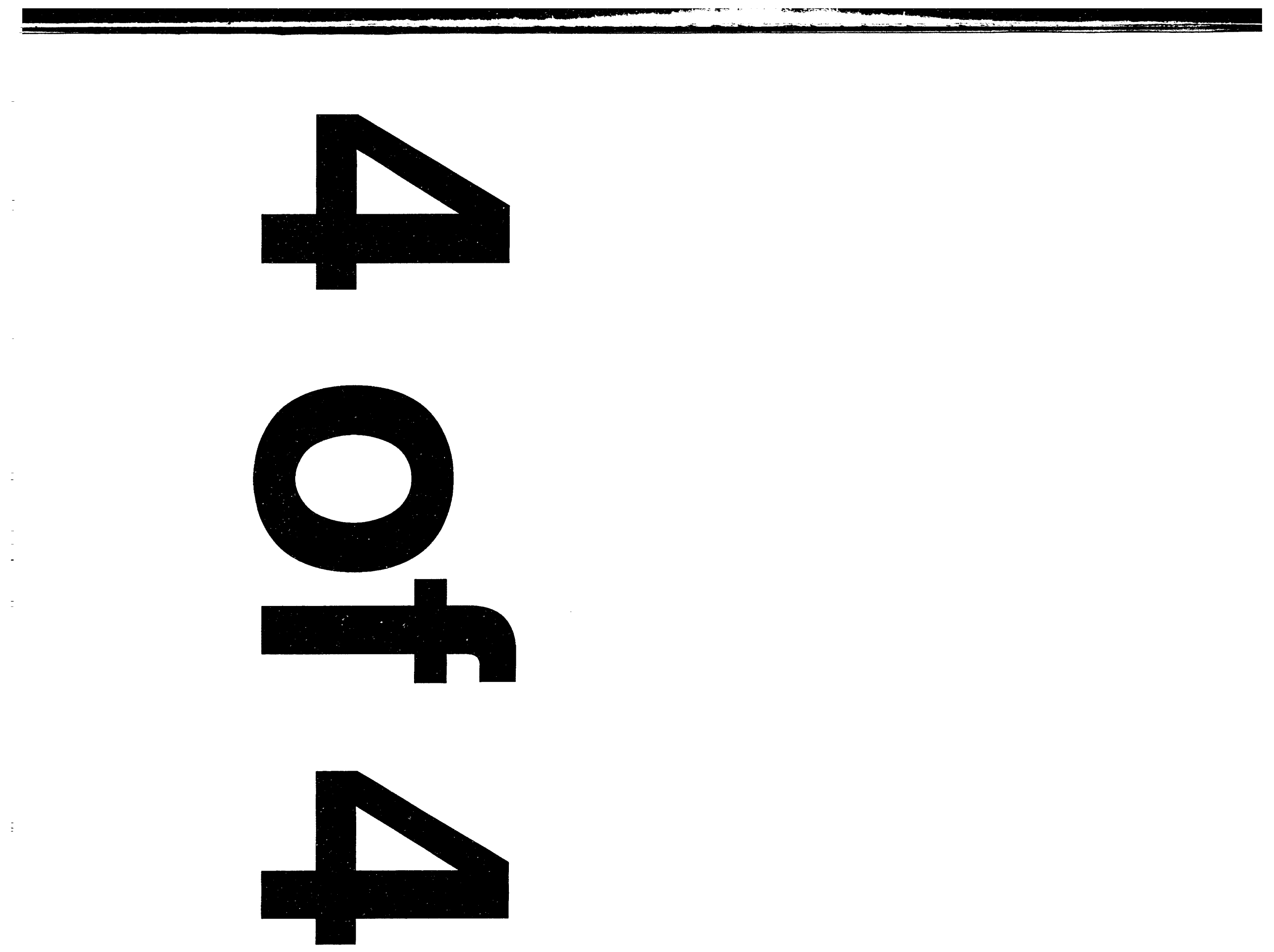


\begin{tabular}{|c|c|}
\hline Interface & Constituents \\
\hline \multicolumn{2}{|l|}{ Deactivation Aqueous Waste Material } \\
\hline $\begin{array}{l}\text { Deactivation Plan } \\
\text { Plan containing the requirements defined in the turnover } \\
\text { specification that must be met before } D \& D \text { will accept the } \\
\text { facility. Certain systems may need to be deactivated. } \\
\text { Contaminated areas will need stabilization. Materials and } \\
\text { equipment may need to be removed, ie. ER. }\end{array}$ & \\
\hline $\begin{array}{l}\text { Deactivation Safety Envelope Constraints } \\
\text { Constraints required to maintain the safety and } \\
\text { compliance envelop. Examples include HVAC system } \\
\text { requirements, configuration, electrical system } \\
\text { requirements/configuration, instrument } \\
\text { requirements/configuration, material storage } \\
\text { configuration/location, fire alarm svstem, etc. }\end{array}$ & \\
\hline \multicolumn{2}{|l|}{ Deactivation Solid Waste Material } \\
\hline \multicolumn{2}{|l|}{$\begin{array}{l}\text { Declaration of Excess Resources (Use) } \\
\text { The paperwork which releases a resource from its current } \\
\text { use by its "owning" organization and allows for new uses } \\
\text { of that resource. }\end{array}$} \\
\hline \multicolumn{2}{|l|}{$\begin{array}{l}\text { Declaration of Excess Resources (ownership) } \\
\text { The paperwork which releases a resource from its current } \\
\text { use by its "owning" organization and allows for it to be } \\
\text { transferred to a new owner. }\end{array}$} \\
\hline $\begin{array}{l}\text { Defined Baseline Information } \\
\text { Controlled program cost, schedule, and technical data that } \\
\text { describe 1) the configurations of the physical and } \\
\text { management systems and 2) the activities for the Hanford } \\
\text { cleanup program. The baseline information provides the } \\
\text { primary basis for formulating the Work Packages. }\end{array}$ & \\
\hline \multicolumn{2}{|l|}{$\begin{array}{l}\text { Defined Deactivation Work Package } \\
\text { A work package containing design media and info on what } \\
\text { needs to be done, the requirements for performance of the } \\
\text { work, directions on performance of the work, inspection } \\
\text { and documentation requirements, description of necessary } \\
\text { tools and type of personnel training required. }\end{array}$} \\
\hline $\begin{array}{l}\text { Defined Work Package } \\
\text { Documentation describing the specific work; work } \\
\text { authorization, description, procedures, resource limits and } \\
\text { schedules; controlling performance of the function }\end{array}$ & $\begin{array}{l}- \text { Defined Deactivation Work } \\
\text { Package }\end{array}$ \\
\hline $\begin{array}{l}\text { Definitive Disposition } \\
\text { The necessary handling, treatment, storage, and/or } \\
\text { disposition actions for a specific shipment of materials. }\end{array}$ & \\
\hline
\end{tabular}




\begin{tabular}{|c|c|}
\hline Interface & Constituents \\
\hline \multicolumn{2}{|l|}{$\begin{array}{l}\text { Demonstrated Technology } \\
\text { Pilot or full-scale demonstration of a new technology or } \\
\text { application of a technology for the Hanford clean-up } \\
\text { mission. }\end{array}$} \\
\hline $\begin{array}{l}\text { Dependent Local Economy } \\
\text { The local and regional economy is highly dependent upon } \\
\text { federal funding of activities at the Hanford Site. } \\
\text { Hanford Site work activity and employment comprise a } \\
\text { substantial portion of the direct economic activity in the } \\
\text { Tri-Cities (communities of Richland, Kennewick, and } \\
\text { Pasco). Other businesses and local infrastructure (e.g., } \\
\text { schools) are highly susceptible to fluctuations in Hanford } \\
\text { Site employment levels. The Hanford Site budget } \\
\text { constitutes a majority of the regions economic base. }\end{array}$ & $\begin{array}{l}\text { - Hanford Payroll } \\
\text { - Secondary Business } \\
\text { - Subsidies }\end{array}$ \\
\hline \multicolumn{2}{|l|}{ Description of Available Budget } \\
\hline \multicolumn{2}{|l|}{$\begin{array}{l}\text { Developed Expertise } \\
\text { Retrained people from within the Hanford work force. }\end{array}$} \\
\hline \multicolumn{2}{|l|}{$\begin{array}{l}\text { Development Needs for Imported Expertise } \\
\text { Expertise development needs for personnel acquired } \\
\text { outside the Hanford work force. }\end{array}$} \\
\hline \begin{tabular}{|l} 
Direction \& Control \\
Management guidance based on the status of \\
implementation of the Defined Work Packages
\end{tabular} & $\begin{array}{l}- \text { Request for Final Disposition } \\
\text { Shipment }\end{array}$ \\
\hline \multicolumn{2}{|l|}{$\begin{array}{l}\text { Disposition Status } \\
\text { Status on physically how complete the disposition of } \\
\text { resources is. }\end{array}$} \\
\hline $\begin{array}{l}\text { Dispositioned Legacy } \\
\text { Legacy materials, facilities, and wastes (including cleanup } \\
\text { byproducts and infrastructure) have been permanently } \\
\text { dispositioned consistent with land use plans and } \\
\text { applicable regulations. } \\
\\
\text { The whole cleanup job is complete. All legacy materials } \\
\text { have been dispositioned in accordance with requirements. } \\
\text { The problem hasn't simply moved from one place to } \\
\text { another or one point in time to another. Radioactive and } \\
\text { hazardous materials are safely managed (e.g., stored, } \\
\text { treated, recycled, disposed, or shipped) on the minimum } \\
\text { required land area. }\end{array}$ & $\begin{array}{l}\text { - Dispositioned Sites, Facilities, \& } \\
\text { Ground Water } \\
\text { - Dispositioned Solid Waste } \\
\text { - Dispositioned Tank Waste } \\
\text { - Releasable Effluents } \\
\text { - Safe Legacy Infrastructure } \\
\text { Conditions } \\
\end{array}$ \\
\hline Dispositioned Sites, Facilities, \& Ground Water & $\begin{array}{l}\text { - Dispositioned Ground Water } \\
\text { - Dispositioned Sites } \\
\end{array}$ \\
\hline
\end{tabular}




\begin{tabular}{|l|l|}
\hline Interface & Constituents \\
\hline Dispositioned Solid Waste \\
Solid waste that is ready for disposal or transfer to a \\
useful function within or outside the Cleanup Hanford \\
mission.
\end{tabular}




Interface
Excess Aqueous Waste Disposition Facilities
The excess facilities and equipment from the disposition
function. Parent arrow is Excess Aqueous Waste
Facilities.

Excess Aqueous Waste Facilities

Excess receiving, treatment, storage, and disposition facilities.

\section{Constituents}

- Excess Aqueous Waste

Disposition Facilities

- Excess Aqueous Waste

Receive/Transport Facilities

- Excess Aqueous Waste Treatment Facilities

Excess Aqueous Waste Receive/Transport Facilities

Excess facilities generated by the Receive/Transport Aqueous Waste function.

Excess Aqueous Waste Storage Facilities

The excess aqueous waste storage facilities which are no longer needed. Parent arrow is Receive/Transport Excess Facilities.

Excess Aqueous Waste Transfer Facilities

The facilities and equipment which can be excessed after the aqueous waste transfers, empty barrels for reuse, as an example. Parent arrow is Receive/Transport Excess Facilities.

Excess Aqueous Waste Treatment Facilities

The excess facilities and equipment from aqueous waste treatment.

Excess Facilities, Equipment \& Materials

Facilities no longer required for performance of Hanford cleanup mission functions

- Excess Aqueous Waste Storage Facilities

- Excess Aqueous Waste Transfer Facilities

\begin{tabular}{|l|l|} 
cleanup mission functions & $\begin{array}{l}\text { - Excess Remedy/Restore Facilities } \\
\text { - Excess Solid Waste Facilities } \\
\text { - Excess Storage Facilities } \\
\text { - Excess Tank Waste Facilities }\end{array}$ \\
\hline Excess Infrastructure Facilities & \\
\hline $\begin{array}{l}\text { Excess Land Transferred } \\
\text { Land and other resources have been transferred for other } \\
\text { socially beneficial uses. }\end{array}$ & \\
$\begin{array}{l}\text { Land and other resources are recovered and returned for } \\
\text { beneficial uses (e.g., caretaker, dual use infrastructure, } \\
\text { and other Hanford Site missions). }\end{array}$ & \\
\hline Excess Remedy/Restore Facilities & - Disposal Facility \\
\hline
\end{tabular}




\begin{tabular}{|c|c|}
\hline Interface & Constituents \\
\hline \multicolumn{2}{|l|}{$\begin{array}{l}\text { Excess Solid Waste Facilities } \\
\text { Facilities that have had a mission in the Hanford Cleanup } \\
\text { Mission. It includes excess sites for disposition (e.g. } \\
\text { potentially contaminated retrieved trench). }\end{array}$} \\
\hline \multicolumn{2}{|l|}{$\begin{array}{l}\text { Excess Solid Waste Samples } \\
\text { These are excess solid waste samples that will be received } \\
\text { as solid waste requiring disposition. }\end{array}$} \\
\hline \multicolumn{2}{|l|}{$\begin{array}{l}\text { Excess Storage Facilities } \\
\text { Facilities that are no longer used to store SNM/NM/NF. }\end{array}$} \\
\hline \multicolumn{2}{|l|}{ Excess Tank Waste Facilities } \\
\hline $\begin{array}{l}\text { Existing Site Resources } \\
\text { The Hanford Site does not presently possess the full set of } \\
\text { capabilities as identified below to efficiently and } \\
\text { completely carry out this mission. } \\
\text { 1. Workforce. There is an existing, multi-contractor, } \\
\text { 16,000-person highly-skilled workforce that faces an } \\
\text { unprecedented technical challenge. Extensive training will } \\
\text { be needed to meet mission needs. Not only is there a need } \\
\text { to retrain staff, but a new mix of skills is required to } \\
\text { efficiently and effectively carry out the mission. } \\
\text { 2. Technology. Appropriate and adequate technical } \\
\text { solutions to all cleanup problems do not presently exist. } \\
\text { Technology development programs are underway outside } \\
\text { of the Hanford Site, but these are not perceived to be } \\
\text { adequate to meet the needs. } \\
\text { 3. Physical Infrastructure. Much of the Hanford Site's } \\
\text { physical infrastructure is aging and deteriorating. Also, } \\
\text { waste storage, treatment, and related support services } \\
\text { (e.g., analytical laboratories) will need to be upgraded or } \\
\text { expanded to support the mission. }\end{array}$ & $\begin{array}{l}\text { - Existing Expertise } \\
\text { - Existing Facilities, Equipment \& } \\
\text { Software } \\
\text { - Existing Infrastructure } \\
\text { - Existing Site Knowledge } \\
\text { - Existing Technologies } \\
\end{array}$ \\
\hline $\begin{array}{l}\text { Existing Tank Waste } \\
\text { Existing tank waste including waste within transfer lines }\end{array}$ & \\
\hline $\begin{array}{l}\text { Expertise Needs for Essential Information } \\
\text { Feedback to the Provide Expertise function from the } \\
\text { Provide Essential Information function describing the } \\
\text { needs for expertise to support this functions, including } \\
\text { number of people and required expertise and experience. }\end{array}$ & \\
\hline
\end{tabular}




\begin{tabular}{|c|c|}
\hline Interface & Constituents \\
\hline $\begin{array}{l}\text { Expertise Needs for Faciliites, Equipment, } \\
\text { Infrastructure, \& Supplies } \\
\text { Feedbark to the Provide Expertise function from the } \\
\text { Provide Facilities, Equipment, Infrastructure, \& Supplies } \\
\text { function describing the needs for expertise to support this } \\
\text { functions, including number of people and required } \\
\text { expertise and experience. }\end{array}$ & \\
\hline $\begin{array}{l}\text { Expertise Needs for Providing Mission Essential } \\
\text { Capabilities } \\
\text { Feedback to the Provide Expertise function from the } \\
\text { Provide Essential Information, Provide Facilities, } \\
\text { Equipment, Infrastructure, \& Supplies, and Provide } \\
\text { Technology functions describing the needs for expertise to } \\
\text { support these functions, including number of people and } \\
\text { required expertise and experience. }\end{array}$ & $\begin{array}{l}\text { - Expertise Needs for Essential } \\
\text { Information } \\
\text { - Expertise Needs for Faciliites, } \\
\text { Equipment, Infrastructure, \& } \\
\text { Supplies } \\
\text { - Expertise Needs for Infrastructure } \\
\text { - Expertise Needs for Technology }\end{array}$ \\
\hline $\begin{array}{l}\text { Expertise Needs for Technology } \\
\text { Feedback to the Provide Expertise function from the } \\
\text { Provide Technology function describing the needs for } \\
\text { expertise to support this functions, including number of } \\
\text { people and required expertise and experience. }\end{array}$ & \\
\hline $\begin{array}{l}\text { Expertise Needs/Information } \\
\text { Feedback to the Manage Program function describing the } \\
\text { status of activities, including problems impeding successful } \\
\text { execution of the work defined in the Work Packages. }\end{array}$ & \\
\hline $\begin{array}{l}\text { Expertise Retention Information } \\
\text { Feedback to the Identify Sources of Expertise function } \\
\text { from the Retain Expertise function describing expertise } \\
\text { retention including data regarding the ability of Hanford } \\
\text { contractors to retain the workforce needed to complete the } \\
\text { clean-up mission. }\end{array}$ & \\
\hline $\begin{array}{l}\text { External Constraints } \\
\text { External Constraints including all system constraints } \\
\text { imposed by agreements or policy decisions made by the } \\
\text { customer or anpropriate legal authority. }\end{array}$ & \\
\hline $\begin{array}{l}\text { External Requirements } \\
\text { External Requirements including all laws, regulations, } \\
\text { orders, and policies that control design solutions or } \\
\text { operations. }\end{array}$ & \\
\hline
\end{tabular}




\begin{tabular}{|c|c|}
\hline Interface & Constituents \\
\hline $\begin{array}{l}\text { Facilities Transfered to D\&D } \\
\text { Facilities, excluding associated materials and equipment, } \\
\text { that have been stabilized and turnover for } D \& D \text { in } \\
\text { accordance with acceptance criteria defined for initiation } \\
\text { of } D \& D \text { activities }\end{array}$ & \\
\hline $\begin{array}{l}\text { Facilities, Equipment, Infrastructure, \& Supplies } \\
\text { Needs for Essential Information } \\
\text { Feedback to the Provide Facilities, Equipment, } \\
\text { Infrastructure \& Supplies function from the Provide } \\
\text { Essential Information function describing the needs for } \\
\text { equipment and infrastructure to support this function, } \\
\text { including computer networking and telecommunications } \\
\text { systems. }\end{array}$ & \\
\hline $\begin{array}{l}\text { Facilities, Equipment, Infrastructure, \& Supplies } \\
\text { Needs/Information } \\
\text { Feedback to the Manage Program function describing the } \\
\text { status of activities, including problems impeding successful } \\
\text { execution of the work defined in the Work Packages. }\end{array}$ & $\begin{array}{l}\text { - Equipment Acquisition } \\
\text { Needs/Information } \\
\text { - Project Acquisition } \\
\text { Needs/Information } \\
\text { - Supplies Acquisition } \\
\text { Needs/Information }\end{array}$ \\
\hline $\begin{array}{l}\text { Facilities/Resources Transferred To Beneficial Uses } \\
\text { Physical and intellectual resources; e.g., facilities, } \\
\text { equipment, materials, infrastructure, land, technology, } \\
\text { scientific data, personnel expertise; that are developed } \\
\text { during facility deactivation and can be transferred to other } \\
\text { areas in the public or private domains }\end{array}$ & \\
\hline $\begin{array}{l}\text { Facility Deactivation Needs/Information } \\
\text { Information, developed during performance of facility } \\
\text { deactivation activities, provided to program management } \\
\text { functions to support decision-making essential to } \\
\text { managing the deactivation process }\end{array}$ & $\begin{array}{l}\text { - Acutely Unsafe Conditions } \\
\text { Information } \\
\text { - Facility Deactivation Status } \\
\text { Information } \\
\text { - NM Information } \\
\text { - Operations \& Capability Needs } \\
\text { - Undocumented Wastes } \\
\text { Information }\end{array}$ \\
\hline $\begin{array}{l}\text { Facility Deactivation Status Information } \\
\text { Information on physically how complete the deactivation } \\
\text { of the facility is. }\end{array}$ & \\
\hline
\end{tabular}




\begin{tabular}{|c|c|}
\hline Interface & Constituents \\
\hline $\begin{array}{l}\text { Facility Materials For Disposition } \\
\text { Includes all materials inside a facility requiring } \\
\text { disposition. Equipment is not included. In addition, type } 3 \\
\text { facilities have hazardous material included. Type } 2 \\
\text { facilities have radioactive materials and possibly } \\
\text { hazardous materials and type I facilities have SNM,NM, } \\
\text { NF and possibly radioactive and hazardous materials. }\end{array}$ & \\
\hline $\begin{array}{l}\text { Findings and Observations } \\
\text { The body of information compiled in, and interpreted from, } \\
\text { program audits and assessments, for use in proparation of } \\
\text { forecasts and trends. }\end{array}$ & \\
\hline $\begin{array}{l}\text { Forecast of Continued SNM/NM/NF Materials Receipts } \\
\text { Forecast information of continued SNM/NM/NF material } \\
\text { receipts. }\end{array}$ & \\
\hline $\begin{array}{l}\text { Forecast of On-Site Nuclear Materials Receipts } \\
\text { Forecast information of on-site SNM/NM/NF material } \\
\text { receipts. }\end{array}$ & \\
\hline $\begin{array}{l}\text { Forecasts } \\
\text { Forecast information of expected handling, storage, } \\
\text { treatment, and disposition activities. }\end{array}$ & \\
\hline $\begin{array}{l}\text { Forecasts and Trends } \\
\text { Projections of technical, budget and schedule progress } \\
\text { based upon analysis of program status information and } \\
\text { upon findings and observations provided by audits and } \\
\text { assessments; includes recommendations for program } \\
\text { redirection to mitigate or reverse unfavorable aspects of } \\
\text { current or projected conditions. }\end{array}$ & \\
\hline
\end{tabular}




\begin{tabular}{|c|c|}
\hline Interface & Constituents \\
\hline $\begin{array}{l}\text { Health'Environmental Non-Compliance Legacy From } \\
\text { Former: Missions } \\
\text { The legacy from the Hanford Site's former missions poses } \\
\text { unacceptable current and future risks to the public, } \\
\text { workers, and environment. } \\
\text { Hanford Site's legacy includes contaminated land, } \\
\text { groundwater, and facilities from prior uses; deteriorating } \\
\text { facilities and infrastructure that pose potential threats to } \\
\text { the Hanford Site's workforce; ongoing releases of } \\
\text { materials to the environment (some controlled and some } \\
\text { uncontrolled) through multiple pathways, including to the } \\
\text { Columbia River; and large inventories of stored and } \\
\text { disposed radioactive wastes, nuclear materials, and other } \\
\text { hazardous wastes, including some wastes that are not well } \\
\text { characterized, thus leading to safety issues and } \\
\text { uncertainties in the selection of appropriate processing } \\
\text { approaches. }\end{array}$ & $\begin{array}{l}\text { - Contaminated Natural Resources } \\
\text { - Existing Tank Waste } \\
\text { - Legacy Facilities, Equipment \& } \\
\text { Non-Nuclear Materials } \\
\text { - Legacy Nuclear Materials } \\
\text { - Legacy Solid Waste } \\
\text { - Unsafe Legacy Infrastructure } \\
\text { Conditions } \\
\end{array}$ \\
\hline \multicolumn{2}{|l|}{ ID Key Issues For PI } \\
\hline \multicolumn{2}{|l|}{$\begin{array}{l}\text { Identification of Potentially Transferrable Resources } \\
\text { Identification of potentially transferable resources which } \\
\text { consists of excess inventory information }\end{array}$} \\
\hline \multicolumn{2}{|l|}{$\begin{array}{l}\text { Identified Need for Inside Information } \\
\text { Identification of the need for mission essential information } \\
\text { that may be obtained from Hanford-internal sources or } \\
\text { activities. }\end{array}$} \\
\hline \multicolumn{2}{|l|}{$\begin{array}{l}\text { Identified Need for Outside Information } \\
\text { Identification of the need for mission essential information } \\
\text { that may be obtained from Hanford-external sources or } \\
\text { suppliers. }\end{array}$} \\
\hline \multicolumn{2}{|l|}{$\begin{array}{l}\text { Identified Need to Develop Expertise } \\
\text { Identification of the need for mission essential expertise } \\
\text { that may be developed from existing Hanford expertise. }\end{array}$} \\
\hline $\begin{array}{l}\text { Identified Need to Import Expertise } \\
\text { Identification of the need for mission essential expertise } \\
\text { that can be obtained only by acquiring the expertise from } \\
\text { outside of the existing Hanford work force. }\end{array}$ & \\
\hline $\begin{array}{l}\text { Identified Need to Retain Expertise } \\
\text { Identification of the need to retain mission essential } \\
\text { expertise from the existing Hanford work force. }\end{array}$ & \\
\hline
\end{tabular}




\begin{tabular}{|c|c|}
\hline Interface & Constituents \\
\hline $\begin{array}{l}\text { Imported Expertise } \\
\text { Expertise that is obtained by acquired people from outside } \\
\text { the existing Hanford work force. }\end{array}$ & \\
\hline $\begin{array}{l}\text { Incidental Waste from Handling } \\
\text { Solid, aqueous, and tank waste generated during the } \\
\text { handling of shipping containers. }\end{array}$ & \\
\hline $\begin{array}{l}\text { Incidental Waste from Radiological Survey } \\
\text { Solid, aqueous, and tank waste generated during } \\
\text { radiological surveys and analysis of the SNM/NM/NF } \\
\text { material packages. }\end{array}$ & \\
\hline $\begin{array}{l}\text { Incidental Waste from Receiving } \\
\text { Solid, aqueous, and tank wastes generated during the } \\
\text { receipt of SNM/NM/NF material shipments. }\end{array}$ & \\
\hline $\begin{array}{l}\text { Incoming Shipment Authorization } \\
\text { The official paperwork giving the authorization or } \\
\text { acceptance of an incoming material shipment request. }\end{array}$ & \\
\hline $\begin{array}{l}\text { Information On Waste Samples Requiring } \\
\text { Characterization } \\
\text { This is information related to solid waste samples that } \\
\text { require more characterization data before final disposition } \\
\text { can be made. }\end{array}$ & \\
\hline Infrastructure Aqueous Waste Documentation & \\
\hline Infrastructure Aqueous Waste Material & \\
\hline Infrastructure Solid Waste Materials & \\
\hline Infrastructure Status Information & \\
\hline Infrastructure Upgrade Needs & \\
\hline $\begin{array}{l}\text { Institutional Information } \\
\text { Institutional information provided to the regulators } \\
\text { concerning planning, progress, and priority actions. }\end{array}$ & \\
\hline $\begin{array}{l}\text { Insufficient Expertise } \\
\text { Identification of insufficient expertise to meet the needs of } \\
\text { the Hanford clean-up mission. }\end{array}$ & \\
\hline $\begin{array}{l}\text { Insufficient Facilities, Equipment, Infrastructure, \& } \\
\text { Supplies } \\
\text { Identification of insufficient facilities, equipment, } \\
\text { infrastructure, \& supplies to meet the needs of the Hanford } \\
\text { clean-up mission. }\end{array}$ & $\begin{array}{l}\text { - Insufficient Equipment } \\
\text { - Insufficient Projects } \\
\text { - Insufficient Supplies }\end{array}$ \\
\hline
\end{tabular}




\begin{tabular}{|c|c|}
\hline Interface & Constituents \\
\hline \multicolumn{2}{|l|}{$\begin{array}{l}\text { Insufficient Integrated Independent Services } \\
\text { Identification of insufficient integrated independent } \\
\text { services to meet the needs of the Hanford clean-up } \\
\text { mission. }\end{array}$} \\
\hline $\begin{array}{l}\text { Insufficient Mission Essential Capabilities } \\
\text { Identification of insufficient expertise, technology, } \\
\text { facilities, equipment, infrastructure, supplies, information, } \\
\text { or integrated independent services to meet the needs of the } \\
\text { Hanford clean-up mission. }\end{array}$ & $\begin{array}{l}\text { - Insufficient Expertise } \\
\text { - Insufficient Facilities, Equipment, } \\
\text { Infrastructure, \& Supplies } \\
\text { - Insufficient Infrastructure } \\
\text { - Insufficient Integrated Independent } \\
\text { Services } \\
\text { - Insufficient Mission Essential } \\
\text { Information } \\
\text { - Insufficient Technology }\end{array}$ \\
\hline \multicolumn{2}{|c|}{$\begin{array}{l}\text { Insufficient Mission Essential Information } \\
\text { Identification of insufficient mission essential information } \\
\text { to meet the needs of the Hanford clean-up mission, } \\
\text { including characteristics of timeliness, accuracy, } \\
\text { completeness, and/or cost-effective availability of } \\
\text { information necessary to perform mission essential } \\
\text { functions and activities. }\end{array}$} \\
\hline $\begin{array}{l}\text { Insufficient Plan And Control } \\
\text { The Hanford Site lacks a comprehensive plan for } \\
\text { controlling cleanup actions. } \\
\text { The whole cleanup job has not been fully specified. The } \\
\text { endpoints for the mission have not been defined and } \\
\text { agreed upon, and the methods or approaches for carrying } \\
\text { out the mission have not been fully defined. Such a plan is } \\
\text { needed to guide and control cleanup activity. The } \\
\text { Tri-Party Agreement (Ecology et al. 1990) drives cleanup } \\
\text { and resource allocation but is not a complete plan for all } \\
\text { mission activities. }\end{array}$ & \\
\hline \multicolumn{2}{|l|}{$\begin{array}{l}\text { Insufficient Technology } \\
\text { Identification of insufficient technology to meet the needs } \\
\text { of the Hanford clean-up mission. }\end{array}$} \\
\hline $\begin{array}{l}\text { Integrated Independent Services Needs/Information } \\
\text { Feedback to the Manage Program function describing the } \\
\text { status of activities, including problems impeding successful } \\
\text { execution of the work defined in the Work Packages. }\end{array}$ & \\
\hline
\end{tabular}




\begin{tabular}{|l|l|}
\hline Interface & Constituents \\
\hline $\begin{array}{l}\text { Internal SNM/NM/NF Safety Operation Constraints } \\
\text { Document } \\
\text { Document defining SNM/NM/NF safety and compliant } \\
\text { operating constraints. }\end{array}$ & \\
\hline $\begin{array}{l}\text { Internal Safety Operations Constraint Documents } \\
\text { Safety analysis reports, radiological safety procedures, } \\
\text { occupational safety codes and standards, DOE safety } \\
\text { orders, etc. }\end{array}$ & \\
\hline Land Requiring Remediation & \\
\hline $\begin{array}{l}\text { Landfill Wastes } \\
\text { Waste suitahle for disposition in a landfill. Does not } \\
\text { include solid, tank, or effluent waste. }\end{array}$ & \\
\hline $\begin{array}{l}\text { Legacy Facilities, Equipment \& Non-Nuclear Materials } \\
\text { Facilities, including all equipment and materiel associated } \\
\text { with those facilities, that supported the Hanford } \\
\text { production mission and are not needed to support the } \\
\text { clcanun mission }\end{array}$ & \\
\hline $\begin{array}{l}\text { Legacy Nuclear Materials } \\
\text { Fissile material and any other isotopes that may have } \\
\text { resource value and that cannot be designated as waste } \\
\text { until assessment of disposition options has been completed }\end{array}$ & \\
\hline $\begin{array}{l}\text { Legacy Solid Waste } \\
\text { It is existing waste resulting from Hanford's defense } \\
\text { material production and energy production missions. }\end{array}$ & - Legacy Waste Information \\
\hline $\begin{array}{l}\text { Legacy Waste Information } \\
\text { The information associated with the legacy solid waste. }\end{array}$ & \\
\hline $\begin{array}{l}\text { Legacy Waste Materials } \\
\text { The solid waste material itself associated with legacy } \\
\text { waste. }\end{array}$ & \\
\hline $\begin{array}{l}\text { Legacy Waste Materials for Packaging } \\
\text { These are removed legacy waste materials that are ready } \\
\text { for packaging. }\end{array}$ & \\
\hline $\begin{array}{l}\text { Legacy Waste Materials for Transport } \\
\text { These are removed legacy waste materials that are ready } \\
\text { or transport to final disposition. }\end{array}$ & \\
\hline $\begin{array}{l}\text { Less Dependent Local Economy } \\
\text { Resources used during cleanup have provided secondary } \\
\text { benefits to enhance the economic viability of the regional } \\
\text { economy. }\end{array}$ & $\bullet$ Transitioned Economy \\
\hline $\begin{array}{l}\text { Liquid Waste Documentation Generated During AW } \\
\text { Cleanup }\end{array}$ & \\
\hline
\end{tabular}




\begin{tabular}{|c|c|}
\hline Interface & Constituents \\
\hline \multicolumn{2}{|l|}{$\begin{array}{l}\text { Liquid Wastes Generated During AW Cleanup } \\
\text { Liquid wastes generated during the cleanup of aqueous } \\
\text { waste facilities as part of maintaining the safety and } \\
\text { compliance envelope. }\end{array}$} \\
\hline $\begin{array}{l}\text { Liquid Wastes and Documentation Generated During } \\
\text { AW Cleanup }\end{array}$ & $\begin{array}{l}\text { - Liquid Waste Documentation } \\
\text { Generated During AW Cleanup } \\
\text { - Liquid Wastes Generated During } \\
\text { AW Cleanup }\end{array}$ \\
\hline \multicolumn{2}{|l|}{$\begin{array}{l}\text { List of Management System Components and } \\
\text { Approaches } \\
\text { A comprehensive list of the candidate policies, procedures, } \\
\text { methods of operation, roles and responsibilies, programs } \\
\text { of rewards and discipline, and interface requirements from } \\
\text { which a preferred set will be selected as the basis for the } \\
\text { management system for performance of the Hanford } \\
\text { cleanup mission. The list will recommend the corporate } \\
\text { culture, the management structure (e.g., line, matrix, team, } \\
\text { or selective combination), and directions for instituting } \\
\text { guality and safety programs. }\end{array}$} \\
\hline \multicolumn{2}{|l|}{$\begin{array}{l}\text { List of Resources Redirected for Mission } \\
\text { The identification of resources which are no longer } \\
\text { available since they have been assigned to support other } \\
\text { activities associated with the cleanup mission. }\end{array}$} \\
\hline \multicolumn{2}{|l|}{$\begin{array}{l}\text { List of Resources Transferred Outside of Mission } \\
\text { The identification of resources which are no longer } \\
\text { available since they have been transferred for } \\
\text { ownership/use outside of the mission. }\end{array}$} \\
\hline \multicolumn{2}{|l|}{ Maintained Infrastructure } \\
\hline \multicolumn{2}{|l|}{$\begin{array}{l}\text { Management System Revision Needs } \\
\text { Description of problems related to formulation of an } \\
\text { acceptable program within the constraints imposed by the } \\
\text { management system structure. }\end{array}$} \\
\hline \multicolumn{2}{|l|}{$\begin{array}{l}\text { Matched User/Resource List } \\
\text { List of all Hanford resources whose use has been matched } \\
\text { with a potential user. }\end{array}$} \\
\hline \multicolumn{2}{|l|}{$\begin{array}{l}\text { Materials For RePackaging } \\
\text { Any materials that are not properly packaged for } \\
\text { transport. }\end{array}$} \\
\hline $\begin{array}{l}\text { Materials Requiring Rework } \\
\text { Materials in storage that need further treatment to ensure } \\
\text { continued storage in safe and compliant manner. }\end{array}$ & \\
\hline
\end{tabular}




\begin{tabular}{|c|c|}
\hline Interface & Constituents \\
\hline $\begin{array}{l}\text { Materials for Disposition } \\
\text { SNM/NM/NF materials that have undergone treatment and } \\
\text { are ready for transfer to final disposition. }\end{array}$ & \\
\hline \multicolumn{2}{|l|}{$\begin{array}{l}\text { Materials for Rework } \\
\text { SNM/NM/NF materials requiring further treatment prior } \\
\text { to their transfer to final disposition. }\end{array}$} \\
\hline Mission Direction & $\begin{array}{l}\text { - Nuclear Materials Disposition } \\
\text { Policy }\end{array}$ \\
\hline $\begin{array}{l}\text { Mission Essential Capabilities } \\
\text { Mission essential capabilities in the form of all physical } \\
\text { resources, manpower, technology, infrastructure, expertise } \\
\text { required by all the other functions to conduct their } \\
\text { submissions. }\end{array}$ & $\begin{array}{l}\text { - Mission Essential Expertise } \\
\text { - Mission Essential Facilities, } \\
\text { Equipment, Infrastructure, \& } \\
\text { Supplies } \\
\text { - Mission Essential Information } \\
\text { - Mission Essential Infrastructure } \\
\text { - Mission Essential Integrated } \\
\text { Independent Services } \\
\text { - Mission Essential Technology }\end{array}$ \\
\hline $\begin{array}{l}\text { Mission Essential Capability Needs/Information } \\
\text { Mission essential capabilities/needs information in terms } \\
\text { of financial and schedular requirements; acquisition } \\
\text { progress information. }\end{array}$ & $\begin{array}{l}\text { - Acquisition Strategy } \\
\text { Needs/Information } \\
\text { - Essential Information } \\
\text { Needs/Information } \\
\text { - Expertise Needs/Information } \\
\text { - Facilities, Equipment, } \\
\text { Infrastructure, \& Supplies } \\
\text { Needs/Information } \\
\text { - Infrastructure Needs/Information } \\
\text { - Integrated Independent Services } \\
\text { Needs/Information } \\
\text { - Technology Needs/Information }\end{array}$ \\
\hline \multicolumn{2}{|l|}{$\begin{array}{l}\text { Mission Essential Equipment } \\
\text { Equipment needed to perform mission essential functions } \\
\text { and activities. }\end{array}$} \\
\hline $\begin{array}{l}\text { Mission Essential Expertise } \\
\text { Expertise needed to perform mission essential functions } \\
\text { and activities. }\end{array}$ & $\begin{array}{l}\text { - Developed Expertise } \\
\text { - Imported Expertise } \\
\text { - Retained and Motivated Expertise }\end{array}$ \\
\hline $\begin{array}{l}\text { Mission Essential Facilities, Equipment, Infrastructure, } \\
\text { \& Supplies } \\
\text { Facilities, equipment, infrastructure, and supplies needed } \\
\text { to perform mission essential functions and activities. }\end{array}$ & $\begin{array}{l}\text { - Mission Essential Equipment } \\
\text { - Mission Essential Projects } \\
\text { - Mission Essential Supplies }\end{array}$ \\
\hline $\begin{array}{l}\text { Mission Essential Information } \\
\text { Essential information needed to perform mission essential } \\
\text { functions and activities. }\end{array}$ & \\
\hline
\end{tabular}




\begin{tabular}{|l|l|}
\hline Interface & Constituents \\
\hline Mission Essential Integrated Independent Services \\
Integrated independent services needed to perform mission \\
essential functions and activities, including services which \\
may be contracted to privately-financed enterprises. The \\
government acquires only the service without any \\
additional financial obligation.
\end{tabular}




\begin{tabular}{|c|c|}
\hline Interface & Constituents \\
\hline $\begin{array}{l}\text { Need for Refinement of Component and Approach List } \\
\text { The description of problems related to formulation of an } \\
\text { optimal management system based upon the list of } \\
\text { candidate components and approaches from which } \\
\text { selections must be made. }\end{array}$ & \\
\hline $\begin{array}{l}\text { Need for Revision of Component and Approach } \\
\text { Information } \\
\text { The description of problems related to formulation of an } \\
\text { optimal management system based upon the information } \\
\text { provided for the list of components and approaches from } \\
\text { which selections must be made. }\end{array}$ & $\begin{array}{l}\text { - Need for Reevaluation of } \\
\text { Components and Approaches } \\
- \text { Need for Refinement of } \\
\text { Component and Approach List }\end{array}$ \\
\hline $\begin{array}{l}\text { Negotiated Requirement Revisions } \\
\text { Revised external requirements agreed upon between } \\
\text { regulators, stakeholders, and the Hanford cleanup } \\
\text { program as replacements for conflicting, ambiguous and } \\
\text { counterproductive external requirements. }\end{array}$ & \\
\hline $\begin{array}{l}\text { Negotiated Turnover State } \\
\text { Turnover is the point when turned over to D \& D } \\
\text { operations after negoations are complete for dormant } \\
\text { state. } \\
\text { Dormant state is when we can lower cost of people and } \\
\text { operaion resources, equals turnover state in final. } \\
\text { minimum survelience. }\end{array}$ & \\
\hline $\begin{array}{l}\text { New Responsibility for Resource } \\
\text { The responsibility for the resource is transferred from its } \\
\text { current site management to a transition management. The } \\
\text { transition management assumes this responsibility until } \\
\text { the final disposition of the resource. }\end{array}$ & \\
\hline Non-Aqueous Wastes Generated During Cleanup & $\begin{array}{l}\text { - Landfill Wastes } \\
\text { - Solid Waste Generated Cleaning } \\
\text { Up Aqueous Waste } \\
\text { - Tank Waste Generated Cleaning } \\
\text { Up Aqueous Waste } \\
\end{array}$ \\
\hline \multicolumn{2}{|l|}{ Non-Compliant Effluent } \\
\hline $\begin{array}{l}\text { Non-Compliant Effluent \& Documentation } \\
\text { The effluent of the aqueous waste treatment which is } \\
\text { non-compliant and may need further treatment. }\end{array}$ & $\begin{array}{l}\text { - Non-Compliant Effluent } \\
\text { - Non-Compliant Effluent } \\
\text { Documentation }\end{array}$ \\
\hline $\begin{array}{l}\text { Non-Compliant Effluent Documentation } \\
\text { The documentation associated with the non-compliant } \\
\text { effluent generated by the aqueous waste facilities. }\end{array}$ & \\
\hline Non-Issue Information & \\
\hline
\end{tabular}




\begin{tabular}{|c|c|}
\hline Interface & Constituents \\
\hline $\begin{array}{l}\text { Non-Solid Wastes Generated During Cleanup } \\
\text { Operations } \\
\text { Incidental gaseous and liquid wastes that are not treated } \\
\text { as part of solid waste treatment operations. }\end{array}$ & $\begin{array}{l}\text { - Aqueous Waste Generated } \\
\text { Cleaning Up Solid Wastes } \\
\text { - Tank Waste Generated Cleaning } \\
\text { Up Solid Wastes } \\
\end{array}$ \\
\hline $\begin{array}{l}\text { Non-Tank Wastes Generated During Cleanup } \\
\text { Operations }\end{array}$ & $\begin{array}{l}\text { - Aqueous Waste Generated } \\
\text { Cleaning Up Tank Waste } \\
\text { - Solid Waste Generated Cleaning } \\
\text { Up Tank Waste } \\
\end{array}$ \\
\hline $\begin{array}{l}\text { NonCompliant Solid Waste } \\
\text { This is solid waste material received from solid waste } \\
\text { operations, cleanup operations, and other missions that is } \\
\text { not in compliance with requirements that allow receipt, } \\
\text { repackaging or disposition. }\end{array}$ & \\
\hline $\begin{array}{l}\text { Nuclear Materials } \\
\text { Accumulated SNM/NM/NF. }\end{array}$ & \\
\hline $\begin{array}{l}\text { Nuclear Materials Transferred to Solid Waste } \\
\text { SNM/NM/NF material disposed of as solid waste. }\end{array}$ & $\begin{array}{l}\text { - Solid Waste Generator Request } \\
\text { Information - Nuclear Materials } \\
\text { - Solid Waste Nuclear Materials } \\
\end{array}$ \\
\hline $\begin{array}{l}\text { Nuclear Materials Transferred to Tank Waste } \\
\text { SNM/NM/NF disposed of as tank waste. }\end{array}$ & \\
\hline $\begin{array}{l}\text { Off-site Certified Solid Waste } \\
\text { This is solid waste that meets the certification } \\
\text { requirements that allow off-site disposition. }\end{array}$ & \\
\hline $\begin{array}{l}\text { Offload Authorization, Manifest, and Materials } \\
\text { Documents } \\
\text { All documentation associated with a specific material } \\
\text { shipment. }\end{array}$ & \\
\hline $\begin{array}{l}\text { Offsite Resources } \\
\text { Expertise, technologies, facilities, equipment, supplies, and } \\
\text { information that may be acquired from resources external } \\
\text { to the Hanford site. }\end{array}$ & \\
\hline $\begin{array}{l}\text { On-Site Nuclear Materials } \\
\text { Nuclear material that is recovered during facility } \\
\text { deactivation and can be transferred to other areas in the } \\
\text { public or private domains }\end{array}$ & $\begin{array}{l}\text { - Forecast of On-Site Nuclear } \\
\text { Materials Receipts } \\
\text { - On-Site Nuclear Materials } \\
\text { (Physical) } \\
\text { - Request to Receive On-Site } \\
\text { Nuclear Materials }\end{array}$ \\
\hline $\begin{array}{l}\text { On-Site Nuclear Materials (Physical) } \\
\text { The actual onsite SNM/NM/NF materials. }\end{array}$ & \\
\hline
\end{tabular}




\begin{tabular}{|l|l|}
\hline Interface & Constituents \\
\hline $\begin{array}{l}\text { On-site Certified Solid Waste } \\
\text { This is solid waste that meets the certification } \\
\text { requirements that allow on-site disposition. }\end{array}$ & \\
\hline $\begin{array}{l}\text { Operations \& Capability Needs } \\
\text { Capabilities and needs discovered while operating the } \\
\text { system. As we operate the system, we get smarter. }\end{array}$ & \\
\hline $\begin{array}{l}\text { Operations \& Maintenance Work Package } \\
\text { Work packages that identify operating and maintenance } \\
\text { activities required to ensure that the facility safety and } \\
\text { compliance envelop and documentation are maintained. }\end{array}$ & \\
\hline $\begin{array}{l}\text { Operations Information } \\
\text { Consists of information on staffing needs, control } \\
\text { information, facility status, required reports, people \& } \\
\text { resource allocation. }\end{array}$ & \\
\hline $\begin{array}{l}\text { Operations Plan } \\
\text { The plans that define and govern all SNM/NM/NF storage, } \\
\text { handling. treatment, and disposition operations. }\end{array}$ & \\
\hline $\begin{array}{l}\text { Other Mission Solid Waste Material } \\
\text { The solid waste material itself that comes from other } \\
\text { missions. }\end{array}$ & \\
\hline $\begin{array}{l}\text { Outgoing Shipment Authorization } \\
\text { Authorization to proceed with outgoing SNM/NM/NF } \\
\text { shipments. }\end{array}$ & \\
\hline $\begin{array}{l}\text { Packaged Resource } \\
\text { Materials that will be transported because there exists a } \\
\text { beneficial use or materials that will be stored. }\end{array}$ & \\
\hline $\begin{array}{l}\text { Packaged Solid Waste } \\
\text { This is solid waste that meets the certification } \\
\text { requirements that allow on-site disposition. }\end{array}$ & \\
\hline Plans \& Activities Accepted By Public & \\
\hline $\begin{array}{l}\text { Potential User List } \\
\text { List of potential users of Hanford resources. }\end{array}$ & \\
\hline $\begin{array}{l}\text { Previously Undefined Use } \\
\text { Information on a new or previously undefined use } \\
\text { resulting from potential user inquiries. }\end{array}$ & \\
\hline $\begin{array}{l}\text { Primary Packages and Material } \\
\text { The SNM/NF/NM material and the associated primary and } \\
\text { possible secondary packaging (does not include the } \\
\text { shipping container). }\end{array}$ \\
\hline
\end{tabular}




\begin{tabular}{|c|c|}
\hline Interface & Constituents \\
\hline $\begin{array}{l}\text { Program Needs And Progress Information } \\
\text { In the course of cleaning up Hanford, needs and } \\
\text { information may be discovered which may impact } \\
\text { direction \& control, mission requirements, and defined } \\
\text { work packages. This information must be fed back to the } \\
\text { MANAGE PROGRAM function for evaluation. }\end{array}$ & $\begin{array}{l}\text { - Mission Essential Capability } \\
\text { Needs/Information } \\
\text { - Public Needs/Information } \\
\text { - Remedy Needs/Information } \\
\text { - Resource Transition } \\
\text { Needs/Information } \\
\text { - Solid Waste Capability Needs } \\
\text { - Waste Disposition Status } \\
\text { Information }\end{array}$ \\
\hline \multicolumn{2}{|l|}{$\begin{array}{l}\text { Program Status Information } \\
\text { The actual technical, cost and schedule state of work } \\
\text { activities against the planned work established in the } \\
\text { defined baseline and the defined work packages. }\end{array}$} \\
\hline \multicolumn{2}{|l|}{$\begin{array}{l}\text { Program Variances } \\
\text { Description of differences between planned and measured } \\
\text { progress relative to technical, schedule and cost baselines; } \\
\text { used as a basis for definition of corrective action. }\end{array}$} \\
\hline \multicolumn{2}{|l|}{$\begin{array}{l}\text { Project Equipment } \\
\text { Equipment needed to perform mission essential functions } \\
\text { and activities that is integrated into a Hanford-internal } \\
\text { project. }\end{array}$} \\
\hline $\begin{array}{l}\text { Project Supplies } \\
\text { Supplies needed to perform mission essential functions and } \\
\text { activities that are integrated into a Hanford-internal } \\
\text { project. }\end{array}$ & \\
\hline \multicolumn{2}{|c|}{\begin{tabular}{|l|l} 
Proposed Turnover State & \\
The turnover endpoint recommended by facility personnel. & \\
\end{tabular}} \\
\hline $\begin{array}{l}\text { Public Acceptance } \\
\text { Public trust and acceptance is obtained. } \\
\text { A prerequisite for successful completion of the cleanup } \\
\text { mission is obtaining public trust. This trust is essential to } \\
\text { gaining the broad public acceptance needed to close the } \\
\text { site. Embedded in this end state is the information } \\
\text { supplied to the public on progress, planning, and unusual } \\
\text { events during mission implementation. }\end{array}$ & $\begin{array}{l}- \text { Plans \& Activities Accepted By } \\
\text { Public }\end{array}$ \\
\hline
\end{tabular}




\begin{tabular}{|c|c|}
\hline Interface & Constituents \\
\hline $\begin{array}{l}\text { Public Distrust } \\
\text { There is a lack of public trust of activities carried out at } \\
\text { the Hanford Site. } \\
\text { The secrecy surrounding the Hanford Site's past } \\
\text { production mission has lead to general mistrust by the } \\
\text { public of the DOE's current activities at the Site. The } \\
\text { complexity of the technical activities and the potential risk } \\
\text { associated with these activities further heighten public } \\
\text { attention and concern. The DOE's traditional } \\
\text { decision-making processes have not effectively involved } \\
\text { the public. Effective public involvement, however, is } \\
\text { essential to making cleanup progress. }\end{array}$ & \\
\hline Public Group Specific PI Plan & \\
\hline $\begin{array}{l}\text { Public Information } \\
\text { Public information to entities outside the mission } \\
\text { consisting of press releases, position papers, educational } \\
\text { matter, policy, strategy, etc. }\end{array}$ & $\begin{array}{l}\text { - Acknowledged Unreconciled } \\
\text { Differences } \\
\text { - Non-Issue Information }\end{array}$ \\
\hline $\begin{array}{l}\text { Public Interaction } \\
\text { Public Interactions from offsite consisting of involvement } \\
\text { with stakeholder groups, Native American Tribes, and the } \\
\text { public at large. This involvement provides feedback on } \\
\text { values systems, cleanup plans, resource allocation, and } \\
\text { public information work. }\end{array}$ & \\
\hline $\begin{array}{l}\text { Public Needs/Information } \\
\text { In the course of acquiring public trust and acceptance, } \\
\text { needs and information may be discovered which may } \\
\text { impact direction \& control, mission requirements, and } \\
\text { defined work packages. This information must be fed back } \\
\text { to the MANAGE PROGRAM function for evaluation. }\end{array}$ & Recommended Changes \\
\hline $\begin{array}{l}\text { Public Values } \\
\text { Public values to the program planning process from the } \\
\text { OBTAIN PUBLIC ACCEPTANCE function. }\end{array}$ & \\
\hline $\begin{array}{l}\text { Qualified AW Staff } \\
\text { Facility staff that have the necessary education, } \\
\text { experience, and training. }\end{array}$ & \\
\hline $\begin{array}{l}\text { Qualified Staff } \\
\text { Staff that has had the necessary training and testing to } \\
\text { pefform the facility deactivation and material disposition } \\
\text { activities in a safe and compliant manner. }\end{array}$ & \\
\hline
\end{tabular}




\begin{tabular}{|l|l|}
\hline Interface & Constituents \\
\hline $\begin{array}{l}\text { Quality Assurance Solid Waste Samples } \\
\text { This is solid waste treatment process quality samples used } \\
\text { to certify that the treated waste product meets the solid } \\
\text { waste disposition requirements. }\end{array}$ & \\
\hline $\begin{array}{l}\text { Ranked Resource Use List } \\
\text { A prioritized list of the potential uses of a specific } \\
\text { resource. This has taken into consideration input from } \\
\text { governmental bodies, special interest groups, and } \\
\text { regulators. }\end{array}$ & \\
\hline Received Aqueous Waste and Documentation & - Untreated Aqueous Waste and \\
\hline $\begin{array}{l}\text { Received Shipment } \\
\text { SNM/NM/NF material shipment accepted for handling, } \\
\text { treatment, storage, and/or disposition. }\end{array}$ & \\
\hline $\begin{array}{l}\text { Received Solid Waste } \\
\text { This is solid waste received from solid waste operations, } \\
\text { cleanup operations, and other missions, including removed } \\
\text { legacy waste, that is now ready for disposition. }\end{array}$ & $\begin{array}{l}\text { Documed } \\
\text { Retrieved Solid Waste }\end{array}$ \\
\hline $\begin{array}{l}\text { Received Solid Waste Documentation } \\
\text { This is documented information associated with solid } \\
\text { waste received from solid waste operations, cleanup } \\
\text { operations, and other missions. }\end{array}$ & \\
\hline $\begin{array}{l}\text { Received Solid Waste Information } \\
\text { This is information associated with solid waste received } \\
\text { from solid waste operations, cleanup operations, and other } \\
\text { missions that is needed for verification. }\end{array}$ & \\
\hline $\begin{array}{l}\text { Received Solid Waste Materials for Transport } \\
\text { This is solid waste received from solid waste operations, } \\
\text { cleanup operations, and other missions that is now ready } \\
\text { for transport to disposition. }\end{array}$ & \\
\hline $\begin{array}{l}\text { Received Solid Waste Safety Documentation } \\
\text { This is documented safety information associated with } \\
\text { solid waste received from solid waste operations, cleanup } \\
\text { operations, and other missions that becomes part of } \\
\text { acutely unsafe conditions information. }\end{array}$ & \\
\hline Recommended Changes & \\
\hline
\end{tabular}




\begin{tabular}{|c|c|}
\hline Interface & Constituents \\
\hline $\begin{array}{l}\text { Recommended Process Corrections } \\
\text { Process corrections recommended based on the sampling } \\
\text { and analysis data generated by the Control Aqueous Waste } \\
\text { Treatment Process function. Recommendations also } \\
\text { include suggestions for increased efficiency or other } \\
\text { purposes. }\end{array}$ & \\
\hline $\begin{array}{l}\text { Recommended Requirement Resolutions } \\
\text { These are recommended resolutions of conflicting } \\
\text { requirements influencing the final disposition of the solid } \\
\text { waste. These resolutions must be approved by the } \\
\text { "Manage Program" function. }\end{array}$ & \\
\hline $\begin{array}{l}\text { Recycled Containers and Transporters } \\
\text { Transporter and shipping containers suitable for reuse. }\end{array}$ & \\
\hline $\begin{array}{l}\text { Releasable Efnuents } \\
\text { Effluent that meets environmental requirements and can be } \\
\text { released to the environment. }\end{array}$ & \\
\hline $\begin{array}{l}\text { Remedy Needs/Information } \\
\text { Remedy Needs/Information consisting of progress and } \\
\text { program evaluation information to assist program } \\
\text { formulation, reporting and resource allocation. }\end{array}$ & $\begin{array}{l}\text { - Aqueous Waste Remedy } \\
\text { Needs/Information } \\
\text { - Facility Deactivation } \\
\text { Needs/Information } \\
\text { - Operations \& Capability Needs } \\
\text { - Restoration Needs/Information } \\
\text { - SNM/NM/NF Needs/Information } \\
\text { - Solid Waste Remedy } \\
\text { Needs/Information } \\
\text { - Tank Waste Remedy } \\
\text { Needs/Information } \\
\text { - Unsafe Infrastructure } \\
\text { Needs/Information } \\
\end{array}$ \\
\hline \multicolumn{2}{|l|}{ Remedy/Restore Aqueous Waste Documentation } \\
\hline \multicolumn{2}{|l|}{ Remedy/Restore Aqueous Waste Material } \\
\hline \multicolumn{2}{|l|}{ Remedy/Restore Solid Waste Material } \\
\hline \multicolumn{2}{|l|}{$\begin{array}{l}\text { Request for Final Disposition Shipment } \\
\text { Programmatic authorization to initiate SNM/NM/NF } \\
\text { material transfer to final disposition. }\end{array}$} \\
\hline $\begin{array}{l}\text { Request to Receive Continued SNM, NM, NF Materials } \\
\text { Requests from offsite generators to send SNM/NM/NF } \\
\text { materials to Hanford for treatment, storage, and/or } \\
\text { disposition. }\end{array}$ & \\
\hline
\end{tabular}




\begin{tabular}{|c|c|}
\hline Interface & Constituents \\
\hline $\begin{array}{l}\text { Request to Receive On-Site Nuclear Materials } \\
\text { Requests from onsite generators to send SNM/NM/NF } \\
\text { materials for treatment. storage, and/or disposition. }\end{array}$ & $\therefore$ \\
\hline $\begin{array}{l}\text { Requests for Additional Aqueous Waste } \\
\text { Characterization Information } \\
\text { Requests sent to the aqueous waste generator for } \\
\text { additional aqueous waste characterization information to } \\
\text { help assess the acceptance capability of the facilities. }\end{array}$ & \\
\hline $\begin{array}{l}\text { Requests for Additional Aqueous Waste Information } \\
\text { Requests for additional information about the waste to be } \\
\text { received from the aqueous waste generator. }\end{array}$ & \\
\hline $\begin{array}{l}\text { Requirement Conflicts and Ambiguities } \\
\text { Conflicts between specific requirements imposed within a } \\
\text { single source or in different sources, and requirements that } \\
\text { are not stated with sufficient clarity or specificity to be } \\
\text { translated into specific constraints on system products or } \\
\text { processes. }\end{array}$ & \\
\hline $\begin{array}{l}\text { Requirement Revision Proposals } \\
\text { Description of the problems related to conflicting, } \\
\text { ambiguous or counterproductive requirements, suggested } \\
\text { revisions, and the rationale for those revisions; prepared } \\
\text { for use in conducting interactions with regulatory and } \\
\text { policy-making external entities. }\end{array}$ & \\
\hline $\begin{array}{l}\text { Requirements Requiring Reconciliation } \\
\text { These are waste disposition requirements that require } \\
\text { reconciliation. }\end{array}$ & $\begin{array}{l}\text { - Recommended Requirement } \\
\text { Resolutions }\end{array}$ \\
\hline Resource In Need Of Maintenance & \\
\hline $\begin{array}{l}\text { Resource List } \\
\text { List of all Hanford resources which have potential for } \\
\text { future uses. }\end{array}$ & \\
\hline $\begin{array}{l}\text { Resource Transfer Procedures } \\
\text { Procedures for redirection of resources for mission use, } \\
\text { transfer of use outside of mission, and transfer of } \\
\text { ownership including definition of the requirements and } \\
\text { constraints on these transfers. }\end{array}$ & \\
\hline $\begin{array}{l}\text { Resource Transition Needs/Information } \\
\text { Program formulation, progress, evaluation, and resource } \\
\text { requirements information }\end{array}$ & \\
\hline
\end{tabular}




\begin{tabular}{|c|c|}
\hline Interface & Constituents \\
\hline Resource Utilization Information & \\
\hline \multicolumn{2}{|l|}{$\begin{array}{l}\text { Resources Redirected for Mission } \\
\text { Resources released from current Hanford use and } \\
\text { reassigned for use in the Hanford clean-up mission. }\end{array}$} \\
\hline $\begin{array}{l}\text { Resources to Be Transferred } \\
\text { Items for which an owner has been found but has not yet } \\
\text { been transferred. }\end{array}$ & \\
\hline $\begin{array}{l}\text { Restoration Needs/Information } \\
\text { In the course of environmental restoration, needs and } \\
\text { information may be discovered which may impact } \\
\text { direction \& control, mission requirements, and defined } \\
\text { work packages. This information must be fed back to the } \\
\text { MANAGE PROGRAM function for evaluation. }\end{array}$ & $\begin{array}{l}\text { - Acutely Unsafe Conditions } \\
\text { Information } \\
\text { - Operations \& Capability Needs } \\
\text { - Undocumented Wastes } \\
\text { Information }\end{array}$ \\
\hline $\begin{array}{l}\text { Restricted Land } \\
\text { Past uses of the Hanford Site restricts land usage and } \\
\text { limits land available for other socially beneficial uses. } \\
\text { The Site consists of } 1,450 \mathrm{~km}^{* *} 2\left(560 \mathrm{mi}^{* *} 2\right) \text { of land of } \\
\text { which roughly } 10 \text { percent has been occupied by past } \\
\text { mission activities. These activities have resulted in } \\
\text { contamination of about } 518 \mathrm{~km} * * 2(200 \text { mi**2) of } \\
\text { underlying groundwater and more than } 1,000 \text { separate } \\
\text { contaminated sites. The Site also contains contaminated } \\
\text { facilities, stored nuclear materials, and previously } \\
\text { disposed radioactive and hazardous materials.Past uses of } \\
\text { the Hanford Site restricts land usage and limits land } \\
\text { available for other socially beneficial uses. }\end{array}$ & $\begin{array}{l}\text { - Administratively Restricted Land } \\
\text { - Land Requiring Remediation }\end{array}$ \\
\hline $\begin{array}{l}\text { The Site consists of } 1,450 \mathrm{~km} * * 2(560 \mathrm{mi} * * 2) \text { of land of } \\
\text { which roughly } 10 \text { percent has been occupied by past } \\
\text { mission activities. These activities have resulted in } \\
\text { contamination of about } 518 \mathrm{~km} * * 2(200 \mathrm{mi} * 2) \text { of } \\
\text { underlying groundwater and more than } 1,000 \text { separate } \\
\text { contaminated sites. The Site also contains contaminated } \\
\text { facilities, stored nuclear materials, and previously } \\
\text { disposed radioactive and hazardous materials. }\end{array}$ & \\
\hline
\end{tabular}




\begin{tabular}{|l|l|}
\hline Interface & Constituents \\
\hline $\begin{array}{l}\text { Retained and Motivated Expertise } \\
\text { Hanford personnel willing and able to complete the } \\
\text { clean-up mission. }\end{array}$ & \\
\hline $\begin{array}{l}\text { Retrieved Solid Waste } \\
\text { Solid waste that has been received or retrieved and is } \\
\text { ready for disposition. }\end{array}$ & \\
\hline $\begin{array}{l}\text { Reuseable Effluents } \\
\text { Effluent that meets reuse requirements and can be reused. }\end{array}$ & \\
\hline SNM/NM/NF Cleanup Aqueous Waste Documentation & \\
\hline SNM/NM/NF Cleanup Aqueous Waste Material & \\
\hline SNM/NM/NF Cleanup Solid Waste Materials & \\
\hline $\begin{array}{l}\text { SNM/NM/NF Container Handling Needs/Information } \\
\text { Information on the material handling status and new } \\
\text { capability needs. }\end{array}$ & \begin{tabular}{l} 
Custody Transfer \\
Acknowledgement \\
SNM/NM/NF Package Handling \\
\hline $\begin{array}{l}\text { SNM/NM/NF Corrective Action Needs } \\
\text { Information on actions required to bring the facility and } \\
\text { facility operations back to a safe and compliant state. }\end{array}$
\end{tabular} \\
\hline $\begin{array}{l}\text { SNM/NM/NF Disposition Treatment Information } \\
\text { Information on the existing facility disposition treatment } \\
\text { capabilities. }\end{array}$ & \\
\hline $\begin{array}{l}\text { SNM/NM/NF Documentation } \\
\text { SNM/NM/NF safety and compliance documentation that is } \\
\text { provided for internal and external safety and compliance } \\
\text { assessments. }\end{array}$ & \\
\hline $\begin{array}{l}\text { SNM/NM/NF Equipment and Structure Information } \\
\text { Facility, equipment and maintenance information that } \\
\text { must be maintained to ensure that adequate safety and } \\
\text { compliance documentation exists. }\end{array}$ & \\
\hline $\begin{array}{l}\text { SNM/NM/NF Materials Transferred To Beneficial Uses } \\
\text { Nuclear material that is recovered during facility } \\
\text { deactivation and transferred to the public or private } \\
\text { domains. }\end{array}$ & Transporters \\
\hline $\begin{array}{l}\text { SNM/NM/NF Materials for Disposition } \\
\text { SNM/NM/NF materials that are being transfer from } \\
\text { storage for disposition. These materials may require } \\
\text { further treatment prior to transfer to final disposition. }\end{array}$ & \\
\hline
\end{tabular}




\begin{tabular}{|c|c|}
\hline Interface & Constituents \\
\hline $\begin{array}{l}\text { SNM/NM/NF Needs/Information } \\
\text { Information and capability needs identified during } \\
\text { performance of SNM/NM/NF handling, treatment, storage, } \\
\text { and/or disposition activities, provided to program } \\
\text { management functions to support decision-making } \\
\text { essential to managing the SNM/NM/NF material } \\
\text { processes. }\end{array}$ & - Compliance Confirmation \\
\hline \multicolumn{2}{|l|}{$\begin{array}{l}\text { SNM/NM/NF Operations Information } \\
\text { Operations information that must be maintained to ensure } \\
\text { adequate safety and compliance documentation exists. } \\
\text { This information is also used to assist in the development } \\
\text { of facility staff training. }\end{array}$} \\
\hline \multicolumn{2}{|l|}{$\begin{array}{l}\text { SNM/NM/NF Operations and Maintenance Work } \\
\text { Package } \\
\text { Work packages that define facility process and } \\
\text { infrastructure maintenance activities. }\end{array}$} \\
\hline \multicolumn{2}{|l|}{$\begin{array}{l}\text { SNM/NM/NF Package Handling Needs//nformation } \\
\text { Information on the status of SNM/NM/NF package } \\
\text { handling and new package handling capability needs. }\end{array}$} \\
\hline \multicolumn{2}{|l|}{$\begin{array}{l}\text { SNM/NM/NF Qualified Staff } \\
\text { A fully trained, qualified, and certified (as required) } \\
\text { facility work staff. }\end{array}$} \\
\hline $\begin{array}{l}\text { SNM/NM/NF Storage Needs/Information } \\
\text { Information on the status of SNM/NM/NF material storage } \\
\text { and new storage capability needs. }\end{array}$ & - \\
\hline \multicolumn{2}{|l|}{$\begin{array}{l}\text { SNM/NM/NF Storage Treatment Information } \\
\text { Information on the existing facility storage treatment } \\
\text { capabilities and SNM/NM/NF material storage } \\
\text { capabilities and status. }\end{array}$} \\
\hline \multicolumn{2}{|l|}{$\begin{array}{l}\text { SNM/NM/NF Transfer Needs/Information } \\
\text { Information on the status of SNM/NM/NF transfers and } \\
\text { new transfer capability needs. }\end{array}$} \\
\hline SW Cleanup Aqueous Waste Documentation & \\
\hline SW Cleanup Aqueous Waste Material & \\
\hline
\end{tabular}




\begin{tabular}{|c|c|}
\hline Interface & Constituents \\
\hline $\begin{array}{l}\text { Safe Legacy Infrastructure Conditions } \\
\text { The conditions that are deemed to be safe even though they } \\
\text { maybe old and are a result of the Legacy Conditions that } \\
\text { exist on the Hanford Site. }\end{array}$ & $\begin{array}{l}\text { - Safe Legacy Emergency Services } \\
\text { Conditions } \\
\text { - Safe Legacy Facility Conditions } \\
\text { - Safe Legacy Technical Services } \\
\text { Conditions } \\
\text { - Safe Legacy Transportation } \\
\text { Conditions } \\
\text { - Safe Legacy Utility Conditions } \\
\end{array}$ \\
\hline \multicolumn{2}{|c|}{ Safety and Compliance Unsafe Conditions Information } \\
\hline \multicolumn{2}{|l|}{$\begin{array}{l}\text { Safety and Security Envelope Constraints } \\
\text { Constraints placed on the receipt, handling, storage, } \\
\text { treatment, and disposition of SNM/NM/NF material, } \\
\text { facility operations and maintenance activities to ensure } \\
\text { safety and compliance are maintained. }\end{array}$} \\
\hline \multicolumn{2}{|l|}{$\begin{array}{l}\text { Safety and Security Needs/Information } \\
\text { Information on safety and security status and new safety } \\
\text { and security capability needs. }\end{array}$} \\
\hline \multicolumn{2}{|l|}{ Sample and Analysis Data } \\
\hline $\begin{array}{l}\text { Samples of Waste Requiring Additional } \\
\text { Characterization } \\
\text { Solid waste that needs additional characterization data } \\
\text { before final disposition can be made. }\end{array}$ & $\begin{array}{l}\text { - Information On Waste Samples } \\
\text { Requiring Characterization }\end{array}$ \\
\hline \multicolumn{2}{|l|}{$\begin{array}{l}\text { Secondary Storage Wastes } \\
\text { Incidental solid, aqueous, or tank waste generated during } \\
\text { the storage of SNM/NM/NF materials. }\end{array}$} \\
\hline \multicolumn{2}{|l|}{$\begin{array}{l}\text { Secondary Treatment Wastes } \\
\text { Incidental solid, aqueous, and tank wastes generated } \\
\text { during the treatments of SNM/NM/NF material to prepare } \\
\text { it for storage. }\end{array}$} \\
\hline \multicolumn{2}{|l|}{$\begin{array}{l}\text { Secondary Waste Documentation } \\
\text { The documentation associated with the secondary aqueous } \\
\text { wastes generated by aqueous waste treatment. }\end{array}$} \\
\hline \multicolumn{2}{|l|}{$\begin{array}{l}\text { Secondary Wastes } \\
\text { The secondary wastes generated by aqueous waste } \\
\text { treatment. }\end{array}$} \\
\hline $\begin{array}{l}\text { Secondary Wastes And Documentation } \\
\text { The secondary wastes generated from aqueous waste } \\
\text { treatment and the associated documentation. }\end{array}$ & $\begin{array}{l}\text { - Secondary Waste Documentation } \\
\text { - Secondary Wastes }\end{array}$ \\
\hline
\end{tabular}




\begin{tabular}{|l|l|}
\hline Interface & Constituents \\
\hline $\begin{array}{l}\text { Selected Management System } \\
\text { The management organization, infrastructure, principles } \\
\text { and policies selected for implementation to formulate and } \\
\text { execute the programs required to complete the Hanford } \\
\text { cleanup mission. }\end{array}$ & \\
\hline $\begin{array}{l}\text { Solid Waste Analysis Information } \\
\text { Information from analysis of the solid waste samples. }\end{array}$ & \\
\hline $\begin{array}{l}\text { Solid Waste Archive Data } \\
\text { Solid waste sample analysis data that is needed to } \\
\text { complete the assessment of characterization information. }\end{array}$ & \\
\hline $\begin{array}{l}\text { Solid Waste Archive Samples } \\
\text { Solid waste samples retrieved from archival storage for } \\
\text { further analysis. }\end{array}$ & \\
\hline $\begin{array}{l}\text { Solid Waste Archive Specifications } \\
\text { Solid waste archive specifications that have been prepared } \\
\text { during disposition specification development. }\end{array}$ & \\
\hline $\begin{array}{l}\text { Solid Waste Capability Needs } \\
\text { These are the resource capability needs that are required } \\
\text { to provide final solid waste dispostion. }\end{array}$ & Needs \\
\hline $\begin{array}{l}\text { Solid Waste Characterization Information } \\
\text { This is characterization data consisting of current or } \\
\text { historical process operation knowledge pertaining to the } \\
\text { solid waste, its current condition, and sampling and } \\
\text { analysis results required to determine its final disposition. }\end{array}$ & \\
\hline $\begin{array}{l}\text { Solid Waste Cleanup Waste Material } \\
\text { The solid waste material itself generated during solid } \\
\text { waste cleanup operations. }\end{array}$ & \\
\hline $\begin{array}{l}\text { Solid Waste Corrective Action Needs } \\
\text { Actions required to bring waste status into compliance } \\
\text { with handling and disposition requirements. }\end{array}$ & \\
\hline $\begin{array}{l}\text { Solid Waste Disposition Capability Needs } \\
\text { These are capability needs to support treatment, storage } \\
\text { and disposal of the solid waste. }\end{array}$ & \\
\hline $\begin{array}{l}\text { Solid Waste Disposition Requirements } \\
\text { These are compiled and sorted solid waste disposition } \\
\text { requirements determined from imposed requirements, } \\
\text { results from proposed solutions and required capabilities, } \\
\text { and operational constraints. }\end{array}$ \\
\hline
\end{tabular}




\begin{tabular}{|c|c|}
\hline Interface & Constituents \\
\hline $\begin{array}{l}\text { Solid Waste Disposition Requirements List } \\
\text { This is a list of disp sition requirements generated during } \\
\text { compilation. This list includes imposed regulatory } \\
\text { requirements and operations requirements, and are listed } \\
\text { by disposition category. }\end{array}$ & \\
\hline $\begin{array}{l}\text { Solid Waste Disposition Specifications } \\
\text { These are disposition specifications for the solid waste. }\end{array}$ & \\
\hline $\begin{array}{l}\text { Solid Waste Documentation } \\
\text { This is documentation that shows that the safety and } \\
\text { compliance envelope of the solid waste is maintained. }\end{array}$ & \\
\hline $\begin{array}{l}\text { Solid Waste Documentation Generated During AW } \\
\text { Cleanup }\end{array}$ & \\
\hline $\begin{array}{l}\text { Solid Waste Equipment and Structure Information } \\
\text { This is information related to the solid waste equipment } \\
\text { and structure safety and maintenance checks, covering } \\
\text { routine maintenance, preventative maintenance, equipment } \\
\text { calibration, and minor facility and equipment } \\
\text { modifications. }\end{array}$ & \\
\hline $\begin{array}{l}\text { Solid Waste Evaluation Information } \\
\text { Information about the solid waste that is required to } \\
\text { identify capability requirements for retrieving the solid } \\
\text { waste that was derived from the evaluation of } \\
\text { characterization information and location information. }\end{array}$ & \\
\hline $\begin{array}{l}\text { Solid Waste From Other Missions } \\
\text { Solid waste that comes from other DOE missions outside } \\
\text { of the Hanford Cleanup Mission. }\end{array}$ & $\begin{array}{l}- \text { Other Mission Solid Waste } \\
\text { Material } \\
\text { - Solid Waste Generator Request } \\
\text { Information - Other Missions } \\
\end{array}$ \\
\hline $\begin{array}{l}\text { Solid Waste Generated Cleaning Up Aqueous Waste } \\
\text { Solid wastes and documentation associated with wastes } \\
\text { generated while maintaining safety and compliance } \\
\text { conditions. Parent function is Wastes and Documentation } \\
\text { Generated During AW Cleanup. }\end{array}$ & $\begin{array}{l}\text { - AW Cleanup Solid Waste Material } \\
\text { - Solid Waste Generator Request } \\
\text { Information - AW Cleanup }\end{array}$ \\
\hline Solid Waste Generated Cleaning Up Tank Waste & $\begin{array}{l}\text { - Solid Waste Generator Request } \\
\text { Information - TW Cleanup } \\
\text { - TW Cleanup Solid Waste } \\
\text { Materials } \\
\end{array}$ \\
\hline Solid Waste Generated Correcting Infrastructure & $\begin{array}{l}\text { - Infrastructure Solid Waste } \\
\text { Materials } \\
\text { - Solid Waste Generator Request } \\
\text { Information - Infrastructure } \\
\end{array}$ \\
\hline
\end{tabular}




\begin{tabular}{|c|c|}
\hline Interface & Constituents \\
\hline $\begin{array}{l}\text { Solid Waste Generated During Deactivation } \\
\text { Very little high level waste, including Legecy Solid Waste, } \\
\text { radioactive \& solid hazardous waste. }\end{array}$ & $\begin{array}{l}\text { - Deactivation Solid Waste Material } \\
\text { - Solid Waste Generator Request } \\
\text { Information - Deactivation }\end{array}$ \\
\hline Solid Waste Generated During Remedy/Restore & $\begin{array}{l}- \text { Remedy/Restore Solid Waste } \\
\text { Material } \\
\text { - Solid Waste Generator Request } \\
\text { Information - Remedy/Restore } \\
\end{array}$ \\
\hline $\begin{array}{l}\text { Solid Waste Generated During Solid Waste Cleanup } \\
\text { Operations } \\
\text { Contaminated tools, equipment and materials generated as } \\
\text { a direct result of cleanup operations; including used } \\
\text { cleaning solvents, rags, stack filters and similar items } \\
\text { requiring processing prior to disposal or transfer to a } \\
\text { useful function within or outside the Cleanup Hanford } \\
\text { mission }\end{array}$ & $\begin{array}{l}\text { - Solid Waste Cleanup Waste } \\
\text { Material } \\
\text { - Solid Waste Generator Request } \\
\text { Information - SW Cleanup }\end{array}$ \\
\hline $\begin{array}{l}\text { Solid Waste Generated Handling SNM/NM/NF } \\
\text { Solid wastes includes hazardous materials and } \\
\text { radioactively contaminated equipment and clothing. }\end{array}$ & $\begin{array}{l}\text { - SNM/NM/NF Cleanup Solid } \\
\text { Waste Materials } \\
\text { - Solid Waste Generator Request } \\
\text { Information - SNM/NM/NF } \\
\end{array}$ \\
\hline $\begin{array}{l}\text { Solid Waste Generator Request Information - AW } \\
\text { Cleanup } \\
\text { Information related to a request from a solid waste } \\
\text { generator for treatment, storage, and/or disposal of a } \\
\text { particular solid waste package generated by aqueous } \\
\text { waste cleanup. }\end{array}$ & \\
\hline $\begin{array}{l}\text { Solid Waste Generator Request Information - } \\
\text { Deactivation } \\
\text { Information related to a request from a solid waste } \\
\text { generator for treatment, storage, and/or disposal of a } \\
\text { particular solid waste package generated by facility } \\
\text { deactivation. }\end{array}$ & \\
\hline $\begin{array}{l}\text { Solid Waste Generator Request Information - } \\
\text { Infrastructure } \\
\text { Information related to a request from a solid waste } \\
\text { generator for treatment, storage, and/or disposal of a } \\
\text { particular solid waste package generated by correcting } \\
\text { infrastructure. }\end{array}$ & \\
\hline
\end{tabular}




\begin{tabular}{|l|l|}
\hline Interface & Constituents \\
\hline $\begin{array}{l}\text { Solid Waste Generator Request Information - Nuclear } \\
\text { Materials } \\
\text { Information related to a request from a solid waste } \\
\text { generator for treatment, storage, and/or disposal of a } \\
\text { particular solid waste package generated by transferring }\end{array}$ & \\
nuclear materials to solid waste. & \\
\hline $\begin{array}{l}\text { Solid Waste Generator Request Information - Other } \\
\text { Missions }\end{array}$ & \\
Information related to a request from a solid waste \\
generator for treatment, storage, and/or disposal of a \\
particular solid waste package generated by other & \\
missions. & \\
\hline $\begin{array}{l}\text { Solid Waste Generator Request Information - } \\
\text { Remedy/Restore } \\
\text { Information related to a request from a solid waste } \\
\text { generator for treatment, storage, and/or disposal of a } \\
\text { particular solid waste package generated by } \\
\text { remedy/restore sites, faciliteis, and ground water. }\end{array}$ & \\
\hline $\begin{array}{l}\text { Solid Waste Generator Request Information - } \\
\text { SNM/NM/NF } \\
\text { Information related to a request from a solid waste } \\
\text { generator for treatment, storage, and/or disposal of a } \\
\text { particular solid waste package generated by handling } \\
\text { SNM/NM/NF. }\end{array}$ & \\
\hline $\begin{array}{l}\text { Solid Waste Generator Request Information - SW } \\
\text { Cleanup } \\
\text { Information related to a request from a solid waste } \\
\text { generator for treatment, storage, and/or disposal of a } \\
\text { particular solid waste package generated by solid waste } \\
\text { operations. }\end{array}$ & \\
\hline $\begin{array}{l}\text { Solid Waste Generator Request Information - TW } \\
\text { Cleanup } \\
\text { Information related to a request from a solid waste } \\
\text { generator for treatment, storage, and/or disposal of a } \\
\text { particular solid waste package generated by cleaning up } \\
\text { tank waste. }\end{array}$ & \\
\hline $\begin{array}{l}\text { Solid Waste Handling Information } \\
\text { This is handling information needed to support transport } \\
\text { of legacy waste materials. }\end{array}$ & \\
\end{tabular}




\begin{tabular}{|c|c|}
\hline Interface & Constituents \\
\hline $\begin{array}{l}\text { Solid Waste Internal Safety Operations Constraint } \\
\text { Documents } \\
\text { These are documents that relate the internal safety } \\
\text { operational constraints for the solid waste systems, } \\
\text { equipment, and structures. }\end{array}$ & \\
\hline $\begin{array}{l}\text { Solid Waste Location Information } \\
\text { This is information on the location of the lesacy waste. }\end{array}$ & \\
\hline Solid Waste Nuclear Matcrials & \\
\hline $\begin{array}{l}\text { Solid Waste Operations \& Maintenance Work Package } \\
\text { This is a work package that relates the solid waste } \\
\text { operations and maintenance work to be performed. }\end{array}$ & \\
\hline $\begin{array}{l}\text { Solid Waste Operations Information } \\
\text { This is information related to the operations of the solid } \\
\text { waste svstems. equipment. and structures. }\end{array}$ & \\
\hline $\begin{array}{l}\text { Solid Waste Process Knowledge } \\
\text { This is knowledge of the process that generated the solid } \\
\text { waste. }\end{array}$ & \\
\hline $\begin{array}{l}\text { Solid Waste Process Quality Data } \\
\text { This is solid waste treatment process quality data resulting } \\
\text { from the solid waste certification function that determines } \\
\text { if the treated waste product meets the solid waste } \\
\text { disposition requirements. This data is provided as } \\
\text { feedback to the treatment process to allow for process } \\
\text { adjustments. }\end{array}$ & \\
\hline $\begin{array}{l}\text { Solid Waste Qualified Staff } \\
\text { These are personnel that have been trained and qualified } \\
\text { to perform the solid waste facility operations. }\end{array}$ & \\
\hline $\begin{array}{l}\text { Solid Waste Receipt Forecast Information } \\
\text { This is forecast information associated with future solid } \\
\text { waste receipts to be used in identifying solid waste } \\
\text { operations and capability needs. }\end{array}$ & \\
\hline $\begin{array}{l}\text { Solid Waste Remedy Needs/Information } \\
\text { In the course of solid waste remediation, needs and } \\
\text { information may be discovered which may impact } \\
\text { direction \& control, mission requirements, and defined } \\
\text { work packages. This information must be fed back to the } \\
\text { MANAGE PROGRAM function for evaluation. }\end{array}$ & $\begin{array}{l}\text { - Acutely Unsafe Conditions } \\
\text { Information } \\
\text { - Operations \& Capability Needs } \\
\text { - Requirements Requiring } \\
\text { Reconciliation } \\
\text { - Solid Waste Capability Needs } \\
\text { - Undocumented Wastes } \\
\text { Information } \\
\text { - Waste Disposition Status } \\
\text { Information }\end{array}$ \\
\hline
\end{tabular}




\begin{tabular}{|c|c|}
\hline Interface & Constituents \\
\hline \multicolumn{2}{|l|}{$\begin{array}{l}\text { Solid Waste Removal Documentation } \\
\text { This is documented information associated with removed } \\
\text { legacy solid waste. }\end{array}$} \\
\hline \multicolumn{2}{|l|}{$\begin{array}{l}\text { Solid Waste Removal Information } \\
\text { This is information obtained during the solid waste } \\
\text { removal process that can be used in its characterization } \\
\text { and disposition. }\end{array}$} \\
\hline \multicolumn{2}{|l|}{$\begin{array}{l}\text { Solid Waste Safety/Compliance Envelope Constraints } \\
\text { These are constraints that follow the solid waste to ensure } \\
\text { that the safety and compliance envelope is maintained } \\
\text { during receipt, characterization, disposition requirement } \\
\text { generation, and final disposition of solid waste. }\end{array}$} \\
\hline \multicolumn{2}{|l|}{$\begin{array}{l}\text { Solid Waste Samples } \\
\text { Solid waste samples that were used to analyze the waste } \\
\text { inventory that now require archiving. }\end{array}$} \\
\hline $\begin{array}{l}\text { Solid Wastes and Documentation Generated During } \\
\text { A W Cleanup } \\
\text { Solid wastes and documentation associated with wastes } \\
\text { generated while maintaining safety and compliance } \\
\text { conditions. }\end{array}$ & $\begin{array}{l}\text { - Solid Waste Documentation } \\
\text { Generated During AW Cleanup } \\
\text { - Solid Wastes Generated During } \\
\text { AW Cleanup }\end{array}$ \\
\hline \multicolumn{2}{|l|}{ Status Capability Information } \\
\hline \multicolumn{2}{|l|}{$\begin{array}{l}\text { Strategy for Acquisition of Capability Classes } \\
\text { Definition of an integrated strategy for the acquisition of } \\
\text { mission essential expertise, technology, facilities, } \\
\text { equipment, infrastructure, supplies, and integrated } \\
\text { independent services. }\end{array}$} \\
\hline \multicolumn{2}{|l|}{$\begin{array}{l}\text { Strategy for Acquisition of Integrated Independent } \\
\text { Services } \\
\text { Definition of a strategy for the acquisition of mission } \\
\text { essential integrated independent services. }\end{array}$} \\
\hline \multicolumn{2}{|l|}{$\begin{array}{l}\text { Strategy for Consolidated Acquisition Opportunities } \\
\text { Definition of a strategy for the consolidated acquisition of } \\
\text { mission essential capabilities including expertise, } \\
\text { technology, facilities, equipment, infrastructure, supplies, } \\
\text { and information. }\end{array}$} \\
\hline \multicolumn{2}{|l|}{ Supplies and Parts } \\
\hline $\begin{array}{l}\text { Survey and Manifest Information } \\
\text { Radiological release of the SNM/NM/NF packaging and } \\
\text { the associated SNM/NM/NF material manifest } \\
\text { information. }\end{array}$ & \\
\hline System Architecture & \\
\hline
\end{tabular}




\begin{tabular}{|c|c|}
\hline Interface & Constituents \\
\hline \multicolumn{2}{|l|}{ System Functional Definition } \\
\hline \multicolumn{2}{|l|}{ System Requirements } \\
\hline \multicolumn{2}{|l|}{ TW Cleanup Aqueous Waste Documentation } \\
\hline \multicolumn{2}{|l|}{ TW Cleanup Aqueous Waste Material } \\
\hline \multicolumn{2}{|l|}{ TW Cleanup Solid Waste Materials } \\
\hline \multicolumn{2}{|l|}{$\begin{array}{l}\text { Tactical Changes } \\
\text { Program redirection of sufficiently linited impact such } \\
\text { that it can be implemented within the framework of the } \\
\text { existing technical, budget and schedule baselines, e.g., } \\
\text { miner reprioritization of activities. }\end{array}$} \\
\hline \multicolumn{2}{|l|}{ Tank Waste Generated Cleaning Un Aqueous Waste } \\
\hline \multicolumn{2}{|l|}{ Tank Waste Generated Cleauning Up Solid Wastes } \\
\hline \multicolumn{2}{|l|}{ Tank Waste Generated Correcting Infrastructure } \\
\hline \multicolumn{2}{|l|}{$\begin{array}{l}\text { Tank Waste Generated During Deactivation } \\
\text { Waste consisting of hazardous mixed, and valid high level } \\
\text { waste contained in tanks, and orphan tanks. }\end{array}$} \\
\hline \multicolumn{2}{|l|}{ Tank Waste Generated During Remedy/Restore } \\
\hline \multicolumn{2}{|l|}{$\begin{array}{l}\text { Tank Waste Generated Handling SNM/NM/NF } \\
\text { High level radioactive liquid waste generated during the } \\
\text { receipt, handling, treatment and disposition of } \\
\text { SNMINM/NE materials. }\end{array}$} \\
\hline $\begin{array}{l}\text { Tank Waste Remedy Needs/Information } \\
\text { In the course of tank waste remediation, needs and } \\
\text { information may be discovered which may impact } \\
\text { direction \& control, mission requirements, and defined } \\
\text { work packages. This information must be fed back to the } \\
\text { MANAGE PROGRAM function for evaluation. }\end{array}$ & $\begin{array}{l}\text { - Acutely Unsafe Conditions } \\
\text { Information } \\
\text { - Operations \& Capability Needs } \\
\text { - Undocumented Wastes } \\
\text { Information }\end{array}$ \\
\hline \multicolumn{2}{|l|}{$\begin{array}{l}\text { Technology Concept } \\
\text { Proven technology concept based on completed research. }\end{array}$} \\
\hline $\begin{array}{l}\text { Technology Needs for Essential Information } \\
\text { Feedback to the Provide Technology function from the } \\
\text { Provide Essential Information function describing the } \\
\text { needs for technologies to support this function, including } \\
\text { advanced information acquisition, networking and } \\
\text { management systems and technologies. }\end{array}$ & \\
\hline
\end{tabular}




\begin{tabular}{|c|c|}
\hline Interface & Constituents \\
\hline $\begin{array}{l}\text { Technology Needs for Facillties, Equipment, \& } \\
\text { Infrastructure } \\
\text { Feedback to the Provide Technology function from the } \\
\text { Provide Facilities, Equipment, Infrastructure, \& Supplies } \\
\text { function describing the needs for technology to support } \\
\text { this function. Needs identify technology gaps needed to } \\
\text { design, procure, and construct facilities or procure } \\
\text { equipment. }\end{array}$ & \\
\hline $\begin{array}{l}\text { Technology Needs for Providing Mission Essential } \\
\text { Capabilities } \\
\text { Parent input to the Provide Technology function from the } \\
\text { constituent outputs from the Provide Essential Information } \\
\text { and Provide Facilities, Equipment. Infrastructure \& } \\
\text { Supplies function. }\end{array}$ & $\begin{array}{l}\text { - Technology Needs for Essential } \\
\text { Information } \\
\text { - Technology Needs for Facilities, } \\
\text { Equipment, \& Infrastructure } \\
\end{array}$ \\
\hline $\begin{array}{l}\text { Technology Needs/nformation } \\
\text { Feedback to the Manage Program function describing the } \\
\text { status of activities, including problems impeding successful } \\
\text { execution of the work defined in the Work Packases. }\end{array}$ & - Identified Existing Technology \\
\hline $\begin{array}{l}\text { Technology Proof-of-Principle } \\
\text { Demonstrated technology based on laboratory-scale } \\
\text { testing. }\end{array}$ & \\
\hline $\begin{array}{l}\text { Training Documentation } \\
\text { Documentation on aqueous waste staff training. } \\
\text { qualification. and certification. }\end{array}$ & \\
\hline $\begin{array}{l}\text { Transfer Agreement } \\
\text { Transfer Agreements consisting of identification of } \\
\text { resources to be used in building the local economy which } \\
\text { essentially causes transferrable items to be removed from } \\
\text { the "disposal" list to the transfer list. }\end{array}$ & \\
\hline $\begin{array}{l}\text { Transfer Request Needs } \\
\text { Needs associated with the aqueous waste transfer request. } \\
\text { Parent arrow is Aqueous Waste Receipt Needs and } \\
\text { Information. }\end{array}$ & \\
\hline
\end{tabular}




\begin{tabular}{|c|c|}
\hline Interface & Constituents \\
\hline $\begin{array}{l}\text { Transferrable Resources } \\
\text { Transferrable Resources consisting of valuable } \\
\text { technologies, materials, and equipment, and infrastructure }\end{array}$ & $\begin{array}{l}\text { - Cultural Resources } \\
\text { - Declaration of Excess Resources } \\
\text { (Use) } \\
\text { - Declaration of Excess Resources } \\
\text { (ownership) } \\
\text { - Facilities } \\
\text { - Facilities/Resources Transferred } \\
\text { To Beneficial Uses } \\
\text { - Infrastructure } \\
\text { - Land } \\
\text { - Materials \& Equipment } \\
\text { - Reuseable Effluents } \\
\text { - SNM/NM/NF Materials } \\
\text { Transferred To Beneficial Uses } \\
\text { - Transferrable Resources (physical) } \\
\text { - Transferrable Technology } \\
\text { - Work Force }\end{array}$ \\
\hline \multicolumn{2}{|l|}{$\begin{array}{l}\text { Transferrable Resources (physical) } \\
\text { Any item having a new owner identified }\end{array}$} \\
\hline $\begin{array}{l}\text { Transferrable Technology } \\
\text { Technologies that are transferrable for uses outside of the } \\
\text { Hanford clean-up mission after modifications/upgrades } \\
\text { are completed to make the technology useable outside of } \\
\text { the Hanford clean-up mission. }\end{array}$ & \\
\hline \multicolumn{2}{|l|}{ Transferred Aqueous Waste } \\
\hline $\begin{array}{l}\text { Transitioned Economy } \\
\text { Resources (e.g., technology, people, expertise) developed } \\
\text { and used during cleanup have been leveraged to enhance } \\
\text { the economic viability of the regional economy. } \\
\text { Cleanup resources will have been expended in a manner to } \\
\text { support independence of the localprivate sector economy. } \\
\text { This can be achieved through development of } \\
\text { public/private sector partnerships, transfer of technologies } \\
\text { for use elsewhere, etc. Also, expenditure of these } \\
\text { resources should, where possible, provide national and } \\
\text { international benefit by making technology and expertise } \\
\text { broadly available. }\end{array}$ & \\
\hline \multicolumn{2}{|l|}{$\begin{array}{l}\text { Treated Effluent } \\
\text { The effluents of aqueous waste treatment. }\end{array}$} \\
\hline Treated Effluent And Documentation & $\begin{array}{l}\text { - Treated Effluent } \\
\text { - Treated Effluent Documentation }\end{array}$ \\
\hline
\end{tabular}




\begin{tabular}{|c|c|}
\hline Interface & Constituents \\
\hline $\begin{array}{l}\text { Treated Effluent Documentation } \\
\text { Documentation associated with the treated effluents from } \\
\text { aqueous waste treatment. }\end{array}$ & \\
\hline $\begin{array}{l}\text { Treated Materials } \\
\text { SNM/NM/NF materials that have been treated to allow for } \\
\text { safe. stable, and compliant storage. }\end{array}$ & \\
\hline $\begin{array}{l}\text { Treated Solid Waste } \\
\text { This is solid waste that has been treated so that it meets } \\
\text { the disposition requirements and is ready for packaging. }\end{array}$ & \\
\hline $\begin{array}{l}\text { Unambiguous, Non-conflicting Requirements } \\
\text { The subset of external requirements that will not require } \\
\text { interactions with external entities to resolve conflicts or } \\
\text { ambiguities. }\end{array}$ & \\
\hline $\begin{array}{l}\text { Undocumented Wastes Information } \\
\text { While doing everyday operations we come upon waste } \\
\text { previously undocumented. Information must be fed back to } \\
\text { the management function. } \\
\text { - liquid tank waste } \\
\text { - liquid non-tank waste } \\
\text { - solid waste } \\
\text { - landfill } \\
\text { - airborne waste } \\
\text { - non-defined waste }\end{array}$ & \\
\hline $\begin{array}{l}\text { Unfunded Work } \\
\text { Work excluded from the current year work plans; despite } \\
\text { its presence in the technical, budget, and schedule } \\
\text { baselines; as a result of a difference between requested } \\
\text { and actual fiscal vear fundine. }\end{array}$ & \\
\hline $\begin{array}{l}\text { Unsafe Infrastructure Needs/Information } \\
\text { In the course of correcting unsafe infrastructure } \\
\text { conditions, needs and information may be discovered } \\
\text { which may impact direction \& control, mission } \\
\text { requirements, and defined work packages. This } \\
\text { information must be fed back to the MANAGE PROGRAM } \\
\text { function for evaluation. }\end{array}$ & $\begin{array}{l}\text { - Infrastructure Upgrade Needs } \\
\text { - Resource Utilization Information } \\
\text { - Status Capability Information }\end{array}$ \\
\hline
\end{tabular}




\begin{tabular}{|c|c|}
\hline Interface & Constituents \\
\hline $\begin{array}{l}\text { Unsafe Legacy Infrastructure Conditions } \\
\text { Those unsafe conditions that exist as a result of inadequate } \\
\text { funding for maintenance and upgrades over the life of the } \\
\text { infrastructure component while it supported the prior } \\
\text { Hanford Mission. }\end{array}$ & $\begin{array}{l}\text { - Unsafe Legacy Emergency Service } \\
\text { Conditions } \\
\text { - Unsafe Legacy Facility Conditions } \\
\text { - Unsafe Legacy Technical Service } \\
\text { Conditions } \\
\text { - Unsafe Legacy Transportation } \\
\text { Conditions } \\
\text { - Unsafe Legacy Utility Conditions }\end{array}$ \\
\hline \multicolumn{2}{|l|}{$\begin{array}{l}\text { Unsolicited Proposals } \\
\text { Unsolicited proposals to conduct Hanford Cleanup } \\
\text { activities in the private sector. }\end{array}$} \\
\hline \multicolumn{2}{|l|}{$\begin{array}{l}\text { Unsolicited Proposals For Use Or Ownership } \\
\text { Potential user interest in resources from outside the } \\
\text { mission. }\end{array}$} \\
\hline \multicolumn{2}{|l|}{$\begin{array}{l}\text { Untreated Aqueous Waste } \\
\text { Untreated aqueous waste received for disposition. }\end{array}$} \\
\hline \multicolumn{2}{|l|}{ Untreated Aqueous Waste Documentation } \\
\hline $\begin{array}{l}\text { Untreated Aqueous Waste and Documentation } \\
\text { The waste and documentation which needs no treatment } \\
\text { and is passed directly to Disposition Aqueous Waste. }\end{array}$ & $\begin{array}{l}\text { - Untreated Aqueous Waste } \\
\text { - Untreated Aqueous Waste } \\
\text { Documentation }\end{array}$ \\
\hline \multicolumn{2}{|l|}{ Value Differences Defined } \\
\hline \multicolumn{2}{|l|}{$\begin{array}{l}\text { Verified Received Waste Materials } \\
\text { These are solid waste materials received from solid waste } \\
\text { operations, cleanup operations, and other missions that } \\
\text { have been verified relative to the information received } \\
\text { from the generator. }\end{array}$} \\
\hline \multicolumn{2}{|l|}{$\begin{array}{l}\text { Waste Disposition Status Information } \\
\text { As the physical status of the solid waste changes, the safety } \\
\text { and compliance envelope changes. This is all information } \\
\text { regarding the disposition state of the solid waste. }\end{array}$} \\
\hline \multicolumn{2}{|l|}{$\begin{array}{l}\text { Waste Documentation } \\
\text { Documentation on the waste to be dispositioned, which } \\
\text { accompanies the waste. }\end{array}$} \\
\hline $\begin{array}{l}\text { Waste Generated Correcting Unsafe Infrastructure } \\
\text { During the course of correcting unsafe conditions some } \\
\text { waste maybe getterated which requires remediation. This } \\
\text { waste will be sent to } 4.5 \text { for proper disposition. }\end{array}$ & $\begin{array}{l}\text { - Aqueous Waste Generated } \\
\text { Correcting Infrastructure } \\
\text { - Solid Waste Generated Correcting } \\
\text { Infrastructure } \\
\text { - Tank Waste Generated Correcting } \\
\text { Infrastructure }\end{array}$ \\
\hline
\end{tabular}




\begin{tabular}{|c|c|}
\hline Interface & Constituents \\
\hline Waste Generated Handling SNM/NM/NF & $\begin{array}{l}\text { - Aqueous Waste Generated } \\
\text { Handling SNM/NM/NF } \\
\text { - Solid Waste Generated Handling } \\
\text { SNM/NM/NF } \\
\text { - Tank Waste Generated Handling } \\
\text { SNM/NM/NF } \\
\end{array}$ \\
\hline $\begin{array}{l}\text { Wastes Generated During Deactivation } \\
\text { Contaminated tools, equipment and materials generated as } \\
\text { a direct result of facility deactivation activities; including } \\
\text { used cleaning solvents, rags, stack filters and similar items } \\
\text { requiring processing prior to disposal or transfer to a } \\
\text { useful function within or outside the Cleanup Hanford } \\
\text { mission }\end{array}$ & $\begin{array}{l}\text { - Aqueous Waste Generated During } \\
\text { Deactivation } \\
\text { - Solid Waste Generated During } \\
\text { Deactivation } \\
\text { - Tank Waste Generated During } \\
\text { Deactivation }\end{array}$ \\
\hline Wastes Generated During Remedy/Restore & $\begin{array}{l}\text { - Aqueous Waste Generated During } \\
\text { Remedy/Restore } \\
\text { - Solid Waste Generated During } \\
\text { Remedy/Restore } \\
\text { - Tank Waste Generated During } \\
\text { Remedy/Restore } \\
\end{array}$ \\
\hline \multicolumn{2}{|c|}{\begin{tabular}{|l|l|} 
Wastes Generated During Transfer \\
Incidental solid, aqueous, and tank waste generated during \\
the transfer of SNM/NM/NF materials to final disposition.
\end{tabular}} \\
\hline \multicolumn{2}{|c|}{\begin{tabular}{|l|l|} 
Wastes Generated During Treatment \\
Incidental solid, aqueous, and tank waste generated during \\
SNM/NM/NF material treatment prior to transfer for final \\
disposition.
\end{tabular}} \\
\hline $\begin{array}{l}\text { Wastes and Documentation Generated During AW } \\
\text { Cleanup }\end{array}$ & $\begin{array}{l}\text { - Liquid Wastes and Documentation } \\
\text { Generated During AW Cleanup } \\
\text { - Solid Wastes and Documentation } \\
\text { Generated During AW Cleanup } \\
\end{array}$ \\
\hline $\begin{array}{l}\text { Worker Safety Information } \\
\text { Facility information on deactivated utilities, deactivated } \\
\text { process systems, and other deactivated facility systems } \\
\text { provided to ensure worker safetv. }\end{array}$ & \\
\hline
\end{tabular}



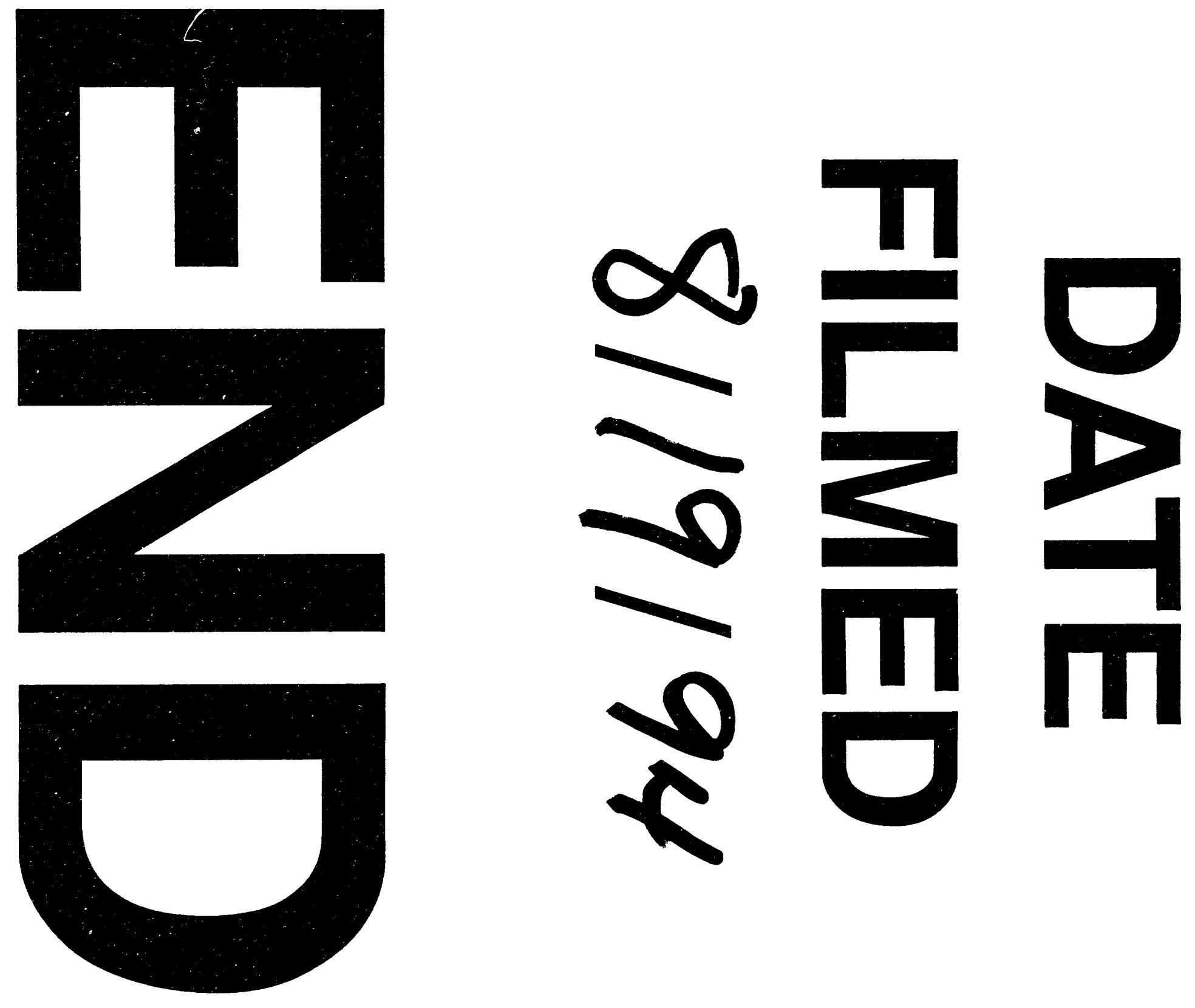
